Supporting Information

\title{
Chiral Iridium Complexes of Anionic NCP Pincer Ligand for Asymmetric Transfer Hydrogenation of 1,1- Diarylethenes with Ethanol
}

Lu Qian, ${ }^{\dagger}$ Xixia Tang, ${ }^{\dagger}$, Zhidao Huang, ${ }^{\dagger}$ Yulei Wang, ${ }^{\dagger}$ Guixia Liu, $,{ }^{*}, \S$ and Zheng Huang* ${ }^{*}, \psi$

${ }^{\dagger}$ The State Key Laboratory of Organometallic Chemistry, Shanghai Institute of Organic Chemistry, University of Chinese Academy of Sciences, Chinese Academy of Sciences, 345 Lingling Road, Shanghai 200032, China

¥School of Physical Science and Technology, Shanghai Tech University, 100 Haike Road, Shanghai 201210, China

${ }^{\S}$ Chang-Kung Chuang Institute, East China Normal University, Shanghai 200062, China

${ }^{\psi}$ School of Chemistry and Material Sciences, Hangzhou Institute of Advanced Study, University of Chinese Academy of Sciences, 1 Sub-lane Xiangshan, Hangzhou 310024, China

\section{Table of content}

I. General Information.......................................... 2

II. Synthesis of Iridium Complexes..............................S3

III. Procedures for Synthesis of Starting Materials ..................S13

IV. Asymmetric Transfer Hydrogenation of 1,1-Diarylethenes.......S24

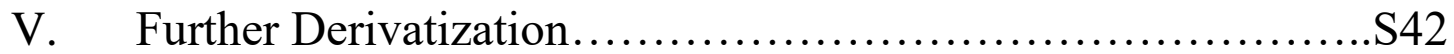

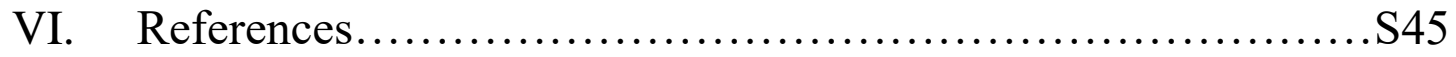

VII. NMR Spectra............................................. 48

VIII. Copies of HPLC and SFC Spectra...........................S161

IX. X-Ray Crystallographic Data.............................S210 


\section{General Information}

All manipulations were carried out using standard Schlenk and glovebox techniques unless otherwise stated. Toluene and THF were distilled from $\mathrm{Na}$ and distilled under argon. Pentane and chlorobenzene were dried over LiAlH4 overnight under an atmosphere of argon, then distilled prior to use and stored in an argon atmosphere glovebox. EtOH was freshly distilled from magnesium under argon. $\mathbf{7 a - 7 f},{ }^{1} \mathbf{7 h},{ }^{1} \mathbf{7 l},{ }^{2}$ $\mathbf{7 m},{ }^{3} \mathbf{7 n},{ }^{4} \mathbf{7 o},{ }^{1} \mathbf{7 q},{ }^{1} \mathbf{7 r}-\mathbf{7 t},{ }^{5} \mathbf{7 u},{ }^{6} \mathbf{7 v},{ }^{5} \mathbf{7 y - 7 z},{ }^{1} \mathbf{7 a b}-\mathbf{7 a c},{ }^{1} \mathbf{7 a h},{ }^{7} \mathbf{7 a k},{ }^{8} \mathbf{7 a l},{ }^{9} \mathbf{7 a o}{ }^{8}$ and $[\operatorname{Ir}(\operatorname{cod}) \mathrm{Cl}]_{2}{ }^{10}$ were prepared according to previously reported procedures. All other reagents and solvents used were purchased from commercial sources and used as received.

NMR spectra were recorded on Agilent $400 \mathrm{MHz}$ or Bruker $400 \mathrm{MHz},{ }^{1} \mathrm{H}$ NMR chemical shifts were referenced to tetramethylsilane signal $(0 \mathrm{ppm})$ or solvent resonance. ${ }^{13} \mathrm{C}$ NMR and ${ }^{19} \mathrm{~F}$ NMR chemical shifts were referenced to the solvent resonance. The following abbreviations were used to explain multiplicities: $\mathrm{s}=$ singlet, $\mathrm{d}=$ doublet, $\mathrm{t}=$ triplet, $\mathrm{m}=$ multiplet, $\mathrm{q}=$ quadruplet, $\mathrm{PE}=$ petroleum ether, $\mathrm{EA}=$ ethyl acetate. Elemental analysis and high resolution mass spectrometer (HR-MS) were carried out by the Analytical Laboratory of Shanghai Institute of Organic Chemistry (CAS) or State Key Laboratory of Organometallic Chemistry of Shanghai Institute of Organic Chemistry (CAS). Enantiomeric excess values were determined by HPLC analysis on a chiral stationary phase on Waters 2489 UV/Visible detector, Waters 1525 binary HPLC pump and Waters 2707 auto sampler, or supercritical fluid chromatography (SFC) analysis using Waters UPC2 instruments. Optical rotations were measured in $\mathrm{CHCl}_{3}$ on a Rudolph Autopol I/II/III or Rudolph APVI polarimeter with a sodium lamp of wavelength $589 \mathrm{~nm}$.

Crystals were mounted on Bruker APEX-II CCD-based diffractometer equipped with an Oxford low-temperature apparatus. Cell parameters were retrieved with SMART, which corrects for Lorentz polarization and decay. Absorption corrections were applied using SADABS. ${ }^{11}$ Space groups were assigned unambiguously by analysis of symmetry and systematic absences determined by XPREP. Using Olex2, ${ }^{12}$ the structures were 
solved with the SHELXT ${ }^{13}$ structure solution program using Intrinsic Phasing and refined with the SHELXT ${ }^{13}$ refinement package using least squares minimization.

\section{Synthesis of Iridium Complexes}

Compounds $\mathbf{1} \mathbf{a}^{14}$ and $\mathbf{1} \mathbf{b}^{15}$ are known compounds and the data match those previously reported.

Procedure for preparation of $\mathrm{N}_{\text {oxa }} \mathrm{C}^{\mathrm{X}} \mathrm{P}$-type ligands

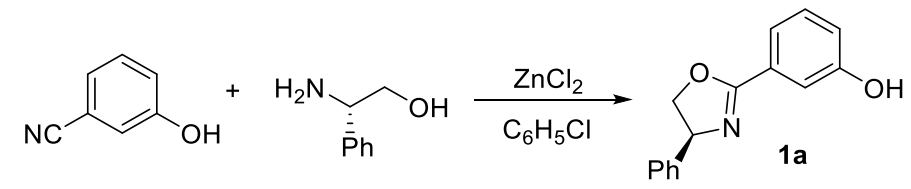

Preparation of (S)-3-(4-phenyl-4, 5-dihydrooxazol-2-yl) phenol (1a) was prepared according to a modified literature procedure. ${ }^{14}$ A $100 \mathrm{~mL}$ oven-dried round-bottom flask was charged with 3-hydroxybenzonitrile (10.0 mmol, $1.19 \mathrm{~g})$, L-phenylglycinol (18 mmol, $2.47 \mathrm{~g}), \mathrm{ZnCl}_{2}(20 \mathrm{mmol}, 2.67 \mathrm{~g})$ and $\mathrm{C}_{6} \mathrm{H}_{5} \mathrm{Cl}(30 \mathrm{~mL})$. The mixture was stirred at $120^{\circ} \mathrm{C}$ in an oil bath for $24 \mathrm{~h}$. After cooling to room temperature, the solvent was removed under vacuo, and the residue was added EtOAc $(50 \mathrm{~mL})$ and water $(50$ $\mathrm{mL}$ ). The organic layer was separated, dried over $\mathrm{Na}_{2} \mathrm{SO}_{4}$, and filtered. The solvent was removed in vacuo and the residue was purified by flash column chromatography (PE/EA $=5: 1-3: 1)$ to give $1 \mathbf{a}(0.64 \mathrm{~g}, 27 \%$ yield $)$ as white amorphous solid. ${ }^{1} \mathrm{H}$ NMR (400 MHz, DMSO) $\delta 9.78(\mathrm{~s}, 1 \mathrm{H}), 7.41-7.23(\mathrm{~m}, 8 \mathrm{H}), 6.96(\mathrm{~d}, J=7.6 \mathrm{~Hz}, 1 \mathrm{H}), 5.37(\mathrm{t}, J=9.1 \mathrm{~Hz}$, $1 \mathrm{H}), 4.81(\mathrm{t}, J=9.3 \mathrm{~Hz}, 1 \mathrm{H}), 4.16(\mathrm{t}, J=8.3 \mathrm{~Hz}, 1 \mathrm{H}) .{ }^{13} \mathrm{C}$ NMR $(101 \mathrm{MHz}, \mathrm{DMSO}) \delta$ $163.3,157.4,142.8,129.8,128.6,128.4,127.4,126.7,118.8,114.6,74.4,69.0$.

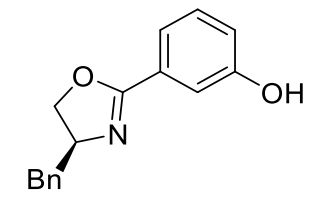

Preparation of (S)-3-(4-benzyl-4,5-dihydrooxazol-2-yl) phenol (1b) $)^{15}$ This compound was afforded as white amorphous solid (1.4 g, 56\% yield) following the similar procedure to 1a with 3-hydroxybenzonitrile (10.0 mmol, $1.19 \mathrm{~g})$, Lphenylalaninol (2.68 g, $18 \mathrm{mmol}), \mathrm{ZnCl}_{2}(20 \mathrm{mmol}, 2.67 \mathrm{~g})$ and $\mathrm{C}_{6} \mathrm{H}_{5} \mathrm{Cl}(30 \mathrm{~mL})$ purified by flash column chromatography $(\mathrm{PE} / \mathrm{EA}=5: 1-3: 1) .{ }^{1} \mathrm{H}$ NMR $(400 \mathrm{MHz}$, $\left.\mathrm{CDCl}_{3}\right) \delta 9.97(\mathrm{~s}, 1 \mathrm{H}), 7.46(\mathrm{~s}, 1 \mathrm{H}), 7.31(\mathrm{~d}, J=7.7 \mathrm{~Hz}, 1 \mathrm{H}), 7.41-7.23(\mathrm{~m}, 6 \mathrm{H}), 6.95$ $(\mathrm{dd}, J=8.1,1.7 \mathrm{~Hz}, 1 \mathrm{H}), 4.61(\mathrm{qd}, J=9.3,4.8 \mathrm{~Hz}, 1 \mathrm{H}), 4.31(\mathrm{t}, J=9.0 \mathrm{~Hz}, 1 \mathrm{H}), 4.17$ 
$-4.10(\mathrm{~m}, 1 \mathrm{H}), 3.21(\mathrm{dd}, J=13.7,4.6 \mathrm{~Hz}, 1 \mathrm{H}), 2.73(\mathrm{dd}, J=13.7,9.4 \mathrm{~Hz}, 1 \mathrm{H})$.

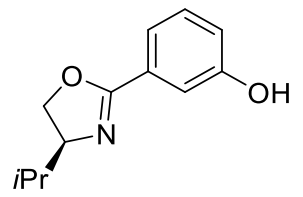

Preparation of (S)-3-(4-benzyl-4,5-dihydrooxazol-2-yl) phenol (1c). This compound was afforded as white amorphous solid (0.939 g, 45.8\% yield) following the similar procedure to 1a with 3-hydroxybenzonitrile (10.0 mmol, $1.19 \mathrm{~g})$, L-valinol (1.85 g, 18 $\mathrm{mmol}), \mathrm{ZnCl}_{2}(20 \mathrm{mmol}, 2.67 \mathrm{~g})$ and $\mathrm{C}_{6} \mathrm{H}_{5} \mathrm{Cl}(30 \mathrm{~mL})$, purified by flash column chromatography $(\mathrm{PE} / \mathrm{EA}=5: 1-3: 1) .{ }^{1} \mathrm{H} \mathrm{NMR}\left(400 \mathrm{MHz}, \mathrm{CDCl}_{3}\right) \delta 9.57(\mathrm{~s}, 1 \mathrm{H}), 7.43$ (s, 1H), $7.36(\mathrm{~d}, J=7.6 \mathrm{~Hz}, 1 \mathrm{H}), 7.18(\mathrm{dd}, J=11.2,4.6 \mathrm{~Hz}, 1 \mathrm{H}), 6.96-6.90(\mathrm{~m}, 1 \mathrm{H})$, $4.40(\mathrm{dd}, J=17.5,6.4 \mathrm{~Hz}, 1 \mathrm{H}), 4.28-4.11(\mathrm{~m}, 2 \mathrm{H}), 1.91(\mathrm{dd}, J=12.3,5.7 \mathrm{~Hz}, 1 \mathrm{H})$, $0.92(\mathrm{~m}, 6 \mathrm{H}) .{ }^{13} \mathrm{C}$ NMR $(101 \mathrm{MHz}, \mathrm{CDCl} 3) \delta 165.1,157.0,129.8,128.2,119.9,119.6$, 115.5, 71.5, 70.0, 32.4, 18.8, 17.5. HRMS (ESI) m/z: $[\mathrm{M}+\mathrm{H}]^{+}$Calcd for $\mathrm{C}_{12} \mathrm{H}_{16} \mathrm{~N}_{2} \mathrm{O}$ 206.1176; Found 206.1179.

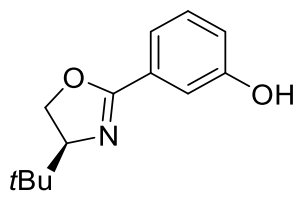

Preparation of (S)-3-(4-benzyl-4,5-dihydrooxazol-2-yl) phenol (1d). This compound was afforded as white amorphous solid $(0.657 \mathrm{~g}, 50 \%$ yield $)$ following the similar procedure to 1a with 3-hydroxybenzonitrile (10.0 mmol, $1.19 \mathrm{~g})$, L-tert-Leucinol (2.1 g, $18 \mathrm{mmol}), \mathrm{ZnCl}_{2}(20 \mathrm{mmol}, 2.67 \mathrm{~g})$ and $\mathrm{C}_{6} \mathrm{H}_{5} \mathrm{Cl}(30 \mathrm{~mL})$, purified by flash column chromatography $(\mathrm{PE} / \mathrm{EA}=5: 1-3: 1) .{ }^{1} \mathrm{H} \mathrm{NMR}\left(400 \mathrm{MHz}, \mathrm{CDCl}_{3}\right) \delta 9.07(\mathrm{~s}, 1 \mathrm{H}), 7.54$ (s, 1H), 7.36 (d, $J=7.7 \mathrm{~Hz}, 1 \mathrm{H}), 7.19$ (t, $J=7.9 \mathrm{~Hz}, 1 \mathrm{H}), 6.92$ (d, $J=8.1 \mathrm{~Hz}, 1 \mathrm{H}), 4.35$ $(\mathrm{dt}, J=15.6,9.2 \mathrm{~Hz}, 2 \mathrm{H}), 4.08(\mathrm{dd}, J=10.0,6.9 \mathrm{~Hz}, 1 \mathrm{H}), 0.93(\mathrm{~s}, 9 \mathrm{H}) .{ }^{13} \mathrm{C} \mathrm{NMR}(101$ $\mathrm{MHz}, \mathrm{CDCl} 3) \delta 165.5,156.9,129.8,128.3,120.0,119.6,115.6,75.2,69.4,34.4,25.8$. HRMS (ESI) m/z: [M+H] $]^{+}$Calcd for $\mathrm{C}_{13} \mathrm{H}_{18} \mathrm{NO}_{2} 220.1336$; Found 220.1332.

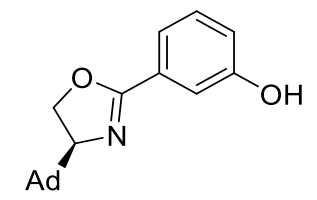

Preparation of 3-((S)-4-((1s,3R,5R,7S)-adamantan-1-yl)-4,5-dihydrooxazol-2-yl) phenol (1e). This compound was afforded as white amorphous solid ( $0.683 \mathrm{~g}, 23 \%$ 
yield) following the similar procedure to 1a with 3-hydroxybenzonitrile (10.0 mmol, $1.19 \mathrm{~g}),(\mathrm{S})-2$-(adamantan-1-yl)-2-aminoethan-1-ol (3.51 g, $18 \mathrm{mmol}), \mathrm{ZnCl}_{2}$ (20 mmol, $2.67 \mathrm{~g})$ and $\mathrm{C}_{6} \mathrm{H}_{5} \mathrm{Cl}(30 \mathrm{~mL})$, purified by flash column chromatography $(\mathrm{PE} / \mathrm{EA}=5: 1-$ 3:1). ${ }^{1} \mathrm{H}$ NMR (400 MHz, DMSO) $\delta 9.66$ (s, 1H), $7.40-7.16$ (m, 3H), 6.90 (d, $J=6.8$ $\mathrm{Hz}, 1 \mathrm{H}), 4.24(\mathrm{dd}, J=18.1,8.4 \mathrm{~Hz}, 2 \mathrm{H}), 3.80(\mathrm{t}, J=8.7 \mathrm{~Hz}, 1 \mathrm{H}), 1.93(\mathrm{~s}, 3 \mathrm{H}), 1.62(\mathrm{~s}$, 9H), $1.37(\mathrm{~d}, J=11.4 \mathrm{~Hz}, 3 \mathrm{H}) .{ }^{13} \mathrm{C}$ NMR (101 MHz, DMSO) $\delta 161.8,157.3,129.6$, $128.8,118.5,118.3,114.4,75.4,66.7,37.9,36.7,35.2,27.6$. HRMS (ESI) m/z : $[\mathrm{M}+\mathrm{H}]^{+}$Calcd for $\mathrm{C}_{19} \mathrm{H}_{24} \mathrm{NO}_{2} 298.1806$; Found 298.1801.

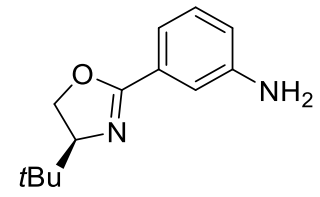

Preparation of (S)-3-(4-(tert-butyl)-4,5-dihydrooxazol-2-yl)aniline (2). This compound was afforded as white amorphous solid $(0.807 \mathrm{~g}, 37 \%$ yield) following the similar procedure to 1a with 3-hydroxybenzonitrile $(10.0 \mathrm{mmol}, 1.19 \mathrm{~g})$, L-tertLeucinol (2.1 g, $18 \mathrm{mmol}), \mathrm{ZnCl}_{2}(20 \mathrm{mmol}, 2.67 \mathrm{~g})$ and $\mathrm{C}_{6} \mathrm{H}_{5} \mathrm{Cl}(30 \mathrm{~mL})$, purified by flash column chromatography $(\mathrm{PE} / \mathrm{EA}=5: 1-3: 1) .{ }^{1} \mathrm{H} \mathrm{NMR}(400 \mathrm{MHz}, \mathrm{CDCl} 3) \delta 7.37$ - $7.30(\mathrm{~m}, 2 \mathrm{H}), 7.21-7.11(\mathrm{~m}, 1 \mathrm{H}), 6.75(\mathrm{dd}, J=7.9,1.5 \mathrm{~Hz}, 1 \mathrm{H}), 4.35-4.28(\mathrm{~m}, 1 \mathrm{H})$, $4.21(\mathrm{t}, J=8.1 \mathrm{~Hz}, 1 \mathrm{H}), 4.03(\mathrm{dd}, J=10.0,7.7 \mathrm{~Hz}, 1 \mathrm{H}), 3.76(\mathrm{~s}, 2 \mathrm{H}), 0.95(\mathrm{~s}, 9 \mathrm{H}) .{ }^{13} \mathrm{C}$ NMR (101 MHz, CDCl3) $\delta 163.5,146.5,129.2,128.8,118.3,117.8,114.6,76.1,68.6$, 34.0, 25.9. HRMS (ESI) m/z: $[\mathrm{M}+\mathrm{H}]^{+}$Calcd for $\mathrm{C}_{13} \mathrm{H}_{19} \mathrm{~N}_{2} \mathrm{O} 219.1495$; Found 219.1492.
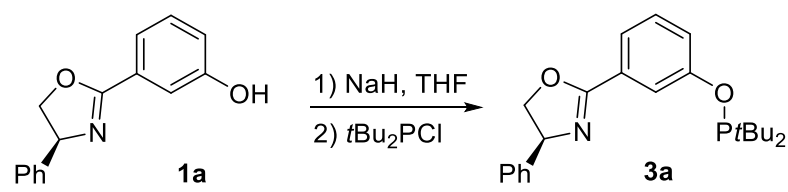

\section{Preparation of (S)-2-(3-((di-tert-butylphosphanyl)oxy)phenyl)-4-phenyl-4,5-}

dihydrooxazole (3a). Under argon atmosphere, a $10 \mathrm{~mL}$ sealed tube was charged with 1a (119.5 mg, $0.5 \mathrm{mmol})$, THF ( $5 \mathrm{~mL}$ ) and $\mathrm{NaH}$ (30 mg, $0.75 \mathrm{mmol}, 60 \%$ purity). After the mixture was stirred for $0.5 \mathrm{~h}$ at room temperature, di-tert-butylchlorophosphine $(0.105 \mathrm{~mL}, 0.55 \mathrm{mmol})$ was added. The tube was sealed with a teflon plug and then stirred at rt overnight. After evaporation of the solvent under vacuum, the residue was extracted with pentane $(30 \mathrm{~mL})$ and the extract was filtered through a pad of Celite. 
After the removal of pentane under vacuum, the flask was heated at $120^{\circ} \mathrm{C}$ for $1 \mathrm{~h}$ under high vacuum to remove the unreacted di-tert-butylchlorophosphine, furnishing $2 \mathbf{a}$ as light yellow oil (186 mg, 97\% yield). ${ }^{1} \mathrm{H}$ NMR (400 MHz, $\left.\mathrm{C}_{6} \mathrm{D}_{6}\right) \delta 8.38$ (dd, $J=3.8$, $2.2 \mathrm{~Hz}, 1 \mathrm{H}), 7.99-7.93(\mathrm{~m}, 1 \mathrm{H}), 7.38-7.32(\mathrm{~m}, 1 \mathrm{H}), 7.18-7.04(\mathrm{~m}, 6 \mathrm{H}), 5.09$ (dd, $J$ $=10.0,8.6 \mathrm{~Hz}, 1 \mathrm{H}), 4.17(\mathrm{dd}, J=10.1,8.3 \mathrm{~Hz}, 1 \mathrm{H}), 3.82(\mathrm{t}, J=8.4 \mathrm{~Hz}, 1 \mathrm{H}), 1.08(\mathrm{dt}$, $J=12.0,2.6 \mathrm{~Hz}, 18 \mathrm{H}) .{ }^{13} \mathrm{C} \mathrm{NMR}\left(101 \mathrm{MHz}, \mathrm{C}_{6} \mathrm{D}_{6}\right) \delta 164.3,160.4(\mathrm{~d}, J=9.8 \mathrm{~Hz}), 143.3$, 130.1, 129.9, 128.8, 127.5, 127.1, 122.2, 121.6 (d, $J=11.1 \mathrm{~Hz}), 118.9$ (d, $J=11.1 \mathrm{~Hz})$, 74.8, 70.6, 35.9 (d, $J=3.9 \mathrm{~Hz}), 35.6(\mathrm{~d}, J=3.8 \mathrm{~Hz}), 27.5$ (d, $J=1.0 \mathrm{~Hz}), 27.4$ (d, $J=$ $1.0 \mathrm{~Hz}) .{ }^{31} \mathrm{P}$ NMR $\left(162 \mathrm{MHz}, \mathrm{C}_{6} \mathrm{D}_{6}\right) \delta$ 154.0. HRMS (DART) m/z: $[\mathrm{M}+\mathrm{H}]^{+}$Calcd for $\mathrm{C}_{23} \mathrm{H}_{31} \mathrm{O}_{2} \mathrm{NP}$ 384.2087; Found 384.2080.

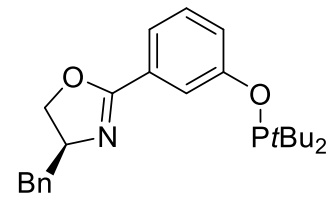

Preparation of (S)-4-benzyl-2-(3-((di-tert-butylphosphanyl)oxy)phenyl)-4,5dihydrooxazole (3b). This compound was afforded as light yellow oil (195 mg, 98\% yield) following the similar procedure to $\mathbf{3 a}$ with $\mathbf{1 b}(125.5 \mathrm{mg}, 0.5 \mathrm{mmol})$, THF (5 $\mathrm{mL}), \mathrm{NaH}$ (30 mg, $0.75 \mathrm{mmol}, 60 \%$ purity) and di-tert-butylchlorophosphine (0.105 mL, $0.55 \mathrm{mmol}) .{ }^{1} \mathrm{H}$ NMR $\left(400 \mathrm{MHz}, \mathrm{C}_{6} \mathrm{D}_{6}\right) \delta 8.28(\mathrm{~d}, J=1.7 \mathrm{~Hz}, 1 \mathrm{H}), 7.92-7.82(\mathrm{~m}$, 1H), $7.32(\mathrm{dt}, J=8.2,2.4 \mathrm{~Hz}, 6 \mathrm{H}), 7.16-7.00(\mathrm{~m}, 1 \mathrm{H}), 4.39-4.27$ (m, 1H), $3.88-$ $3.80(\mathrm{~m}, 1 \mathrm{H}), 3.73(\mathrm{dd}, J=17.2,9.3 \mathrm{~Hz}, 1 \mathrm{H}), 3.03(\mathrm{dd}, J=13.7,5.7 \mathrm{~Hz}, 1 \mathrm{H}), 2.47(\mathrm{dd}$, $J=13.7,8.2 \mathrm{~Hz}, 1 \mathrm{H}), 1.13-1.05(\mathrm{~m}, J=11.7 \mathrm{~Hz}, 18 \mathrm{H}) .{ }^{13} \mathrm{C} \mathrm{NMR}\left(101 \mathrm{MHz}, \mathrm{C}_{6} \mathrm{D}_{6}\right)$ $\delta 163.4,160.3(\mathrm{~d}, J=9.8 \mathrm{~Hz}), 138.7,130.3,129.8,129.6,128.7,126.6,122.0,121.4$ $(\mathrm{d}, J=11.1 \mathrm{~Hz}), 118.7$ (d, $J=11.1 \mathrm{~Hz}), 71.8,68.4,42.1,35.9,35.6,27.5,27.4 .{ }^{31} \mathrm{P}$ NMR $\left(162 \mathrm{MHz}, \mathrm{C}_{6} \mathrm{D}_{6}\right) \delta$ 153.8. HRMS (DART) m/z: $[\mathrm{M}+\mathrm{H}]^{+}$Calcd for $\mathrm{C}_{24} \mathrm{H}_{33} \mathrm{O}_{2} \mathrm{NP}$ 398.2243; Found 398.2236.

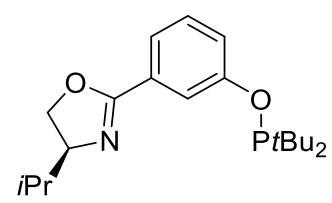

Preparation of (S)-2-(3-((di-tert-butylphosphanyl)oxy)phenyl)-4-isopropyl-4,5dihydrooxazole (3c). This compound was afforded as white amorphous solid (171 mg, 
98\% yield) following the similar procedure to $3 \mathbf{a}$ with $\mathbf{1 c}(102.5 \mathrm{mg}, 0.5 \mathrm{mmol})$, THF (5 mL), NaH (30 mg, $0.75 \mathrm{mmol}, 60 \%$ purity) and di-tert-butylchlorophosphine (0.105 $\mathrm{mL}, 0.55 \mathrm{mmol}) .{ }^{1} \mathrm{H} \mathrm{NMR}\left(400 \mathrm{MHz}, \mathrm{C}_{6} \mathrm{D}_{6}\right) \delta 8.20(\mathrm{~d}, J=1.6 \mathrm{~Hz}, 1 \mathrm{H}), 7.85(\mathrm{~d}, J=7.7$ $\mathrm{Hz}, 1 \mathrm{H}), 7.31$ (d, $J=8.1 \mathrm{~Hz}, 1 \mathrm{H}), 7.08$ (t, $J=8.0 \mathrm{~Hz}, 1 \mathrm{H}), 3.97$ (dd, $J=9.3,7.8 \mathrm{~Hz}$, 1H), $3.88-3.79(\mathrm{~m}, 1 \mathrm{H}), 3.75(\mathrm{t}, J=7.9 \mathrm{~Hz}, 1 \mathrm{H}), 1.65-1.55(\mathrm{~m}, 1 \mathrm{H}), 1.07$ (d, $J=11.8$ $\mathrm{Hz}, 18 \mathrm{H}), 0.94(\mathrm{~d}, J=6.7 \mathrm{~Hz}, 3 \mathrm{H}), 0.76(\mathrm{~d}, J=6.7 \mathrm{~Hz}, 3 \mathrm{H}) .{ }^{13} \mathrm{C} \mathrm{NMR}\left(101 \mathrm{MHz}, \mathrm{C}_{6} \mathrm{D}_{6}\right)$ $\delta 162.84,160.26(\mathrm{~d}, J=9.8 \mathrm{~Hz}), 130.4,129.7,122.0,121.1(\mathrm{~d}, J=11.7 \mathrm{~Hz}), 118.7(\mathrm{~d}$, $J=10.4 \mathrm{~Hz}), 73.3,70.4,35.9(\mathrm{~d}, J=4.1 \mathrm{~Hz}), 35.6(\mathrm{~d}, J=3.8 \mathrm{~Hz}), 33.4,27.6,27.4,19.1$, 18.8. ${ }^{31} \mathrm{P}$ NMR (162 MHz, $\left.\mathrm{C}_{6} \mathrm{D}_{6}\right) \delta$ 153.3. HRMS (DART) m/z: $[\mathrm{M}+\mathrm{H}]^{+}$Calcd for $\mathrm{C}_{20} \mathrm{H}_{33} \mathrm{O}_{2} \mathrm{NP} 350.2243$; Found 350.2243.

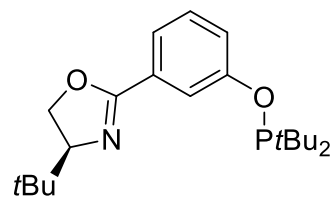

Preparation of (S)-4-(tert-butyl)-2-(3-((di-tert-butylphosphanyl)oxy)phenyl)-4,5dihydrooxazole (3d). This compound was afforded as white amorphous solid (180 mg, 99\% yield) following the similar procedure to $\mathbf{3 a}$ with $\mathbf{1 d}(109.5 \mathrm{mg}, 0.5 \mathrm{mmol})$, THF (5 mL), NaH (30 mg, $0.75 \mathrm{mmol}, 60 \%$ purity) and di-tert-butylchlorophosphine $(0.105$ mL, $0.55 \mathrm{mmol}) .{ }^{1} \mathrm{H}$ NMR (400 MHz, $\left.\mathrm{C}_{6} \mathrm{D}_{6}\right) \delta 8.26(\mathrm{~s}, 1 \mathrm{H}), 7.90(\mathrm{~d}, J=7.7 \mathrm{~Hz}, 1 \mathrm{H})$, $7.40-7.31(\mathrm{~m}, 1 \mathrm{H}), 7.07$ (t, $J=8.0 \mathrm{~Hz}, 1 \mathrm{H}), 3.93-3.80(\mathrm{~m}, 3 \mathrm{H}), 1.07(\mathrm{~d}, J=11.7 \mathrm{~Hz}$, $18 \mathrm{H}), 0.85(\mathrm{~s}, 9 \mathrm{H}) .{ }^{13} \mathrm{C} \mathrm{NMR}\left(101 \mathrm{MHz}, \mathrm{C}_{6} \mathrm{D}_{6}\right) \delta 162.9,160.3(\mathrm{~d}, J=9.8 \mathrm{~Hz}), 130.4$, 129.7, 122.0, 121.1 (d, $J=12.0 \mathrm{~Hz}), 118.7$ (d, $J=10.2 \mathrm{~Hz}), 76.7,68.6,35.9$ (d, $J=6.3$ $\mathrm{Hz}), 35.6(\mathrm{~d}, J=6.1 \mathrm{~Hz}), 34.0,27.5,27.4,26.1 .{ }^{31} \mathrm{P} \mathrm{NMR}\left(162 \mathrm{MHz}, \mathrm{C}_{6} \mathrm{D}_{6}\right) \delta 153.2$. HRMS (DART) m/z: [M+H] $]^{+}$calcd for $\mathrm{C}_{21} \mathrm{H}_{35} \mathrm{O}_{2} \mathrm{NP}$ 364.2400; Found 364.2395.

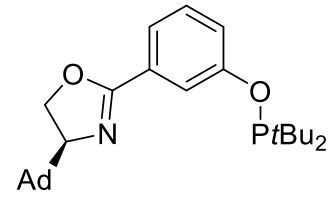

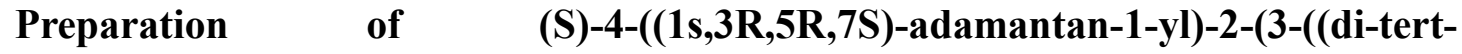
butylphosphanyl)oxy)phenyl)-4,5-dihydrooxazole (3e). This compound was afforded as white amorphous solid (214 mg, 99\% yield) following the similar procedure to 3a with 1e (148.5 mg, $0.5 \mathrm{mmol})$, THF ( $5 \mathrm{~mL}), \mathrm{NaH}$ (30 mg, $0.75 \mathrm{mmol}, 60 \%$ purity) 
and di-tert-butylchlorophosphine (0.105 mL, $0.55 \mathrm{mmol}) .{ }^{1} \mathrm{H}$ NMR (400 MHz, $\left.\mathrm{C}_{6} \mathrm{D}_{6}\right)$ $\delta 8.18(\mathrm{~d}, J=8.3 \mathrm{~Hz}, 1 \mathrm{H}), 7.87(\mathrm{t}, J=7.6 \mathrm{~Hz}, 1 \mathrm{H}), 7.32$ (d, $J=8.2 \mathrm{~Hz}, 1 \mathrm{H}), 7.09$ (t, $J$ $=8.0 \mathrm{~Hz}, 1 \mathrm{H}), 4.03(\mathrm{t}, J=8.4 \mathrm{~Hz}, 1 \mathrm{H}), 3.97-3.83(\mathrm{~m}, 1 \mathrm{H}), 3.72(\mathrm{t}, J=9.2 \mathrm{~Hz}, 1 \mathrm{H})$, $1.87(\mathrm{~s}, 3 \mathrm{H}), 1.58(\mathrm{dd}, J=30.4,12.0 \mathrm{~Hz}, 9 \mathrm{H}), 1.36(\mathrm{~d}, J=12.1 \mathrm{~Hz}, 3 \mathrm{H}), 1.09$ (s, 9H), $1.06(\mathrm{~s}, 9 \mathrm{H}) .{ }^{13} \mathrm{C} \mathrm{NMR}\left(101 \mathrm{MHz}, \mathrm{C}_{6} \mathrm{D}_{6}\right) \delta 162.7,160.3(\mathrm{~d}, J=9.8 \mathrm{~Hz}), 130.4,129.6$, 122.0, $121.0(\mathrm{~d}, J=12.3 \mathrm{~Hz}), 118.6(\mathrm{~d}, J=10.0 \mathrm{~Hz}), 76.9,67.2,38.8,37.5,36.0,35.9$ $(\mathrm{d}, J=7.5 \mathrm{~Hz}), 35.6$ (d, $J=7.2 \mathrm{~Hz}), 28.7,27.6,27.4 .{ }^{31} \mathrm{P} \mathrm{NMR}\left(162 \mathrm{MHz}, \mathrm{C}_{6} \mathrm{D}_{6}\right) \delta$ 153.1. HRMS (DART) m/z: [M+H] $]^{+}$Calcd for $\mathrm{C}_{27} \mathrm{H}_{41} \mathrm{O}_{2} \mathrm{NP} 442.2869$; Found 442.2860.

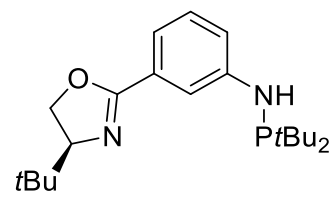

\section{Preparation of (S)-1,1-di-tert-butyl-N-(3-(4-(tert-butyl)-4,5-dihydrooxazol-2-} yl)phenyl)phosphanamine (4a). This compound was afforded as white amorphous solid (177 mg, 98\% yield) following the similar procedure to 3a with 2 (109 $\mathrm{mg}, 0.5$ mmol), THF (5 mL), NaH (30 mg, $0.75 \mathrm{mmol}, 60 \%$ purity) and di-tertbutylchlorophosphine (0.105 mL, $0.55 \mathrm{mmol}) .{ }^{1} \mathrm{H}$ NMR $\left(400 \mathrm{MHz}, \mathrm{C}_{6} \mathrm{D}_{6}\right) \delta 7.96(\mathrm{~d}, J$ $=1.8 \mathrm{~Hz}, 1 \mathrm{H}), 7.70(\mathrm{~d}, J=7.6 \mathrm{~Hz}, 1 \mathrm{H}), 7.29-7.23(\mathrm{~m}, 1 \mathrm{H}), 7.10(\mathrm{t}, J=7.9 \mathrm{~Hz}, 1 \mathrm{H})$, $4.00-3.82(\mathrm{~m}, 4 \mathrm{H}), 0.96$ (s, 9H), 0.94 (s, 9H), 0.88 (s, 9H). ${ }^{13} \mathrm{C} \mathrm{NMR}(101 \mathrm{MHz}, \mathrm{C6D} 6)$ $\delta 163.5,149.9(\mathrm{~d}, J=17.7 \mathrm{~Hz}), 129.8,129.5$ (d, $J=1.0 \mathrm{~Hz}), 119.1$ (d, $J=1.1 \mathrm{~Hz}), 118.7$ (d, $J=15.7 \mathrm{~Hz}), 116.5(\mathrm{~d}, J=9.0 \mathrm{~Hz}), 76.7,68.5,34.2(\mathrm{~d}, J=8.7 \mathrm{~Hz}), 34.03$ (d, $J=8.4$ $\mathrm{Hz}), 34.02,28.2,28.1,26.1 .{ }^{31} \mathrm{P}$ NMR $\left(162 \mathrm{MHz}, \mathrm{C}_{6} \mathrm{D}_{6}\right) \delta 58.1$. HRMS (DART) m/z: $[\mathrm{M}+\mathrm{H}]^{+}$Calcd for $\mathrm{C}_{21} \mathrm{H}_{36} \mathrm{ON}_{2} \mathrm{P}$ 363.2560; Found 363.2554.

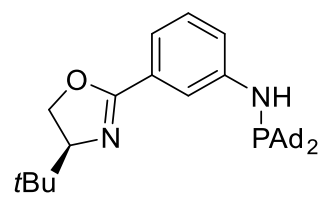

\section{Preparation of 1,1-di((3S,5S,7S)-adamantan-1-yl)-N-(3-((S)-4-(tert-butyl)-4,5-} dihydrooxazol-2-yl)phenyl)phosphanamine (4b). This compound was afforded as white amorphous solid (227 mg, 88\% yield) following the similar procedure to 3a with 2 (109 mg, $0.5 \mathrm{mmol})$, THF (5 mL), NaH (30 mg, $0.75 \mathrm{mmol}, 60 \%$ purity) and $\operatorname{di}((3 \mathrm{~S}, 5 \mathrm{~S}, 7 \mathrm{~S})$-adamantan-1-yl)chlorophosphane (168.5 mg, $0.5 \mathrm{mmol}) .{ }^{1} \mathrm{H}$ NMR (400 
$\left.\mathrm{MHz}, \mathrm{C}_{6} \mathrm{D}_{6}\right) \delta 8.10(\mathrm{~s}, 1 \mathrm{H}), 7.78(\mathrm{~d}, J=7.6 \mathrm{~Hz}, 1 \mathrm{H}), 7.37-7.31(\mathrm{~m}, 1 \mathrm{H}), 7.14(\mathrm{t}, J=$ $5.4 \mathrm{~Hz}, 1 \mathrm{H}), 4.01(\mathrm{~d}, J=10.6 \mathrm{~Hz}, 1 \mathrm{H}), 3.95-3.84(\mathrm{~m}, 3 \mathrm{H}), 1.80(\mathrm{q}, J=11.5 \mathrm{~Hz}, 18 \mathrm{H})$, $1.63-1.54(\mathrm{~m}, 12 \mathrm{H}), 0.88(\mathrm{~s}, 9 \mathrm{H}) .{ }^{13} \mathrm{C}$ NMR $\left(101 \mathrm{MHz}, \mathrm{C}_{6} \mathrm{D}_{6}\right) \delta 163.6,150.7$ (d, $J=$ $18.0 \mathrm{~Hz}), 129.8,129.5,118.9,118.7$ (d, $J=15.4 \mathrm{~Hz}), 116.5$ (d, $J=9.3 \mathrm{~Hz}), 76.7,68.5$, $39.8(\mathrm{~d}, J=12.7 \mathrm{~Hz}), 38.9(\mathrm{~d}, J=9.2 \mathrm{~Hz}), 38.7(\mathrm{~d}, J=8.8 \mathrm{~Hz}), 37.3,34.0,28.8(\mathrm{~d}, J=$ 8.6 Hz), 26.1. ${ }^{31} \mathrm{P}$ NMR (162 MHz, $\left.\mathrm{C}_{6} \mathrm{D}_{6}\right) \delta 54.1$. HRMS (DART) m/z: $[\mathrm{M}+\mathrm{H}]^{+} \mathrm{Calcd}$ for $\mathrm{C}_{33} \mathrm{H}_{48} \mathrm{ON}_{2} \mathrm{P}$ 519.3499; Found 519.3487.

\section{Procedure for preparation of $\mathrm{N}_{\mathrm{oxa}} \mathrm{C}^{\mathrm{X}} \mathrm{P}$-type complexes}

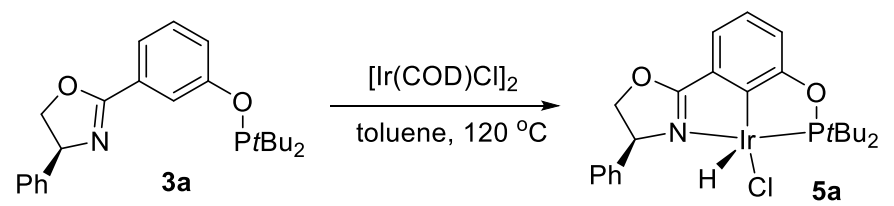

Preparation of Iridium Complex 5a. Compound 3a (191.5 mg, $0.5 \mathrm{mmol})$, $[\operatorname{Ir}(\mathrm{COD}) \mathrm{Cl}]_{2}(161 \mathrm{mg}, 0.24 \mathrm{mmol})$ and toluene $(20 \mathrm{~mL})$ were added to a $100 \mathrm{~mL}$ Kontes flask. The flask was sealed with a teflon plug and then heated at $120{ }^{\circ} \mathrm{C}$ in an oil bath for $12 \mathrm{~h}$ under hydrogen atmosphere. After the reaction mixture was cooled to room temperature, the volatiles were removed under high vacuum. The residue was extracted with pentane $(15 \mathrm{~mL} \times 3)$ and the extract was dried under vacuum to give $5 \mathbf{a}$ (128.0 mg, 42\% yield) as wine-red amorphous solid. ${ }^{1} \mathrm{H}$ NMR (400 MHz, $\left.\mathrm{C}_{6} \mathrm{D}_{6}\right) \delta 7.32$ $-7.26(\mathrm{~m}, J=7.7,6.3 \mathrm{~Hz}, 2 \mathrm{H}), 7.14(\mathrm{~s}, 1 \mathrm{H}), 7.12-6.94(\mathrm{~m}, 3 \mathrm{H}), 6.92-6.83$ (m, $J=$ 19.2, $7.8 \mathrm{~Hz}, 1 \mathrm{H}), 6.77$ (t, $J=7.7 \mathrm{~Hz}, 1 \mathrm{H}), 5.40$ (dd, $J=9.7,5.4 \mathrm{~Hz}, 1 \mathrm{H}), 4.11(\mathrm{dd}, J=$

8.8, 5.4 Hz, 1H), $4.02(\mathrm{dd}, J=11.5,7.8 \mathrm{~Hz}, 1 \mathrm{H}), 1.32(\mathrm{~d}, J=14.4 \mathrm{~Hz}, 9 \mathrm{H}), 1.04$ (d, $J$ $=15.0 \mathrm{~Hz}, 9 \mathrm{H}),-38.12(\mathrm{~s}, 1 \mathrm{H}) .{ }^{13} \mathrm{C} \mathrm{NMR}\left(101 \mathrm{MHz}, \mathrm{C}_{6} \mathrm{D}_{6}\right) \delta 179.6,165.3(\mathrm{~d}, J=2.8$ Hz), 144.2, 140.6, 130.6 (d, $J=1.7 \mathrm{~Hz}), 128.6,127.8,127.3,122.4,120.4,112.8$ (d, $J$ $=9.8 \mathrm{~Hz}), 78.4(\mathrm{~d}, J=2.2 \mathrm{~Hz}), 64.5,41.6(\mathrm{~d}, J=27.2 \mathrm{~Hz}), 38.2(\mathrm{~d}, J=30.6 \mathrm{~Hz}), 27.4$, 27.4. ${ }^{31} \mathrm{P}$ NMR (162 MHz, $\left.\mathrm{C}_{6} \mathrm{D}_{6}\right) \delta$ 162.5. Anal. Calcd for $\mathrm{C}_{23} \mathrm{H}_{30} \mathrm{ClIrNO}_{2} \mathrm{P}: \mathrm{C}, 45.20$; H, 4.95; N, 2.29. Found: C, 45.52; H, 5.42; N, 2.11.

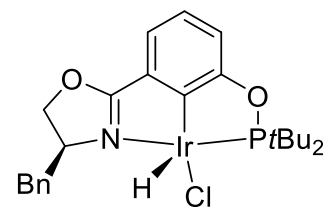

Preparation of Iridium Complex 5b. This compound was afforded as wine-red 
amorphous solid (203.2 $\mathrm{mg}, 65 \%$ yield) following the similar procedure to $\mathbf{5 a}$ with $\mathbf{3 b}$ (199.0 mg, $0.5 \mathrm{mmol}),[\operatorname{Ir}(\mathrm{COD}) \mathrm{Cl}]_{2}(161 \mathrm{mg}, 0.24 \mathrm{mmol})$ and toluene $(20 \mathrm{~mL}) .{ }^{1} \mathrm{H}$ NMR (400 MHz, C6 $\left.\mathrm{D}_{6}\right) \delta 7.15(\mathrm{dd}, J=15.0,7.6 \mathrm{~Hz}, 3 \mathrm{H}), 7.10-7.03(\mathrm{~m}, 2 \mathrm{H}), 7.01-$ $6.97(\mathrm{~m}, 1 \mathrm{H}), 6.84(\mathrm{dd}, J=7.6,4.0 \mathrm{~Hz}, 1 \mathrm{H}), 6.71(\mathrm{t}, J=7.7 \mathrm{~Hz}, 1 \mathrm{H}), 4.80$ (s, 1H), 4.20 $-4.04(\mathrm{~m}, 2 \mathrm{H}), 3.80(\mathrm{dd}, J=11.1,7.8 \mathrm{~Hz}, 1 \mathrm{H}), 2.60(\mathrm{dd}, J=13.4,9.6 \mathrm{~Hz}, 1 \mathrm{H}), 1.34$ (d, $J=14.3 \mathrm{~Hz}, 9 \mathrm{H}), 1.26(\mathrm{~d}, J=14.7 \mathrm{~Hz}, 9 \mathrm{H}),-35.27$ (s, 1H). ${ }^{13} \mathrm{C} \mathrm{NMR}\left(101 \mathrm{MHz}, \mathrm{C}_{6} \mathrm{D}_{6}\right)$ $\delta 179.6,165.8(\mathrm{~d}, J=3.1 \mathrm{~Hz}), 145.6,137.9,131.3,129.8,128.9,126.8,122.7,120.3$, $112.8(\mathrm{~d}, J=9.8 \mathrm{~Hz}), 75.2,63.3,41.8(\mathrm{~d}, J=26.0 \mathrm{~Hz}), 40.4,39.4(\mathrm{~d}, J=31.1 \mathrm{~Hz}), 28.1$ $(\mathrm{d}, J=4.3 \mathrm{~Hz}), 28.0(\mathrm{~d}, J=4.2 \mathrm{~Hz}) .{ }^{31} \mathrm{P} \mathrm{NMR}\left(162 \mathrm{MHz}, \mathrm{C}_{6} \mathrm{D}_{6}\right) \delta 160.9(\mathrm{~d}, J=12.2$ Hz). Anal. Calcd for $\mathrm{C}_{24} \mathrm{H}_{32} \mathrm{ClIrNO}_{2} \mathrm{P}: \mathrm{C}, 46.11 ; \mathrm{H}, 5.16$; N, 2.24. Found: C, 46.24; H, $5.45 ; \mathrm{N}, 2.02$.

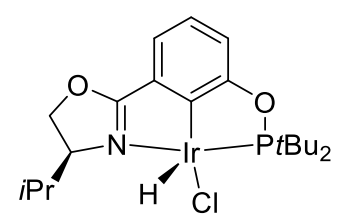

Preparation of Iridium Complex 5c. This compound was afforded as wine-red amorphous solid (170.2 $\mathrm{mg}, 59 \%$ yield) following the similar procedure to 5a with $\mathbf{3 c}$ (174.5 mg, $0.5 \mathrm{mmol}),[\operatorname{Ir}(\mathrm{COD}) \mathrm{Cl}]_{2}(161 \mathrm{mg}, 0.24 \mathrm{mmol})$ and toluene $(20 \mathrm{~mL}) .1 \mathrm{H}$ NMR $\left(400 \mathrm{MHz}, \mathrm{C}_{6} \mathrm{D}_{6}\right) \delta 7.23-7.11(\mathrm{~m}, 1 \mathrm{H}), 6.85(\mathrm{~d}, J=7.7 \mathrm{~Hz}, 1 \mathrm{H}), 6.72(\mathrm{t}, J=7.7$ Hz, 1H), 4.37 (ddd, $J=9.5,5.4,3.8 \mathrm{~Hz}, 1 \mathrm{H}), 4.06$ (dd, $J=8.9,5.6 \mathrm{~Hz}, 1 \mathrm{H}), 3.79$ (t, $J$ $=9.1 \mathrm{~Hz}, 1 \mathrm{H}), 2.82-2.64(\mathrm{~m}, 1 \mathrm{H}), 1.34(\mathrm{~d}, J=14.0 \mathrm{~Hz}, 9 \mathrm{H}), 1.19(\mathrm{~d}, J=14.9 \mathrm{~Hz}, 9 \mathrm{H})$, $0.64(\mathrm{t}, J=6.9 \mathrm{~Hz}, 6 \mathrm{H}),-38.56(\mathrm{~d}, J=20.8 \mathrm{~Hz}, 1 \mathrm{H}) .13 \mathrm{C} \mathrm{NMR}\left(101 \mathrm{MHz}, \mathrm{C}_{6} \mathrm{D}_{6}\right) \delta$ 179.2, $165.8(\mathrm{~d}, J=3.1 \mathrm{~Hz}), 145.1,131.2,122.7,120.3,112.8$ (d, J = 10.0 Hz), 71.4, 66.6, $41.8(\mathrm{~d}, J=26.2 \mathrm{~Hz}), 39.2(\mathrm{~d}, J=31.1 \mathrm{~Hz}), 29.5,28.02,27.98,18.7,14.5 .{ }^{31} \mathrm{P}$ NMR (162 MHz, $\left.\mathrm{C}_{6} \mathrm{D}_{6}\right) \delta$ 161.4. Anal. Calcd for $\mathrm{C}_{20} \mathrm{H}_{32} \mathrm{ClIrNO}_{2} \mathrm{P}: \mathrm{C}, 41.62 ; \mathrm{H}, 5.59$; N, 2.43. Found: C, 41.76; H, 5.50; N, 2.13.

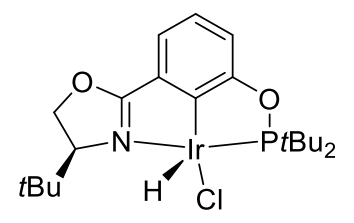

Preparation of Iridium Complex 5d. This compound was afforded as wine-red amorphous solid (209.8 mg, 71\% yield) following the similar procedure to 5a with $\mathbf{3 d}$ 
(181.5 mg, $0.5 \mathrm{mmol}),[\operatorname{Ir}(\mathrm{COD}) \mathrm{Cl}]_{2}(161 \mathrm{mg}, 0.24 \mathrm{mmol})$ and toluene $(20 \mathrm{~mL}) .{ }^{1} \mathrm{H}$ NMR (400 MHz, $\left.\mathrm{C}_{6} \mathrm{D}_{6}\right) \delta 7.21(\mathrm{~d}, J=7.5 \mathrm{~Hz}, 1 \mathrm{H}), 6.80$ (d, $\left.J=7.7 \mathrm{~Hz}, 1 \mathrm{H}\right), 6.72$ (t, $J$ $=7.7 \mathrm{~Hz}, 1 \mathrm{H}), 4.29(\mathrm{dd}, J=9.4,3.5 \mathrm{~Hz}, 1 \mathrm{H}), 4.20(\mathrm{dd}, J=9.1,3.5 \mathrm{~Hz}, 1 \mathrm{H}), 3.63(\mathrm{t}, J$ $=9.2 \mathrm{~Hz}, 1 \mathrm{H}), 1.37$ (d, $J=14.6 \mathrm{~Hz}, 9 \mathrm{H}), 1.22(\mathrm{~d}, J=14.9 \mathrm{~Hz}, 9 \mathrm{H}), 0.90$ (s, 9H), -36.60 $(\mathrm{d}, J=20.2 \mathrm{~Hz}, 1 \mathrm{H}) .{ }^{13} \mathrm{C} \mathrm{NMR}\left(101 \mathrm{MHz}, \mathrm{C}_{6} \mathrm{D}_{6}\right) \delta 178.8,165.3(\mathrm{~d}, J=2.8 \mathrm{~Hz}), 141.1$, $130.4(\mathrm{~d}, J=1.9 \mathrm{~Hz}), 122.7,120.6,113.0$ (d, $J=9.8 \mathrm{~Hz}), 73.1$ (d, $J=2.8 \mathrm{~Hz}), 69.5$ (d, $J=2.3 \mathrm{~Hz}), 41.8(\mathrm{~d}, J=27.4 \mathrm{~Hz}), 38.7(\mathrm{~d}, J=30.8 \mathrm{~Hz}), 35.3,28.2(\mathrm{~d}, J=4.5 \mathrm{~Hz}), 27.7$ $(\mathrm{d}, J=4.2 \mathrm{~Hz}), 26.6 .{ }^{31} \mathrm{P}$ NMR $\left(162 \mathrm{MHz}, \mathrm{C}_{6} \mathrm{D}_{6}\right) \delta$ 161.4. Anal. Calcd for $\mathrm{C}_{21} \mathrm{H}_{34} \mathrm{ClIINO}_{2} \mathrm{P}: \mathrm{C}, 42.67 ; \mathrm{H}, 5.80 ; \mathrm{N}, 2.37$. Found: C, 43.02; H, 5.92; N, 2.05.

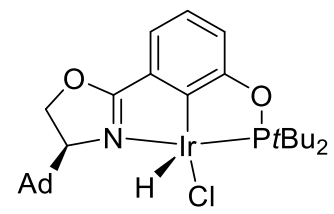

Preparation of Iridium Complex 5e. This compound was afforded as wine-red amorphous solid (190.7 mg, 57\% yield). following the similar procedure to $\mathbf{5 a}$ with $\mathbf{3 e}$ (220.5 mg, $0.5 \mathrm{mmol}),[\operatorname{Ir}(\mathrm{COD}) \mathrm{Cl}]_{2}(161 \mathrm{mg}, 0.24 \mathrm{mmol})$ and toluene $(20 \mathrm{~mL})$. The product $(20 \mathrm{mg})$ was dissolved in toluene $(2 \mathrm{~mL})$, then pentane $(1.0 \mathrm{~mL})$ was added slowly. The resulting mixture was kept at ambient temperature in the glovebox. After a few days, red crystals of 5e suitable for X-ray analysis were obtained. ${ }^{1} \mathrm{H}$ NMR (400 $\left.\mathrm{MHz}, \mathrm{C}_{6} \mathrm{D}_{6}\right) \delta 7.23(\mathrm{~d}, J=7.5 \mathrm{~Hz}, 1 \mathrm{H}), 6.82(\mathrm{~d}, J=7.6 \mathrm{~Hz}, 1 \mathrm{H}), 6.76$ (t, $J=7.7 \mathrm{~Hz}$, $1 \mathrm{H}), 4.36(\mathrm{dd}, J=9.1,3.4 \mathrm{~Hz}, 1 \mathrm{H}), 4.19(\mathrm{dd}, J=9.3,3.2 \mathrm{~Hz}, 1 \mathrm{H}), 3.64$ (t, $J=9.2 \mathrm{~Hz}$, 1H), $1.83(\mathrm{~s}, 3 \mathrm{H}), 1.64(\mathrm{~d}, J=12.3 \mathrm{~Hz}, 3 \mathrm{H}), 1.52(\mathrm{dd}, J=22.9,14.5 \mathrm{~Hz}, 9 \mathrm{H}), 1.37$ (d, $J=14.5 \mathrm{~Hz}, 9 \mathrm{H}), 1.23(\mathrm{~d}, J=14.9 \mathrm{~Hz}, 9 \mathrm{H}),-36.57(\mathrm{~d}, J=21.3 \mathrm{~Hz}, 1 \mathrm{H}) .{ }^{13} \mathrm{C} \mathrm{NMR}$ $\left(101 \mathrm{MHz}, \mathrm{C}_{6} \mathrm{D}_{6}\right) \delta 178.7,165.3(\mathrm{~d}, J=2.8 \mathrm{~Hz}), 141.2,130.4(\mathrm{~d}, J=1.9 \mathrm{~Hz}), 122.7$, 120.5, $113.0(\mathrm{~d}, J=9.8 \mathrm{~Hz}), 71.7(\mathrm{~d}, J=2.7 \mathrm{~Hz}), 69.6(\mathrm{~d}, J=2.2 \mathrm{~Hz}), 41.8(\mathrm{~d}, J=27.4$ Hz), 38.9, 38.6 (d, $J=30.7 \mathrm{~Hz}), 37.3,37.2,28.5,28.2$ (d, $J=4.5 \mathrm{~Hz}), 27.7$ (d, $J=4.2$ Hz). ${ }^{31} \mathrm{P}$ NMR $\left(162 \mathrm{MHz}, \mathrm{C}_{6} \mathrm{D}_{6}\right) \delta 161.3(\mathrm{~d}, \mathrm{~J}=13.5 \mathrm{~Hz})$. Anal Calcd for $\mathrm{C}_{27} \mathrm{H}_{40} \mathrm{ClIINO}_{2} \mathrm{P}: \mathrm{C}, 48.46 ; \mathrm{H}, 6.02 ; \mathrm{N}, 2.09$. Found: C, 49.02; H, 6.38; N, 1.90. 


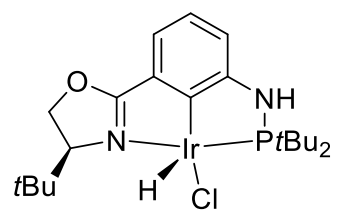

Preparation of Iridium Complex 6a. This compound was afforded as wine-red amorphous solid (177.0 mg, $60 \%$ yield) following the similar procedure to $\mathbf{5 a}$ with $\mathbf{4 a}$ (181.0 mg, $0.5 \mathrm{mmol}),[\operatorname{Ir}(\mathrm{COD}) \mathrm{Cl}]_{2}(161 \mathrm{mg}, 0.24 \mathrm{mmol})$ and toluene $(20 \mathrm{~mL}) .{ }^{1} \mathrm{H}$ NMR (400 MHz, C $\left.6 \mathrm{D}_{6}\right) \delta 7.18(\mathrm{~d}, J=7.8 \mathrm{~Hz}, 1 \mathrm{H}), 6.84(\mathrm{t}, J=7.6 \mathrm{~Hz}, 1 \mathrm{H}), 6.37$ (d, $J$ $=7.6 \mathrm{~Hz}, 1 \mathrm{H}), 4.36(\mathrm{dd}, J=9.3,3.4 \mathrm{~Hz}, 1 \mathrm{H}), 4.23(\mathrm{dd}, J=9.1,3.5 \mathrm{~Hz}, 1 \mathrm{H}), 3.93(\mathrm{~d}, J$ $=2.6 \mathrm{~Hz}, 1 \mathrm{H}), 3.67(\mathrm{t}, J=9.2 \mathrm{~Hz}, 1 \mathrm{H}), 1.28-1.11(\mathrm{~m}, 18 \mathrm{H}), 0.93(\mathrm{~s}, 9 \mathrm{H}),-36.89(\mathrm{~d}, J$ $=20.9 \mathrm{~Hz}, 1 \mathrm{H}) .{ }^{13} \mathrm{C} \mathrm{NMR}\left(101 \mathrm{MHz}, \mathrm{C}_{6} \mathrm{D}_{6}\right) \delta 178.8,165.3(\mathrm{~d}, J=2.8 \mathrm{~Hz}), 141.1,130.4$ (d, $J=1.9 \mathrm{~Hz}), 122.7,120.6,113.0(\mathrm{~d}, J=9.8 \mathrm{~Hz}), 73.1$ (d, $J=2.8 \mathrm{~Hz}), 69.5$ (d, $J=2.3$ $\mathrm{Hz}), 41.8(\mathrm{~d}, J=27.4 \mathrm{~Hz}), 38.7(\mathrm{~d}, J=30.8 \mathrm{~Hz}), 35.3,28.2(\mathrm{~d}, J=4.5 \mathrm{~Hz}), 27.7$ (d, $J=$ 4.2 Hz), 26.6. ${ }^{31} \mathrm{P}$ NMR (162 MHz, $\left.\mathrm{C}_{6} \mathrm{D}_{6}\right) \delta 97.4(\mathrm{~d}, J=14.6 \mathrm{~Hz})$. Anal. Calcd for $\mathrm{C}_{21} \mathrm{H}_{35} \mathrm{ClIIN}_{2} \mathrm{OP}: \mathrm{C}, 42.74 ; \mathrm{H}, 5.98 ; \mathrm{N}, 4.75$. Found: C, 42.37; H, 6.02; N, 4.34.

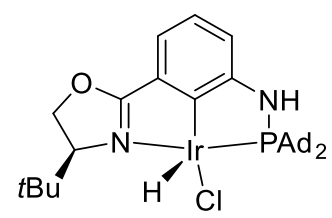

Preparation of Iridium Complex 6b. This compound was afforded as wine-red amorphous solid (175.3 mg, 47\% yield) following the similar procedure to $\mathbf{5 a}$ with $\mathbf{4 b}$ (259.0 mg, $0.5 \mathrm{mmol}),[\operatorname{Ir}(\mathrm{COD}) \mathrm{Cl}]_{2}(161 \mathrm{mg}, 0.24 \mathrm{mmol})$ and toluene $(20 \mathrm{~mL}) .{ }^{1} \mathrm{H}$ NMR (400 MHz, C $\left.6 \mathrm{D}_{6}\right) \delta 7.22(\mathrm{~d}, J=7.7 \mathrm{~Hz}, 1 \mathrm{H}), 6.88$ (t, $\left.J=7.6 \mathrm{~Hz}, 1 \mathrm{H}\right), 6.50(\mathrm{~d}, J$ $=7.5 \mathrm{~Hz}, 1 \mathrm{H}), 4.44-4.37(\mathrm{~m}, 1 \mathrm{H}), 4.26(\mathrm{dd}, J=9.0,2.9 \mathrm{~Hz}, 1 \mathrm{H}), 4.04(\mathrm{~s}, 1 \mathrm{H}), 3.71(\mathrm{t}$, $J=9.2 \mathrm{~Hz}, 1 \mathrm{H}), 2.42(\mathrm{~d}, J=10.6 \mathrm{~Hz}, 3 \mathrm{H}), 2.23$ (d, $J=10.5 \mathrm{~Hz}, 3 \mathrm{H}), 2.09$ (dd, $J=20.7$, $10.6 \mathrm{~Hz}, 6 \mathrm{H}), 1.79$ (s, 6H), 1.58 (dt, $J=26.5,11.8 \mathrm{~Hz}, 12 \mathrm{H}), 0.95$ (s, 9H), -37.78 (d, $J$ $=20.2 \mathrm{~Hz}, 1 \mathrm{H}) .{ }^{13} \mathrm{C} \mathrm{NMR}\left(101 \mathrm{MHz}, \mathrm{C}_{6} \mathrm{D}_{6}\right) \delta 179.5,156.2(\mathrm{~d}, J=11.6 \mathrm{~Hz}), 140.9$, 129.9, 122.0, 117.4, 110.0 (d, $J=9.9 \mathrm{~Hz}), 73.0$ (d, $J=2.6 \mathrm{~Hz}), 69.2(\mathrm{~d}, J=2.6 \mathrm{~Hz})$, $45.8(\mathrm{~d}, J=26.7 \mathrm{~Hz}), 41.5(\mathrm{~d}, J=31.3 \mathrm{~Hz}), 40.2,38.6,37.0,36.9,35.4,28.7$ (d, $J=5.2$ $\mathrm{Hz}), 28.6(\mathrm{~d}, J=5.0 \mathrm{~Hz}), 26.7 .{ }^{31} \mathrm{P}$ NMR $\left(162 \mathrm{MHz}, \mathrm{C}_{6} \mathrm{D}_{6}\right) \delta 91.3(\mathrm{~d}, J=14.5 \mathrm{~Hz})$. Anal Calcd for $\mathrm{C}_{33} \mathrm{H}_{47} \mathrm{ClIrN}_{2} \mathrm{OP}: \mathrm{C}, 53.10 ; \mathrm{H}, 6.35 ; \mathrm{N}, 3.75$. Found: C, 53.71; H, 6.61; N, 3.11 . 


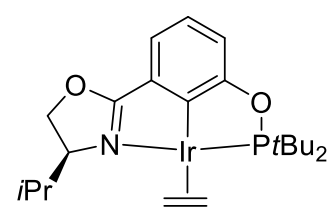

Preparation of Iridium Complex 5c'. In an argon-filled glovebox, a $50 \mathrm{~mL}$ flamedried Schlenk flask was charged with complex 5c (114 mg, $0.2 \mathrm{mmol})$ and $\mathrm{NaO} t \mathrm{Bu}(24$ $\mathrm{mg}, 0.4 \mathrm{mmol})$. After the argon was replaced by ethylene and toluene $(10 \mathrm{~mL})$ was injected to Schlenk flask by syringe. The mixture was stirred at $25^{\circ} \mathrm{C}$ for $1 \mathrm{~h}$ and then volatiles were removed under high vacuum. The residue was extracted with pentane (15 $\mathrm{mL} \times 3$ ) and the extract was dried under vacuum to give 5c' (99 mg, 87\% yield) as black amorphous solid. ${ }^{1} \mathrm{H}$ NMR (400 MHz, $\left.\mathrm{C}_{6} \mathrm{D}_{6}\right) \delta 7.22-7.13(\mathrm{~m}, J=15.4,6.2 \mathrm{~Hz}, 2 \mathrm{H})$, $6.91(\mathrm{t}, J=7.6 \mathrm{~Hz}, 1 \mathrm{H}), 3.93(\mathrm{dd}, J=8.7,4.5 \mathrm{~Hz}, 1 \mathrm{H}), 3.82-3.74(\mathrm{~m}, 1 \mathrm{H}), 3.70-3.63$ (m, 3H), $3.58-3.48(\mathrm{~m}, 2 \mathrm{H}), 2.04-1.92(\mathrm{~m}, 1 \mathrm{H}), 1.34(\mathrm{dd}, J=19.1,13.4 \mathrm{~Hz}, 18 \mathrm{H})$, $0.62(\mathrm{~d}, J=6.8 \mathrm{~Hz}, 3 \mathrm{H}), 0.45(\mathrm{~d}, J=7.1 \mathrm{~Hz}, 3 \mathrm{H}) .{ }^{13} \mathrm{C} \mathrm{NMR}\left(101 \mathrm{MHz}, \mathrm{C}_{6} \mathrm{D}_{6}\right) \delta 181.4$ $(\mathrm{d}, J=1.3 \mathrm{~Hz}), 166.0$ (d, $J=8.3 \mathrm{~Hz}), 165.2$ (d, $J=7.8 \mathrm{~Hz}), 132.4,124.6,118.4,113.4$ $(\mathrm{d}, J=11.5 \mathrm{~Hz}), 70.1(\mathrm{~d}, J=2.7 \mathrm{~Hz}), 67.9(\mathrm{~d}, J=2.1 \mathrm{~Hz}), 49.0,41.7(\mathrm{~d}, J=27.1 \mathrm{~Hz})$, $41.3(\mathrm{~d}, J=27.1 \mathrm{~Hz}), 30.3,29.4(\mathrm{~d}, J=4.7 \mathrm{~Hz}), 28.7(\mathrm{~d}, J=4.8 \mathrm{~Hz}), 18.5,14.1 .{ }^{31} \mathrm{P}$ NMR (162 MHz, $\left.\mathrm{CDCl}_{3}\right) \delta 173.8$.

\section{Procedures for Synthesis of Starting Materials}

\section{General procedure for synthesis of ketones}

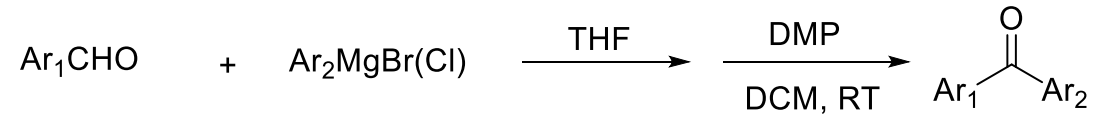

A $100 \mathrm{~mL}$ flame-dried Schlenk flask was charged with the corresponding benzaldehyde $(10 \mathrm{mmol})$ and THF $(30 \mathrm{~mL})$ under argon atmosphere. The corresponding aryl magnesium bromide ( 1.5 equiv) was added dropwisely at $0{ }^{\circ} \mathrm{C}$. The mixture was then warmed to room temperature, stirred about 3 to 10 hours and monitored by TLC. After the corresponding benzaldehyde was fully consumed, $15 \mathrm{~mL}$ of saturated $\mathrm{NH}_{4} \mathrm{Cl}$ (aq) was added, and the resulting mixture was extracted with EA (30 $\mathrm{mL} \times 3)$. The combined organic layers were washed with saturated brine, dried over $\mathrm{Na}_{2} \mathrm{SO}_{4}$, filtered, concentrated by rotary evaporation and further purified by flash chromatography 
$(\mathrm{PE} / \mathrm{EA}=8: 1-4: 1)$ on silica gel to afford the corresponding alcohol. In a $100 \mathrm{~mL}$ roundbottom flask, a solution of the corresponding alcohol in DCM (30 mL) was added DessMartin periodinane (DMP) $(15 \mathrm{mmol})$ at $0{ }^{\circ} \mathrm{C}$. The mixture was stirred at room temperature and monitored by TLC. After the corresponding alcohol was fully consumed, the reaction mixture was filtered through a pad of silica gel and washed with DCM. The solvent was removed by rotary evaporation and the residue was further purified by flash chromatography $(\mathrm{PE} / \mathrm{EA}=20: 1-10: 1)$ on silica gel to afford the corresponding ketone.

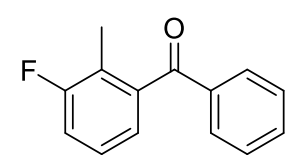

(3-fluoro-2-methylphenyl)(phenyl)methanone (S7i). This compound was afforded as colorless oil (1.90 g, 89\% yield) using 3-fluoro-2-methylbenzaldehyde (1.38 g, 10 mmol) and phenylmagnesium bromide (6 mL, $15 \mathrm{mmol}, 2.5 \mathrm{M}$ in THF) as starting materials, purified by flash chromatography (PE/EA = 8:1). ${ }^{1} \mathrm{H} \mathrm{NMR}\left(400 \mathrm{MHz}, \mathrm{CDCl}_{3}\right)$ $\delta 7.80(\mathrm{dt}, J=8.4,1.5 \mathrm{~Hz}, 2 \mathrm{H}), 7.62-7.57(\mathrm{~m}, 1 \mathrm{H}), 7.49-7.44(\mathrm{~m}, 2 \mathrm{H}), 7.25-7.20$ (m, 1H), $7.18-7.08(\mathrm{~m}, 2 \mathrm{H}), 2.21(\mathrm{~d}, J=2.2 \mathrm{~Hz}, 3 \mathrm{H}) .{ }^{13} \mathrm{C} \mathrm{NMR}\left(101 \mathrm{MHz}, \mathrm{CDCl}_{3}\right) \delta$ 197.3, $161.5(\mathrm{~d}, J=246.1 \mathrm{~Hz}), 141.1(\mathrm{~d}, J=3.8 \mathrm{~Hz}), 137.3,133.6,130.2,128.7,126.7$ $(\mathrm{d}, J=8.5 \mathrm{~Hz}), 124.1,123.9(\mathrm{~d}, J=3.6 \mathrm{~Hz}), 117.0(\mathrm{~d}, J=23.1 \mathrm{~Hz}), 11.8(\mathrm{~d}, J=5.0 \mathrm{~Hz})$. ${ }^{19} \mathrm{~F}$ NMR (377 MHz, $\left.\mathrm{CDCl}_{3}\right) \delta$-114.2. HRMS (EI) m/z: $[\mathrm{M}]^{+}$Calcd for $\mathrm{C}_{14} \mathrm{H}_{10} \mathrm{FO}$ 213.0712; Found 213.0710.<smiles>Cc1cc(F)ccc1C(=O)c1ccccc1</smiles>

(4-fluoro-2-methylphenyl(phenyl)methanone (S7j). This compound was afforded as colorless oil (1.90 g, 89\% yield) using 4-fluoro-2-methylbenzaldehyde (1.38 g, 10 mmol) and phenylmagnesium bromide (6 mL, $15 \mathrm{mmol}, 2.5 \mathrm{M}$ in THF) as starting materials, purified by flash chromatography (PE/EA = 8:1). ${ }^{1} \mathrm{H} \mathrm{NMR}\left(400 \mathrm{MHz}, \mathrm{CDCl}_{3}\right)$ $\delta 7.80(\mathrm{dd}, J=7.2,1.2 \mathrm{~Hz}, 2 \mathrm{H}), 7.62-7.56(\mathrm{~m}, 1 \mathrm{H}), 7.50-7.42(\mathrm{~m}, 2 \mathrm{H}), 7.25-7.07$ (m, 3H), $2.21(\mathrm{~d}, J=2.2 \mathrm{~Hz}, 3 \mathrm{H}) .{ }^{13} \mathrm{C} \mathrm{NMR}\left(101 \mathrm{MHz}, \mathrm{CDCl}_{3}\right) \delta 197.3,161.5(\mathrm{~d}, J=$ $246.1 \mathrm{~Hz}), 141.1$ (d, $J=3.8 \mathrm{~Hz}), 137.3,133.6,130.2,128.7,126.7$ (d, $J=8.5 \mathrm{~Hz}), 124.1$, 
$123.9(\mathrm{~d}, J=3.5 \mathrm{~Hz}), 117.0(\mathrm{~d}, J=23.1 \mathrm{~Hz}), 11.8(\mathrm{~d}, J=5.0 \mathrm{~Hz}) .{ }^{19} \mathrm{~F}$ NMR $(377 \mathrm{MHz}$, CDCl3) $\delta$-114.6. HRMS (EI) m/z: [M] $]^{+}$Calcd for $\mathrm{C}_{14} \mathrm{H}_{10} \mathrm{FO}$ 213.0712; Found 213.0710.<smiles>Cc1ccc(F)cc1C(=O)c1ccccc1</smiles>

(5-fluoro-2-methylphenyl)(phenyl)methanone (S7k). This compound was afforded as colorless oil (1.93 g, 90\% yield) using 5-fluoro-2-methylbenzaldehyde (1.38 g, 10 mmol) and phenylmagnesium bromide (6 mL, $15 \mathrm{mmol}, 2.5 \mathrm{M}$ in THF) as starting materials, purified by flash chromatography (PE/EA = 8:1). ${ }^{1} \mathrm{H} \mathrm{NMR}\left(400 \mathrm{MHz}, \mathrm{CDCl}_{3}\right)$ $\delta 7.82-7.75(\mathrm{~m}, 2 \mathrm{H}), 7.62-7.55(\mathrm{~m}, 1 \mathrm{H}), 7.50-7.41(\mathrm{~m}, 2 \mathrm{H}), 7.24(\mathrm{dd}, J=7.7,4.5$ $\mathrm{Hz}, 1 \mathrm{H}), 7.13-6.98(\mathrm{~m}, 2 \mathrm{H}), 2.26(\mathrm{~d}, J=2.6 \mathrm{~Hz}, 3 \mathrm{H}) .{ }^{13} \mathrm{C} \mathrm{NMR}\left(101 \mathrm{MHz}, \mathrm{CDCl}_{3}\right)$ $\delta 197.3,160.4(\mathrm{~d}, J=245.9 \mathrm{~Hz}), 140.1(\mathrm{~d}, J=5.9 \mathrm{~Hz}), 137.1,133.6,132.6(\mathrm{~d}, J=7.4$ $\mathrm{Hz}), 132.3(\mathrm{~d}, J=3.4 \mathrm{~Hz}), 130.2,128.7,117.1(\mathrm{~d}, J=20.9 \mathrm{~Hz}), 115.2(\mathrm{~d}, J=22.6 \mathrm{~Hz})$, 19.2 ${ }^{19} \mathrm{~F}$ NMR (377 MHz, $\left.\mathrm{CDCl}_{3}\right) \delta$-118.5. HRMS (EI) m/z: $[\mathrm{M}]^{+}$Calcd for $\mathrm{C}_{14} \mathrm{H}_{10} \mathrm{FO}$ 213.0712; Found 213.0710.<smiles>O=C(c1ccc(Br)cc1)c1ccccc1Cl</smiles>

(4-(tert-butyl)phenyl)(2-chlorophenyl)methanone (S7ae). This compound was afforded as white amorphous solid (2.53 g, 93\% yield) using 2-chlorobenzaldehyde (1.41 g, $10 \mathrm{mmol})$ and (4-(tert-butyl)phenyl)magnesium bromide (7.5 mL, $15 \mathrm{mmol}, 2$ $\mathrm{M}$ in THF) as starting materials, purified by flash chromatography $(\mathrm{PE} / \mathrm{EA}=8: 1) .{ }^{1} \mathrm{H}$ NMR (400 MHz, $\left.\mathrm{CDCl}_{3}\right) \delta 7.80-7.73(\mathrm{~m}, 2 \mathrm{H}), 7.51-7.39(\mathrm{~m}, 4 \mathrm{H}), 7.35$ (d, J=3.8 $\mathrm{Hz}, 2 \mathrm{H}), 1.35$ (s, 9H). ${ }^{13} \mathrm{C} \mathrm{NMR}\left(101 \mathrm{MHz}, \mathrm{CDCl}_{3}\right) \delta 194.9,157.8,139.0,133.9,131.3$, 131.0, 130.2, 130.1, 129.1, 126.7, 125.7, 35.3, 31.2. HRMS (EI) m/z: [M] Calcd for $\mathrm{C}_{17} \mathrm{H}_{17} \mathrm{ClO} 272.0967$; Found 272.0962.<smiles>Cc1ccc(Br)c(C(=O)c2ccccc2)c1</smiles> 
(2-bromo-5-methylphenyl)(phenyl)methanone (S7ai). This compound was afforded as colorless oil (2.41 g, 88\% yield) using 2-bromo-5-methylbenzaldehyde (1.99 g, 10 $\mathrm{mmol}$ ) and phenylmagnesium bromide $(6 \mathrm{~mL}, 15 \mathrm{mmol}, 2.5 \mathrm{M}$ in THF) as starting materials purified by flash chromatography $(\mathrm{PE} / \mathrm{EA}=8: 1) .{ }^{1} \mathrm{H} \mathrm{NMR}\left(400 \mathrm{MHz}, \mathrm{CDCl}_{3}\right)$ $\delta 7.79(\mathrm{~d}, J=7.2 \mathrm{~Hz}, 2 \mathrm{H}), 7.54(\mathrm{t}, J=7.4 \mathrm{~Hz}, 1 \mathrm{H}), 7.42(\mathrm{dd}, J=13.5,5.8 \mathrm{~Hz}, 3 \mathrm{H}), 7.19$ $(\mathrm{dd}, J=16.4,7.7 \mathrm{~Hz}, 2 \mathrm{H}), 2.35$ (s, 3H). ${ }^{13} \mathrm{C} \mathrm{NMR}\left(101 \mathrm{MHz}, \mathrm{CDCl}_{3}\right) \delta 195.8,141.8$, 137.5, 136.3, 133.6, 133.5, 130.1, 129.1, 128.5, 127.9, 119.5, 21.0. HRMS (EI) m/z: $[\mathrm{M}]^{+}$Calcd for $\mathrm{C}_{14} \mathrm{H}_{11} \mathrm{BrO} 273.9989$; Found 273.9988.

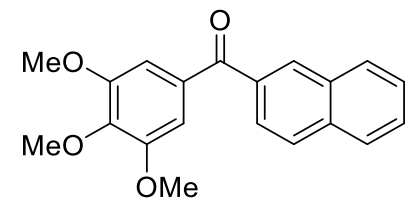

naphthalen-2-yl(3,4,5-trimethoxyphenyl)methanone (SS7am) This compound was afforded as yellow oil (2.99 g, 93\% yield) using 3,4,5-trimethoxybenzaldehyde (1.96 g, $10 \mathrm{mmol}$ ) and naphthalen-2-ylmagnesium bromide (15 mL, $15 \mathrm{mmol}, 1.0 \mathrm{M}$ in THF) as starting materials, purified by flash chromatography (PE/EA $=8: 1) .{ }^{1} \mathrm{H}$ NMR (400 $\left.\mathrm{MHz}, \mathrm{CDCl}_{3}\right) \delta 8.28(\mathrm{~s}, 1 \mathrm{H}), 7.93(\mathrm{q}, J=8.4 \mathrm{~Hz}, 4 \mathrm{H}), 7.64-7.53(\mathrm{~m}, 2 \mathrm{H}), 7.13(\mathrm{~s}, 2 \mathrm{H})$, $3.96(\mathrm{~s}, 3 \mathrm{H}), 3.87(\mathrm{~s}, 6 \mathrm{H}) .{ }^{13} \mathrm{C} \mathrm{NMR}\left(101 \mathrm{MHz}, \mathrm{CDCl}_{3}\right) \delta 195.9,153.0,142.1,135.2$, 135.1, 133.0, 132.3, 131.5, 129.4, 128.4, 128.3, 127.9, 127.0, 125.9, 107.8, 61.1, 56.4. HRMS (EI) m/z: [M] Calcd for $\mathrm{C}_{20} \mathrm{H}_{18} \mathrm{O}_{4}$ 322.1207; Found 322.1200 .<smiles>COc1cc(C(=O)c2ccc3ccccc3c2)c(Br)c(OC)c1OC</smiles>

(2-bromo-3,4,5-trimethoxyphenyl)(naphthalen-2-yl)methanone (S7am). To a solution of SS7am (966 mg, $3 \mathrm{mmol})$ in $\mathrm{CHCl}_{3}(20 \mathrm{~mL})$ was added $\mathrm{N}$ bromosuccinimide (5.44 g, $3.6 \mathrm{mmol})$. After reaction was heated at reflux temperature in an oil bath for $4 \mathrm{~h}$, the reaction mixture was cooled to room temperature, and the reaction mixture was washed with water and extracted with diethyl ether $\left(\mathrm{Et}_{2} \mathrm{O}\right)$. The combined extracts were dried with anhydrous $\mathrm{Na}_{2} \mathrm{SO}_{4}$ and concentrated, the residue was purified by flash column chromatography $(\mathrm{PE} / \mathrm{EA}=5: 1)$ to give S7am $(970 \mathrm{mg}$, $81 \%$ yield) as white amorphous solid. ${ }^{1} \mathrm{H} \mathrm{NMR}\left(400 \mathrm{MHz}, \mathrm{CDCl}_{3}\right) \delta 8.21(\mathrm{~s}, 1 \mathrm{H}), 8.01$ 
$(\mathrm{dd}, J=8.6,1.4 \mathrm{~Hz}, 1 \mathrm{H}), 7.95-7.86(\mathrm{~m}, 3 \mathrm{H}), 7.61(\mathrm{t}, J=7.5 \mathrm{~Hz}, 1 \mathrm{H}), 7.53(\mathrm{t}, J=7.6$ $\mathrm{Hz}, 1 \mathrm{H}), 6.77$ (s, 1H), 3.98 (d, $J=12.0 \mathrm{~Hz}, 6 \mathrm{H}), 3.85$ (s, 3H). ${ }^{13} \mathrm{C}$ NMR (101 MHz, $\left.\mathrm{CDCl}_{3}\right) \delta 195.6,153.2,151.2,144.5,136.3,136.0,133.5,133.1,132.5,129.9,129.0$, 128.8, 128.0, 127.0, 124.9, 108.0, 106.6, 61.4, 61.3, 56.4. HRMS (EI) m/z: [M] ${ }^{+}$Calcd for $\mathrm{C}_{20} \mathrm{H}_{17} \mathrm{BrO}_{4} 400.0316$; Found 400.0318 .<smiles>O=C(c1ncco1)c1ccccc1Br</smiles>

(2-bromophenyl)(oxazol-2-yl)methanone (S7ap). A flame-dried flask was charged with oxazole (690 mg, $10 \mathrm{mmol}$ ), 2-bromobenzoyl chloride (4.36 g, 20mmol,), 4dimethylaminopyridine (366 mg, $3 \mathrm{mmol}$ ) and anhydrous acetonitrile $(60 \mathrm{~mL})$. To this solution, triethylamine $(3.03 \mathrm{~g}, 30 \mathrm{mmol})$ were added. A reflux condenser was attached to the flask, and the reaction mixture heated at $70{ }^{\circ} \mathrm{C}$ in an oil bath for 24 hours. Upon completion, the reaction mixture was cooled to room temperature, and diluted with ethyl acetate and saturated $\mathrm{NaHCO}_{3}$. Ethyl acetate was used to extract the aqueous layer three times. The combined organic layers were washed with brine, dried over $\mathrm{Na}_{2} \mathrm{SO}_{4}$, and evaporated under reduced pressure. The resulting residue was purified by column chromatography yielding S7ap $\left(1.1 \mathrm{mg}, 48 \%\right.$ yield) as yellow amorphous solid. ${ }^{1} \mathrm{H}$ NMR $\left(400 \mathrm{MHz}, \mathrm{CDCl}_{3}\right) \delta 7.90(\mathrm{~s}, 1 \mathrm{H}), 7.69-7.61(\mathrm{~m}, 2 \mathrm{H}), 7.47-7.37(\mathrm{~m}, 3 \mathrm{H}) .{ }^{13} \mathrm{C}$ NMR (101 MHz, $\left.\mathrm{CDCl}_{3}\right) \delta 181.1,157.7,142.2,137.8,133.8,132.8,130.5,130.0,127.3$, 120.6. HRMS (EI) m/z: $[\mathrm{M}+\mathrm{H}]^{+}$Calcd for $\mathrm{C}_{10} \mathrm{H}_{7} \mathrm{NO}_{2} \mathrm{Br} 251.9655$; Found 251.9663.<smiles>O=C(c1cccs1)c1ccccc1Br</smiles>

(2-bromophenyl)(thiazol-2-yl)methanone (S7aq). This compound was afforded as yellow liquid ( $2 \mathrm{~g}, 75 \%$ yield) following the similar procedure to S7ap with thiazole (850 mg, $10 \mathrm{mmol}$ ), 2-bromobenzoyl chloride (4.36 mg, 20mmol), 4dimethylaminopyridine (366 mg, $3 \mathrm{mmol})$, anhydrous acetonitrile $(60 \mathrm{~mL})$, and triethylamine $(3.03 \mathrm{~g}, 30 \mathrm{mmol})$, purified by flash column chromatography $(\mathrm{PE} / \mathrm{EA}=$ 10:1-5:1). ${ }^{1} \mathrm{H}$ NMR $\left(400 \mathrm{MHz}, \mathrm{CDCl}_{3}\right) \delta 8.04(\mathrm{~d}, J=3.0 \mathrm{~Hz}, 1 \mathrm{H}), 7.77(\mathrm{~d}, J=3.0 \mathrm{~Hz}$, 1H), $7.69-7.65(\mathrm{~m}, 1 \mathrm{H}), 7.61(\mathrm{dd}, J=7.5,1.8 \mathrm{~Hz}, 1 \mathrm{H}), 7.46-7.41(\mathrm{~m}, 1 \mathrm{H}), 7.38(\mathrm{td}$, $J=7.7,1.8 \mathrm{~Hz}, 1 \mathrm{H}) .{ }^{13} \mathrm{C} \mathrm{NMR}\left(101 \mathrm{MHz}, \mathrm{CDCl}_{3}\right) \delta 186.0,165.4,144.5,137.5,132.6$, 
131.2, 129.3, 126.2, 126.1, 120.0. HRMS (EI) m/z: $[\mathrm{M}-\mathrm{H}]^{+}$Calcd for $\mathrm{C}_{10} \mathrm{H}_{5} \mathrm{BrNOS}$ 265.9258; Found 265.9270.

\section{Procedures for the synthesis of diarylalkenes}

All the alkenes were prepared by Wittig olefination of the ketones (in $5 \mathrm{mmol}$ scale) according to previously reported procedures ${ }^{16}$ except $\mathbf{7 g}$.

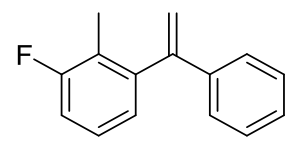

1-fluoro-2-methyl-3-(1-phenylvinyl)benzene (7i). This compound was afforded as colorless oil (901 mg, 85\% yield), purified by flash column chromatography (PE/EA = 50:1). ${ }^{1} \mathrm{H}$ NMR (400 MHz, $\left.\mathrm{CDCl}_{3}\right) \delta 7.30-7.21(\mathrm{~m}, 5 \mathrm{H}), 7.19-7.11(\mathrm{~m}, 1 \mathrm{H}), 7.03-$ $6.96(\mathrm{~m}, 2 \mathrm{H}), 5.78(\mathrm{~s}, 1 \mathrm{H}), 5.19(\mathrm{~s}, 1 \mathrm{H}), 1.97(\mathrm{~s}, 3 \mathrm{H}) .{ }^{13} \mathrm{C} \mathrm{NMR}\left(101 \mathrm{MHz}, \mathrm{CDCl}_{3}\right) \delta$ $161.5(\mathrm{~d}, J=243.9 \mathrm{~Hz}), 148.4(\mathrm{~d}, J=2.7 \mathrm{~Hz}), 144.1$ (d, $J=4.5 \mathrm{~Hz}), 140.3,128.6,127.9$, 126.7 (d, $J=9.0 \mathrm{~Hz}), 126.5,125.7$ (d, $J=3.0 \mathrm{~Hz}), 123.6$ (d, $J=16.6 \mathrm{~Hz}), 115.5,114.2$ $(\mathrm{d}, J=23.1 \mathrm{~Hz}), 12.0(\mathrm{~d}, J=5.0 \mathrm{~Hz}) .{ }^{19} \mathrm{~F}$ NMR $\left(377 \mathrm{MHz}, \mathrm{CDCl}_{3}\right) \delta-116.6$. HRMS (EI) m/z: [M] ${ }^{+}$Calcd for $\mathrm{C}_{15} \mathrm{H}_{13} \mathrm{~F} 212.0998$; found 212.0996.<smiles>C=C(c1ccccc1)c1ccc(F)cc1C</smiles>

1-fluoro-2-methyl-3-(1-phenylvinyl)benzene (7j). This compound was afforded as colorless oil (1.02 g, 96\% yield), purified by flash column chromatography (PE/EA = 50:1). ${ }^{1} \mathrm{H}$ NMR (400 MHz, $\left.\mathrm{CDCl}_{3}\right) \delta 7.29-7.11(\mathrm{~m}, 6 \mathrm{H}), 7.03-6.95(\mathrm{~m}, 2 \mathrm{H}), 5.79-$ $5.78(\mathrm{~m}, 1 \mathrm{H}), 5.21-5.18(\mathrm{~m}, 1 \mathrm{H}), 1.97(\mathrm{~d}, J=2.5 \mathrm{~Hz}, 3 \mathrm{H}) .{ }^{13} \mathrm{C} \mathrm{NMR}\left(101 \mathrm{MHz}, \mathrm{CDCl}_{3}\right)$ $\delta 161.5(\mathrm{~d}, J=243.9 \mathrm{~Hz}), 148.4(\mathrm{~d}, J=2.6 \mathrm{~Hz}), 144.1(\mathrm{~d}, J=4.4 \mathrm{~Hz}), 140.3,128.6$, 127.9, 126.7 (d, $J=9.0 \mathrm{~Hz},), 126.5,125.7$ (d, $J=2.9 \mathrm{~Hz}), 123.6$ (d, $J=16.5 \mathrm{~Hz}), 115.5$, $114.2(\mathrm{~d}, J=23.1 \mathrm{~Hz}), 12.0(\mathrm{~d}, J=5.0 \mathrm{~Hz}) .{ }^{19} \mathrm{~F}$ NMR $\left(377 \mathrm{MHz}, \mathrm{CDCl}_{3}\right) \delta-117.3$. HRMS (EI) m/z: [M] ${ }^{+}$Calcd for $\mathrm{C}_{15} \mathrm{H}_{13} \mathrm{~F} 212.0998$; Found, 212.0996.

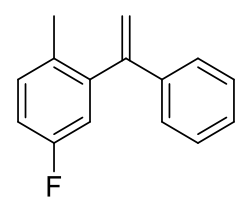


4-fluoro-1-methyl-2-(1-phenylvinyl)benzene (7k). This compound was afforded as colorless oil (1.02 g, 96\% yield), purified by flash column chromatography (PE/EA = 50:1). ${ }^{1} \mathrm{H}$ NMR (400 MHz, $\left.\mathrm{CDCl}_{3}\right) \delta 7.32$ - $7.22(\mathrm{~m}, 5 \mathrm{H}), 7.14-7.09(\mathrm{~m}, 1 \mathrm{H}), 6.97-$ $6.90(\mathrm{~m}, 2 \mathrm{H}), 5.78(\mathrm{~d}, J=1.1 \mathrm{~Hz}, 1 \mathrm{H}), 5.20(\mathrm{~d}, J=1.1 \mathrm{~Hz}, 1 \mathrm{H}), 2.00(\mathrm{~s}, 3 \mathrm{H}) .{ }^{13} \mathrm{C}$ NMR $\left(101 \mathrm{MHz}, \mathrm{CDCl}_{3}\right) \delta 161.1(\mathrm{~d}, J=243.7 \mathrm{~Hz}), 148.7,143.4(\mathrm{~d}, J=7.4 \mathrm{~Hz}), 140.0,131.9$ $(\mathrm{d}, J=3.2 \mathrm{~Hz}, 4 \mathrm{H}), 131.4(\mathrm{~d}, J=7.9 \mathrm{~Hz}), 128.6,127.9,126.6,116.8(\mathrm{~d}, J=21.2 \mathrm{~Hz})$, 115.5, $114.2(\mathrm{~d}, J=20.6 \mathrm{~Hz}), 19.4 .{ }^{19} \mathrm{~F} \mathrm{NMR}\left(377 \mathrm{MHz}, \mathrm{CDCl}_{3}\right) \delta-118.5$. HRMS (EI) $\mathrm{m} / \mathrm{z}:[\mathrm{M}]^{+}$Calcd for $\mathrm{C}_{15} \mathrm{H}_{13} \mathrm{~F} 212.0998$; Found 212.0996.

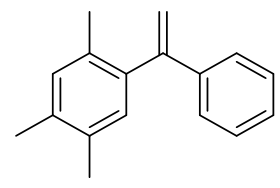

1,2,4-trimethyl-5-(1-phenylvinyl)benzene (7p). This compound was afforded as colorless oil ( $899 \mathrm{mg}, 81 \%$ yield), purified by flash column chromatography (PE/EA = 50:1). ${ }^{1} \mathrm{H}$ NMR $\left(400 \mathrm{MHz}, \mathrm{CDCl}_{3}\right) \delta 7.43-7.31(\mathrm{~m}, 5 \mathrm{H}), 7.10(\mathrm{~d}, J=17.0 \mathrm{~Hz}, 2 \mathrm{H})$, $5.84(\mathrm{~s}, 1 \mathrm{H}), 5.29(\mathrm{~s}, 1 \mathrm{H}), 2.37$ (d, J=6.2 Hz, 6H), 2.11 (s, 3H). ${ }^{13} \mathrm{C}$ NMR (101 MHz, $\left.\mathrm{CDCl}_{3}\right) \delta 149.6,141.1,139.2,135.8,133.7,133.4,131.6,131.4,128.4,127.6,126.7$, 114.7, 19.7, 19.5, 19.3. HRMS (EI) m/z: [M] $]^{+}$Calcd for $\mathrm{C}_{17} \mathrm{H}_{18}$ 224.1400; Found 222.1403 .

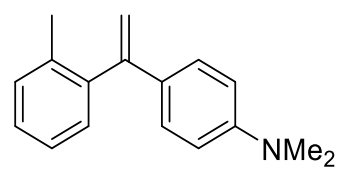

N,N-dimethyl-4-(1-(o-tolyl)vinyl)aniline (7s). This compound was afforded as yellow oil (356 mg, 30\% yield), purified by flash column chromatography (PE/EA = 10:1). ${ }^{1} \mathrm{H}$ NMR (400 MHz, $\left.\mathrm{CDCl}_{3}\right) \delta 7.23-7.09(\mathrm{~m}, 6 \mathrm{H}), 6.62(\mathrm{~d}, J=8.5 \mathrm{~Hz}, 2 \mathrm{H}), 5.63(\mathrm{~s}, 1 \mathrm{H})$, 4.97 (s, 1H), 2.93 (s, 6H), 2.09 (s, 3H). $\left.{ }^{13} \mathrm{C} \mathrm{NMR} \mathrm{(101} \mathrm{MHz,} \mathrm{CDCl}_{3}\right) \delta$ 150.1, 149.0, 142.4 136.3, 130.1, 130.0, 128.8, 127.4, 127.3, 125.6, 112.2, 111.0, 40.6, 20.1. HRMS (ESI) $\mathrm{m} / \mathrm{z}:[\mathrm{M}+\mathrm{H}]^{+}$Calcd for $\mathrm{C}_{17} \mathrm{H}_{20} \mathrm{~N} 238.1590$; Found 238.1596.

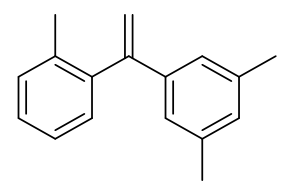


1,3-dimethyl-5-(1-(o-tolyl)vinyl)benzene (7w). This compound was afforded as colorless oil (932 mg, 84\% yield), purified by flash column chromatography (PE/EA = 50:1). ${ }^{1} \mathrm{H}$ NMR $\left(400 \mathrm{MHz}, \mathrm{CDCl}_{3}\right) \delta 7.26-7.15(\mathrm{~m}, 4 \mathrm{H}), 6.88(\mathrm{~d}, J=4.7 \mathrm{~Hz}, 3 \mathrm{H}), 5.72$ (s, 1H), $5.14(\mathrm{~s}, 1 \mathrm{H}), 2.25(\mathrm{~s}, 6 \mathrm{H}), 2.07(\mathrm{~s}, 3 \mathrm{H}) .{ }^{13} \mathrm{C} \mathrm{NMR}\left(101 \mathrm{MHz}, \mathrm{CDCl}_{3}\right) \delta$ 149.7, 142.0, 140.7, 137.9, 136.3, 130.1, 129.4, 127.5, 125.7, 124.5, 114.8, 21.5, 20.3. HRMS (EI) m/z: [M] ${ }^{+}$Calcd for $\mathrm{C}_{17} \mathrm{H}_{18}$ 224.1400; Found 222.1403.<smiles>C=C(c1ccccc1)c1c(Cl)cccc1Cl</smiles>

1,3-dichloro-2-(1-phenylvinyl)benzene (7aa). This compound was afforded as colorless oil (657 mg, 53\% yield), purified by flash column chromatography (PE/EA = 50:1). ${ }^{1} \mathrm{H}$ NMR (400 MHz, CDCl3) $\delta 7.39-7.27$ (m, 6H), $7.25-7.17(\mathrm{~m}, 2 \mathrm{H}), 6.04$ (d, $J=1.7 \mathrm{~Hz}, 1 \mathrm{H}), 5.27$ (d, $J=1.7 \mathrm{~Hz}, 1 \mathrm{H}) .13 \mathrm{C} \mathrm{NMR}(101 \mathrm{MHz}, \mathrm{CDCl} 3) \delta$ 144.0, 139.5, 138.1, 135.3, 129.2, 128.6, 128.2, 128.1, 126.0, 117.3. HRMS (EI) m/z: [M] ${ }^{+}$ Calcd for $\mathrm{C}_{14} \mathrm{H}_{10} \mathrm{C}_{12}$ 248.0158; Found 248.0154.<smiles>C=C(c1ccccc1)c1cc(C(F)(F)F)ccc1Cl</smiles>

1-chloro-2-(1-phenylvinyl)-4-(trifluoromethyl)benzene (7ab). This compound was afforded as colorless oil (1.27 g, 90\% yield), purified by flash column chromatography $(\mathrm{PE} / \mathrm{EA}=50: 1) .{ }^{1} \mathrm{H} \mathrm{NMR}\left(400 \mathrm{MHz}, \mathrm{CDCl}_{3}\right) \delta 7.61(\mathrm{~s}, 1 \mathrm{H}), 7.49(\mathrm{dd}, J=18.8,8.4 \mathrm{~Hz}$, 2H), $7.26(\mathrm{dt}, J=7.7,6.3 \mathrm{~Hz}, 5 \mathrm{H}), 5.86(\mathrm{~s}, 1 \mathrm{H}), 5.30(\mathrm{~s}, 1 \mathrm{H}) .{ }^{13} \mathrm{C} \mathrm{NMR}(101 \mathrm{MHz}$, $\mathrm{CDCl} 3) \delta 146.7,141.7,139.1,137.5,130.5,129.5$ (q, J = 65.8), 128.7, 128.5 (q, J = 7.4), 128.3, 126.5, 125.8 (q, J = 7.3), 123.9 (q, J = 272.2 Hz), 117.4. ${ }^{19} \mathrm{~F}$ NMR (377 $\mathrm{MHz}, \mathrm{CDCl}_{3}$ ) $\delta$-62.4. HRMS (EI) m/z: [M] $]^{+}$Calcd for $\mathrm{C}_{15} \mathrm{H}_{10} \mathrm{ClF}_{3}$ 282.0414; Found 282.0418 .

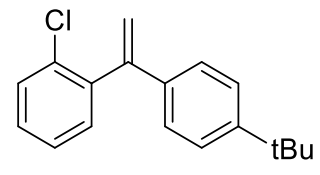

1-(1-(4-(tert-butyl)phenyl)vinyl)-2-chlorobenzene (7ae). This compound was afforded as white amorphous solid (1.27 g, 96\% yield), purified by flash column 
chromatography $(\mathrm{PE} / \mathrm{EA}=50: 1) .{ }^{1} \mathrm{H}$ NMR $\left(400 \mathrm{MHz}, \mathrm{CDCl}_{3}\right) \delta 7.41-7.36(\mathrm{~m}, 1 \mathrm{H})$, $7.33-7.19(\mathrm{~m}, 7 \mathrm{H}), 5.82(\mathrm{~d}, J=1.0 \mathrm{~Hz}, 1 \mathrm{H}), 5.22(\mathrm{~d}, J=1.0 \mathrm{~Hz}, 1 \mathrm{H}), 1.29$ (d, $J=6.0$ $\mathrm{Hz}, 9 \mathrm{H}) .{ }^{13} \mathrm{C} \mathrm{NMR}\left(101 \mathrm{MHz}, \mathrm{CDCl}_{3}\right) \delta 150.9,147.1,141.0,136.8,133.4,131.7,129.8$, 128.9, 126.8, 126.2, 125.4, 115.5, 34.7, 31.4. HRMS (EI) m/z: $[M]^{+}$Calcd for $\mathrm{C}_{18} \mathrm{H}_{19} \mathrm{Cl} 270.1177$; Found 270.1170.<smiles>C=C(c1ccc(F)cc1)c1ccccc1Cl</smiles>

1-chloro-2-(1-(4-fluorophenyl)vinyl)benzene (7ag). This compound was afforded as white amorphous solid (1.07 g, 92\% yield), purified by flash column chromatography $(\mathrm{PE} / \mathrm{EA}=50: 1) .{ }^{1} \mathrm{H} \mathrm{NMR}\left(400 \mathrm{MHz}, \mathrm{CDCl}_{3}\right) \delta 7.42-7.19(\mathrm{~m}, 6 \mathrm{H}), 6.96$ (dd, $J=12.2$, $5.1 \mathrm{~Hz}, 2 \mathrm{H}), 5.75(\mathrm{~s}, 1 \mathrm{H}), 5.25(\mathrm{~s}, 1 \mathrm{H}) .{ }^{13} \mathrm{C} \mathrm{NMR}\left(101 \mathrm{MHz}, \mathrm{CDCl}_{3}\right) \delta 162.6(\mathrm{~d}, J=$ $247.1 \mathrm{~Hz}), 146.6,140.6,136.1$ (d, $J=3.3 \mathrm{~Hz}), 133.3,131.6,129.9,129.2,128.2$ (d, $J$ $=8.1 \mathrm{~Hz}), 126.9,116.2(\mathrm{~d}, J=1.3 \mathrm{~Hz}), 115.3(\mathrm{~d}, J=21.5 \mathrm{~Hz}) \cdot{ }^{19} \mathrm{~F}$ NMR $(377 \mathrm{MHz}$, $\left.\mathrm{CDCl}_{3}\right) \delta$-114.6. HRMS (EI) m/z: [M] ${ }^{+}$Calcd for $\mathrm{C}_{14} \mathrm{H}_{10} \mathrm{ClF} 232.0454$; found 232.0450.<smiles>C=C(c1ccccc1)c1cc(C)ccc1Br</smiles>

1-bromo-4-methyl-2-(1-phenylvinyl)benzene (7ai). This compound was afforded as colorless oil (1.21 g, 89\% yield), purified by flash column chromatography (PE/EA = 50:1). ${ }^{1} \mathrm{H}$ NMR (400 MHz, $\left.\mathrm{CDCl}_{3}\right) \delta 7.42(\mathrm{~s}, 1 \mathrm{H}), 7.31-7.25(\mathrm{~m}, 5 \mathrm{H}), 7.19(\mathrm{~d}, J=7.7$ $\mathrm{Hz}, 1 \mathrm{H}), 7.13(\mathrm{dd}, J=7.7,0.9 \mathrm{~Hz}, 1 \mathrm{H}), 5.80(\mathrm{~d}, J=0.9 \mathrm{~Hz}, 1 \mathrm{H}), 5.24(\mathrm{~d}, J=0.9 \mathrm{~Hz}$, 1H), 2.35 (s, 3H). ${ }^{13} \mathrm{C}$ NMR (101 MHz, $\left.\mathrm{CDCl}_{3}\right) \delta 148.9,139.9,139.8,139.3,133.5$, 131.5, 128.4, 128.2 127.8, 126.7, 123.1, 116.1, 20.9. HRMS (EI) m/z: [M] Calcd for $\mathrm{C}_{15} \mathrm{H}_{13} \mathrm{Br} 272.0196$; Found 272.0195.<smiles>C=C(c1ccccc1)c1cc(F)ccc1Br</smiles>

1-bromo-4-fluoro-2-(1-phenylvinyl)benzene (7aj). This compound was afforded as colorless oil (1.34 g, 97\% yield), purified by flash column chromatography (PE/EA = 50:1). ${ }^{1} \mathrm{H}$ NMR (400 MHz, $\left.\mathrm{CDCl}_{3}\right) \delta 7.51(\mathrm{dd}, J=8.8,5.3 \mathrm{~Hz}, 1 \mathrm{H}), 7.32-7.23(\mathrm{~m}$, 
5H), $7.05(\mathrm{dd}, J=8.9,3.1 \mathrm{~Hz}, 1 \mathrm{H}), 6.95-6.87(\mathrm{~m}, 1 \mathrm{H}), 5.83(\mathrm{~s}, 1 \mathrm{H}), 5.26(\mathrm{~s}, 1 \mathrm{H}) .{ }^{13} \mathrm{C}$ NMR (101 MHz, $\left.\mathrm{CDCl}_{3}\right) \delta 161.9(\mathrm{~d}, J=247.7 \mathrm{~Hz}), 148.2(\mathrm{~d}, J=1.3 \mathrm{~Hz}), 144.6(\mathrm{~d}, J$ = 7.7 Hz), 139.0, $134.3(\mathrm{~d}, J=8.1 \mathrm{~Hz}), 128.6,128.1,126.6,118.7$ (d, $J=22.6 \mathrm{~Hz})$, $117.7(\mathrm{~d}, J=3.3 \mathrm{~Hz}), 116.7,116.3(\mathrm{~d}, J=22.3 \mathrm{~Hz}) .{ }^{19} \mathrm{~F} \mathrm{NMR}\left(377 \mathrm{MHz}, \mathrm{CDCl}_{3}\right) \delta-$ 115.3. HRMS (EI) m/z: [M] $]^{+}$Calcd for $\mathrm{C}_{14} \mathrm{H}_{10} \mathrm{BrF} 275.9945$; Found 275.9944 .<smiles>C=C(c1ccc2ccccc2c1)c1cc(OC)c(OC)c(OC)c1Br</smiles>

2-(1-(2-bromo-3,4,5-trimethoxyphenyl)vinyl)naphthalene (7am). This compound was afforded as colorless oil (1.69 g, 85\% yield), purified by flash column chromatography $(\mathrm{PE} / \mathrm{EA}=10: 1) .{ }^{1} \mathrm{H} \mathrm{NMR}\left(400 \mathrm{MHz}, \mathrm{CDCl}_{3}\right) \delta 7.82-7.70(\mathrm{~m}, 3 \mathrm{H})$, $7.56(\mathrm{~d}, J=7.2 \mathrm{~Hz}, 2 \mathrm{H}), 7.45-7.39(\mathrm{~m}, 2 \mathrm{H}), 6.74(\mathrm{~s}, 1 \mathrm{H}), 5.95(\mathrm{~s}, 1 \mathrm{H}), 5.34(\mathrm{~s}, 1 \mathrm{H})$, $3.94(\mathrm{~d}, J=14.8 \mathrm{~Hz}, 6 \mathrm{H}), 3.85(\mathrm{~s}, 3 \mathrm{H}) .{ }^{13} \mathrm{C} \mathrm{NMR}\left(101 \mathrm{MHz}, \mathrm{CDCl}_{3}\right) \delta$ 152.7, 151.2, $149.0,142.6,138.3,136.8,133.4,133.0,128.5,128.1,127.7,126.3,126.2,126.0,124.4$, 116.4, 110.4, 109.9, 61.3, 61.1, 56.3. HRMS (EI) m/z: [M] ${ }^{+}$Calcd for $\mathrm{C}_{21} \mathrm{H}_{19} \mathrm{O}_{3} \mathrm{Br}$ 398.0527; Found 398.0512.

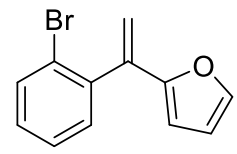

2-(1-(2-bromophenyl)vinyl)furan (7an). This compound was afforded as colorless oil (902 mg, 73\% yield), purified by flash column chromatography $(\mathrm{PE} / \mathrm{EA}=10: 1) .{ }^{1} \mathrm{H}$ NMR (400 MHz, $\left.\mathrm{CDCl}_{3}\right) \delta 7.60(\mathrm{~d}, J=8.0 \mathrm{~Hz}, 1 \mathrm{H}), 7.39$ (d, $\left.J=1.3 \mathrm{~Hz}, 1 \mathrm{H}\right), 7.33-$ $7.26(\mathrm{~m}, 2 \mathrm{H}), 7.22-7.17(\mathrm{~m}, 1 \mathrm{H}), 6.32(\mathrm{dd}, J=3.3,1.8 \mathrm{~Hz}, 1 \mathrm{H}), 5.90(\mathrm{~s}, 1 \mathrm{H}), 5.87(\mathrm{~d}$, $J=3.3 \mathrm{~Hz}, 1 \mathrm{H}), 5.10(\mathrm{~d}, J=0.8 \mathrm{~Hz}, 1 \mathrm{H}) .{ }^{13} \mathrm{C} \mathrm{NMR}\left(101 \mathrm{MHz}, \mathrm{CDCl}_{3}\right) \delta 153.3,142.6$, 140.4, 138.4, 133.0, 131.3, 129.4, 127.3, 123.3, 113.3, 111.5, 109.1. HRMS (EI) m/z: $[\mathrm{M}]^{+}$Calcd for $\mathrm{C}_{12} \mathrm{H}_{9} \mathrm{BrO} 247.9830$; Found 247.9831.<smiles>C=C(c1ncco1)c1ccccc1Br</smiles>

2-(1-(2-bromophenyl)vinyl)oxazole (7ap). This compound was afforded as yellow oil (248 mg, 20\% yield), purified by flash column chromatography $(\mathrm{PE} / \mathrm{EA}=6: 1) .{ }^{1} \mathrm{H}$ NMR (400 MHz, $\left.\mathrm{CDCl}_{3}\right) \delta 7.79(\mathrm{~d}, J=3.2 \mathrm{~Hz}, 1 \mathrm{H}), 7.62(\mathrm{~d}, J=7.9 \mathrm{~Hz}, 1 \mathrm{H}), 7.39-$ 
$7.35(\mathrm{~m}, 2 \mathrm{H}), 7.28-7.21(\mathrm{~m}, 2 \mathrm{H}), 6.33(\mathrm{~s}, 1 \mathrm{H}), 5.45(\mathrm{~s}, 1 \mathrm{H}) .{ }^{13} \mathrm{C}$ NMR (101 MHz, $\left.\mathrm{CDCl}_{3}\right) \delta 168.2,143.7,142.5,140.6,133.1,131.4,129.9,127.6,123.4,119.8,119.7$. HRMS (EI) m/z: [M-H] ${ }^{+}$Calcd for $\mathrm{C}_{11} \mathrm{H}_{7} \mathrm{BrO} 247.9830$; Found 247.9831.<smiles>C=C(c1nccs1)c1ccccc1Br</smiles>

2-(1-(2-bromophenyl)vinyl)thiazole (7aq). This compound was afforded as yellow oil (334 mg, 25\% yield), purified by flash column chromatography (PE/EA $=6: 1) .{ }^{1} \mathrm{H}$ NMR (400 MHz, $\left.\mathrm{CDCl}_{3}\right) \delta 7.80(\mathrm{~d}, J=3.2 \mathrm{~Hz}, 1 \mathrm{H}), 7.62$ (d, $\left.J=7.8 \mathrm{~Hz}, 1 \mathrm{H}\right), 7.40-$ $7.36(\mathrm{~m}, 2 \mathrm{H}), 7.29-7.23(\mathrm{~m}, 2 \mathrm{H}), 6.33(\mathrm{~s}, 1 \mathrm{H}), 5.46(\mathrm{~s}, 1 \mathrm{H}) .{ }^{13} \mathrm{C}$ NMR (101 MHz, $\left.\mathrm{CDCl}_{3}\right) \delta 168.2,143.7,142.5,140.6,133.1,131.4,123.0,127.6,123.4,119.8,119.7$. HRMS (EI) m/z: [M-H] ${ }^{+}$Calcd for $\mathrm{C}_{11} \mathrm{H}_{7} \mathrm{BrNS}$ 263.9476; Found 263.9477.

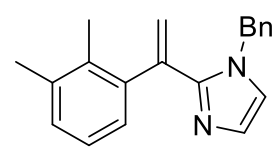

1-benzyl-2-(1-(2,3-dimethylphenyl)vinyl)-1H-imidazole (7ar). This compound was afforded as white amorphous solid (332 mg, 23\% yield), purified by flash column chromatography (PE/EA = 3:1). ${ }^{1} \mathrm{H} \mathrm{NMR}\left(400 \mathrm{MHz}, \mathrm{CDCl}_{3}\right) \delta 7.24(\mathrm{~d}, J=5.5 \mathrm{~Hz}, 3 \mathrm{H})$, $7.14-7.01(\mathrm{~m}, 4 \mathrm{H}), 6.83(\mathrm{~d}, J=5.9 \mathrm{~Hz}, 3 \mathrm{H}), 5.90(\mathrm{~s}, 1 \mathrm{H}), 5.41(\mathrm{~s}, 1 \mathrm{H}), 4.77(\mathrm{~s}, 2 \mathrm{H})$, 2.19 (s, 3H), 1.89 (s, 3H). ${ }^{13} \mathrm{C}$ NMR (101 MHz, $\left.\mathrm{CDCl}_{3}\right) \delta 148.2,140.1,139.7,137.4$, $136.8,134.7,129.9,128.7,128.7,127.7,127.6,126.6,125.7,121.7,121.6,50.3,20.5$, 16.2. HRMS (ESI) m/z: [M+H] $]^{+}$Calcd for $\mathrm{C}_{20} \mathrm{H}_{21} \mathrm{~N}_{2}$ 289.1709; Found 289.1699.

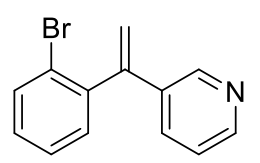

3-(1-(2-bromophenyl)vinyl)pyridine (7as). This compound was afforded as yellow oil (903 mg, 70\% yield), purified by flash column chromatography $(\mathrm{PE} / \mathrm{EA}=6: 1) .{ }^{1} \mathrm{H}$ $\operatorname{NMR}\left(400 \mathrm{MHz}, \mathrm{CDCl}_{3}\right) \delta 8.57(\mathrm{~d}, J=1.9 \mathrm{~Hz}, 1 \mathrm{H}), 8.50(\mathrm{dd}, J=4.8,1.4 \mathrm{~Hz}, 1 \mathrm{H}), 7.59$ $(\mathrm{dd}, J=8.0,0.8 \mathrm{~Hz}, 1 \mathrm{H}), 7.53-7.48(\mathrm{~m}, 1 \mathrm{H}), 7.38-7.30(\mathrm{~m}, 2 \mathrm{H}), 7.21$ (ddd, $J=7.9$, 6.6, 2.8 Hz, 2H), 5.87 (s, 1H), $5.37(\mathrm{~s}, 1 \mathrm{H}) .{ }^{13} \mathrm{C} \mathrm{NMR}\left(101 \mathrm{MHz}, \mathrm{CDCl}_{3}\right) \delta 148.8,148.1$, 146.3 141.5, 135.3, 133.7, 133.2, 131.6, 129.5, 127.6, 123.2, 123.1, 117.9. HRMS (EI) m/z: $[\mathrm{M}+\mathrm{H}]^{+}$Calcd for $\mathrm{C}_{13} \mathrm{H}_{10} \mathrm{BrN}$ 259.0001; Found 258.9991. 


\section{Procedure for synthesis of $7 \mathrm{~g}$}

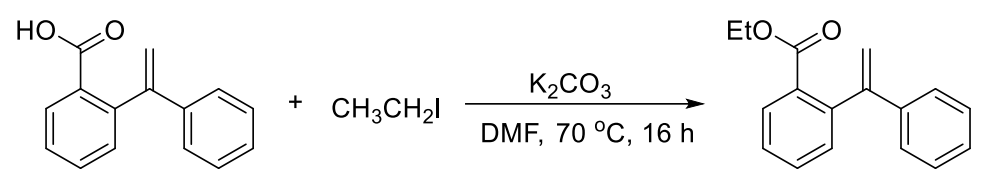

Ethyl 2-(1-phenylvinyl)benzoate (7g). A flame-dried flask was charged with 2-(1phenylvinyl)benzoic acid (2.13 g, $9.5 \mathrm{mmol})$, iodoethane (2.25 g, $14.25 \mathrm{mmol})$, potassium carbonate $(2.6 \mathrm{~g}, 19 \mathrm{mmol})$ and DMF $(50 \mathrm{~mL})$. The mixture was stirred at $70^{\circ} \mathrm{C}$ in an oil bath for $16 \mathrm{~h}$. After the reaction was completed by TLC, the reaction mixture was cooled to room temperature, the mixture was partitioned between EtOAc $(100 \mathrm{~mL})$ and aqueous $\mathrm{HCl}(100 \mathrm{~mL}, 1 \mathrm{M})$. The layers were separated and the organic layer was washed successively with saturated aqueous $\mathrm{NaHCO}_{3}$ and water. The organic layer was dried over $\mathrm{MgSO}_{4}$, filtered and the solvent evaporated invacuo. The residue was purified by silica gel chromatography $(\mathrm{PE} / \mathrm{EA}=10: 1-4: 1)$ yielding $7 \mathbf{g}(573 \mathrm{mg}$, $24 \%$ yield) as yellow liquid. ${ }^{1} \mathrm{H} \mathrm{NMR}\left(400 \mathrm{MHz}, \mathrm{CDCl}_{3}\right) \delta 7.81(\mathrm{dd}, J=7.7,1.2 \mathrm{~Hz}$, 1H), $7.50(\mathrm{td}, J=7.5,1.3 \mathrm{~Hz}, 1 \mathrm{H}), 7.39(\mathrm{ddd}, J=11.3,8.9,4.3 \mathrm{~Hz}, 2 \mathrm{H}), 7.28-7.20$ (m, 5H), 5.69 (d, $J=0.6 \mathrm{~Hz}, 1 \mathrm{H}), 5.25(\mathrm{~d}, J=0.5 \mathrm{~Hz}, 1 \mathrm{H}), 3.96(\mathrm{q}, J=7.1 \mathrm{~Hz}, 2 \mathrm{H})$, $1.06(\mathrm{t}, J=7.1 \mathrm{~Hz}, 3 \mathrm{H}) .{ }^{13} \mathrm{C} \mathrm{NMR}\left(101 \mathrm{MHz}, \mathrm{CDCl}_{3}\right) \delta 167.9,149.7,142.5,140.9$, 131.6, 131.5, 131.4, 129.8, 128.2, 127.7, 127.6, 126.8, 114.3, 61.0, 13.9. HRMS (EI) $\mathrm{m} / \mathrm{z}:[\mathrm{M}]^{+}$Calcd for $\mathrm{C}_{17} \mathrm{H}_{16} \mathrm{O}_{2} 252.1151$; Found 252.1145 .

\section{Asymmetric Transfer Hydrogenation of 1,1-Diarylethenes}

General Procedure for Asymmetric Transfer Hydrogenation of 1,1-Diarylethenes with EtOH: In an argon filled glovebox, catalyst $6 \mathbf{b}(10 \mu \mathrm{mol}, 7.46 \mathrm{mg}), \mathrm{NaO} t \mathrm{Bu}(15$ $\mu \mathrm{mol}, 1.44 \mathrm{mg})$, alkene $(0.25 \mathrm{mmol})$ and $\mathrm{EtOH}(0.2 \mathrm{~mL})$ were added to a $10 \mathrm{~mL}$ dried Schlenk tube. The tube was sealed with a Teflon plug and the mixture was stirred at room temperature for $36 \mathrm{~h}$. the reaction was quenched by exposure to air. The resulting mixture was concentrated in vacuum and the residue was purified by chromatography $(\mathrm{PE} / \mathrm{EA}=50: 1-10: 1)$ on silica gel. 


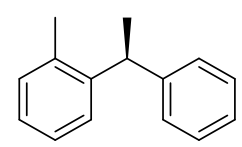

(S)-1-methyl-2-(1-phenylethyl)benzene $(\mathbf{8 a}) .{ }^{1}$ This compound was afforded as colorless oil (48 mg, 98\% yield) after flash column chromatography eluting with

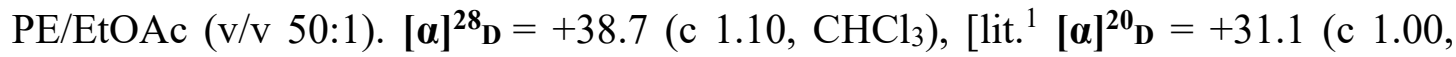
$\mathrm{CHCl}_{3}$ ), 80\% ee]; 93\% ee, determined by HPLC, HPLC conditions: Chiralcel OJ-H, nhexane $/ i-\mathrm{PrOH}=90 / 10,1.0 \mathrm{~mL} / \mathrm{min}, \mathrm{n}=214 \mathrm{~nm}, \mathrm{t}_{\mathrm{r}} 6.8$ (minor), 7.8 (major); ${ }^{1} \mathrm{H}$ NMR $\left(400 \mathrm{MHz}, \mathrm{CDCl}_{3}\right) \delta 7.28-7.10(\mathrm{~m}, 9 \mathrm{H}), 4.31(\mathrm{q}, J=7.1 \mathrm{~Hz}, 1 \mathrm{H}), 2.22(\mathrm{~s}, 3 \mathrm{H}), 1.60$ $(\mathrm{d}, J=7.2 \mathrm{~Hz}, 3 \mathrm{H}) .{ }^{13} \mathrm{C} \mathrm{NMR}\left(101 \mathrm{MHz}, \mathrm{CDCl}_{3}\right) \delta 146.4,144.0,136.2,130.5,128.4$, $127.8,126.8,126.2,126.2,125.9,41.1,22.2,19.9$. These spectroscopic data correspond to reported data. ${ }^{1}$

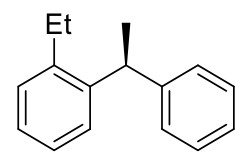

(S)-1-ethyl-2-(1-phenylethyl)benzene $(\mathbf{8 b}) .^{1}$ This compound was afforded as colorless oil (42.4 mg, 80\% yield) after flash column chromatography eluting with PE/EtOAc (v/v 50:1). $[\boldsymbol{\alpha}]^{25} \mathrm{D}=-4.1\left(\mathrm{c} 1.30, \mathrm{CHCl}_{3}\right),\left[\right.$ lit. $^{1}[\boldsymbol{\alpha}]^{\mathbf{2 0}} \mathrm{D}=-2.7\left(\mathrm{c} 1.40, \mathrm{CHCl}_{3}\right)$, 84\% ee]; 92\% ee, determined by HPLC, HPLC conditions: Chiralcel OJ-H, n-hexane/ $i$ $\mathrm{PrOH}=90 / 10,1.0 \mathrm{~mL} / \mathrm{min}, \mathrm{n}=214 \mathrm{~nm}, \mathrm{t}_{\mathrm{r}} 4.5$ (minor), 5.7 (major); ${ }^{1} \mathrm{H} \mathrm{NMR}(400 \mathrm{MHz}$, $\left.\mathrm{CDCl}_{3}\right) \delta 7.27-7.21(\mathrm{~m}, 3 \mathrm{H}), 7.19-7.11(\mathrm{~m}, 6 \mathrm{H}), 4.40(\mathrm{q}, J=7.2 \mathrm{~Hz}, 1 \mathrm{H}), 2.75-$ $2.50(\mathrm{~m}, 2 \mathrm{H}), 1.61(\mathrm{~d}, J=7.2 \mathrm{~Hz}, 3 \mathrm{H}), 1.14(\mathrm{t}, J=7.6 \mathrm{~Hz}, 3 \mathrm{H}) .{ }^{13} \mathrm{C} \mathrm{NMR}(101 \mathrm{MHz}$, $\left.\mathrm{CDCl}_{3}\right) \delta 146.7,143.6,141.9,128.7,128.4,127.7,127.3,126.4,126.1,125.9,40.1$, 25.7, 22.6, 15.5. These spectroscopic data correspond to reported data. ${ }^{1}$

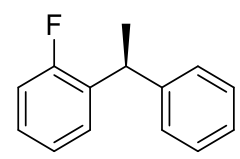

(S)-1-fluoro-2-(1-phenylethyl)benzene (8c). ${ }^{1}$ This compound was afforded as Colorless oil (48.5 mg, 97\% yield) after flash column chromatography eluting with

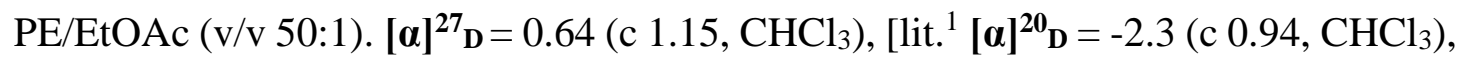
59\% ee]; 71\% ee, determined by HPLC, HPLC conditions: Chiralcel OJ-H, n-hexane/i$\mathrm{PrOH}=99.9 / 0.1,1.0 \mathrm{~mL} / \mathrm{min}, \mathrm{n}=214 \mathrm{~nm}, \mathrm{t}_{\mathrm{r}} 25.6$ (major), 28.9 (minor); ${ }^{1} \mathrm{H}$ NMR (400 
$\left.\mathrm{MHz}, \mathrm{CDCl}_{3}\right) \delta 7.35-7.06(\mathrm{~m}, 9 \mathrm{H}), 4.65(\mathrm{q}, J=7.2 \mathrm{~Hz}, 1 \mathrm{H}), 1.59(\mathrm{t}, J=7.9 \mathrm{~Hz}, 3 \mathrm{H})$. ${ }^{13} \mathrm{C}$ NMR (101 MHz, CDCl3) $\delta 145.1,143.8,134.0,129.7,128.7,128.5,127.9,127.5$, 127.0, 126.3, 41.1, 21.3. ${ }^{19} \mathrm{~F}$ NMR (377 MHz, CDC13) $\delta$-117.9. These spectroscopic data correspond to reported data. ${ }^{1}$

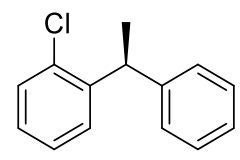

(S)-1-chloro-2-(1-phenylethyl)benzene (8d). ${ }^{1}$ This compound was afforded as colorless oil (53.4 mg, 99\% yield) after flash column chromatography eluting with PE/EtOAc (v/v 50:1). $[\boldsymbol{\alpha}]^{30} \mathbf{D}=-29.5\left(\mathrm{c} 1.10, \mathrm{CHCl}_{3}\right),\left[\right.$ lit. ${ }^{1}[\boldsymbol{\alpha}]^{\mathbf{2 0}} \mathbf{D}=-30.0\left(\mathrm{c} 1.39, \mathrm{CHCl}_{3}\right)$, 90\% ee]; 92\% ee, determined by HPLC, HPLC conditions: Chiralcel OJ-H, n-hexane/ $i$ $\mathrm{PrOH}=95 / 5,1.0 \mathrm{~mL} / \mathrm{min}, \mathrm{n}=214 \mathrm{~nm}, \mathrm{t}_{\mathrm{r}} 8.2$ (minor), 12.8 (major); ${ }^{1} \mathrm{H}$ NMR $(400 \mathrm{MHz}$, $\left.\mathrm{CDCl}_{3}\right) \delta 7.36-7.09(\mathrm{~m}, 9 \mathrm{H}), 4.65(\mathrm{q}, J=7.2 \mathrm{~Hz}, 1 \mathrm{H}), 1.61(\mathrm{~d}, J=7.2 \mathrm{~Hz}, 3 \mathrm{H}) .{ }^{13} \mathrm{C}$ NMR (101 MHz, $\left.\mathrm{CDCl}_{3}\right) \delta 145.1,143.8,134.0,129.7,128.7,128.5,127.9,127.5,127.0$, 126.3, 41.1, 21.3. These spectroscopic data correspond to reported data. ${ }^{1}$

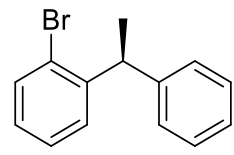

(S)-1-bromo-2-(1-phenylethyl)benzene (8e). ${ }^{17}$ This compound was afforded as Colorless oil (73.7 mg, 98\% yield) after flash column chromatography eluting with

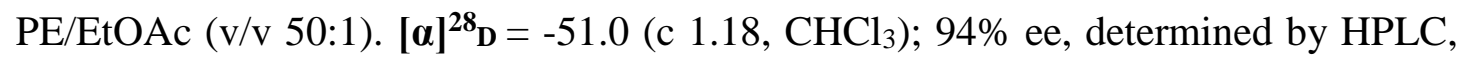
HPLC conditions: Chiralcel OJ-H, n-hexane $/ i-\mathrm{PrOH}=95 / 5,1.0 \mathrm{~mL} / \mathrm{min}, \mathrm{n}=214 \mathrm{~nm}$, t 7.9 (minor), 16.4 (major); ${ }^{1} \mathrm{H}$ NMR (400 MHz, $\left.\mathrm{CDCl}_{3}\right) \delta 7.54(\mathrm{t}, J=8.0 \mathrm{~Hz}, 1 \mathrm{H})$, $7.33-7.16(\mathrm{~m}, 7 \mathrm{H}), 7.04(\mathrm{dt}, J=8.9,3.4 \mathrm{~Hz}, 1 \mathrm{H}), 4.63(\mathrm{dd}, J=14.3,7.1 \mathrm{~Hz}, 1 \mathrm{H}), 1.62$ $(\mathrm{dd}, J=11.2,4.4 \mathrm{~Hz}, 3 \mathrm{H}) .{ }^{13} \mathrm{C} \mathrm{NMR}\left(101 \mathrm{MHz}, \mathrm{CDCl}_{3}\right) \delta 145.5,145.0,133.1,129.0$, $128.5,128.0,127.8,127.7,126.3,124.9,43.7,21.5$. These spectroscopic data correspond to reported data. ${ }^{17}$

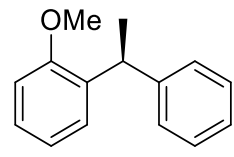

(S)-1-methoxy-2-(1-phenylethyl)benzene (8f). ${ }^{1}$ This compound was afforded as colorless oil (51.4 mg, 97\% yield) after flash column chromatography eluting with PE/EtOAc (v/v 10:1). $[\alpha]^{24} \mathrm{D}=-21.6\left(\mathrm{c} 1.10, \mathrm{CHCl}_{3}\right),\left[\right.$ lit. ${ }^{1}[\boldsymbol{\alpha}]^{\mathbf{2 0}} \mathrm{D}=-24.5\left(\mathrm{c} \mathrm{0.69}, \mathrm{CHCl}_{3}\right)$, 
58\% ee]; 58\% ee, determined by HPLC, HPLC conditions: Chiralcel OJ-H, n-hexane/ $i$ $\mathrm{PrOH}=98.5 / 1.5,1.0 \mathrm{~mL} / \mathrm{min}, \mathrm{n}=214 \mathrm{~nm}, \mathrm{t}_{\mathrm{r}} 11.0$ (minor), 12.4 (major); ${ }^{1} \mathrm{H}$ NMR (400 $\left.\mathrm{MHz}, \mathrm{CDCl}_{3}\right) \delta 7.27-7.23(\mathrm{~m}, 3 \mathrm{H}), 7.20-7.11(\mathrm{~m}, 4 \mathrm{H}), 6.89(\mathrm{td}, J=7.5,1.0 \mathrm{~Hz}, 1 \mathrm{H})$, $6.82(\mathrm{~d}, J=8.1 \mathrm{~Hz}, 1 \mathrm{H}), 4.57$ (q, $J=7.3 \mathrm{~Hz}, 1 \mathrm{H}), 3.74(\mathrm{~s}, 3 \mathrm{H}), 1.57$ (d, $J=7.3 \mathrm{~Hz}, 3 \mathrm{H})$. ${ }^{13} \mathrm{C}$ NMR $\left(101 \mathrm{MHz}, \mathrm{CDCl}_{3}\right) \delta 156.9,146.5,135.0,128.2,127.9,127.8,127.2,125.8$, 120.6, 110.7, 55.5, 37.5, 21.0. These spectroscopic data correspond to reported data. ${ }^{1}$

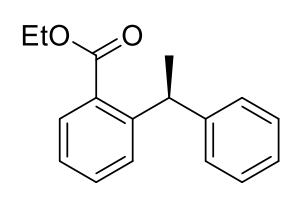

Ethyl (S)-2-(1-phenylethyl)benzoate (8g). This compound was afforded as colorless oil (40.0 mg, 63\% yield) after flash column chromatography eluting with PE/EtOAc (v/v 20:1). $[\alpha]^{28} \mathbf{D}=-35.2\left(\mathrm{c} 1.10, \mathrm{CHCl}_{3}\right) ; 40 \%$ ee, determined by SFC, SFC conditions: Chiralcel OJ-3, $\mathrm{CO}_{2} / \mathrm{MeOH}=90 / 10,1.0 \mathrm{~mL} / \mathrm{min}, \mathrm{n}=214 \mathrm{~nm}, \mathrm{t}_{\mathrm{r}} 1.6$ (minor), 2.2 (major); ${ }^{1} \mathrm{H}$ NMR (400 MHz, $\left.\mathrm{CDCl}_{3}\right) \delta 7.74(\mathrm{dd}, J=7.7,1.2 \mathrm{~Hz}, 1 \mathrm{H}), 7.42-7.37$ (m, 1H), $7.29-7.20(\mathrm{~m}, 6 \mathrm{H}), 7.19-7.14(\mathrm{~m}, 1 \mathrm{H}), 5.10$ (q, $J=7.1 \mathrm{~Hz}, 1 \mathrm{H}), 4.30$ (q, $J=7.1$ $\mathrm{Hz}, 2 \mathrm{H}), 1.63(\mathrm{~d}, J=7.2 \mathrm{~Hz}, 3 \mathrm{H}), 1.32(\mathrm{t}, J=7.1 \mathrm{~Hz}, 3 \mathrm{H}) .{ }^{13} \mathrm{C} \mathrm{NMR}\left(101 \mathrm{MHz}, \mathrm{CDCl}_{3}\right)$ $\delta 168.5,147.2,146.2,131.7,130.8,129.9,128.6,128.3,128.0,126.0,125.9,61.1,39.9$, 22.1, 14.3; HRMS (EI) m/z: [M] ${ }^{+}$Calcd for $\mathrm{C}_{17} \mathrm{H}_{18} \mathrm{O}_{2}$ 254.1295; Found 254.1301.

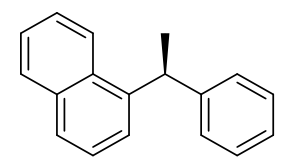

(S)-1-(1-phenylethyl)naphthalene (8h). ${ }^{1}$ This compound was afforded as colorless oil (56.8 mg, 98\% yield) after flash column chromatography eluting with PE/EtOAc (v/v 50:1). $[\boldsymbol{\alpha}]^{27} \mathbf{D}=-15.7\left(\mathrm{c} \mathrm{0.80}, \mathrm{CHCl}_{3}\right),\left[\right.$ lit. $^{1}[\boldsymbol{\alpha}]^{20} \mathrm{D}=-29.5$ (c 0.59, $\left.\mathrm{CHCl} 3\right), 77 \%$ ee]; $97 \%$ ee, determined by HPLC, HPLC conditions: Chiralcel OJ-H, n-hexane/i-PrOH = 96/4, $1.0 \mathrm{~mL} / \mathrm{min}, \mathrm{n}=214 \mathrm{~nm}, \mathrm{t}_{\mathrm{r}} 10.8$ (minor), 11.8 (major); ${ }^{1} \mathrm{H} \mathrm{NMR}\left(400 \mathrm{MHz}, \mathrm{CDCl}_{3}\right.$ ) $\delta$ $8.07-7.99(\mathrm{~m}, 1 \mathrm{H}), 7.86-7.79(\mathrm{~m}, 1 \mathrm{H}), 7.73(\mathrm{~d}, J=7.5 \mathrm{~Hz}, 1 \mathrm{H}), 7.49-7.36(\mathrm{~m}, 4 \mathrm{H})$, $7.27-7.19(\mathrm{~m}, 4 \mathrm{H}), 7.18-7.11(\mathrm{~m}, 1 \mathrm{H}), 4.91(\mathrm{q}, J=7.1 \mathrm{~Hz}, 1 \mathrm{H}), 1.75(\mathrm{~d}, J=7.1 \mathrm{~Hz}$, $3 \mathrm{H}) .{ }^{13} \mathrm{C} \mathrm{NMR}\left(101 \mathrm{MHz}, \mathrm{CDCl}_{3}\right) \delta 146.8,141.7,134.1,131.8,128.9,128.6,127.8$, $127.1,126.1,126.0,125.6,125.5,124.5,124.1,40.7,22.7$. These spectroscopic data correspond to reported data. ${ }^{1}$ 


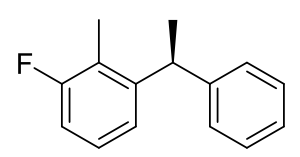

(S)-1-fluoro-2-methyl-3-(1-phenylethyl)benzene (8i). This compound was afforded as colorless oil (52.2 mg, 97\% yield) after flash column chromatography eluting with PE/EtOAc (v/v 50:1). [ $[\boldsymbol{\alpha}]^{27} \mathbf{D}=+30.0\left(\mathrm{c} 1.32, \mathrm{CHCl}_{3}\right) ; 94 \%$ ee, determined by HPLC, HPLC conditions: Chiralcel OJ-H, n-hexane $/ i-\mathrm{PrOH}=99 / 1,1.0 \mathrm{~mL} / \mathrm{min}, \mathrm{n}=214 \mathrm{~nm}$, $\mathrm{t}_{\mathrm{r}} 5.1$ (minor), 8.3 (major); ${ }^{1} \mathrm{H}$ NMR (400 MHz, $\left.\mathrm{CDCl}_{3}\right) \delta 7.31-7.22(\mathrm{~m}, 2 \mathrm{H}), 7.14$ (s, 4H), $7.06(\mathrm{~d}, J=2.9 \mathrm{~Hz}, 1 \mathrm{H}), 6.96-6.85(\mathrm{~m}, 1 \mathrm{H}), 4.31(\mathrm{dd}, J=6.8,3.1 \mathrm{~Hz}, 1 \mathrm{H}), 2.13$ (s, 3H), $1.59(\mathrm{dd}, J=6.8,3.6 \mathrm{~Hz}, 3 \mathrm{H}) .{ }^{13} \mathrm{C} \mathrm{NMR}\left(101 \mathrm{MHz}, \mathrm{CDCl}_{3}\right) \delta 161.5(\mathrm{~d}, J=$ 243.3 Hz), 146.6, 145.8, 128.5, 127.7, 126.6 (d, $J=9.1 \mathrm{~Hz}), 126.2,123.3$ (d, $J=15.7$ $\mathrm{Hz}), 122.4,113.0(\mathrm{~d}, J=23.5 \mathrm{~Hz}), 41.2,22.3,10.5$ (d, $J=6.3 \mathrm{~Hz}) .{ }^{19} \mathrm{~F}$ NMR $(377 \mathrm{MHz}$, $\left.\mathrm{CDCl}_{3}\right) \delta$-116.2; HRMS (EI) m/z: [M] ${ }^{+}$Calcd for $\mathrm{C}_{15} \mathrm{H}_{15} \mathrm{~F}$ 214.1151; Found 214.1152.<smiles>Cc1cc(F)ccc1C(C)c1ccccc1</smiles>

(S)-4-fluoro-2-methyl-1-(1-phenylethyl)benzene (8j). This compound was afforded as colorless oil (51.9 mg, 98\% yield) after flash column chromatography eluting with PE/EtOAc (v/v 50:1). [ $\boldsymbol{\alpha}]^{28} \mathbf{D}=+20.8\left(\mathrm{c} 1.20, \mathrm{CHCl}_{3}\right) ; 93 \%$ ee, determined by HPLC, HPLC conditions: Chiralcel OJ-H, n-hexane/i-PrOH = 99/1, $1.0 \mathrm{~mL} / \mathrm{min}, \mathrm{n}=214 \mathrm{~nm}$, t 5.1 (minor), 8.2 (major); ${ }^{1} \mathrm{H}$ NMR (400 MHz, $\left.\mathrm{CDCl}_{3}\right) \delta 7.27-7.21$ (m, 2H), $7.18-$ 7.09 (m, 4H), 7.04 (d, $J=7.4 \mathrm{~Hz}, 1 \mathrm{H}), 6.88$ (dd, $J=13.4,5.0 \mathrm{~Hz}, 1 \mathrm{H}), 4.30$ (q, $J=7.2$ $\mathrm{Hz}, 1 \mathrm{H}), 2.12(\mathrm{~d}, J=2.2 \mathrm{~Hz}, 3 \mathrm{H}), 1.59(\mathrm{~d}, J=7.2 \mathrm{~Hz}, 3 \mathrm{H}) .{ }^{13} \mathrm{C} \mathrm{NMR}\left(101 \mathrm{MHz}, \mathrm{CDCl}_{3}\right)$ $\delta 161.5(\mathrm{~d}, J=242.9 \mathrm{~Hz}), 146.6(\mathrm{~d}, J=3.6 \mathrm{~Hz}), 145.8,128.5,127.7,126.6(\mathrm{~d}, J=9.1$ Hz), 126.2, 123.3 (d, $J=15.4 \mathrm{~Hz}), 122.4$ (d, $J=3.0 \mathrm{~Hz}), 113.0(\mathrm{~d}, J=23.5 \mathrm{~Hz}), 41.2$ $(\mathrm{d}, J=2.6 \mathrm{~Hz}), 22.3,10.5(\mathrm{~d}, J=6.4 \mathrm{~Hz}) .{ }^{19} \mathrm{~F} \mathrm{NMR}\left(377 \mathrm{MHz}, \mathrm{CDCl}_{3}\right) \delta-116.2$. HRMS (EI) m/z: [M] $]^{+}$Calcd for $\mathrm{C}_{15} \mathrm{H}_{15} \mathrm{~F} 214.1151$; Found 214.1152.

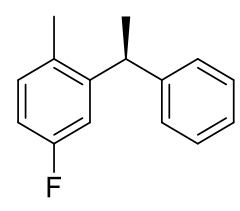

(S)-4-fluoro-1-methyl-2-(1-phenylethyl)benzene (8k). This compound was afforded as colorless oil (52.4 mg, 98\% yield) after flash column chromatography eluting with 
PE/EtOAc (v/v 50:1). $[\alpha]^{29} \mathrm{D}=+25.4\left(\mathrm{c} 1.38, \mathrm{CHCl}_{3}\right)$; 95\% ee, determined by HPLC, HPLC conditions: Chiralcel OJ-H, n-hexane $/ i-\mathrm{PrOH}=99 / 1,1.0 \mathrm{~mL} / \mathrm{min}, \mathrm{n}=214 \mathrm{~nm}$, tr 4.9 (minor), 5.1 (major); ${ }^{1} \mathrm{H}$ NMR $\left(400 \mathrm{MHz}, \mathrm{CDCl}_{3}\right) \delta 7.30-7.22$ (m, 2H), 7.15 (dd, $J=18.1,7.3 \mathrm{~Hz}, 3 \mathrm{H}), 7.06(\mathrm{dd}, J=8.1,6.2 \mathrm{~Hz}, 1 \mathrm{H}), 6.97(\mathrm{dd}, J=10.6,2.7 \mathrm{~Hz}, 1 \mathrm{H})$, $6.81(\mathrm{td}, J=8.3,2.7 \mathrm{~Hz}, 1 \mathrm{H}), 4.26(\mathrm{q}, J=7.1 \mathrm{~Hz}, 1 \mathrm{H}), 2.17(\mathrm{~s}, 3 \mathrm{H}), 1.58(\mathrm{~d}, J=7.2 \mathrm{~Hz}$, $3 \mathrm{H}) .{ }^{13} \mathrm{C} \mathrm{NMR}\left(101 \mathrm{MHz}, \mathrm{CDCl}_{3}\right) \delta 161.7(\mathrm{~d}, J=242.6 \mathrm{~Hz}), 146.2(\mathrm{~d}, J=6.5 \mathrm{~Hz})$, 145.6, $131.7(\mathrm{~d}, J=3.1 \mathrm{~Hz}), 131.6(\mathrm{~d}, J=7.8 \mathrm{~Hz}), 128.6,127.7,126.2,113.8(\mathrm{~d}, J=$ 21.7 Hz), $112.7(\mathrm{~d}, J=20.7 \mathrm{~Hz}), 41.3(\mathrm{~d}, J=1.5 \mathrm{~Hz}), 22.1,19.1 .{ }^{19} \mathrm{~F}$ NMR $(377 \mathrm{MHz}$, $\left.\mathrm{CDCl}_{3}\right) \delta$-117.3. HRMS (EI) m/z: [M] ${ }^{+}$Calcd for $\mathrm{C}_{15} \mathrm{H}_{15} \mathrm{~F}$ 214.1151; Found 214.1152.

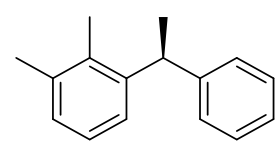

(S)-1,2-dimethyl-3-(1-phenylethyl)benzene (81). ${ }^{18}$ This compound was afforded as colorless oil (40.9 mg, 78\% yield) after flash column chromatography eluting with PE/EtOAc (v/v 50:1). $[\alpha]^{26} \mathrm{D}=+7.3\left(\mathrm{c} 0.86, \mathrm{CHCl}_{3}\right) ; 95 \%$ ee, determined by HPLC, HPLC conditions: Chiralcel OJ-H, n-hexane $/ i-\mathrm{PrOH}=99.5 / 0.5,1.0 \mathrm{~mL} / \mathrm{min}, \mathrm{n}=214$ nm, $\mathrm{t}_{\mathrm{r}} 7.9$ (minor), 8.3 (major); ${ }^{1} \mathrm{H}$ NMR (400 MHz, $\left.\mathrm{CDCl}_{3}\right) \delta 7.25-7.20$ (m, 2H), 7.16 $-7.05(\mathrm{~m}, 5 \mathrm{H}), 7.02(\mathrm{~d}, J=7.1 \mathrm{~Hz}, 1 \mathrm{H}), 4.36(\mathrm{q}, J=7.2 \mathrm{~Hz}, 1 \mathrm{H}), 2.25(\mathrm{~s}, 3 \mathrm{H}), 2.11(\mathrm{~s}$, $3 \mathrm{H}), 1.58(\mathrm{~d}, J=7.2 \mathrm{~Hz}, 3 \mathrm{H}) .{ }^{13} \mathrm{C} \mathrm{NMR}\left(101 \mathrm{MHz}, \mathrm{CDCl}_{3}\right) \delta 146.8,143.8,136.9,134.8$, $128.4,128.1,127.8,125.7,125.5,124.8,41.4,22.5,21.1,15.2$. These spectroscopic data correspond to reported data. ${ }^{18}$

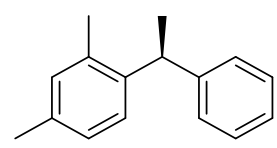

(S)-2,4-dimethyl-1-(1-phenylethyl)benzene (8m). ${ }^{19}$ This compound was afforded as colorless oil (50.4 mg, 96\% yield) after flash column chromatography eluting with PE/EtOAc (v/v 50:1). $[\boldsymbol{\alpha}]^{27} \mathrm{D}=+54.4\left(\mathrm{c} 1.09, \mathrm{CHCl}_{3}\right) ; 91 \%$ ee, determined by HPLC, HPLC conditions: Chiralcel OJ-H, n-hexane $/ i-\mathrm{PrOH}=99 / 1,1.0 \mathrm{~mL} / \mathrm{min}, \mathrm{n}=214 \mathrm{~nm}$, $\mathrm{t}_{\mathrm{r}} 6.5$ (minor), 8.4 (major); ${ }^{1} \mathrm{H} \mathrm{NMR}\left(400 \mathrm{MHz}, \mathrm{CDCl}_{3}\right) \delta 7.26-7.21$ (m, 2H), 7.13 (dd, $J=10.5,4.1 \mathrm{~Hz}, 4 \mathrm{H}), 7.00(\mathrm{~d}, J=7.8 \mathrm{~Hz}, 1 \mathrm{H}), 6.95(\mathrm{~s}, 1 \mathrm{H}), 4.27(\mathrm{q}, J=7.2 \mathrm{~Hz}, 1 \mathrm{H})$, $2.28(\mathrm{~s}, 3 \mathrm{H}), 2.19(\mathrm{~s}, 3 \mathrm{H}), 1.58(\mathrm{~d}, J=7.2 \mathrm{~Hz}, 3 \mathrm{H}) .{ }^{13} \mathrm{C} \mathrm{NMR}\left(101 \mathrm{MHz}, \mathrm{CDCl}_{3}\right) \delta$ 
146.6, 141.1, 136.0, 135.6, 131.4, 128.4, 127.8, 126.8, 126.7, 125.9, 40.8, 22.3, 21.0, 19.8. These spectroscopic data correspond to reported data. ${ }^{19}$

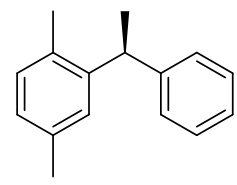

(S)-1,4-dimethyl-2-(1-phenylethyl)benzene (8n). ${ }^{20}$ This compound was afforded as colorless oil (50.4 mg, 96\% yield) after flash column chromatography eluting with PE/EtOAc (v/v 50:1). [a] $]^{25} \mathrm{D}=+26.7\left(\mathrm{c} 1.03, \mathrm{CHCl}_{3}\right) ; 94 \%$ ee, determined by HPLC, HPLC conditions: Chiralcel OJ-H, n-hexane $/ i-\mathrm{PrOH}=99.5 / 0.5,1.0 \mathrm{~mL} / \mathrm{min}, \mathrm{n}=214$ nm, tr 12.0 (major), 14.4 (minor); ${ }^{1} \mathrm{H}$ NMR (400 MHz, $\left.\mathrm{CDCl}_{3}\right) \delta 7.32-6.80(\mathrm{~m}, 8 \mathrm{H})$, $4.27(\mathrm{q}, J=7.0 \mathrm{~Hz}, 1 \mathrm{H}), 2.30(\mathrm{~s}, 3 \mathrm{H}), 2.17(\mathrm{~s}, 3 \mathrm{H}), 1.58(\mathrm{~d}, J=7.1 \mathrm{~Hz}, 3 \mathrm{H}) .{ }^{13} \mathrm{C} \mathrm{NMR}$ $\left(101 \mathrm{MHz}, \mathrm{CDCl}_{3}\right) \delta 146.4,143.8,135.4,133.0,130.4,128.4,127.8,127.6,126.9$, $125.9,41.0,22.2,21.4,19.4$. These spectroscopic data correspond to reported data. ${ }^{20}$<smiles>Cc1cc(Cl)ccc1C(C)c1ccccc1</smiles>

(S)-4-chloro-2-methyl-1-(1-phenylethyl)benzene (8o). ${ }^{1}$ This compound was afforded as colorless oil (55.8 mg, 97\% yield) after flash column chromatography eluting with

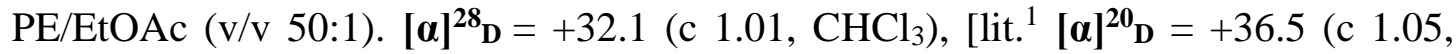
$\mathrm{CHCl}_{3}$ ), $89 \%$ ee]; 93\% ee, determined by HPLC, HPLC conditions: Chiralcel OJ-H, nhexane $/ i-\mathrm{PrOH}=90 / 10,1.0 \mathrm{~mL} / \mathrm{min}, \mathrm{n}=214 \mathrm{~nm}, \mathrm{t}_{\mathrm{r}} 6.1$ (minor), 6.4 (major); ${ }^{1} \mathrm{H}$ NMR $\left(400 \mathrm{MHz}, \mathrm{CDCl}_{3}\right) \delta 7.29-7.08(\mathrm{~m}, 8 \mathrm{H}), 4.25(\mathrm{q}, J=7.0 \mathrm{~Hz}, 1 \mathrm{H}), 2.19(\mathrm{~s}, 3 \mathrm{H}), 1.57$ $(\mathrm{d}, J=7.1 \mathrm{~Hz}, 3 \mathrm{H}) .{ }^{13} \mathrm{C} \mathrm{NMR}\left(101 \mathrm{MHz}, \mathrm{CDCl}_{3}\right) \delta 145.8,142.6,138.2,131.7,130.3$, 128.6, 128.2, 127.7, 126.2, 126.1, 40.7, 22.2, 19.7. These spectroscopic data correspond to reported data. ${ }^{1}$<smiles>Cc1cc(C)c(C(C)c2ccccc2)cc1C</smiles>

(S)-1,2,4-trimethyl-5-(1-phenylethyl)benzene (8p). This compound was afforded as colorless oil (53.8 mg, 96\% yield) after flash column chromatography eluting with PE/EtOAc (v/v 50:1). $[\boldsymbol{\alpha}]^{29} \mathbf{D}=+18.1\left(\mathrm{c} 1.23, \mathrm{CHCl}_{3}\right) ; 94 \%$ ee, determined by SFC, SFC 
conditions: Chiralcel OJ-3, $\mathrm{CO}_{2} / \mathrm{MeOH}=98 / 2,1.0 \mathrm{~mL} / \mathrm{min}, \mathrm{n}=214 \mathrm{~nm}, \mathrm{t}_{\mathrm{r}} 3.1$ (major), 3.5 (minor); ${ }^{1} \mathrm{H}$ NMR (400 MHz, $\left.\mathrm{CDCl}_{3}\right) \delta 7.28-7.09(\mathrm{~m}, 5 \mathrm{H}), 7.01$ (s, 1H), 6.90 (s, $1 \mathrm{H}), 4.25(\mathrm{q}, J=7.1 \mathrm{~Hz}, 1 \mathrm{H}), 2.26-2.12(\mathrm{~m}, 9 \mathrm{H}), 1.58(\mathrm{~d}, J=7.2 \mathrm{~Hz}, 3 \mathrm{H}) .{ }^{13} \mathrm{C} \mathrm{NMR}$ $\left(101 \mathrm{MHz}, \mathrm{CDCl}_{3}\right) \delta 146.7,141.4,134.1,133.9,133.3,132.0,128.4,128.2,127.8$, 125.8, 40.7, 22.3, 19.7, 19.3, 19.2; HRMS (EI) m/z: [M] $]^{+}$Calcd for $\mathrm{C}_{17} \mathrm{H}_{20}$ 224.1561; Found 224.1560.<smiles>COc1cccc(C(C)c2ccccc2C)c1</smiles>

(S)-1-(1-(3-methoxyphenyl)ethyl)-2-methylbenzene (8q). ${ }^{1}$ This compound was afforded as colorless oil (52.5 mg, 93\% yield) after flash column chromatography eluting with PE/EtOAc (v/v 10:1). $[\boldsymbol{\alpha}]^{27} \mathbf{D}=+28.9\left(\mathrm{c} 1.08, \mathrm{CHCl}_{3}\right),\left[\right.$ lit. $^{1}[\boldsymbol{\alpha}]^{\mathbf{2 0}} \mathbf{D}=+28.4$ (c 1.06, $\mathrm{CHCl}_{3}$ ), 91\% ee]; $93 \%$ ee, determined by HPLC, HPLC conditions: Chiralcel OJ-H, n-hexane $/ i-\mathrm{PrOH}=90 / 10,1.0 \mathrm{~mL} / \mathrm{min}, \mathrm{n}=214 \mathrm{~nm}, \mathrm{t}_{\mathrm{r}} 7.5$ (minor), 10.1 (major); ${ }^{1} \mathrm{H}$ NMR $\left(400 \mathrm{MHz}, \mathrm{CDCl}_{3}\right) \delta 7.26(\mathrm{~d}, J=7.6 \mathrm{~Hz}, 1 \mathrm{H}), 7.21-7.0(\mathrm{~m}, 4 \mathrm{H}), 6.77-6.66$ (m, 3H), 4.27 (q, $J=7.2 \mathrm{~Hz}, 1 \mathrm{H}), 3.72(\mathrm{~s}, 3 \mathrm{H}), 2.22$ (s, 3H), 1.59 (d, $J=7.2 \mathrm{~Hz}, 3 \mathrm{H})$. ${ }^{13} \mathrm{C}$ NMR (101 MHz, CDCl3) $\delta$ 159.7, 148.1, 143.8, 136.2, 130.5, 129.3, 126.7, 126.2, 126.2, 120.4, 114.0, 110.7, 55.2, 41.1, 22.2, 19.9. These spectroscopic data correspond to reported data. ${ }^{1}$

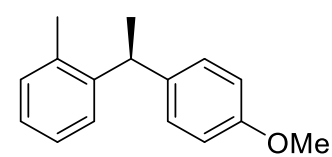

(S)-1-(1-(4-methoxyphenyl)ethyl)-2-methylbenzene (8r). ${ }^{21}$ This compound was afforded as colorless oil $(54.8 \mathrm{mg}, 97 \%$ yield) after flash column chromatography

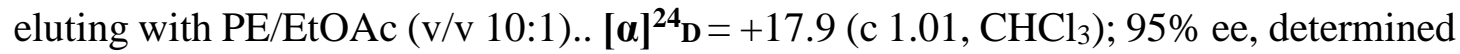
by HPLC, HPLC conditions: Chiralcel OJ-H, n-hexane $/ i$-PrOH $=90 / 10,1.0 \mathrm{~mL} / \mathrm{min}, \mathrm{n}$ $=214 \mathrm{~nm}, \mathrm{t}_{\mathrm{r}} 13.4$ (minor), 15.4 (major); ${ }^{1} \mathrm{H} \mathrm{NMR}\left(400 \mathrm{MHz}, \mathrm{CDCl}_{3}\right) \delta 7.19-7.08(\mathrm{~m}$, 2H), $7.03(\mathrm{~d}, J=4.1 \mathrm{~Hz}, 2 \mathrm{H}), 6.98(\mathrm{~d}, J=8.6 \mathrm{~Hz}, 2 \mathrm{H}), 6.71(\mathrm{~d}, J=8.6 \mathrm{~Hz}, 2 \mathrm{H}), 4.18$ $(\mathrm{q}, J=7.1 \mathrm{~Hz}, 1 \mathrm{H}), 3.67(\mathrm{~s}, 3 \mathrm{H}), 2.15(\mathrm{~s}, 3 \mathrm{H}), 1.49(\mathrm{~d}, J=7.2 \mathrm{~Hz}, 3 \mathrm{H}) .{ }^{13} \mathrm{C}$ NMR $(101$ $\left.\mathrm{MHz}, \mathrm{CDCl}_{3}\right) \delta 157.8,144.4,138.5,136.1,130.5,128.7,126.7,126.1,126.1,113.8$, $55.3,40.2,22.3,19.8$. These spectroscopic data correspond to reported data. ${ }^{21}$ 


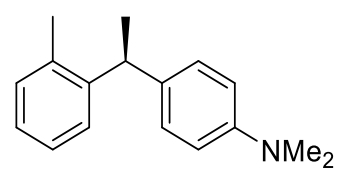

(S)-N,N-dimethyl-4-(1-(o-tolyl)ethyl)aniline (8s). This compound was afforded as colorless oil (58.0 mg, 97\% yield) after flash column chromatography eluting with

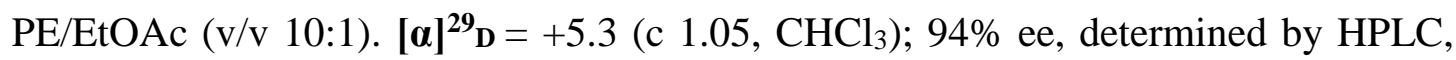
HPLC conditions: Chiralcel OJ-H, n-hexane/i-PrOH = 92/8, $1.0 \mathrm{~mL} / \mathrm{min}, \mathrm{n}=214 \mathrm{~nm}$, t 19.4 (minor), 25.9 (maJor); ${ }^{1} \mathrm{H}$ NMR (400 MHz, $\left.\mathrm{CDCl}_{3}\right) \delta 7.28-7.24$ (m, 1H), 7.20 $-7.08(\mathrm{~m}, 3 \mathrm{H}), 7.02(\mathrm{~d}, J=8.6 \mathrm{~Hz}, 2 \mathrm{H}), 6.66(\mathrm{~d}, J=8.6 \mathrm{~Hz}, 2 \mathrm{H}), 4.23(\mathrm{q}, J=7.1 \mathrm{~Hz}$, 1H), $2.89(\mathrm{~s}, 6 \mathrm{H}), 2.25(\mathrm{~s}, 3 \mathrm{H}), 1.57(\mathrm{~d}, J=7.2 \mathrm{~Hz}, 3 \mathrm{H}) .{ }^{13} \mathrm{C} \mathrm{NMR}\left(101 \mathrm{MHz}, \mathrm{CDCl}_{3}\right)$ $\delta$ 197.0, 153.7, 140.3, 135.7, 132.6, 130.6, 129.2, 127.6, 125.2, 125.2, 110.7, 40.1, 19.7. HRMS (ESI) m/z: [M+H] $]^{+}$Calcd for $\mathrm{C}_{21} \mathrm{H}_{20}$ 240.1677; Found 240.1673.

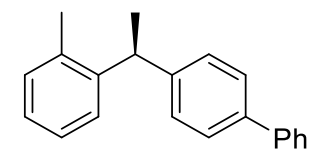

(S)-4-(1-(o-tolyl)ethyl)-1,1'-biphenyl (8t). This compound was afforded as colorless oil (63.9 mg, 94\% yield) after flash column chromatography eluting with PE/EtOAc $(\mathrm{v} / \mathrm{v} 50: 1) .[\alpha]^{29} \mathbf{D}=+54.1\left(\mathrm{c} 1.35, \mathrm{CHCl}_{3}\right) ; 92 \%$ ee, determined by HPLC, HPLC conditions: Chiralcel OJ-H, n-hexane $/ i-\mathrm{PrOH}=99 / 1,1.0 \mathrm{~mL} / \mathrm{min}, \mathrm{n}=214 \mathrm{~nm}, \mathrm{t}_{\mathrm{r}} 8.9$ (minor), 9.9 (major); ${ }^{1} \mathrm{H}$ NMR (400 MHz, $\left.\mathrm{CDCl}_{3}\right) \delta 7.57-7.53(\mathrm{~m}, 2 \mathrm{H}), 7.50-7.46$ (m, 2H), $7.41(\mathrm{dd}, J=10.3,4.8 \mathrm{~Hz}, 2 \mathrm{H}), 7.33-7.28(\mathrm{~m}, 2 \mathrm{H}), 7.24-7.18(\mathrm{~m}, 3 \mathrm{H}), 7.16$ $-7.12(\mathrm{~m}, 2 \mathrm{H}), 4.35(\mathrm{q}, J=7.1 \mathrm{~Hz}, 1 \mathrm{H}), 2.26(\mathrm{~s}, 3 \mathrm{H}), 1.64(\mathrm{~d}, J=7.2 \mathrm{~Hz}, 3 \mathrm{H}) .{ }^{13} \mathrm{C}$ NMR (101 MHz, $\left.\mathrm{CDCl}_{3}\right) \delta 145.5,144.0,141.1,138.8,136.2,130.6,128.8,128.2,127.2$, 127.1, 126.8, 126.3, 126.2, 40.8, 22.2, 20.0. HRMS (EI) m/z: [M] Calcd for $\mathrm{C}_{21} \mathrm{H}_{20}$ 272.1557; Found 272.1560.<smiles>Cc1ccccc1C(C)c1ccc(Cl)cc1</smiles>

(S)-1-(1-(4-chlorophenyl)ethyl)-2-methylbenzene $\quad(\mathbf{8 u}){ }^{22}$ This compound was afforded as colorless oil (56.3 mg, 98\% yield) after flash column chromatography

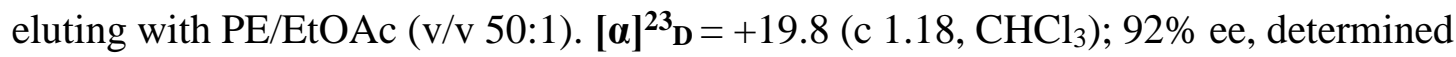
by HPLC, HPLC conditions: Chiralcel OJ-H, n-hexane $/ i$-PrOH $=90 / 10,1.0 \mathrm{~mL} / \mathrm{min}, \mathrm{n}$ 
$=214 \mathrm{~nm}, \mathrm{t}_{\mathrm{r}} 15.7$ (minor), 16.2 (major); ${ }^{1} \mathrm{H} \mathrm{NMR}\left(400 \mathrm{MHz}, \mathrm{CDCl}_{3}\right) \delta 7.25-7.04(\mathrm{~m}$, $8 \mathrm{H}), 4.27(\mathrm{q}, J=7.2 \mathrm{~Hz}, 1 \mathrm{H}), 2.20(\mathrm{~s}, 3 \mathrm{H}), 1.58(\mathrm{~d}, J=7.2 \mathrm{~Hz}, 3 \mathrm{H}) .{ }^{13} \mathrm{C}$ NMR $(101$ $\left.\mathrm{MHz}, \mathrm{CDCl}_{3}\right) \delta 144.9,143.5,136.2,131.7,130.7,129.2,128.6,126.7,126.5,126.3$, 40.6, 22.2, 19.8. These spectroscopic data correspond to reported data. ${ }^{22}$

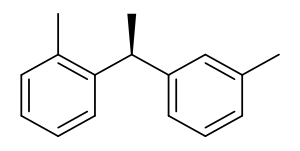

(S)-1-methyl-2-(1-(m-tolyl)ethyl)benzene (8v). ${ }^{1}$ This compound was afforded as colorless oil (52.0 mg, 99\% yield) after flash column chromatography eluting with PE/EtOAc (v/v 50:1). $[\boldsymbol{\alpha}]^{27} \mathbf{D}=+24.8\left(\mathrm{c} 1.45, \mathrm{CHCl}_{3}\right),\left[\mathrm{lit}^{1}{ }^{1}[\boldsymbol{\alpha}]^{\mathbf{2 0}} \mathbf{D}=+32\left(\mathrm{c} \mathrm{0.98}, \mathrm{CHCl}_{3}\right)\right.$, 86\% ee]; 93\% ee, determined by HPLC, HPLC conditions: Chiralcel OJ-H, n-hexane/ $i$ $\mathrm{PrOH}=99.5 / 0.5,1.0 \mathrm{~mL} / \mathrm{min}, \mathrm{n}=214 \mathrm{~nm}, \mathrm{t}_{\mathrm{r}} 7.3$ (major), 9.7 (minor); ${ }^{1} \mathrm{H} \mathrm{NMR}$ (400 $\left.\mathrm{MHz}, \mathrm{CDCl}_{3}\right) \delta 7.26(\mathrm{~d}, J=7.6 \mathrm{~Hz}, 1 \mathrm{H}), 7.22-7.10(\mathrm{~m}, 4 \mathrm{H}), 6.95(\mathrm{t}, J=7.1 \mathrm{~Hz}, 3 \mathrm{H})$, $4.27(\mathrm{q}, J=7.2 \mathrm{~Hz}, 1 \mathrm{H}), 2.26(\mathrm{~d}, J=19.3 \mathrm{~Hz}, 6 \mathrm{H}), 1.58(\mathrm{~d}, J=7.2 \mathrm{~Hz}, 3 \mathrm{H}) .{ }^{13} \mathrm{C} \mathrm{NMR}$ $\left(101 \mathrm{MHz}, \mathrm{CDCl}_{3}\right) \delta 146.3,144.2,137.9,136.2,130.5,128.6,128.3,126.8,126.7$, $126.1,124.9,41.1,22.3,21.6,19.9$. These spectroscopic data correspond to reported data. ${ }^{1}$

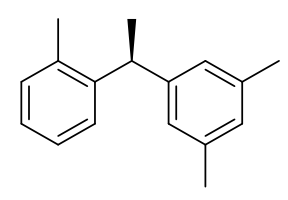

(S)-1,3-dimethyl-5-(1-(o-tolyl)ethyl)benzene (8w). This compound was afforded as colorless oil (53.8 mg, 96\% yield) after flash column chromatography eluting with

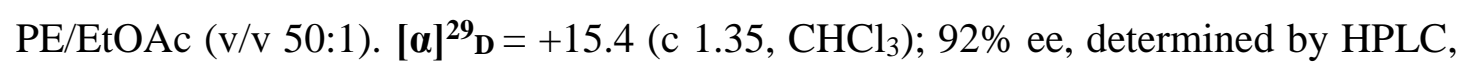
HPLC conditions: Chiralcel OJ-H, n-hexane $/ i-\mathrm{PrOH}=100 / 0,1.0 \mathrm{~mL} / \mathrm{min}, \mathrm{n}=214 \mathrm{~nm}$, $\mathrm{t}_{\mathrm{r}} 10.3$ (minor), 11.0 (major); ${ }^{1} \mathrm{H} \mathrm{NMR}\left(400 \mathrm{MHz}, \mathrm{CDCl}_{3}\right) \delta 7.26(\mathrm{~d}, J=7.5 \mathrm{~Hz}, 1 \mathrm{H})$, $7.18(\mathrm{dt}, J=7.9,3.9 \mathrm{~Hz}, 1 \mathrm{H}), 7.11(\mathrm{~d}, J=3.9 \mathrm{~Hz}, 2 \mathrm{H}), 6.78(\mathrm{~d}, J=10.7 \mathrm{~Hz}, 3 \mathrm{H}), 4.23$ $(\mathrm{q}, J=7.1 \mathrm{~Hz}, 1 \mathrm{H}), 2.24(\mathrm{~s}, 9 \mathrm{H}), 1.57(\mathrm{~d}, J=7.2 \mathrm{~Hz}, 3 \mathrm{H}) .{ }^{13} \mathrm{C} \mathrm{NMR}\left(101 \mathrm{MHz}, \mathrm{CDCl}_{3}\right)$ $\delta 146.2,144.3,137.8,136.3,130.4,127.7,126.8,126.1,126.1,125.7,41.0,22.4,21.5$, 20.0; HRMS (EI) m/z: [M] $]^{+}$Calcd for $\mathrm{C}_{17} \mathrm{H}_{20}$ 224.1558; Found 224.1560. 


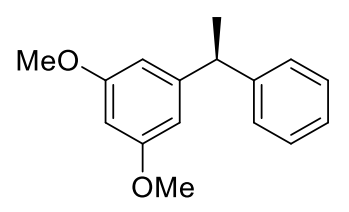

(S)-1,3-dimethoxy-5-(1-phenylethyl)benzene (8x). ${ }^{23}$ This compound was afforded as colorless oil (59.3 mg, 98\% yield) after flash column chromatography eluting with PE/EtOAc (v/v 10:1). $[\boldsymbol{\alpha}]^{\mathbf{2 6}} \mathbf{D}=+0.89\left(\mathrm{c} 1.30, \mathrm{CHCl}_{3}\right),\left[\mathrm{lit}^{23}[\mathbf{\alpha}]^{\mathbf{2 1}} \mathbf{D}=+0.3\left(\mathrm{c} 0.7, \mathrm{CHCl}_{3}\right)\right.$, 94\% ee]; $19 \%$ ee, determined by HPLC, HPLC conditions: Chiralcel OJ-H, n-hexane/i$\mathrm{PrOH}=90 / 10,0.7 \mathrm{~mL} / \mathrm{min}, \mathrm{n}=214 \mathrm{~nm}, \mathrm{t}_{\mathrm{r}} 20.5$ (minor), 22.9 (major); ${ }^{1} \mathrm{H}$ NMR (400 $\left.\mathrm{MHz}, \mathrm{CDCl}_{3}\right) \delta 7.29-7.21(\mathrm{~m}, 4 \mathrm{H}), 7.19-7.14(\mathrm{~m}, 1 \mathrm{H}), 6.39(\mathrm{~d}, J=2.2 \mathrm{~Hz}, 2 \mathrm{H}), 6.29$ $(\mathrm{t}, J=2.3 \mathrm{~Hz}, 1 \mathrm{H}), 4.07(\mathrm{q}, J=7.2 \mathrm{~Hz}, 1 \mathrm{H}), 3.74(\mathrm{~s}, 6 \mathrm{H}), 1.61(\mathrm{~d}, J=7.2 \mathrm{~Hz}, 3 \mathrm{H}) .{ }^{13} \mathrm{C}$ NMR (101 MHz, $\left.\mathrm{CDCl}_{3}\right) \delta 160.8,149.0,146.1,128.5,127.6,126.2,106.1,97.8,55.3$, 45.1, 21.7. These spectroscopic data correspond to reported data. ${ }^{23}$<smiles>CC(c1ccccc1)c1ccc(Cl)cc1Cl</smiles>

(S)-2,4-dichloro-1-(1-phenylethyl)benzene (8y). ${ }^{1}$ This compound was afforded as colorless oil (61.25 mg, 98\% yield) after flash column chromatography eluting with PE/EtOAc (v/v 50:1). $[\boldsymbol{\alpha}]^{30} \mathbf{D}=-22.6\left(\mathrm{c} 1.05, \mathrm{CHCl}_{3}\right),\left[\right.$ lit. ${ }^{1}[\boldsymbol{\alpha}]^{20} \mathbf{D}=-25.8\left(\mathrm{c} 0.95, \mathrm{CHCl}_{3}\right)$, 93\% ee]; $91 \%$ ee, determined by HPLC, HPLC conditions: Chiralcel OJ-H, n-hexane/ $i$ $\mathrm{PrOH}=95 / 5,1.0 \mathrm{~mL} / \mathrm{min}, \mathrm{n}=214 \mathrm{~nm}, \mathrm{t}_{\mathrm{r}} 4.9$ (minor), 5.8 (major); ${ }^{1} \mathrm{H} \mathrm{NMR}(400 \mathrm{MHz}$, $\left.\mathrm{CDCl}_{3}\right) \delta 7.36(\mathrm{~d}, J=2.1 \mathrm{~Hz}, 1 \mathrm{H}), 7.31-7.26(\mathrm{~m}, 2 \mathrm{H}), 7.22-7.10(\mathrm{~m}, 5 \mathrm{H}), 4.59(\mathrm{q}, J$ $=7.2 \mathrm{~Hz}, 1 \mathrm{H}), 1.59(\mathrm{~d}, J=7.2 \mathrm{~Hz}, 3 \mathrm{H}) .{ }^{13} \mathrm{C} \mathrm{NMR}\left(101 \mathrm{MHz}, \mathrm{CDCl}_{3}\right) \delta 144.5,142.5$, 134.6, 132.4, 129.6, 129.5, 128.6, 127.8, 127.3, 126.5, 40.7, 21.2. These spectroscopic data correspond to reported data. ${ }^{1}$<smiles>CC(c1ccccc1)c1cc(Cl)ccc1Cl</smiles>

(S)-1,4-dichloro-2-(1-phenylethyl)benzene (8z). ${ }^{1}$ This compound was afforded as colorless oil (61.9 mg, 99\% yield) after flash column chromatography eluting with

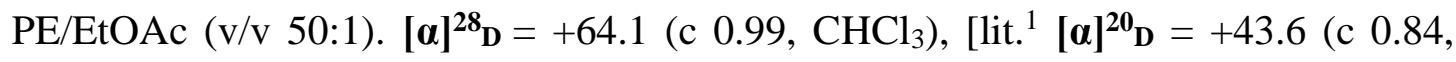
$\mathrm{CHCl}_{3}$ ), 86\% ee]; 95\% ee, determined by HPLC, HPLC conditions: Chiralcel OJ-H, n- 
hexane $/ i-\mathrm{PrOH}=95 / 5,1.0 \mathrm{~mL} / \mathrm{min}, \mathrm{n}=214 \mathrm{~nm}, \mathrm{t}_{\mathrm{r}} 4.7$ (minor), 5.1 (major); ${ }^{1} \mathrm{H} \mathrm{NMR}$ $\left(400 \mathrm{MHz} \mathrm{CDCl}_{3}\right) \delta 7.32-7.17(\mathrm{~m}, 7 \mathrm{H}), 7.10(\mathrm{dd}, J=8.5,2.5 \mathrm{~Hz}, 1 \mathrm{H}), 4.59(\mathrm{q}, J=$ $7.2 \mathrm{~Hz}, 1 \mathrm{H}), 1.59$ (d, $J=7.2 \mathrm{~Hz}, 3 \mathrm{H}) .{ }^{13} \mathrm{C} \mathrm{NMR}\left(101 \mathrm{MHz}, \mathrm{CDCl}_{3}\right) \delta 145.6,144.2$, 132.9, 132.3, 130.8, 128.8, 128.6, 127.8, 127.6, 126.6, 41.2, 21.2. These spectroscopic data correspond to reported data. ${ }^{1}$

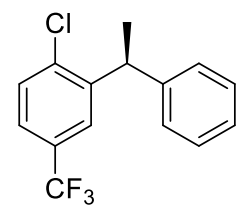

(S)-1-chloro-2-(1-phenylethyl)-4-(trifluoromethyl)benzene (8ab). This compound was afforded as colorless oil $(68.9 \mathrm{mg}, 97 \%$ yield) after flash column chromatography

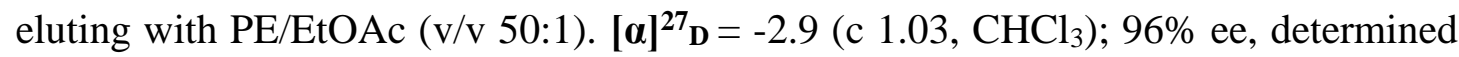
by HPLC, HPLC conditions: Chiralcel OJ-H, n-hexane/i-PrOH =99.9/0.1, 1.0 mL/min, $\mathrm{n}=214 \mathrm{~nm}, \mathrm{t}_{\mathrm{r}} 5.6$ (minor), 6.7 (major); ${ }^{1} \mathrm{H} \mathrm{NMR}\left(400 \mathrm{MHz}, \mathrm{CDCl}_{3}\right) \delta 7.50(\mathrm{~d}, J=1.3$ $\mathrm{Hz}, 1 \mathrm{H}), 7.45(\mathrm{~d}, J=8.3 \mathrm{~Hz}, 1 \mathrm{H}), 7.39(\mathrm{dd}, J=8.3,1.8 \mathrm{~Hz}, 1 \mathrm{H}), 7.29(\mathrm{t}, J=7.4 \mathrm{~Hz}$, 2H), $7.20(\mathrm{dd}, J=9.1,7.5 \mathrm{~Hz}, 3 \mathrm{H}), 4.66(\mathrm{q}, J=7.2 \mathrm{~Hz}, 1 \mathrm{H}), 1.63(\mathrm{~d}, J=7.2 \mathrm{~Hz}, 3 \mathrm{H})$. ${ }^{13} \mathrm{C} \mathrm{NMR}\left(101 \mathrm{MHz}, \mathrm{CDCl}_{3}\right) \delta 144.7,143.9,137.7,130.2,129.4(\mathrm{q}, J=32.6 \mathrm{~Hz}), 128.6$, 127.7, 126.6, 125.4 (q, $J=3.8 \mathrm{~Hz}), 124.3(\mathrm{q}, J=3.7 \mathrm{~Hz}), 122.5,41.1,21.1 .{ }^{19} \mathrm{~F}$ NMR (377 MHz, CDCl3) $\delta$-62.5; HRMS (EI) m/z: [M] $]^{+}$Calcd for $\mathrm{C}_{15} \mathrm{H}_{12} \mathrm{ClF}_{3}$ 284.0574; Found 284.0574.<smiles>COc1cccc(C(C)c2ccccc2Cl)c1</smiles>

(S)-1-chloro-2-(1-(3-methoxyphenyl)ethyl)benzene (8ac). ${ }^{1}$ This compound was afforded as colorless oil (60.2 mg, 98\% yield) after flash column chromatography eluting with PE/EtOAc (v/v 20:1). $[\boldsymbol{\alpha}]^{28} \mathbf{D}=-50.6\left(\mathrm{c} 1.13, \mathrm{CHCl}_{3}\right),\left[\right.$ lit. ${ }^{1}[\boldsymbol{\alpha}]^{\mathbf{2 0}} \mathbf{D}=-34.0\left(\mathrm{c} 0.73, \mathrm{CHCl}_{3}\right)$, 95\% ee]; 90\% ee, determined by HPLC, HPLC conditions: Chiralcel OJ-H, n-hexane/ $i$ $\mathrm{PrOH}=90 / 10,1.0 \mathrm{~mL} / \mathrm{min}, \mathrm{n}=214 \mathrm{~nm}, \mathrm{t}_{\mathrm{r}} 9.0$ (minor), 10.0 (major); ${ }^{1} \mathrm{H}$ NMR (400 $\left.\mathrm{MHz}, \mathrm{CDCl}_{3}\right) \delta 7.33(\mathrm{~d}, J=8.0 \mathrm{~Hz}, 1 \mathrm{H}), 7.23-7.16(\mathrm{~m}, 3 \mathrm{H}), 7.15-7.09(\mathrm{~m}, 1 \mathrm{H}), 6.85$ $-6.71(\mathrm{~m}, 3 \mathrm{H}), 4.62(\mathrm{q}, J=7.2 \mathrm{~Hz}, 1 \mathrm{H}), 3.76(\mathrm{~s}, 3 \mathrm{H}), 1.60(\mathrm{~d}, J=7.2 \mathrm{~Hz}, 3 \mathrm{H}) .{ }^{13} \mathrm{C}$ $\operatorname{NMR}\left(101 \mathrm{MHz} \mathrm{CDCl}_{3}\right) \delta 159.7,146.8,143.7,134.0,129.7,130.0,128.7,127.5,127.1$, $120.4,114.2,111.2,55.3,41.0,21.2$. These spectroscopic data correspond to reported 
data. ${ }^{1}$

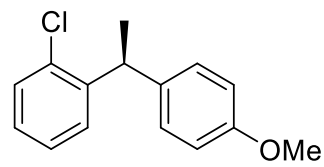

(S)-1-chloro-2-(1-(4-methoxyphenyl)ethyl)benzene (8ad). ${ }^{1}$ This compound was afforded as colorless oil (60.9 mg, 99\% yield) after flash column chromatography eluting with PE/EtOAc (v/v 20:1). $[\boldsymbol{\alpha}]^{\mathbf{2 6}} \mathbf{D}=-55.5\left(\mathrm{c} 0.73, \mathrm{CHCl}_{3}\right)$, $\left[\right.$ lit. $^{1}[\boldsymbol{\alpha}]^{\mathbf{2 0}} \mathbf{D}=-43.3$ (c $0.75, \mathrm{CHCl}_{3}$ ), 90\% ee]; 92\% ee, determined by HPLC, HPLC conditions: Chiralcel OJ-H, n-hexane $/ i-\mathrm{PrOH}=90 / 10,1.0 \mathrm{~mL} / \mathrm{min}, \mathrm{n}=214 \mathrm{~nm}, \mathrm{t}_{\mathrm{r}} 10.6$ (minor), 10.7 (major); ${ }^{1} \mathrm{H}$ NMR $\left(400 \mathrm{MHz}, \mathrm{CDCl}_{3}\right) \delta 7.33(\mathrm{~d}, J=7.8 \mathrm{~Hz}, 1 \mathrm{H}), 7.21-7.07(\mathrm{~m}, 5 \mathrm{H}), 6.82(\mathrm{~d}, J$ $=8.6 \mathrm{~Hz}, 2 \mathrm{H}), 4.60(\mathrm{q}, J=7.2 \mathrm{~Hz}, 1 \mathrm{H}), 3.77(\mathrm{~s}, 3 \mathrm{H}), 1.58(\mathrm{~d}, J=7.2 \mathrm{~Hz}, 3 \mathrm{H}) .{ }^{13} \mathrm{C} \mathrm{NMR}$ $\left(101 \mathrm{MHz}, \mathrm{CDCl}_{3}\right) \delta 158.0,144.2,137.2,133.9,129.7,128.8,128.6,127.4,127.0$, $113.8,55.3,40.2,21.4$. These spectroscopic data correspond to reported data. ${ }^{1}$

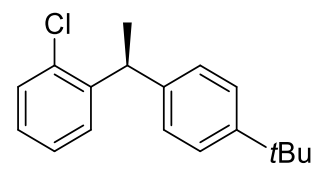

(S)-1-(1-(4-(tert-butyl)phenyl)ethyl)-2-chlorobenzene (8ae). This compound was afforded as Colorless oil (66.6 mg, 98\% yield) after flash column chromatography

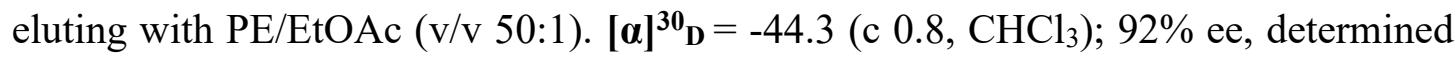
by HPLC, HPLC conditions: Chiralcel OJ-H, n-hexane $/ i-\mathrm{PrOH}=100 / 0,1.0 \mathrm{~mL} / \mathrm{min}, \mathrm{n}$ $=214 \mathrm{~nm}, \mathrm{t}_{\mathrm{r}} 10.6$ (major), 12.3 (minor); ${ }^{1} \mathrm{H} \mathrm{NMR}\left(400 \mathrm{MHz}, \mathrm{CDCl}_{3}\right) \delta 7.31$ (dd, $J=$ 14.1, 8.1 Hz, 3H), $7.23-7.14(\mathrm{~m}, 4 \mathrm{H}), 7.13-7.07$ (m, 1H), 4.63 (q, $J=7.2 \mathrm{~Hz}, 1 \mathrm{H})$, $1.60(\mathrm{~d}, J=7.2 \mathrm{~Hz}, 3 \mathrm{H}), 1.29(\mathrm{~s}, 9 \mathrm{H}) .{ }^{13} \mathrm{C} \mathrm{NMR}\left(101 \mathrm{MHz}, \mathrm{CDCl}_{3}\right) \delta$ 149.0, 144.1, 141.9, 133.9, 129.7, 128.8, 127.5, 127.3, 127.0, 125.3, 40.5, 34.5, 31.5, 21.3; HRMS (EI) $\mathrm{m} / \mathrm{z}:[\mathrm{M}]^{+}$Calcd for $\mathrm{C}_{18} \mathrm{H}_{21} \mathrm{Cl} 272.1324$; Found 272.1326.<smiles>CC(c1ccc(Cl)cc1)c1ccccc1Cl</smiles>

(S)-1-chloro-2-(1-(4-chlorophenyl)ethyl)benzene (8af). ${ }^{24}$ This compound was afforded as colorless oil (60.0 mg, 96\% yield) after flash column chromatography eluting with PE/EtOAc (v/v 50:1). $[\boldsymbol{\alpha}]^{30} \mathrm{D}=-28.4\left(\mathrm{c} 1.27, \mathrm{CHCl}_{3}\right) ; 91 \%$ ee, determined 
by HPLC, HPLC conditions: Chiralcel OJ-H, n-hexane $/ i-\mathrm{PrOH}=95 / 5,1.0 \mathrm{~mL} / \mathrm{min}, \mathrm{n}$ $=214 \mathrm{~nm}, \mathrm{t}_{\mathrm{r}} 5.2$ (minor), 5.7 (major); ${ }^{1} \mathrm{H}$ NMR (400 MHz, $\left.\mathrm{CDCl}_{3}\right) \delta 7.37-7.32$ (m, 1H), $7.27-7.11(\mathrm{~m}, 7 \mathrm{H}), 4.61(\mathrm{q}, J=7.2 \mathrm{~Hz}, 1 \mathrm{H}), 1.59(\mathrm{~d}, J=7.2 \mathrm{~Hz}, 3 \mathrm{H}) .{ }^{13} \mathrm{C} \mathrm{NMR}$ $\left(101 \mathrm{MHz}, \mathrm{CDCl}_{3}\right) \delta 143.6,143.2,134.0,132.0,129.8,129.2,128.6,128.5,127.7$, 127.1, 40.6, 21.2. These spectroscopic data correspond to reported data. ${ }^{24}$<smiles>CC(c1ccc(F)cc1)c1ccccc1Cl</smiles>

(S)-1-chloro-2-(1-(4-fluorophenyl)ethyl)benzene (8ag). ${ }^{25}$ This compound was afforded as colorless oil (57.3 mg, 98\% yield) after flash column chromatography

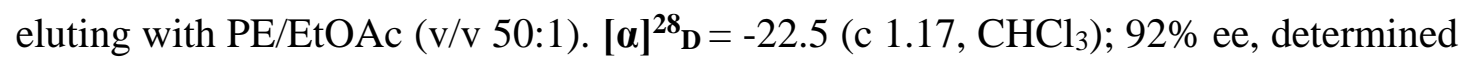
by HPLC, HPLC conditions: Chiralcel OJ-H, n-hexane/i-PrOH = 90/10, $1.0 \mathrm{~mL} / \mathrm{min}, \mathrm{n}$ $=214 \mathrm{~nm}, \mathrm{t}_{\mathrm{r}} 5.8$ (minor), 7.0 (major); ${ }^{1} \mathrm{H} \mathrm{NMR}\left(400 \mathrm{MHz}, \mathrm{CDCl}_{3}\right) \delta 7.33(\mathrm{~d}, J=7.7$ $\mathrm{Hz}, 1 \mathrm{H}), 7.23-7.09$ (m, 5H), $6.99-6.90$ (m, 2H), 4.62 (q, J=7.2 Hz, 1H), 1.58 (d, J $=7.2 \mathrm{~Hz}, 3 \mathrm{H}) .{ }^{13} \mathrm{C}$ NMR $\left(101 \mathrm{MHz}, \mathrm{CDCl}_{3}\right) \delta 161.4(\mathrm{~d}, J=244.2 \mathrm{~Hz}), 143.6,140.8(\mathrm{~d}$, $J=3.2 \mathrm{~Hz}), 134.0,129.8,129.3(\mathrm{~d}, J=7.8 \mathrm{~Hz}), 128.5,127.6,127.1,115.2$ (d, $J=21.1$ $\mathrm{Hz}), 40.4,21.4 .{ }^{19} \mathrm{~F}$ NMR $(377 \mathrm{MHz}, \mathrm{CDCl} 3) \delta-117.1$. These spectroscopic data correspond to reported data. ${ }^{25}$

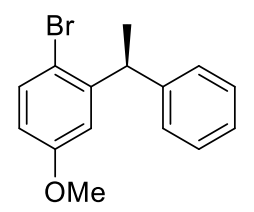

(S)-1-bromo-4-methoxy-2-(1-phenylethyl)benzene (8ah). This compound was afforded as colorless oil (68.8 mg, 95\% yield) after flash column chromatography eluting with PE/EtOAc (v/v 20:1). [ $[\boldsymbol{\alpha}]^{24} \mathrm{D}=+0.32$ (c 1.17, $\mathrm{CHCl}_{3}$ ); $95 \%$ ee, determined by HPLC, HPLC conditions: Chiralcel OJ-3, n-hexane/i-PrOH = 90/10, 0.5 mL/min, $\mathrm{n}$ $=230 \mathrm{~nm}, \mathrm{t}_{\mathrm{r}} 8.8$ (minor), 9.6 (major); ${ }^{1} \mathrm{H}$ NMR (400 MHz, $\left.\mathrm{CDCl}_{3}\right) \delta 7.41$ (d, $J=8.7$ $\mathrm{Hz}, 1 \mathrm{H}), 7.22(\mathrm{qd}, J=14.9,7.3 \mathrm{~Hz}, 5 \mathrm{H}), 6.75(\mathrm{~d}, J=1.9 \mathrm{~Hz}, 1 \mathrm{H}), 6.60$ (dd, $J=8.7,2.9$ $\mathrm{Hz}, 1 \mathrm{H}), 4.57$ (q, $J=7.1 \mathrm{~Hz}, 1 \mathrm{H}), 3.69$ (s, 3H), $1.58(\mathrm{~d}, J=7.1 \mathrm{~Hz}, 3 \mathrm{H}) .{ }^{13} \mathrm{C} \mathrm{NMR}(101$ $\mathrm{MHz}, \mathrm{CDC13}) \delta 159.2,146.5,144.8,133.5,128.5,127.9,126.3,115.4,115.3,112.8$, 55.5, 43.8, 21.4; HRMS (EI) m/z: [M] $]^{+}$Calcd for $\mathrm{C}_{15} \mathrm{H}_{15} \mathrm{OBr}$ 290.0297; Found 290.0301 . 


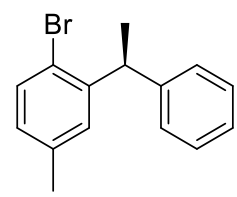

1-bromo-4-methyl-2-(1-phenylethyl)benzene (8ai). This compound was afforded as colorless oil (65.1 mg, 95\% yield) after flash column chromatography eluting with

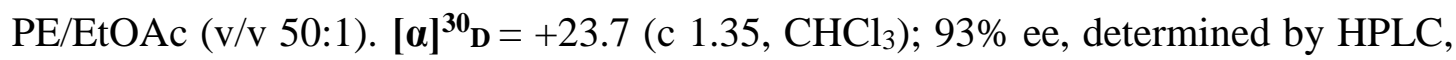
HPLC conditions: Chiralcel OJ-H, n-hexane/i-PrOH =99/1, $1 \mathrm{~mL} / \mathrm{min}, \mathrm{n}=214 \mathrm{~nm}, \mathrm{t}_{\mathrm{r}}$ 10.4 (minor), 14.8 (major); ${ }^{1} \mathrm{H}$ NMR (400 MHz, $\left.\mathrm{CDCl}_{3}\right) \delta 7.37$ (s, 1H), 7.30 - 7.14 (m, $5 \mathrm{H}), 7.11-7.01(\mathrm{~m}, 2 \mathrm{H}), 4.59(\mathrm{q}, J=7.1 \mathrm{~Hz}, 1 \mathrm{H}), 2.27(\mathrm{~s}, 3 \mathrm{H}), 1.58(\mathrm{~d}, J=7.2 \mathrm{~Hz}$, 3H). ${ }^{13} \mathrm{C}$ NMR $\left(101 \mathrm{MHz}, \mathrm{CDCl}_{3}\right) \delta 145.3,142.4,137.7,133.4,128.6,128.5,128.4$, 127.9, 126.2, 124.6, 43.2, 21.5, 20.7. HRMS (EI) m/z: $[\mathrm{M}]^{+}$Calcd for $\mathrm{C}_{15} \mathrm{H}_{15} \mathrm{Br}$ 274.0352; Found 274.0352.

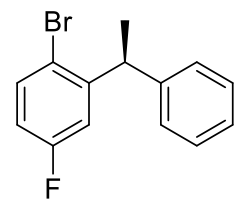

(S)-1-bromo-4-fluoro-2-(1-phenylethyl)benzene (8aj). This compound was afforded as colorless oil (66.7 mg, 96\% yield) after flash column chromatography eluting with PE/EtOAc (v/v 50:1). [a $]^{28} \mathbf{D}=-16.4\left(\mathrm{c} 1.45, \mathrm{CHCl}_{3}\right) ; 94 \%$ ee, determined by HPLC, HPLC conditions: Chiralcel OJ-3, n-hexane $/ i-\mathrm{PrOH}=90 / 10,0.5 \mathrm{~mL} / \mathrm{min}, \mathrm{n}=230 \mathrm{~nm}$, t 4.1 (minor), 4.8 (major); ${ }^{1} \mathrm{H}$ NMR $\left(400 \mathrm{MHz}, \mathrm{CDCl}_{3}\right) \delta 7.47$ (dd, $\left.J=8.7,5.5 \mathrm{~Hz}, 1 \mathrm{H}\right)$, $7.24(\mathrm{dq}, J=14.8,7.5 \mathrm{~Hz}, 5 \mathrm{H}), 6.96-6.89(\mathrm{~m}, 1 \mathrm{H}), 6.77(\mathrm{td}, J=8.3,3.0 \mathrm{~Hz}, 1 \mathrm{H}), 4.58$ $(\mathrm{q}, J=7.0 \mathrm{~Hz}, 1 \mathrm{H}), 1.57(\mathrm{~d}, J=7.2 \mathrm{~Hz}, 3 \mathrm{H}) .{ }^{13} \mathrm{C} \mathrm{NMR}\left(101 \mathrm{MHz}, \mathrm{CDCl}_{3}\right) \delta 162.3(\mathrm{~d}$, $J=246.3 \mathrm{~Hz}), 147.8(\mathrm{~d}, J=6.8 \mathrm{~Hz}), 144.2,134.1(\mathrm{~d}, J=8.0 \mathrm{~Hz}), 128.6,127.9,126.6$, $118.7(\mathrm{~d}, J=3.1 \mathrm{~Hz}), 116.0(\mathrm{~d}, J=23.2 \mathrm{~Hz}), 115.0(\mathrm{~d}, J=22.5 \mathrm{~Hz}), 43.9,21.3 ;{ }^{19} \mathrm{~F}$ NMR (377 MHz, CDCl3) $\delta$-114.2; HRMS (EI) m/z: $[\mathrm{M}]^{+}$Calcd for $\mathrm{C}_{14} \mathrm{H}_{12} \mathrm{BrF}$ 278.0102; Found 278.0101.<smiles>COc1ccc(C(C)c2ccccc2Br)cc1</smiles> 
(S)-1-bromo-2-(1-(4-methoxyphenyl)ethyl)benzene (8ak). ${ }^{18}$ This compound was afforded as colorless oil (69.6 mg, 96\% yield) after flash column chromatography eluting with PE/EtOAc (v/v 20:1). $[\boldsymbol{\alpha}]^{29} \mathrm{D}=-63.8\left(\mathrm{c} 1.16, \mathrm{CHCl}_{3}\right) ; 94 \%$ ee, determined by HPLC, HPLC conditions: Chiralcel OJ-H, n-hexane $/ i$-PrOH $=90 / 10,1 \mathrm{~mL} / \mathrm{min}, \mathrm{n}$ $=214 \mathrm{~nm}, \mathrm{t}_{\mathrm{r}} 9.1$ (minor), 11.8 (major); ${ }^{1} \mathrm{H}$ NMR (400 MHz, $\left.\mathrm{CDCl}_{3}\right) \delta 7.53(\mathrm{~d}, J=7.9$ $\mathrm{Hz}, 1 \mathrm{H}), 7.26-7.11(\mathrm{~m}, 4 \mathrm{H}), 7.06-6.99(\mathrm{~m}, 1 \mathrm{H}), 6.82(\mathrm{~d}, J=8.6 \mathrm{~Hz}, 2 \mathrm{H}), 4.58$ (q, $J$ $=7.1 \mathrm{~Hz}, 1 \mathrm{H}), 3.76(\mathrm{~s}, 3 \mathrm{H}), 1.57(\mathrm{~d}, J=7.2 \mathrm{~Hz}, 3 \mathrm{H}) .{ }^{13} \mathrm{C} \mathrm{NMR}(101 \mathrm{MHz}, \mathrm{CDCl} 3) \delta$ 158.0, 145.8, 137.2, 133.0, 128.9, 128.8, 127.7, 124.8, 113.8, 55.3, 42.8, 21.6. These spectroscopic data correspond to reported data. ${ }^{18}$<smiles>COc1ccccc1C(C)c1ccccc1Br</smiles>

(S)-1-bromo-2-(1-(2-methoxyphenyl)ethyl)benzene (8al). ${ }^{18}$ This compound was afforded as white amorphous solid $(57.3 \mathrm{mg}, 79 \%$ yield) after flash column chromatography eluting with PE/EtOAc (v/v 20:1). $[\boldsymbol{\alpha}]^{26} \mathrm{D}=-49.6\left(\mathrm{c} 1.10, \mathrm{CHCl}_{3}\right) ; 95 \%$ ee, determined by HPLC, HPLC conditions: Chiralcel OJ-3, n-hexane/i-PrOH = 95/5, $0.7 \mathrm{~mL} / \mathrm{min}, \mathrm{n}=230 \mathrm{~nm}, \mathrm{t}_{\mathrm{r}} 7.4$ (minor), 7.8 (major). The product $(25 \mathrm{mg}$ ) was dissolved in pentane $(5.0 \mathrm{~mL})$ and the solvent evaporated slowly at $0{ }^{\circ} \mathrm{C}$. After a few days, white crystals of 8al suitable for X-ray analysis were obtained. ${ }^{1} \mathrm{H}$ NMR (400 MHz, $\left.\mathrm{CDCl}_{3}\right)$ $\delta 7.52(\mathrm{dd}, J=7.9,0.7 \mathrm{~Hz}, 1 \mathrm{H}), 7.23-7.16(\mathrm{~m}, 2 \mathrm{H}), 7.12(\mathrm{dd}, J=10.4,4.4 \mathrm{~Hz}, 2 \mathrm{H})$, $7.02(\mathrm{td}, J=7.9,1.7 \mathrm{~Hz}, 1 \mathrm{H}), 6.91(\mathrm{t}, J=7.4 \mathrm{~Hz}, 1 \mathrm{H}), 6.83(\mathrm{~d}, J=8.1 \mathrm{~Hz}, 1 \mathrm{H}), 4.86(\mathrm{q}$, $J=7.1 \mathrm{~Hz}, 1 \mathrm{H}), 3.74(\mathrm{~s}, 3 \mathrm{H}), 1.52(\mathrm{~d}, J=7.2 \mathrm{~Hz}, 3 \mathrm{H}) .{ }^{13} \mathrm{C} \mathrm{NMR}\left(101 \mathrm{MHz}, \mathrm{CDCl}_{3}\right) \delta$ 157.3, 145.7, 133.8, 132.9, 128.6, 127.5, 127.5, 127.4, 127.3, 125.1, 120.4, 110.8, 55.6, $37.7,20.2$. These spectroscopic data correspond to reported data. ${ }^{18}$

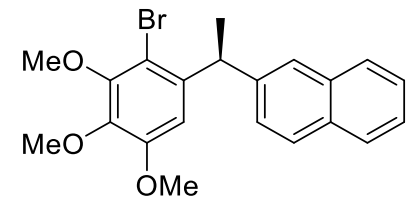

(S)-2-(1-(2-bromo-3,4,5-trimethoxyphenyl)ethyl)naphthalene (8am). This compound was afforded as yellow oil (96.0 mg, 96\% yield) after flash column chromatography eluting with PE/EtOAc (v/v 10:1). $[\alpha]^{26} \mathbf{D}=106.6\left(\mathrm{c} 1.30, \mathrm{CHCl}_{3}\right) ; 95 \%$ 
ee, determined by HPLC, HPLC conditions: Chiralcel OJ-H, n-hexane $/ i$-PrOH = 99.5/0.5, $1 \mathrm{~mL} / \mathrm{min}, \mathrm{n}=214 \mathrm{~nm}, \mathrm{t}_{\mathrm{r}} 19.7$ (major), 22.2 (minor); ${ }^{1} \mathrm{H} \mathrm{NMR}$ (400 MHz, $\left.\mathrm{CDCl}_{3}\right) \delta 7.86-7.71(\mathrm{~m}, 4 \mathrm{H}), 7.46(\mathrm{qd}, J=6.8,3.3 \mathrm{~Hz}, 2 \mathrm{H}), 7.35(\mathrm{dd}, J=8.5,1.5 \mathrm{~Hz}$, 1H), $6.55(\mathrm{~s}, 1 \mathrm{H}), 4.85(\mathrm{q}, J=7.1 \mathrm{~Hz}, 1 \mathrm{H}), 3.92(\mathrm{~d}, J=13.1 \mathrm{~Hz}, 6 \mathrm{H}), 3.73(\mathrm{~s}, 3 \mathrm{H}), 1.71$ $(\mathrm{d}, J=7.1 \mathrm{~Hz}, 3 \mathrm{H}) .{ }^{13} \mathrm{C} \mathrm{NMR}\left(101 \mathrm{MHz}, \mathrm{CDCl}_{3}\right) \delta 152.8,150.8,142.4,141.6,141.1$, 133.5, 132.2, 128.0, 127.9, 127.7, 127.1, 126.1, 125.6, 125.5, 111.3, 107.9, 61.1, 61.0, 56.2, 43.7, 21.2. HRMS (EI) m/z: [M] ${ }^{+}$Calcd for $\mathrm{C}_{21} \mathrm{H}_{21} \mathrm{O}_{3} \mathrm{Br}$ 400.0670; Found 400.0669 .

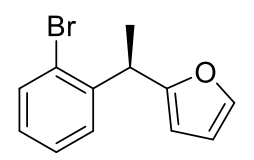

(R)-2-(1-(2-bromophenyl)ethyl)furan (8an). This compound was afforded as colorless oil (52.4 mg, 98\% yield) after flash column chromatography eluting with PE/EtOAc (v/v 20:1). [ $[\boldsymbol{\alpha}]^{\mathbf{2 6}} \mathbf{D}=-75.1$ (c 1.07, $\mathrm{CHCl}_{3}$ ); 95\% ee, determined by HPLC, HPLC conditions: Chiralcel OJ-H, n-hexane $/ i$-PrOH $=99.9 / 0.1,0.8 \mathrm{~mL} / \mathrm{min}, \mathrm{n}=214$ nm, $\mathrm{t}_{\mathrm{r}} 9.5$ (major), 10.8 (minor); ${ }^{1} \mathrm{H}$ NMR (400 MHz, $\left.\mathrm{CDCl}_{3}\right) \delta 7.54(\mathrm{~d}, J=7.5 \mathrm{~Hz}, 1 \mathrm{H})$, $7.32(\mathrm{~s}, 1 \mathrm{H}), 7.21(\mathrm{t}, J=7.0 \mathrm{~Hz}, 1 \mathrm{H}), 7.05(\mathrm{t}, J=7.3 \mathrm{~Hz}, 2 \mathrm{H}), 6.31(\mathrm{dd}, J=2.6,1.8 \mathrm{~Hz}$, $1 \mathrm{H}), 6.13(\mathrm{~d}, J=3.0 \mathrm{~Hz}, 1 \mathrm{H}), 4.63(\mathrm{q}, J=7.1 \mathrm{~Hz}, 1 \mathrm{H}), 1.55(\mathrm{~d}, J=7.2 \mathrm{~Hz}, 3 \mathrm{H}) .{ }^{13} \mathrm{C}$ NMR (101 MHz, $\left.\mathrm{CDCl}_{3}\right) \delta$ 157.8, 143.4, 141.7, 133.0, 128.6, 128.1, 127.8, 124.1 110.1, 106.0, 38.4, 19.5. HRMS (EI) m/z: $[\mathrm{M}]^{+}$Calcd for $\mathrm{C}_{12} \mathrm{H}_{11} \mathrm{BrO}$ 249.9990; Found 249.9988 .

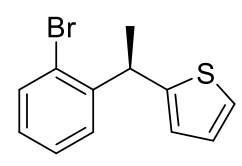

(R)-2-(1-(2-bromophenyl)ethyl)thiophene (8ao). ${ }^{26}$ This compound was afforded as colorless oil (63.8 mg, 96\% yield) after flash column chromatography eluting with PE/EtOAc (v/v 20:1). $[\boldsymbol{\alpha}]^{\mathbf{2 6}} \mathrm{D}=-56.4\left(\mathrm{c} 1.18, \mathrm{CHCl}_{3}\right) .89 \%$ ee, determined by HPLC, HPLC conditions: Chiralcel OJ-H, n-hexane/i-PrOH = 99.9/0.1, $1 \mathrm{~mL} / \mathrm{min}, \mathrm{n}=214 \mathrm{~nm}$, $\mathrm{t}_{\mathrm{r}} 9.7$ (major), 12.8 (minor); ${ }^{1} \mathrm{H} \mathrm{NMR}\left(400 \mathrm{MHz}, \mathrm{CDCl}_{3}\right) \delta 7.54(\mathrm{~d}, J=7.8 \mathrm{~Hz}, 1 \mathrm{H})$, $7.22(\mathrm{~d}, J=3.4 \mathrm{~Hz}, 3 \mathrm{H}), 7.15(\mathrm{dd}, J=5.1,1.0 \mathrm{~Hz}, 1 \mathrm{H}), 7.08-7.02(\mathrm{~m}, 1 \mathrm{H}), 6.92(\mathrm{dd}$, $J=5.1,3.5 \mathrm{~Hz}, 1 \mathrm{H}), 6.84(\mathrm{dd}, J=2.4,1.1 \mathrm{~Hz}, 1 \mathrm{H}), 4.86(\mathrm{q}, J=7.1 \mathrm{~Hz}, 1 \mathrm{H}), 1.67(\mathrm{~d}, J$ $=7.1 \mathrm{~Hz}, 3 \mathrm{H}) .{ }^{13} \mathrm{C} \mathrm{NMR}\left(101 \mathrm{MHz}, \mathrm{CDCl}_{3}\right) \delta 149.2,145.2,133.0,128.7,128.1,127.9$, 
$126.7,124.2,124.1,123.8,39.6,22.5$. These spectroscopic data correspond to reported data. $^{26}$<smiles>CC(c1ncco1)c1ccccc1Br</smiles>

(R)-2-(1-(2-bromophenyl)ethyl)oxazole (8ap). This compound was afforded as colorless oil (61.5 mg, 98\% yield) after flash column chromatography eluting with PE/EtOAc (v/v 10:1). $[\boldsymbol{\alpha}]^{28} \mathbf{D}=-28.9\left(\mathrm{c} 1.0, \mathrm{CHCl}_{3}\right) ; 37 \%$ ee, determined by HPLC, HPLC conditions: Chiralcel OD-H, n-hexane $/ i-\mathrm{PrOH}=99 / 10,0.7 \mathrm{~mL} / \mathrm{min}, \mathrm{n}=230 \mathrm{~nm}$, t 7.8 (minor), 8.7 (major); ${ }^{1} \mathrm{H}$ NMR (400 MHz, $\left.\mathrm{CDCl}_{3}\right) \delta 7.57$ (d, J=7.1 Hz, 2H), 7.24 (t, $J=7.7 \mathrm{~Hz}, 1 \mathrm{H}), 7.18-7.04(\mathrm{~m}, 3 \mathrm{H}), 4.78(\mathrm{q}, J=7.1 \mathrm{~Hz}, 1 \mathrm{H}), 1.68(\mathrm{~d}, J=7.2 \mathrm{~Hz}$, $3 \mathrm{H}) .{ }^{13} \mathrm{C} \mathrm{NMR}\left(101 \mathrm{MHz}, \mathrm{CDCl}_{3}\right) \delta 166.1,141.3,138.7,133.1,128.6,128.5,128.0$, 127.1, 123.9, 38.8, 19.3. HRMS (EI) m/z: [M] ${ }^{+}$Calcd for $\mathrm{C}_{11} \mathrm{H}_{9} \mathrm{BrNO} 249.9857$; Found 249.9862 .

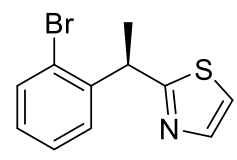

(R)-2-(1-(2-bromophenyl)ethyl)thiazole (8aq). This compound was afforded as colorless oil (65.4 mg, 98\% yield) after flash column chromatography eluting with

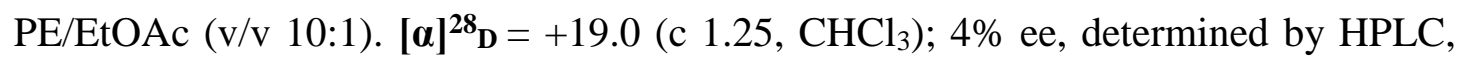
HPLC conditions: Chiralcel OJ-H, n-hexane $/ i-\mathrm{PrOH}=99 / 10,0.7 \mathrm{~mL} / \mathrm{min}, \mathrm{n}=230 \mathrm{~nm}$, t 9.8 (minor), 10.5 (major); ${ }^{1} \mathrm{H} \mathrm{NMR}\left(400 \mathrm{MHz}, \mathrm{CDCl}_{3}\right) \delta 7.73(\mathrm{~d}, J=3.3 \mathrm{~Hz}, 1 \mathrm{H})$, $7.57(\mathrm{dd}, J=8.0,1.0 \mathrm{~Hz}, 1 \mathrm{H}), 7.34-7.27(\mathrm{~m}, 2 \mathrm{H}), 7.21(\mathrm{~d}, J=3.3 \mathrm{~Hz}, 1 \mathrm{H}), 7.14-7.07$ $(\mathrm{m}, 1 \mathrm{H}), 5.01(\mathrm{q}, J=7.1 \mathrm{~Hz}, 1 \mathrm{H}), 1.77(\mathrm{~d}, J=7.1 \mathrm{~Hz}, 3 \mathrm{H}) .{ }^{13} \mathrm{C} \mathrm{NMR}\left(101 \mathrm{MHz}, \mathrm{CDCl}_{3}\right)$ $\delta 174.1,143.1,142.5,133.1,128.9,128.7,128.0,124.3,118.7,42.7,21.1$. HRMS (EI) m/z: [M] $]^{+}$Calcd for $\mathrm{C}_{11} \mathrm{H}_{9}$ BrNS 265.9627; Found 265.9634.<smiles>Cc1cccc(C(C)c2nccn2Cc2ccccc2)c1C</smiles>

(R)-1-benzyl-2-(1-(2,3-dimethylphenyl)ethyl)-1H-imidazole (8ar). This compound was afforded as yellow amorphous solid (71.1 mg, 98\% yield) after flash column chromatography eluting with PE/EtOAc (v/v 10:1). $[\alpha]^{28} \mathrm{D}=-1.60\left(\mathrm{c} 1.05, \mathrm{CHCl}_{3}\right) ; 11 \%$ ee, determined by HPLC, HPLC conditions: Chiralcel OD-H, n-hexane/i-PrOH = 60/40, 
$0.7 \mathrm{~mL} / \mathrm{min}, \mathrm{n}=230 \mathrm{~nm}, \mathrm{t}_{\mathrm{r}} 3.7$ (minor), 4.7 (major); ${ }^{1} \mathrm{H} \mathrm{NMR}\left(400 \mathrm{MHz}, \mathrm{CDCl}_{3}\right) \delta$ $7.28-7.23(\mathrm{~m}, 3 \mathrm{H}), 7.08(\mathrm{~d}, J=1.1 \mathrm{~Hz}, 1 \mathrm{H}), 7.03-6.95(\mathrm{~m}, 1 \mathrm{H}), 6.88-6.80(\mathrm{~m}, 3 \mathrm{H})$, $6.74-6.69(\mathrm{~m}, 1 \mathrm{H}), 4.67(\mathrm{dd}, J=48.3,15.8 \mathrm{~Hz}, 2 \mathrm{H}), 4.24(\mathrm{q}, J=7.0 \mathrm{~Hz}, 1 \mathrm{H}), 2.28(\mathrm{~s}$, $3 \mathrm{H}), 2.17(\mathrm{~s}, 3 \mathrm{H}), 1.63(\mathrm{~d}, J=7.0 \mathrm{~Hz}, 3 \mathrm{H}) .{ }^{13} \mathrm{C} \mathrm{NMR}\left(101 \mathrm{MHz}, \mathrm{CDCl}_{3}\right) \delta 150.9,142.0$, 137.1, 136.4, 133.2, 128.9, 128.4, 127.9, 127.2, 126.9, 126.2, 125.0, 120.6, 49.4, 34.9, 21.0, 20.4, 14.6. HRMS (ESI) m/z: $[\mathrm{M}+\mathrm{H}]^{+}$Calcd for $\mathrm{C}_{20} \mathrm{H}_{23} \mathrm{~N}_{2}$ 291.1856; Found 291.1856.

\section{Further Derivatization}
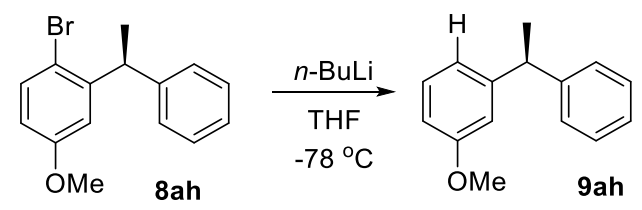

(R)-1-methoxy-3-(1-phenylethyl)benzene (9ah). ${ }^{27}$ A $50 \mathrm{~mL}$ flame-dried Schlenk flask was charged with $\mathbf{8 a h}(65 \mathrm{mg}, 0.25 \mathrm{mmol})$, THF (10mL) Under inert atmosphere. $n$-BuLi $\left(0.5 \mathrm{mmol}, 0.2 \mathrm{~mL}, 2.5 \mathrm{M}\right.$ in hexane) was added dropwise at $-78{ }^{\circ} \mathrm{C}$. After stirred at $-78^{\circ} \mathrm{C}$ for 3 hours, the mixture was quenched with water $(5 \mathrm{~mL})$ and extracted with EA $(3 \times 5 \mathrm{~mL})$. The combined organic layers were dried over anhydrous $\mathrm{Na}_{2} \mathrm{SO}_{4}$, filtered, concentrated by rotary evaporation and further purified by flash chromatography $(\mathrm{PE} / \mathrm{EA}=10 / 1)$ to afford 9ah $(47.1 \mathrm{mg}, 89 \%$ yield $)$ as colorless oil. $[\alpha]^{28} \mathrm{D}=+1.52\left(\mathrm{c} 1.58, \mathrm{CHCl}_{3}\right) ; 95 \%$ ee, determined by HPLC, HPLC conditions: Chiralcel OJ-H, n-hexane/i-PrOH = 99.7/0.3, $1 \mathrm{~mL} / \mathrm{min}, \mathrm{n}=214 \mathrm{~nm}, \mathrm{t}_{\mathrm{r}} 46.7$ (minor), 51.2 (major); ${ }^{1} \mathrm{H}$ NMR (400 MHz, $\left.\mathrm{CDCl}_{3}\right) \delta 7.29-7.14$ (m, 6H), $6.84-6.69$ (m, 3H), $4.11(\mathrm{q}, J=7.2 \mathrm{~Hz}, 1 \mathrm{H}), 3.74(\mathrm{~d}, J=1.6 \mathrm{~Hz}, 3 \mathrm{H}), 1.62(\mathrm{dd}, J=7.2,2.0 \mathrm{~Hz}, 3 \mathrm{H}) .{ }^{13} \mathrm{C}$ NMR $\left(101 \mathrm{MHz}, \mathrm{CDCl}_{3}\right) \delta 159.7,148.2,146.3,129.4,128.5,127.7,126.2,120.2,113.9$, 111.0, 55.2, 44.9, 21.9. These spectroscopic data correspond to reported data. ${ }^{27}$

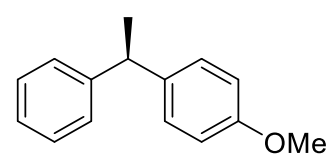


(R)-1-methoxy-4-(1-phenylethyl)benzene (9ak). ${ }^{1}$ This compound was afforded as colorless oil (50.3 mg, 95\% yield) following the similar procedure to 9ah with 8ak (65 $\mathrm{mg}, 0.25 \mathrm{mmol})$, THF (10 mL) and $n$-BuLi (0.5 mmol, $0.2 \mathrm{~mL}, 2.5 \mathrm{M}$ in hexane) purified by flash column chromatography (PE/EA $=10: 1) .[\alpha]^{29} \mathbf{D}=-11.3$ (c 0.50 , $\left.\mathrm{CHCl}_{3}\right)$, [lit. ${ }^{1}[\alpha]^{20} \mathbf{D}=+8.2\left(\mathrm{c} 0.72, \mathrm{CHCl}_{3}\right), 88 \%$ ee]; 93\% ee, determined by HPLC, HPLC conditions: Chiralcel OJ-H, n-hexane $/ i-\mathrm{PrOH}=99 / 1,1 \mathrm{~mL} / \mathrm{min}, \mathrm{n}=214 \mathrm{~nm}, \mathrm{t}_{\mathrm{r}}$ 16.7 (minor), 19.1 (major); ${ }^{1} \mathrm{H}$ NMR (400 MHz, $\left.\mathrm{CDCl}_{3}\right) \delta 7.29-7.11$ (m, 7H), $6.84-$ $6.80(\mathrm{~m}, 2 \mathrm{H}), 4.10(\mathrm{q}, J=7.2 \mathrm{~Hz}, 1 \mathrm{H}), 3.76(\mathrm{~s}, 3 \mathrm{H}), 1.61(\mathrm{~d}, J=7.2 \mathrm{~Hz}, 3 \mathrm{H}) .{ }^{13} \mathrm{C} \mathrm{NMR}$ $\left(101 \mathrm{MHz} \mathrm{CDCl}_{3}\right) \delta 158.0,146.9,138.7,128.6,128.5,127.7,126.1,113.9,55.4,44.1$, 22.2. These spectroscopic data correspond to reported data. ${ }^{1}$<smiles>COc1cc(C(C)c2ccc3ccccc3c2)cc(OC)c1OC</smiles>

(S)-2-(1-(3,4,5-trimethoxyphenyl)ethyl)naphthalene (9am). ${ }^{28}$ This compound was afforded as colorless oil (78.1 mg, 97\% yield) following the similar procedure to 9ah with 8am (100 mg, $0.25 \mathrm{mmol})$, THF (10mL) and $n$-BuLi (0.5 mmol, 0.2 mL, $2.5 \mathrm{M}$ in hexane) purified by flash column chromatography $(\mathrm{PE} / \mathrm{EA}=10: 1) \cdot[\boldsymbol{\alpha}]^{27} \mathbf{D}=-26.2(\mathrm{c}$ 1.00, $\left.\mathrm{CHCl}_{3}\right),\left[\mathrm{lit}^{28}{ }^{28}[\boldsymbol{\alpha}]^{23} \mathrm{D}=+24.9\left(\mathrm{c} 0.78, \mathrm{CHCl}_{3}\right), 96 \%\right.$ ee]; $94 \%$ ee, determined by HPLC, HPLC conditions: Chiralcel OJ-H, n-hexane/i-PrOH = 92/8, $1 \mathrm{~mL} / \mathrm{min}, \mathrm{n}=214$ nm, $\mathrm{t}_{\mathrm{r}} 8.1$ (major), 8.9 (minor); ${ }^{1} \mathrm{H} \mathrm{NMR}\left(400 \mathrm{MHz}, \mathrm{CDCl}_{3}\right) \delta 7.86-7.76$ (m, 3H), 7.72 (s, 1H), $7.47(\mathrm{td}, J=6.8,1.3 \mathrm{~Hz}, 2 \mathrm{H}), 7.36(\mathrm{dd}, J=8.5,1.6 \mathrm{~Hz}, 1 \mathrm{H}), 6.51(\mathrm{~s}, 2 \mathrm{H}), 4.28$ $(\mathrm{q}, J=7.1 \mathrm{~Hz}, 1 \mathrm{H}), 3.86(\mathrm{~s}, 3 \mathrm{H}), 3.82(\mathrm{~s}, 6 \mathrm{H}), 1.75(\mathrm{~d}, J=7.2 \mathrm{~Hz}, 3 \mathrm{H}) .{ }^{13} \mathrm{C} \mathrm{NMR}(101$ $\left.\mathrm{MHz}, \mathrm{CDCl}_{3}\right) \delta 153.2,143.7,142.0,136.3,133.6,132.2,128.0,127.8,127.7,126.8$, $126.1,125.5,125.3,104.9,60.9,56.2,45.2,22.0$. These spectroscopic data correspond to reported data. ${ }^{28}$

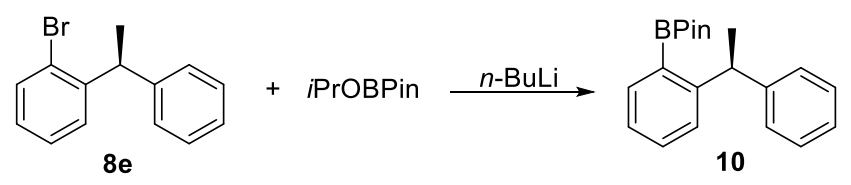

(S)-4,4,5,5-tetramethyl-2-(2-(1-phenylethyl)phenyl)-1,3,2-dioxaborolane

(10).

After a solution of $\mathbf{8 e}(65 \mathrm{mg}, 0.25 \mathrm{mmol})$ in $\mathrm{THF}(10 \mathrm{~mL})$ was cooled to $-78{ }^{\circ} \mathrm{C}, \mathrm{n}$ - 
$\operatorname{BuLi}(0.5 \mathrm{mmol}, 0.2 \mathrm{~mL}, 2.5 \mathrm{M}$ in hexane $)$ was added dropwise. After stirring at $-78^{\circ} \mathrm{C}$ for $1 \mathrm{~h}, 2$-isopropoxy-4,4,5,5-tetramethyl-1,3,2-dioxaborolane (98 mg, $0.525 \mathrm{mmol}$,) was added dropwise. After $10 \mathrm{~min}$, the reaction was quenched at $-78^{\circ} \mathrm{C}$ with sat. $\mathrm{NH}_{4} \mathrm{Cl}$ (aq, $10 \mathrm{~mL}$ ) and $\mathrm{H}_{2} \mathrm{O}(10 \mathrm{~mL})$. After warming to room temperature, mixture was extracted with $\mathrm{Et}_{2} \mathrm{O}(3 \times 60 \mathrm{~mL})$. The combined organic extracts were washed with brine $(30 \mathrm{~mL})$, dried over anhydrous $\mathrm{Na}_{2} \mathrm{SO}_{4}$, and filtered. The filtrate was concentrated under reduced pressure and purified by flash chromatography $(\mathrm{PE} / \mathrm{EA}=10 / 1)$ to afford $10(72.0 \mathrm{mg}, 93 \%$ yield $)$ as a slightly yellow oil. $[\boldsymbol{\alpha}]^{\mathbf{2 6}} \mathrm{D}=-59.6\left(\mathrm{c} 1.00, \mathrm{CHCl}_{3}\right) ; 94 \%$ ee, determined by HPLC, HPLC conditions: Chiralcel OJ-H, n-hexane/i-PrOH = 95/5, $1 \mathrm{~mL} / \mathrm{min}, \mathrm{n}=214 \mathrm{~nm}, \mathrm{t}_{\mathrm{r}} 5.2$ (major), 5.7 (minor); ${ }^{1} \mathrm{H} \mathrm{NMR}\left(400 \mathrm{MHz}, \mathrm{CDCl}_{3}\right) \delta 7.69$ $(\mathrm{dd}, J=7.4,1.2 \mathrm{~Hz}, 1 \mathrm{H}), 7.27(\mathrm{td}, J=7.8,1.5 \mathrm{~Hz}, 1 \mathrm{H}), 7.20-7.14(\mathrm{~m}, 4 \mathrm{H}), 7.13-$ $7.02(\mathrm{~m}, 3 \mathrm{H}), 4.99(\mathrm{q}, J=7.2 \mathrm{~Hz}, 1 \mathrm{H}), 1.52(\mathrm{~d}, J=7.2 \mathrm{~Hz}, 3 \mathrm{H}), 1.22(\mathrm{~d}, J=6.8 \mathrm{~Hz}$, 12H). ${ }^{13} \mathrm{C}$ NMR (101 MHz, CDCl3) $\delta$ 153.1, 147.5, 136.0, 131.1, 128.1, 127.0, 125.7, 125.3, 83.6, 42.0, 25.0, 24.9, 22.6. HRMS (EI) m/z: $[\mathrm{M}]^{+}$Calcd for $\mathrm{C}_{20} \mathrm{H}_{25} \mathrm{BO}_{2}$ 308.1953; Found 308.1942.

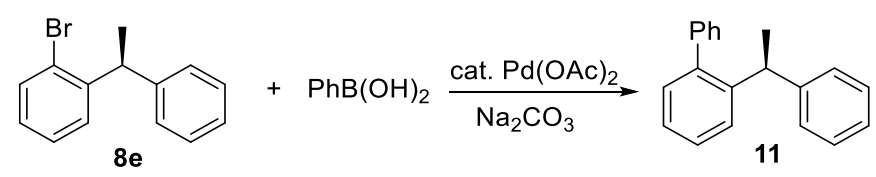

(S)-2-(1-phenylethyl)-1,1'-biphenyl (11). Prepared according to previously reported procedure with some modifications. ${ }^{29}$ A $50 \mathrm{~mL}$ of flame-dried Schlenk flask was charged with 8e (65mg, $0.25 \mathrm{mmol}), \mathrm{Pd}(\mathrm{OAc})_{2}(0.6 \mathrm{mg}, 0.25 \mathrm{~mol} \%)$ phenylboronic acid (45 mg, $0.375 \mathrm{mmol}$ ), $\mathrm{Na}_{2} \mathrm{CO}_{3}(106 \mathrm{mg}, 0.5 \mathrm{mmol}), \mathrm{H}_{2} \mathrm{O}(2 \mathrm{~mL})$ and DMF (2 mL). After the mixture was stirred at $35{ }^{\circ} \mathrm{C}$ in an oil bath for 1 hour, water $(10 \mathrm{~mL})$ was added, the resulting mixture was extracted with EA $(3 \times 5 \mathrm{~mL})$. The combined organic layers were dried over anhydrous $\mathrm{Na}_{2} \mathrm{SO}_{4}$, filtered, concentrated by rotary evaporation and further purified by flash chromatography $(\mathrm{PE} / \mathrm{EA}=10 / 1)$ to afford $11(56.7 \mathrm{mg}$, $88 \%$ yield) as colorless oil. $[\alpha]^{27} \mathbf{D}=-43.4\left(\right.$ c $\left.1.20, \mathrm{CHCl}_{3}\right) ; 94 \%$ ee, determined by HPLC, HPLC conditions: Chiralcel OJ-H, n-hexane/i-PrOH = 99.2/0.8, $1 \mathrm{~mL} / \mathrm{min}, \mathrm{n}=$ $214 \mathrm{~nm}, \mathrm{t}_{\mathrm{r}} 8.8$ (major), 11.9 (minor); ${ }^{1} \mathrm{H}$ NMR (400 MHz, CDCl3) $\delta 7.43-6.99$ (m, $14 \mathrm{H}), 4.28(\mathrm{q}, J=7.1 \mathrm{~Hz}, 1 \mathrm{H}), 1.53(\mathrm{~d}, J=7.0 \mathrm{~Hz}, 3 \mathrm{H}) .{ }^{13} \mathrm{C} \mathrm{NMR}\left(101 \mathrm{MHz}, \mathrm{CDCl}_{3}\right)$ 
$\delta$ 146.6, 144.1, 142.0, 141.9, 130.1, 129.5, 128.3, 128.1, 127.8, 127.7, 127.7, 127.0, 125.8, 125.8, 40.3, 22.4. HRMS (EI) m/z: [M] Calcd for $\mathrm{C}_{20} \mathrm{H}_{18}$ 258.1406; Found 258.1403.

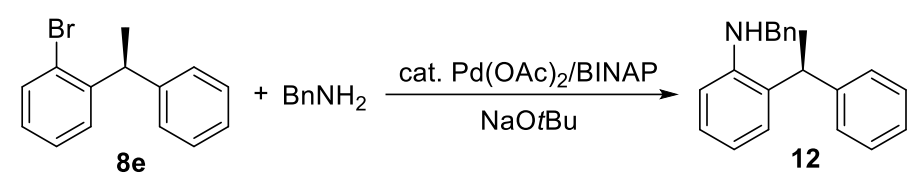

(S)-N-benzyl-2-(1-phenylethyl)aniline (12). Prepared according to previously reported procedure with some modifications. ${ }^{30}$ Under inert atmosphere, palladium acetate $(4 \mathrm{mg}, 0.0175 \mathrm{mmol})$, BINAP $(24 \mathrm{mg}, 0.034 \mathrm{mmol})$, and sodium $t$-butoxide (104 mg, $1.08 \mathrm{mmol})$ were added to toluene $(25 \mathrm{~mL})$. After the mixture was stirred for $25 \mathrm{~min}$. benzylamine $(43 \mathrm{mg}, 0.398 \mathrm{mmol})$ and 8 e $(65 \mathrm{mg}, 0.25 \mathrm{mmol})$ were added. After stirring at $100{ }^{\circ} \mathrm{C}$ in an oil bath for 16 hours, the reaction mixture was quenched with water $(5 \mathrm{~mL})$ and extracted with EA $(3 \times 5 \mathrm{~mL})$. The combined organic layers were dried over anhydrous $\mathrm{Na}_{2} \mathrm{SO}_{4}$, filtered, concentrated by rotary evaporation and further purified by flash chromatography $(\mathrm{PE} / \mathrm{EA}=5 / 1)$ to afford $12(68 \mathrm{mg}, 95 \%$ yield $)$ as yellow oil. $[\alpha]^{\mathbf{2 6}} \mathbf{D}=+5.19\left(\mathrm{c} 1.00, \mathrm{CHCl}_{3}\right) ; 94 \%$ ee, determined by HPLC, HPLC conditions: Chiralcel OD-H, n-hexane $/ i-\mathrm{PrOH}=90 / 10,0.7 \mathrm{~mL} / \mathrm{min}, \mathrm{n}=254 \mathrm{~nm}, \mathrm{t}_{\mathrm{r}} 6.3$ (major), 6.7 (minor); ${ }^{1} \mathrm{H}$ NMR (400 MH z, $\left.\mathrm{CDCl}_{3}\right) \delta 7.31$ (d, $\left.J=7.4 \mathrm{~Hz}, 1 \mathrm{H}\right), 7.27-$ 7.09 (m, 9H), $7.00-6.88(\mathrm{~m}, 2 \mathrm{H}), 6.84-6.76(\mathrm{~m}, 1 \mathrm{H}), 6.57$ (d, J= $7.8 \mathrm{~Hz}, 1 \mathrm{H}), 4.21$ $-4.10(\mathrm{~m}, 2 \mathrm{H}), 4.03(\mathrm{q}, J=7.1 \mathrm{~Hz}, 1 \mathrm{H}), 3.86(\mathrm{~s}, 1 \mathrm{H}), 1.62(\mathrm{~d}, J=7.1 \mathrm{~Hz}, 3 \mathrm{H}) .{ }^{13} \mathrm{C}$ $\mathrm{NMR}\left(101 \mathrm{MHz}, \mathrm{CDCl}_{3}\right) \delta 145.8,145.5,139.3,129.2,128.9,128.6,127.6,127.6,127.2$, 127.1, 127.0, 126.5, 117.3, 111.1, 48.1, 40.3, 22.1. HRMS (EI) m/z: [M] Calcd for $\mathrm{C}_{21} \mathrm{H}_{21} \mathrm{~N} 287.1672$; Found 287.1669.

\section{References}

(1) Chen, J.; Chen, C.; Ji, C.; Lu, Z. Cobalt-Catalyzed Asymmetric Hydrogenation of 1,1-Diarylethenes. Org. Lett. 2016, 18, 1594-1597.

(2) Wang, X.; Zhao, Z. Spherical hollow mesoporous silica supported phosphotungstic 
acid as a promising catalyst for $\alpha$-arylstyrenes synthesis via Friedel-Crafts alkenylation. Chin. Chem. Lett. 2019, 30, 729-734.

(3) Sartori, G.; Bigi, F.; Pastorio, A.; Porta, C.; Arienti, A.; Maggi, R.; Moretti, N.; Gnappi, G. Electrophilic Alkenylation of Aromatics with Phenylacetylene over Zeolite HSZ-360. Tetrahedron Lett. 1995, 36, 9177-9180.

(4) Song, C. E.; Jung, D. U.; Choung, S. Y.; Roh, E. J.; Lee, S. G. Dramatic enhancement of catalytic activity in an ionic liquid: highly practical Friedel-Crafts alkenylation of arenes with alkynes catalyzed by metal triflates. Angew. Chem. Int. Ed. 2004, 43, 6183-6185.

(5) Sinai, Á.; Simkó, D. C.; Szabó, F.; Paczal, A.; Gáti, T.; Bényei, A.; Novák, Z.; Kotschy, A. Aryl-Diadamantyl Phosphine Ligands in Palladium-Catalyzed CrossCoupling Reactions: Synthesis, Structural Analysis, and Application. Eur. J. Org. Chem. 2020, 2020, 1122-1128.

(6) Dai, Q. P.; Zhao, B. G.; Yang, Y. H.; Shi, Y. A. Pd-Catalyzed Oxidative Heck Reaction of Grignard Reagents with Diaziridinone as Oxidant. Org. Lett. 2019, 21, 5157-5161.

(7) Kazuhiro, K.; Seiki, F.; Masanori, H.; Osamu, M.; Hisatoshi, K. Synthesis of 1(3H)Imino-2-benzothiophene and 1-Imino-1H-2-benzothiopyran Derivatives by Reactions of Secondary o-(Vinyl)thiobenzamide Derivatives with Iodine. Bull. Chem. Soc. Jpn. 2007, 80, 763-767.

(8) Hu, T. J.; Zhang, G.; Chen, Y. H.; Feng, C. G.; Lin, G.Q. Borylation of Olefin C-H Bond via Aryl to Vinyl Palladium 1,4-Migration. J. Am. Chem. Soc. 2016, 138, 2897-2900.

(9) Ogawa, N.; Yamaoka, Y.; Yamada, K.I.; Takasu, K. Synthesis of $\pi$-Extended Fluoranthenes via a KHMDS-Promoted Anionic-Radical Reaction Cascade. Org. Lett. 2017, 19, 3327-3330.

(10) Yang, D.; Long, Y.; Zhang, J.; Zeng, H.; Wang, S.; Li, C. Organometallics 2010 , 29, 3477.

(11)Sheldrick, G. M. SADABS: Program for Empirical Absorption Correction of Area Detector Data; University of Gottingen: Germany, 1996.

(12)Dolomanov, O.V., Bourhis, L.J., Gildea, R.J, Howard, J.A.K. \& Puschmann, H. OLEX2: A Complete Structure Solution, Refinement and Analysis Program. J. Appl. Cryst. 2009, 42, 339-341.

(13)Sheldrick, G. M. SHELXT-Integrated Space-Group and Crystal-Structure Determination. Acta Cryst. 2015, A71, 3-8.

(14) Ando, A. (Pfizer Inc). U.S. Patent 5,814,648, 1998.

(15)Myllymaki, M. J.; Kasnanen, H.; Kataja, A. O.; Lahtela-Kakkonen, M.; Saario, S. M.; Poso, A.; Koskinen, A. M. Chiral 3-(4,5-dihydrooxazol-2-yl)phenyl alkylcarbamates as novel FAAH inhibitors: Insight into FAAH enantioselectivity by molecular docking and interaction fields. Eur. J. Med. Chem. 2009, 44, 4179-91.

(16) Yilmaz, M. Synthesis of dihydrofurans containing trifluoromethyl ketone and heterocycles by radical cyclization of fluorinated 1,3-dicarbonyl compounds with 2-thienyl and 2-furyl substituted alkenes. Tetrahedron 2011, 67, 8255.

(17) Jongsma, C.; Bickelhaupt, F. Nucleophilic substitution of aromatic bromine in 2- 
bromobenzophenone by Grignard reagents. Recl. Trav. Chim. Pays-Bas. 1973, 92, 1143-1151.

(18) Chu, C. M.; Huang, W. J.; Liu, J. T.; Yao, C. F. Highly efficient iodine-catalyzed hydroarylation of arenes with styrenes. Tetrahedron Lett. 2007, 48, 6881-6885.

(19) Tang, R. J.; Milcent, T.; Crousse, B. Bisulfate Salt-Catalyzed Friedel-Crafts Benzylation of Arenes with Benzylic Alcohols. J. Org. Chem. 2018, 83, 1400114009.

(20) Ang, H. T.; Rygus, J. P. G.; Hall, D.G. Two-component boronic acid catalysis for increased reactivity in challenging Friedel-Crafts alkylations with deactivated benzylic alcohols. Org. Biomol. Chem. 2019, 17, 6007-6014.

(21)Chen, Y. G.; Shuai, B.; Xu, X. T.; Li, Y. Q.; Yang, Q. L.; Qiu, H.; Zhang, K.; Fang, P.; Mei, T. S. Nickel-catalyzed Enantioselective Hydroarylation and Hydroalkenylation of Styrenes. J. Am. Chem. Soc. 2019, 141, 3395-3399.

(22)Dai, Q. P.; Zhao, B. G.; Yang, Y. H.; Shi, Y. A. Pd-Catalyzed Oxidative Heck Reaction of Grignard Reagents with Diaziridinone as Oxidant. Org. Lett. 2019, 21, 5157-5161.

(23)Dine, T. M. E.; Erb, W.; Berhault, Y.; Rouden, J.; Blanchet, J. Catalytic Chemical Amide Synthesis at Room Temperature: One More Step Toward Peptide Synthesis. J. Org. Chem. 2015, 80, 4532-4544.

(24) Yuen, K.; Cheng, Z.; Cheng, C. C. Synthesis of Compounds Related to 2-(ochlorophenyl)-2-(p-chlorophenyl)-1,1-dichloroethanea. J. Med. Chem. 1962, 5, 1008-1015.

(25) Jones. (Imperial Chemical Industries PLC). U.S. Patent 4,98,997, 1990.

(26) Odachowski, M.; Bonet, A.; Essafi, S.; Conti-Ramsden, P.; Harvey, J. N. Daniele Leonori, V.; Aggarwal, K. Development of Enantiospecific Coupling of Secondary and Tertiary Boronic Esters with Aromatic Compounds. J. Am. Chem. Soc. 2016, 138, 9521-9532.

(27)Bess, E. N.; Sigman, M. S. Distinctive Meta-Directing Group Effect for IridiumCatalyzed 1,1-Diarylalkene Enantioselective Hydrogenation. Org. Lett. 2013, 15, 646-649.

(28) Buck L. H.; Taylor, E. C. S.; Joshua D. Waetzig.; Elizabeth R. Jarvo. Stereospecific Nickel-Catalyzed Cross-Coupling Reactions of Alkyl Ethers: Enantioselective Synthesis of Diarylethanes. J. Am. Chem. Soc. 2010. 133, 389-391.

(29) Liu, L. F.; Zhang, Y. H.; Xin, B. W. Synthesis of Biaryls and Polyaryls by LigandFree Suzuki Reaction in Aqueous Phase. J. Org. Chem. 2006, 71, 3994-3997.

(30) Seiders, T. J.; Ward, D. W.; Grubbs, R. H. Enantioselective Ruthenium-Catalyzed Ring-Closing Metathesis. Org. Lett. 2001, 3, 3225-3228. 


\section{NMR Spectra}

$\stackrel{9}{9}$

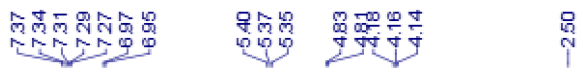<smiles>Oc1cccc(C2=NC(c3ccccc3)CO2)c1</smiles>
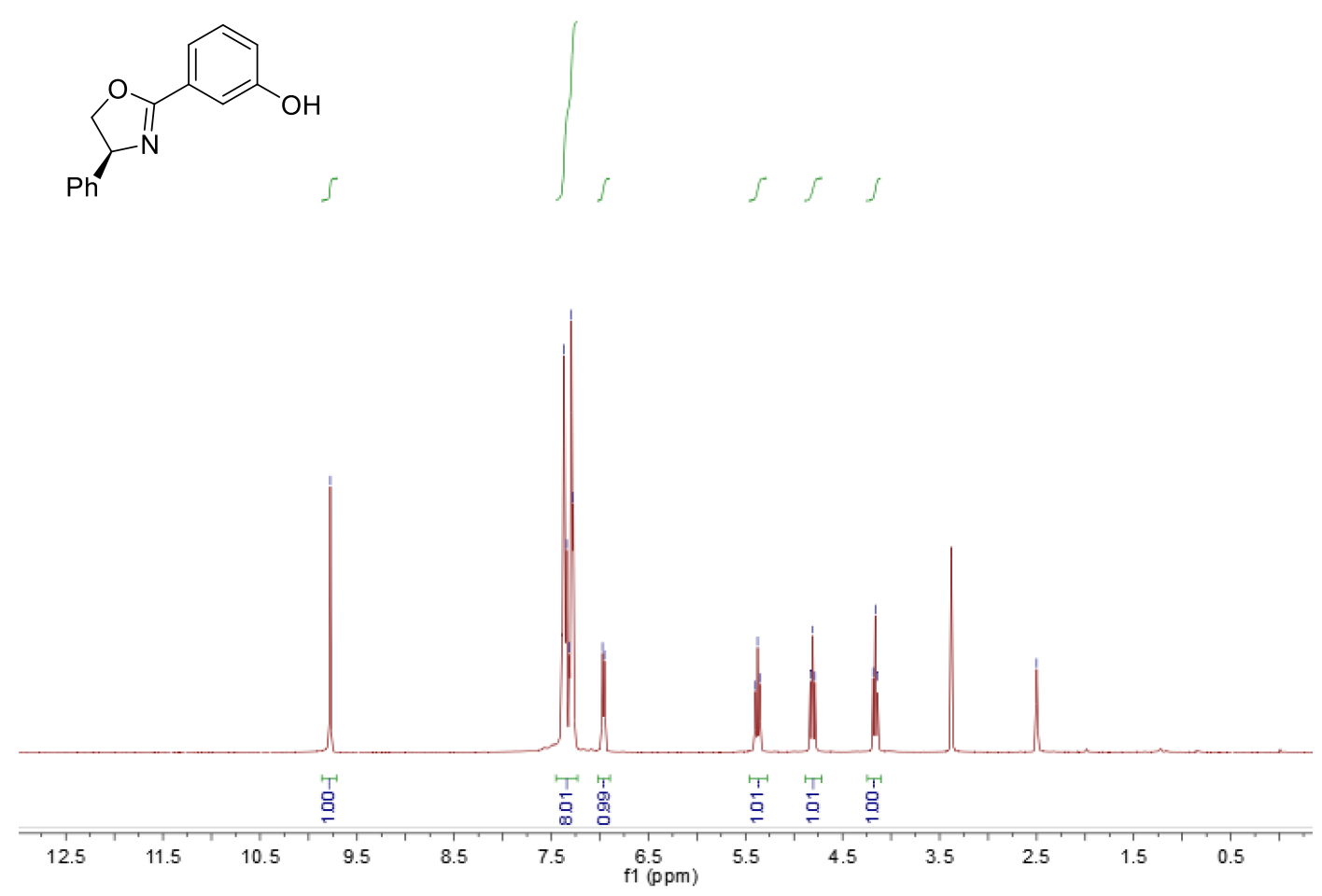

${ }^{1} \mathrm{H}$ NMR (400M, DMSO) spectrum of $\mathbf{1 a}$

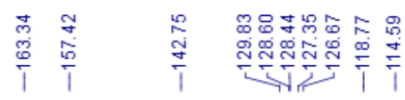

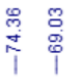

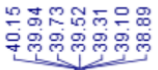

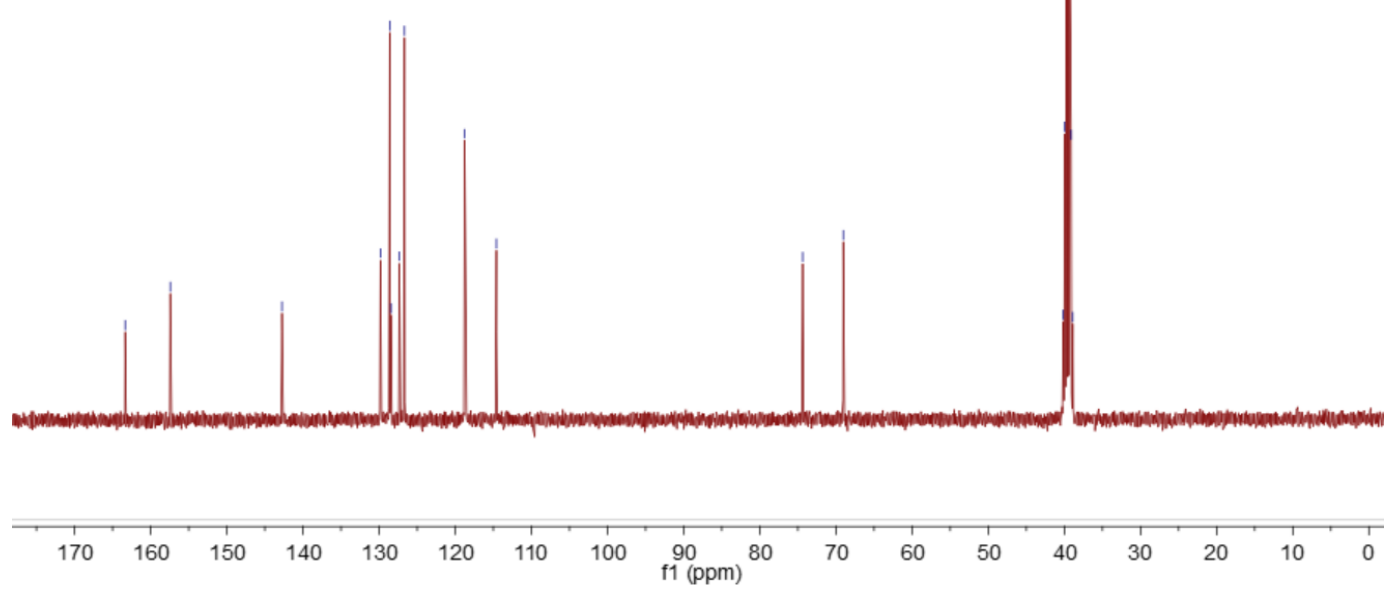

${ }^{13} \mathrm{C}$ NMR (101M, DMSO) spectrum of 1a 


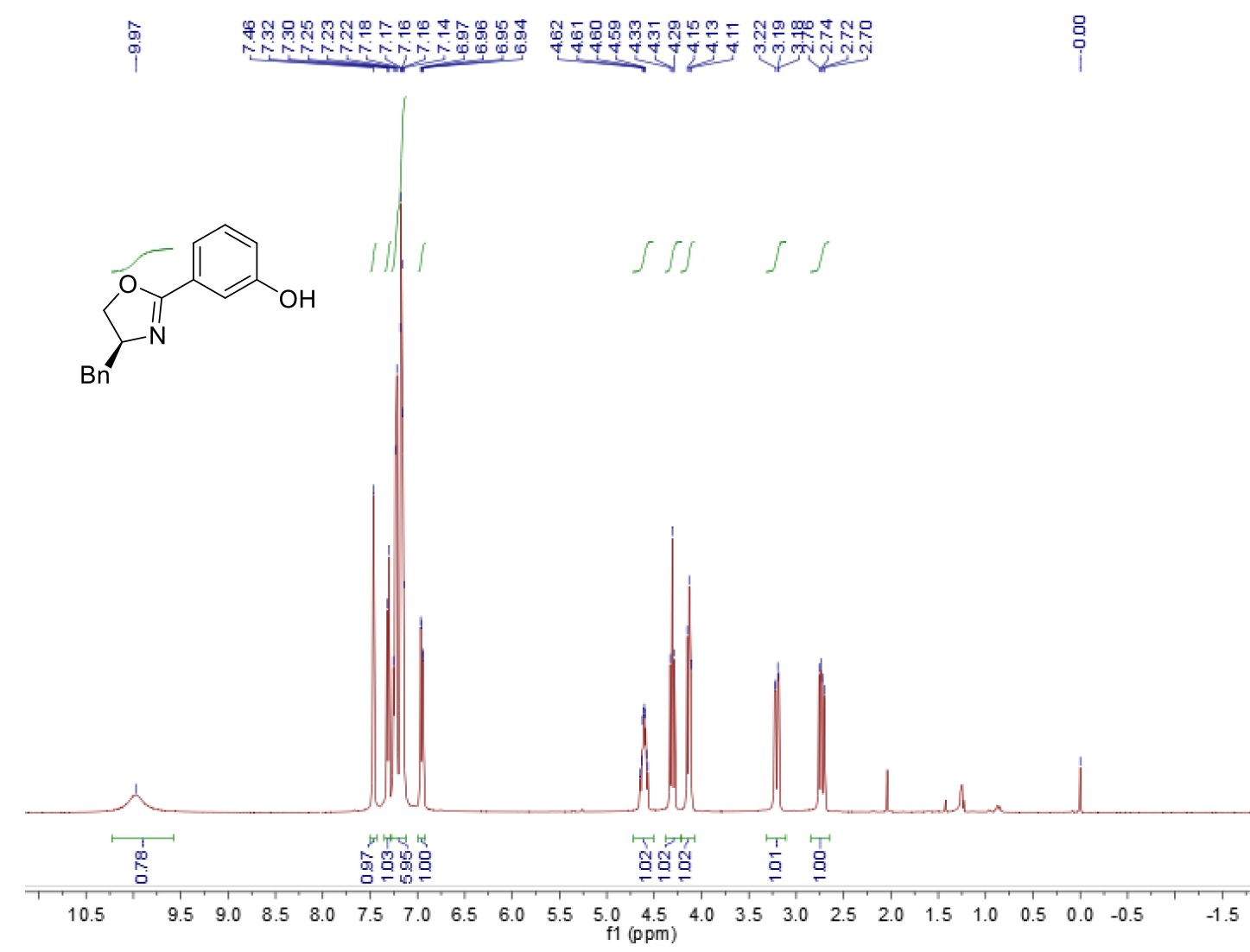

${ }^{1} \mathrm{H}$ NMR (400M, $\mathrm{CHCl}_{3}$ ) spectrum of $\mathbf{1 b}$

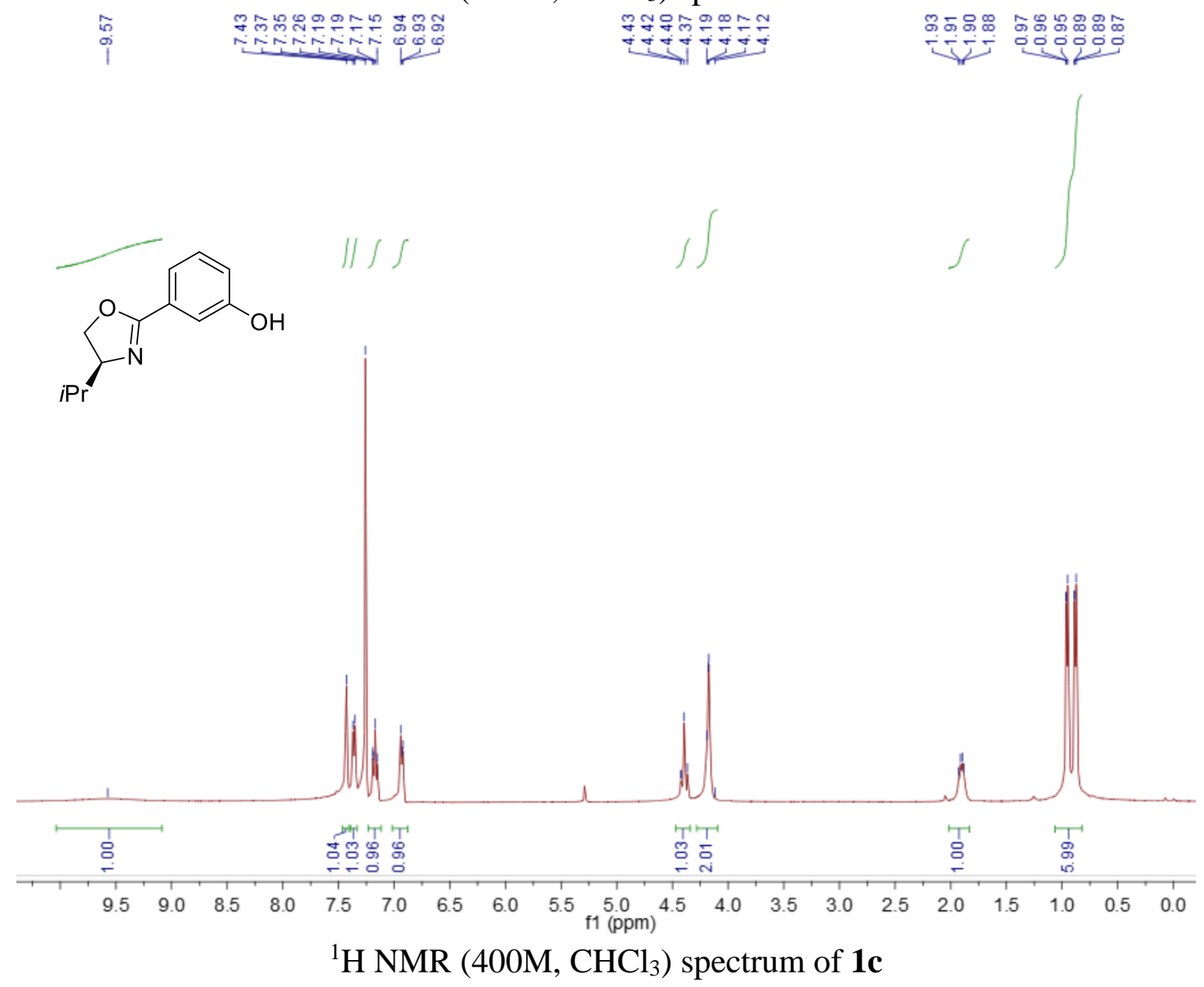



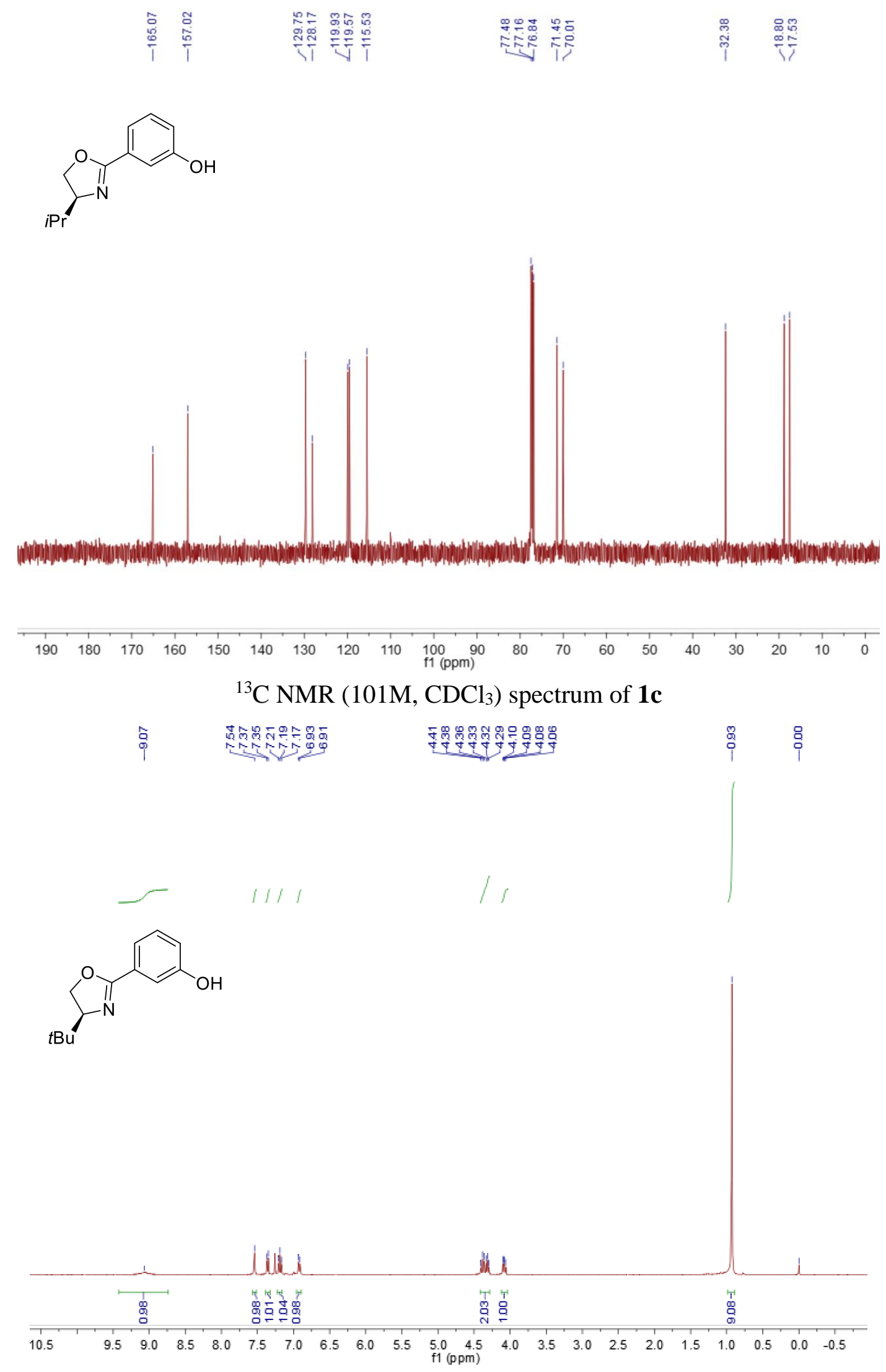

${ }^{1} \mathrm{H}$ NMR (400M, $\mathrm{CHCl}_{3}$ ) spectrum of $\mathbf{1 d}$ 


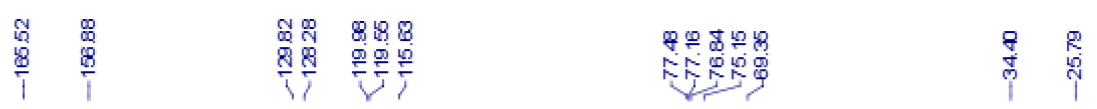<smiles>[13CH3]C1COC(c2cccc(O)c2)=N1</smiles>

\begin{tabular}{|c|c|c|c|c|c|c|c|c|c|c|c|c|c|c|c|c|c|}
\hline 180 & 170 & 160 & 150 & 140 & 130 & 120 & 110 & 100 & $\begin{array}{c}90 \\
\mathrm{f} 1(\mathrm{ppm})\end{array}$ & 80 & 70 & 60 & & & & & 10 \\
\hline
\end{tabular}

${ }^{13} \mathrm{C}$ NMR $\left(101 \mathrm{M}, \mathrm{CDCl}_{3}\right)$ spectrum of $\mathbf{1 d}$

\begin{tabular}{|c|}
\hline 崽 \\
\hline
\end{tabular}

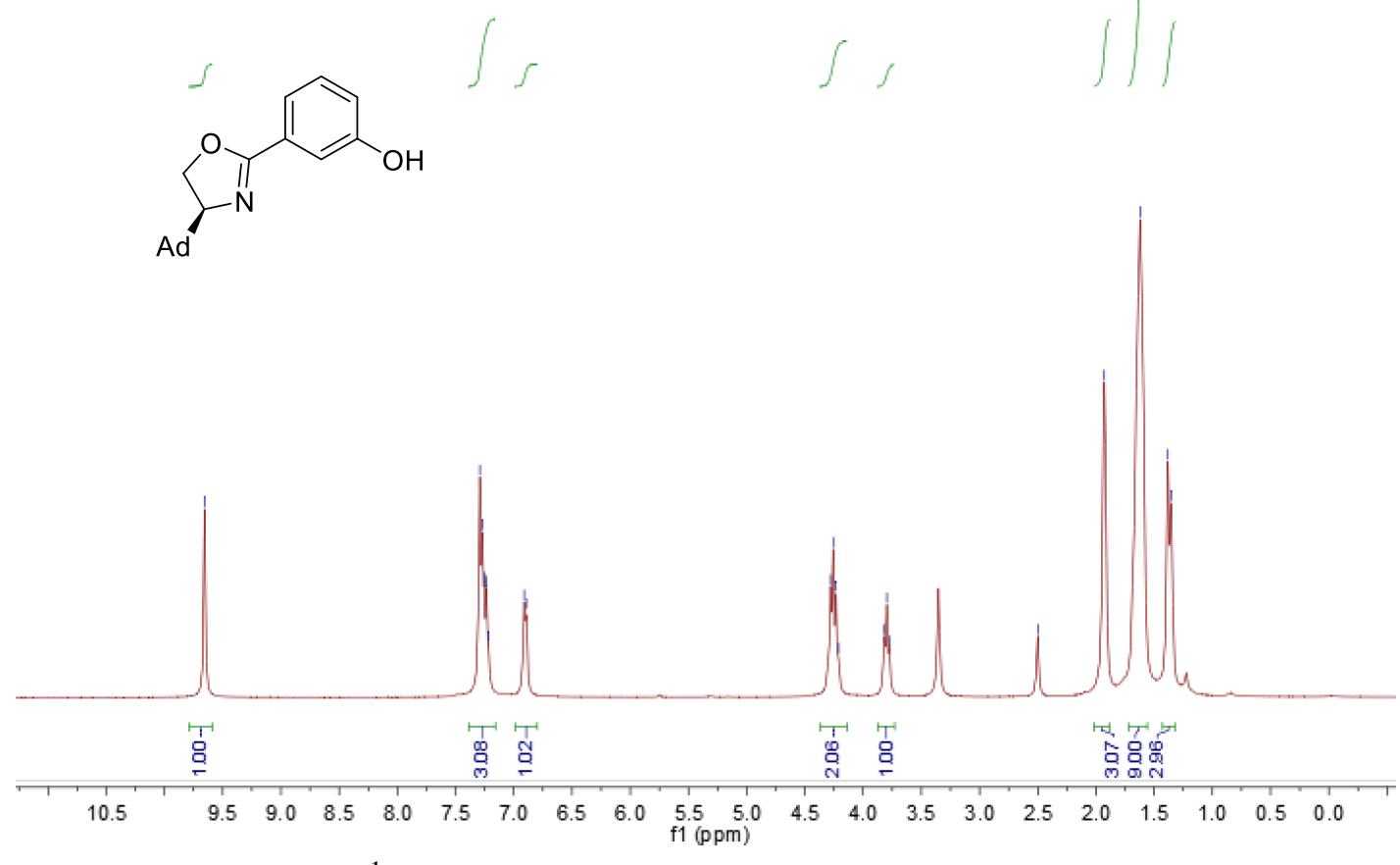

${ }^{1} \mathrm{H}$ NMR (400M, DMSO) spectrum of $\mathbf{1 e}$ 


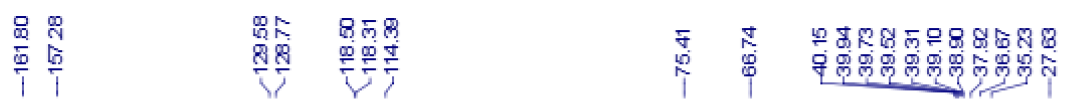<smiles>Oc1cccc(C2=NC([Ge])CO2)c1</smiles>

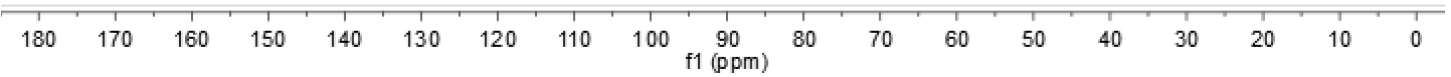

${ }^{13} \mathrm{C}$ NMR (101M, DMSO) spectrum of $\mathbf{1 e}$

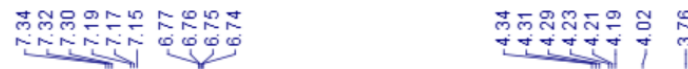<smiles>Nc1cccc(C2=NC(Cc3ccccc3)CO2)c1</smiles>

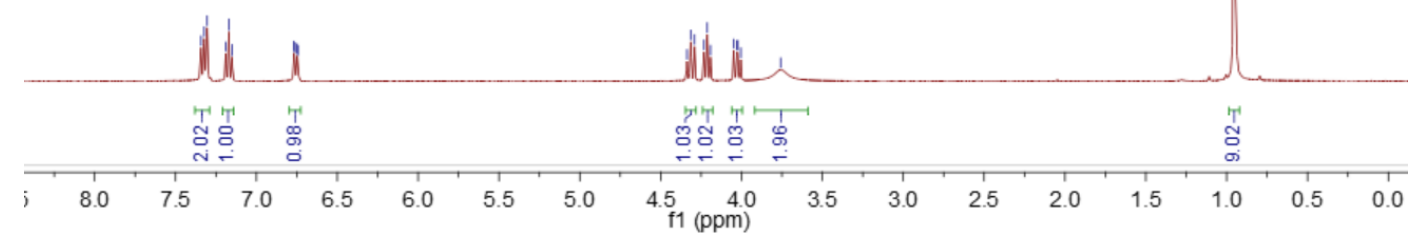

${ }^{1} \mathrm{H}$ NMR (400M, $\mathrm{CHCl}_{3}$ ) spectrum of 2 
<smiles>[13CH3]C1COC(c2cccc(N)c2)=N1</smiles>
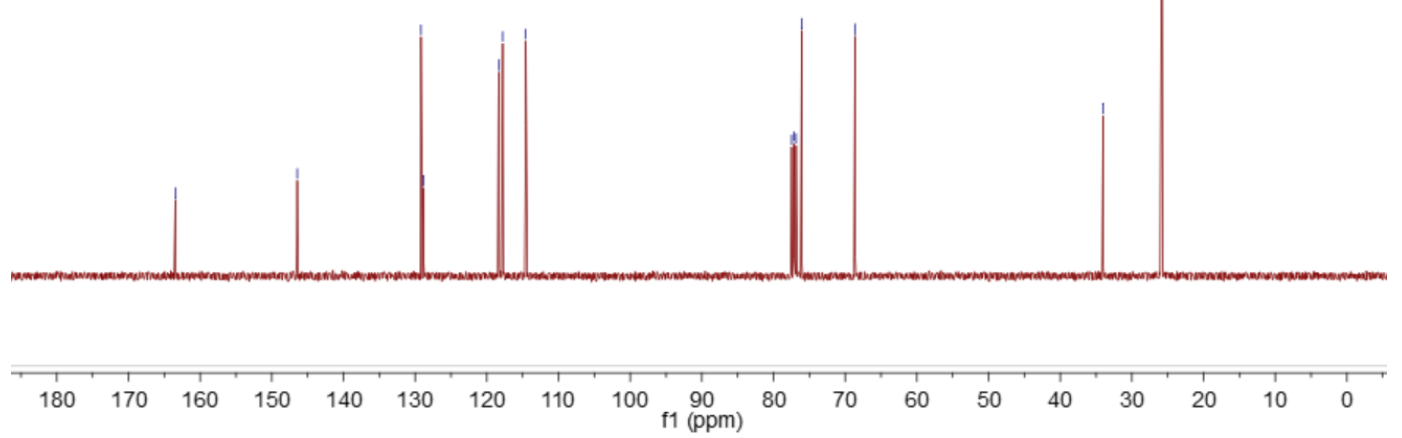

${ }^{13} \mathrm{C} \mathrm{NMR}\left(101 \mathrm{M}, \mathrm{CDCl}_{3}\right)$ spectrum of 2

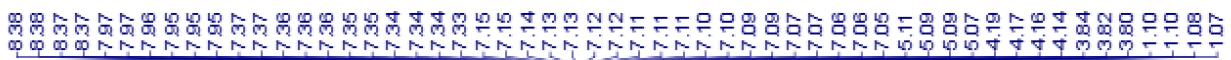<smiles>CCCCOc1cccc(C2=NC(c3ccccc3)CO2)c1</smiles>
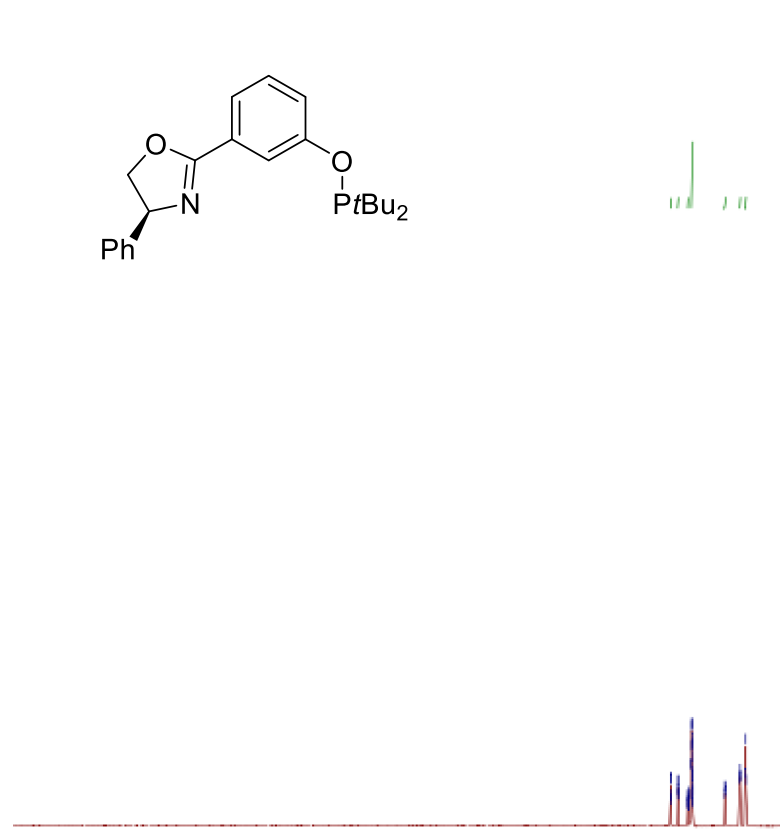

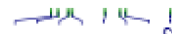
薄8 8 8

$\begin{array}{lllllllll}45 & 40 & 35 & 30 & 25 & 20 & 15 & 10 & \left.\begin{array}{c}5 \\ \mathrm{f} 1(\mathrm{ppm})\end{array}\right)\end{array}$

${ }^{1} \mathrm{H}$ NMR (400M, $\left.\mathrm{C}_{6} \mathrm{D}_{6}\right)$ spectrum of $\mathbf{3 a}$ 


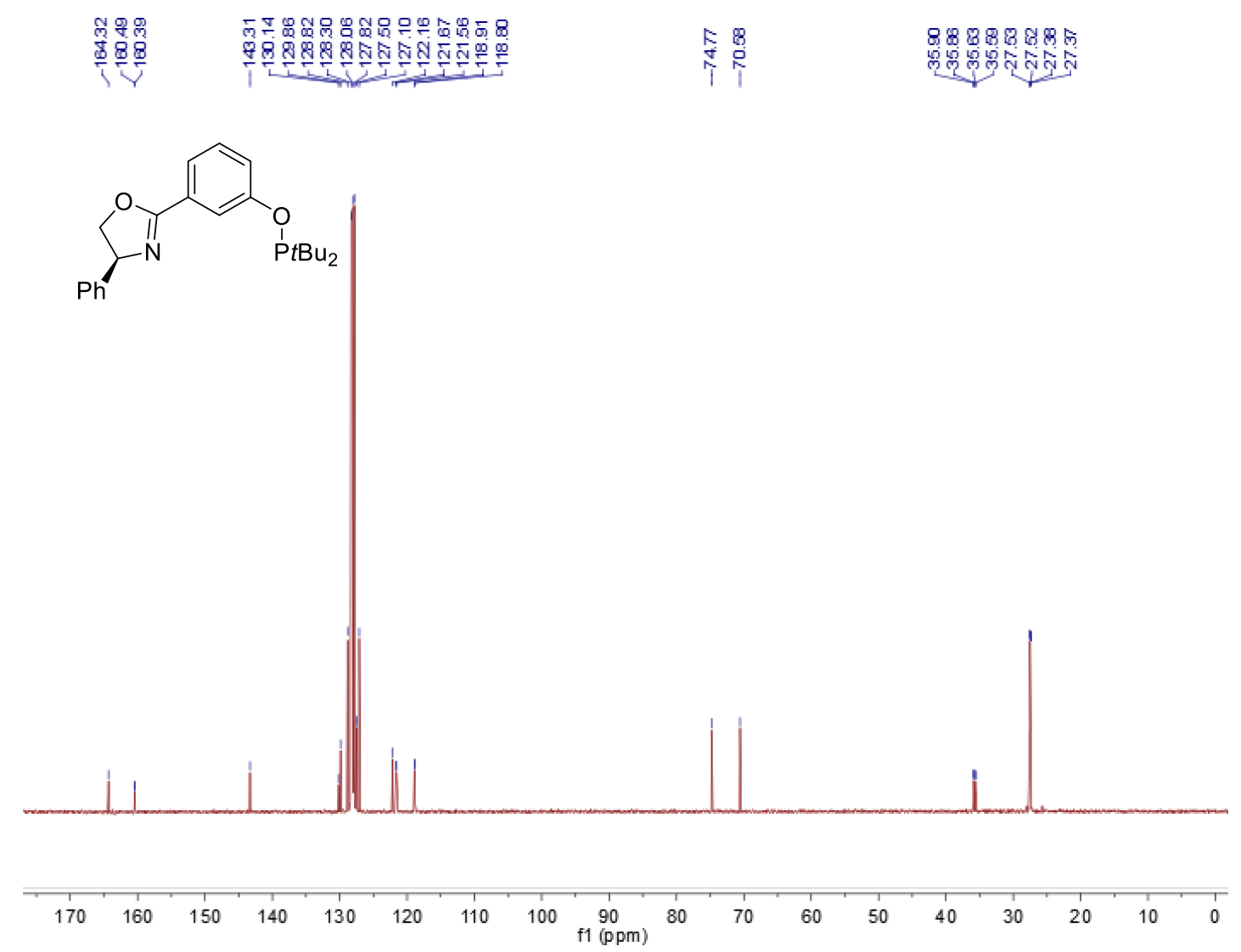

${ }^{13} \mathrm{C}$ NMR (162M, $\left.\mathrm{C}_{6} \mathrm{D}_{6}\right)$ spectrum of $\mathbf{3 a}$

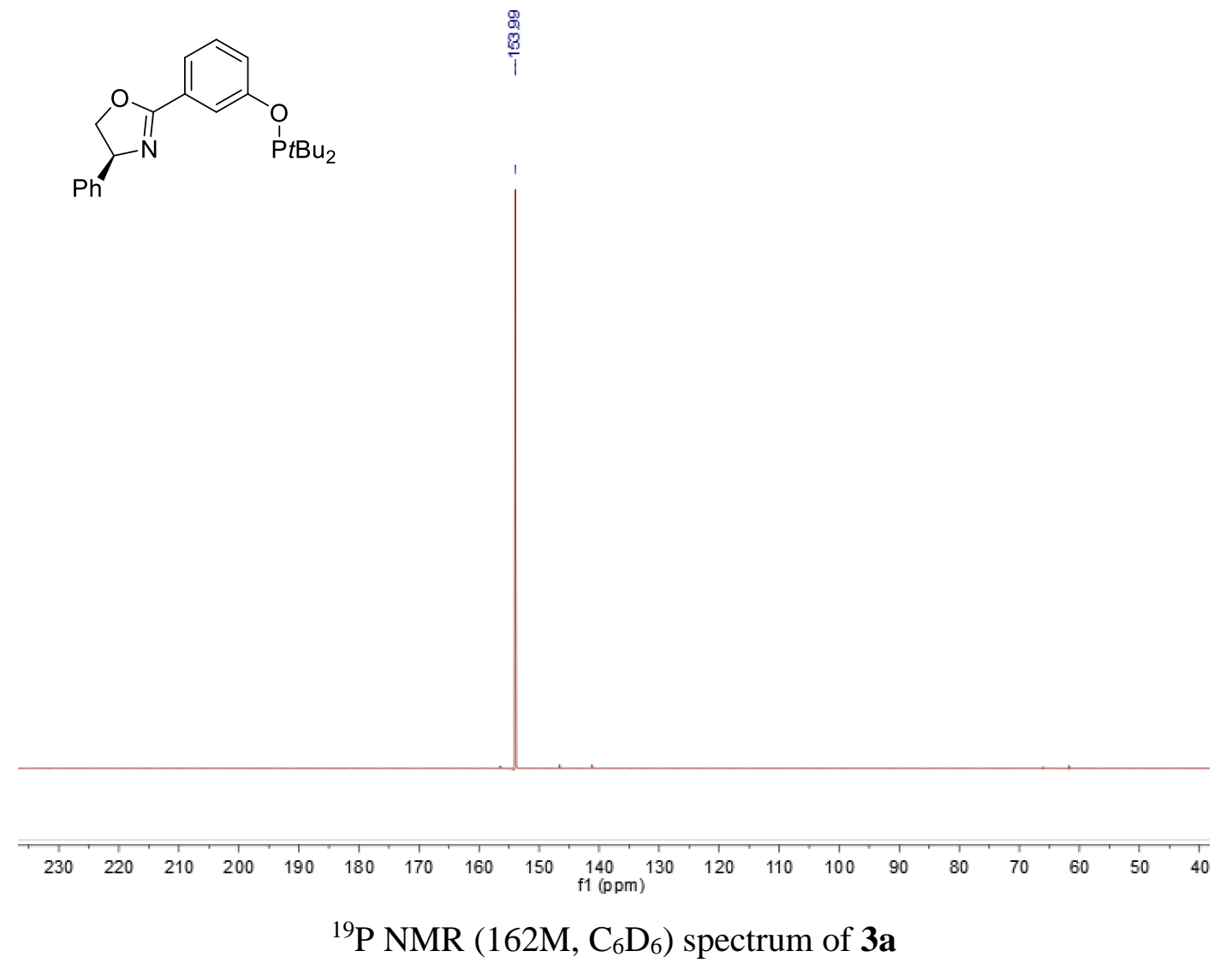


<smiles>CCCCOc1cccc(C2=NC(Cc3ccccc3)CO2)c1</smiles>

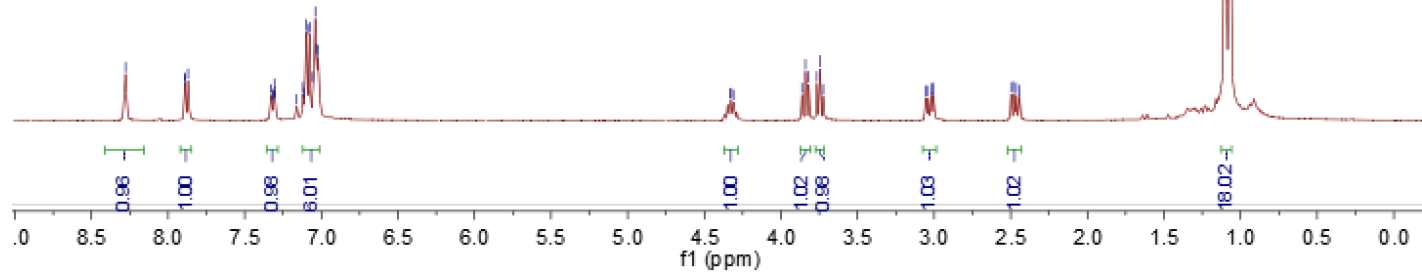

${ }^{1} \mathrm{H}$ NMR (400M, $\mathrm{C}_{6} \mathrm{D}_{6}$ ) spectrum of $\mathbf{3 b}$

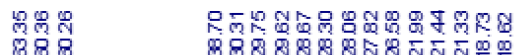

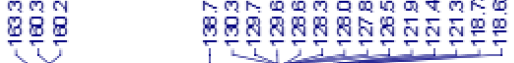
急 㝵

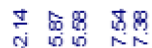

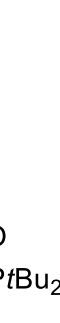<smiles>CCCCOc1cccc(C2=N[C@H](Cc3ccccc3)CO2)c1</smiles>
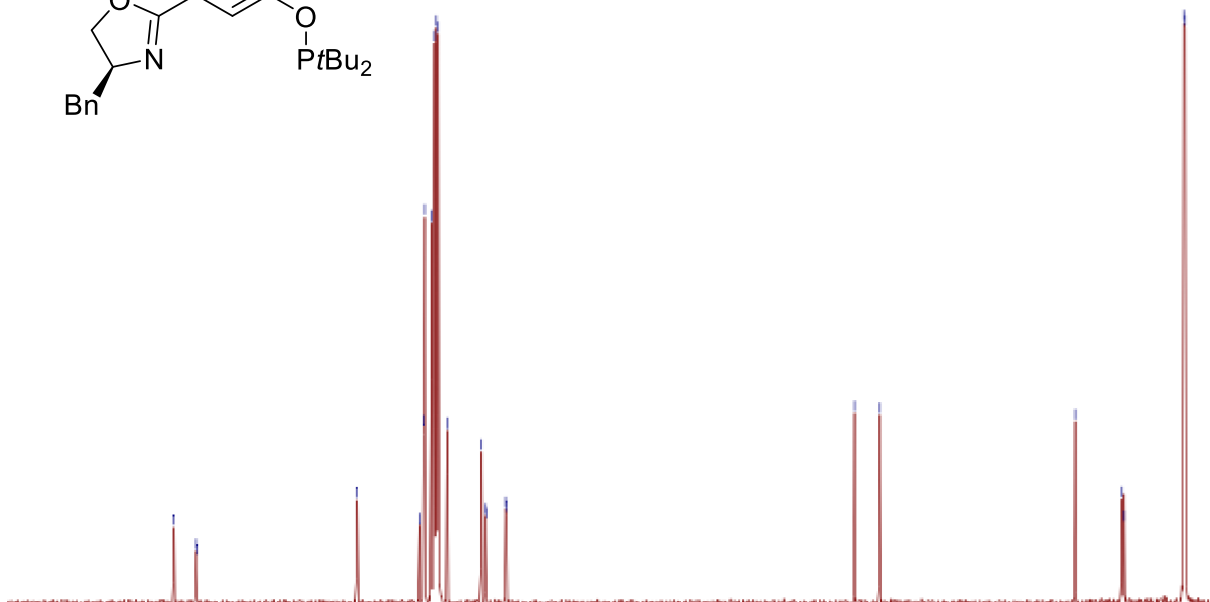

$180 \quad 170 \quad 160$
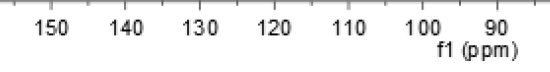

${ }^{13} \mathrm{C}$ NMR $\left(162 \mathrm{M}, \mathrm{C}_{6} \mathrm{D}_{6}\right)$ spectrum of $\mathbf{3 b}$ 

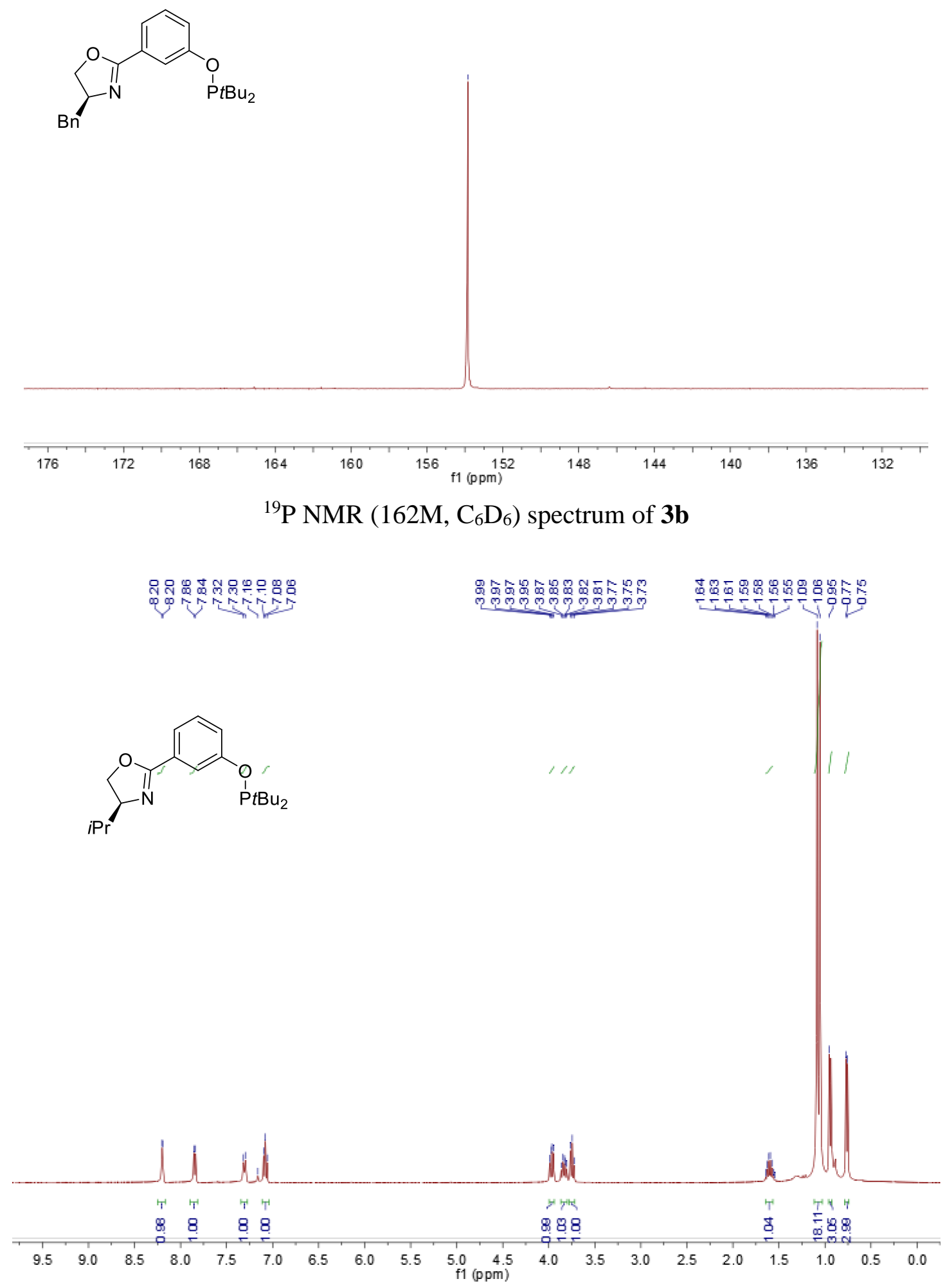

${ }^{1} \mathrm{H}$ NMR (400M, $\mathrm{C}_{6} \mathrm{D}_{6}$ ) spectrum of $\mathbf{3 c}$ 

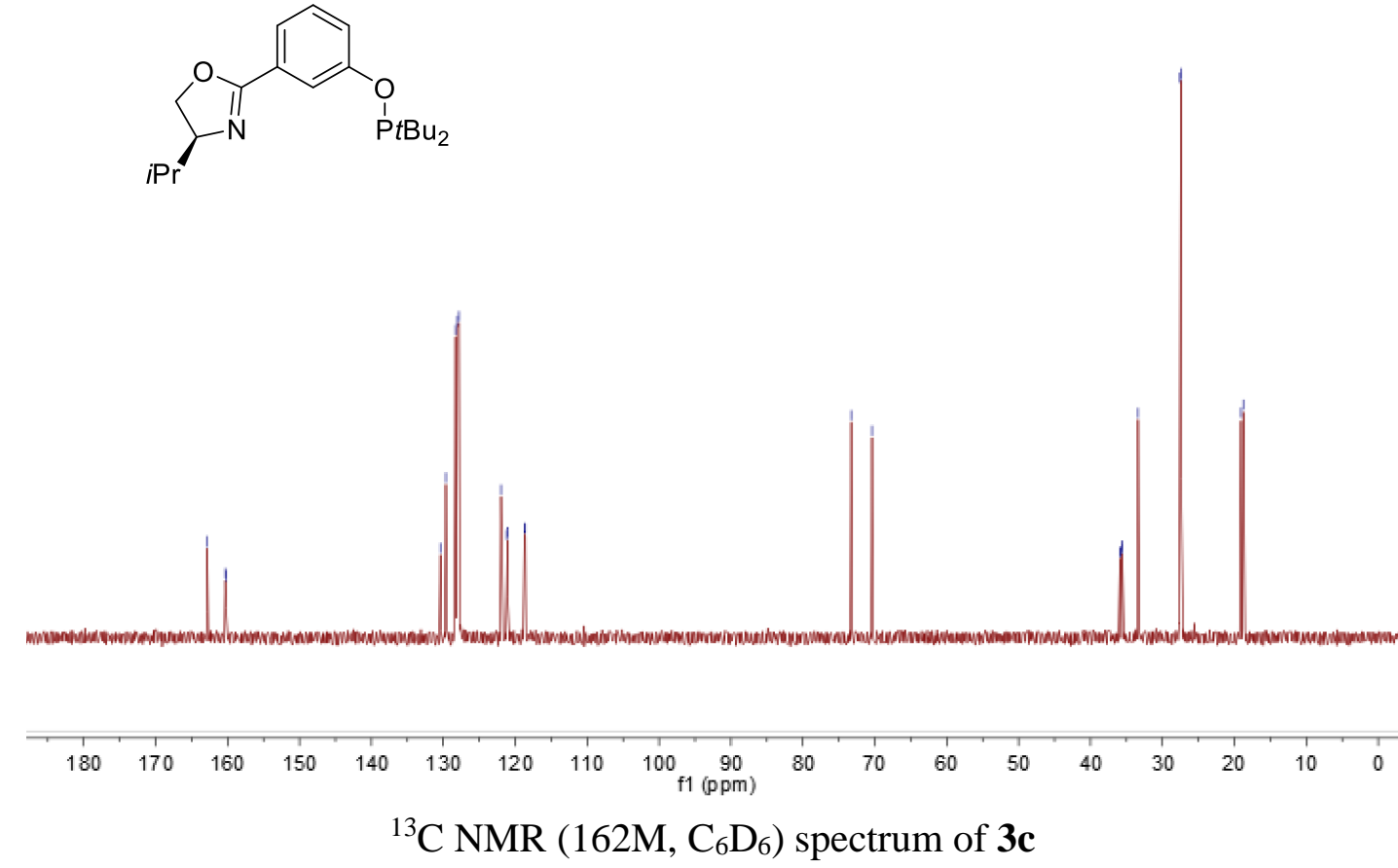

$\stackrel{\frac{0}{8}}{\frac{8}{1}}$<smiles>CCCCOc1cccc(C2=NC(C(C)C)CO2)c1</smiles>

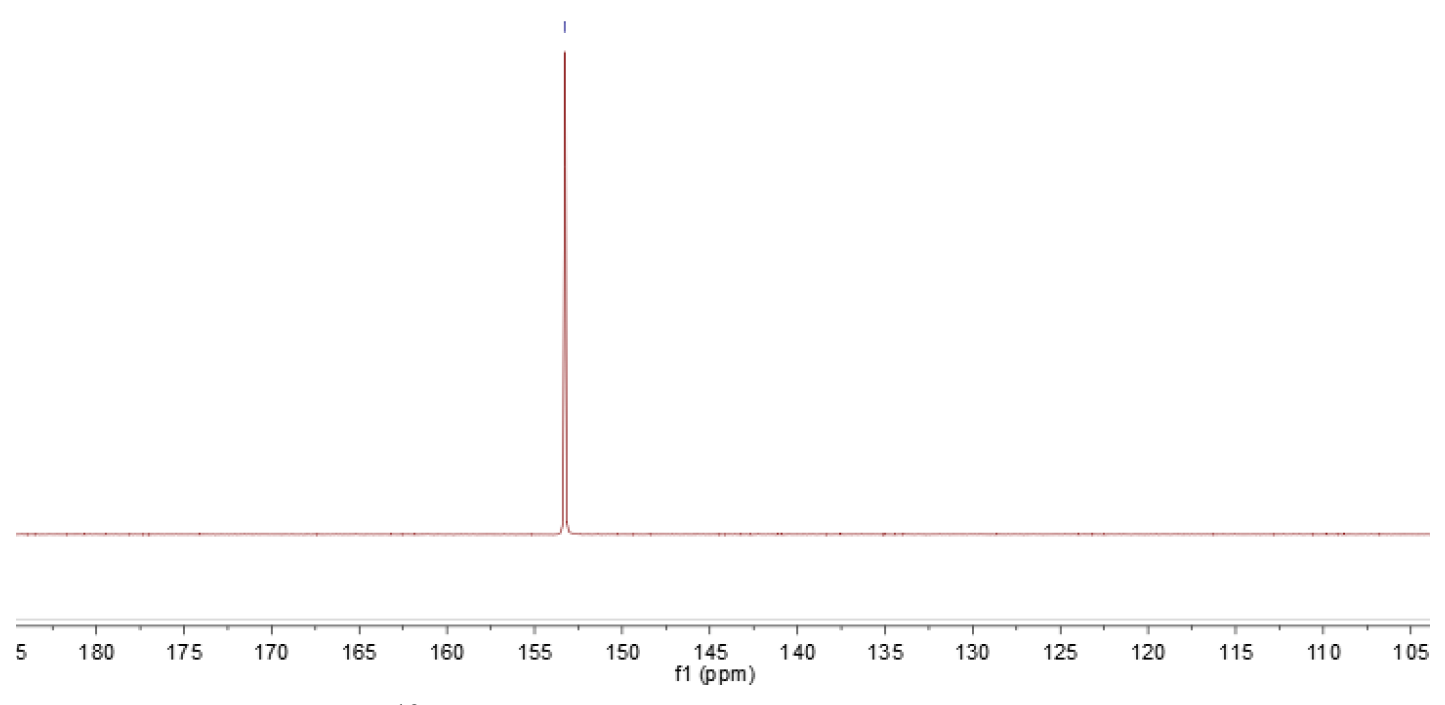

${ }^{19} \mathrm{P}$ NMR $\left(162 \mathrm{M}, \mathrm{C}_{6} \mathrm{D}_{6}\right)$ spectrum of $\mathbf{3 c}$ 


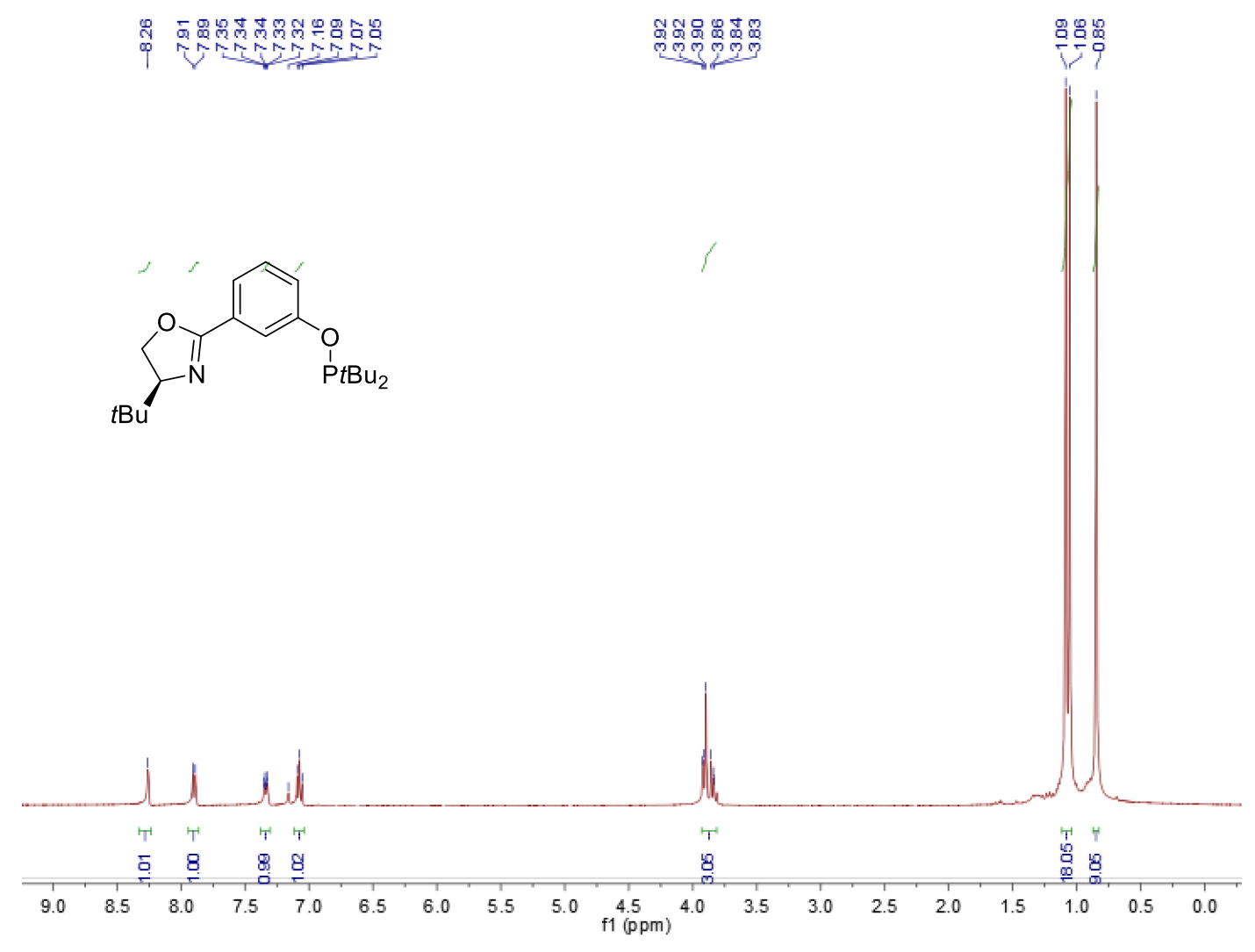

${ }^{1} \mathrm{H}$ NMR (400M, $\mathrm{C}_{6} \mathrm{D}_{6}$ ) spectrum of $\mathbf{3 d}$

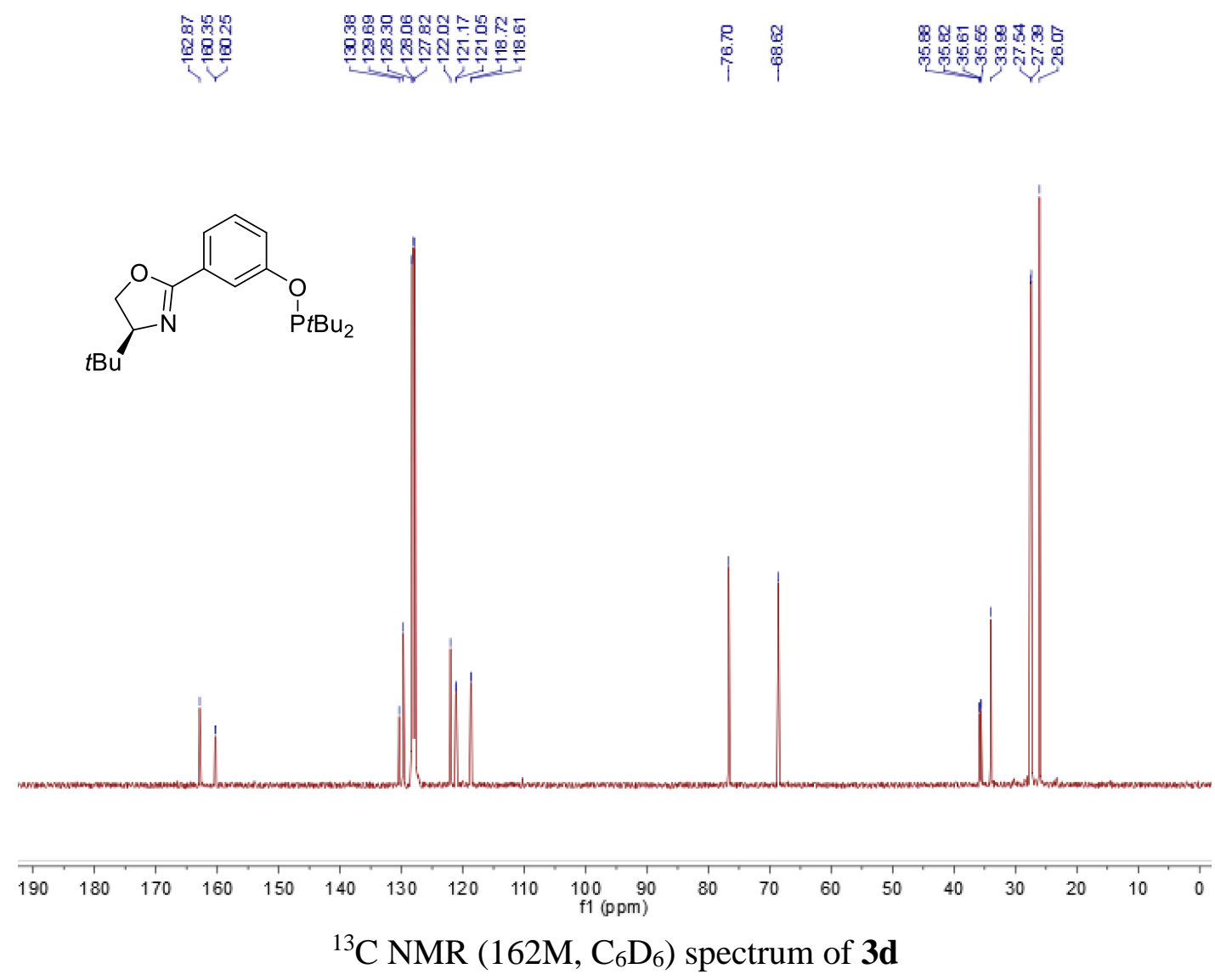



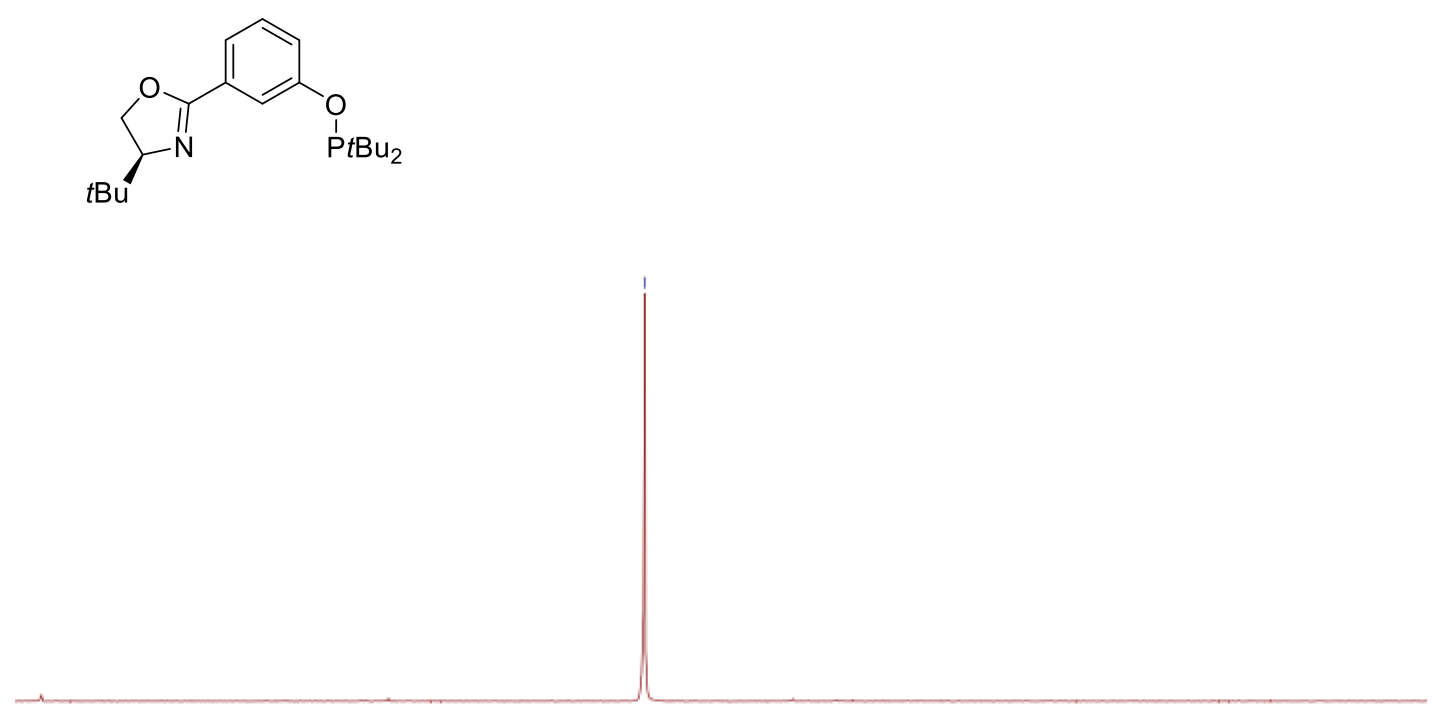

\begin{tabular}{|c|c|c|c|c|c|c|c|c|c|c|c|c|}
\hline 180 & 175 & 170 & 165 & 160 & 155 & $\begin{array}{l}150 \\
\text { f1 (ppm) }\end{array}$ & 145 & 140 & 135 & 130 & 125 & 120 \\
\hline
\end{tabular}

${ }^{19} \mathrm{P}$ NMR (162M, $\left.\mathrm{C}_{6} \mathrm{D}_{6}\right)$ spectrum of $\mathbf{3 d}$

䓦<smiles>CCCCOc1cccc(C2=NC([Ge])CO2)c1</smiles>

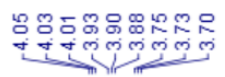

//
†

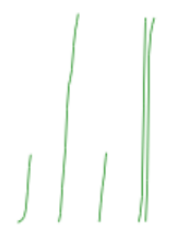

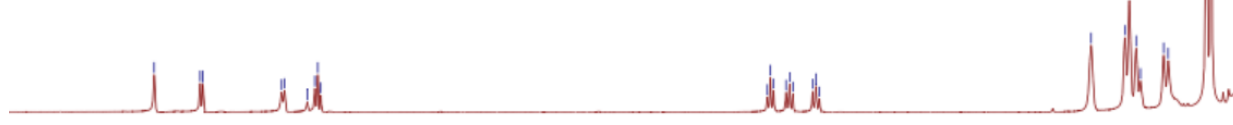

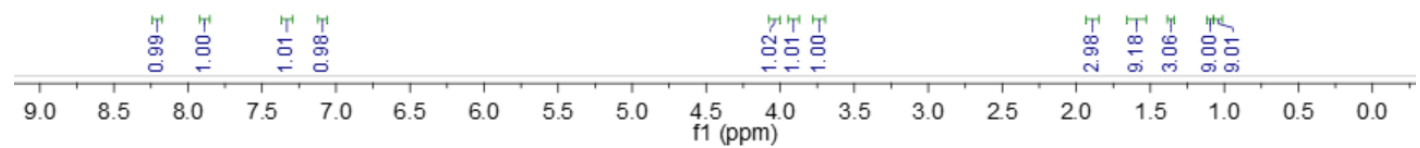

${ }^{1} \mathrm{H}$ NMR (400M, $\mathrm{C}_{6} \mathrm{D}_{6}$ ) spectrum of $\mathbf{3 e}$ 


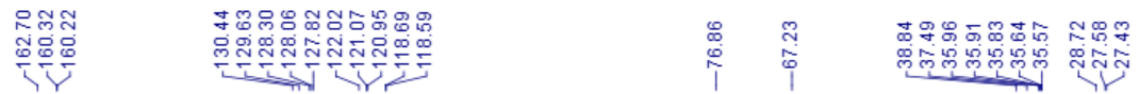<smiles>CCCCOc1cccc(C2=NC([Ge])CO2)c1</smiles>
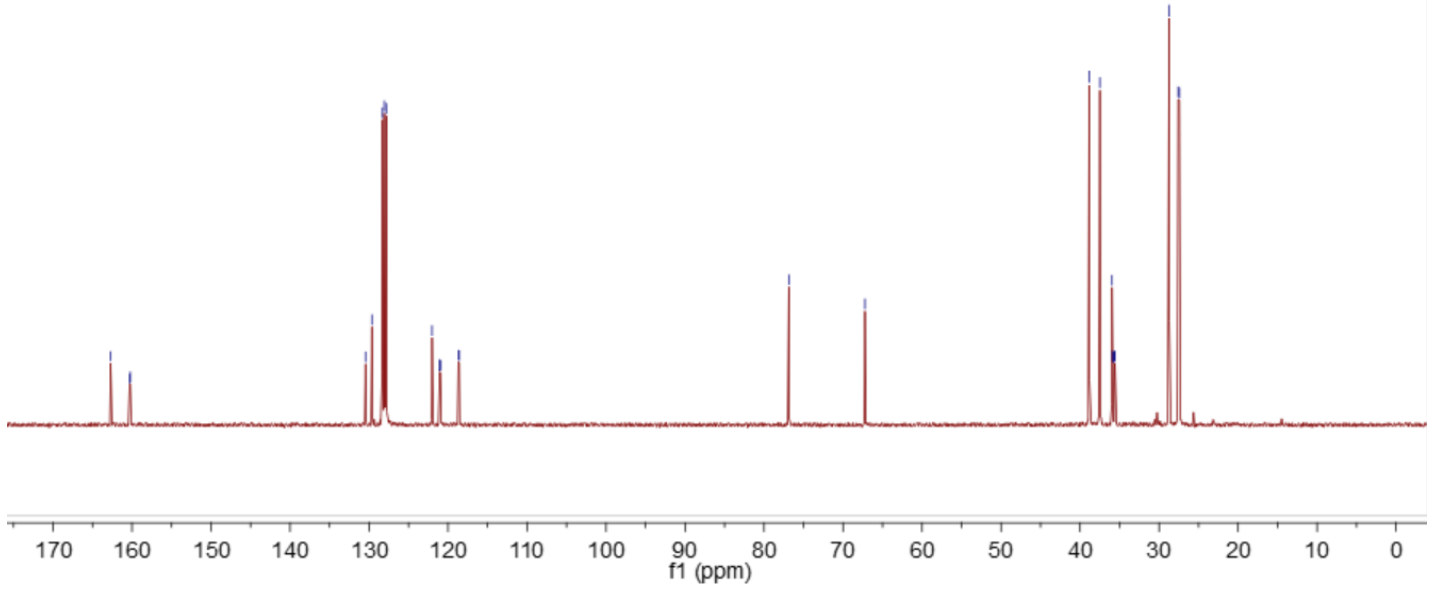

${ }^{13} \mathrm{C}$ NMR $\left(162 \mathrm{M}, \mathrm{C}_{6} \mathrm{D}_{6}\right)$ spectrum of $\mathbf{3 e}$

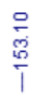<smiles>CCCCOc1cccc(C2=N[C@@H](Cl)CO2)c1</smiles>
${ }^{19} \mathrm{P}$ NMR $\left(162 \mathrm{M}, \mathrm{C}_{6} \mathrm{D}_{6}\right)$ spectrum of $\mathbf{3 e}$ 


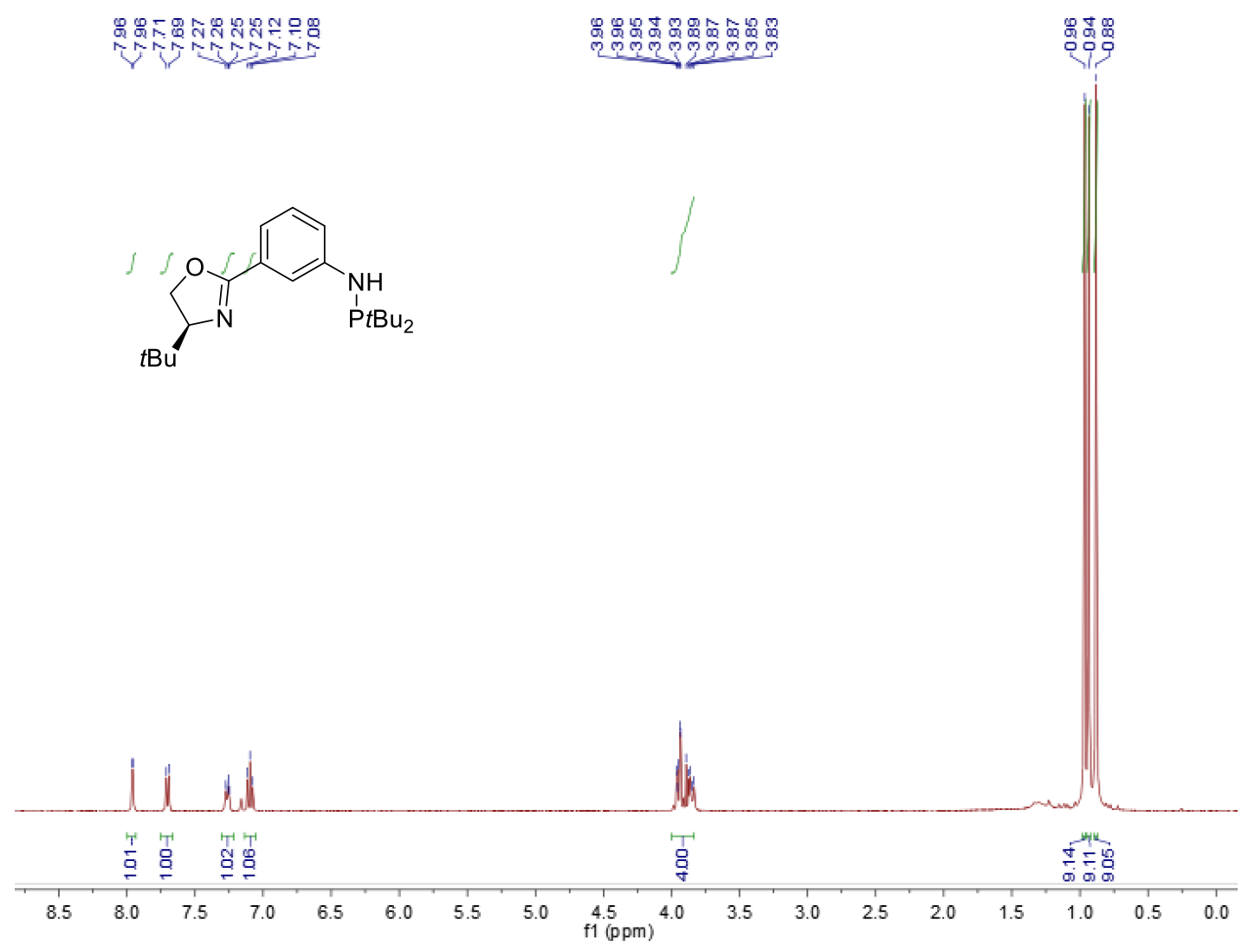

${ }^{1} \mathrm{H}$ NMR (400M, $\mathrm{C}_{6} \mathrm{D}_{6}$ ) spectrum of $\mathbf{4 a}$
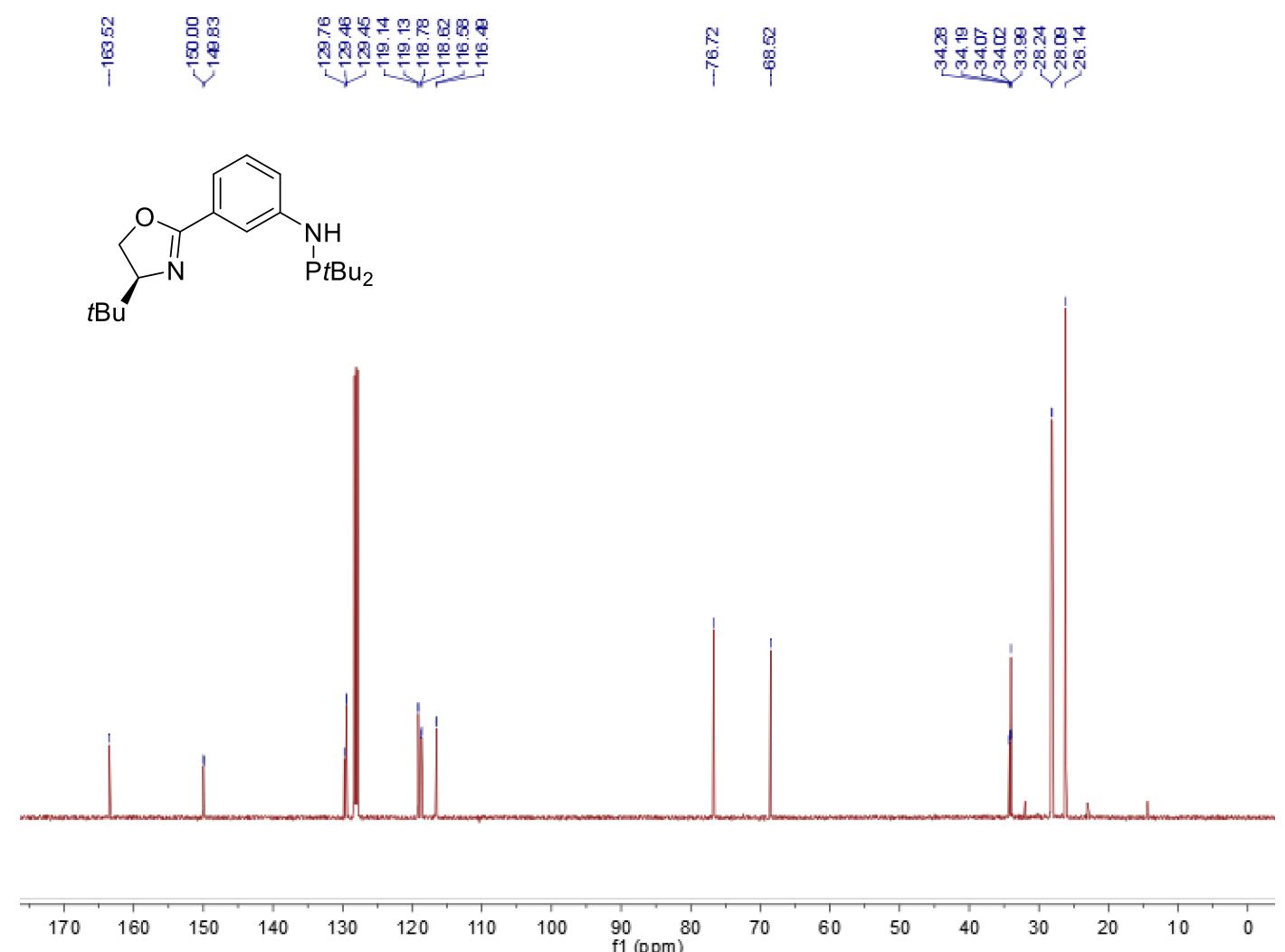

${ }^{13} \mathrm{C}$ NMR $\left(162 \mathrm{M}, \mathrm{C}_{6} \mathrm{D}_{6}\right)$ spectrum of $\mathbf{4 a}$ 


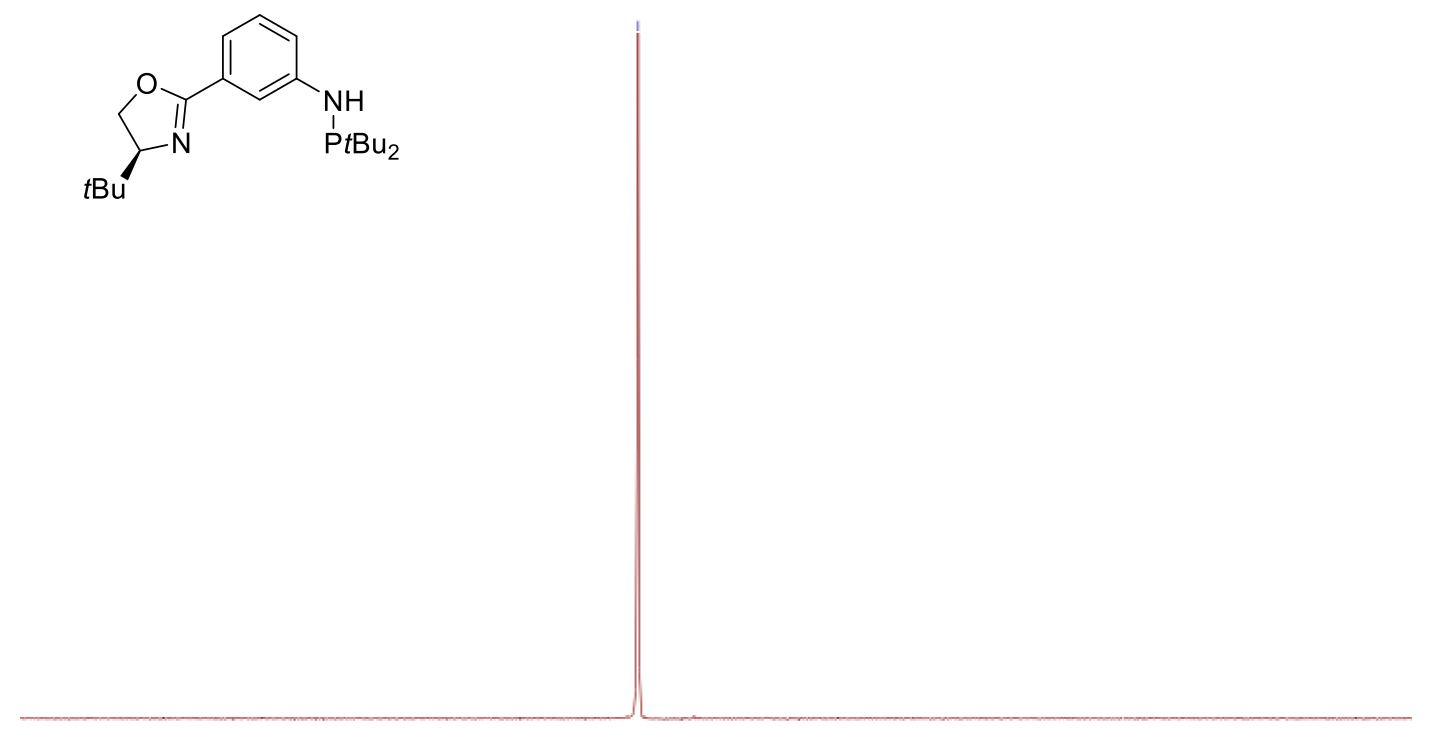

${ }^{19} \mathrm{P}$ NMR $\left(162 \mathrm{M}, \mathrm{C}_{6} \mathrm{D}_{6}\right)$ spectrum of $\mathbf{4 a}$

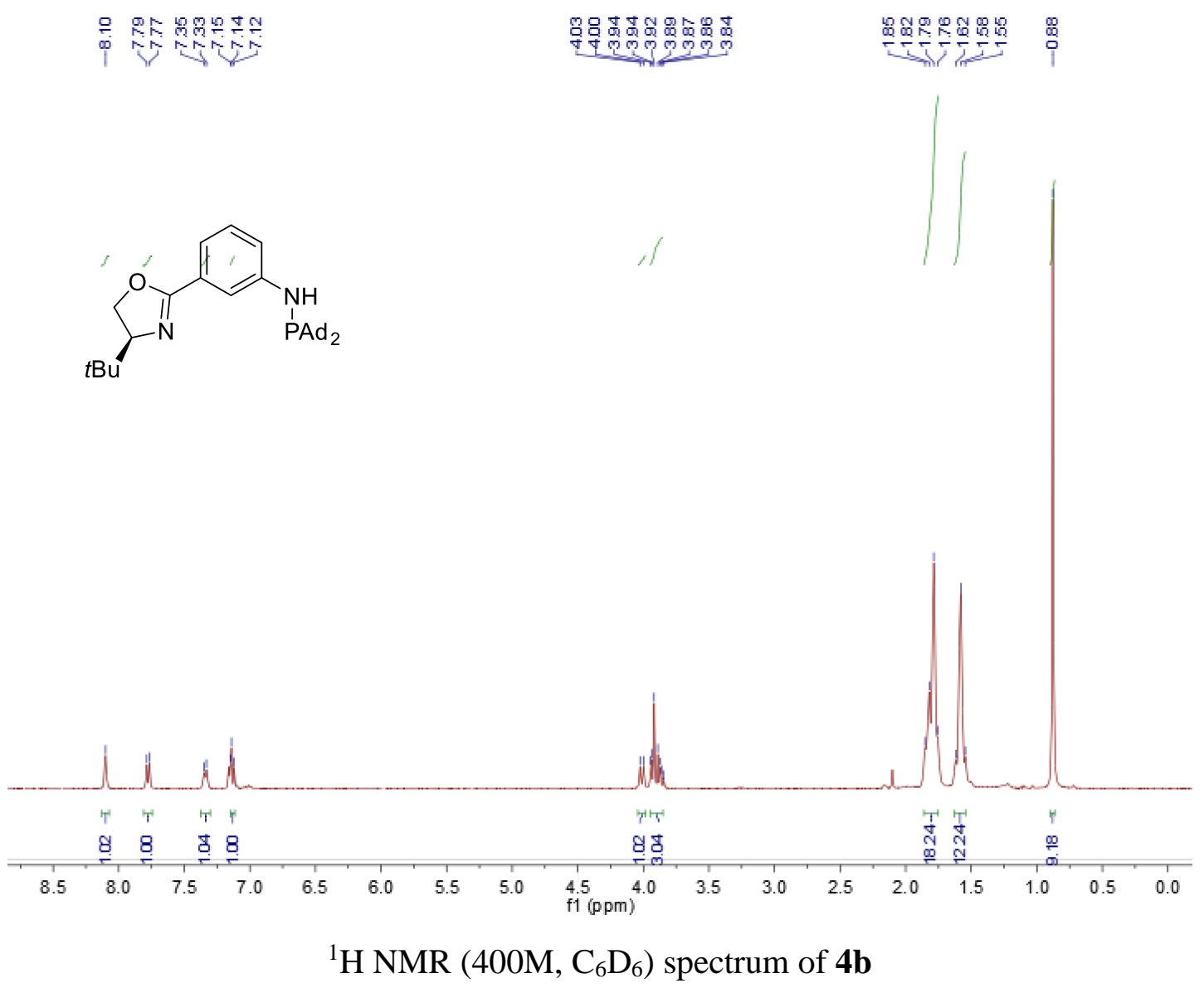




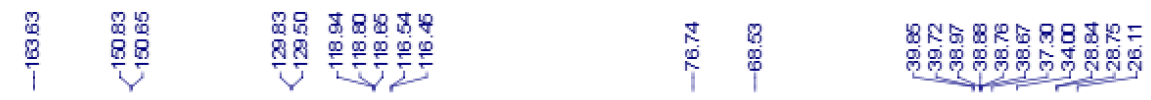
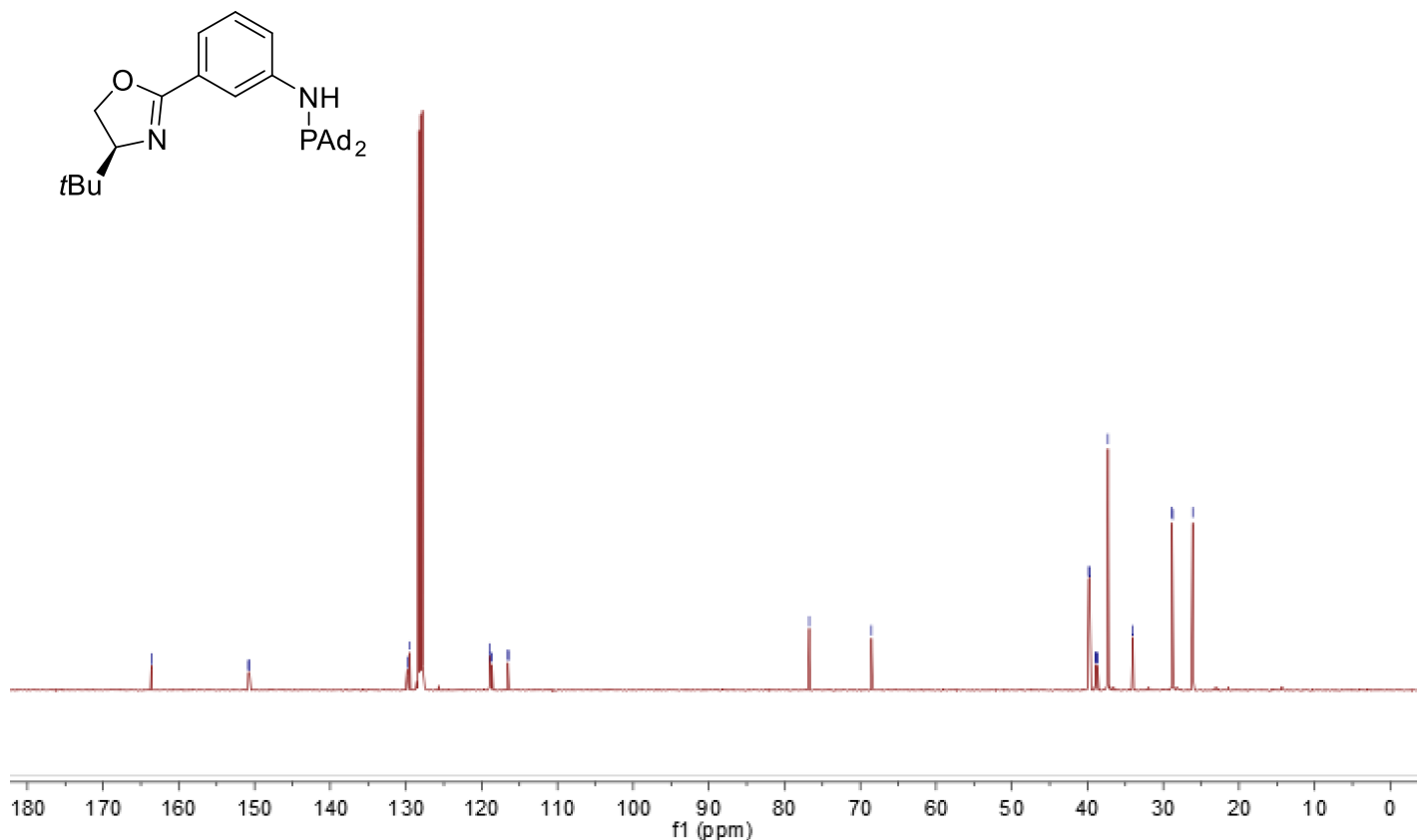

${ }^{13} \mathrm{C}$ NMR $\left(162 \mathrm{M}, \mathrm{C}_{6} \mathrm{D}_{6}\right)$ spectrum of $\mathbf{4 b}$

$\stackrel{\frac{m}{5}}{\frac{9}{0}}$<smiles>CC(C)CC1COC(c2cccc(NC(=O)OCc3ccccc3)c2)=N1</smiles>

\begin{tabular}{llllllllllllllllllllllllllllllllllll}
\hline 66 & 65 & 64 & 63 & 62 & 61 & 60 & 59 & 58 & 57 & 56 & 55 & 54 & 53 & 52 & 51 & 50 & 49 & 48 & 47 & 46 & 45 & 44 & 43 & 42 & 41 & 40 & 39 & 38
\end{tabular}

${ }^{19} \mathrm{P}$ NMR $\left(162 \mathrm{M}, \mathrm{C}_{6} \mathrm{D}_{6}\right)$ spectrum of $\mathbf{4 b}$ 

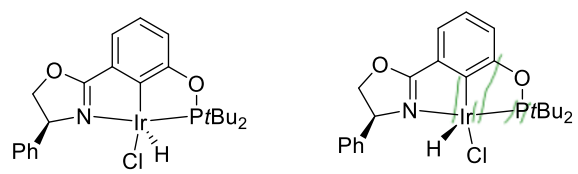

$\underset{\substack{n \\ i}}{\stackrel{\infty}{\infty}}$
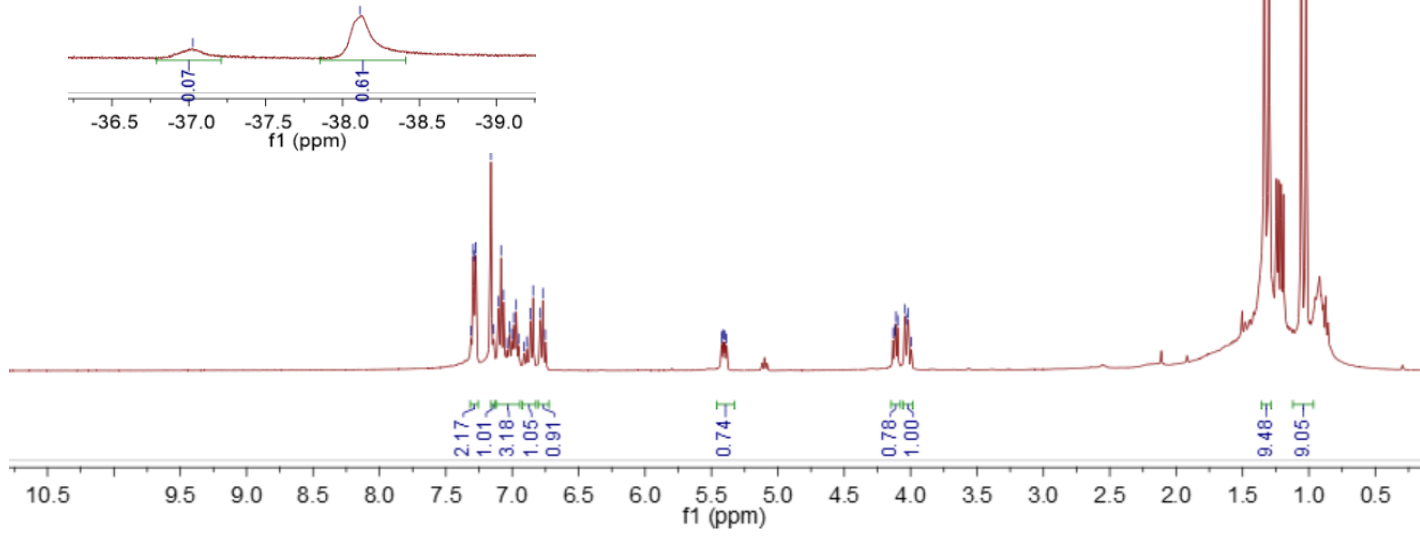

${ }^{1} \mathrm{H}$ NMR (400M, $\mathrm{C}_{6} \mathrm{D}_{6}$ ) spectrum of $\mathbf{5 a}$

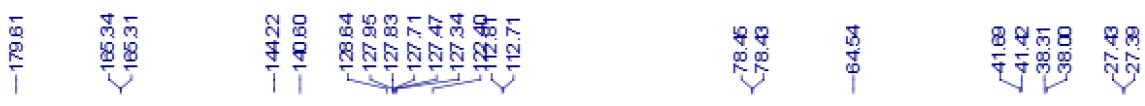
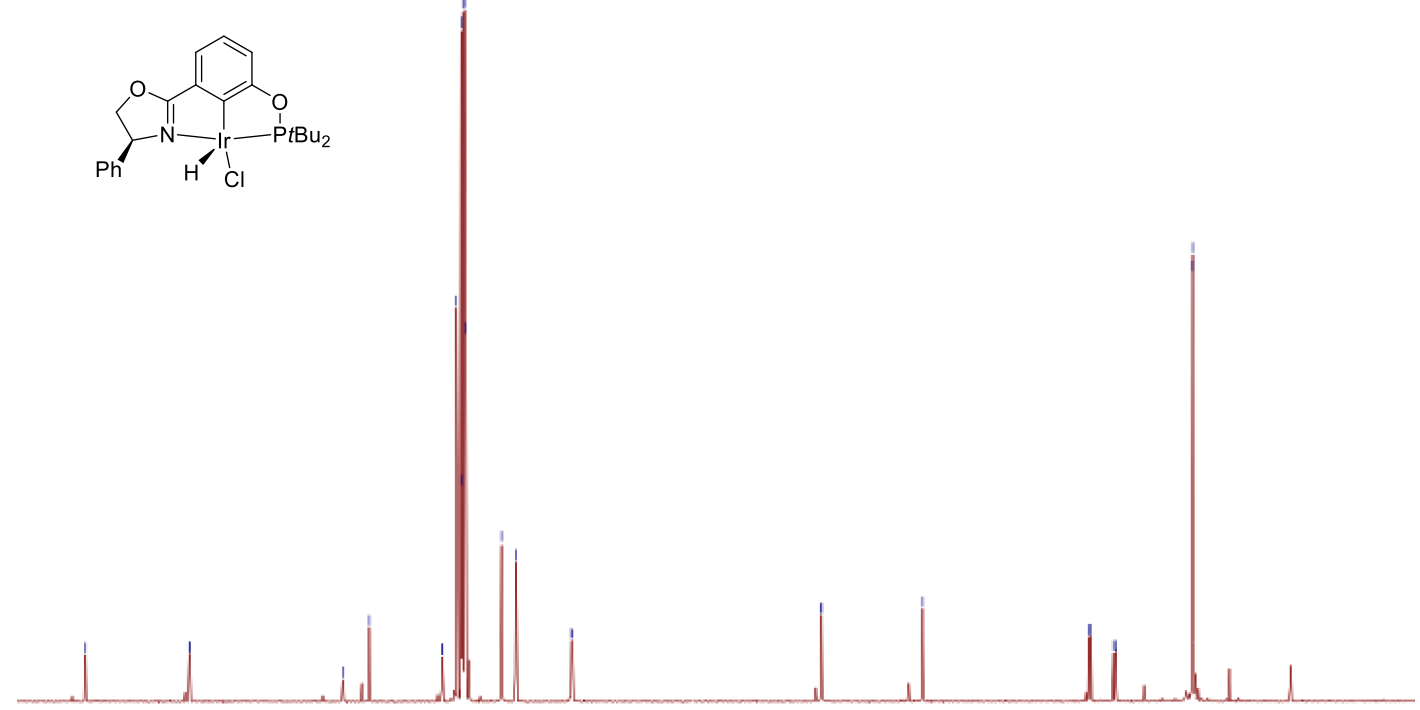

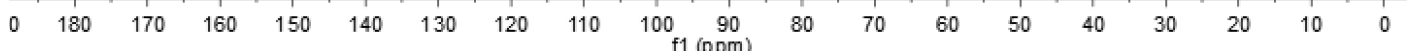

${ }^{13} \mathrm{C}$ NMR (162M, $\left.\mathrm{C}_{6} \mathrm{D}_{6}\right)$ spectrum of $\mathbf{5 a}$ 

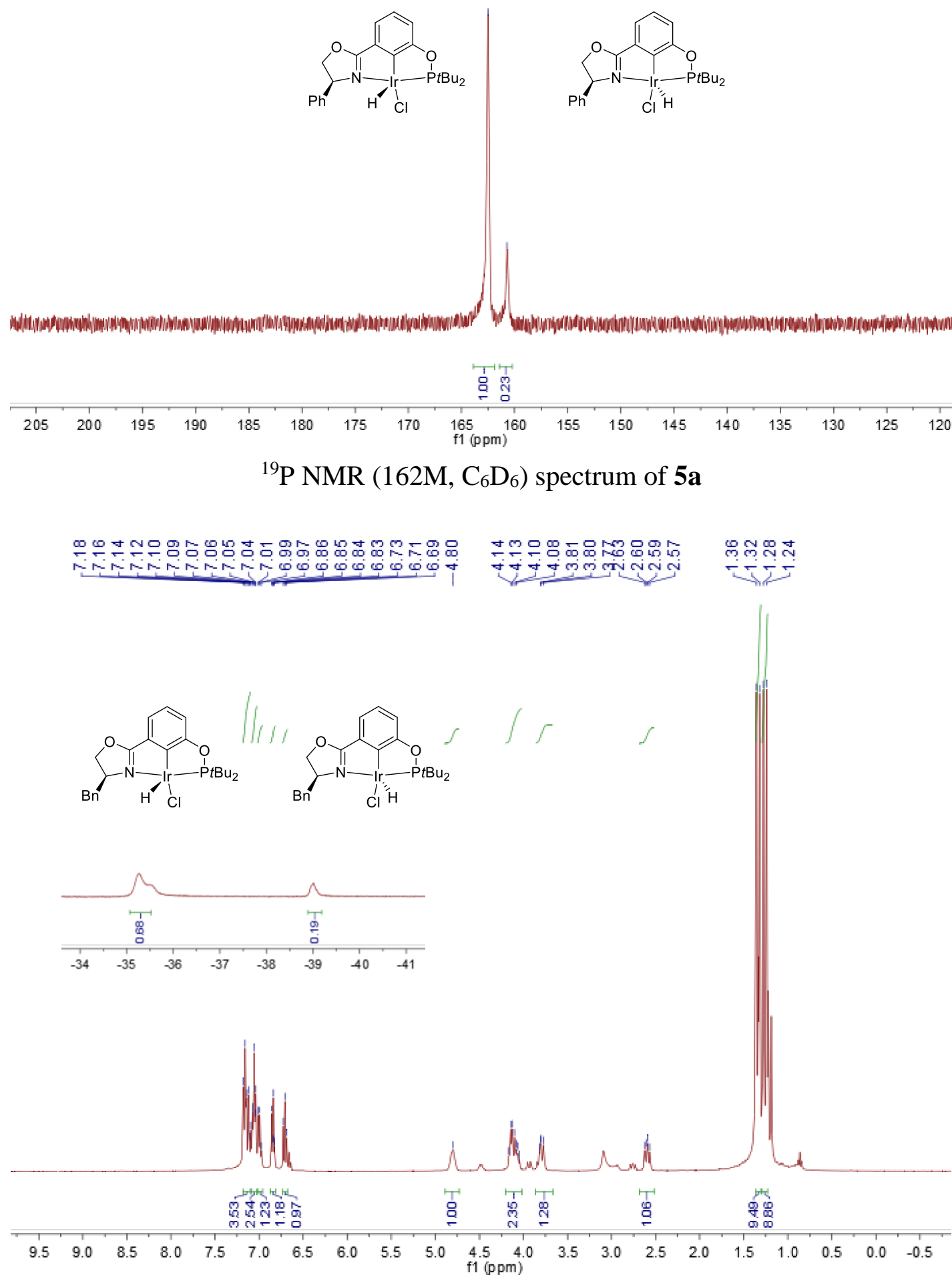

${ }^{1} \mathrm{H}$ NMR (400M, $\mathrm{C}_{6} \mathrm{D}_{6}$ ) spectrum of $\mathbf{5 b}$ 

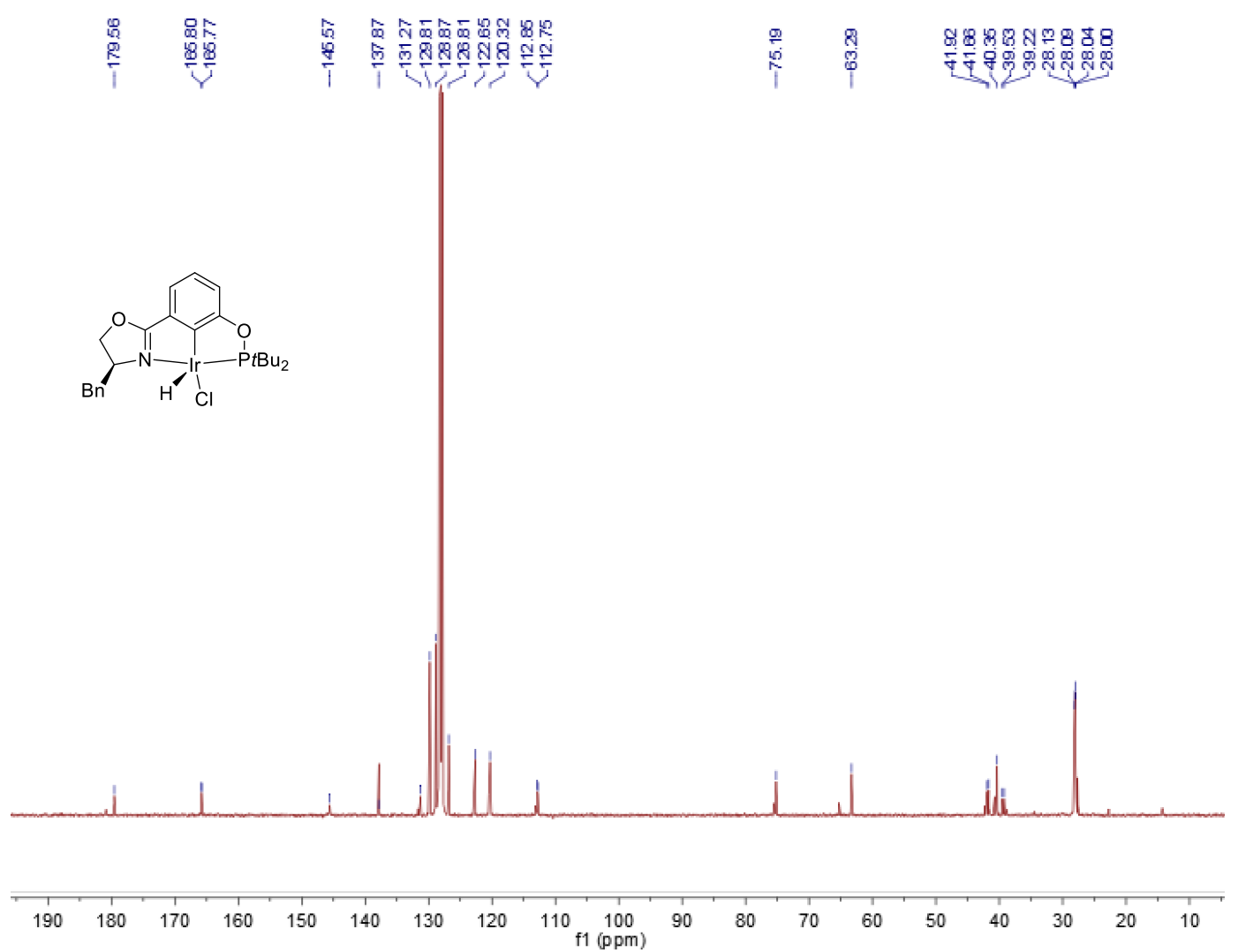

${ }^{13} \mathrm{C}$ NMR $\left(162 \mathrm{M}, \mathrm{C}_{6} \mathrm{D}_{6}\right)$ spectrum of $\mathbf{5 b}$

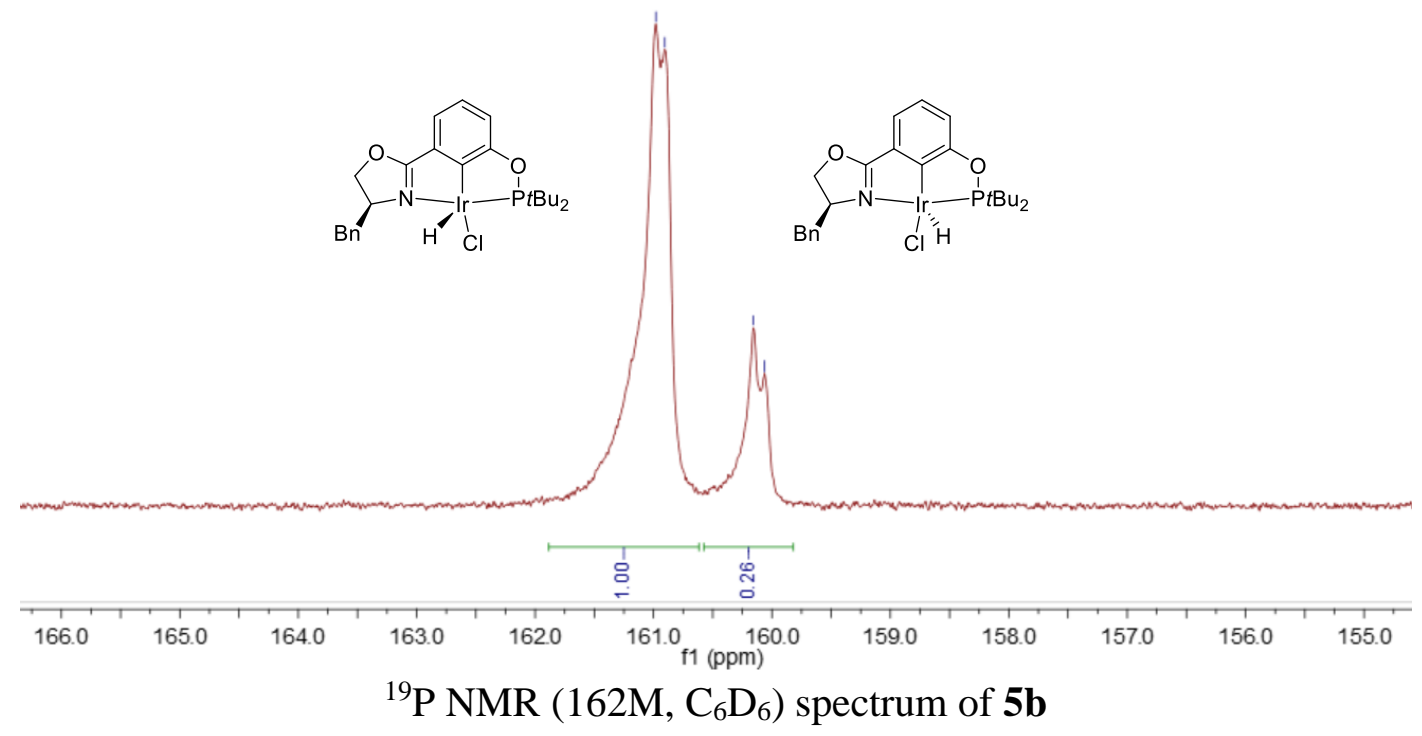




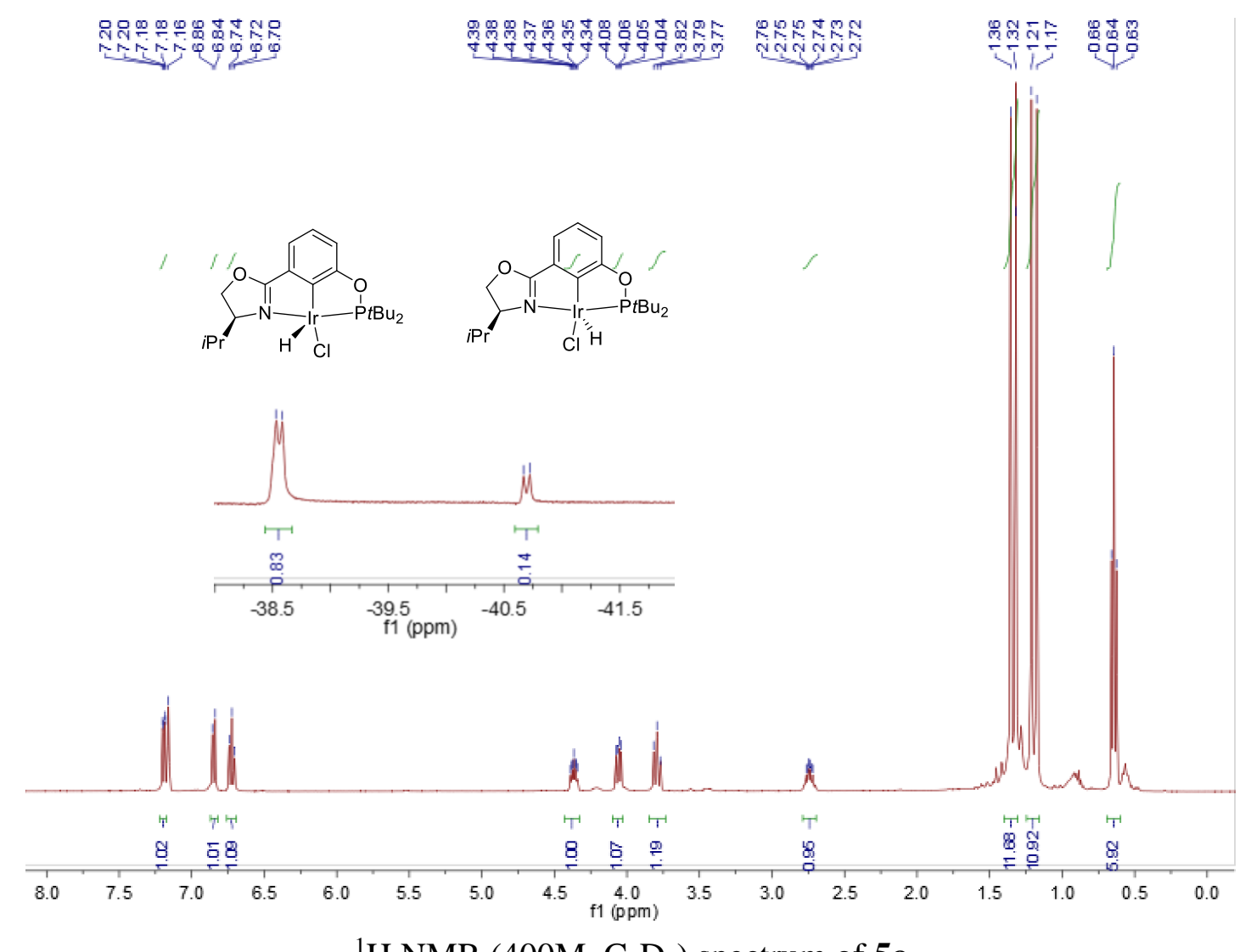

${ }^{1} \mathrm{H}$ NMR $\left(400 \mathrm{M}, \mathrm{C}_{6} \mathrm{D}_{6}\right)$ spectrum of $\mathbf{5 c}$

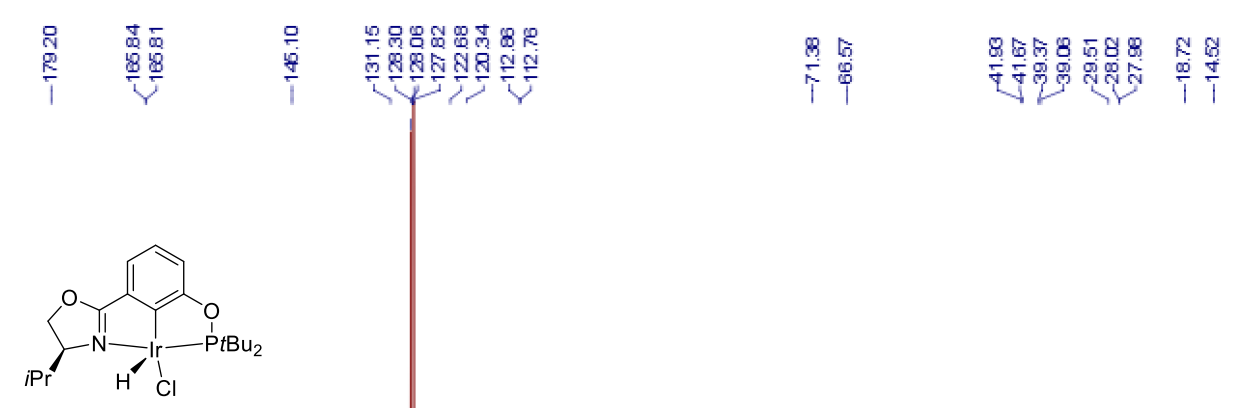

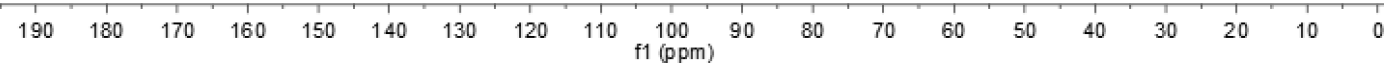

${ }^{13} \mathrm{C}$ NMR $\left(162 \mathrm{M}, \mathrm{C}_{6} \mathrm{D}_{6}\right)$ spectrum of $\mathbf{5 c}$ 


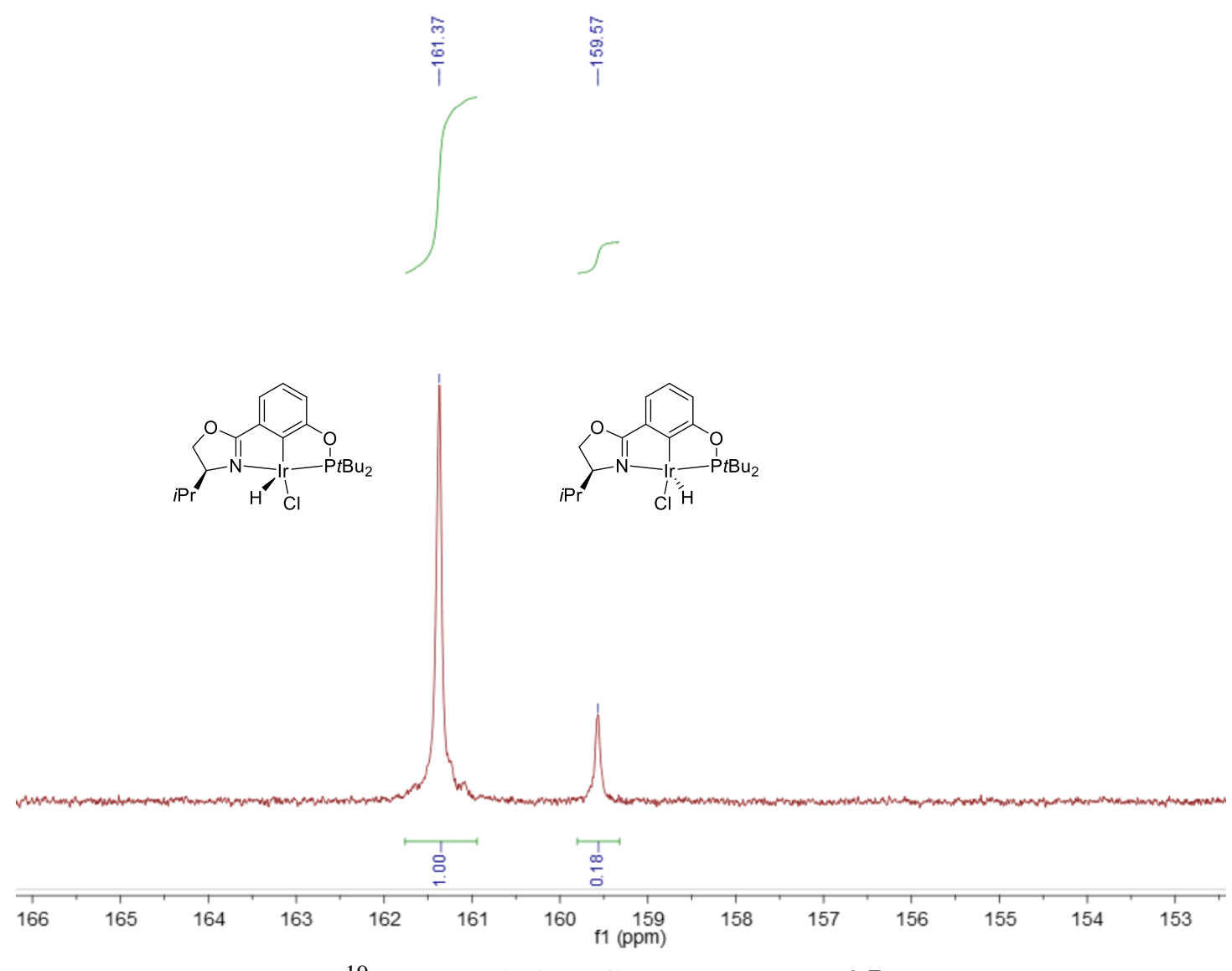

${ }^{19} \mathrm{P}$ NMR $\left(162 \mathrm{M}, \mathrm{C}_{6} \mathrm{D}_{6}\right)$ spectrum of $\mathbf{5 c}$

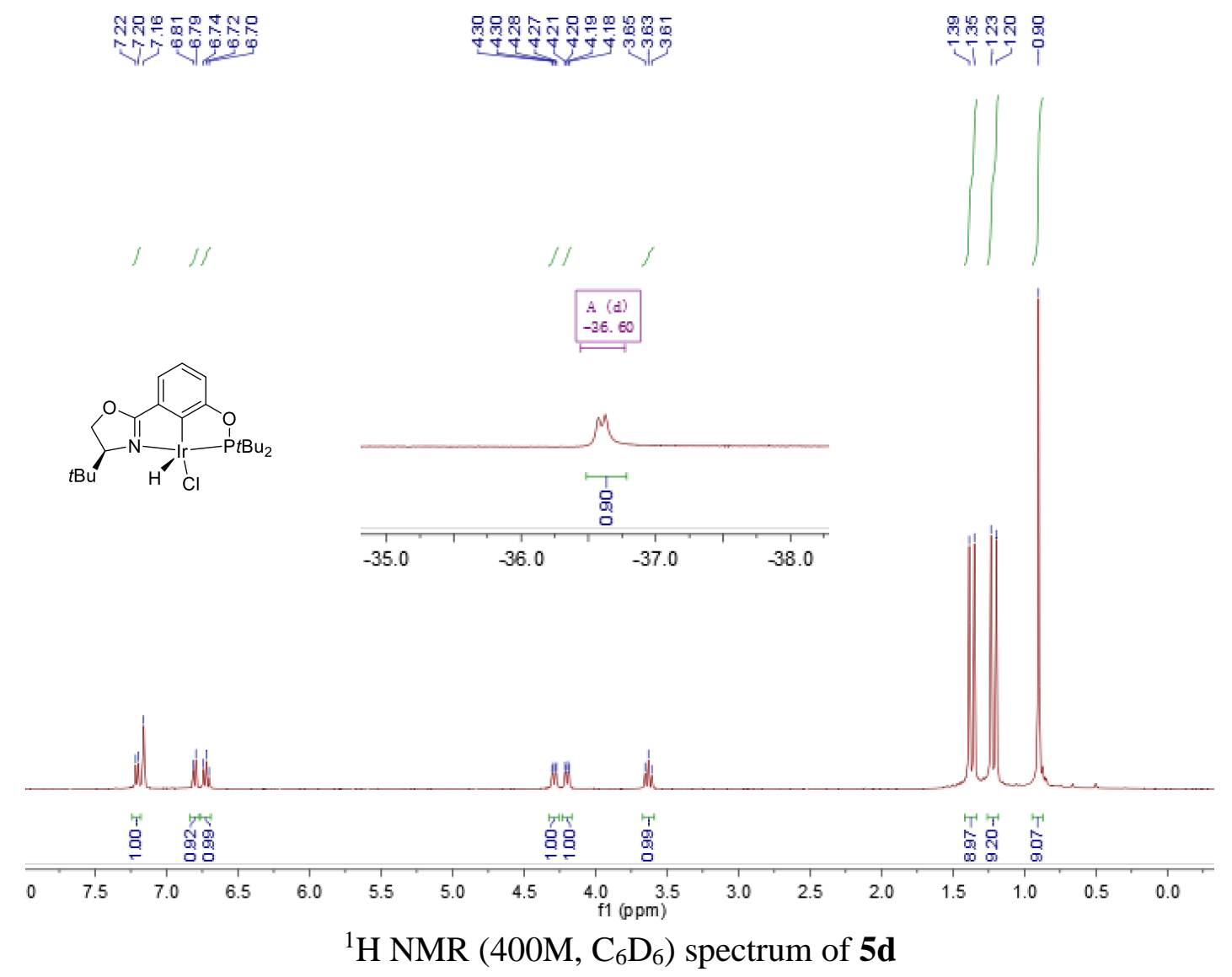



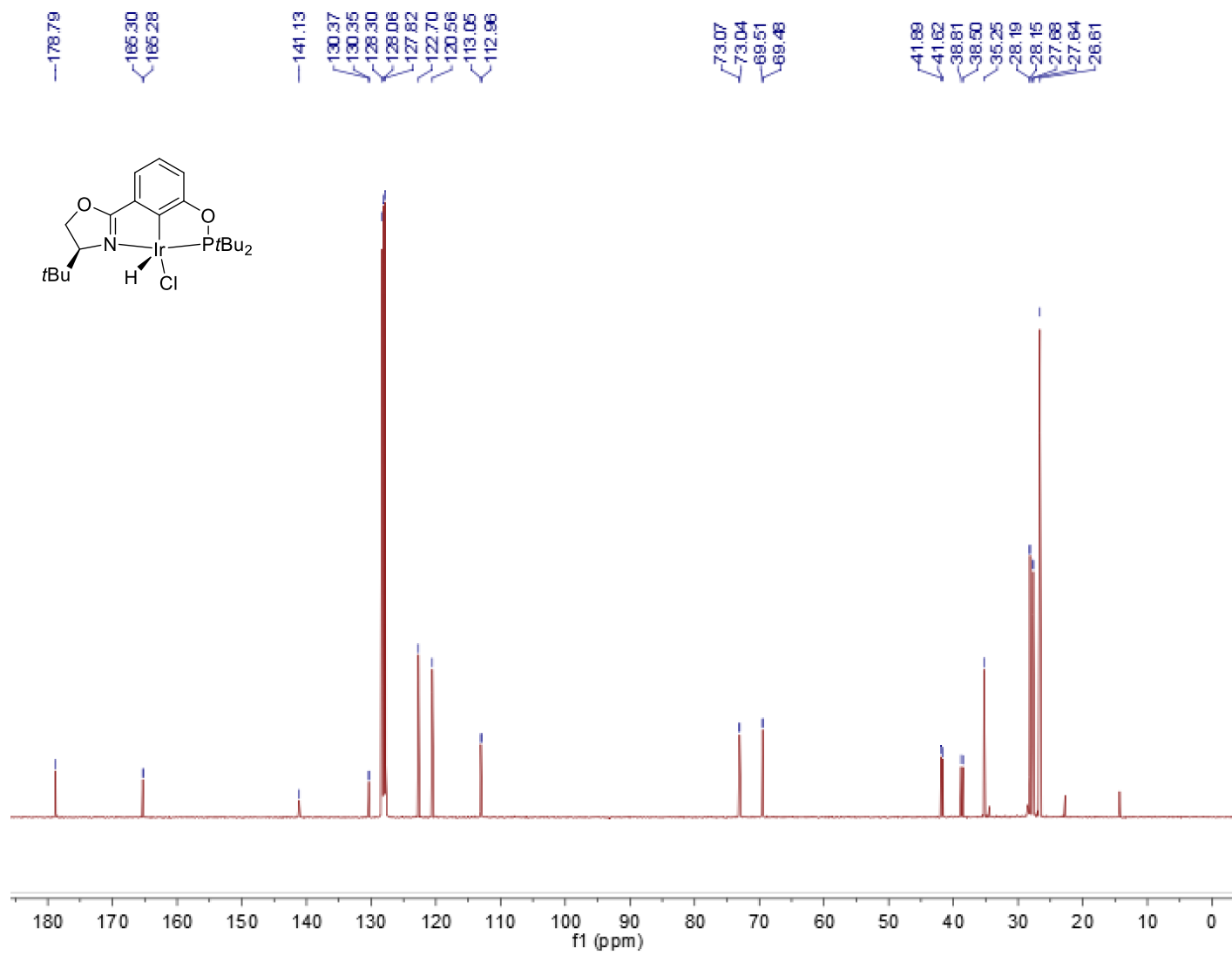

${ }^{13} \mathrm{C}$ NMR $\left(162 \mathrm{M}, \mathrm{C}_{6} \mathrm{D}_{6}\right)$ spectrum of $\mathbf{5 d}$

$\frac{7}{\frac{1}{1}}$
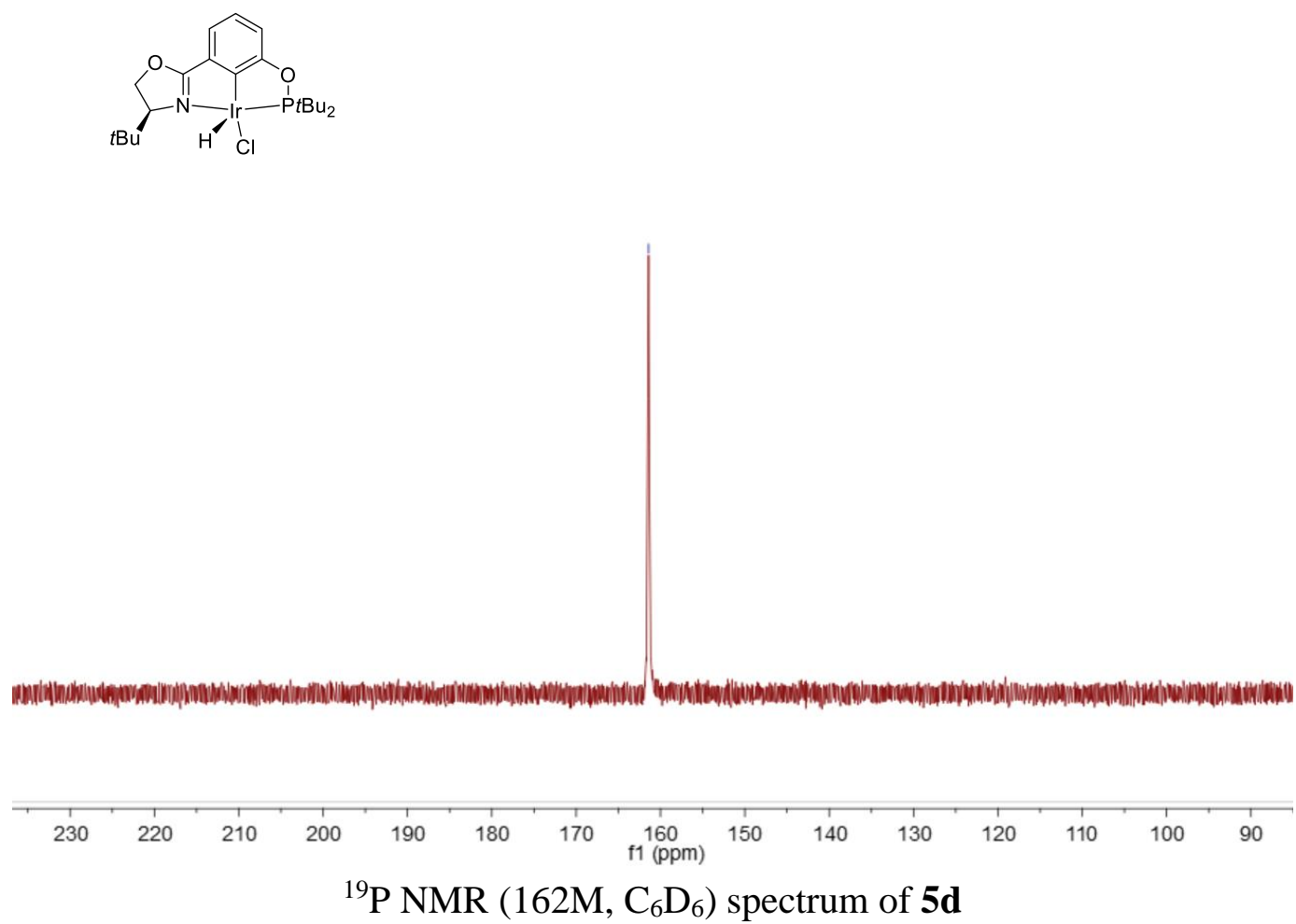


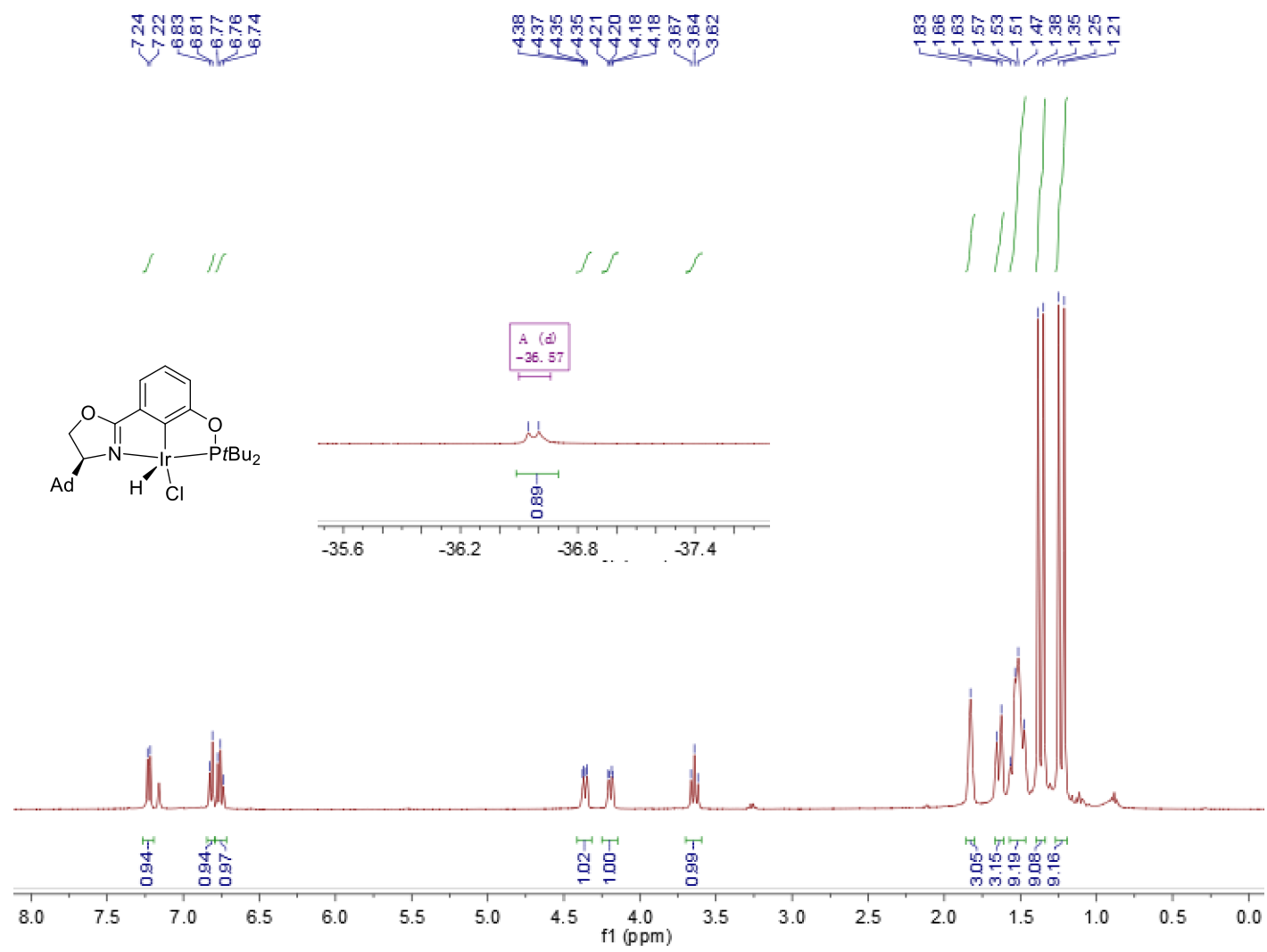

${ }^{1} \mathrm{H}$ NMR (400M, $\mathrm{C}_{6} \mathrm{D}_{6}$ ) spectrum of $\mathbf{5 e}$
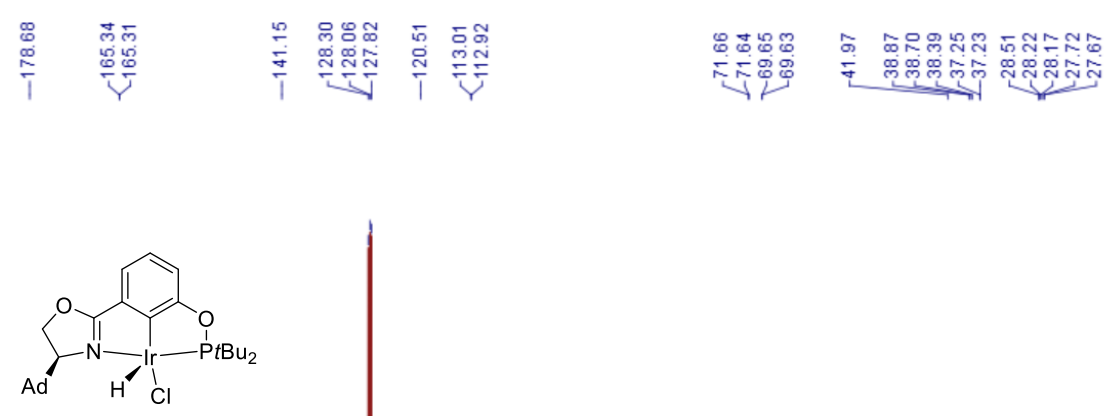

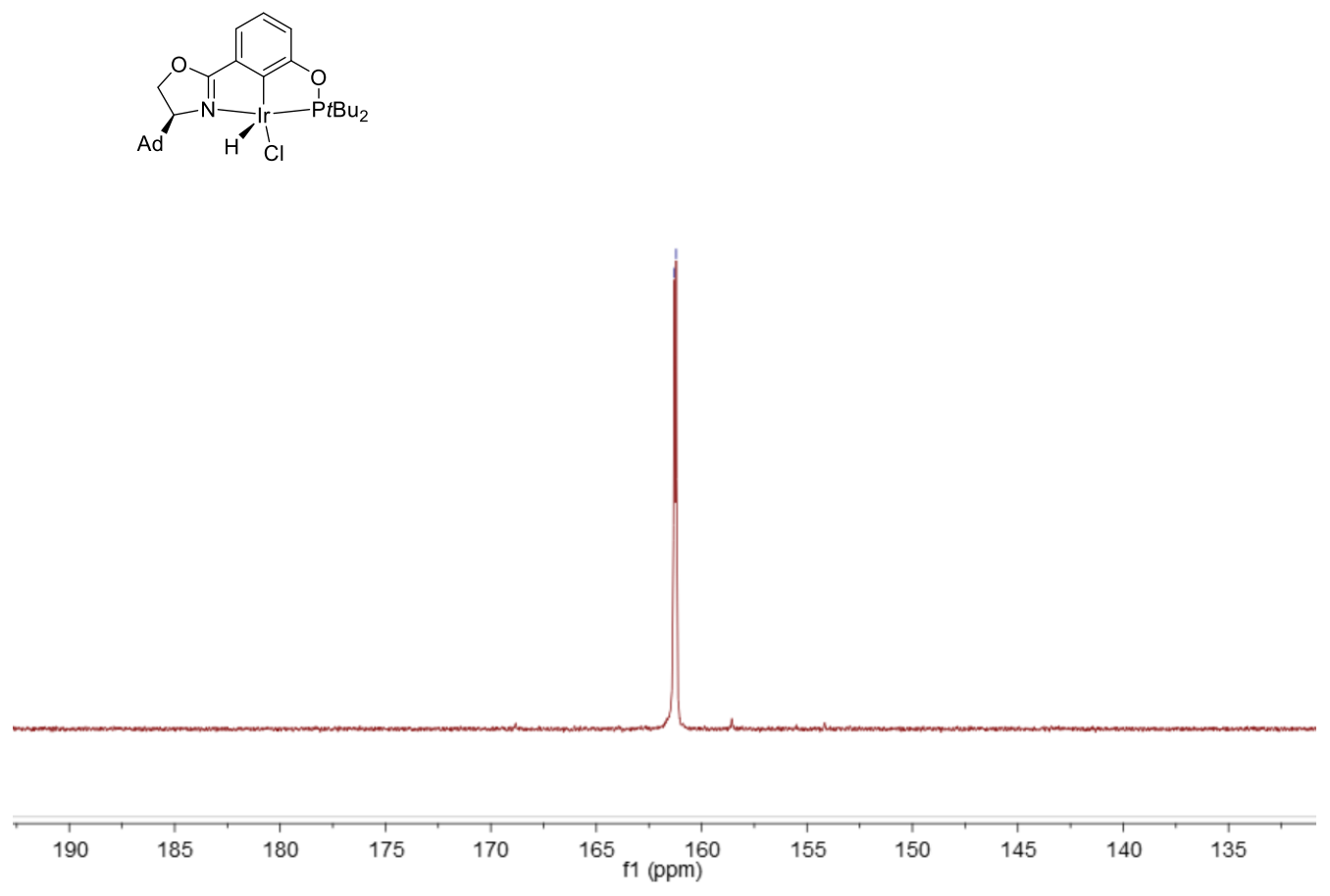

${ }^{19} \mathrm{P}$ NMR (162M, C6 $\left.\mathrm{D}_{6}\right)$ spectrum of $\mathbf{5 e}$

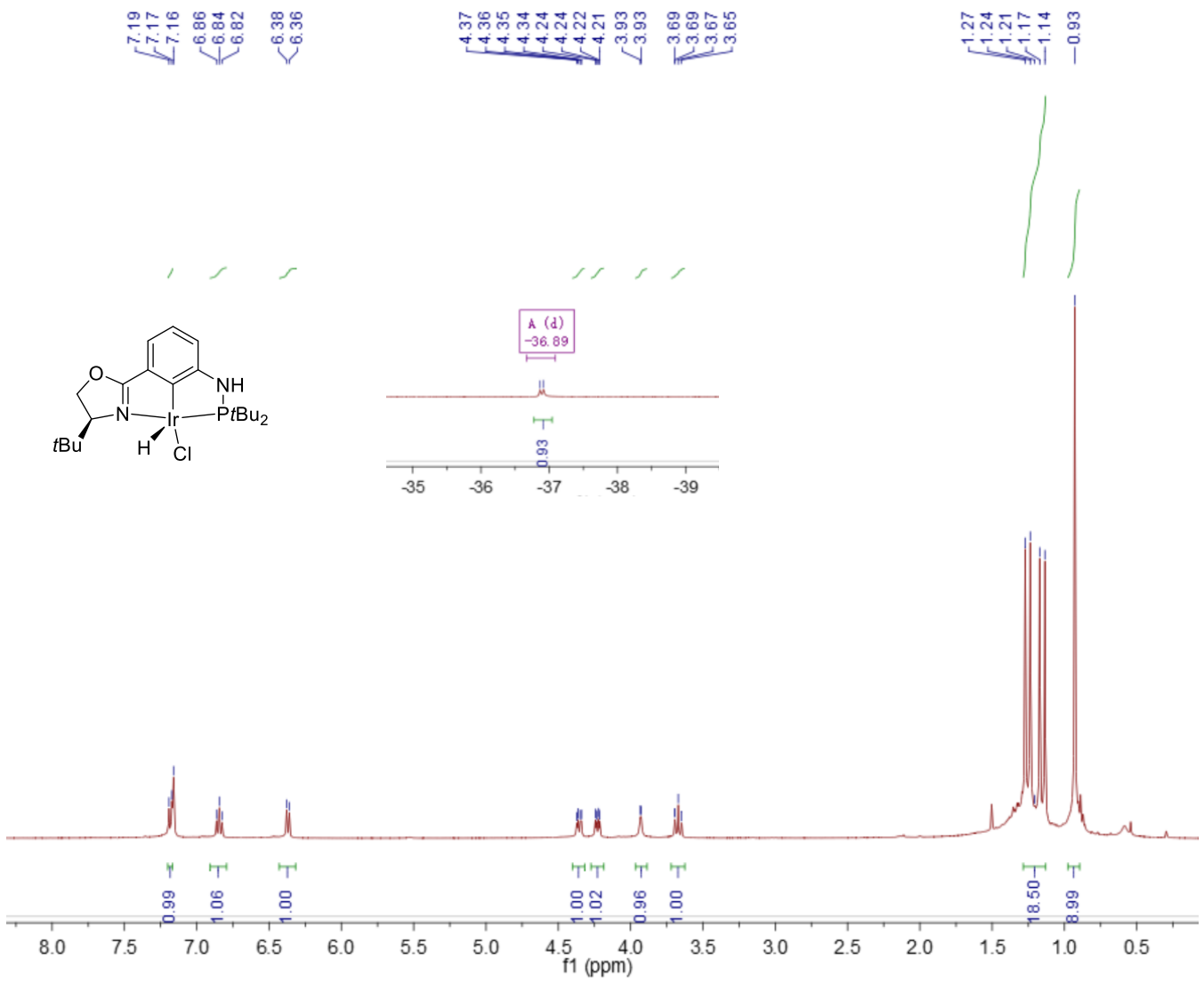

${ }^{1} \mathrm{H}$ NMR (400M, $\mathrm{C}_{6} \mathrm{D}_{6}$ ) spectrum of $\mathbf{6 a}$ 


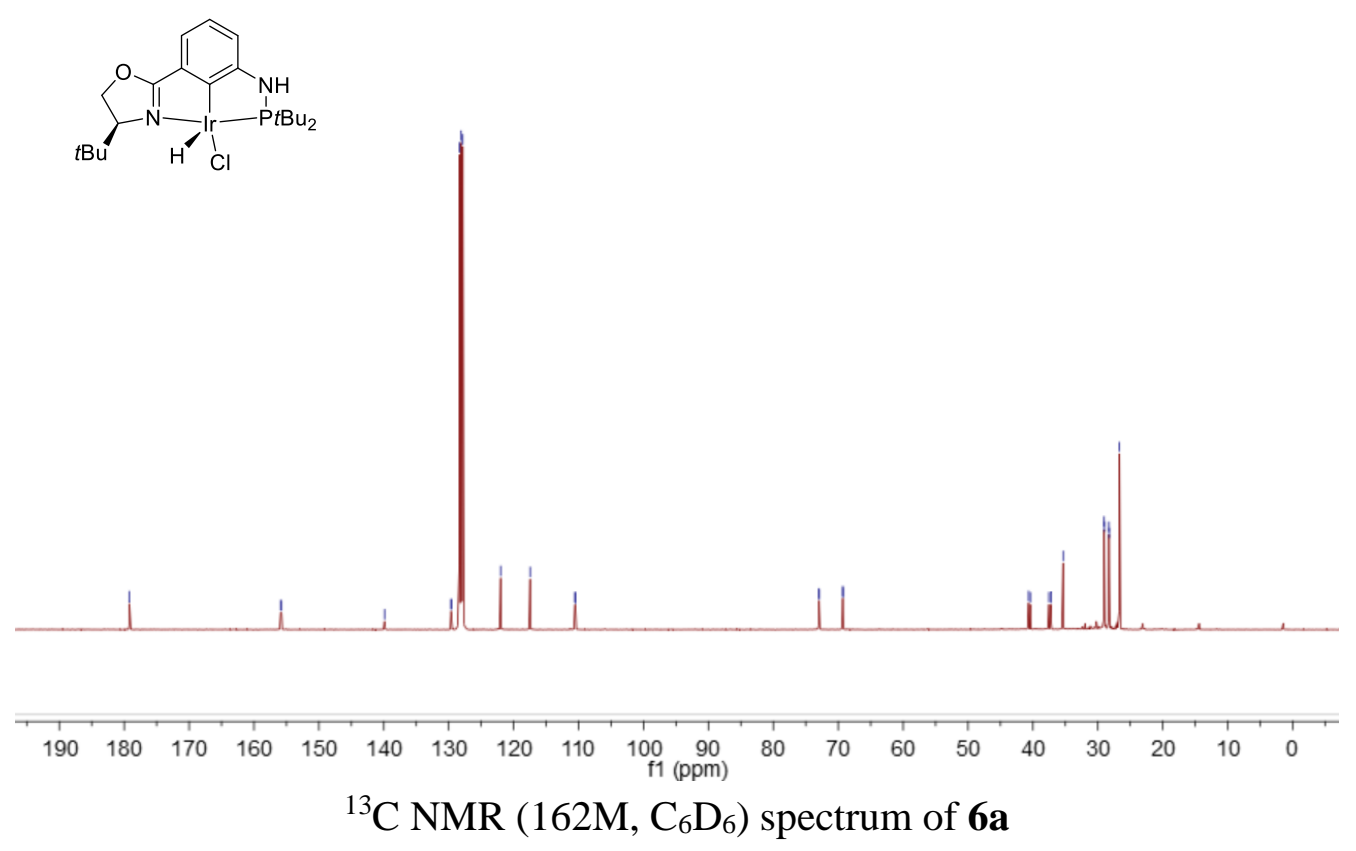

亭管
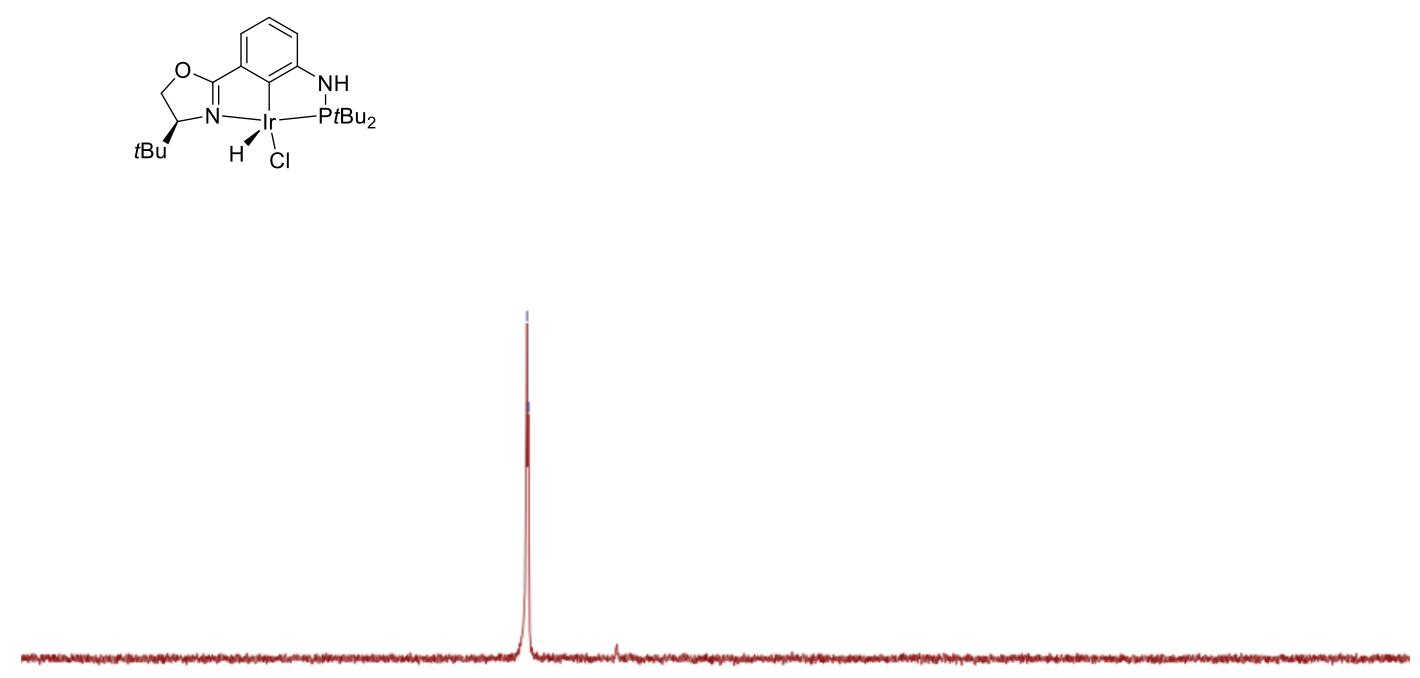

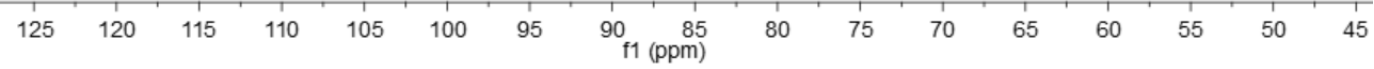

${ }^{19} \mathrm{P}$ NMR $\left(162 \mathrm{M}, \mathrm{C}_{6} \mathrm{D}_{6}\right)$ spectrum of $\mathbf{6 a}$ 

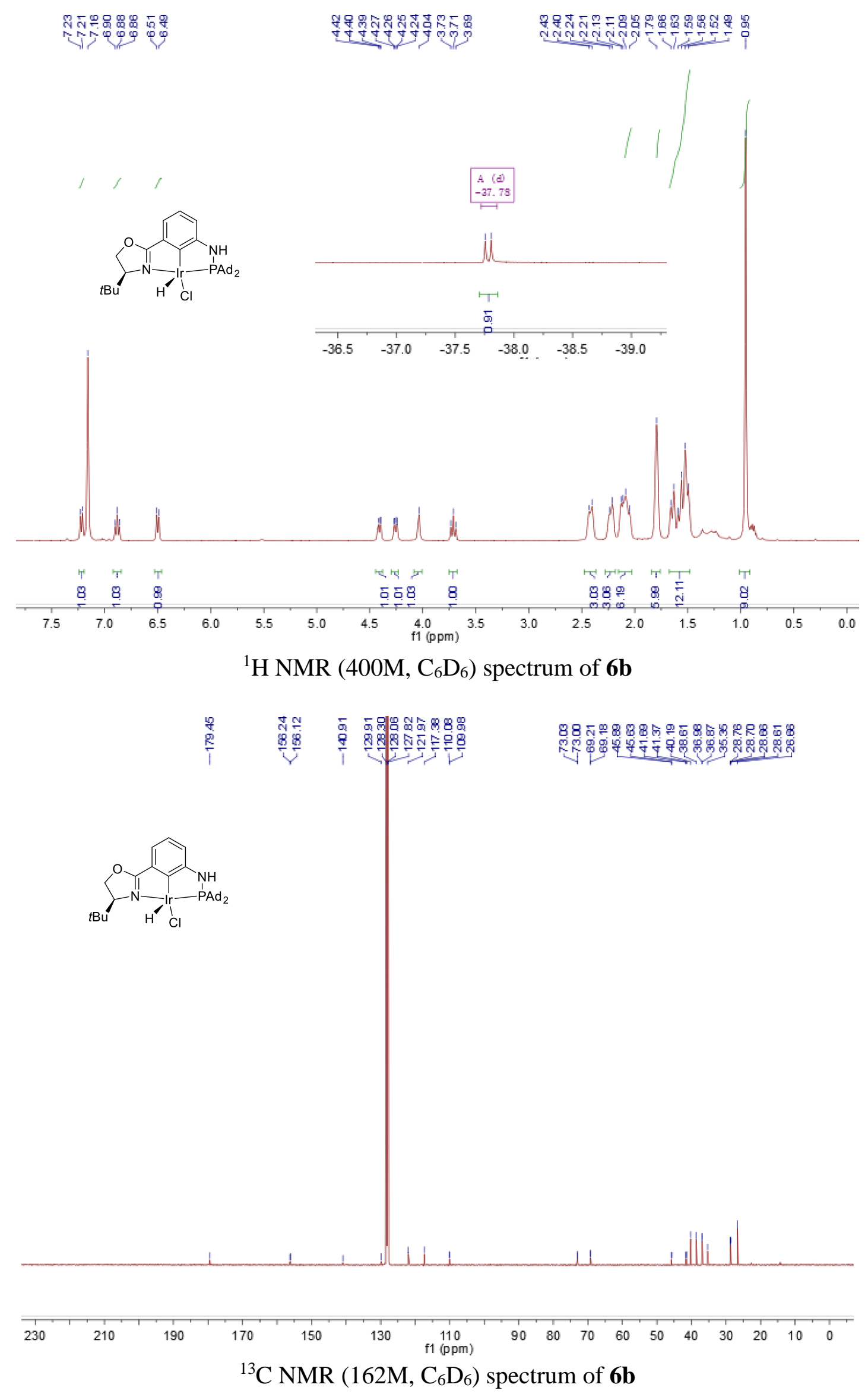


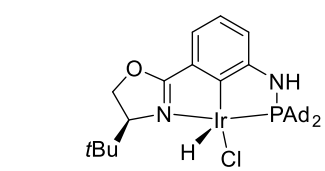

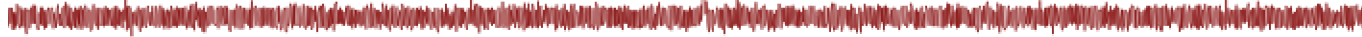

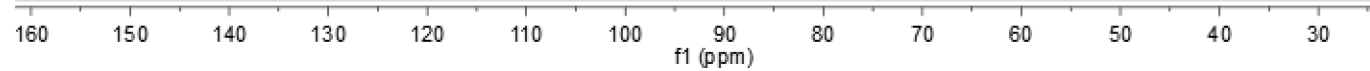

${ }^{19} \mathrm{P}$ NMR $\left(162 \mathrm{M}, \mathrm{C}_{6} \mathrm{D}_{6}\right)$ spectrum of $\mathbf{6 b}$

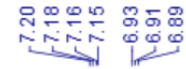

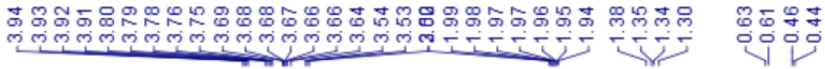
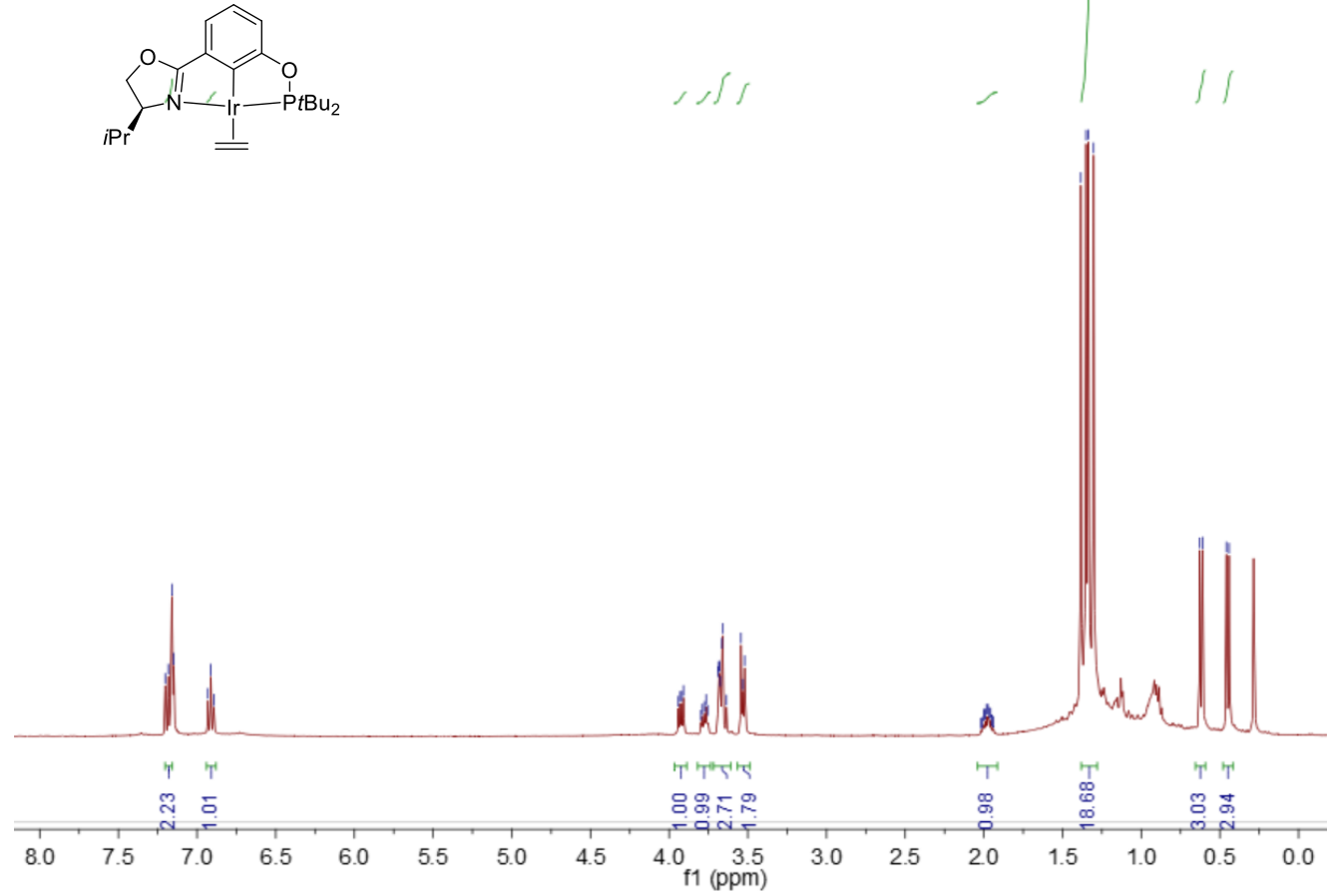

${ }^{1} \mathrm{H}$ NMR $\left(400 \mathrm{M}, \mathrm{C}_{6} \mathrm{D}_{6}\right)$ spectrum of $\mathbf{5 e}$ ' 


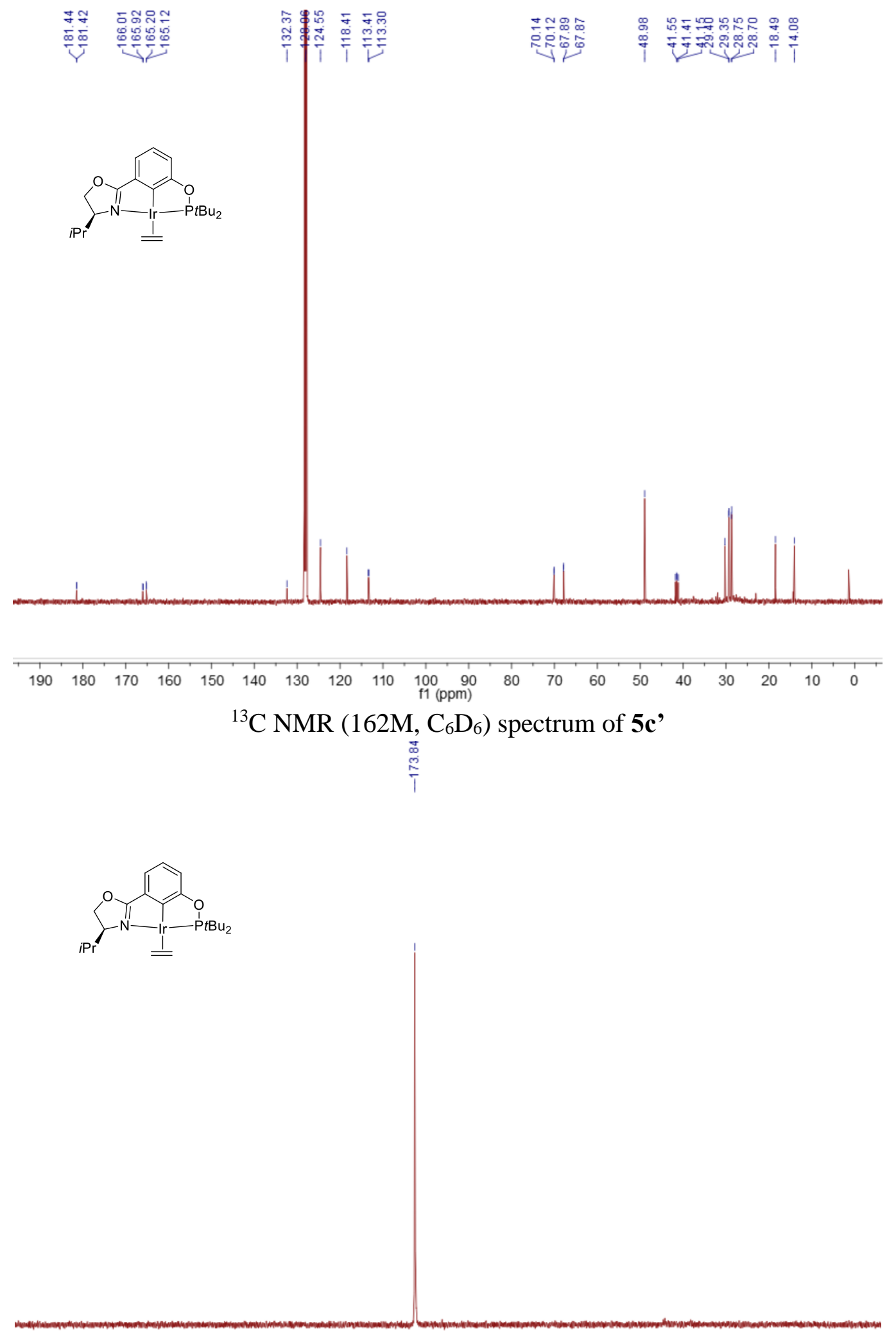

$\begin{array}{lllllllllllllllllllll}220 & 215 & 210 & 205 & 200 & 195 & 190 & 185 & 180 & \begin{array}{r}175 \\ \mathrm{f} 1(\mathrm{ppm})\end{array} & 165 & 160 & 155 & 150 & 145 & 140 & 135 & 130 & 125 & 120\end{array}$ ${ }^{19} \mathrm{P}$ NMR (162M, $\left.\mathrm{C}_{6} \mathrm{D}_{6}\right)$ spectrum of $\mathbf{5 e}$ ' 


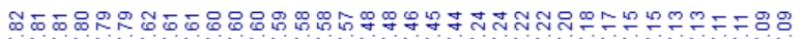
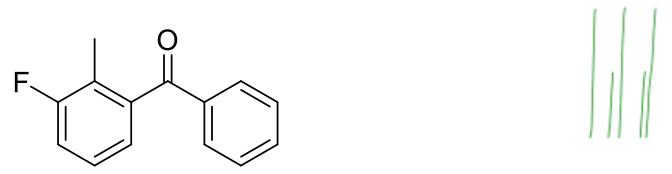

:

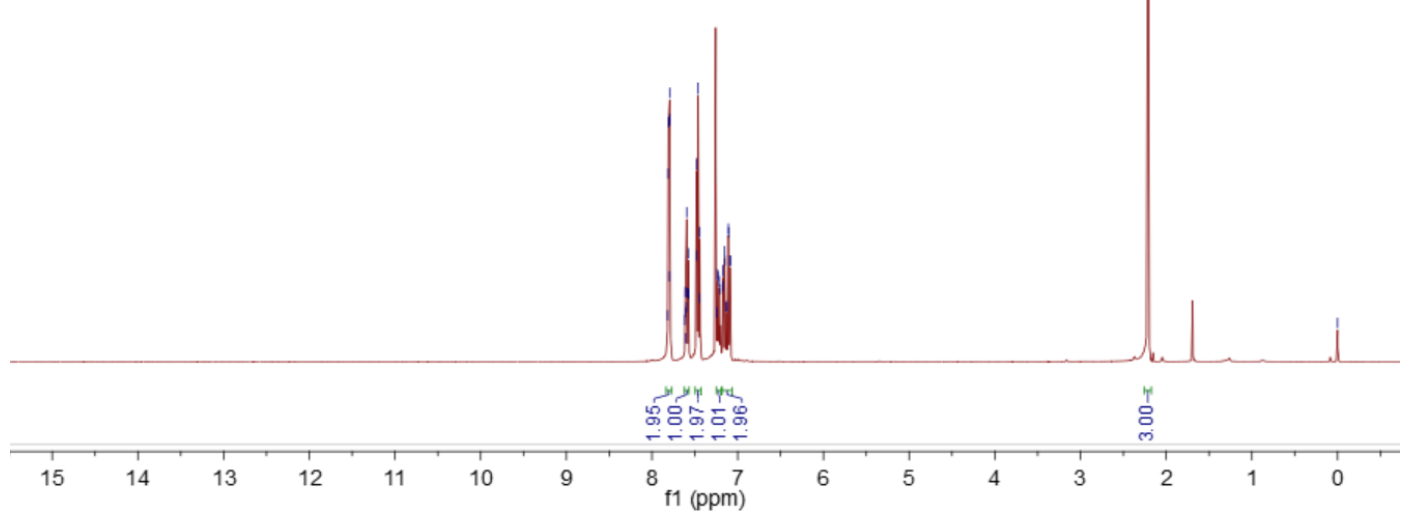

${ }^{1} \mathrm{H}$ NMR (400M, $\mathrm{CDCl}_{3}$ ) spectrum of $\mathbf{S 7 i}$

高

蕰

905
55

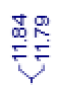
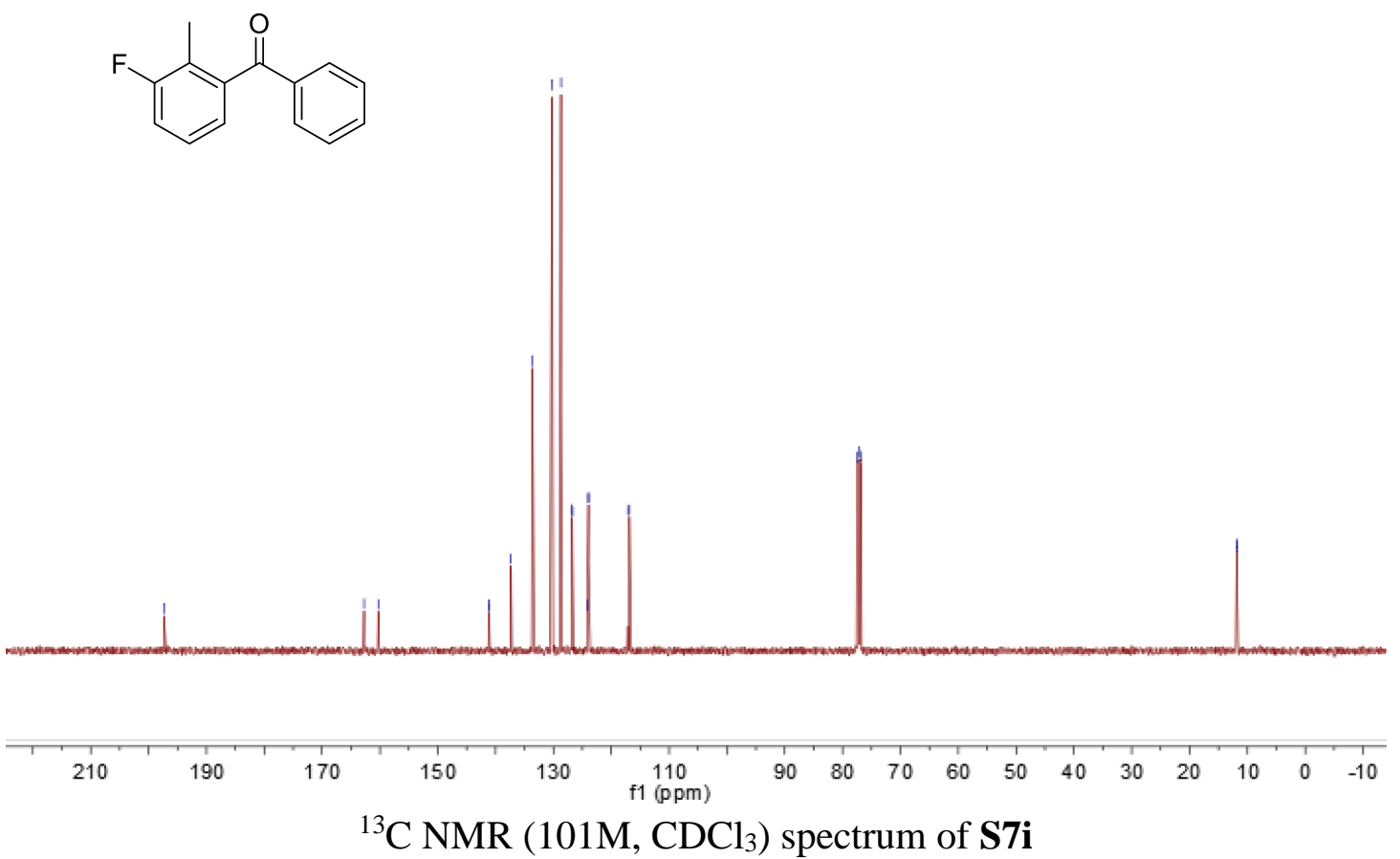

S76 
$\frac{i}{i}$
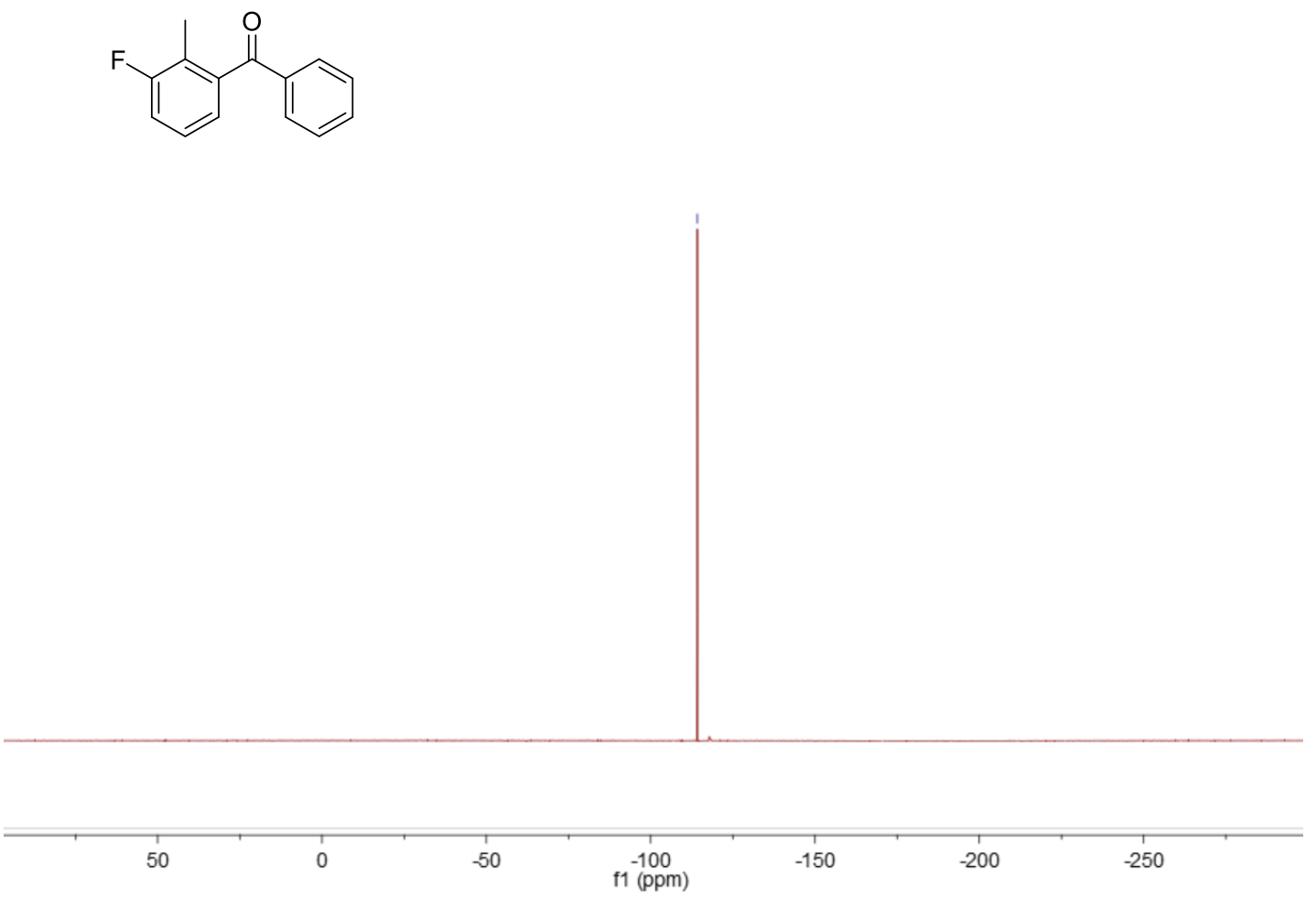

${ }^{19} \mathrm{~F}$ NMR $\left(377 \mathrm{MHz}, \mathrm{CDCl}_{3}\right)$ spectrum of $\mathbf{S 7 i}$
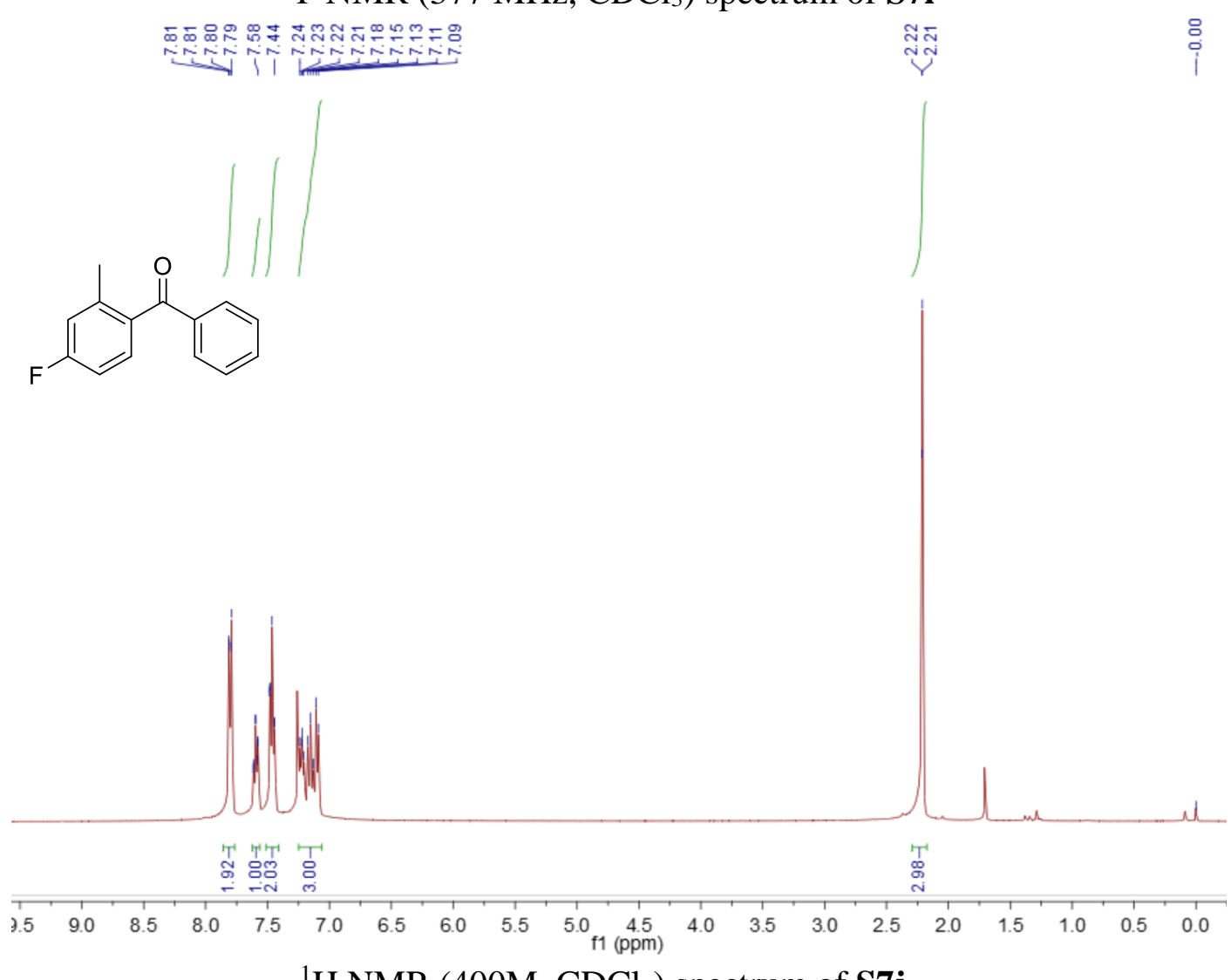

${ }^{1} \mathrm{H}$ NMR (400M, $\mathrm{CDCl}_{3}$ ) spectrum of $\mathbf{S 7} \mathbf{j}$

S77 


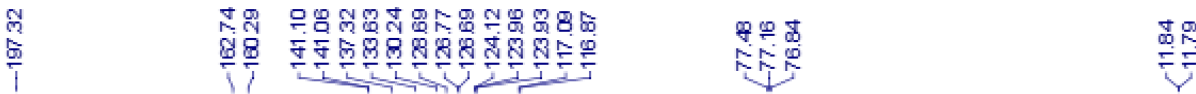<smiles>Cc1cc(F)ccc1C(=O)c1ccccc1</smiles>

21 190 150 110 $\begin{array}{lllllllllll}90 & 80 & 70 & 60 & 50 & 40 & 30 & 20 & 10 & 0 & -10\end{array}$

${ }^{13} \mathrm{C}$ NMR $\left(101 \mathrm{M}, \mathrm{CDCl}_{3}\right)$ spectrum of $\mathbf{S} \mathbf{j}$

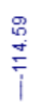<smiles>Cc1cc(F)ccc1C(=O)c1ccccc1</smiles>

50

${ }^{19} \mathrm{~F}$ NMR (377 MHz, $\mathrm{CDCl}_{3}$ ) spectrum of $\mathbf{S} \mathbf{7} \mathbf{j}$ 


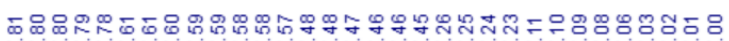
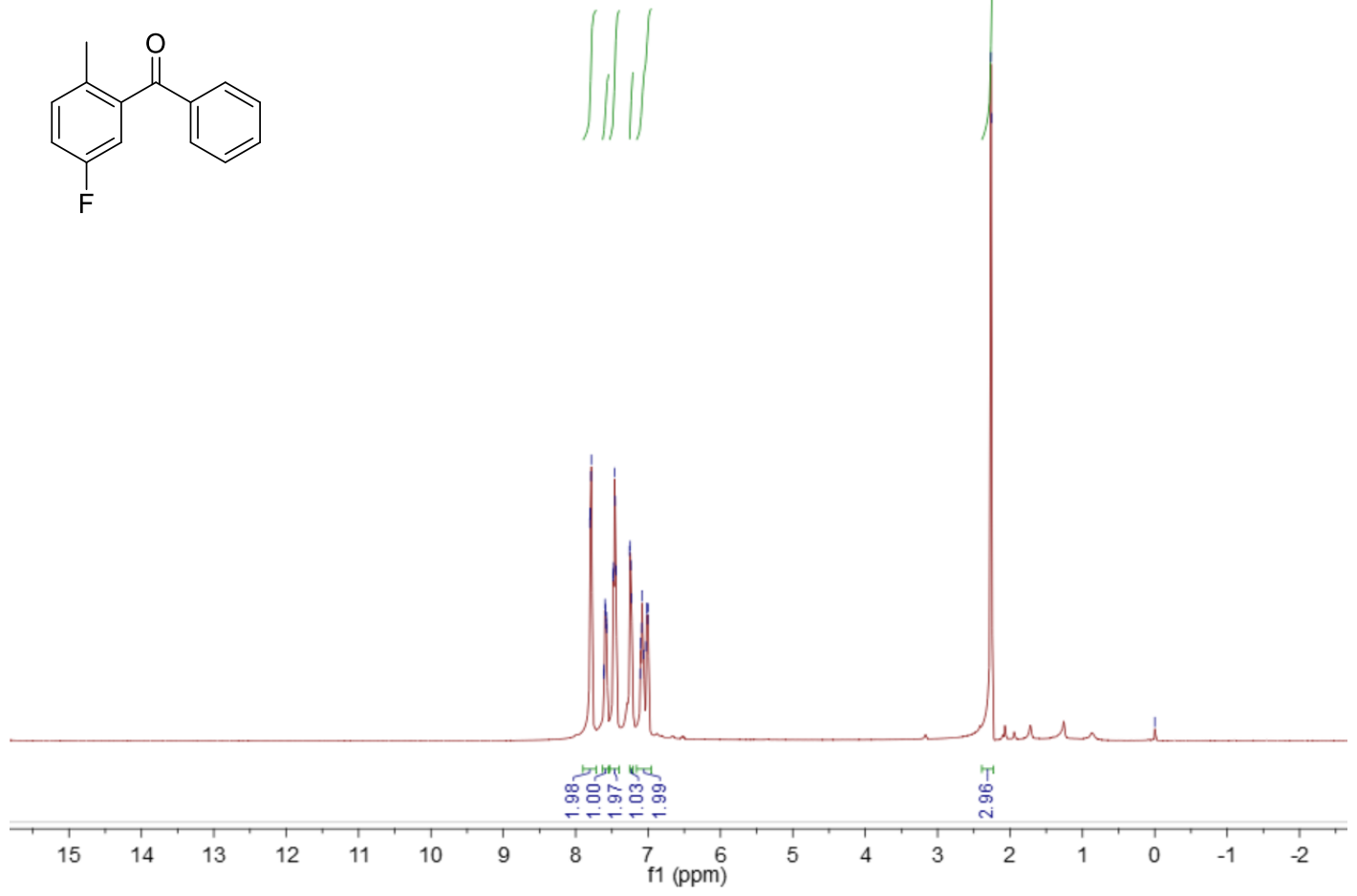

${ }^{1} \mathrm{H}$ NMR $\left(400 \mathrm{M}, \mathrm{CDCl}_{3}\right)$ spectrum of $\mathbf{S 7 k}$

$\stackrel{9}{\stackrel{9}{1}}$

문

哭吉

$\underset{\substack{\mathrm{g} \\ \mathrm{I}}}{\mathrm{d}}$
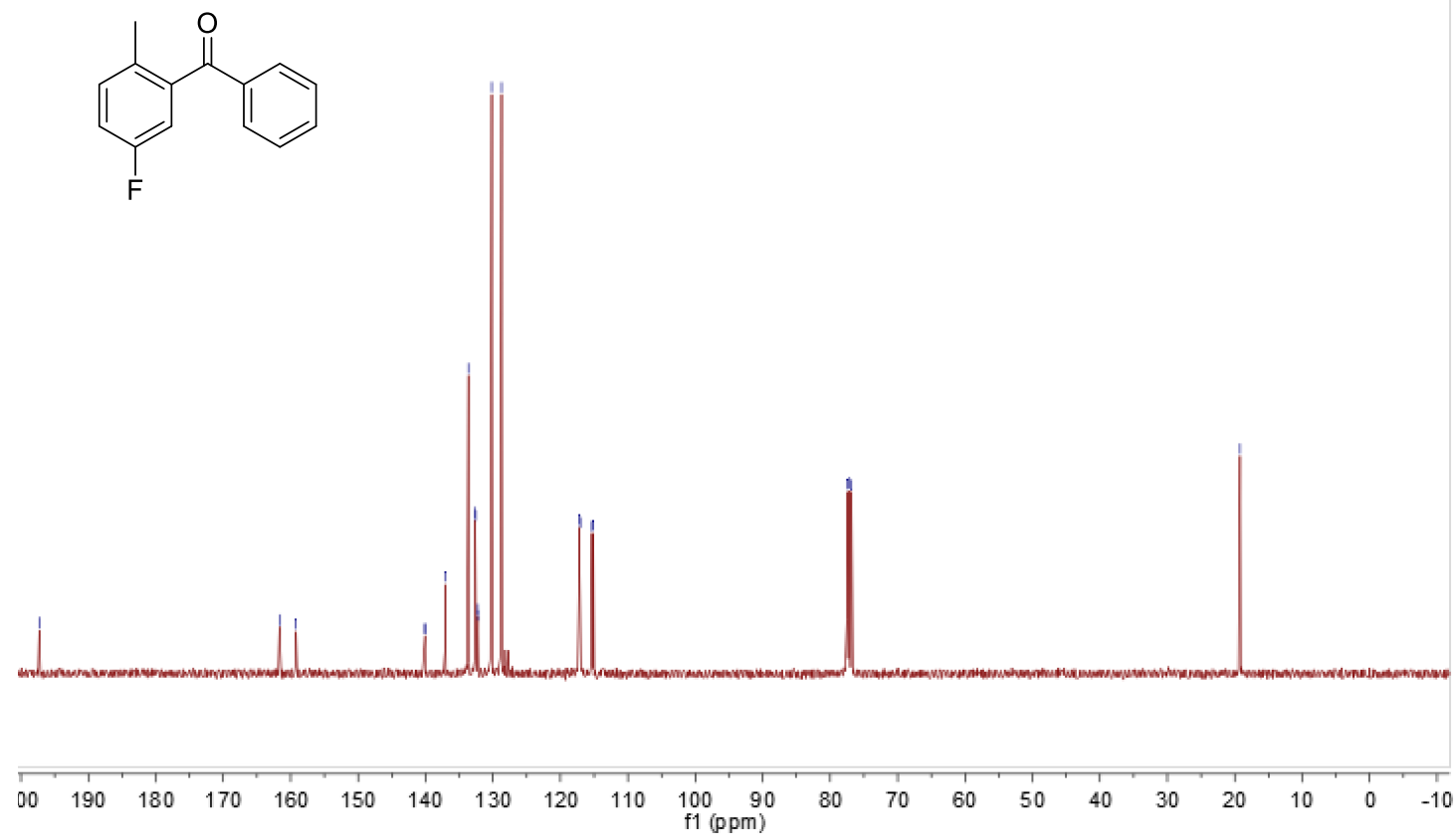

${ }^{13} \mathrm{C}$ NMR $\left(101 \mathrm{M}, \mathrm{CDCl}_{3}\right)$ spectrum of $\mathbf{S 7 k}$

S79 


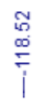
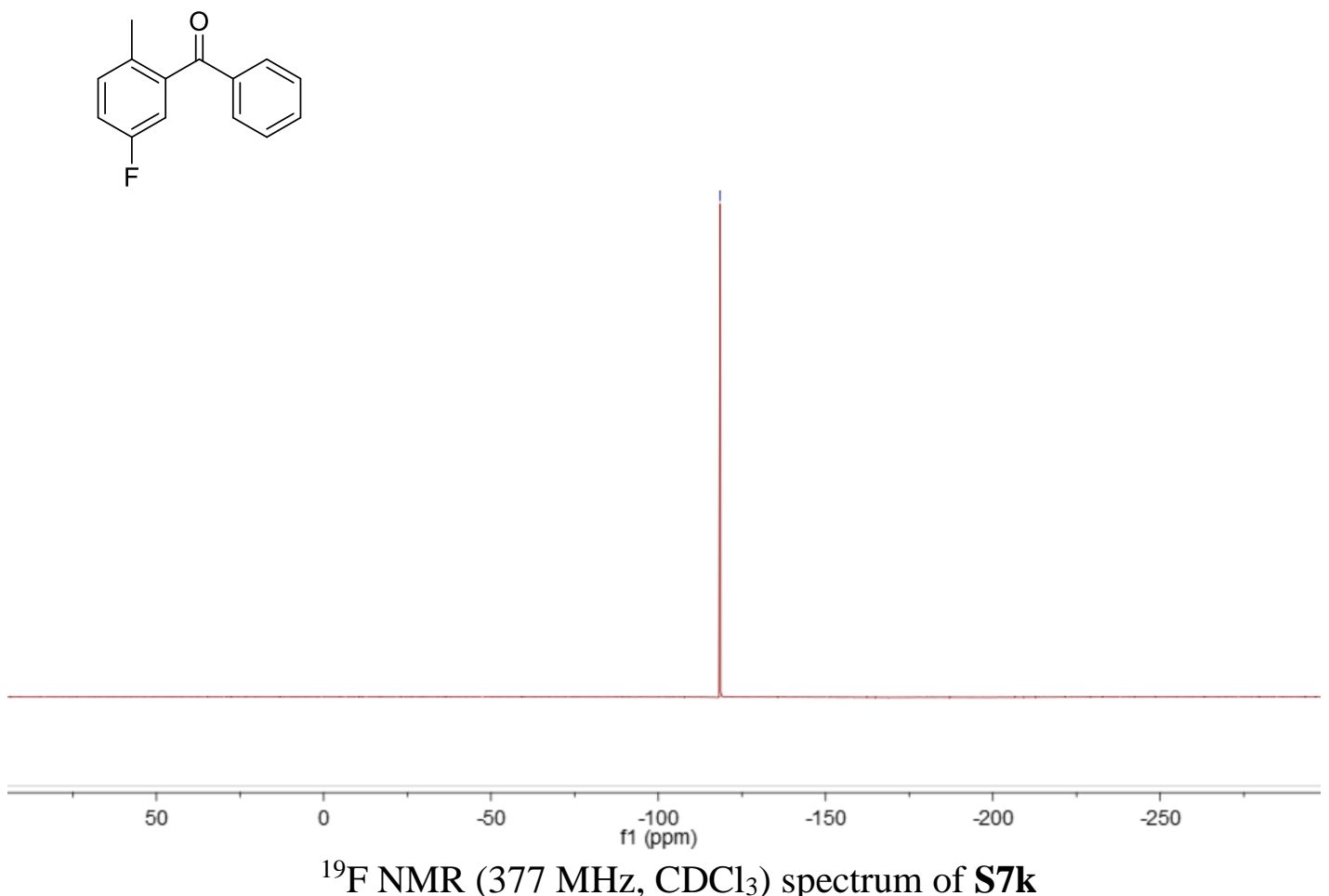

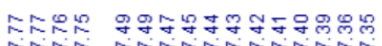
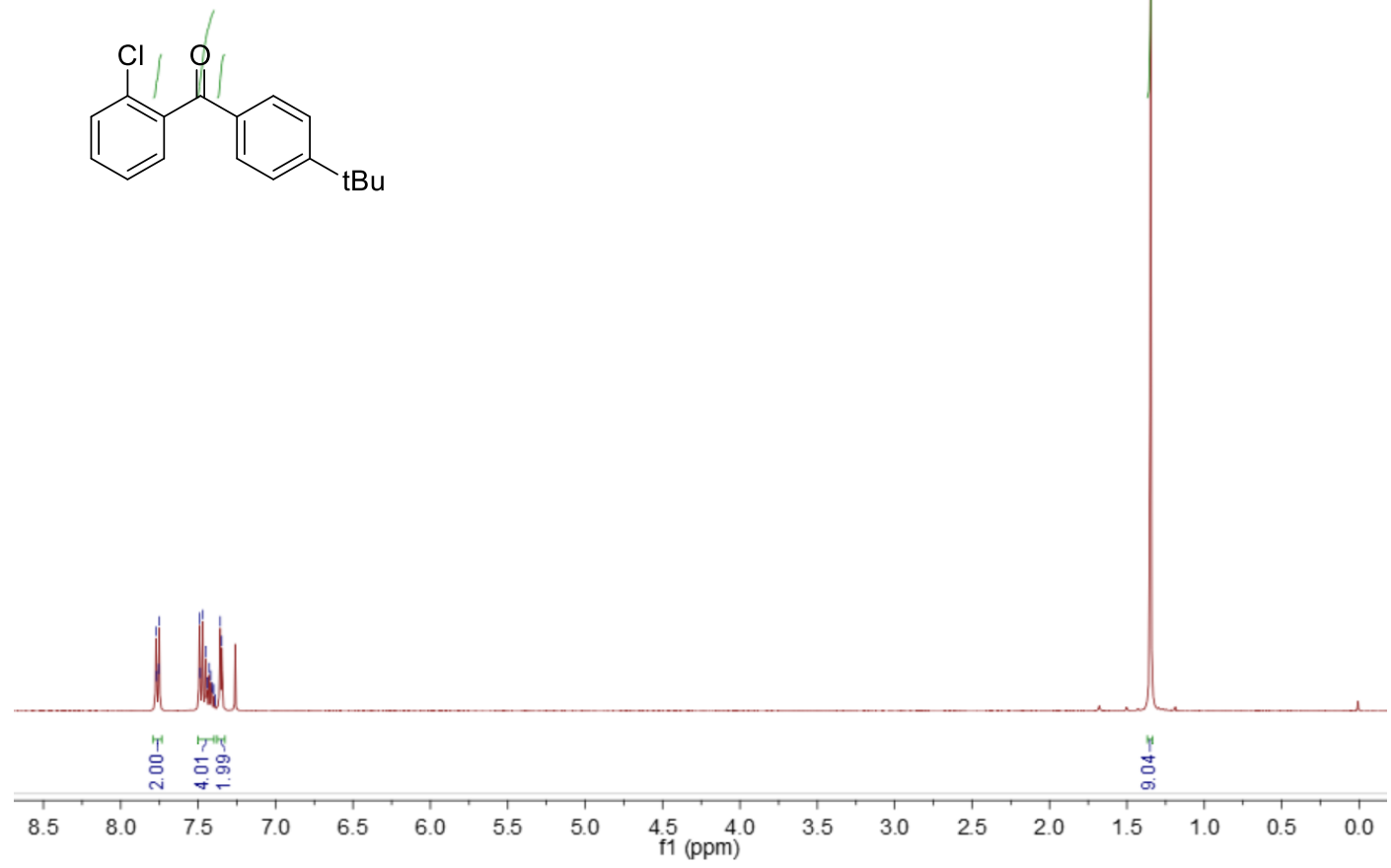

${ }^{1} \mathrm{H}$ NMR (400M, $\mathrm{CDCl}_{3}$ ) spectrum of $\mathbf{S 7 a e}$

$\mathrm{S} 80$ 
浑

些垔

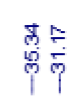
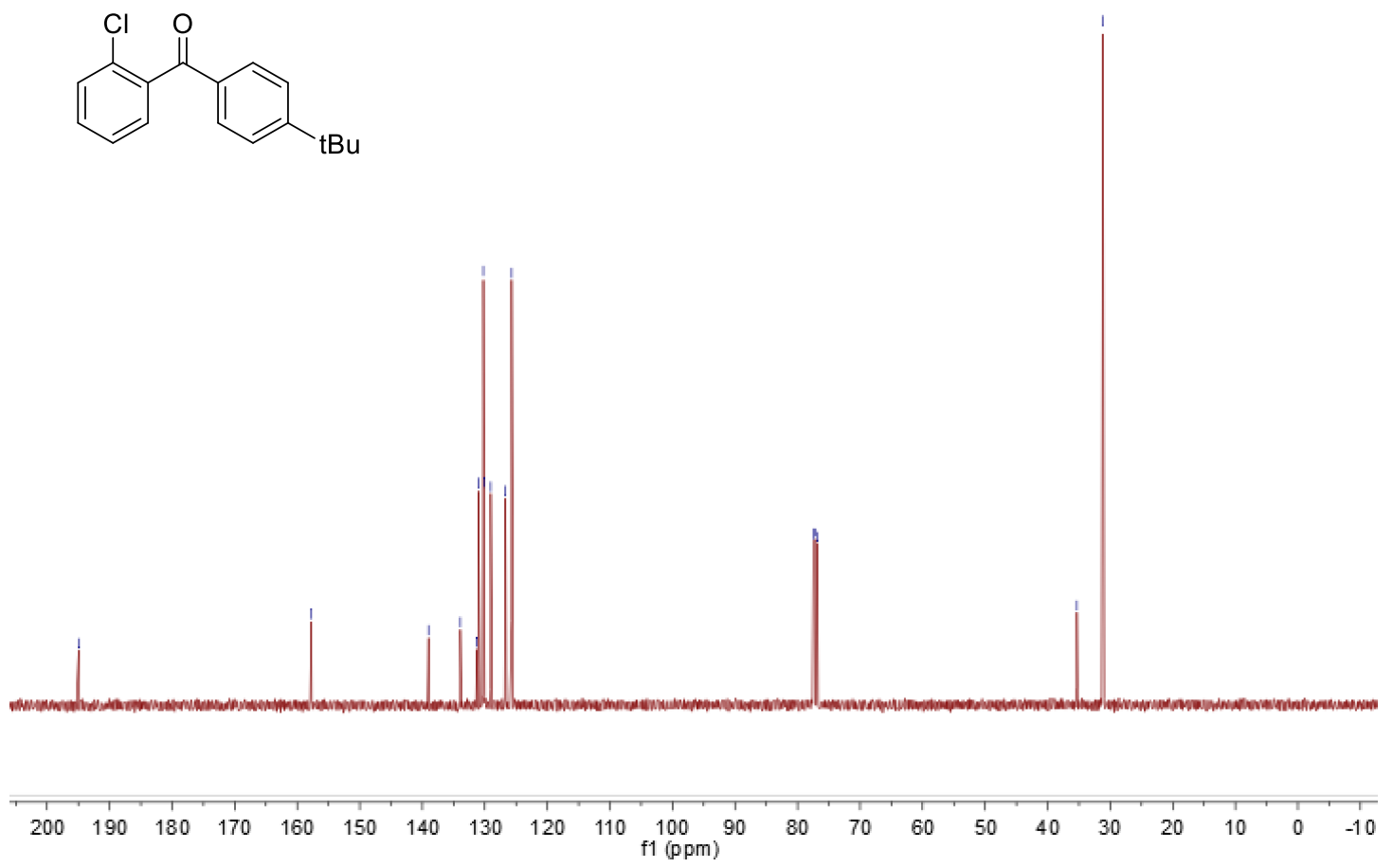

${ }^{13} \mathrm{C}$ NMR $\left(101 \mathrm{M}, \mathrm{CDCl}_{3}\right)$ spectrum of $\mathbf{S 7 a e}$

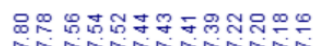

:
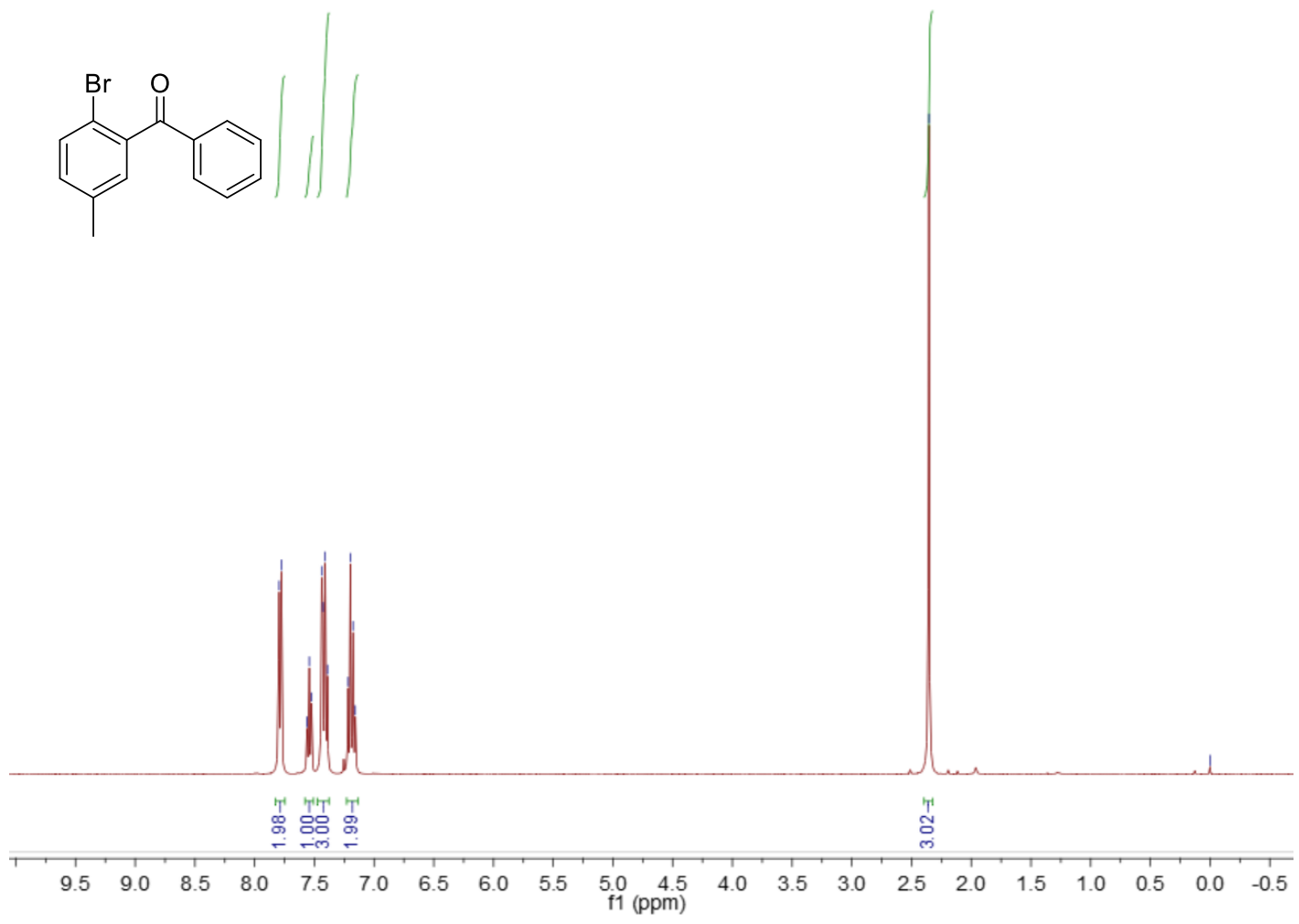

${ }^{1} \mathrm{H}$ NMR (400M, $\mathrm{CDCl}_{3}$ ) spectrum of S7ai

$\mathrm{S} 81$ 


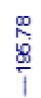

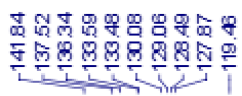

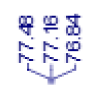

$\stackrel{8}{\circ}$
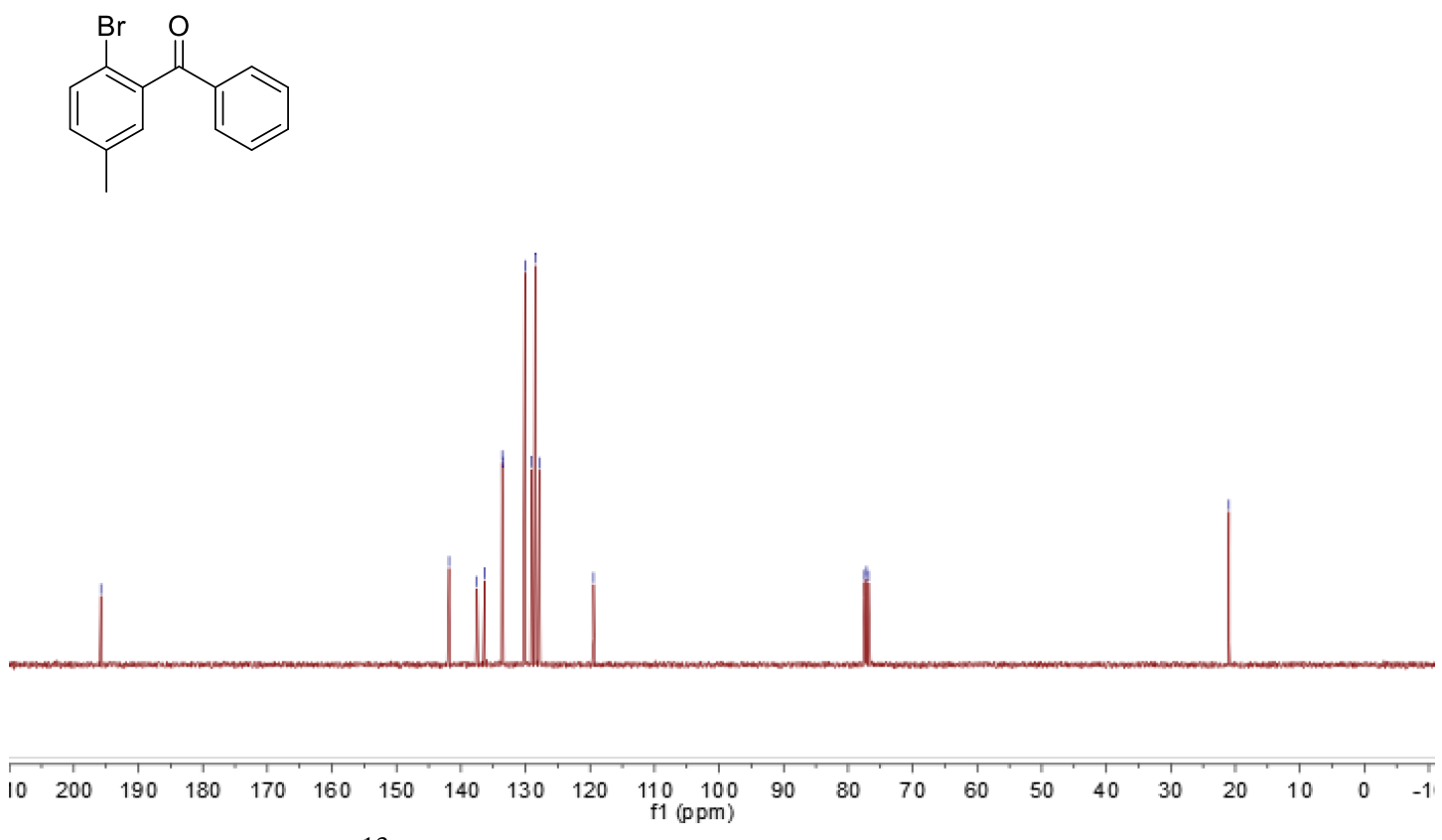

${ }^{13} \mathrm{C}$ NMR $\left(101 \mathrm{M}, \mathrm{CDCl}_{3}\right)$ spectrum of S7ai

$\underset{0}{\infty} \underset{1}{\infty}$

$\underset{\substack{\infty \\ j i j}}{\infty}$

$\stackrel{\circ}{i}$
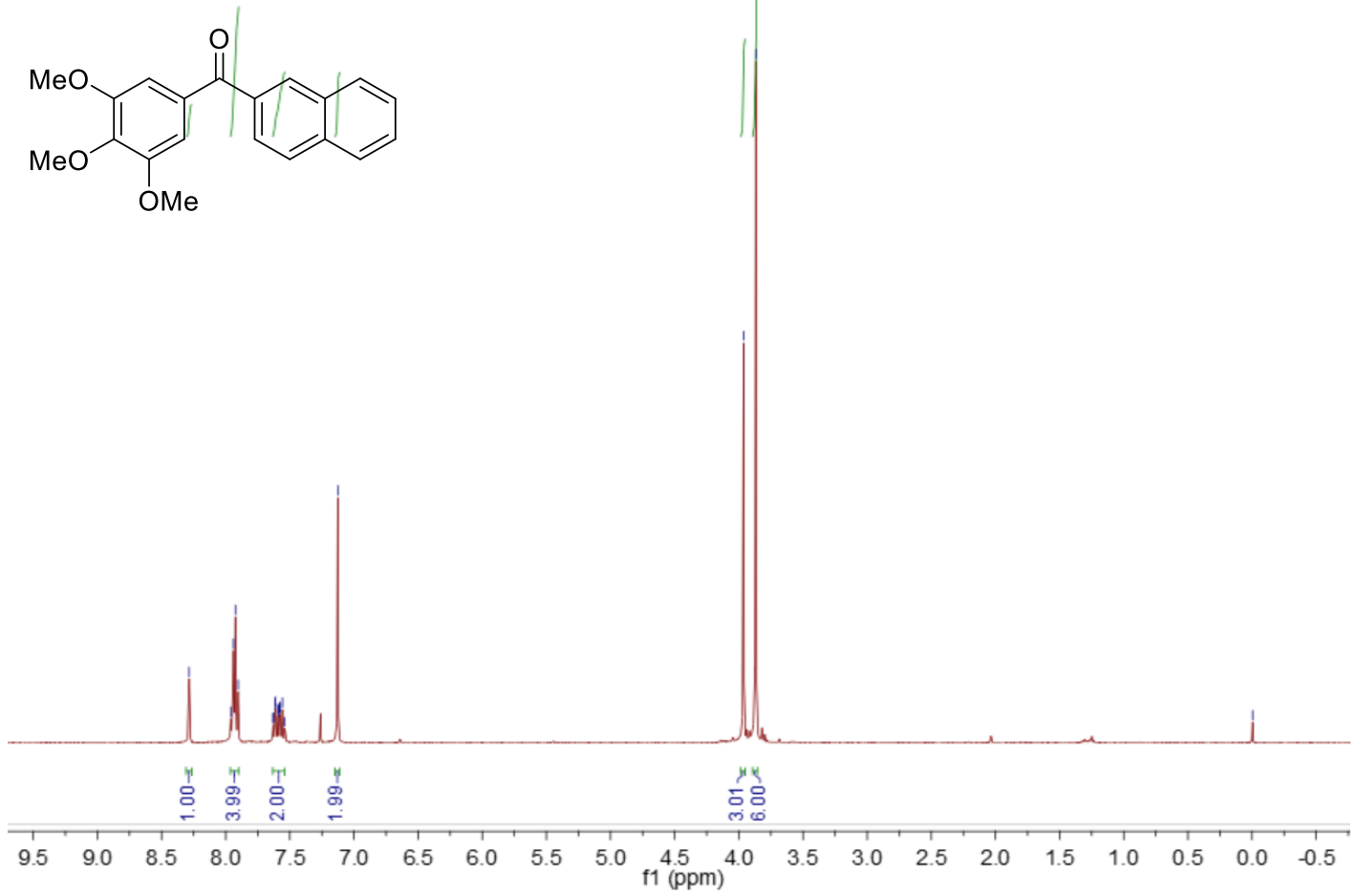

${ }^{1} \mathrm{H}$ NMR (400M, $\mathrm{CDCl}_{3}$ ) spectrum of SS7am

S82 
<smiles>COc1cc(C(=O)c2ccc3ccccc3c2)cc(OC)c1OC</smiles>
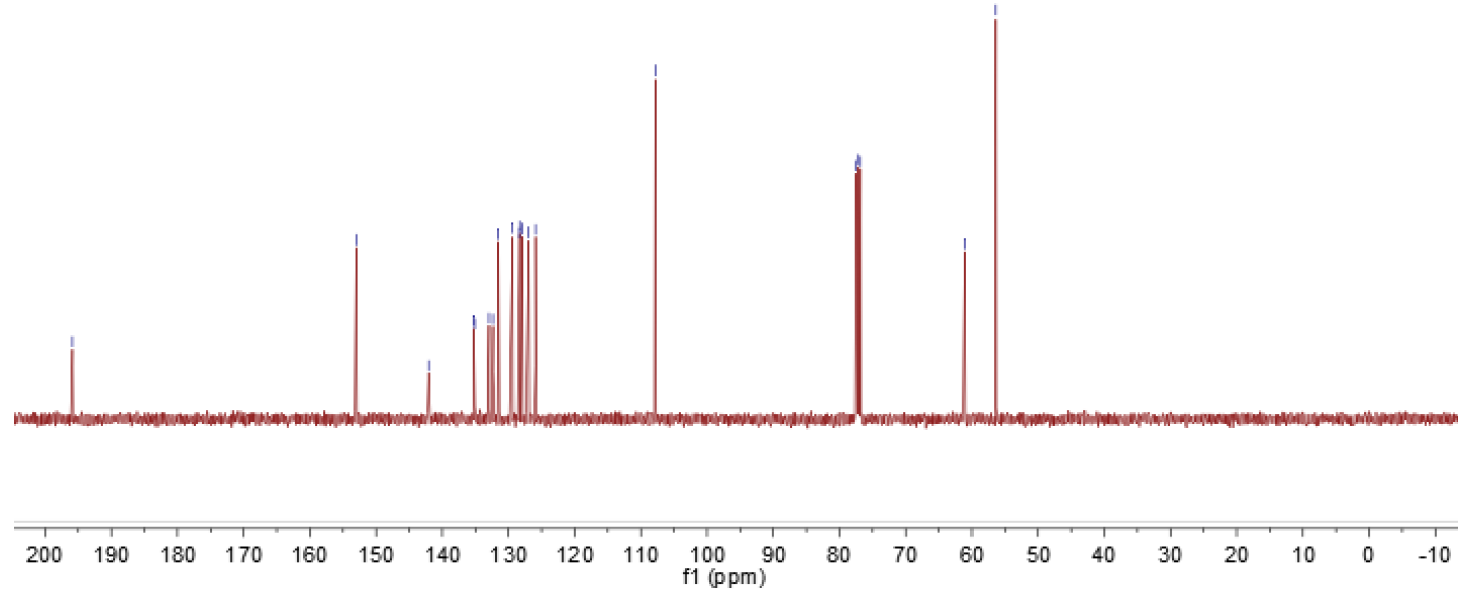

${ }^{13} \mathrm{C}$ NMR $\left(101 \mathrm{M}, \mathrm{CDCl}_{3}\right)$ spectrum of SS7am

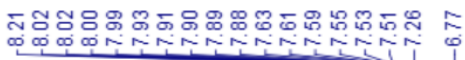
宓禺<smiles>COc1cc(C(=O)c2ccc3ccccc3c2)c(Br)c(OC)c1OC</smiles>
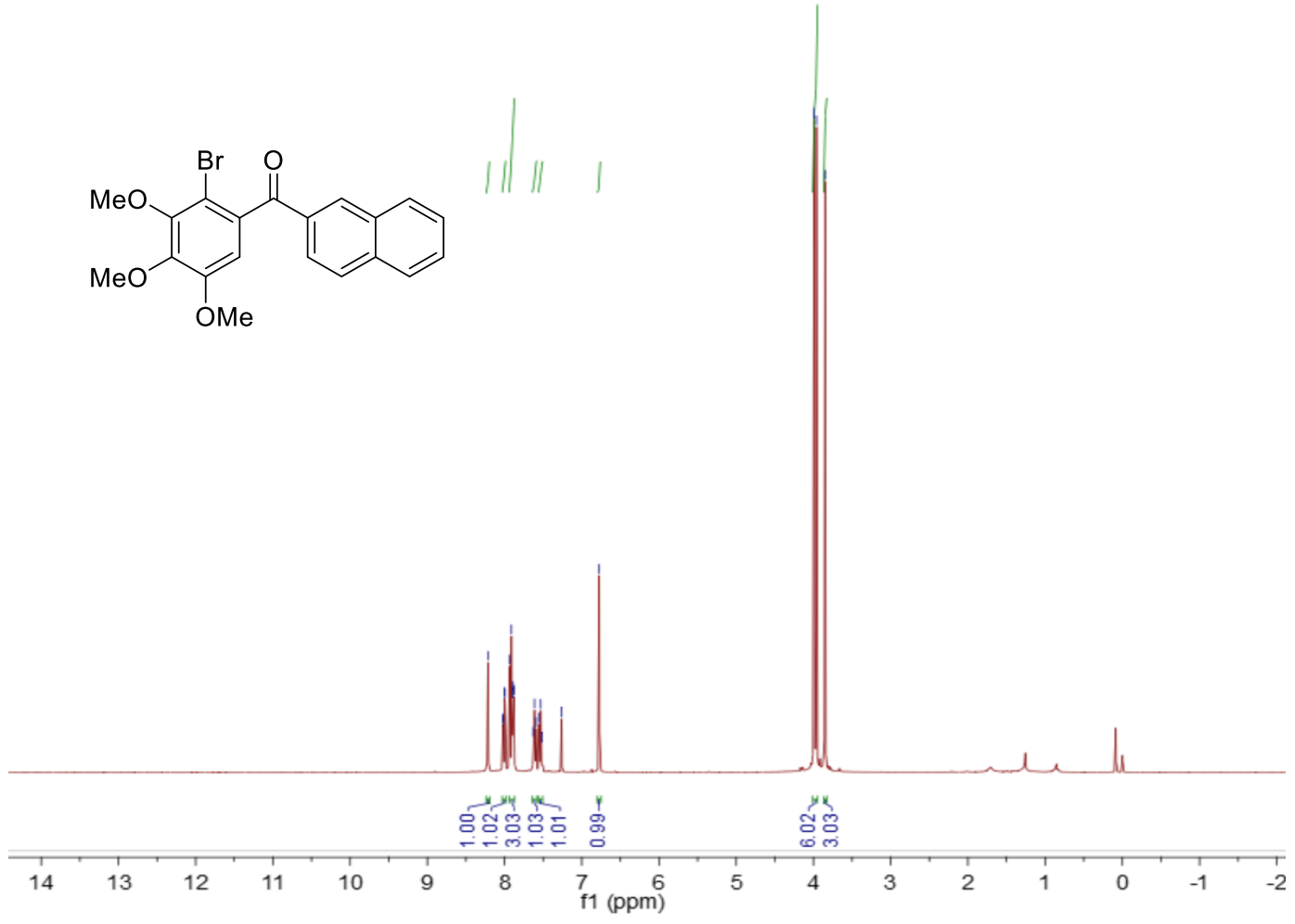

${ }^{1} \mathrm{H}$ NMR (400M, $\mathrm{CDCl}_{3}$ ) spectrum of S7am 

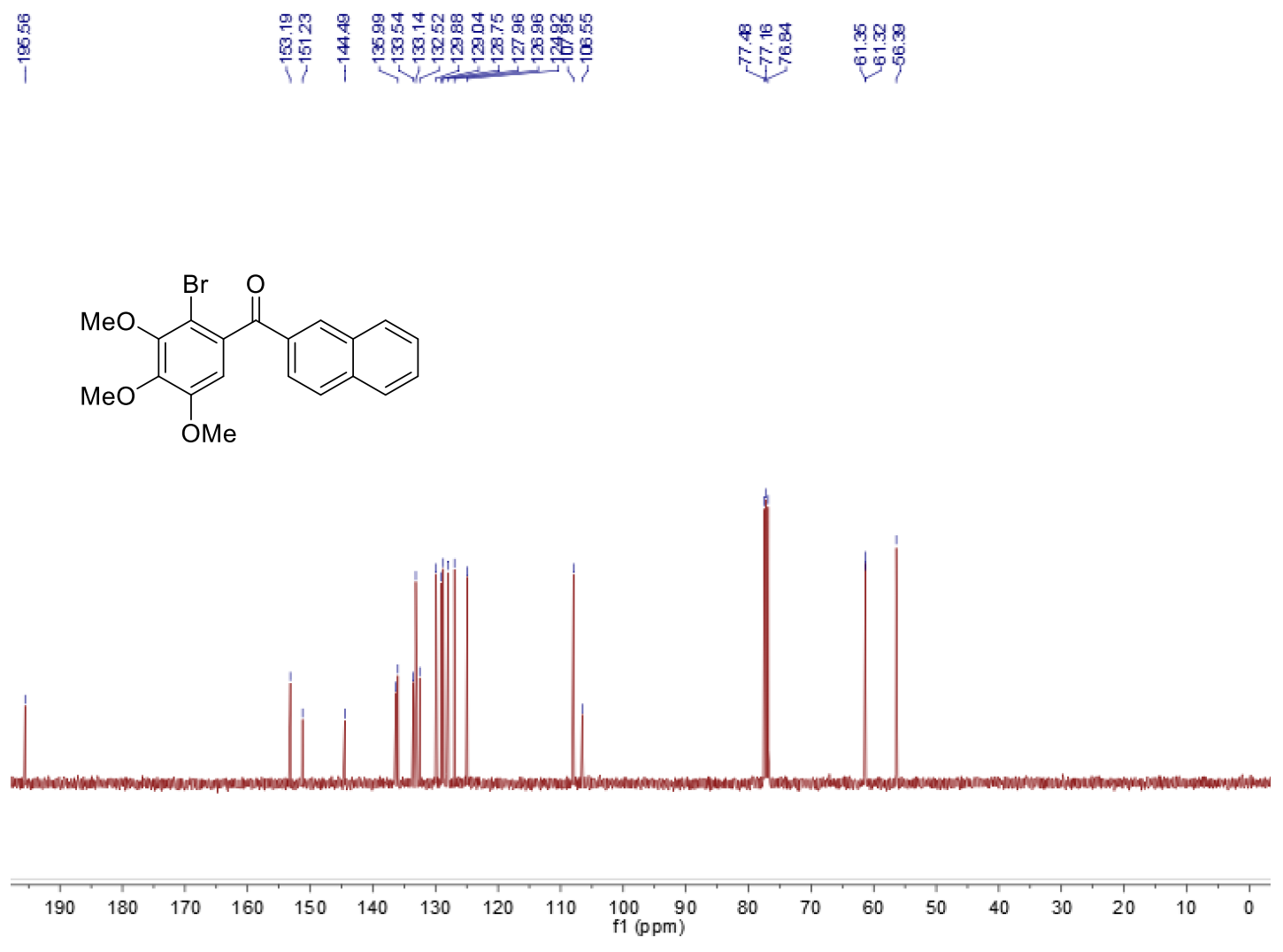

${ }^{13} \mathrm{C}$ NMR $\left(101 \mathrm{M}, \mathrm{CDCl}_{3}\right)$ spectrum of S7am

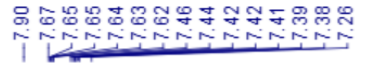

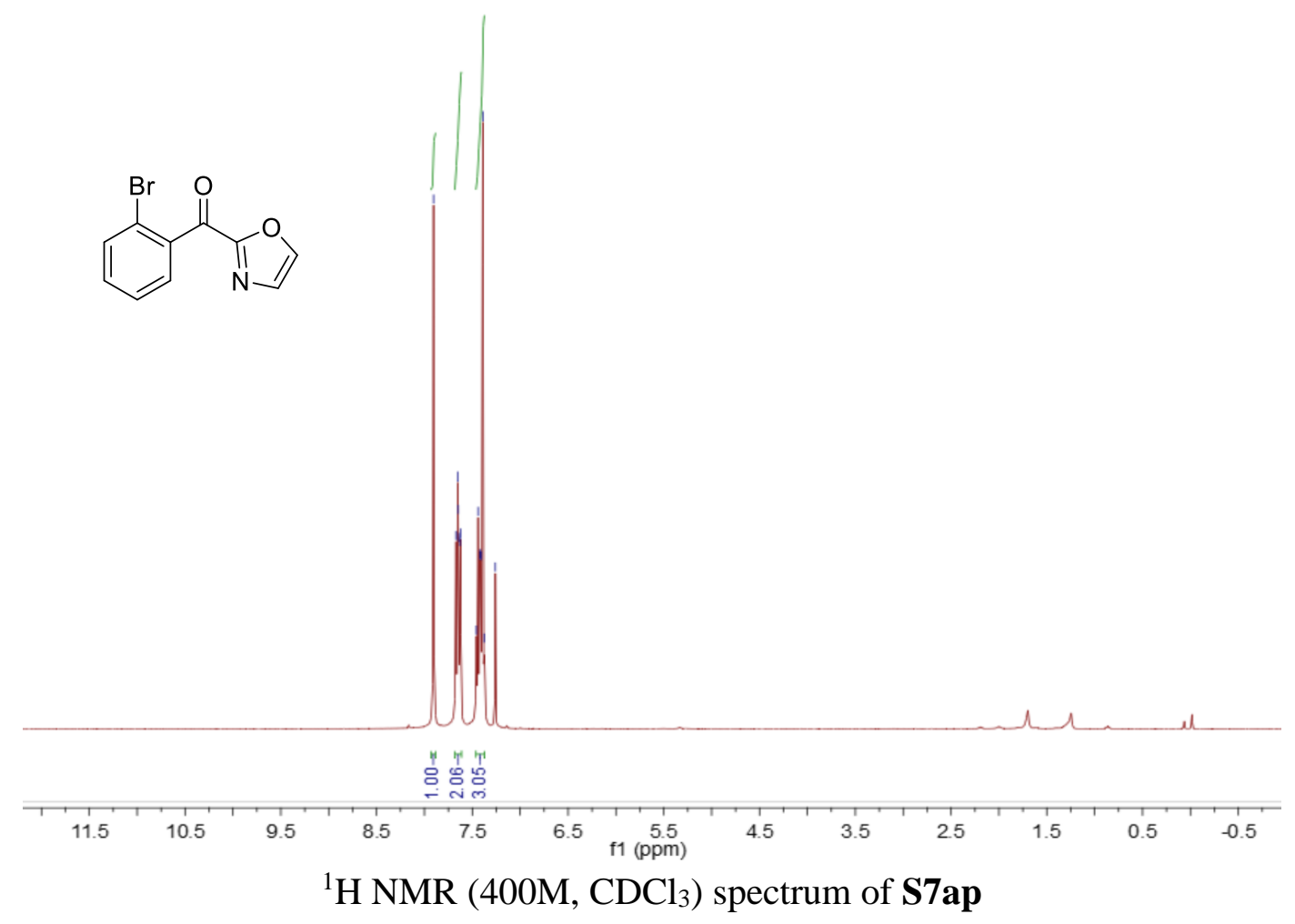



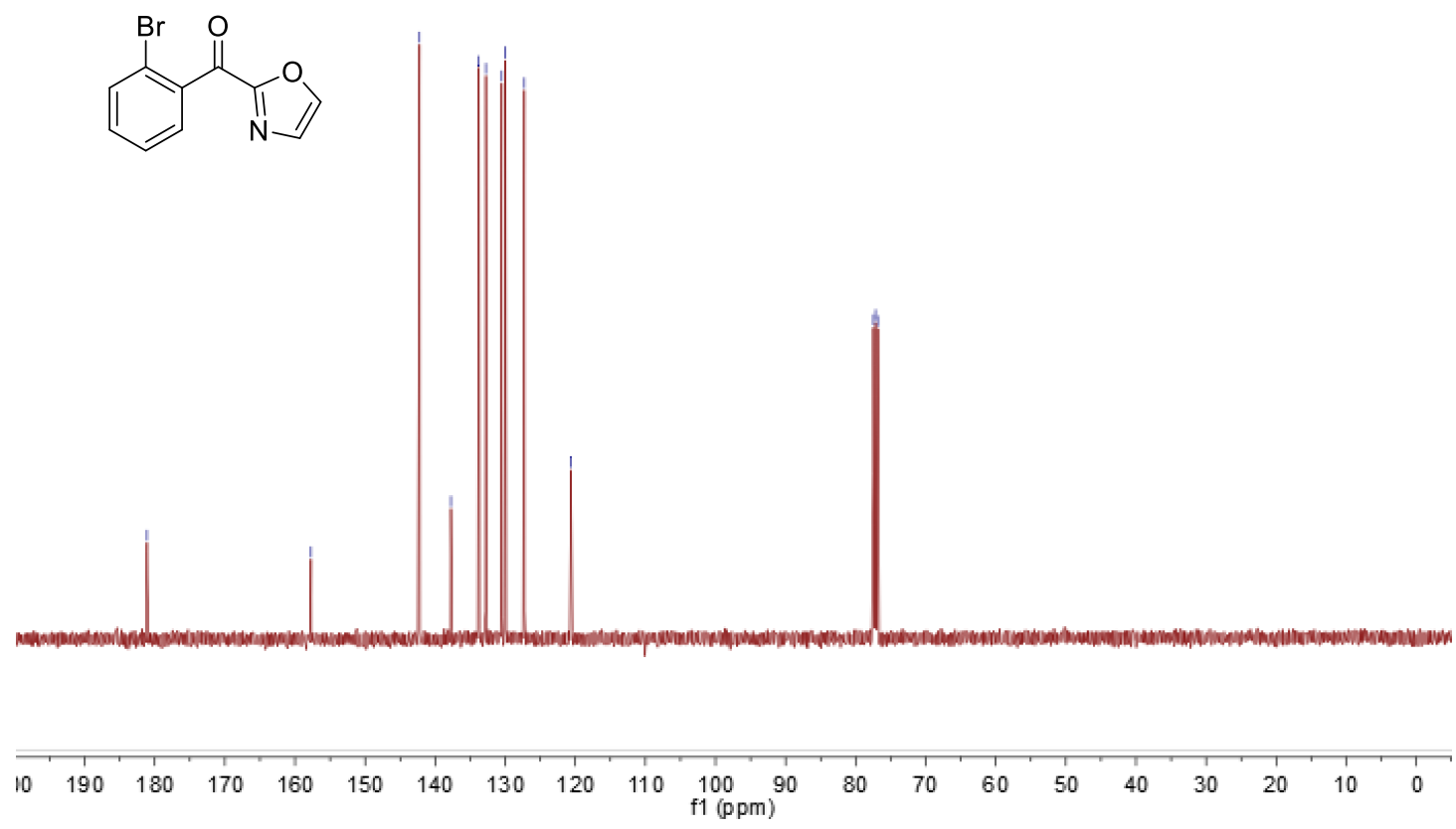

${ }^{13} \mathrm{C}$ NMR $\left(101 \mathrm{M}, \mathrm{CDCl}_{3}\right)$ spectrum of S7ap

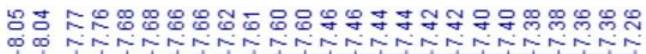

i

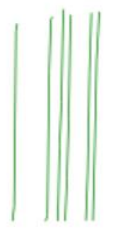

$\underbrace{B r}_{N}$

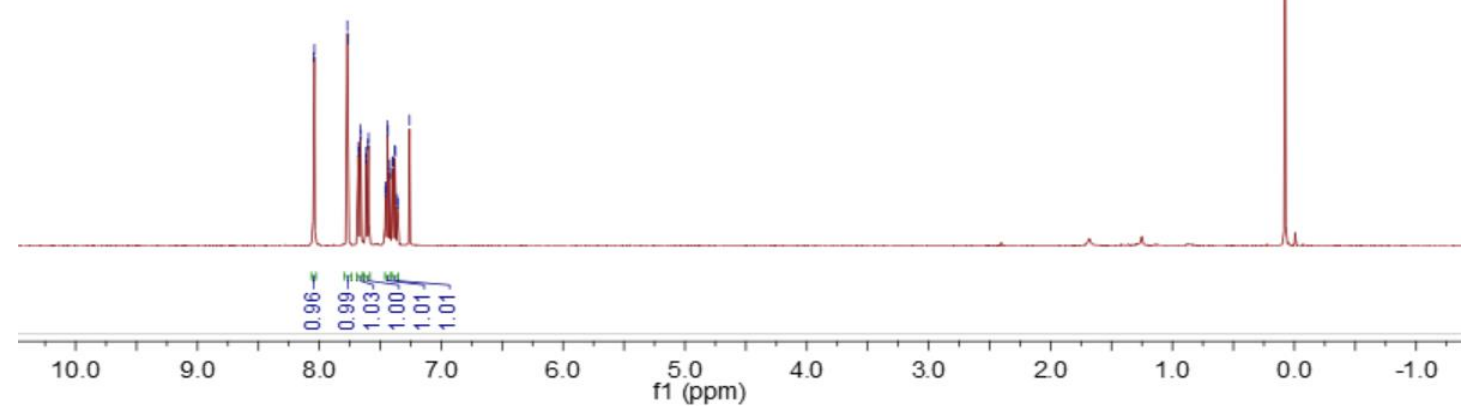

${ }^{1} \mathrm{H}$ NMR (400M, $\mathrm{CDCl}_{3}$ ) spectrum of S7aq 
<smiles>O=C(c1nccs1)c1ccccc1Br</smiles>
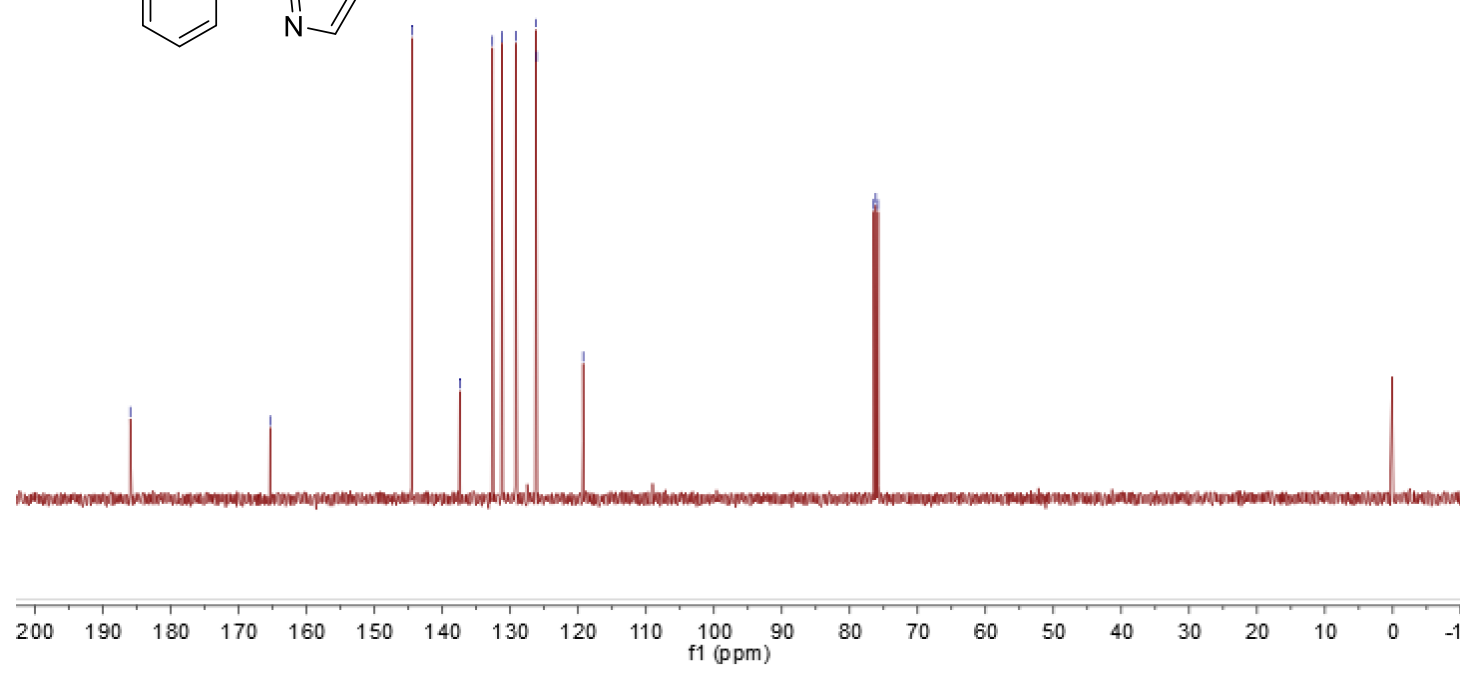

${ }^{13} \mathrm{C}$ NMR $\left(101 \mathrm{M}, \mathrm{CDCl}_{3}\right)$ spectrum of S7aq

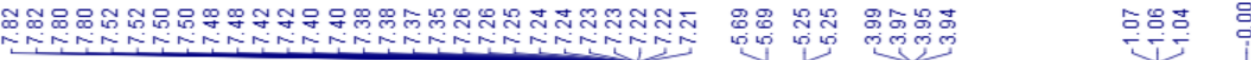

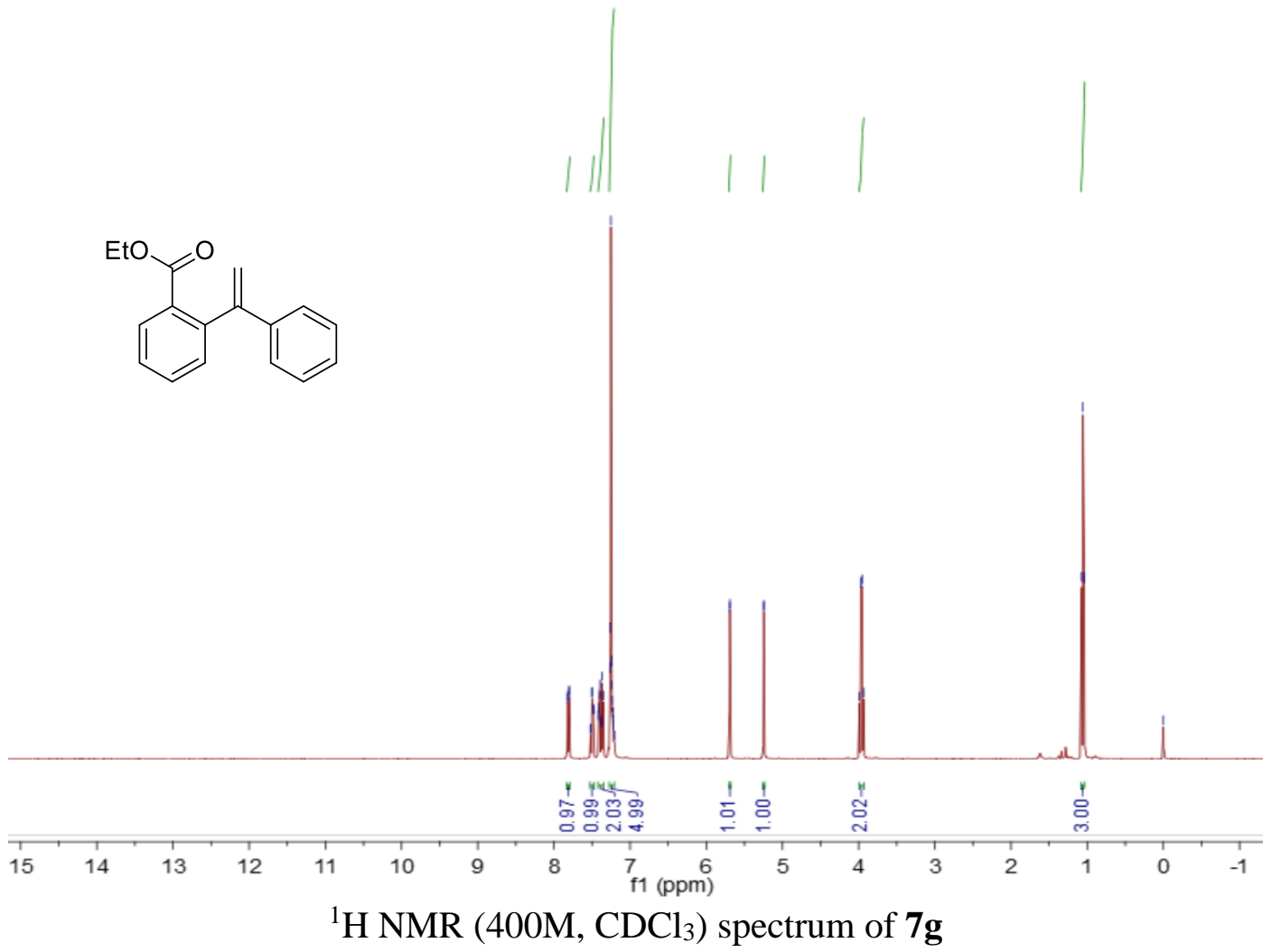



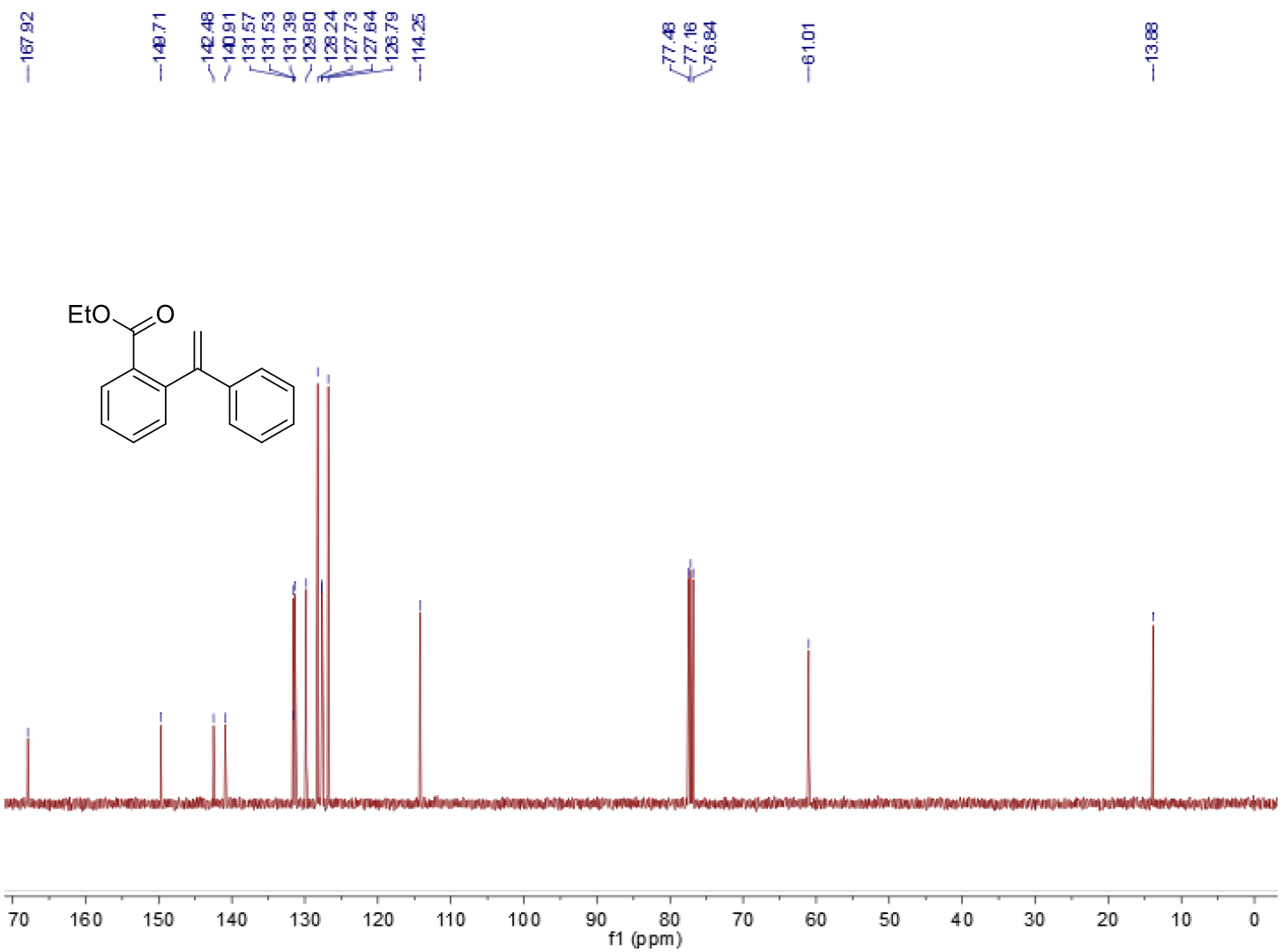

${ }^{13} \mathrm{C} \mathrm{NMR}\left(101 \mathrm{M}, \mathrm{CDCl}_{3}\right)$ spectrum of $\mathbf{7 g}$

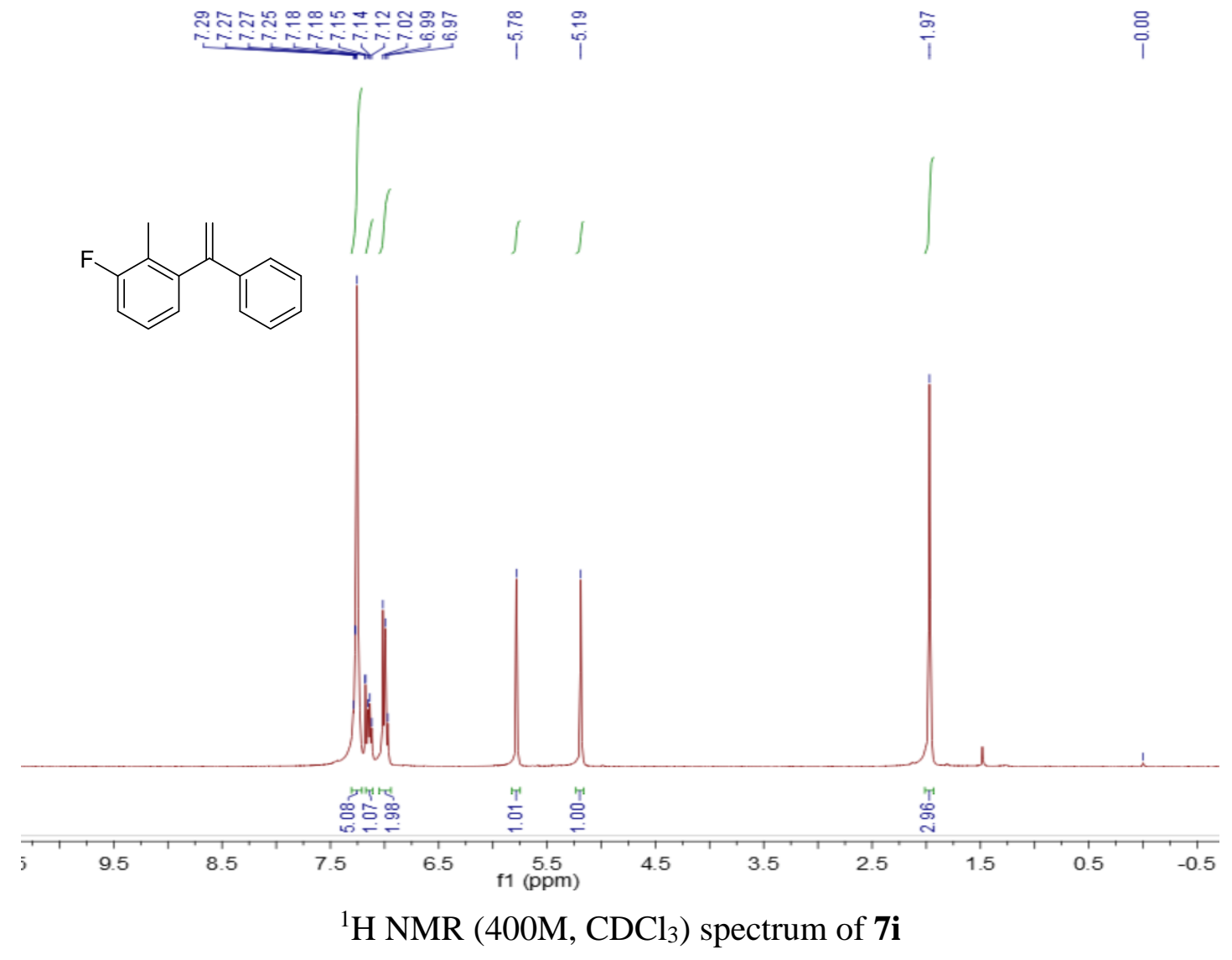



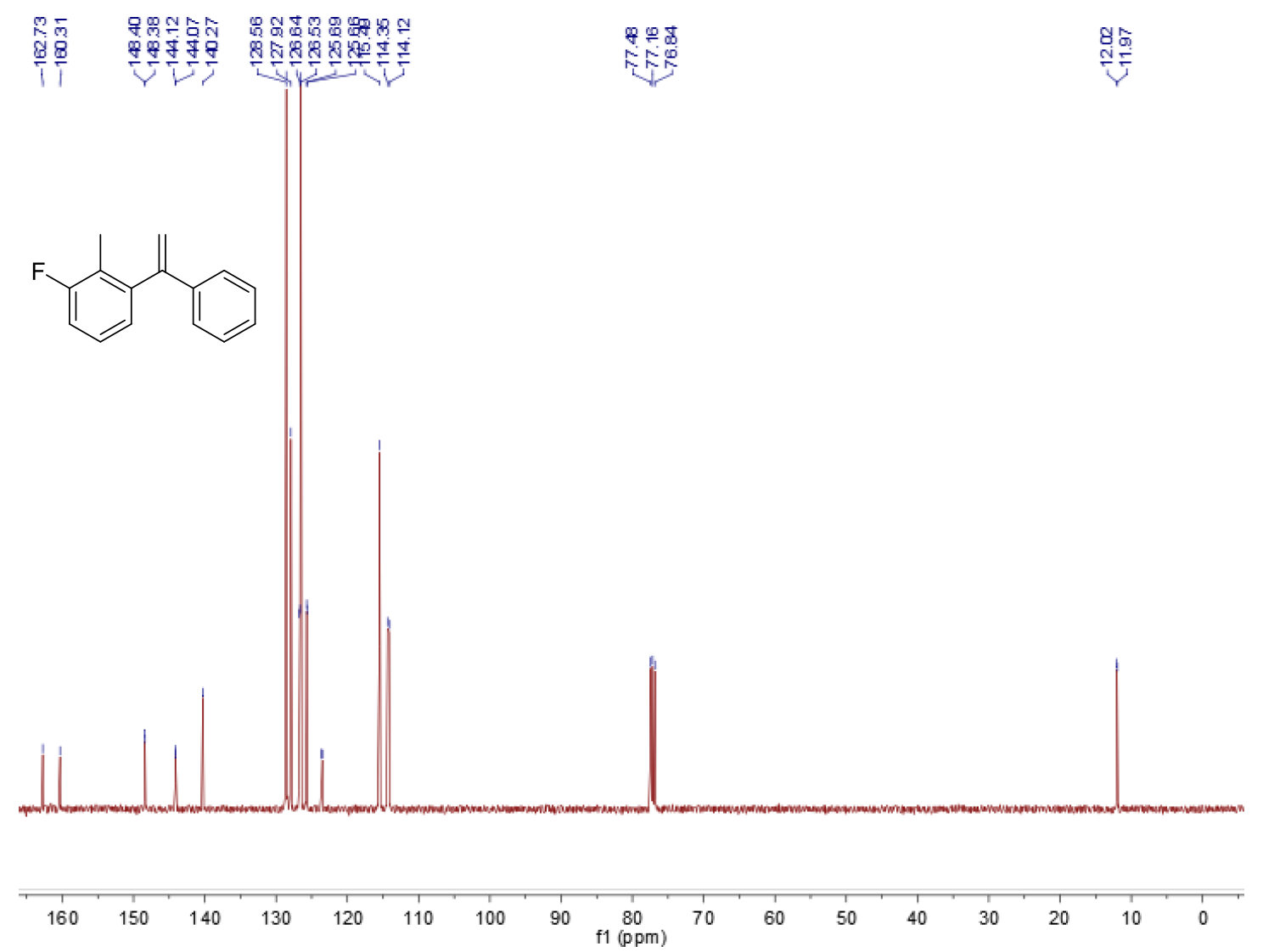

${ }^{13} \mathrm{C} \mathrm{NMR}\left(101 \mathrm{M}, \mathrm{CDCl}_{3}\right)$ spectrum of $7 \mathbf{i}$

$\stackrel{\circ}{\stackrel{0}{\oplus}}$<smiles>C=C(c1ccccc1)c1cccc(F)c1C</smiles>

${ }^{19} \mathrm{~F}$ NMR (377 MHz, $\left.\mathrm{CDCl}_{3}\right)$ spectrum of $7 \mathbf{i}$ 


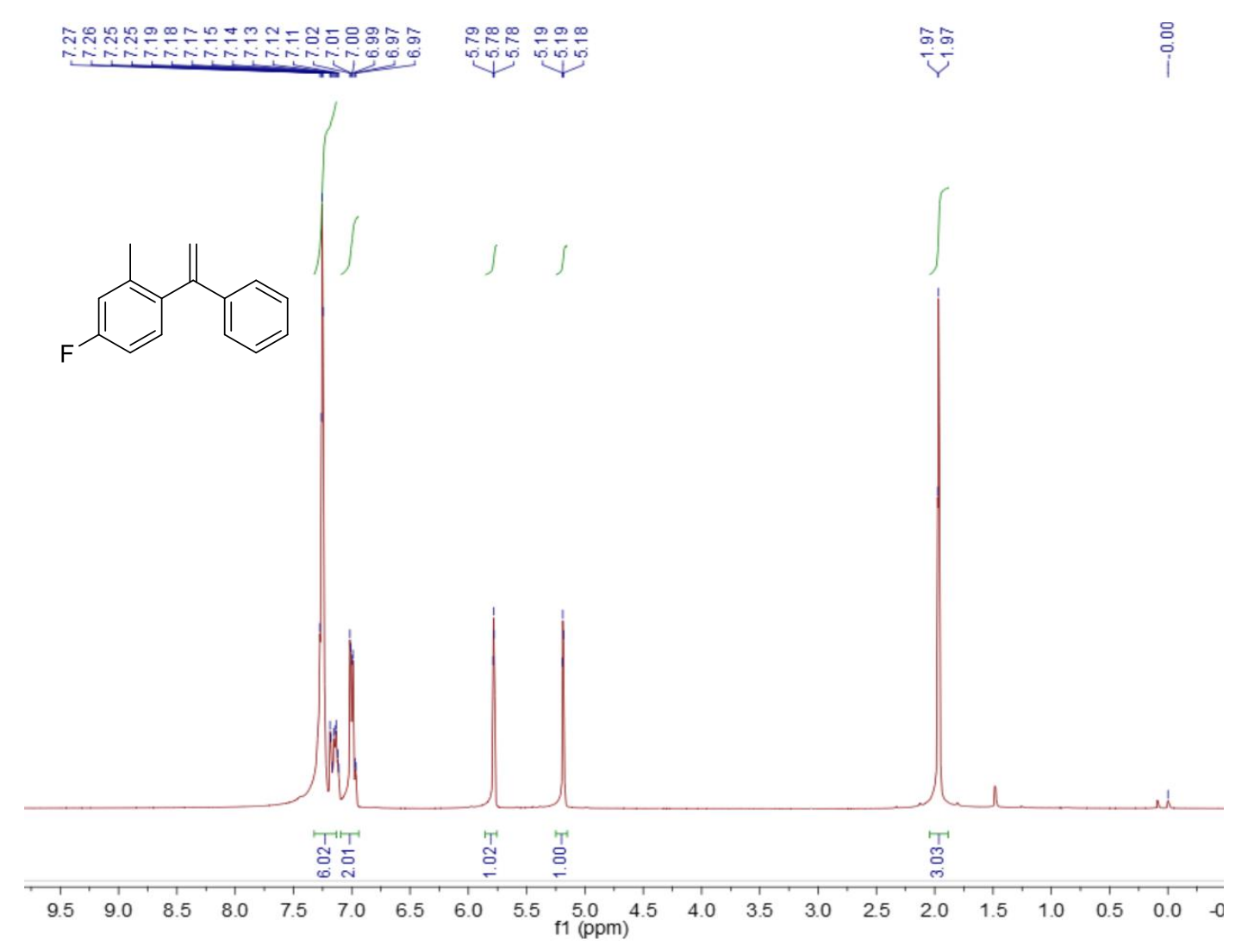

${ }^{1} \mathrm{H}$ NMR (400M, $\mathrm{CDCl}_{3}$ ) spectrum of $\mathbf{7 j}$

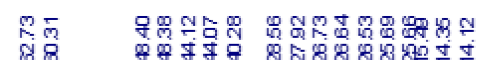

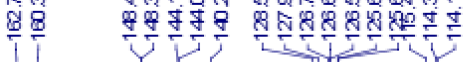

T

要蕰

85

몷

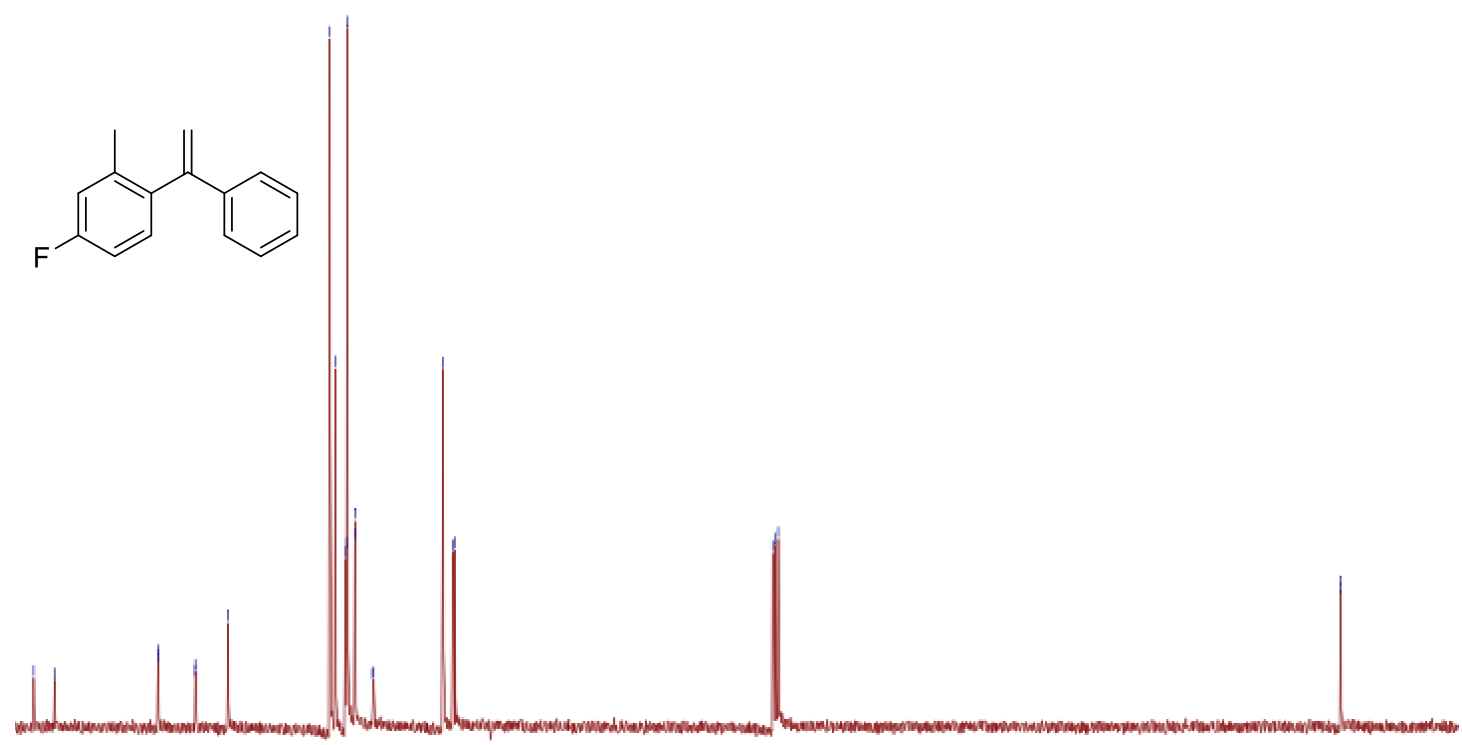

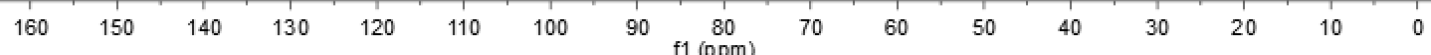

${ }^{13} \mathrm{C}$ NMR (101M, $\left.\mathrm{CDCl}_{3}\right)$ spectrum of $\mathbf{7} \mathbf{j}$ 
$\stackrel{\text { ले }}{\stackrel{\text { i }}{\bar{i}}}$
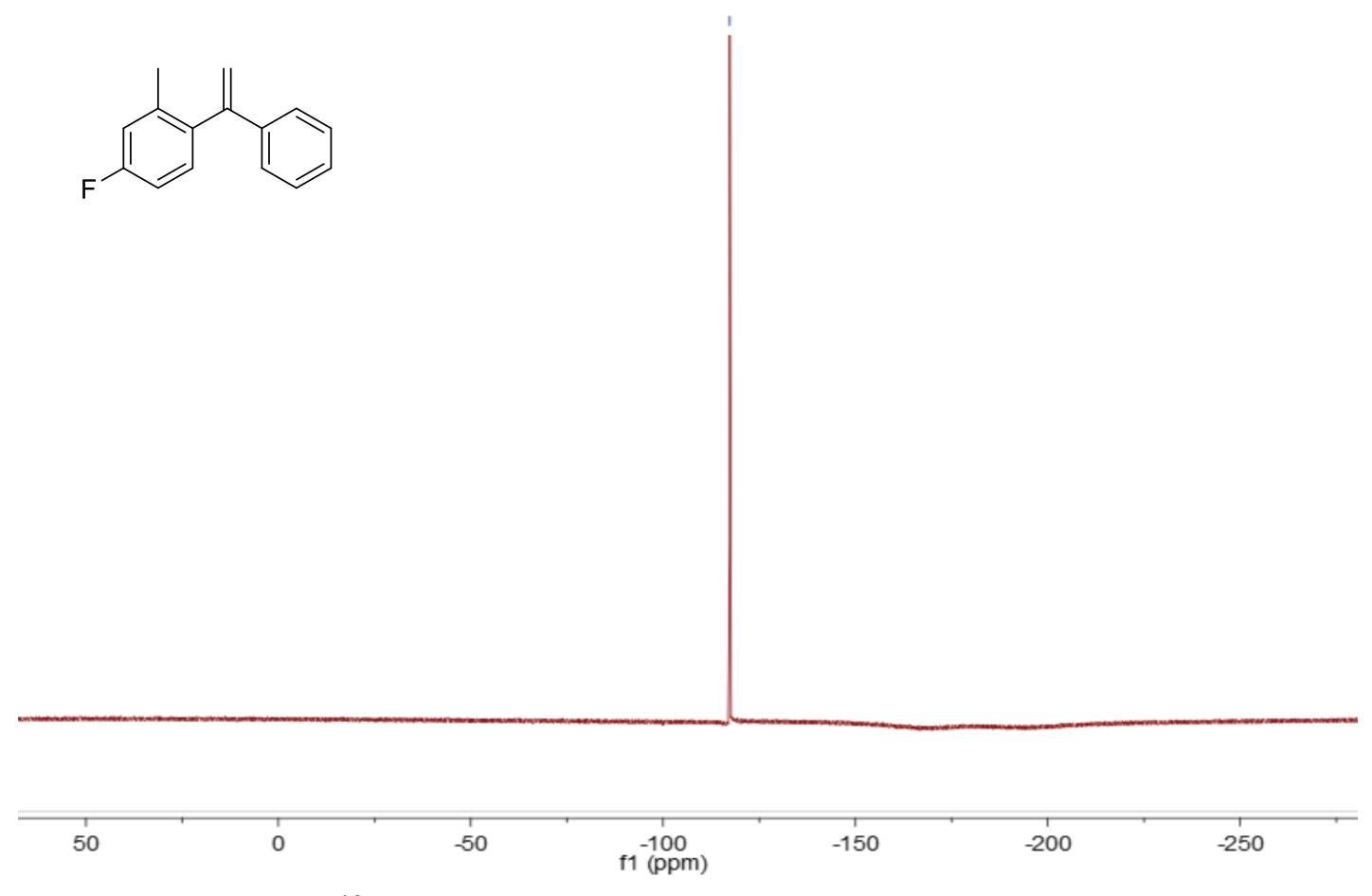

${ }^{19} \mathrm{~F}$ NMR (377 MHz, $\left.\mathrm{CDCl}_{3}\right)$ spectrum of $7 \mathbf{j}$

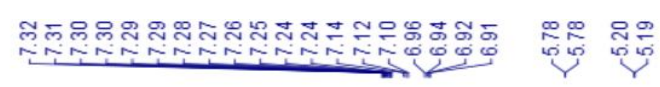

$\stackrel{\text { i }}{1}$

:

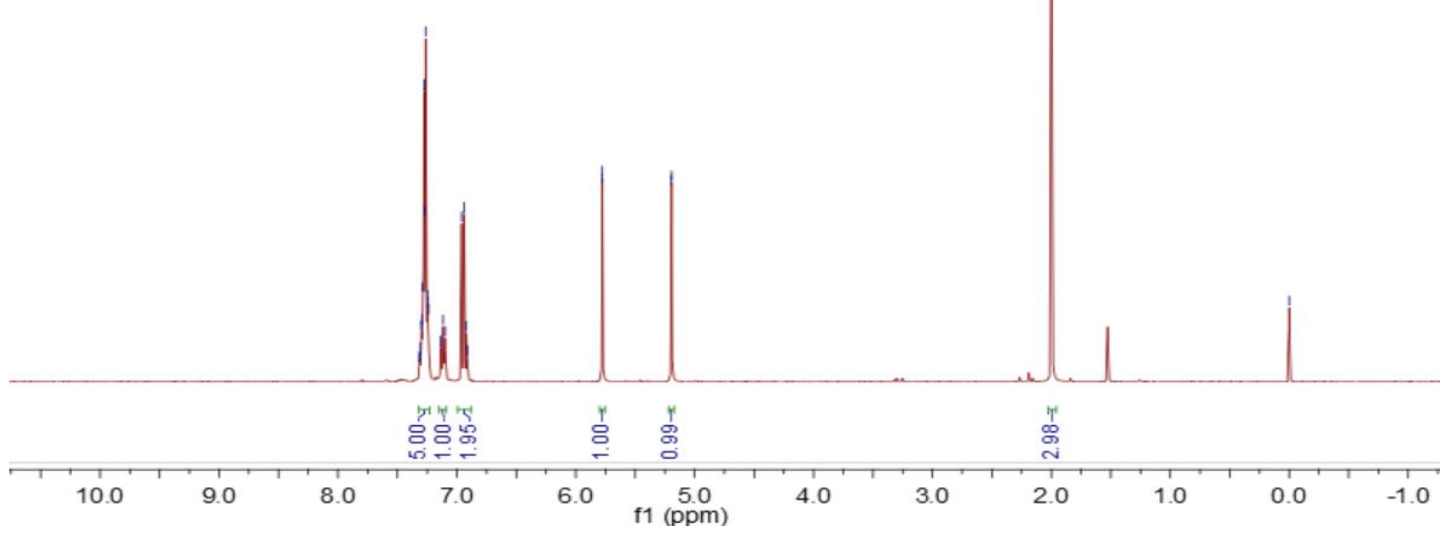

${ }^{1} \mathrm{H}$ NMR (400M, $\mathrm{CDCl}_{3}$ ) spectrum of $\mathbf{7 k}$

S90 

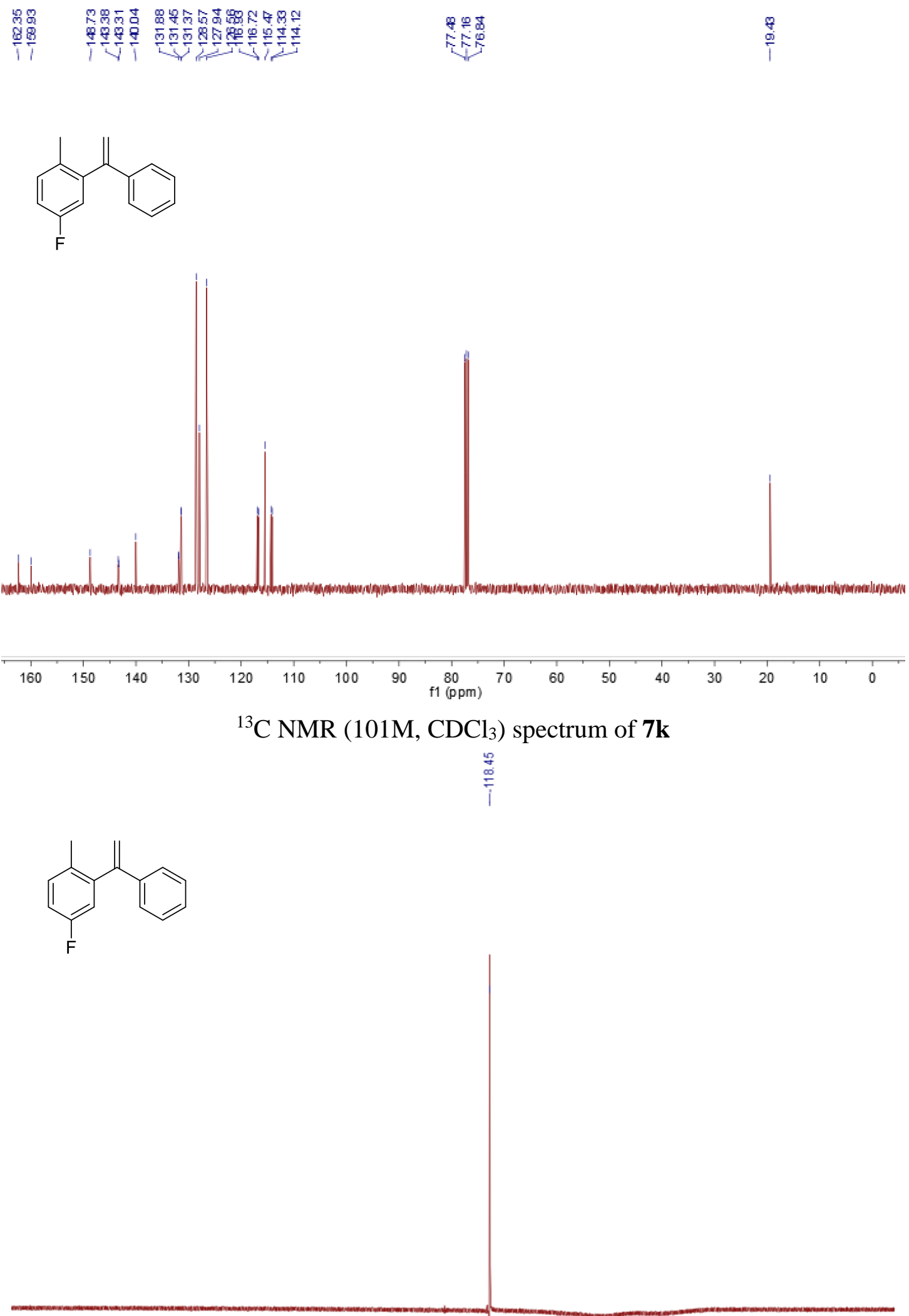

50

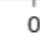

$-50$

-100
$\mathrm{f} 1$ (ppm)

$-150$

$-200$

$-250$

${ }^{19} \mathrm{~F}$ NMR (377 MHz, $\mathrm{CDCl}_{3}$ ) spectrum of $\mathbf{7 k}$ 


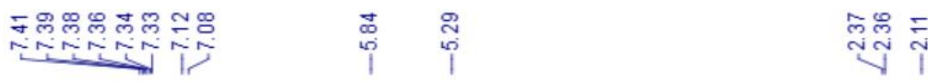<smiles>CCCC</smiles><smiles>C=C(c1ccccc1)c1cc(C)c(C)cc1C</smiles>
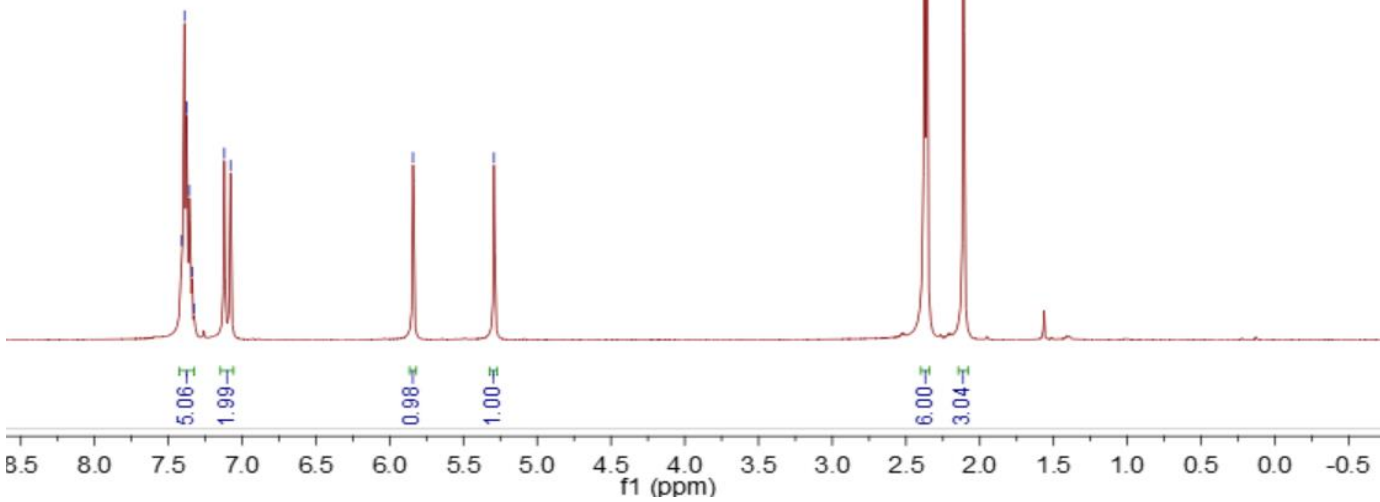

${ }^{1} \mathrm{H}$ NMR $\left(400 \mathrm{M}, \mathrm{CDCl}_{3}\right)$ spectrum of $\mathbf{7 p}$

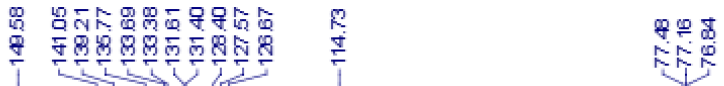

舟需宁<smiles>C=C(c1ccccc1)c1cc(C)c(C)cc1C</smiles>
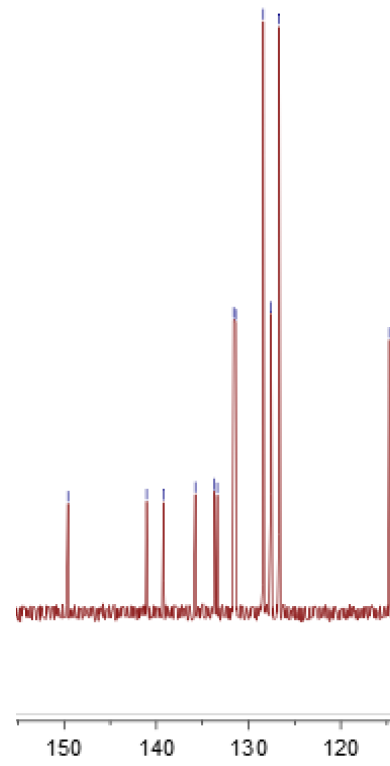

$110 \quad 100$

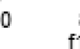

80

$70 \quad 60$

${ }^{13} \mathrm{C}$ NMR $\left(101 \mathrm{M}, \mathrm{CDCl}_{3}\right)$ spectrum of $\mathbf{7 p}$ 

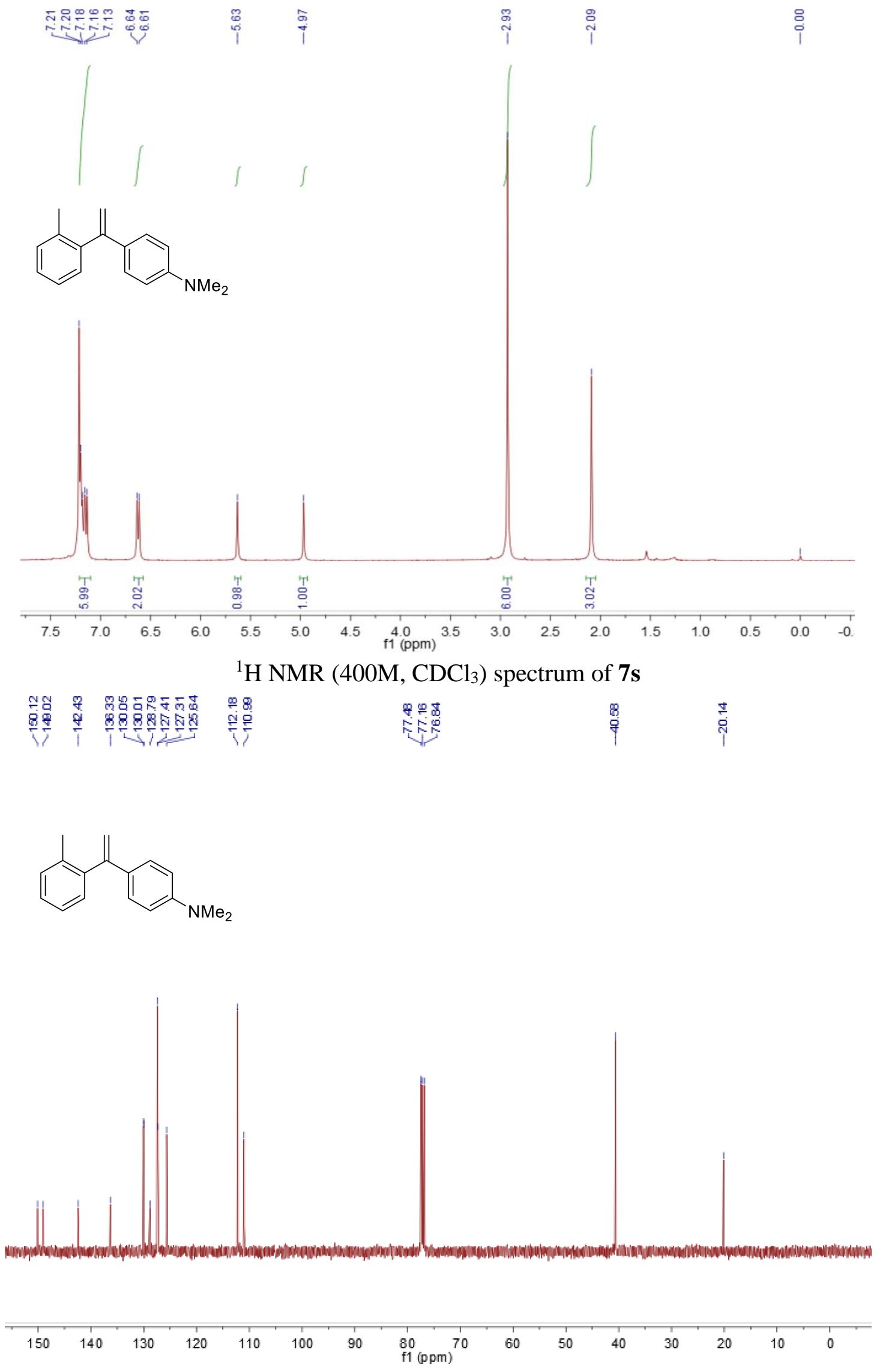

${ }^{13} \mathrm{C}$ NMR $\left(101 \mathrm{M}, \mathrm{CDCl}_{3}\right)$ spectrum of $\mathbf{7 s}$

S93 


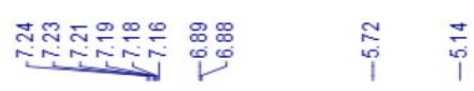<smiles>C=C(c1cc(C)cc(C)c1)c1ccccc1C</smiles>
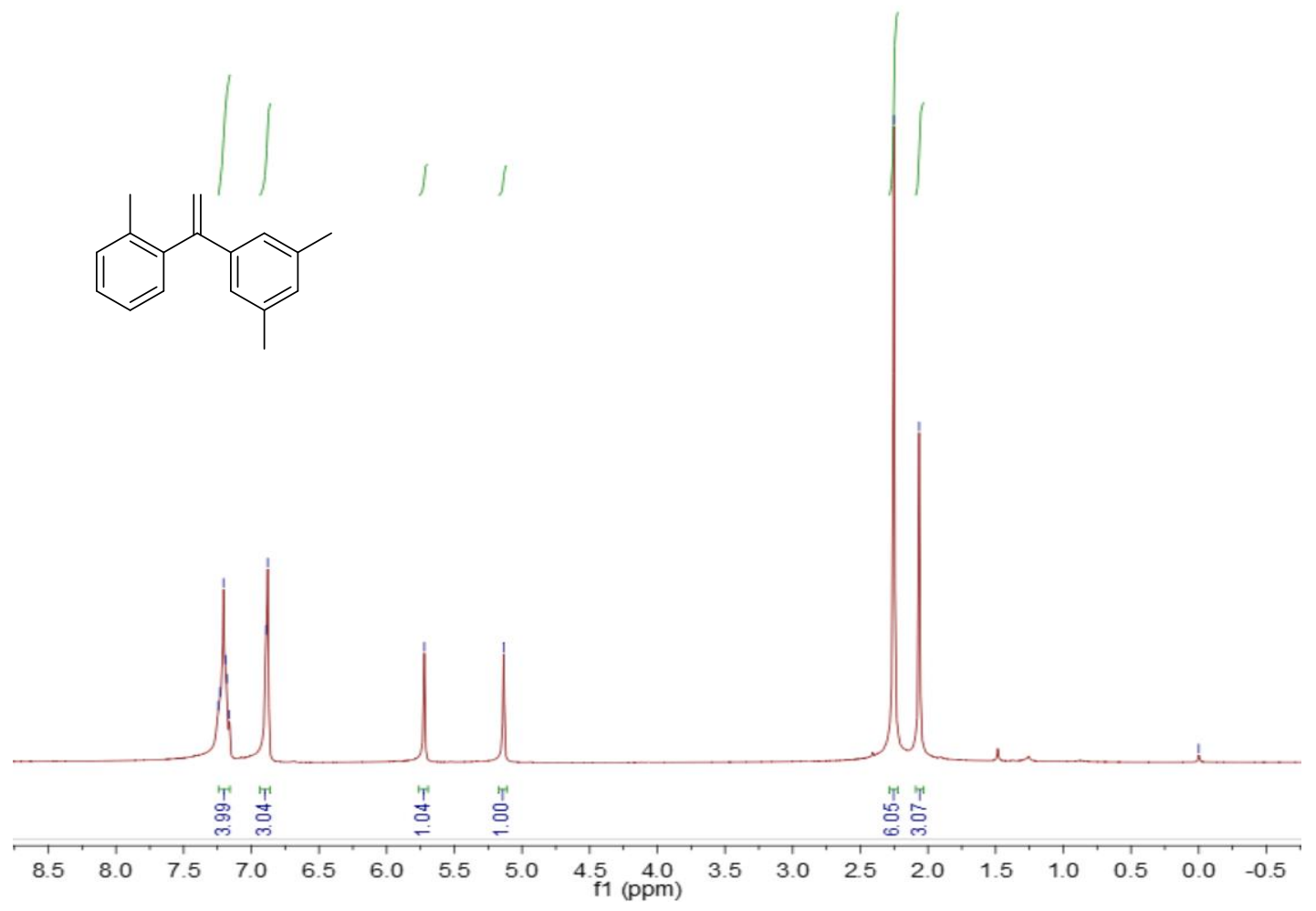

${ }^{1} \mathrm{H}$ NMR (400M, $\mathrm{CDCl}_{3}$ ) spectrum of $\mathbf{7 w}$

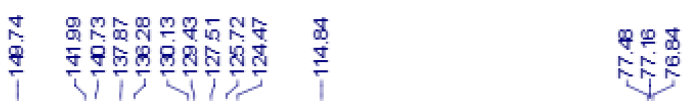<smiles>C=C(c1cc(C)cc(C)c1)c1ccccc1C</smiles>
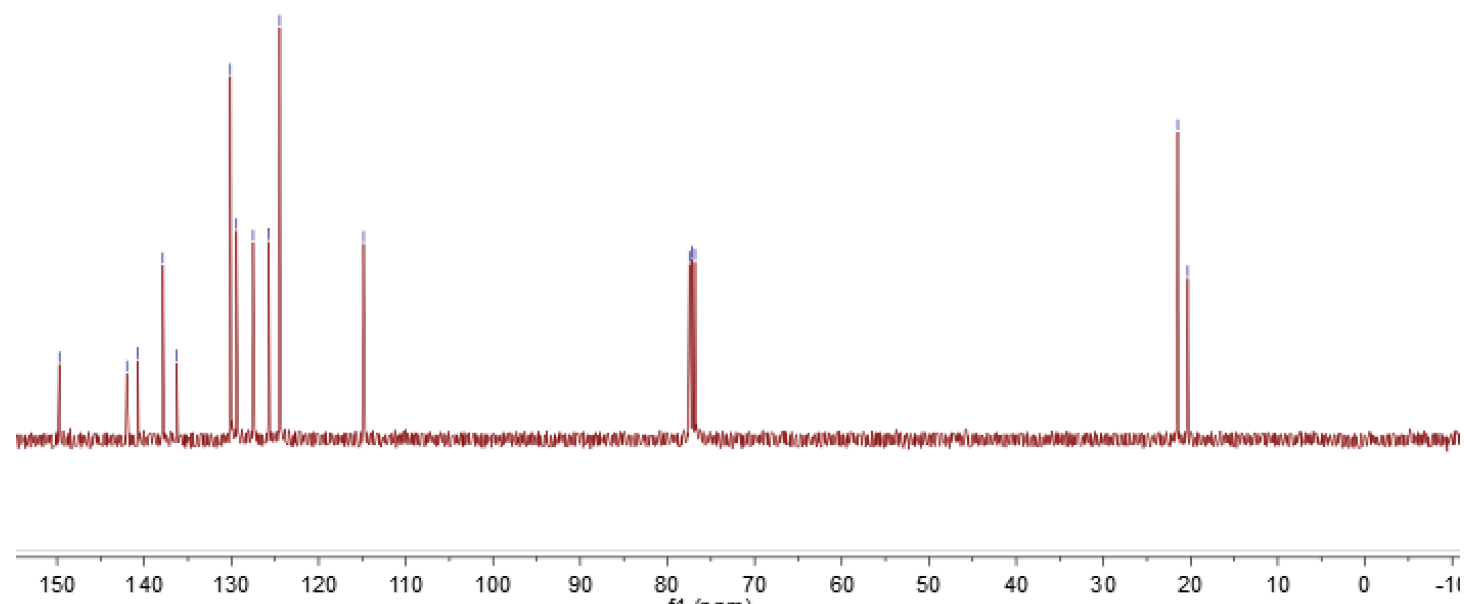

$130 \quad 120$

${ }^{13} \mathrm{C} \mathrm{NMR}\left(101 \mathrm{M}, \mathrm{CDCl}_{3}\right)$ spectrum of $\mathbf{7 w}$ 


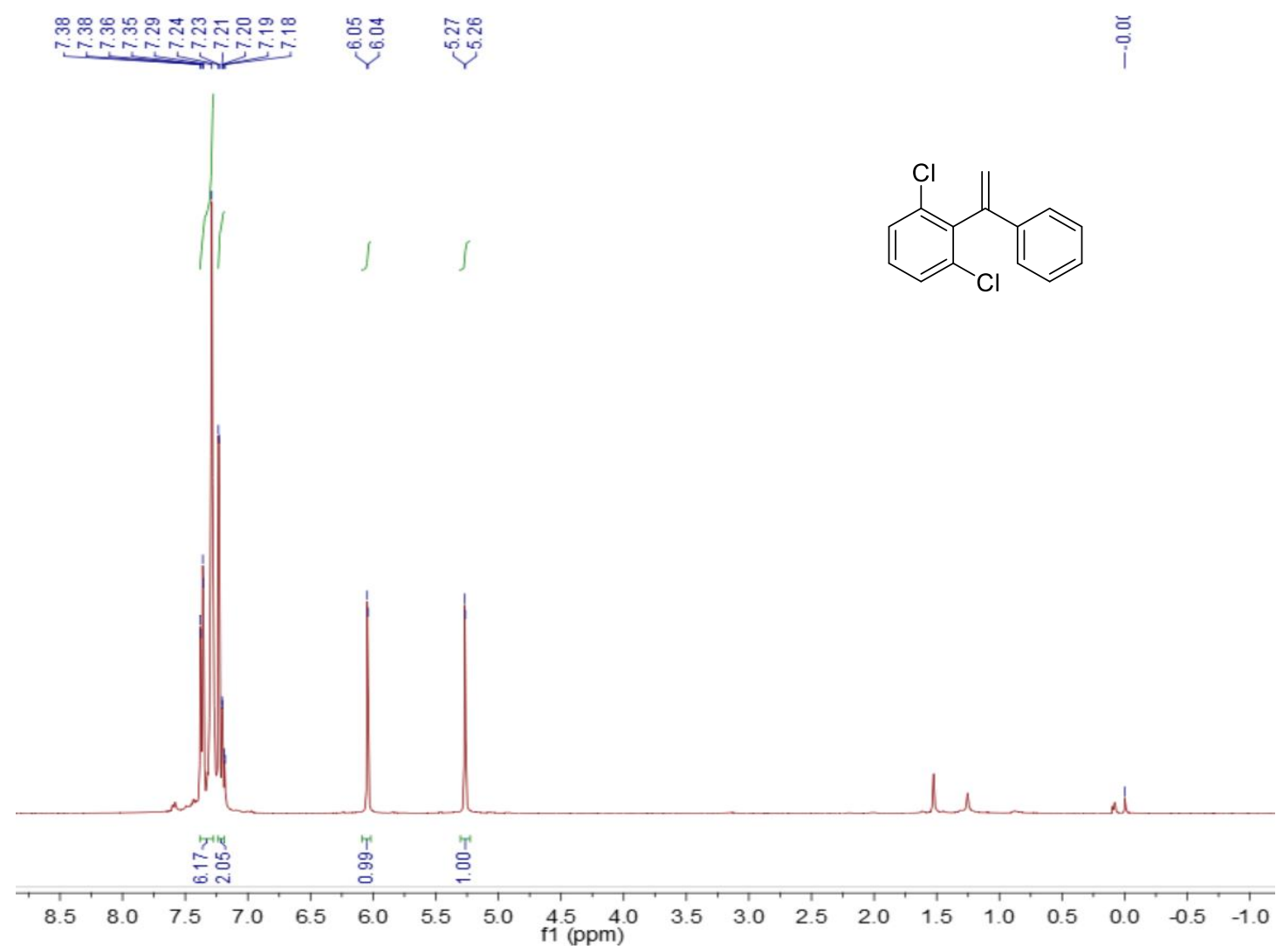

${ }^{1} \mathrm{H}$ NMR (400M, $\mathrm{CDCl}_{3}$ ) spectrum of 7aa

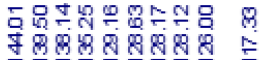

舟
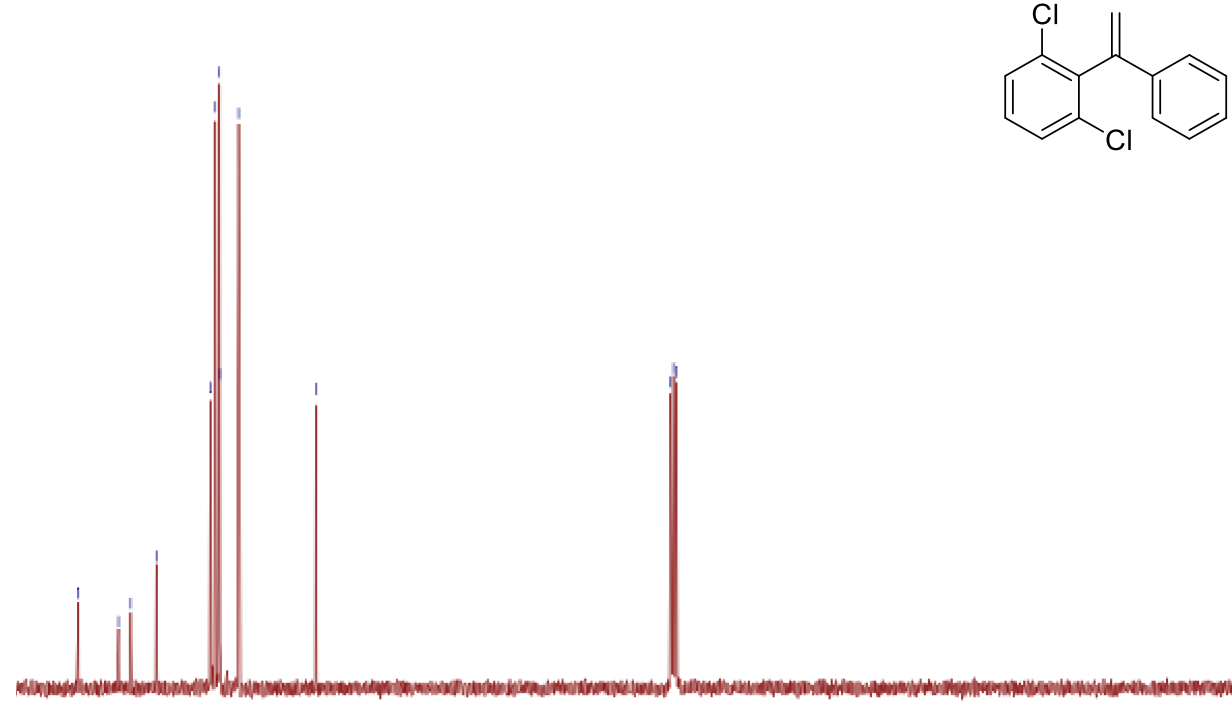

$50 \quad 140 \quad 130$

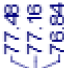



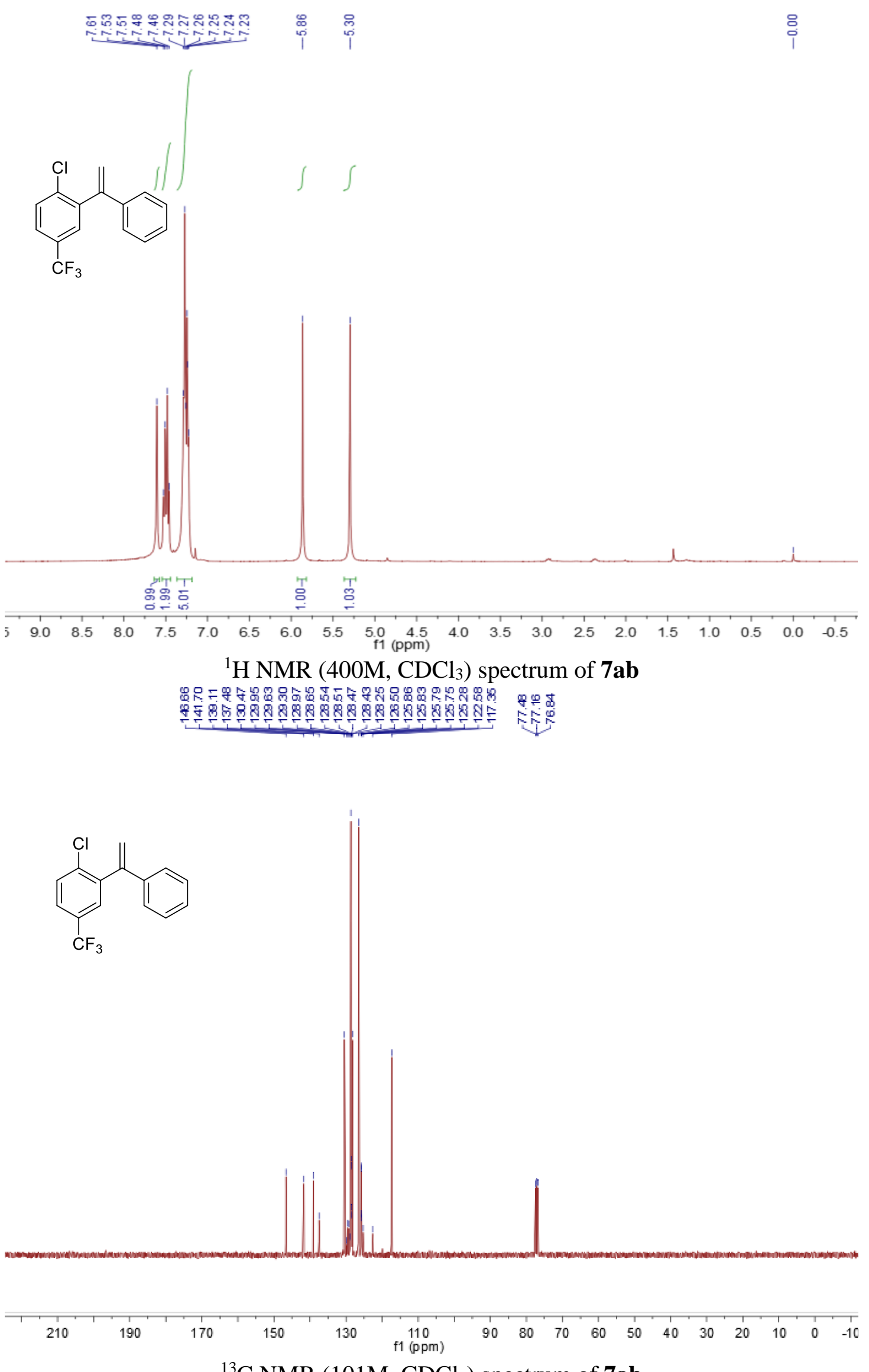

${ }^{13} \mathrm{C}$ NMR $\left(101 \mathrm{M}, \mathrm{CDCl}_{3}\right)$ spectrum of $\mathbf{7 a b}$

S96 
$\underset{\substack{\text { ¿ } \\ 1}}{1}$
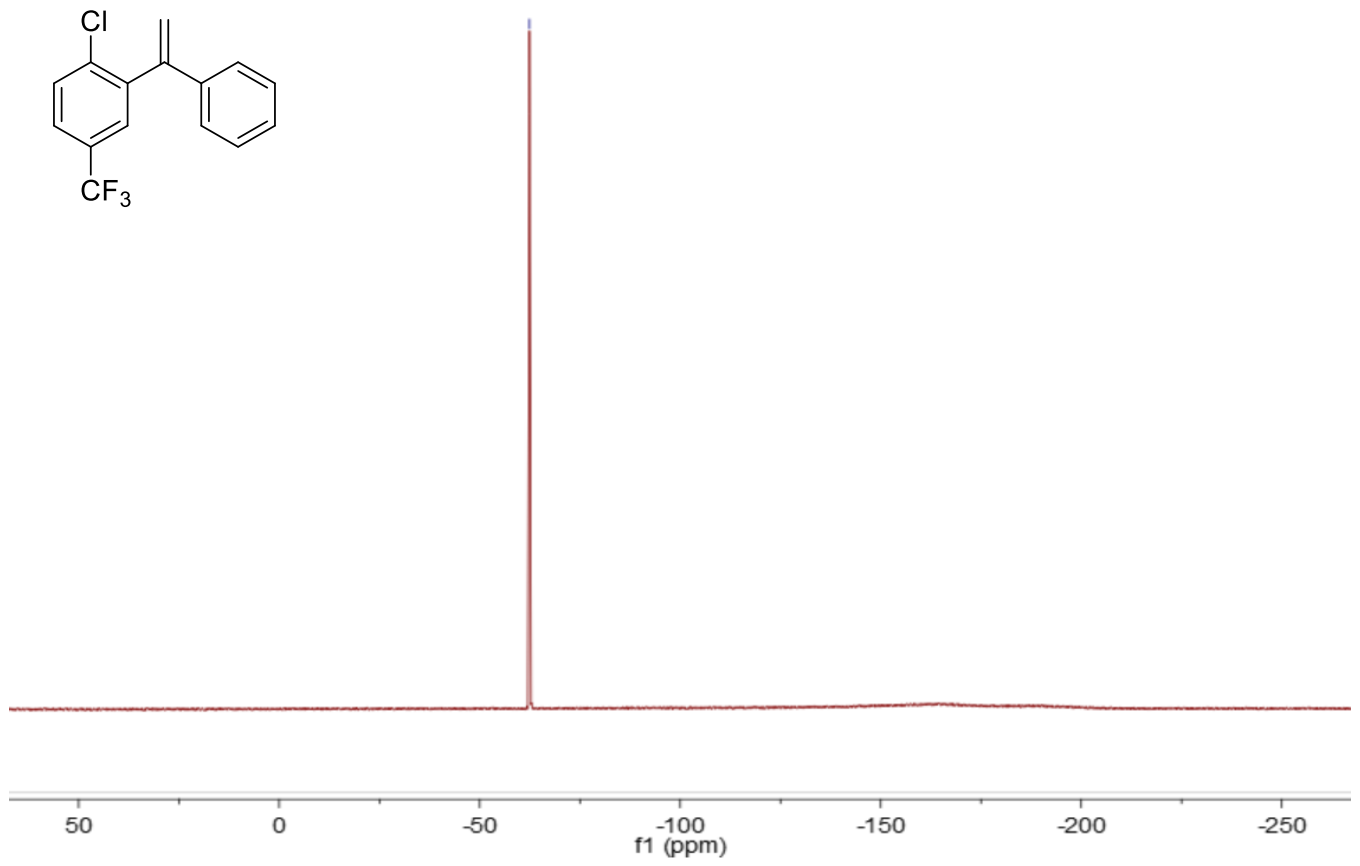

${ }^{19} \mathrm{~F}$ NMR (377 MHz, $\mathrm{CDCl}_{3}$ ) spectrum of 7ab
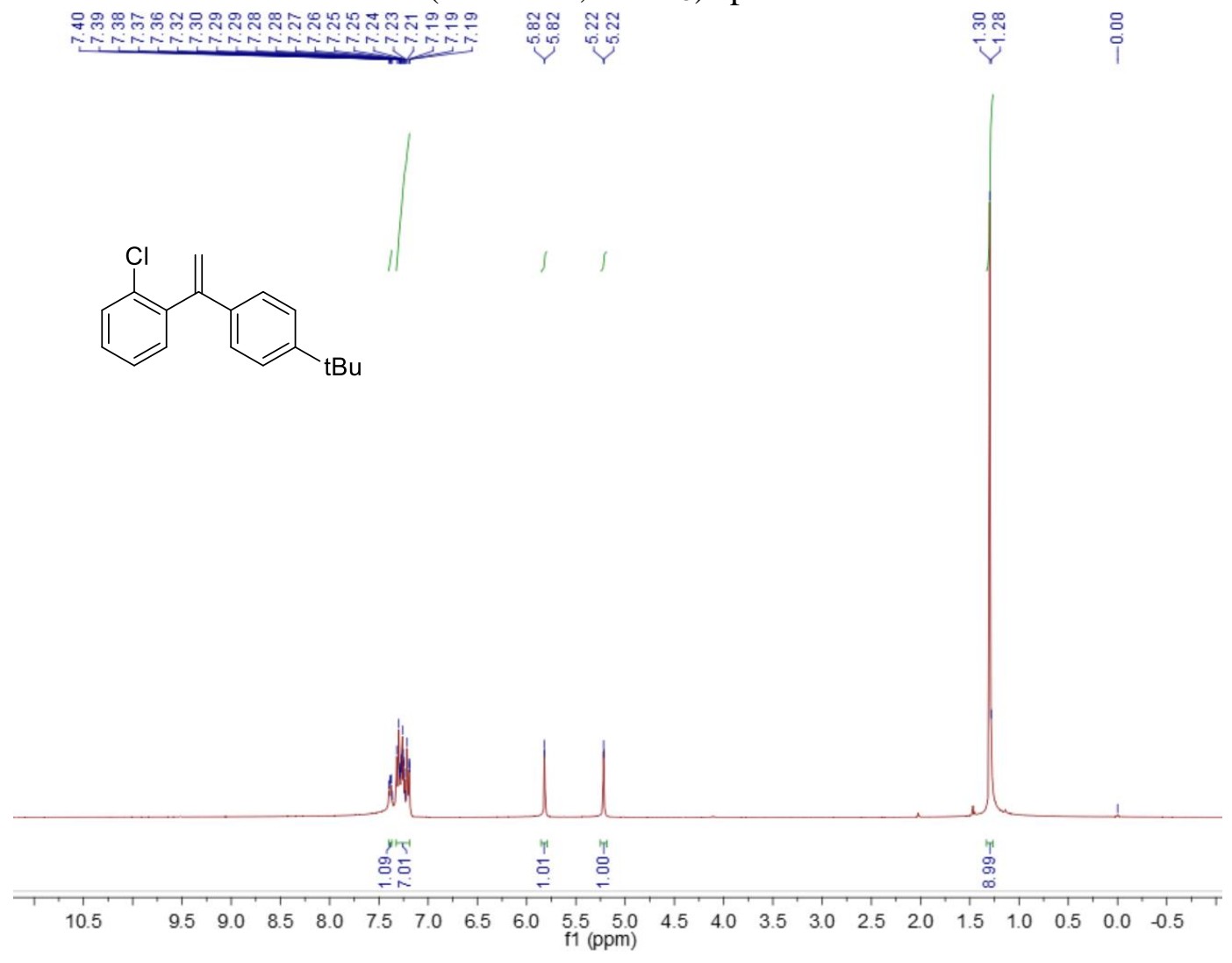

${ }^{1} \mathrm{H}$ NMR (400M, $\mathrm{CDCl}_{3}$ ) spectrum of 7ae

S97 


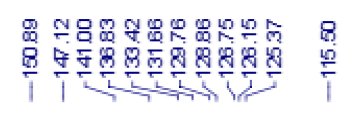

紫要

黑管
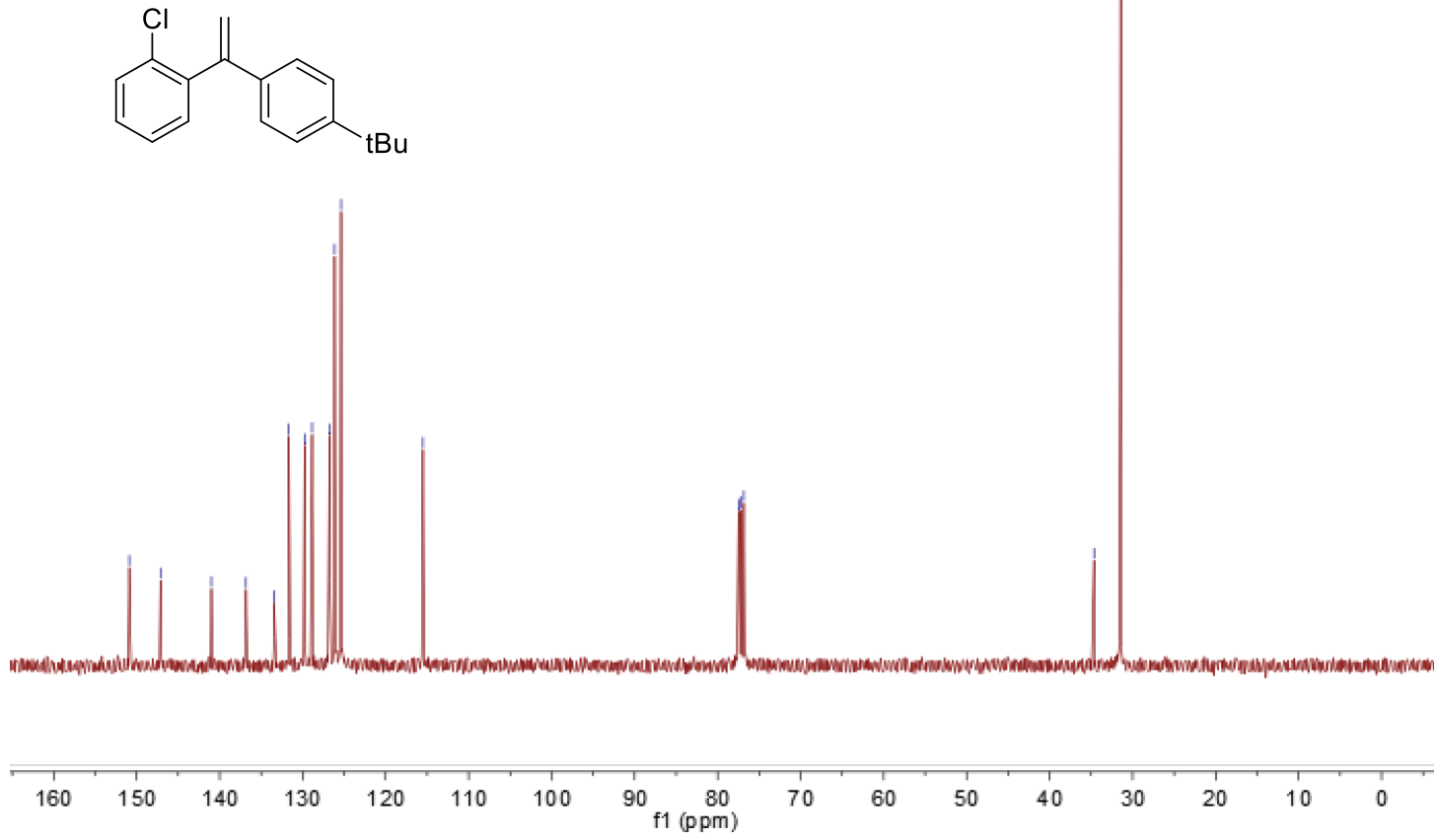

${ }^{13} \mathrm{C}$ NMR $\left(101 \mathrm{M}, \mathrm{CDCl}_{3}\right)$ spectrum of $7 \mathbf{a e}$

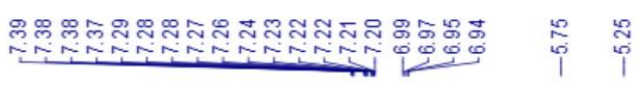

:
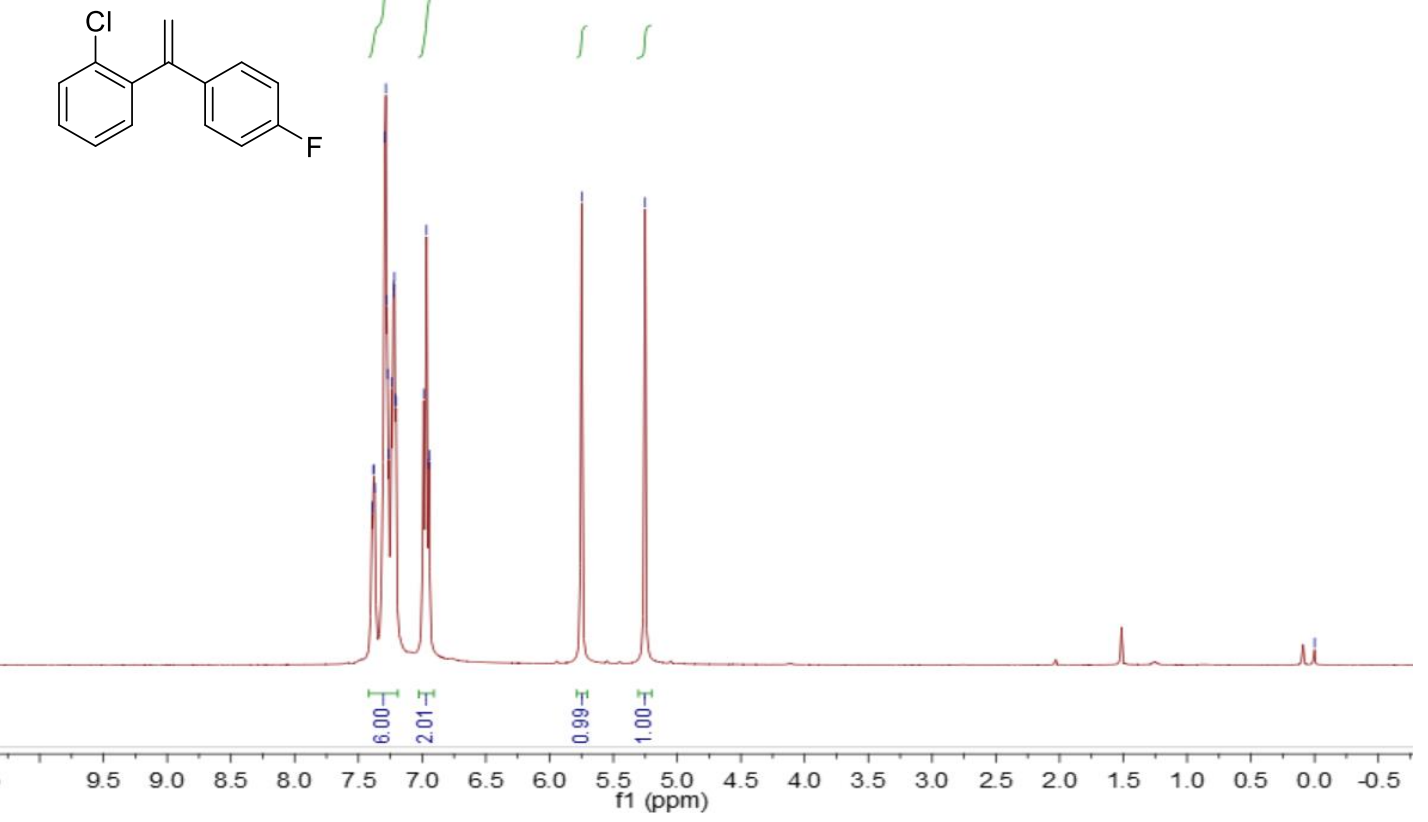

${ }^{1} \mathrm{H}$ NMR $\left(400 \mathrm{M}, \mathrm{CDCl}_{3}\right)$ spectrum of $7 \mathbf{a g}$

S98 


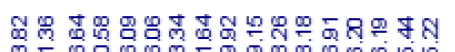

哭哭
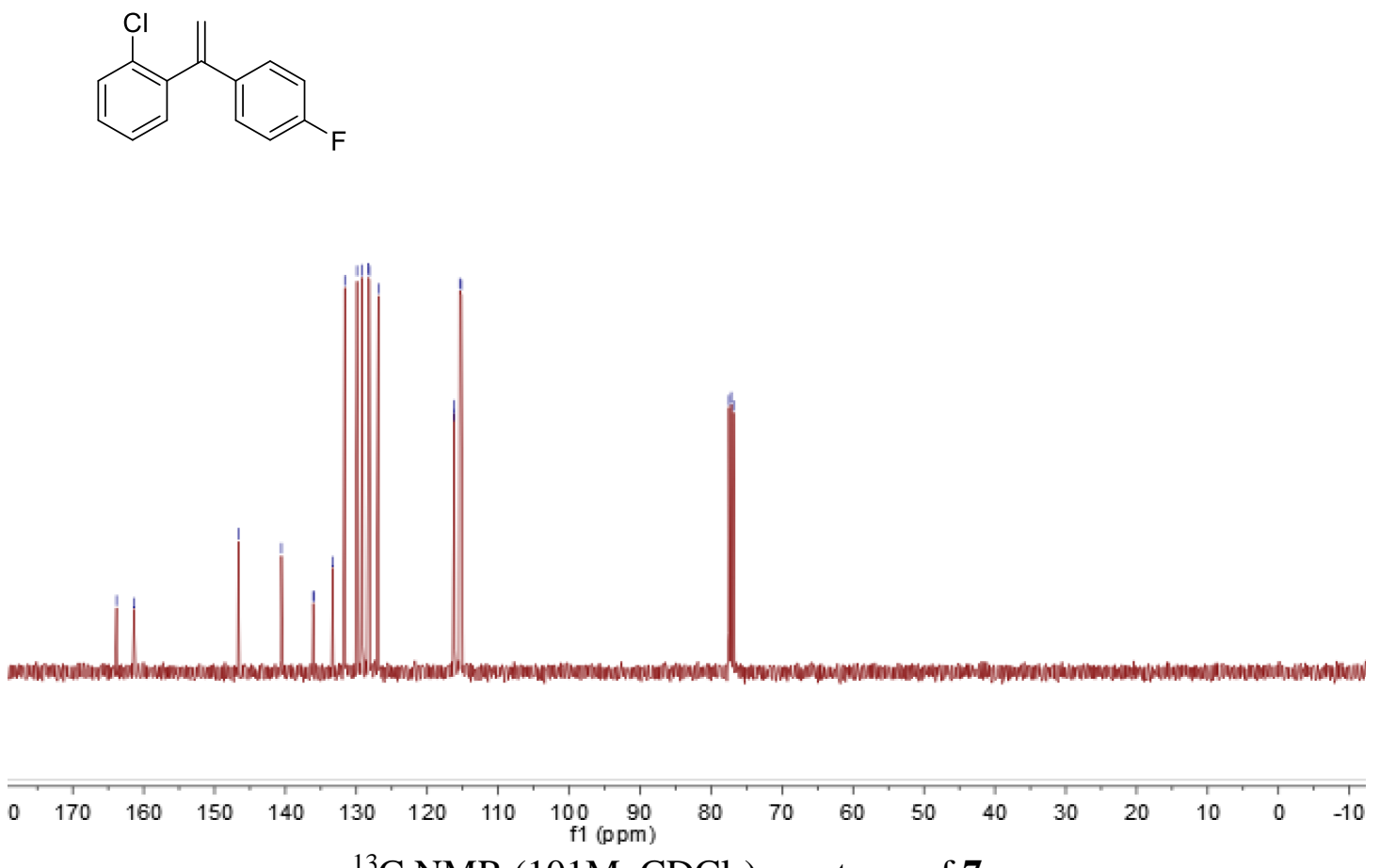

${ }^{13} \mathrm{C}$ NMR $\left(101 \mathrm{M}, \mathrm{CDCl}_{3}\right)$ spectrum of 7ag

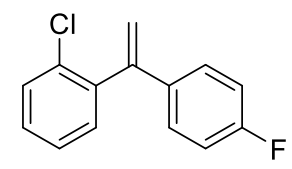

傽

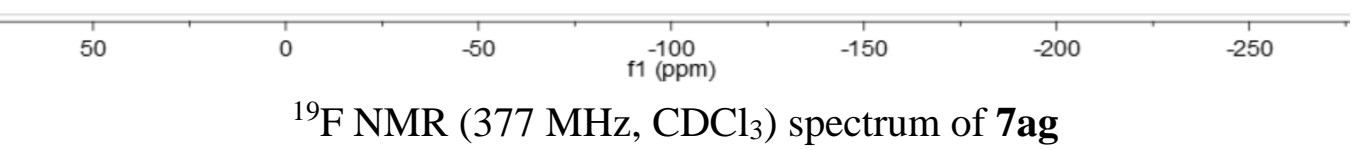

S99 


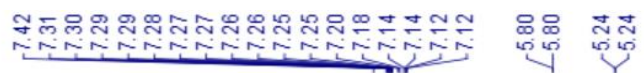

$\stackrel{\infty}{i}$

응
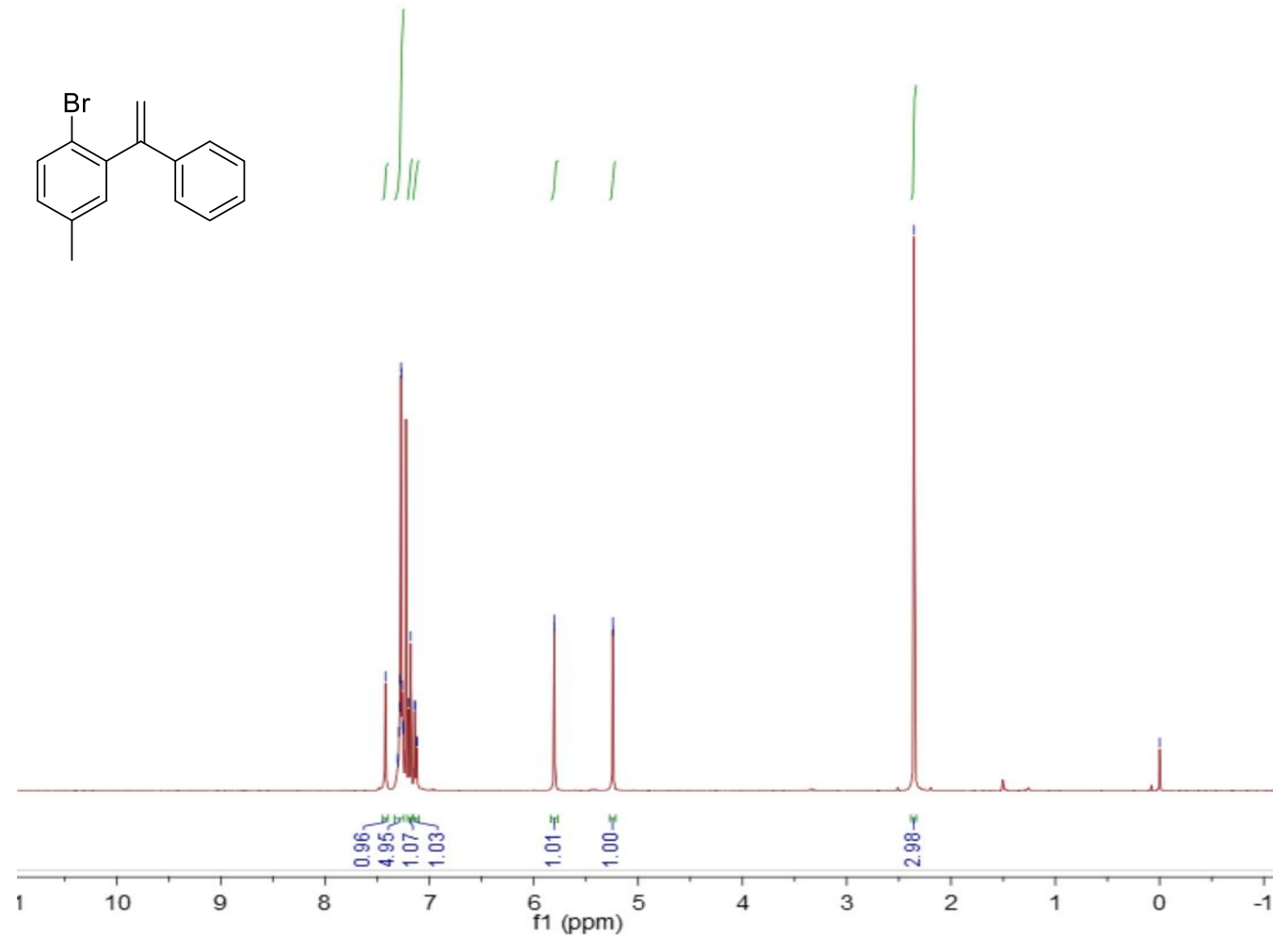

${ }^{1} \mathrm{H}$ NMR $\left(400 \mathrm{M}, \mathrm{CDCl}_{3}\right.$ ) spectrum of 7ai

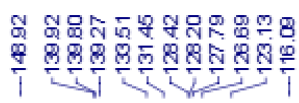

然惪

จึ่
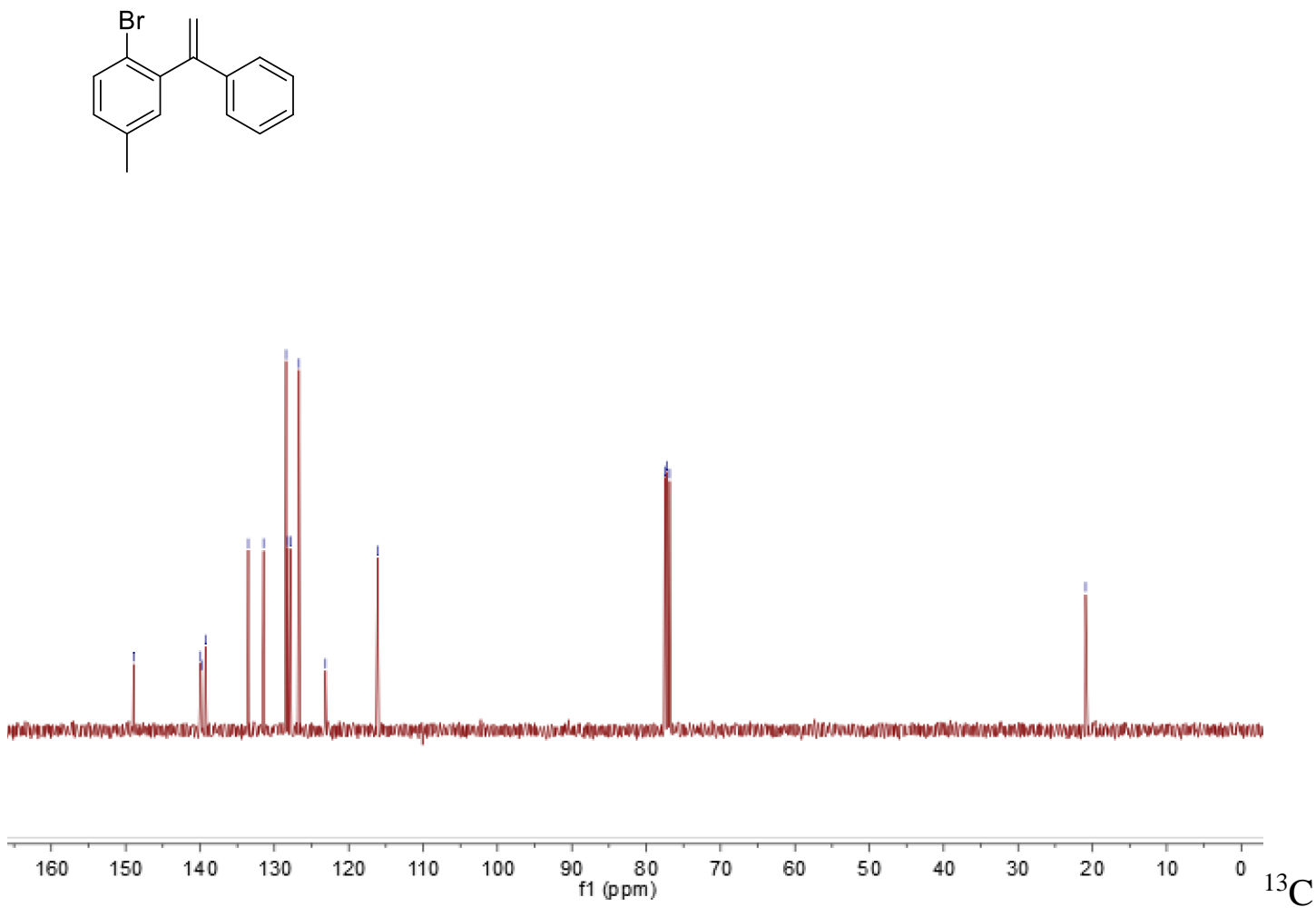

NMR (101M, $\left.\mathrm{CDCl}_{3}\right)$ spectrum of 7ai

S100 
ஜำ

$\stackrel{\breve{c}}{i}$
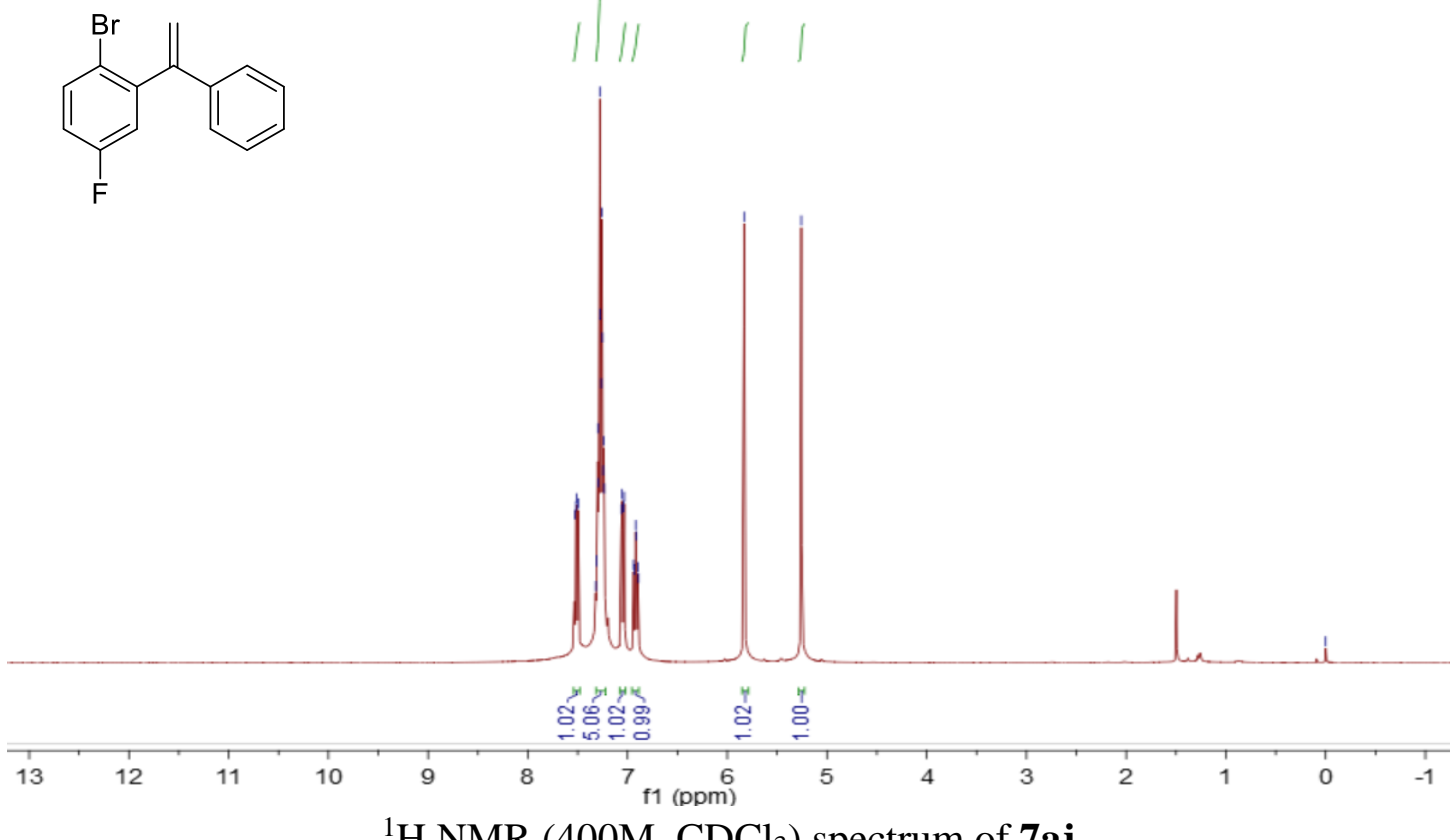

${ }^{1} \mathrm{H}$ NMR $\left(400 \mathrm{M}, \mathrm{CDCl}_{3}\right)$ spectrum of 7aj

贺

然耐
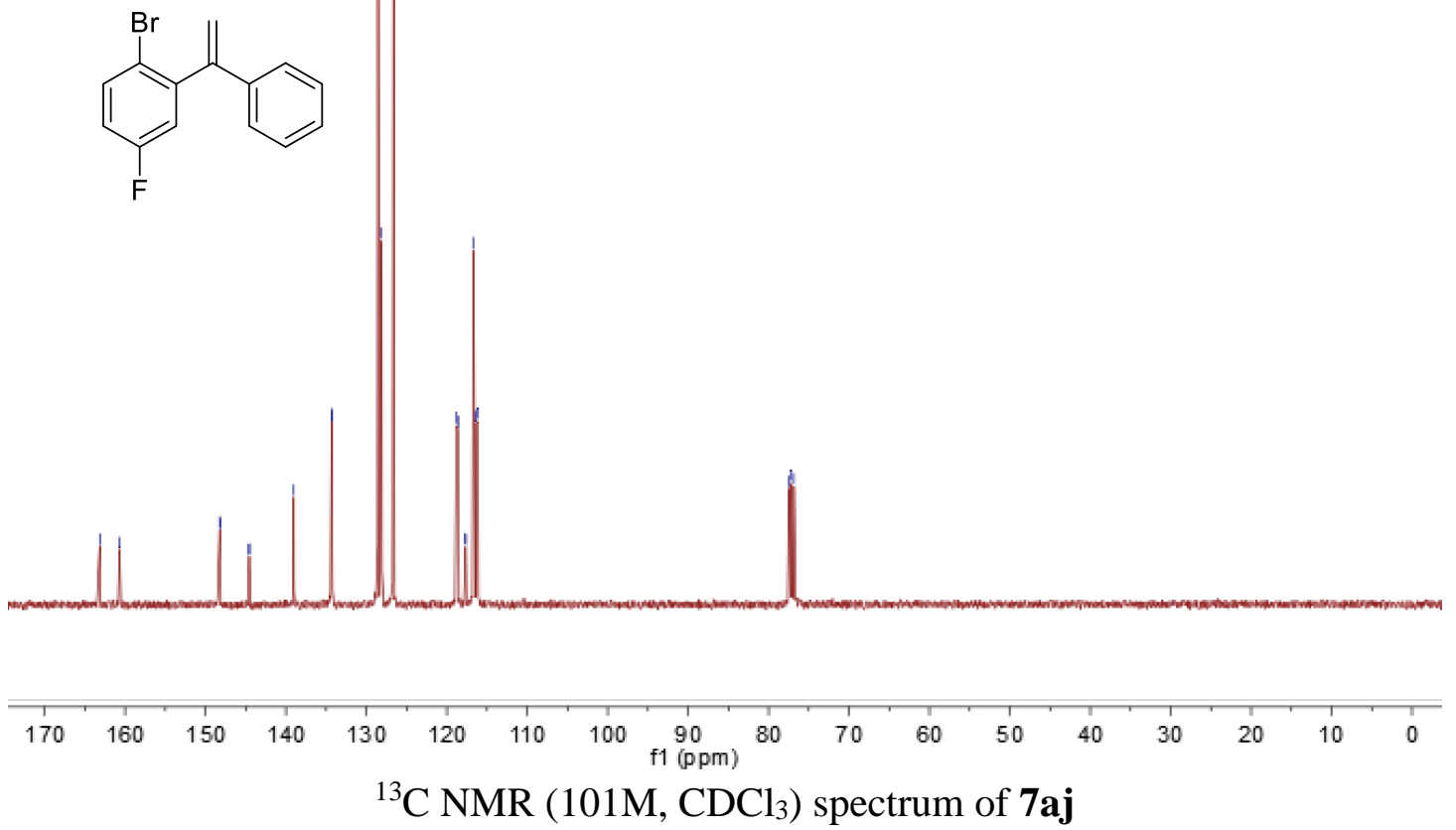

S101 
<smiles>C=C(c1ccccc1)c1cc(F)ccc1Br</smiles>

\begin{tabular}{|c|c|c|c|c|c|c|}
\hline 50 & 0 & -50 & $\begin{array}{c}-100 \\
f 1(\mathrm{ppm})\end{array}$ & -150 & -200 & -250 \\
\hline \multicolumn{7}{|c|}{${ }^{19} \mathrm{~F} \mathrm{NMR}\left(377 \mathrm{MHz}, \mathrm{CDCl}_{3}\right)$ spectrum of 7aj } \\
\hline 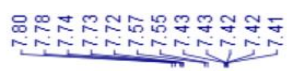 & $\underset{\substack{ \pm 0 \\
\text { i }}}{1}$ & 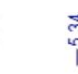 & 象尔 & & & 兽 \\
\hline
\end{tabular}<smiles>C=C(c1ccc2ccccc2c1)c1cc(OC)c(OC)c(OC)c1Br</smiles>

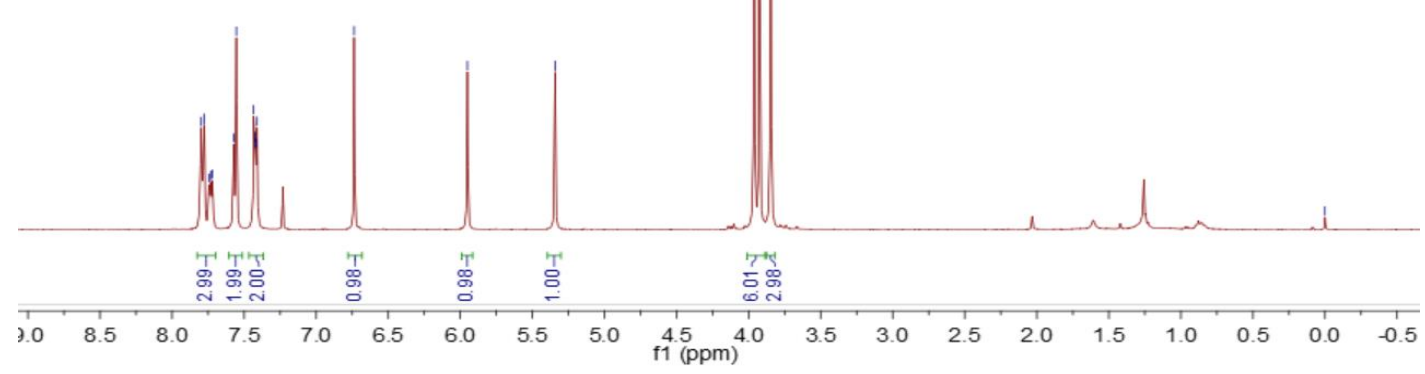

${ }^{1} \mathrm{H}$ NMR (400M, $\mathrm{CDCl}_{3}$ ) spectrum of 7am 
<smiles>C=C(c1ccc2ccccc2c1)c1cc(OC)c(OC)c(OC)c1Br</smiles>
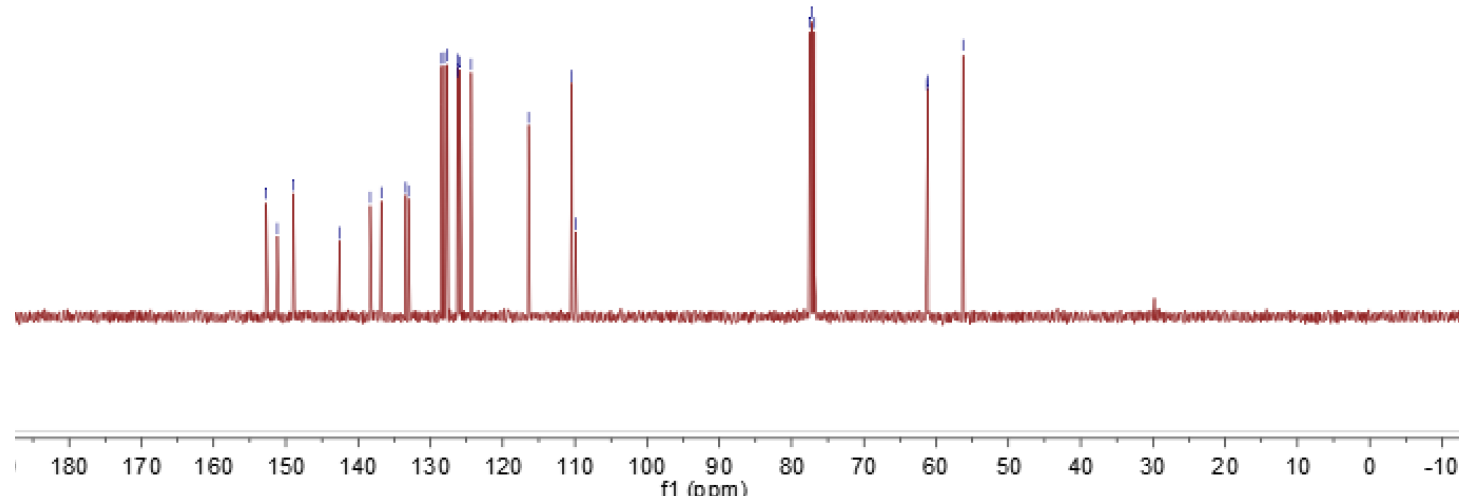

${ }^{13} \mathrm{C}$ NMR $\left(101 \mathrm{M}, \mathrm{CDCl}_{3}\right)$ spectrum of 7am

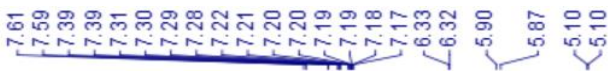

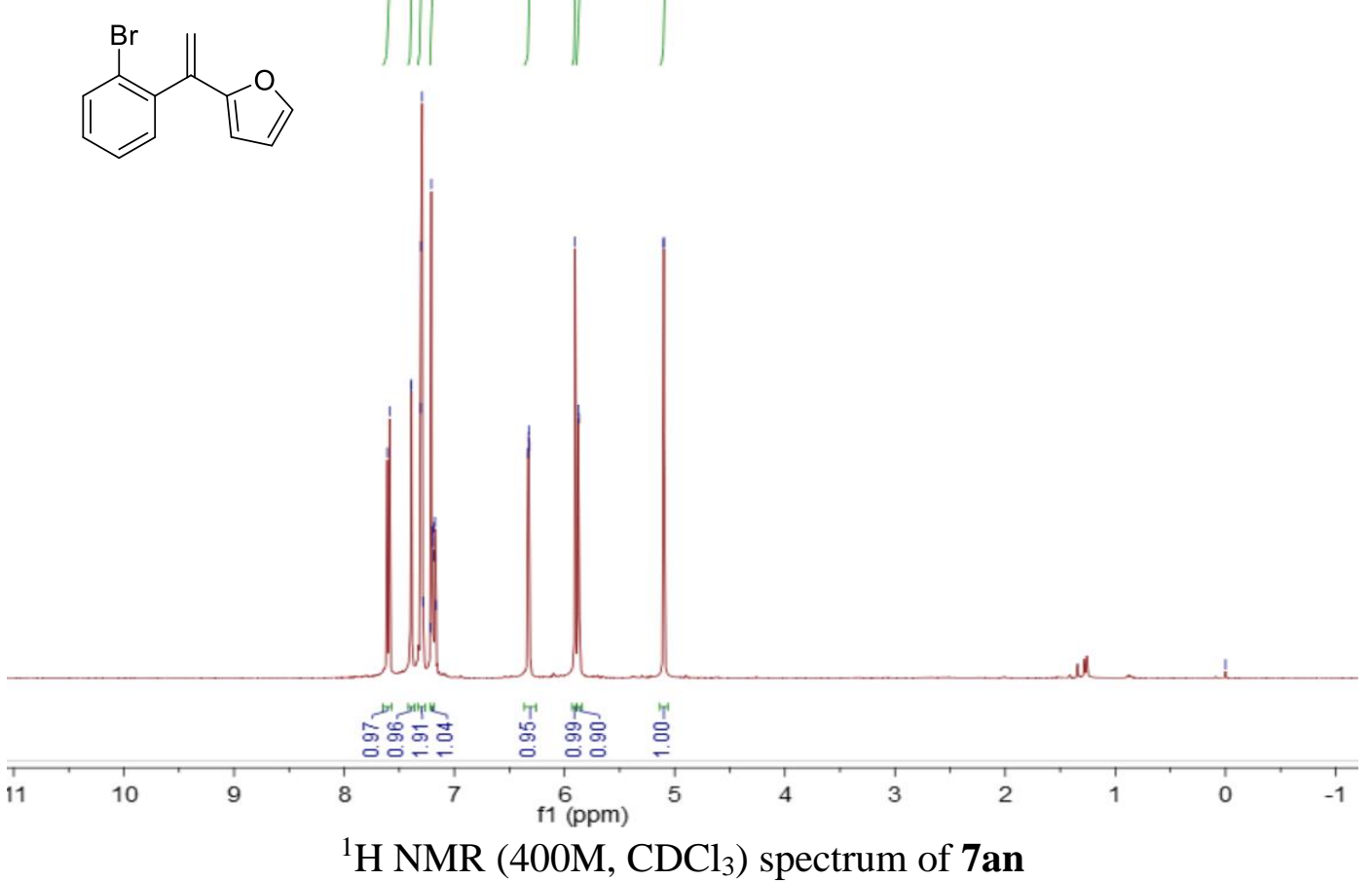


(1)
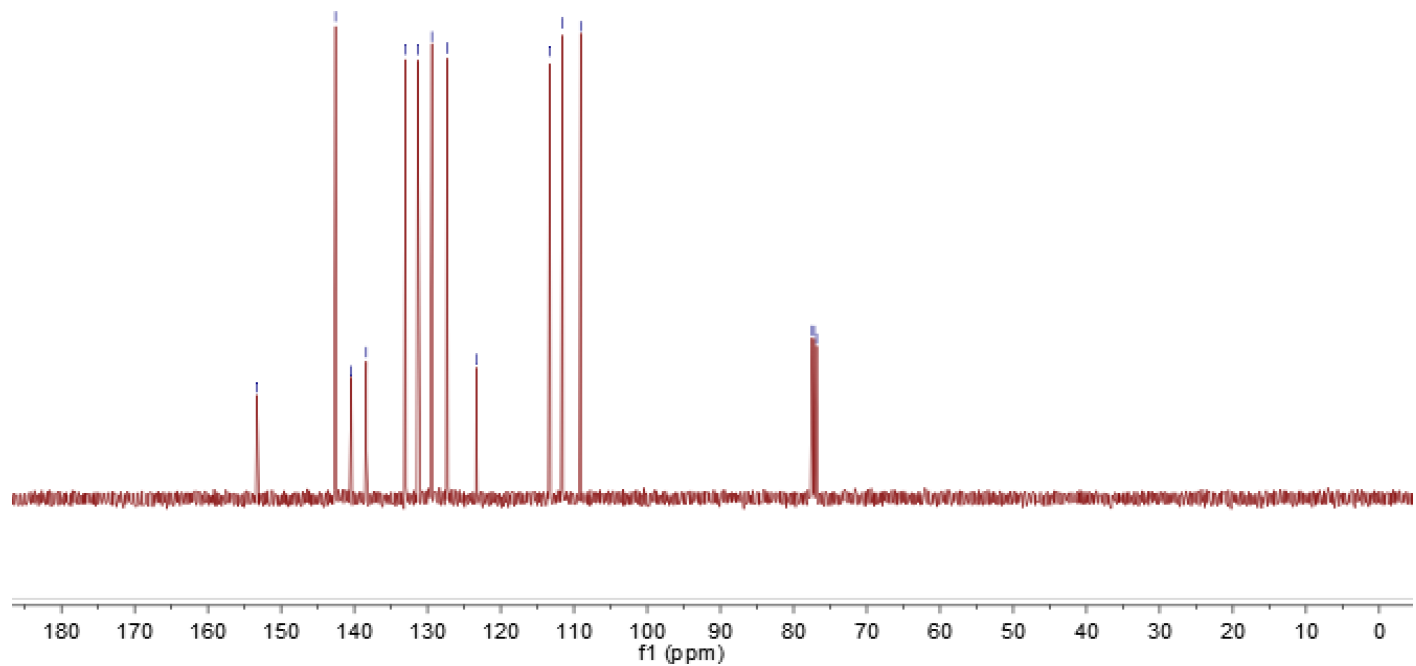

${ }^{13} \mathrm{C} \mathrm{NMR}\left(101 \mathrm{M}, \mathrm{CDCl}_{3}\right)$ spectrum of 7an

व.⿻口卄.

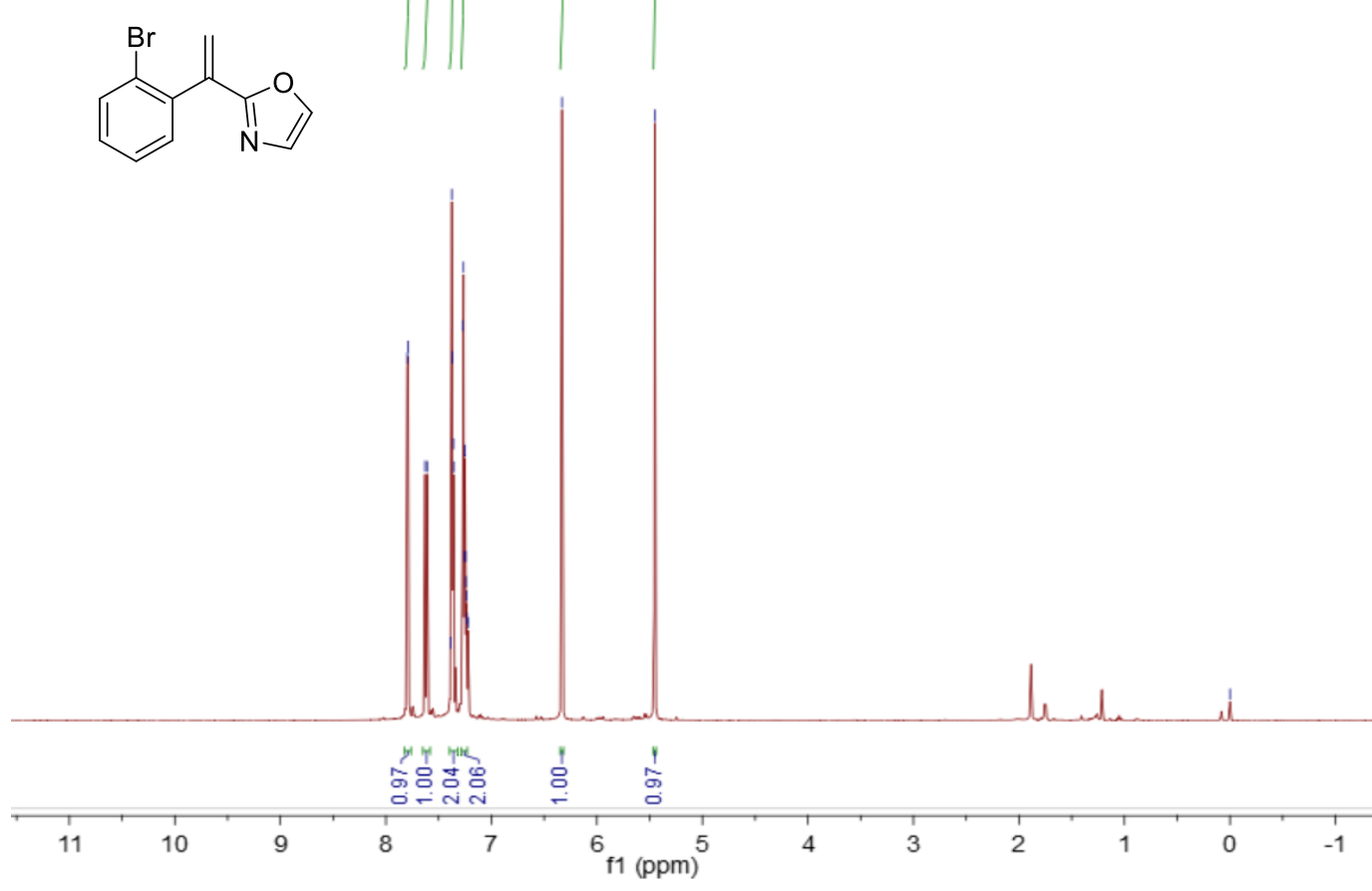

${ }^{1} \mathrm{H}$ NMR (400M, $\mathrm{CDCl}_{3}$ ) spectrum of 7ap 
$\underbrace{B r}_{N}$
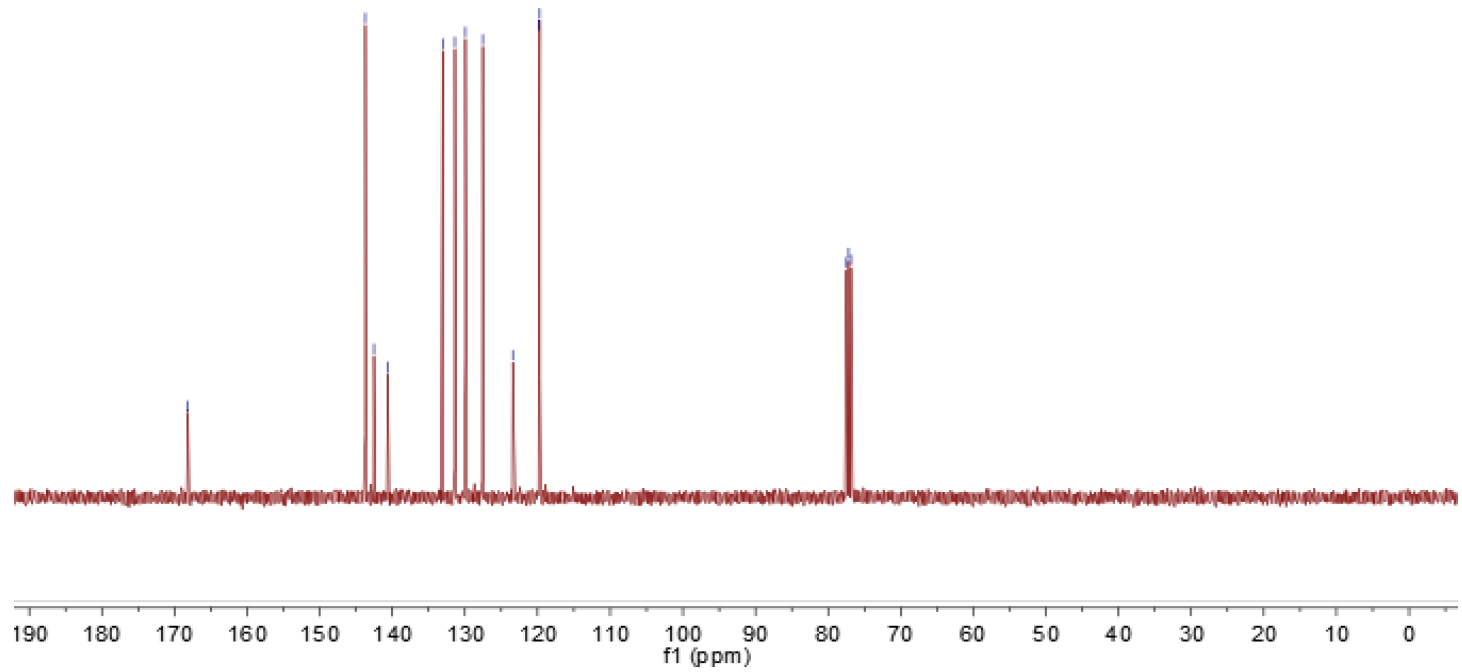

${ }^{13} \mathrm{C}$ NMR $\left(101 \mathrm{M}, \mathrm{CDCl}_{3}\right)$ spectrum of 7ap

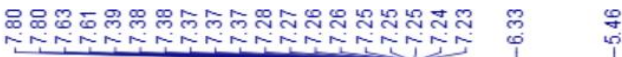<smiles>C=C(c1nccs1)c1ccccc1Br</smiles>
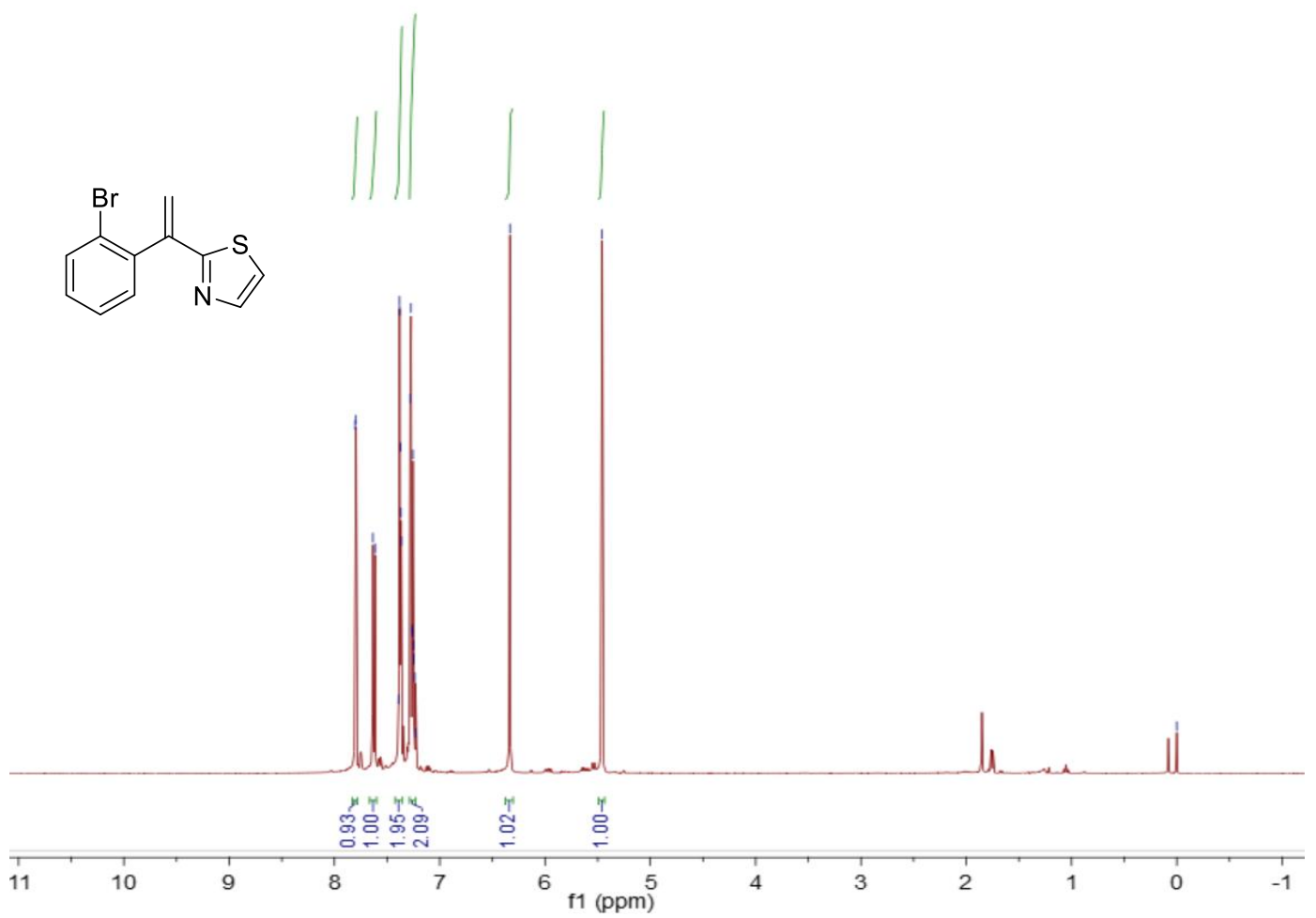

${ }^{1} \mathrm{H}$ NMR (400M, $\left.\mathrm{CDCl}_{3}\right)$ spectrum of 7aq 
<smiles>C=C(c1nccs1)c1ccccc1Br</smiles>
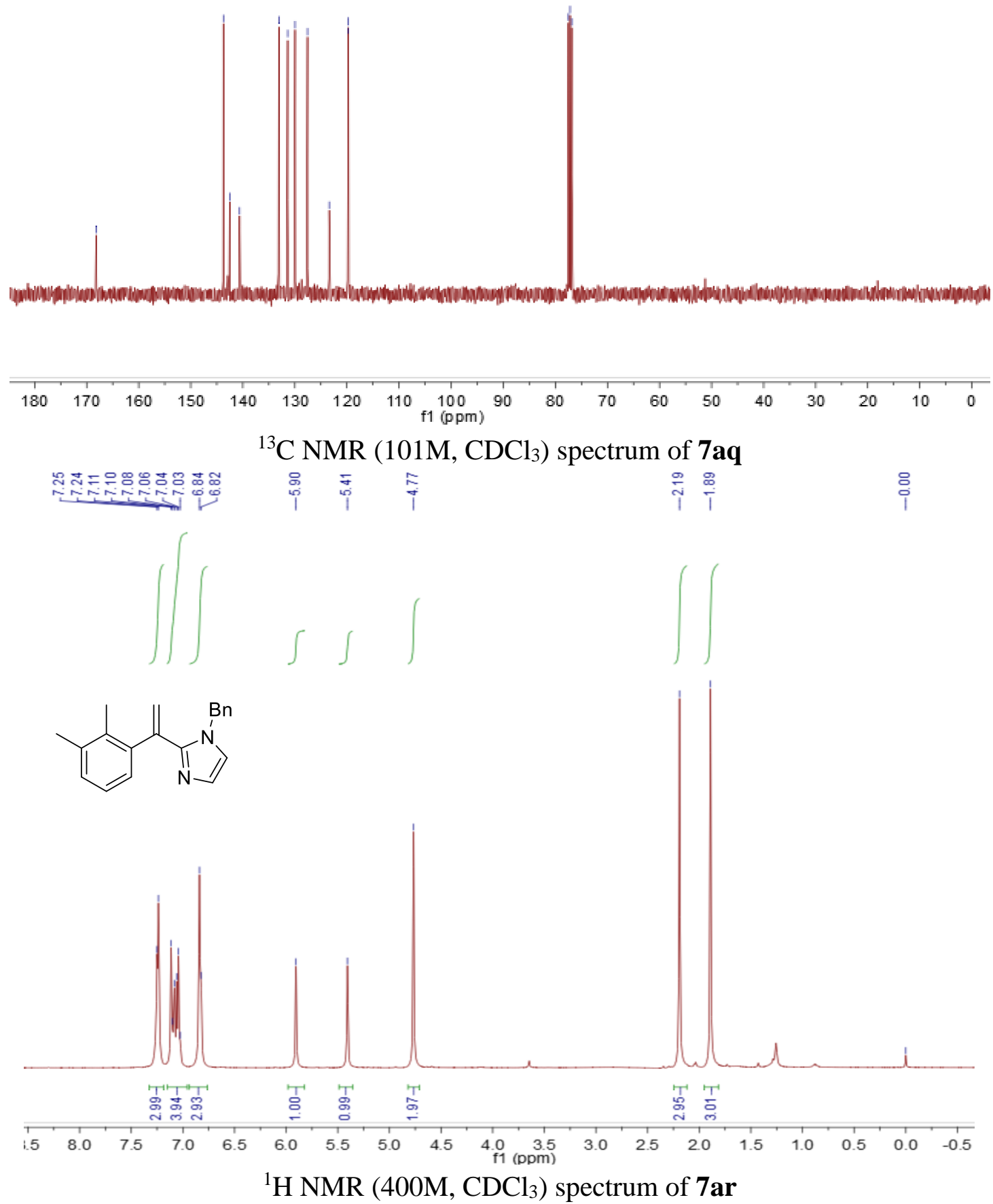
<smiles>C=C(c1cccc(C)c1C)c1nccn1Cc1ccccc1</smiles>
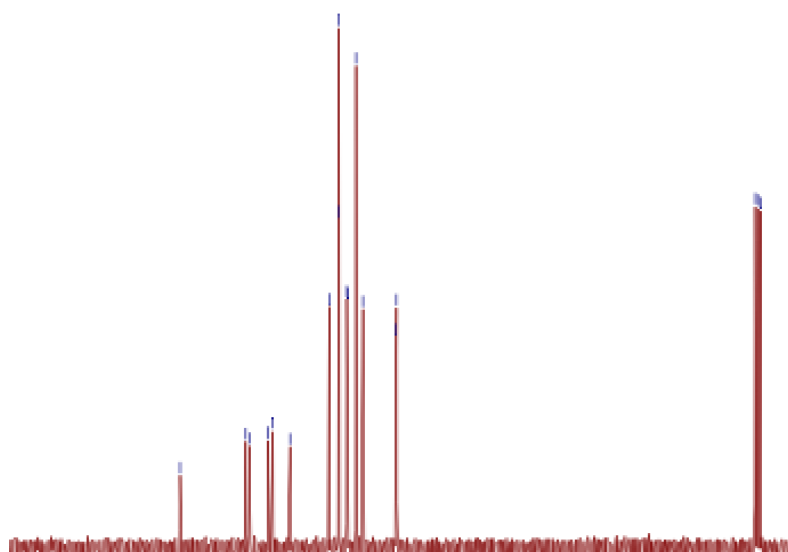

$\begin{array}{lllll}0 & 160 & 150 & 140 & 130\end{array}$

${ }^{13} \mathrm{C}$ NMR (101M, $\left.\mathrm{CDCl}_{3}\right)$ spectrum of 7ar

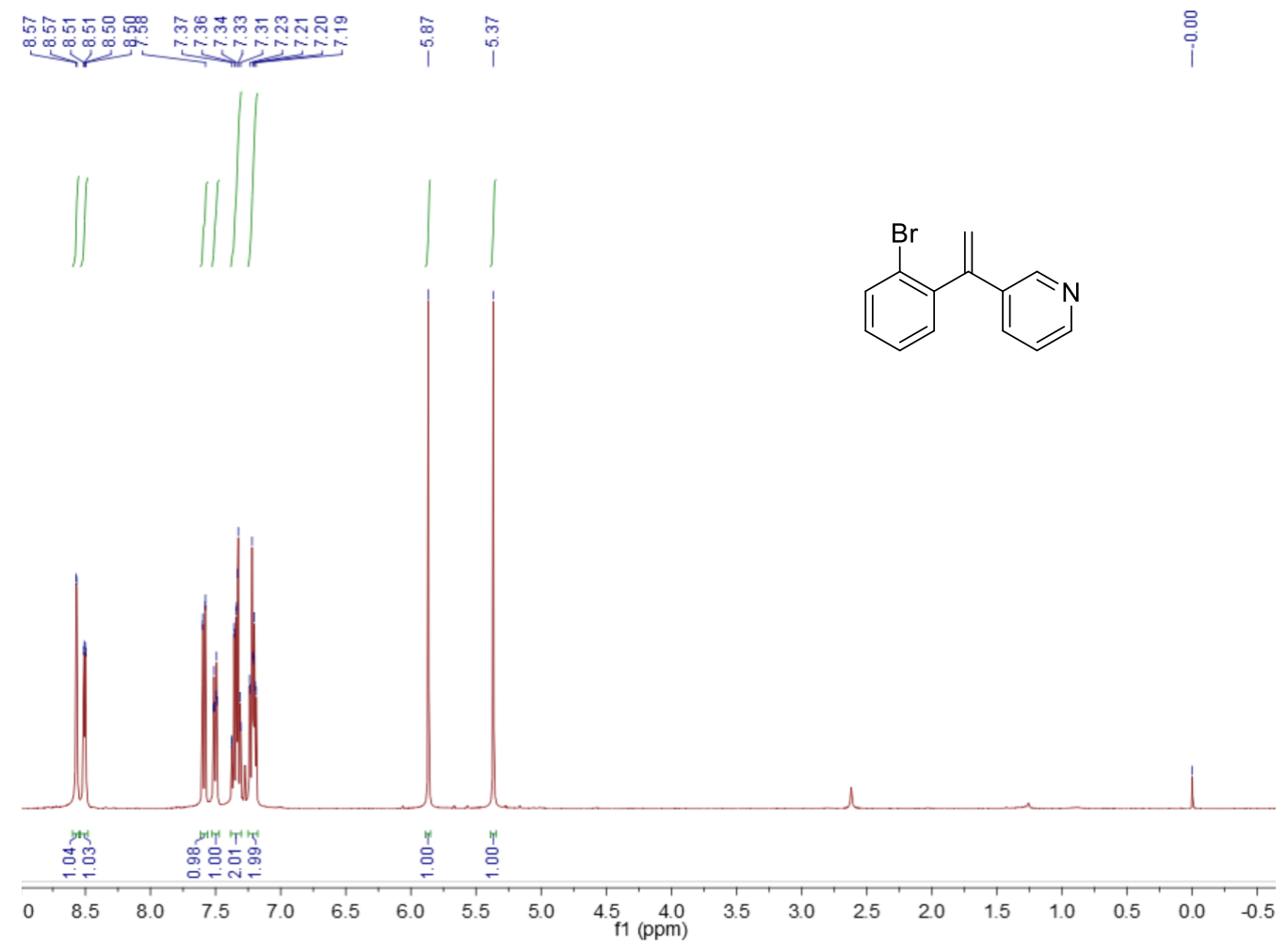

${ }^{1} \mathrm{H}$ NMR $\left(400 \mathrm{M}, \mathrm{CDCl}_{3}\right)$ spectrum of 7as 
<smiles>C=C(c1cccnc1)c1ccccc1Br</smiles>

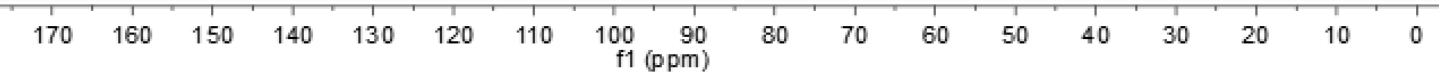

${ }^{13} \mathrm{C}$ NMR $\left(101 \mathrm{M}, \mathrm{CDCl}_{3}\right)$ spectrum of $7 \mathbf{a s}$

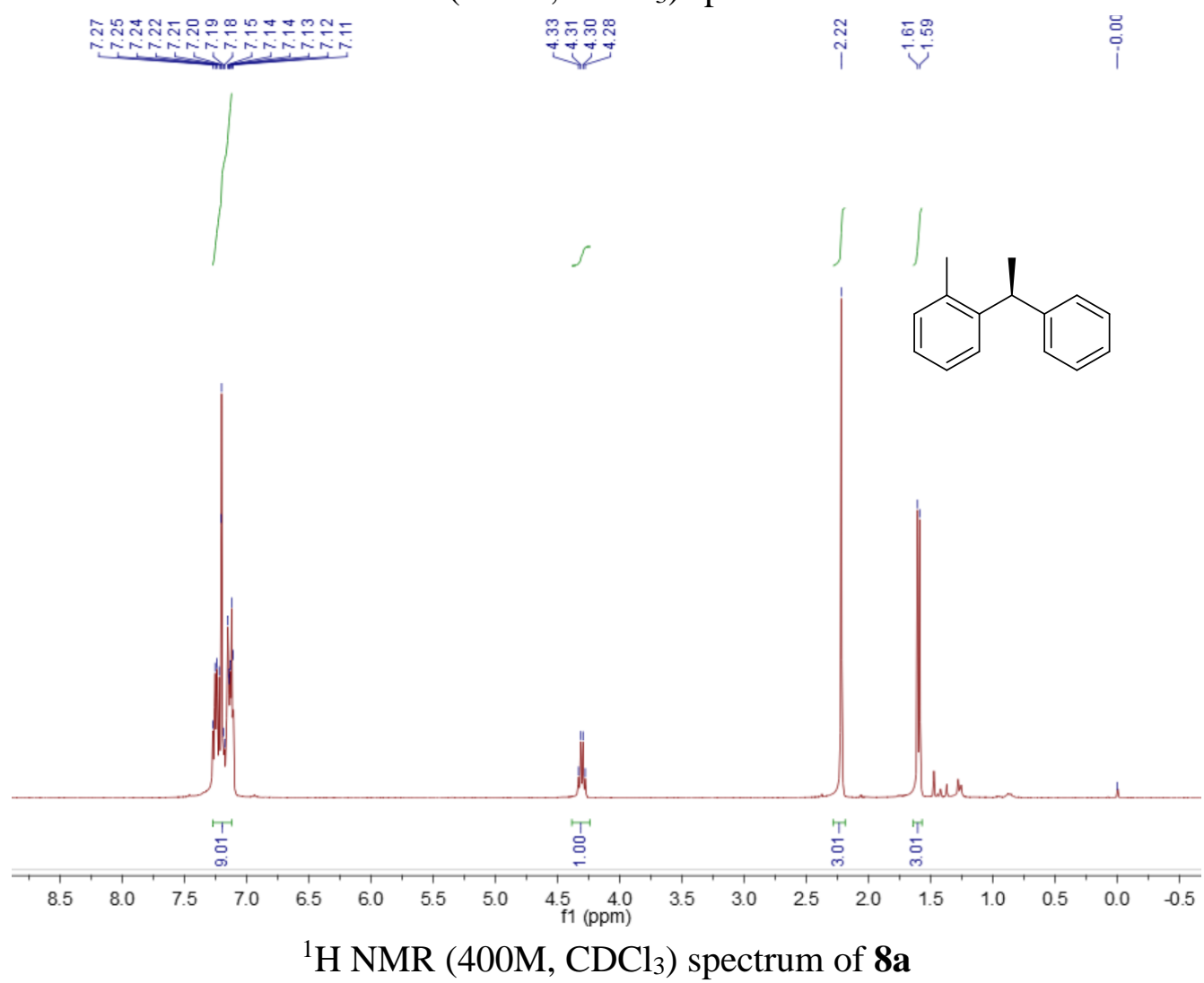




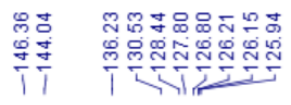
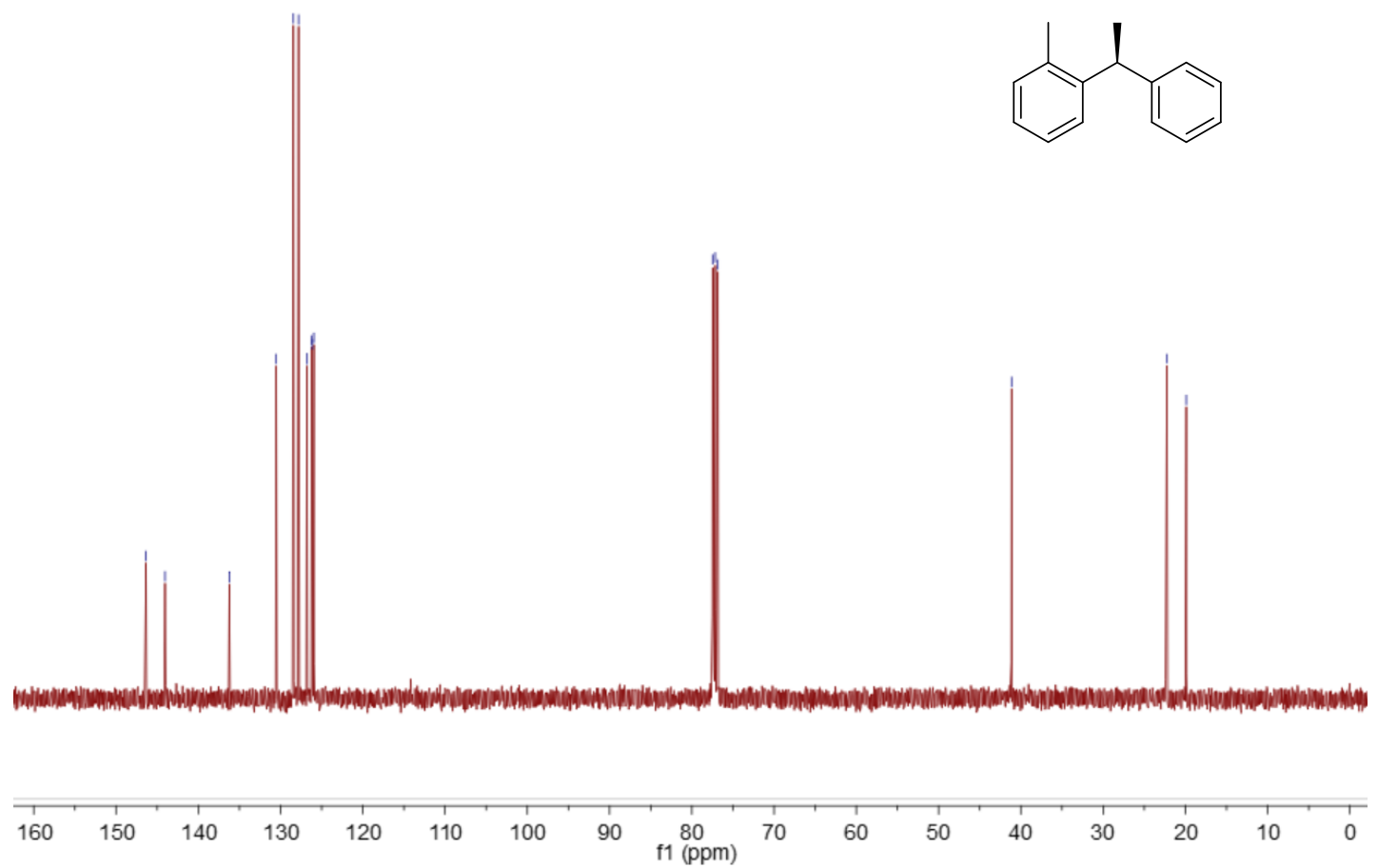

${ }^{13} \mathrm{C}$ NMR $\left(101 \mathrm{M}, \mathrm{CDCl}_{3}\right)$ spectrum of $\mathbf{8 a}$
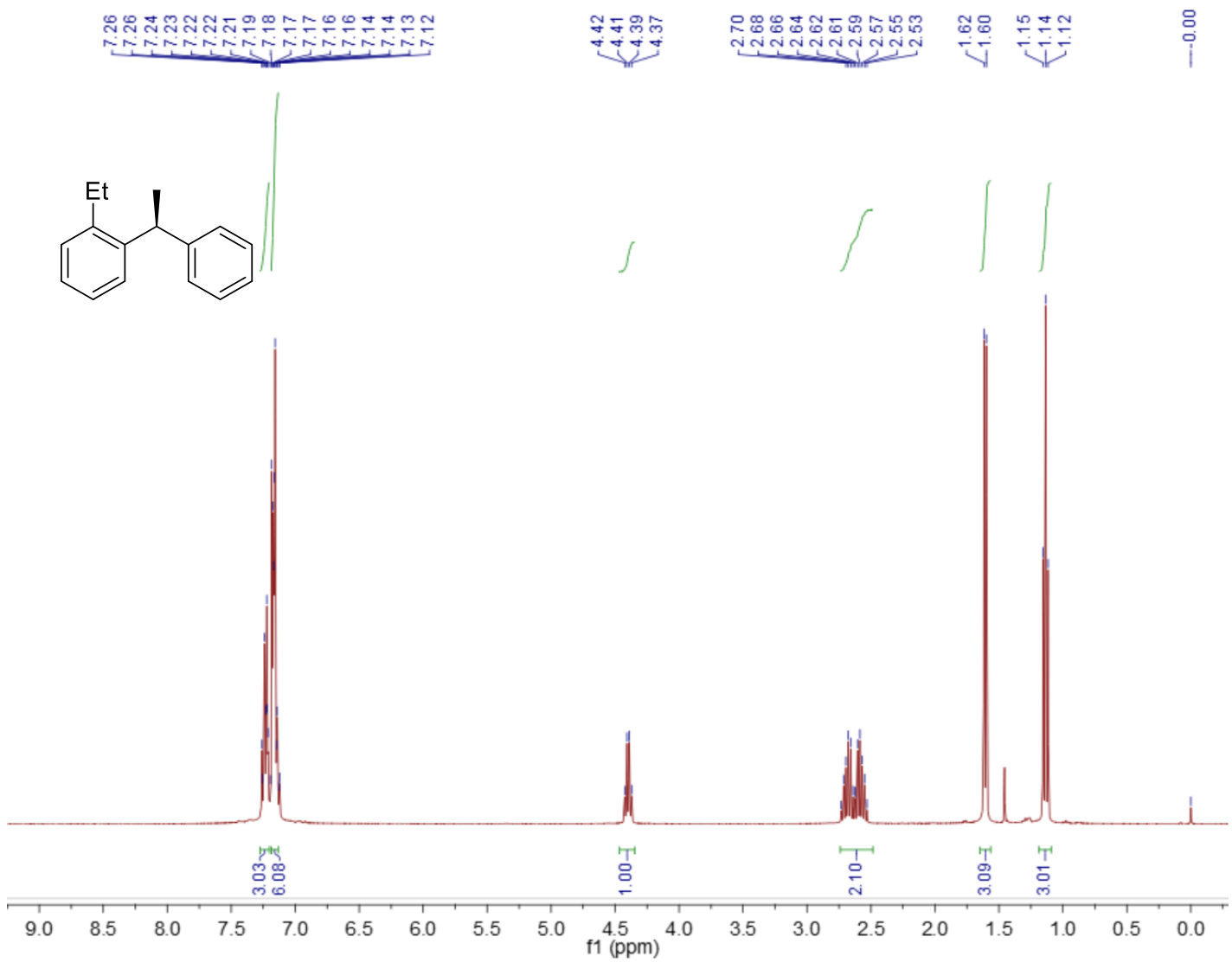

${ }^{1} \mathrm{H}$ NMR $\left(400 \mathrm{M}, \mathrm{CDCl}_{3}\right)$ spectrum of $\mathbf{8 b}$

S109 
<smiles>CCc1ccccc1C(C)c1ccccc1</smiles>
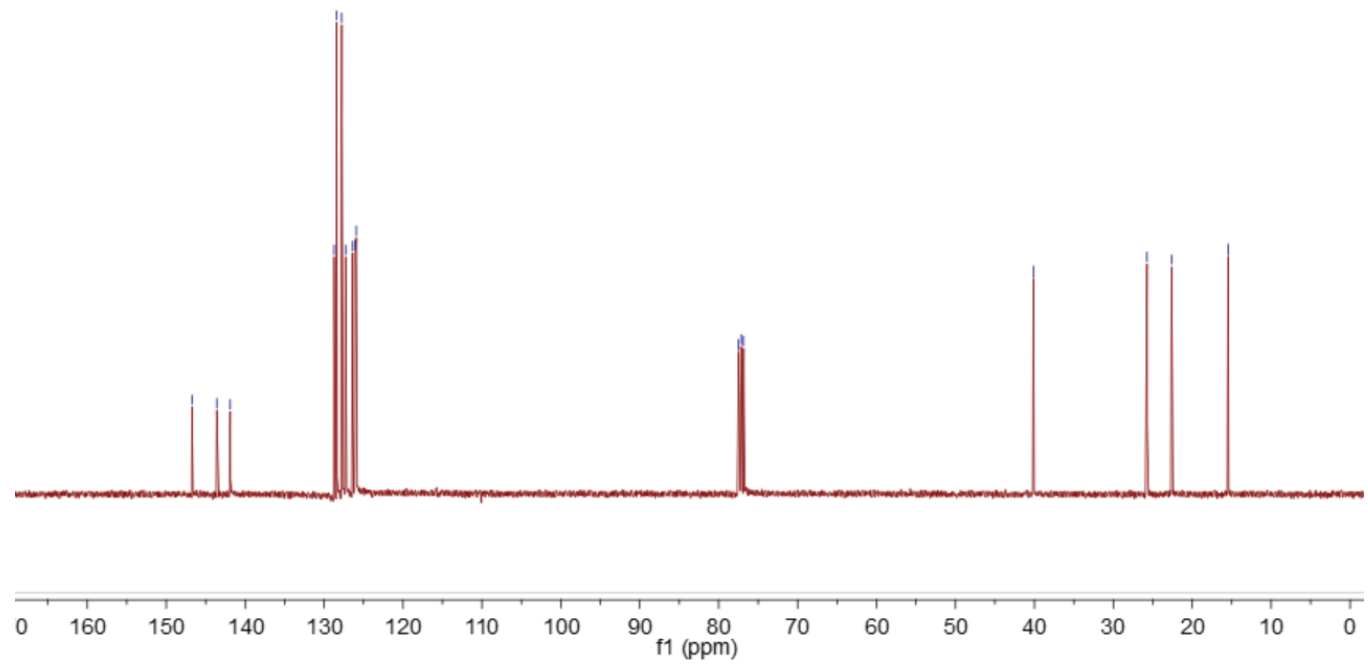

${ }^{13} \mathrm{C}$ NMR $\left(101 \mathrm{M}, \mathrm{CDCl}_{3}\right)$ spectrum of $\mathbf{8 b}$ 等<smiles>C[C@H](c1ccccc1)c1ccccc1F</smiles>
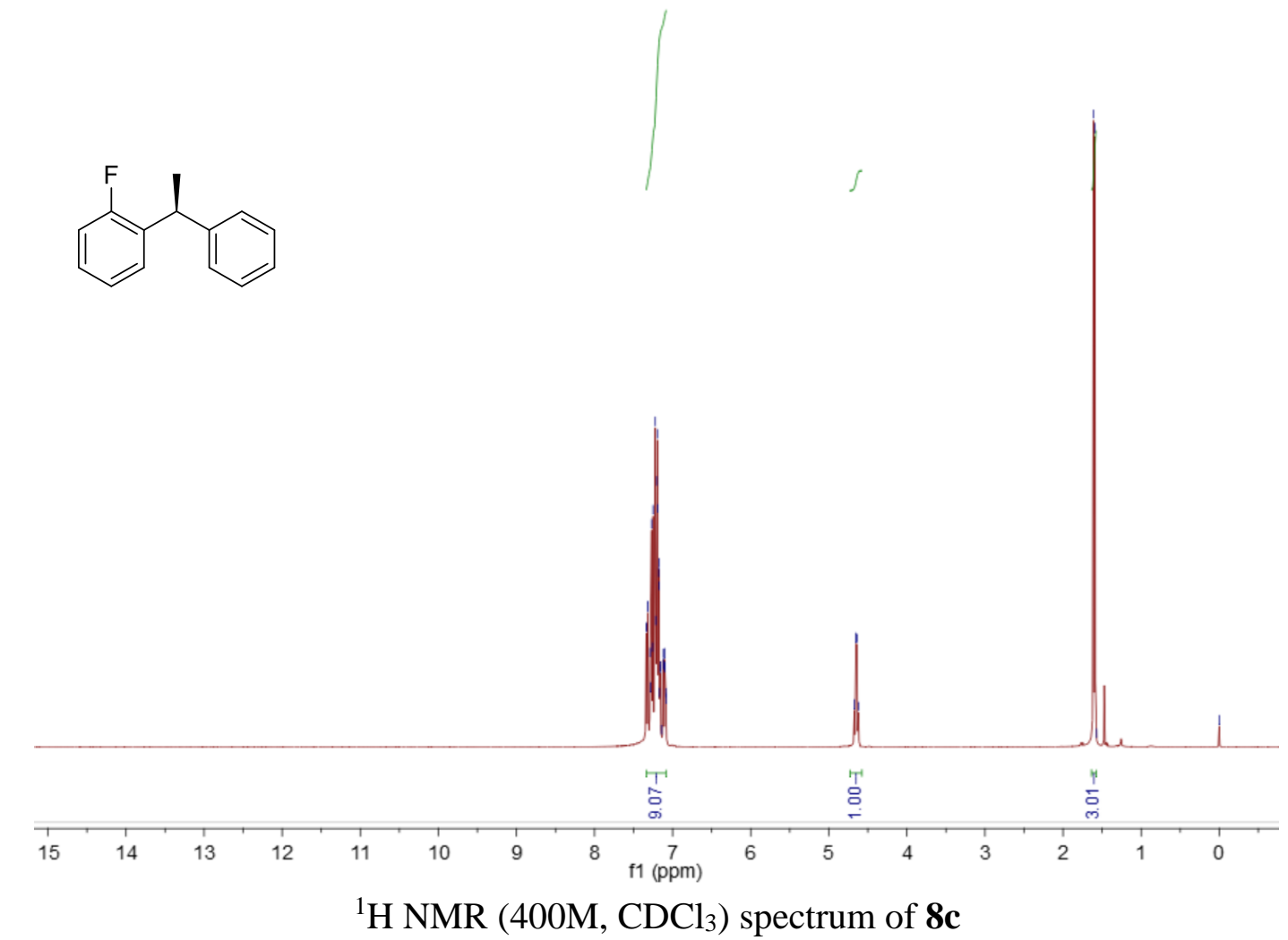


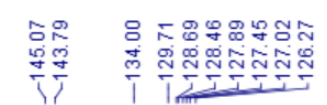

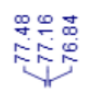

$\stackrel{8}{\mathrm{j}}$

$\stackrel{\stackrel{1}{\top}}{i}$
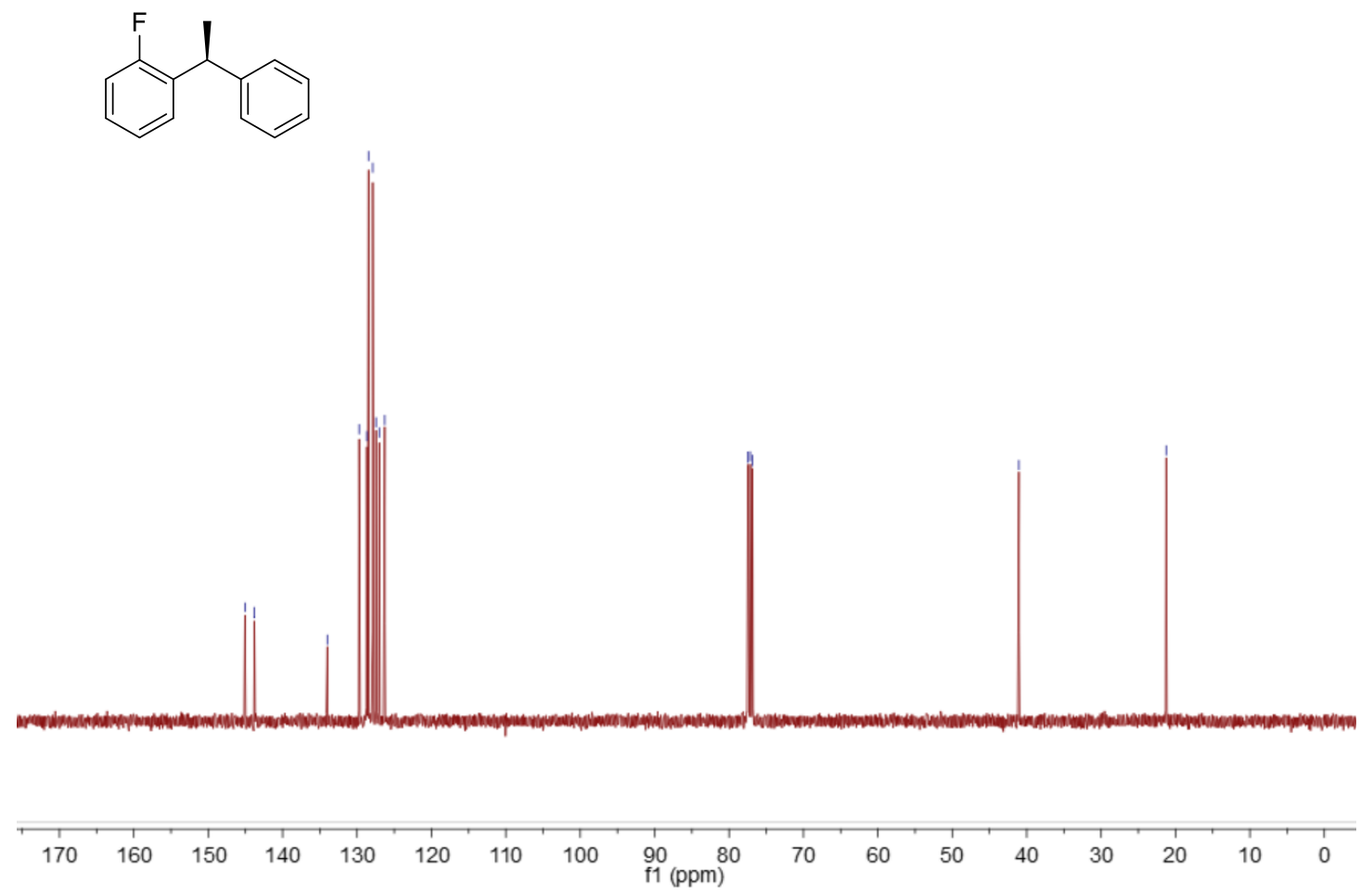

${ }^{13} \mathrm{C}$ NMR $\left(101 \mathrm{M}, \mathrm{CDCl}_{3}\right)$ spectrum of $\mathbf{8 c}$

$\stackrel{\text { }}{\stackrel{\text { }}{i}}$
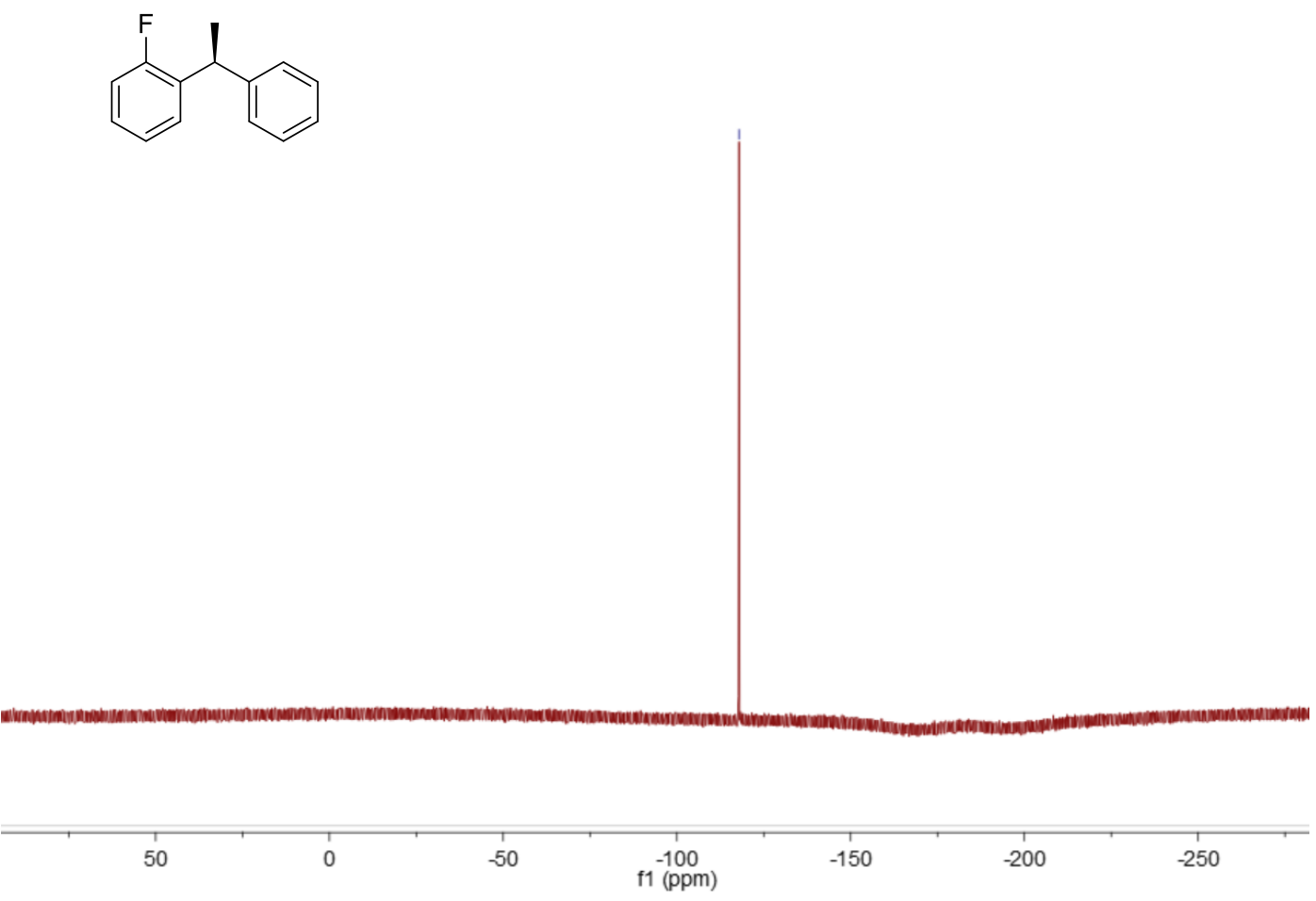

${ }^{19}$ F NMR (377 MHz, $\left.\mathrm{CDCl}_{3}\right)$ spectrum of $\mathbf{8 c}$

S111 
్ㅐㅆ

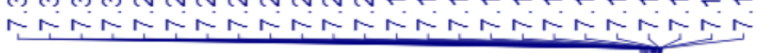
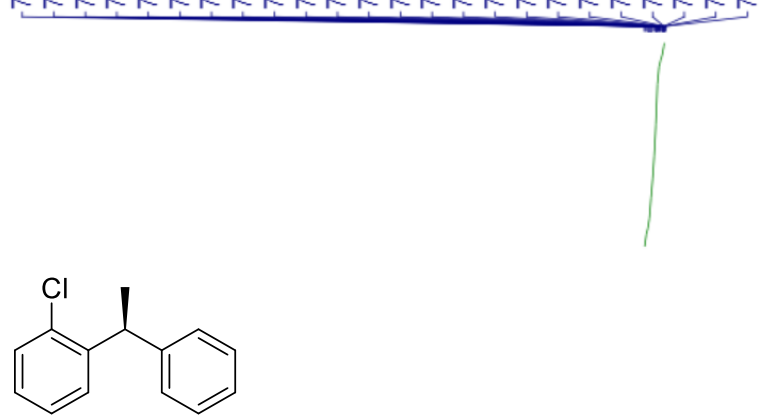

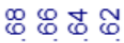

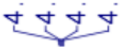
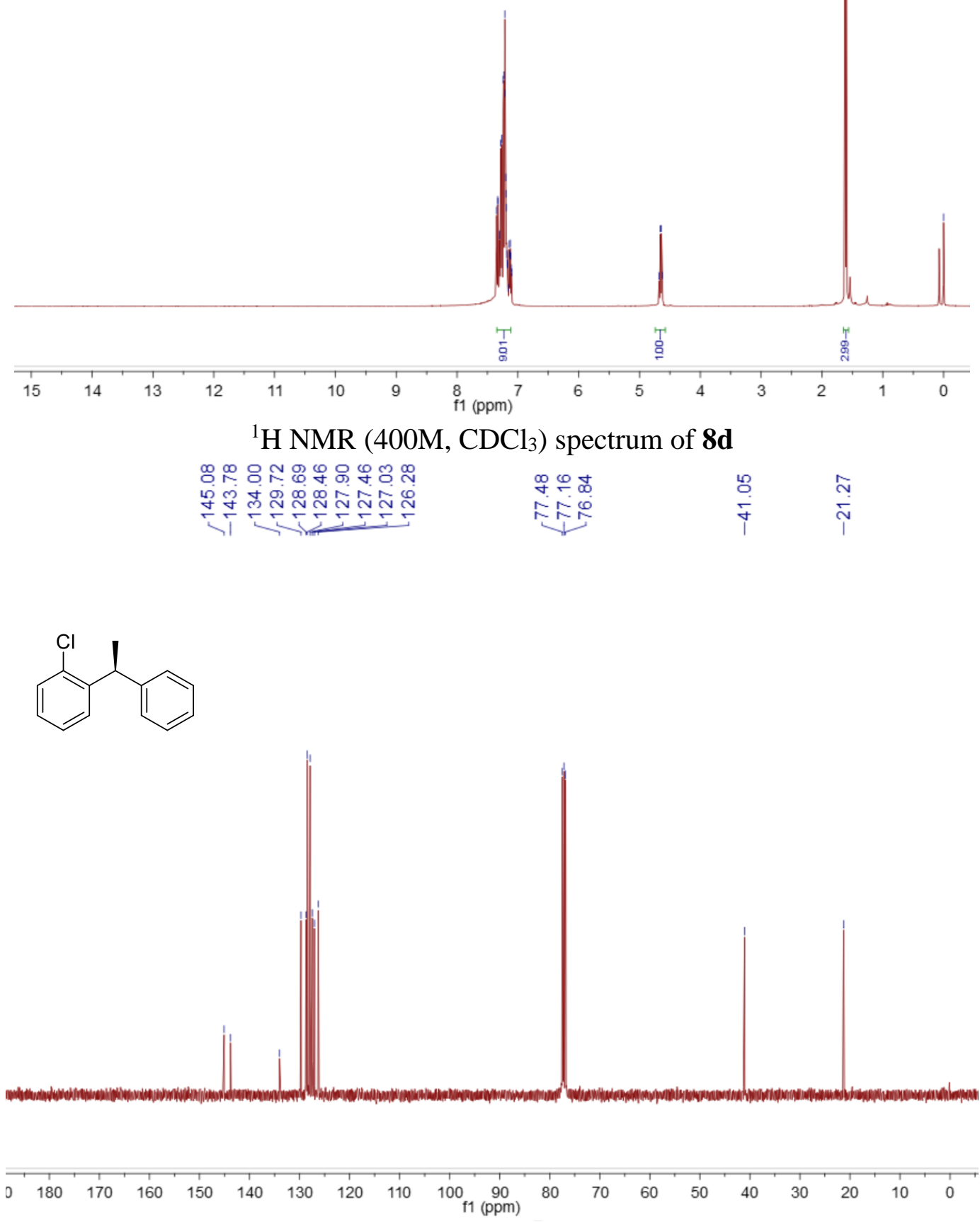

${ }^{13} \mathrm{C}$ NMR (101M, $\left.\mathrm{CDCl}_{3}\right)$ spectrum of $\mathbf{8 d}$

S112 


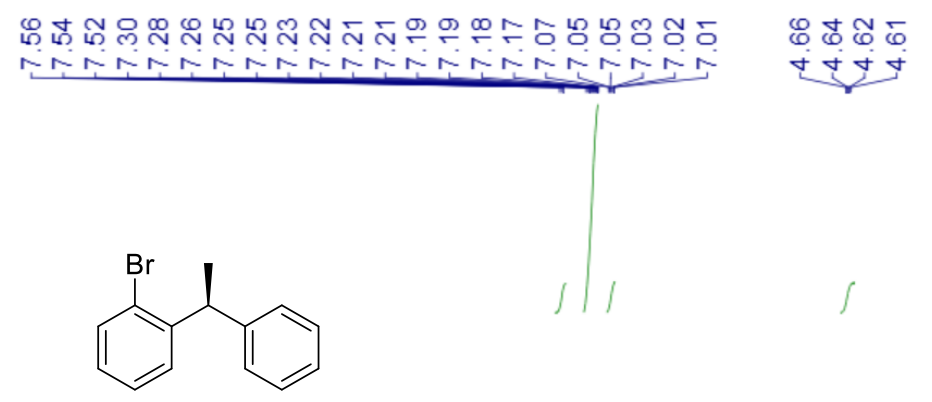

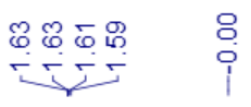

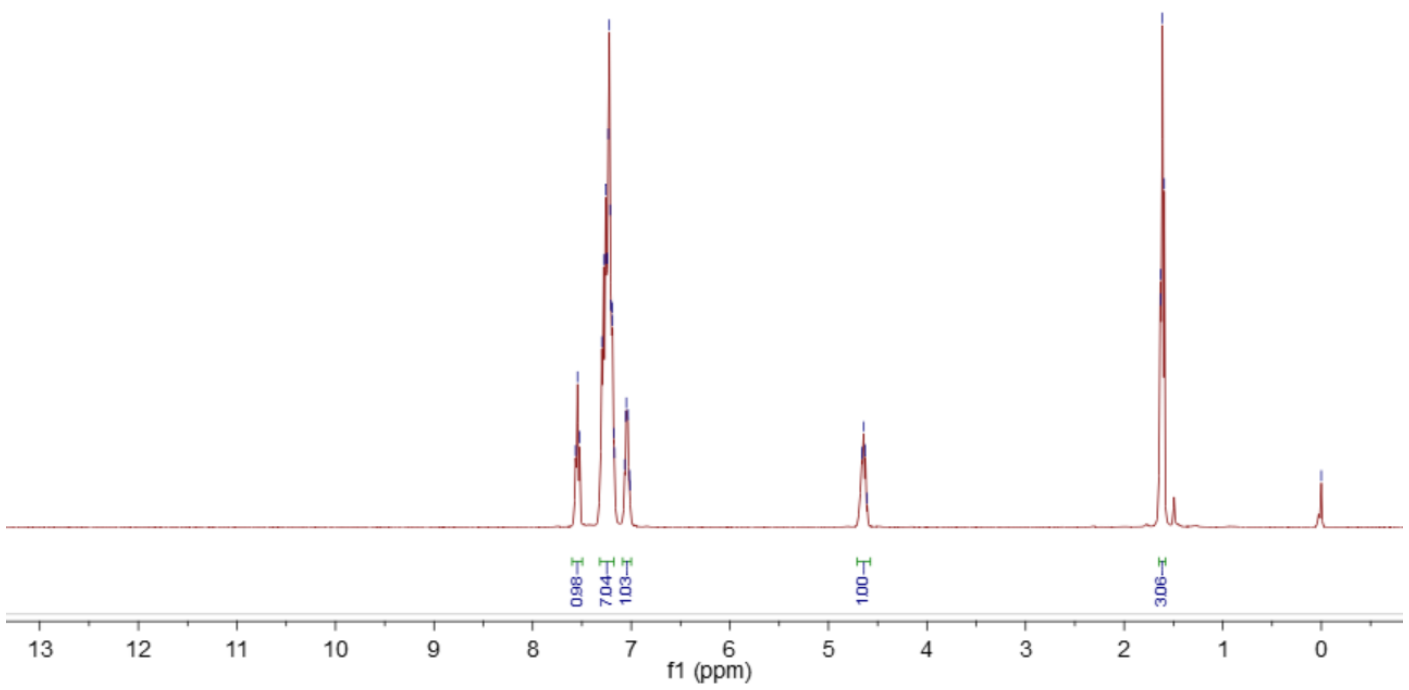

${ }^{1} \mathrm{H}$ NMR (400M, $\mathrm{CDCl}_{3}$ ) spectrum of $\mathbf{8 e}$

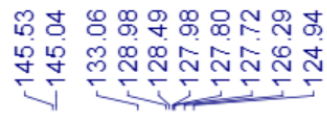

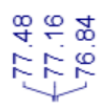

ڤิ)

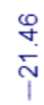
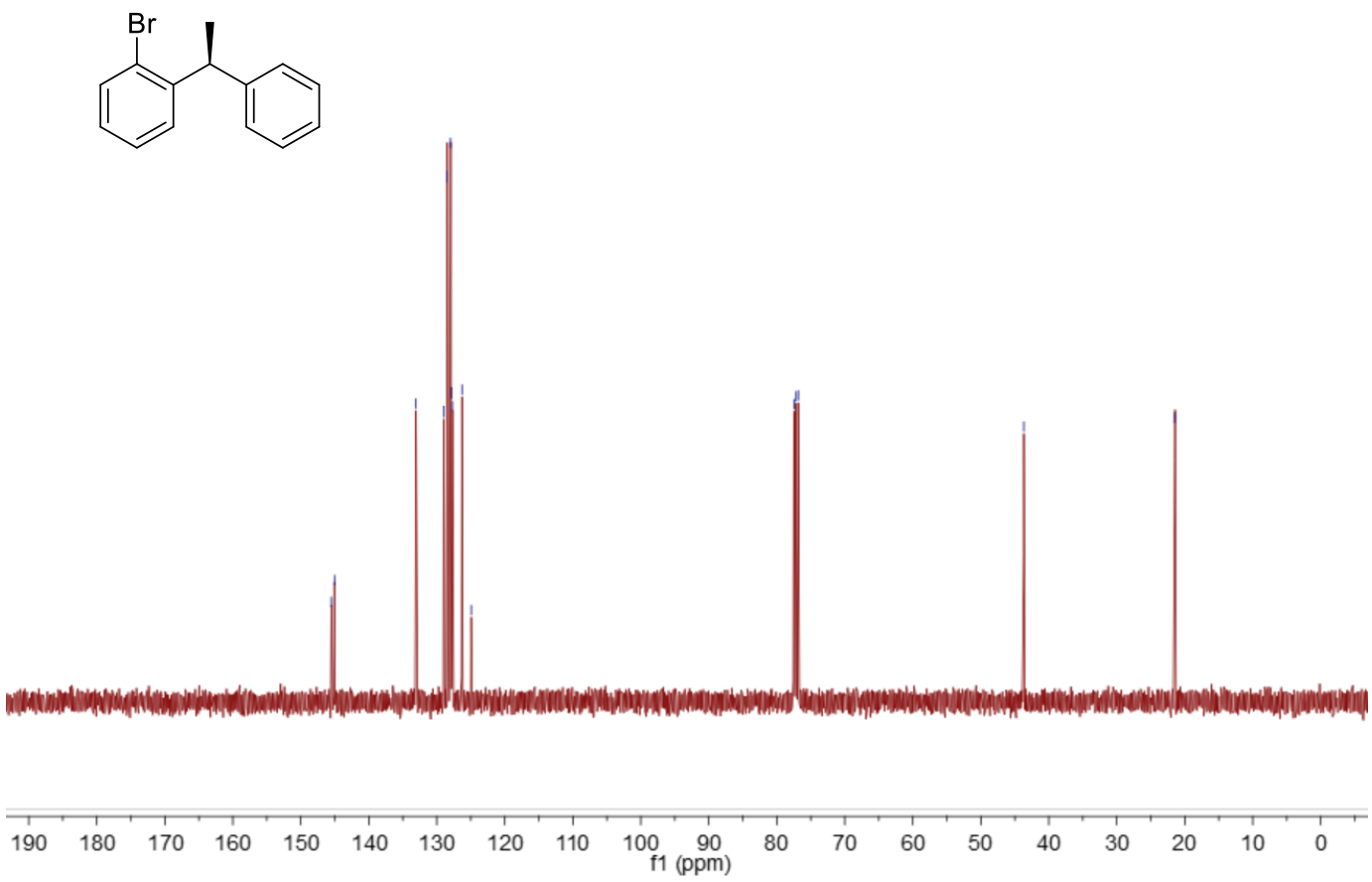

${ }^{13} \mathrm{C}$ NMR $\left(101 \mathrm{M}, \mathrm{CDCl}_{3}\right)$ spectrum of $\mathbf{8 e}$

S113 


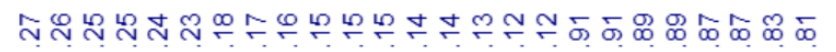

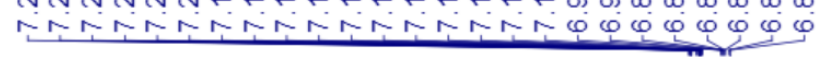

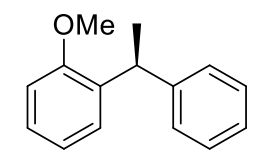

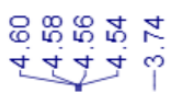

帒

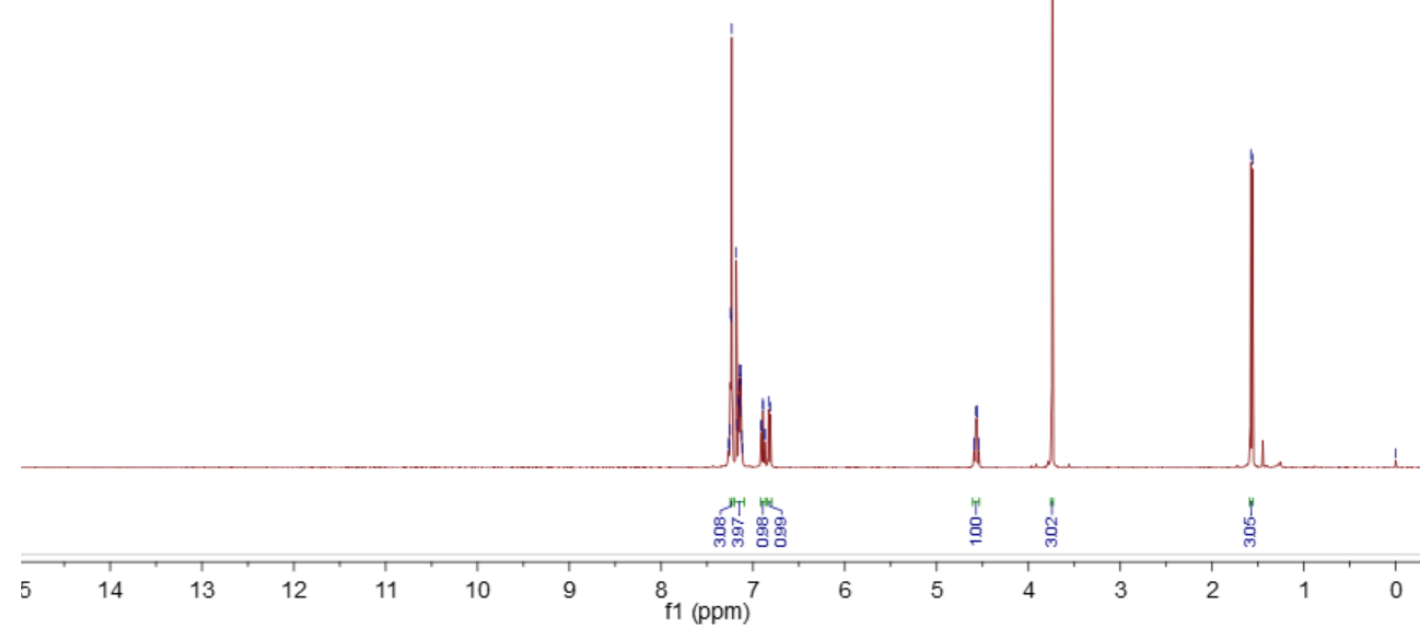

${ }^{1} \mathrm{H}$ NMR (400M, $\mathrm{CDCl}_{3}$ ) spectrum of $\mathbf{8 f}$

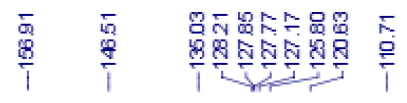

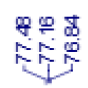

8

$\frac{\mathbb{q}}{\mathrm{q}}$
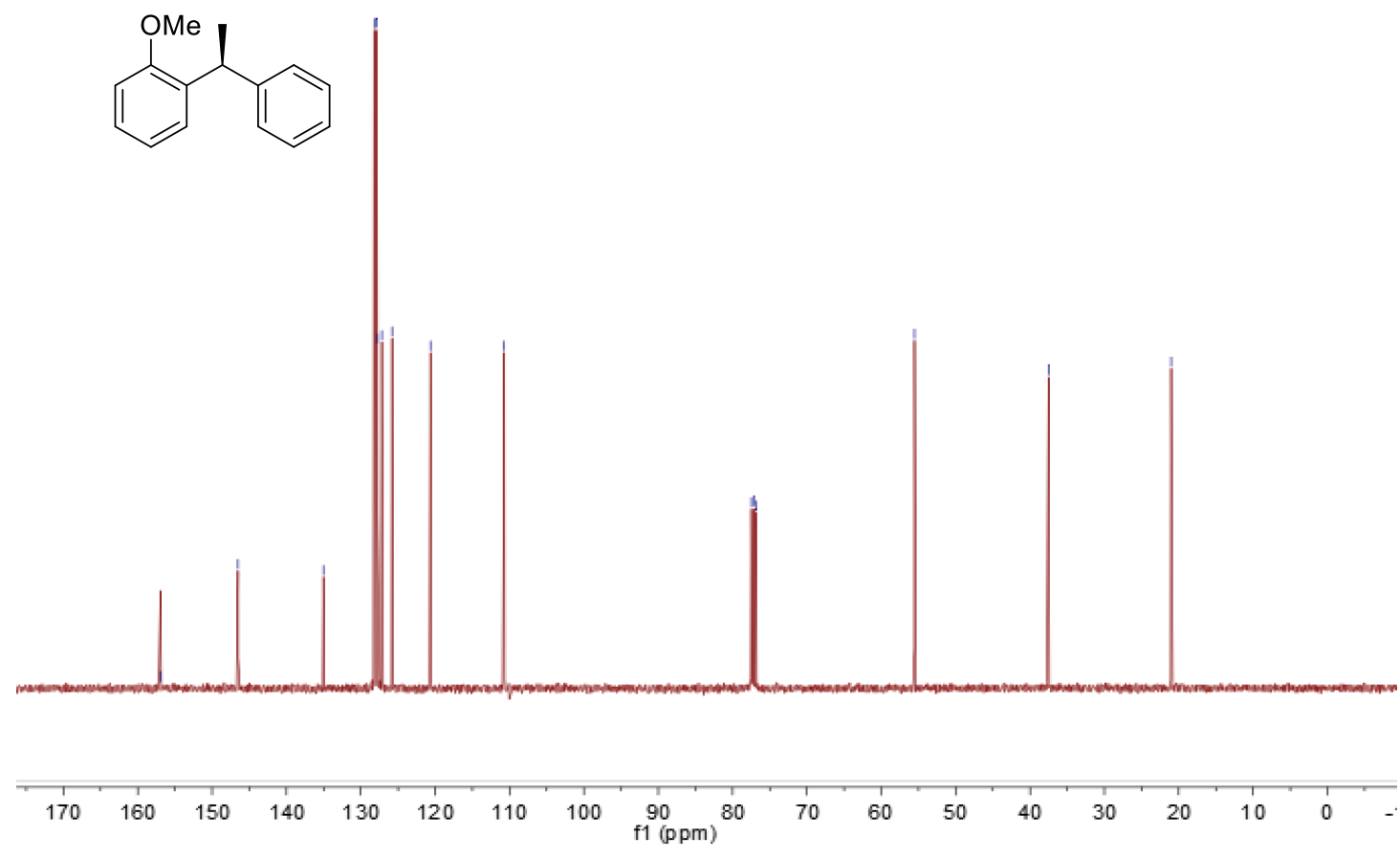

${ }^{13} \mathrm{C}$ NMR (101M, $\left.\mathrm{CDCl}_{3}\right)$ spectrum of $\mathbf{8 f}$

S114 


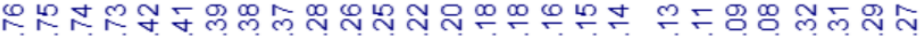

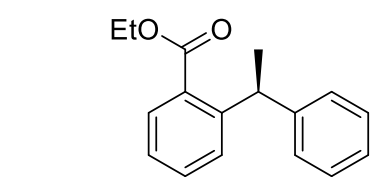

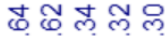

i

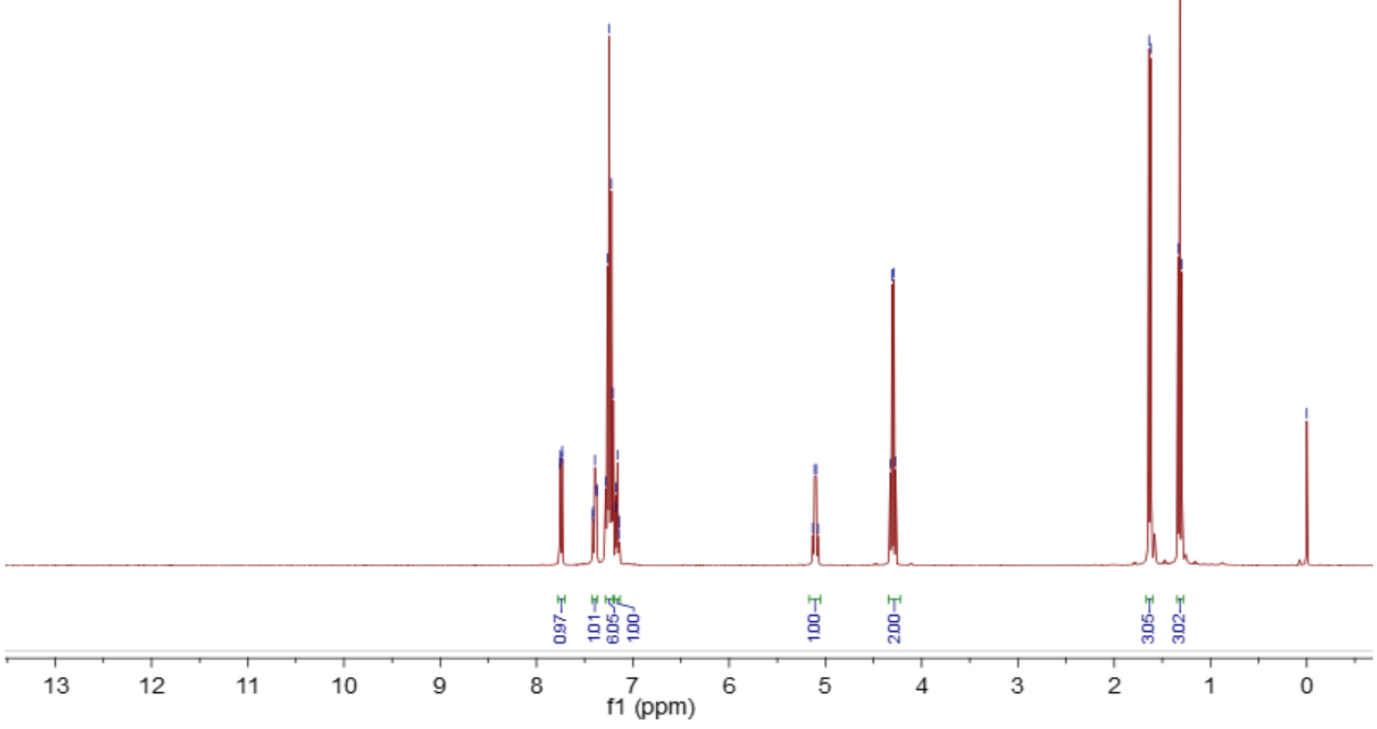

${ }^{1} \mathrm{H}$ NMR (400M, $\mathrm{CDCl}_{3}$ ) spectrum of $\mathbf{8 g}$
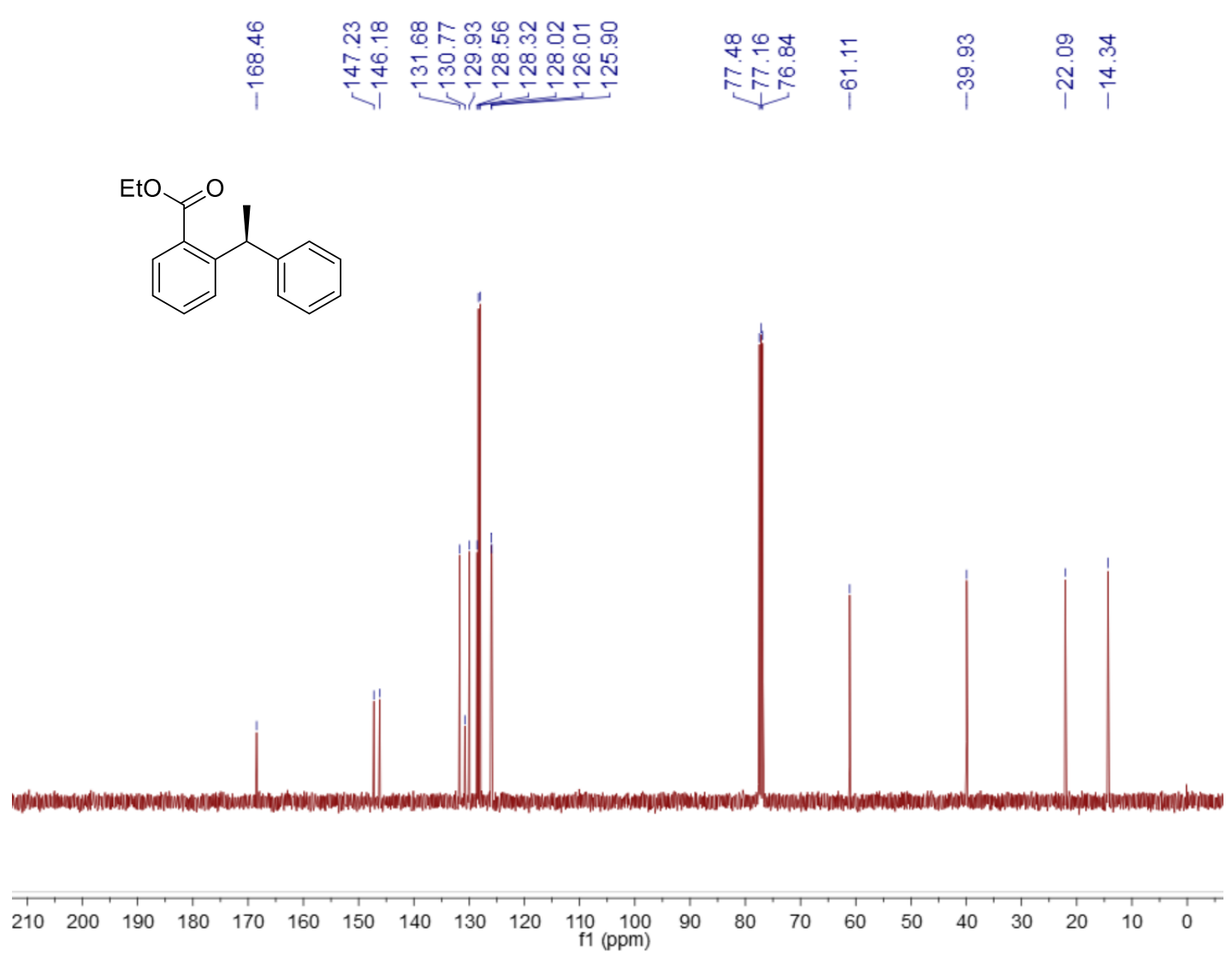

${ }^{13} \mathrm{C}$ NMR (101M, $\mathrm{CDCl}_{3}$ ) spectrum of $\mathbf{8 g}$

S115 

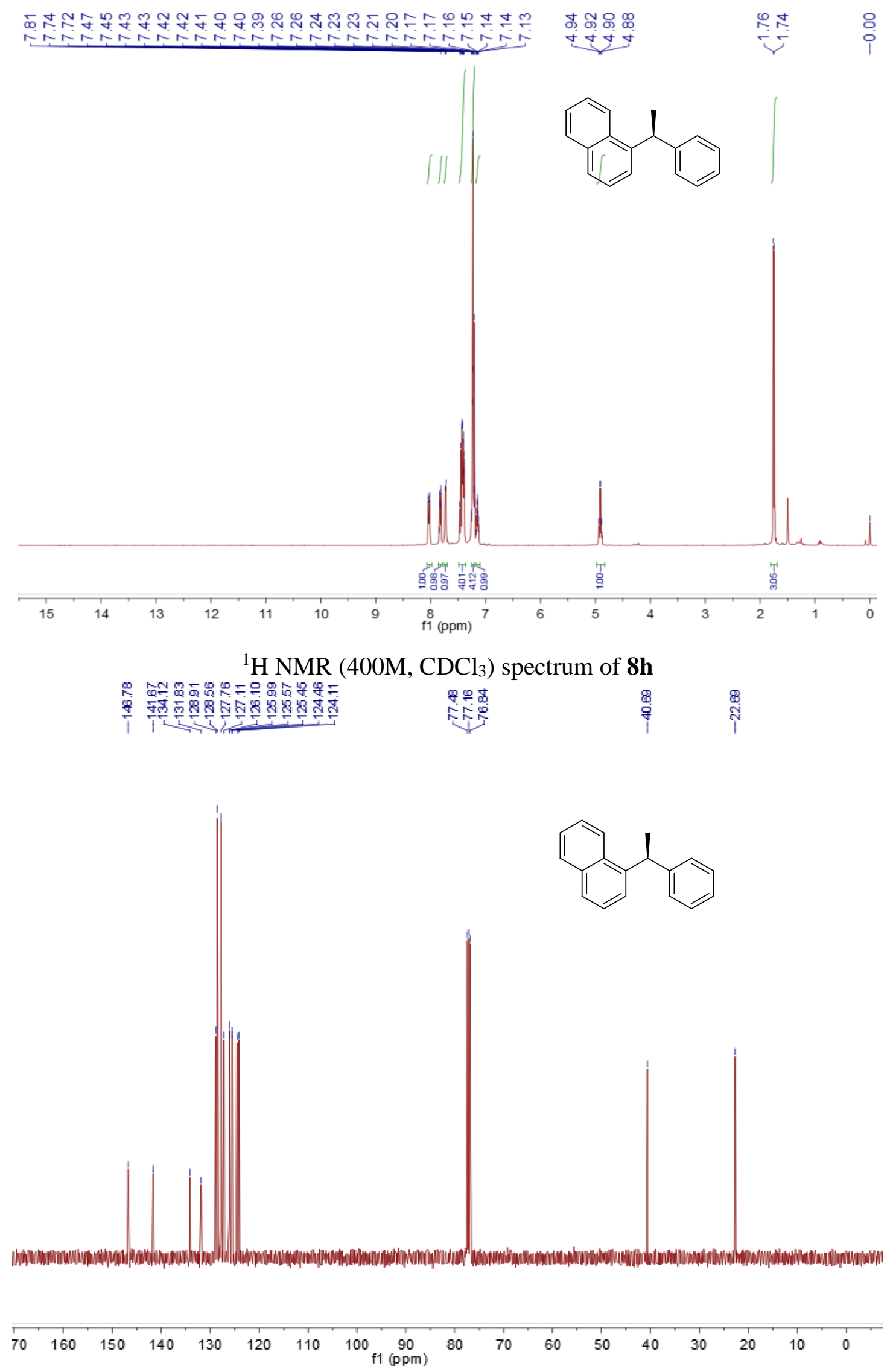

${ }^{13} \mathrm{C}$ NMR $\left(101 \mathrm{M}, \mathrm{CDCl}_{3}\right)$ spectrum of $\mathbf{8 h}$

S116 

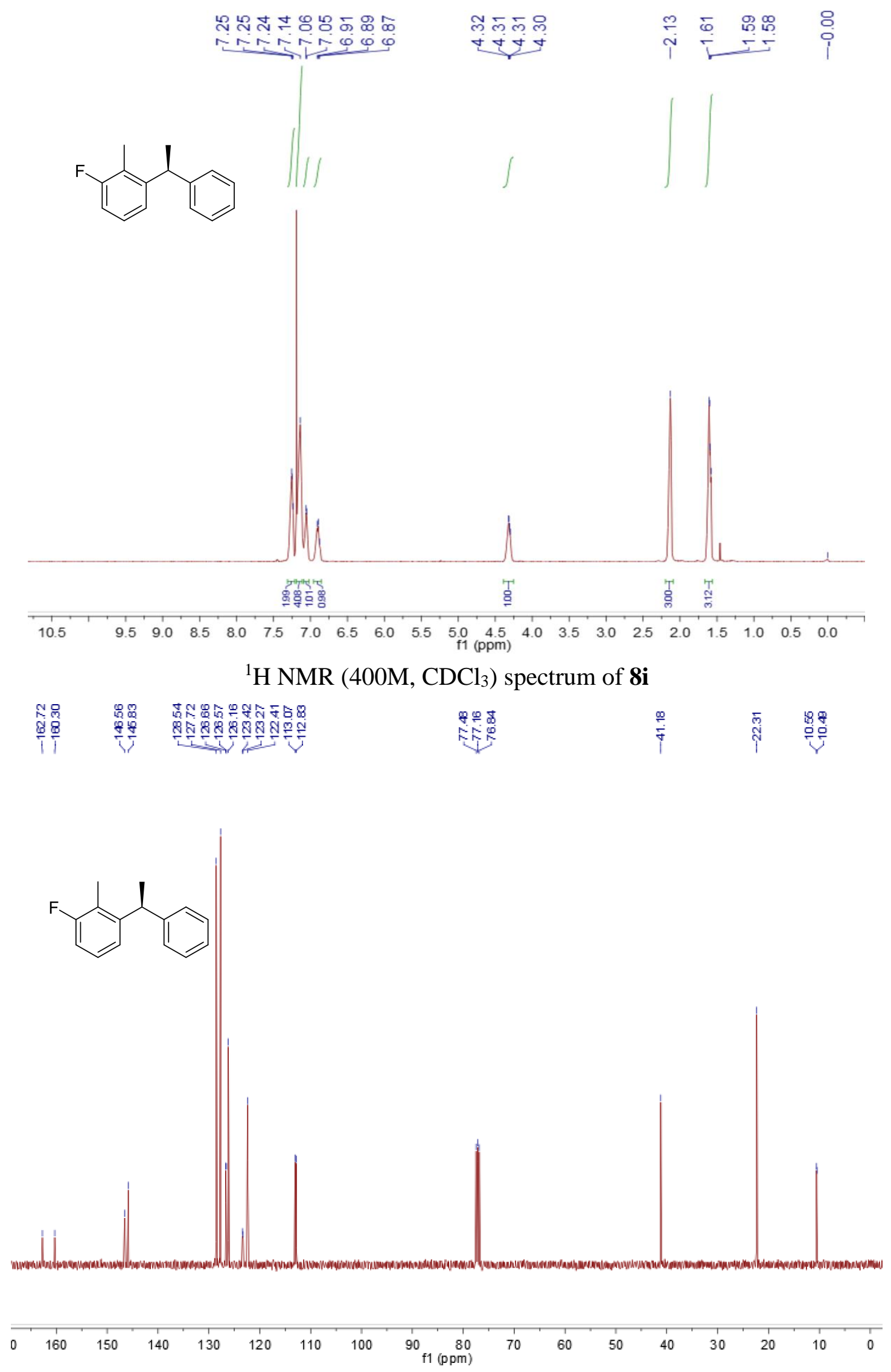

${ }^{13} \mathrm{C}$ NMR (101M, $\left.\mathrm{CDCl}_{3}\right)$ spectrum of $\mathbf{8 i}$

S117 
$\stackrel{\infty}{\stackrel{0}{\circ}}$
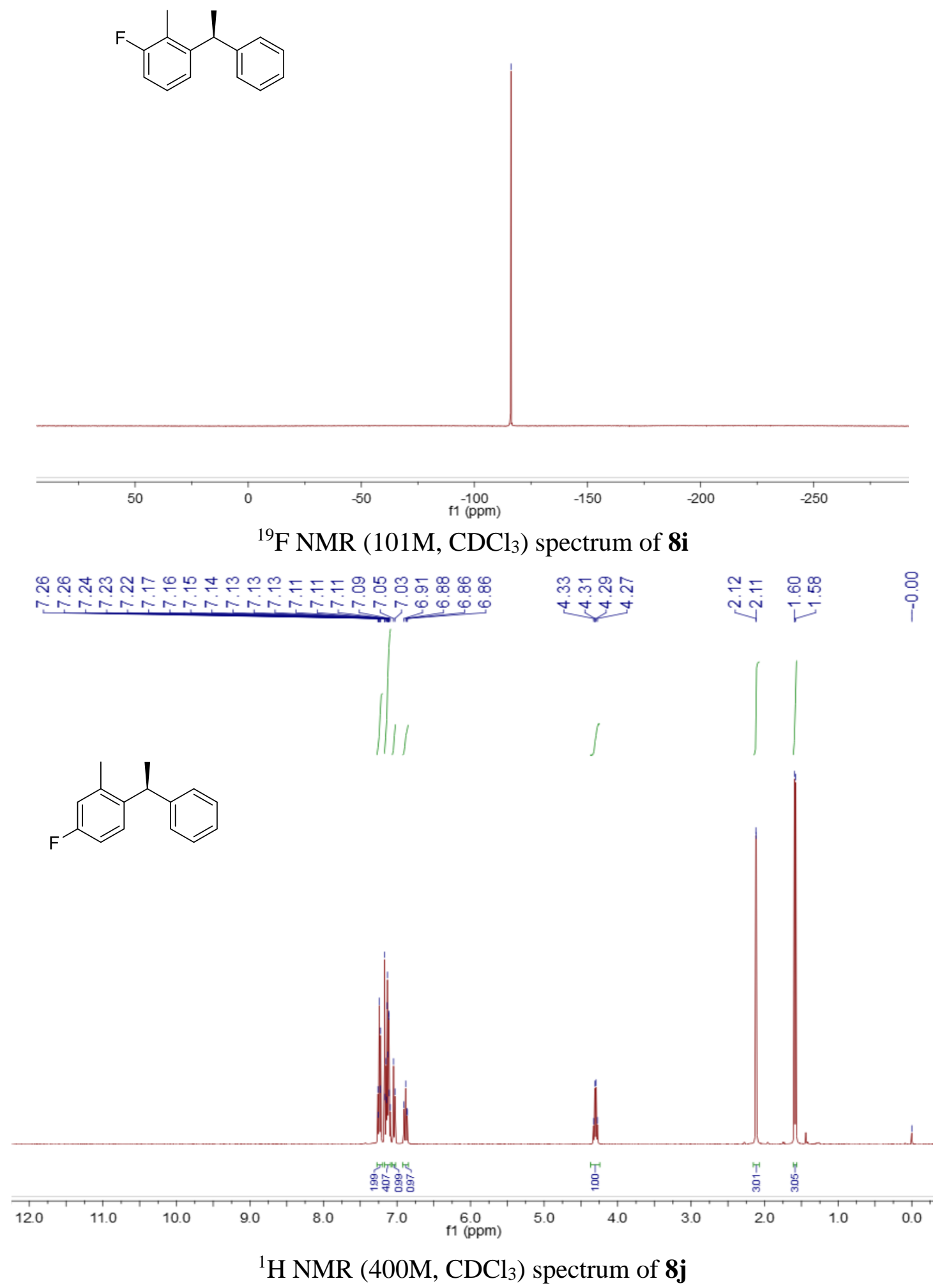

S118 


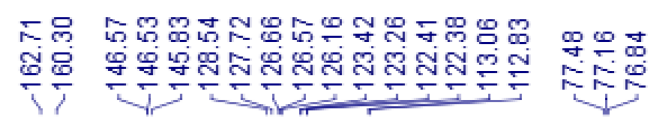

过 寽
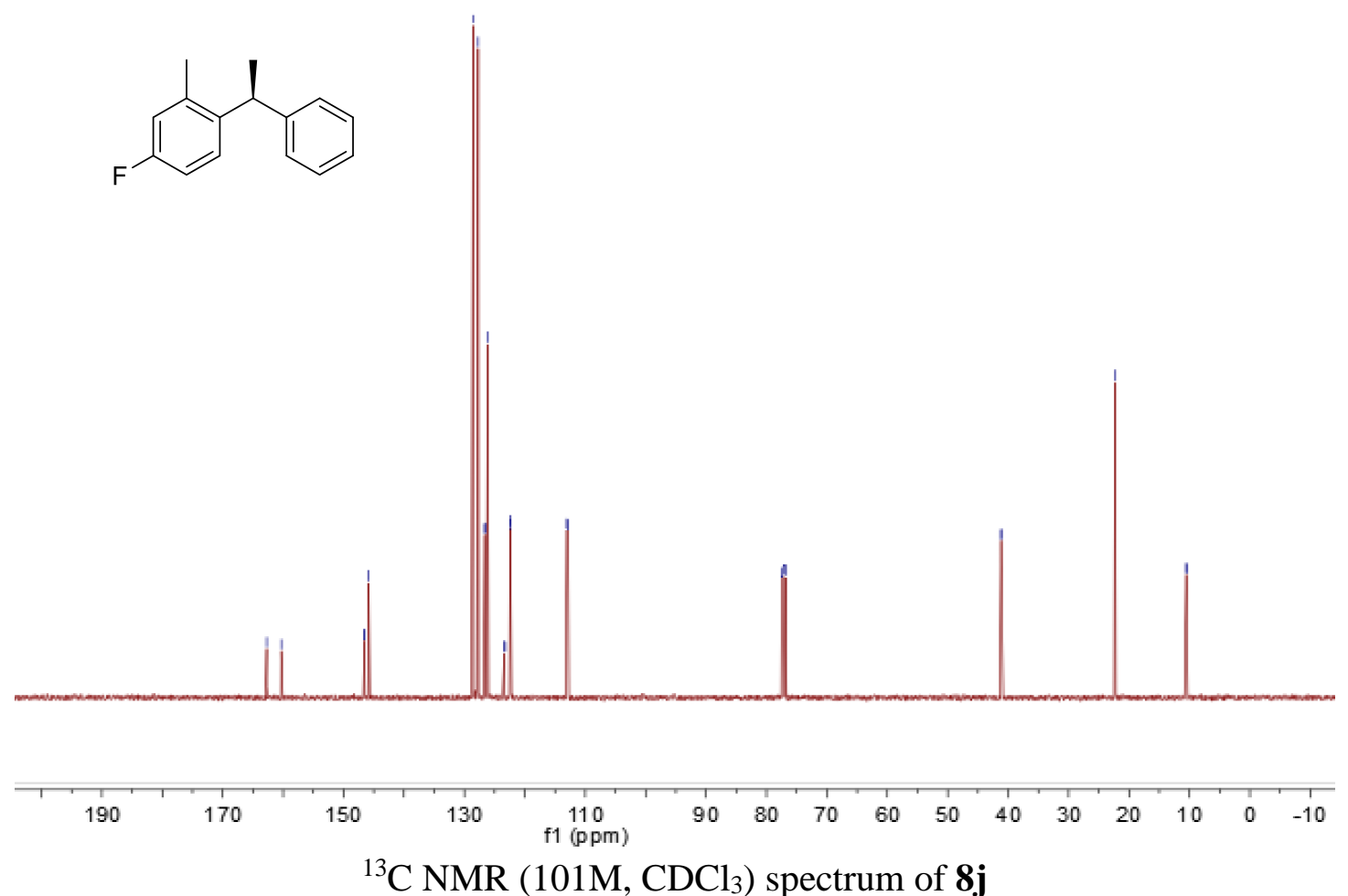

${ }^{13} \mathrm{C}$ NMR (101M, $\left.\mathrm{CDCl}_{3}\right)$ spectrum of $\mathbf{8 j}$

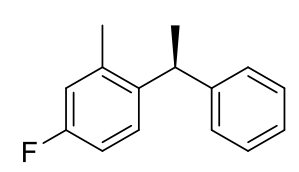

$\stackrel{\overbrace{}}{\stackrel{\circ}{\circ}}$
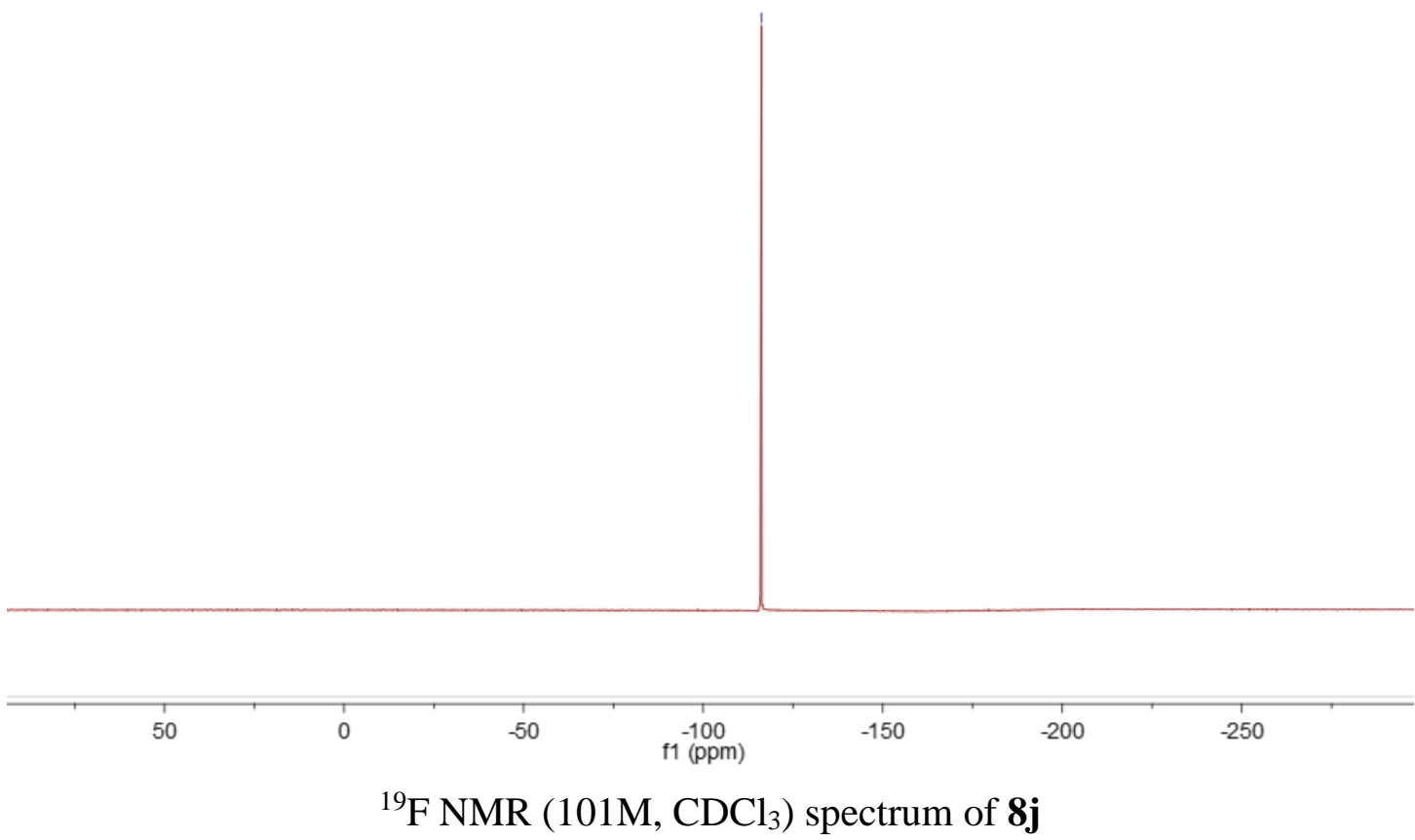

S119 


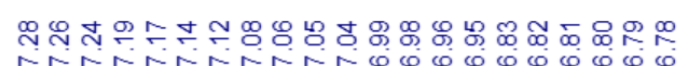

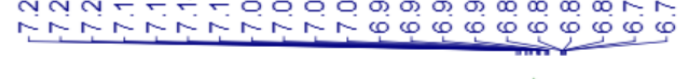

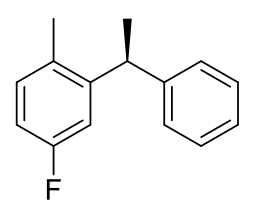

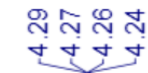

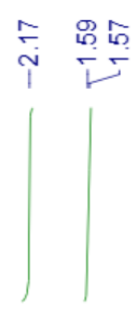

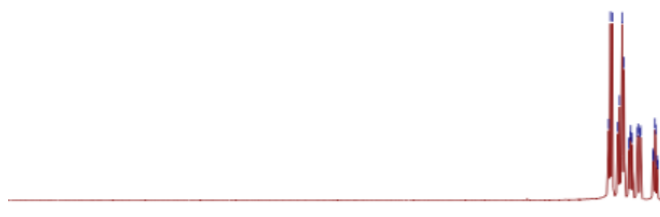

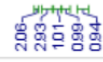

罗

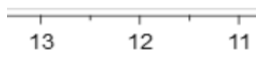

10

7

${ }^{1} \mathrm{H}$ NMR (400M, $\left.\mathrm{CDCl}_{3}\right)$ spectrum of $\mathbf{8 k}$

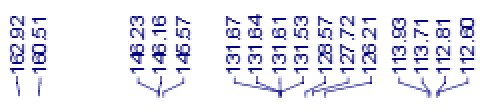

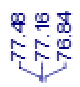

$\frac{88}{2}$

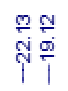
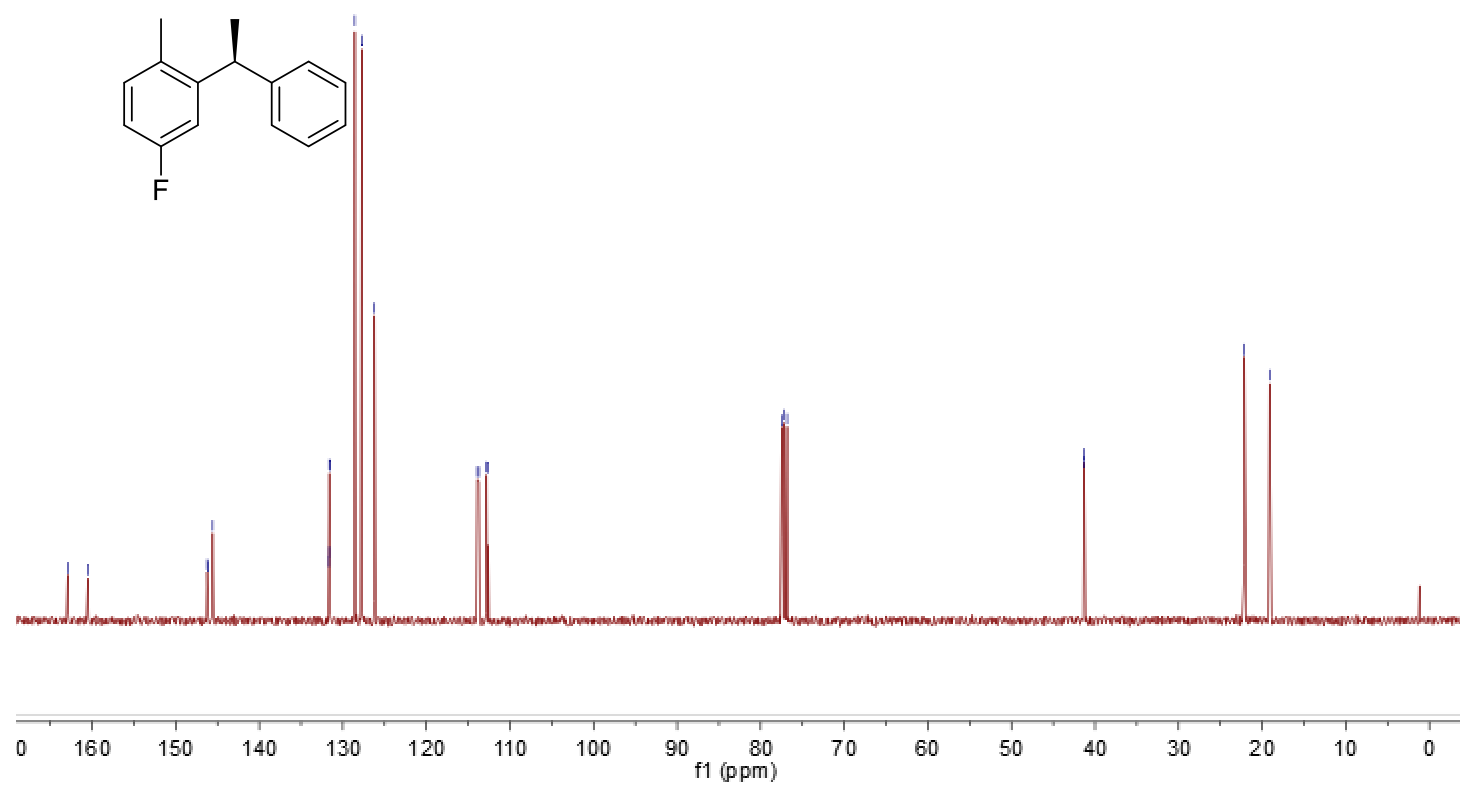

${ }^{13} \mathrm{C}$ NMR $\left(101 \mathrm{M}, \mathrm{CDCl}_{3}\right)$ spectrum of $\mathbf{8 k}$

S120 


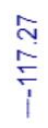
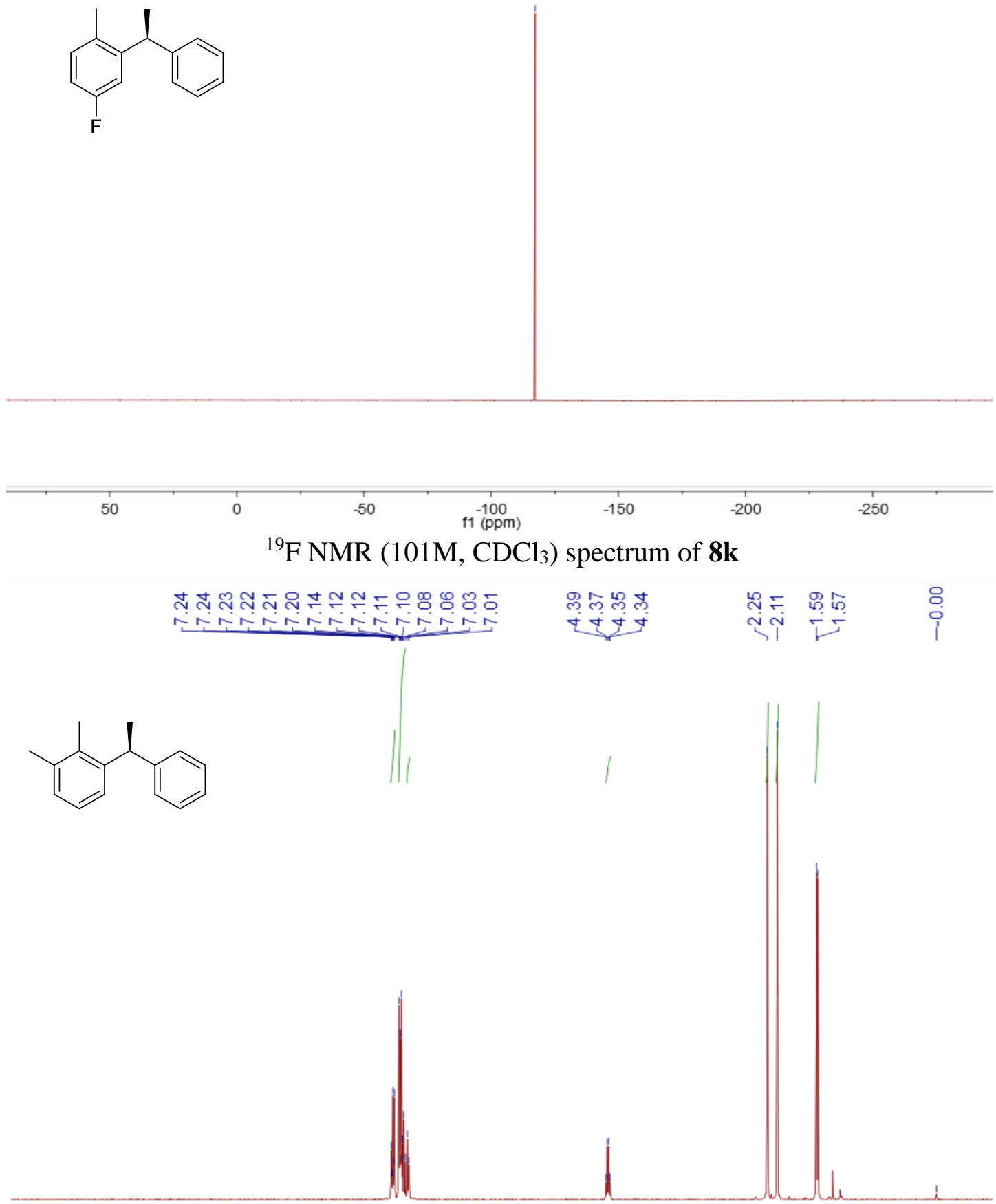

客然哭

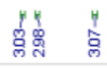

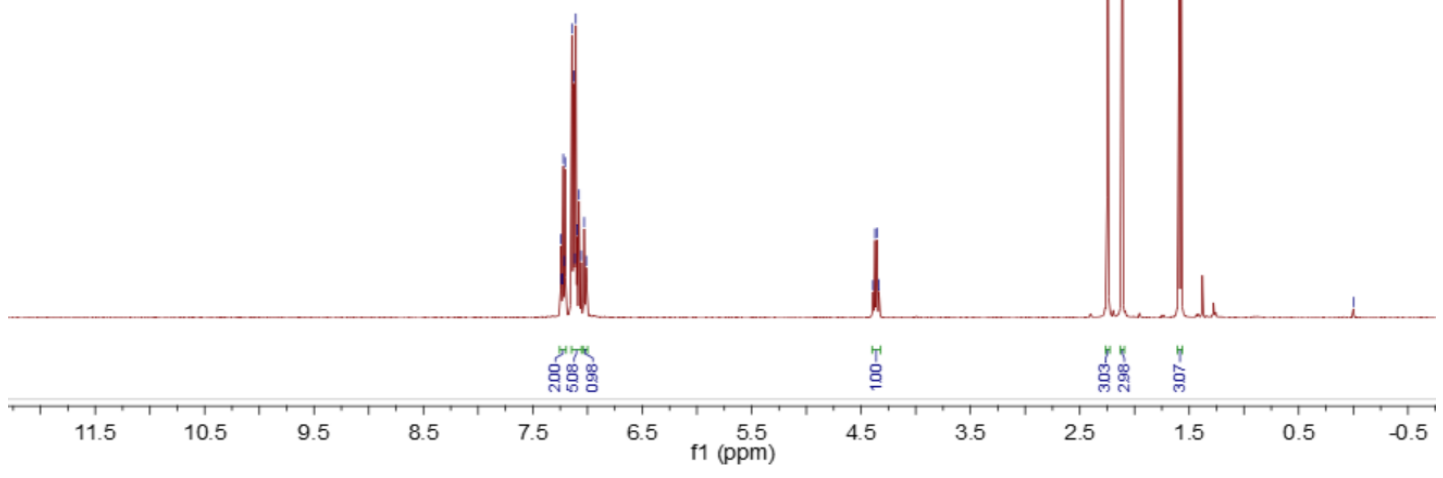

${ }^{1} \mathrm{H}$ NMR (400M, $\mathrm{CDCl}_{3}$ ) spectrum of $\mathbf{8 I}$

S121 

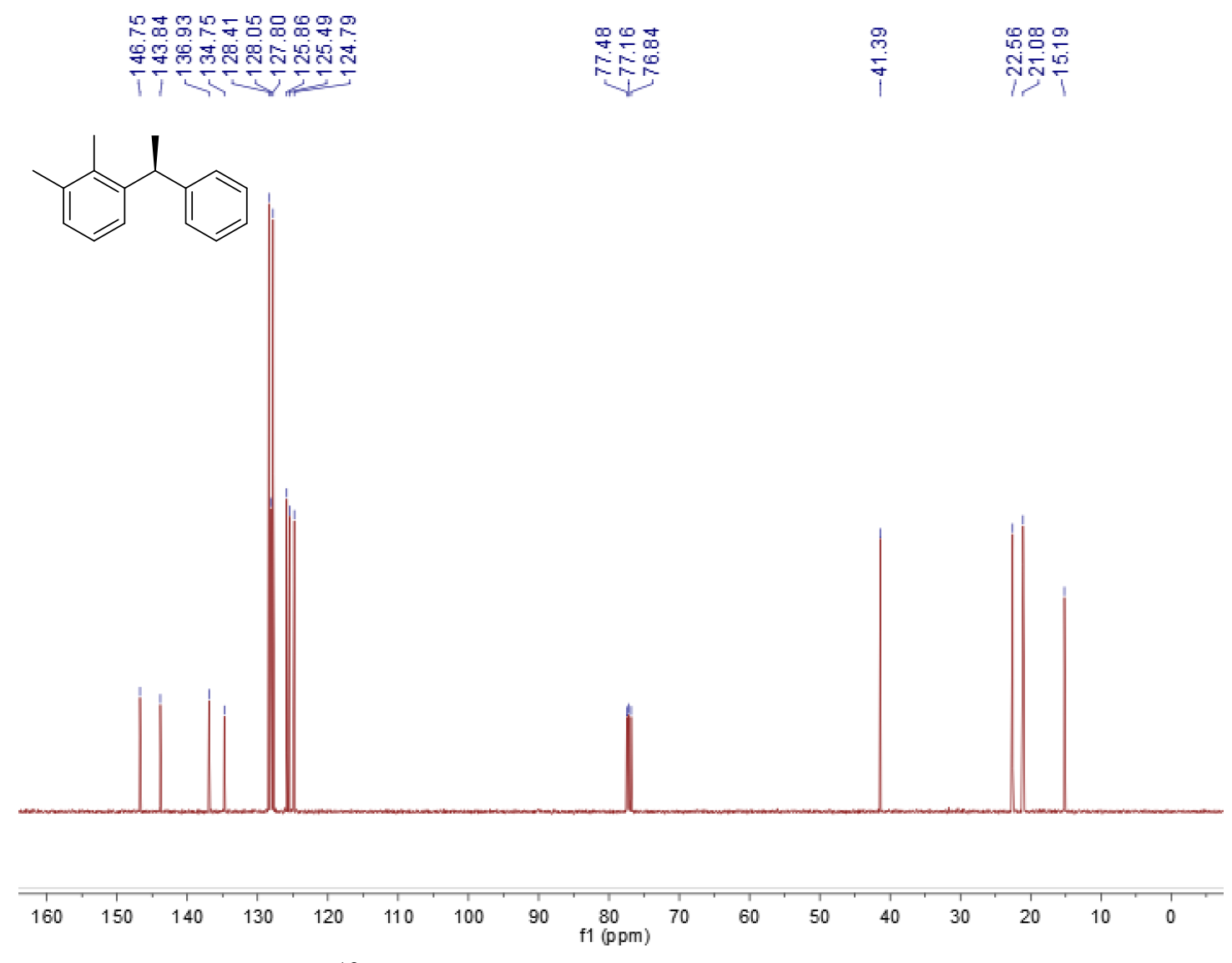

${ }^{13} \mathrm{C}$ NMR $\left(101 \mathrm{M}, \mathrm{CDCl}_{3}\right)$ spectrum of $\mathbf{8 1}$

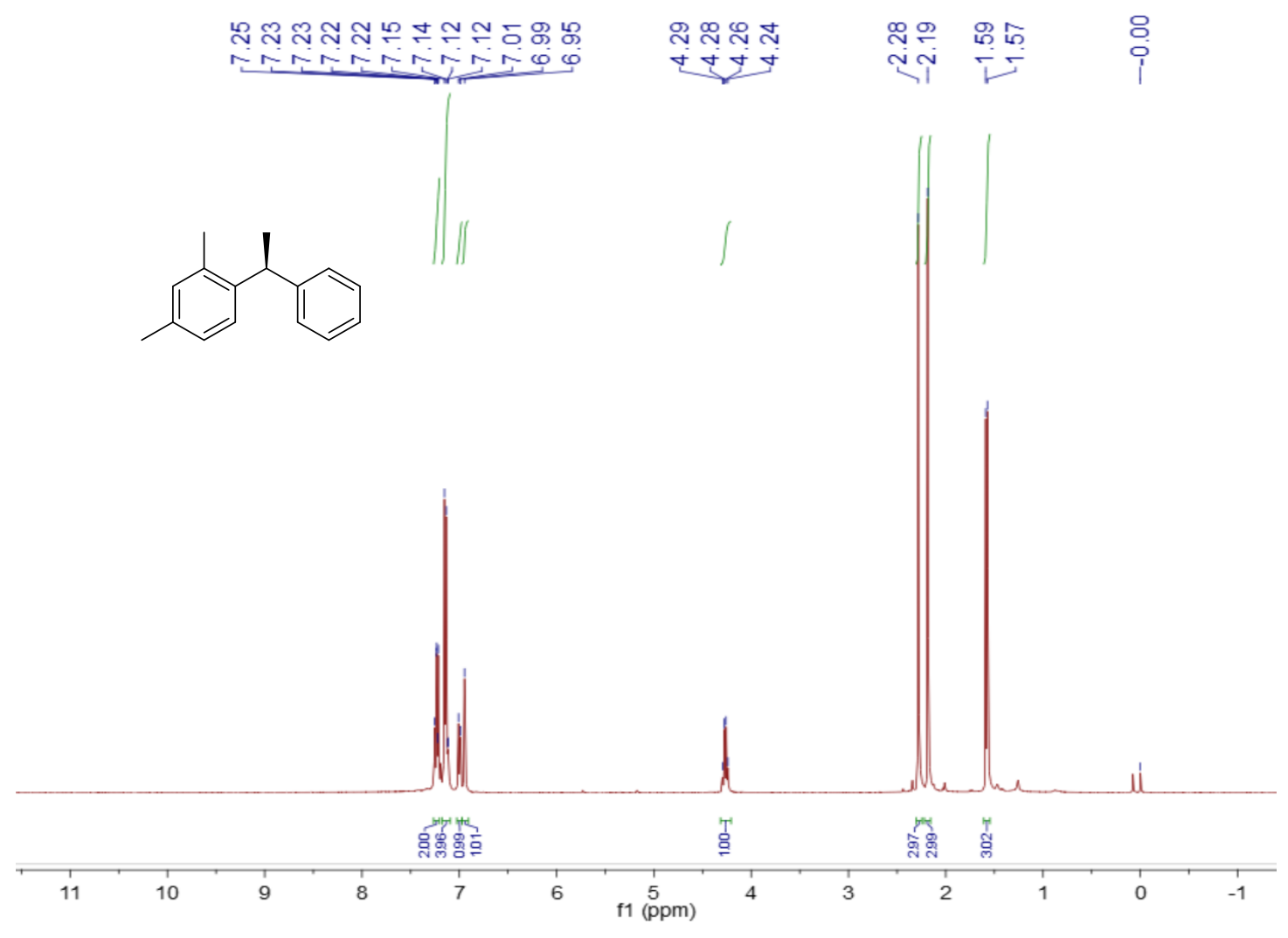

${ }^{1} \mathrm{H}$ NMR (400M, $\mathrm{CDCl}_{3}$ ) spectrum of $\mathbf{8 m}$ 
<smiles>Cc1ccc(C(C)c2ccccc2)c(C)c1</smiles>

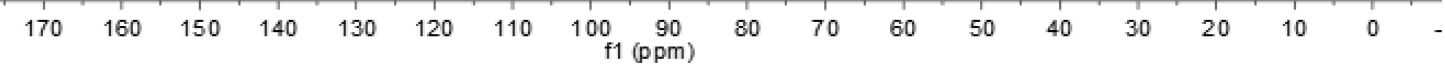

${ }^{13} \mathrm{C}$ NMR $\left(101 \mathrm{M}, \mathrm{CDCl}_{3}\right)$ spectrum of $\mathbf{8 m}$

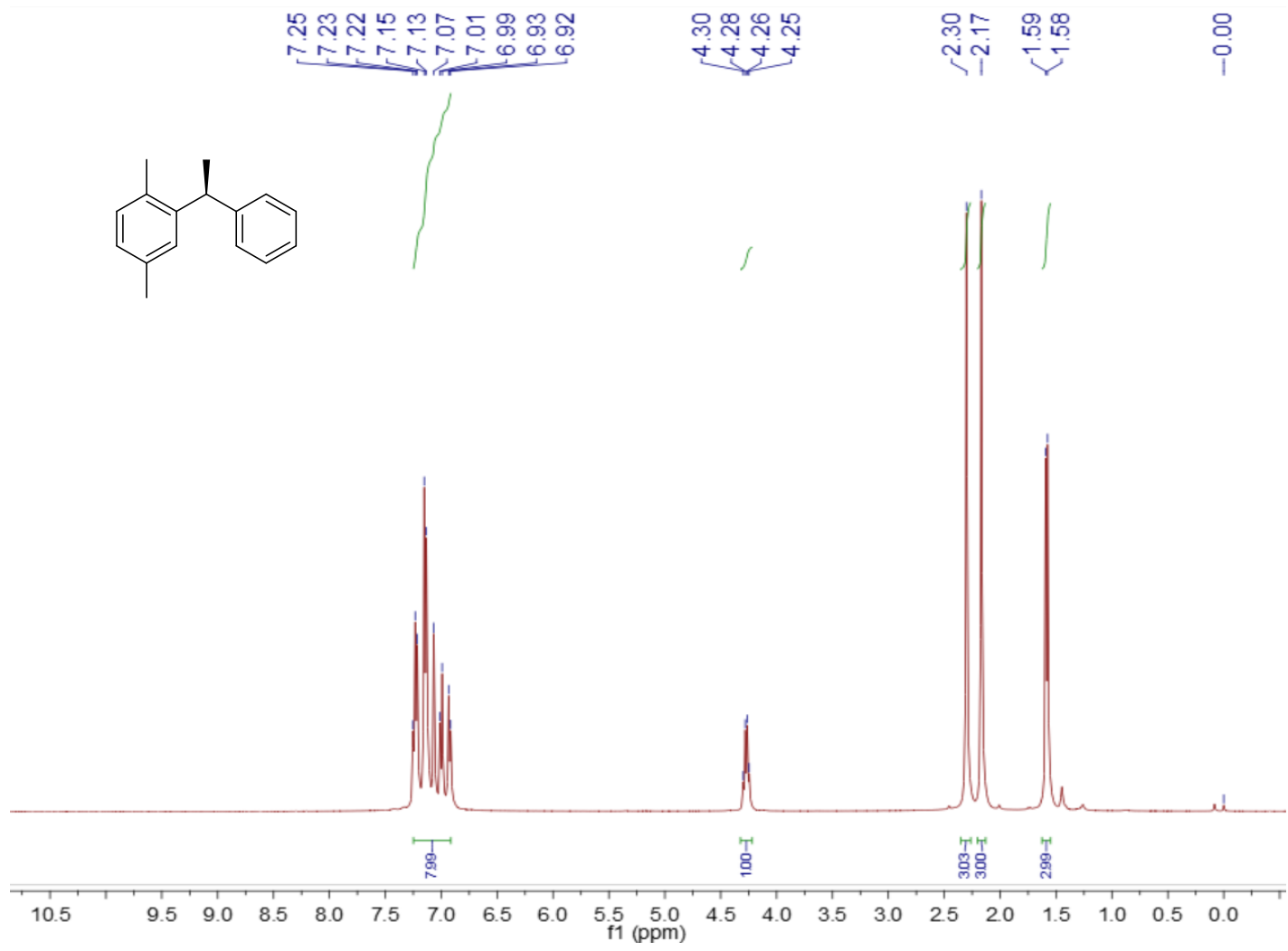

${ }^{1} \mathrm{H}$ NMR (400M, $\mathrm{CDCl}_{3}$ ) spectrum of $\mathbf{8 n}$ 
<smiles>Cc1ccc(C)c(C(C)c2ccccc2)c1</smiles>
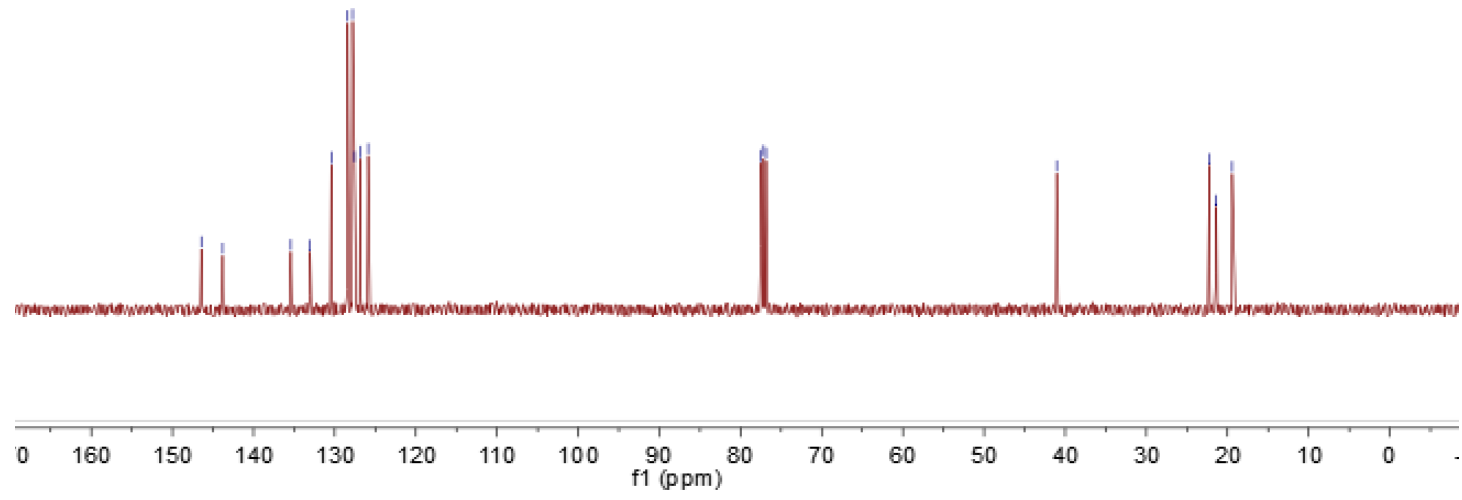

${ }^{13} \mathrm{C}$ NMR $\left(101 \mathrm{M}, \mathrm{CDCl}_{3}\right)$ spectrum of $\mathbf{8 n}$

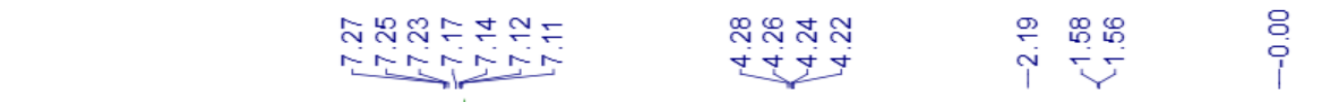<smiles>Cc1cc(Cl)ccc1C(C)c1ccccc1</smiles>
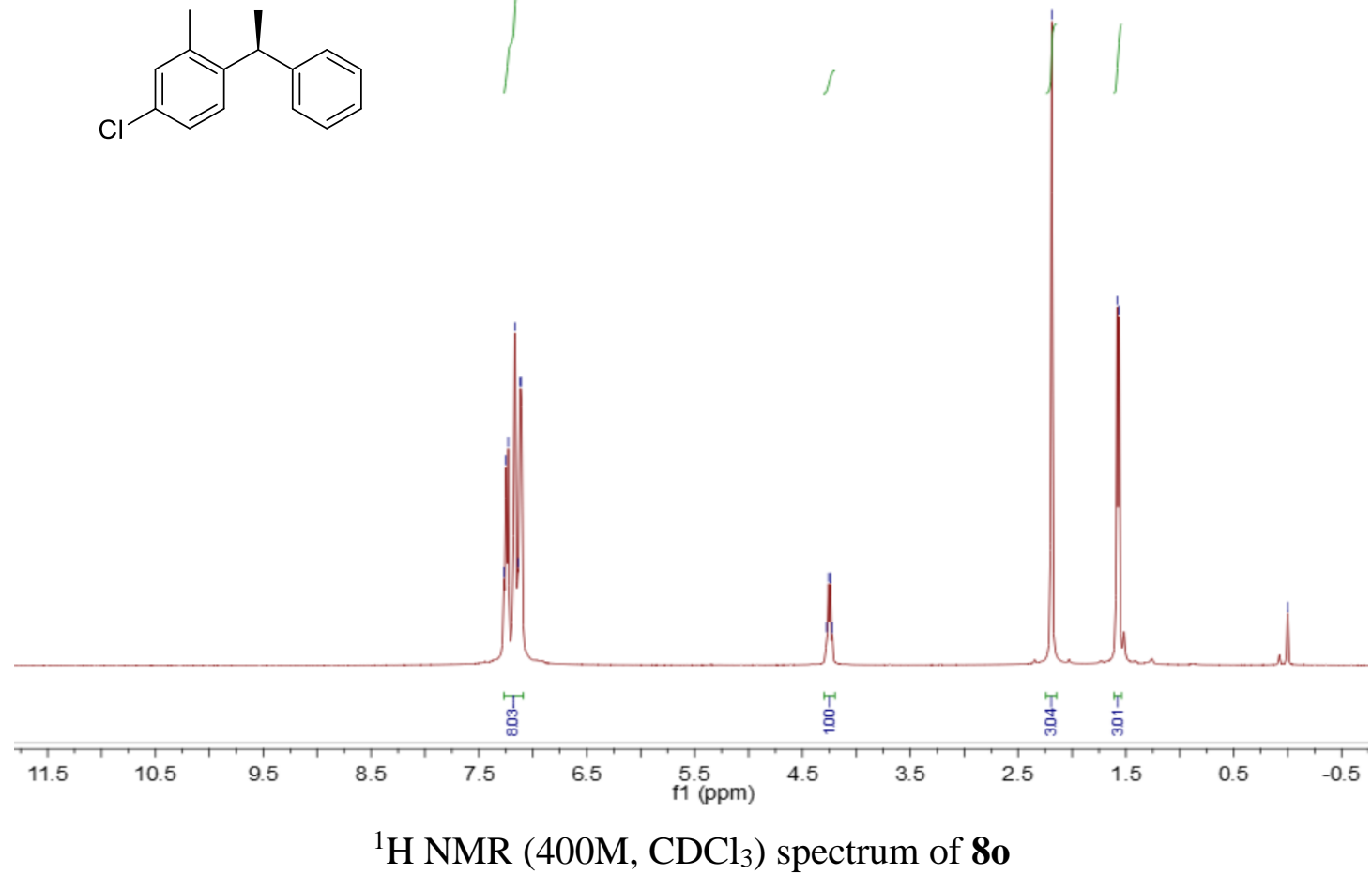

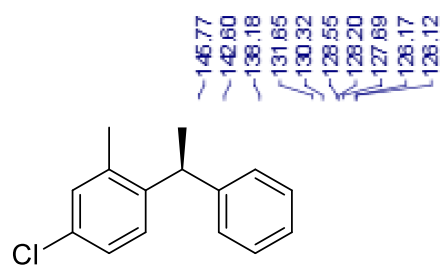

些弯

章

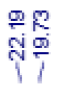
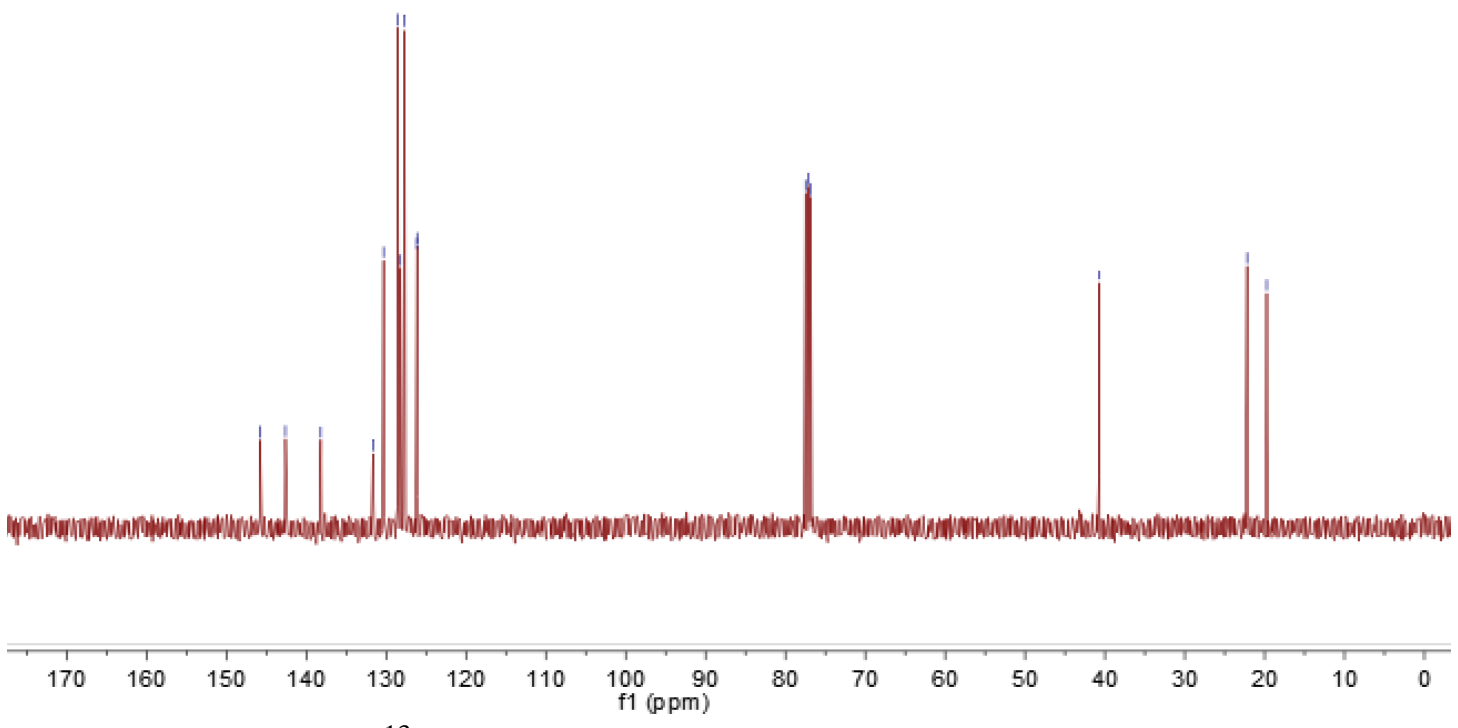

${ }^{13} \mathrm{C}$ NMR $\left(101 \mathrm{M}, \mathrm{CDCl}_{3}\right)$ spectrum of 80

$\sqrt{3}$
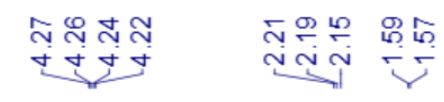

$\stackrel{\circ}{i}$
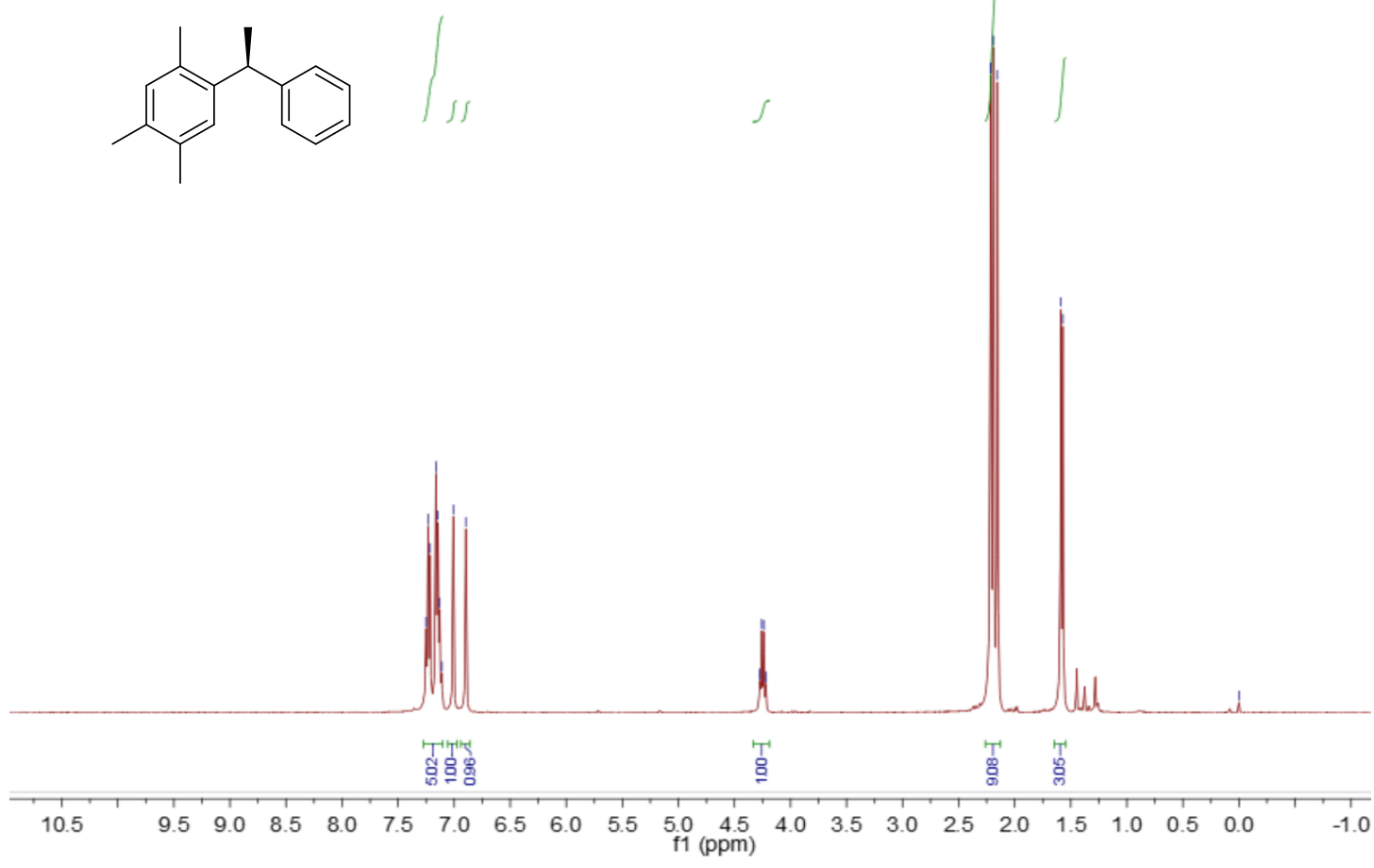

${ }^{1} \mathrm{H}$ NMR (400M, $\mathrm{CDCl}_{3}$ ) spectrum of $\mathbf{8 p}$

S125 
<smiles>Cc1cc(C)c(C(C)c2ccccc2)cc1C</smiles>

$\begin{array}{lllllllllllllllll}60 & 150 & 140 & 130 & 120 & 110 & 100 & 90 & \begin{array}{c}80 \\ \mathrm{f} 1\end{array}(\mathrm{ppm}) & 70 & 60 & 50 & 40 & 30 & 20 & 10 & 0\end{array}$

${ }^{13} \mathrm{C} \mathrm{NMR}\left(101 \mathrm{M}, \mathrm{CDCl}_{3}\right)$ spectrum of $\mathbf{8 p}$

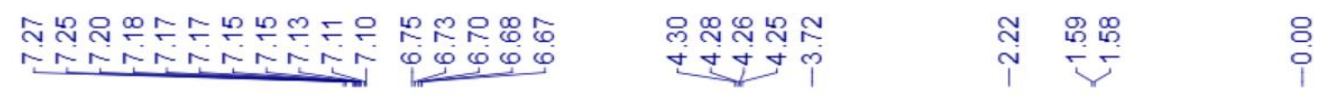

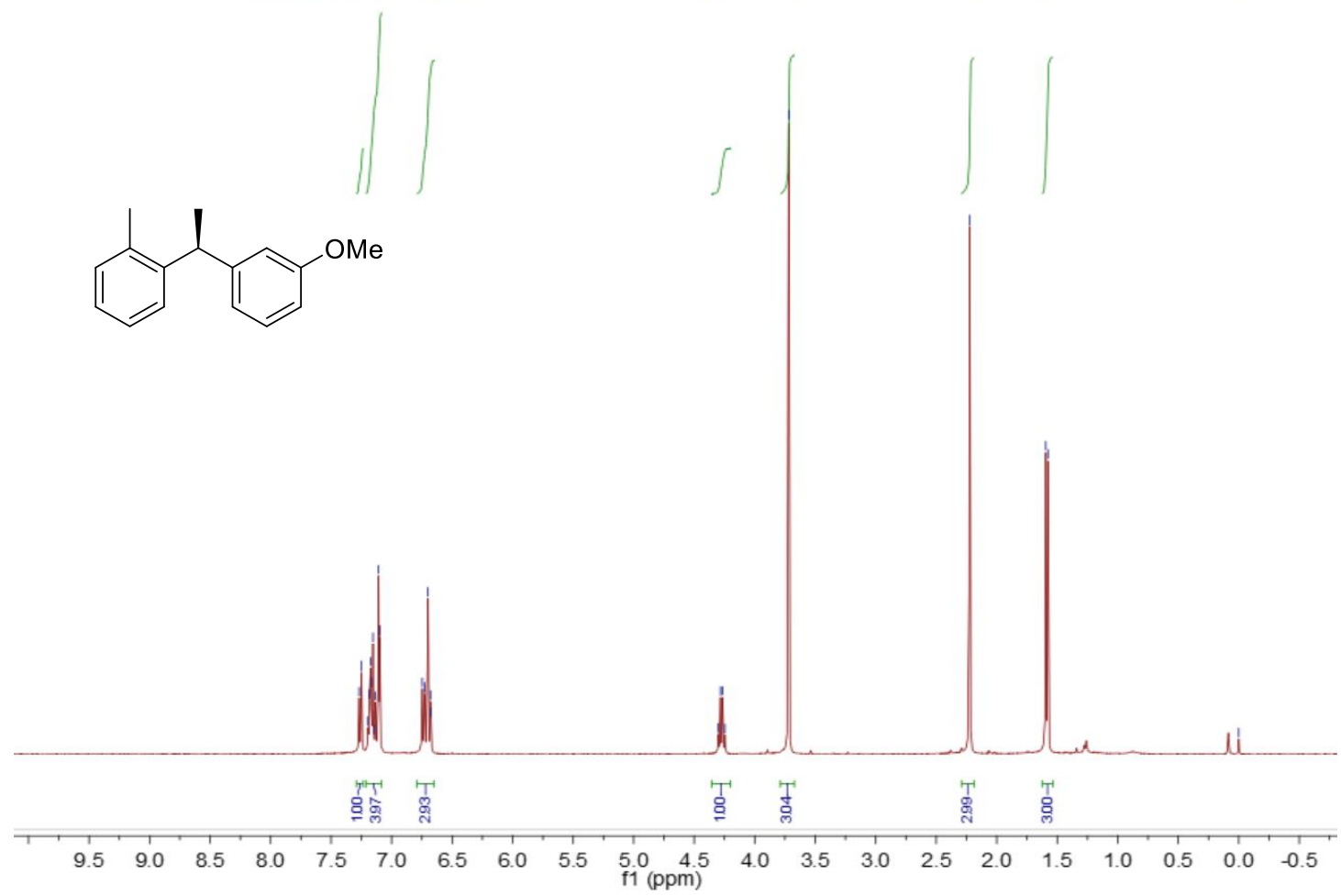

${ }^{1} \mathrm{H}$ NMR $\left(400 \mathrm{M}, \mathrm{CDCl}_{3}\right)$ spectrum of $\mathbf{8 q}$ 


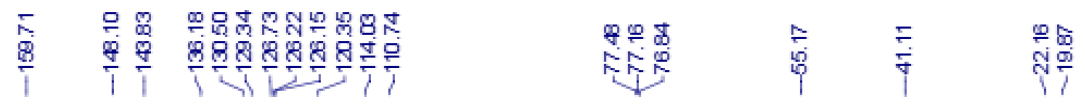<smiles>COc1cccc(C(C)c2ccccc2C)c1</smiles>
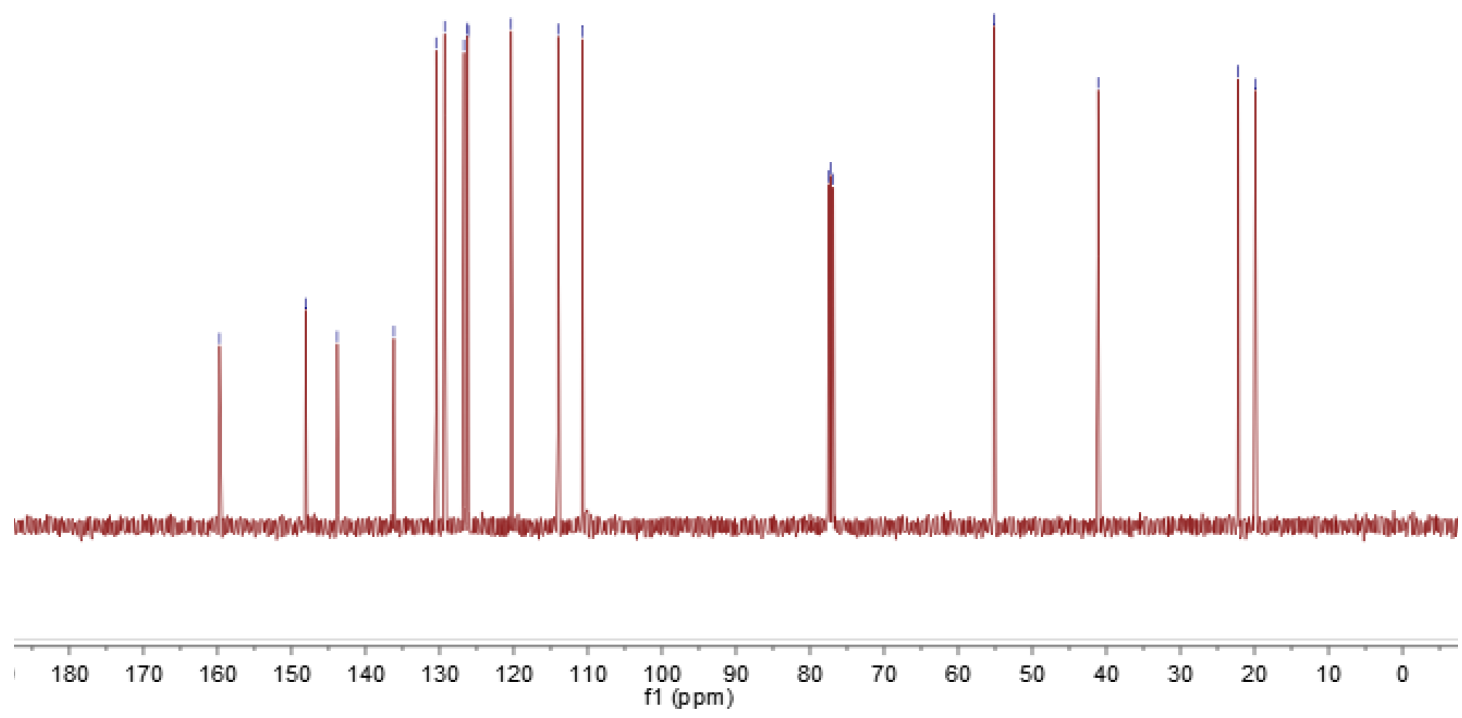

${ }^{13} \mathrm{C}$ NMR (101M, $\left.\mathrm{CDCl}_{3}\right)$ spectrum of $\mathbf{8 q}$

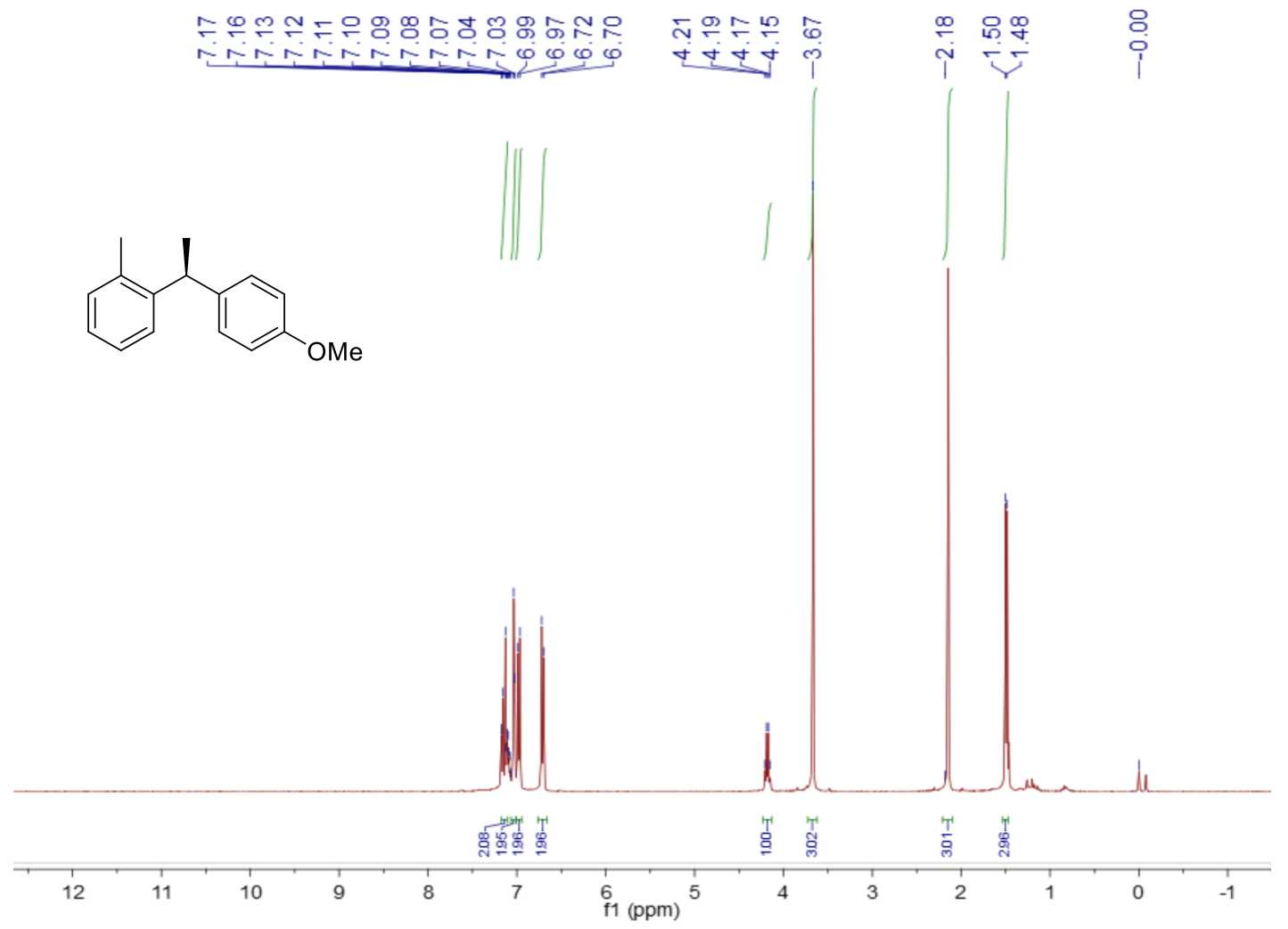

${ }^{1} \mathrm{H}$ NMR (400M, $\mathrm{CDCl}_{3}$ ) spectrum of $\mathbf{8 r}$ 


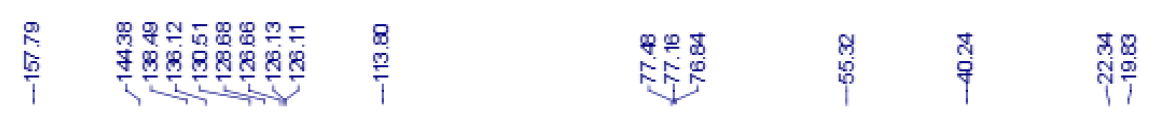
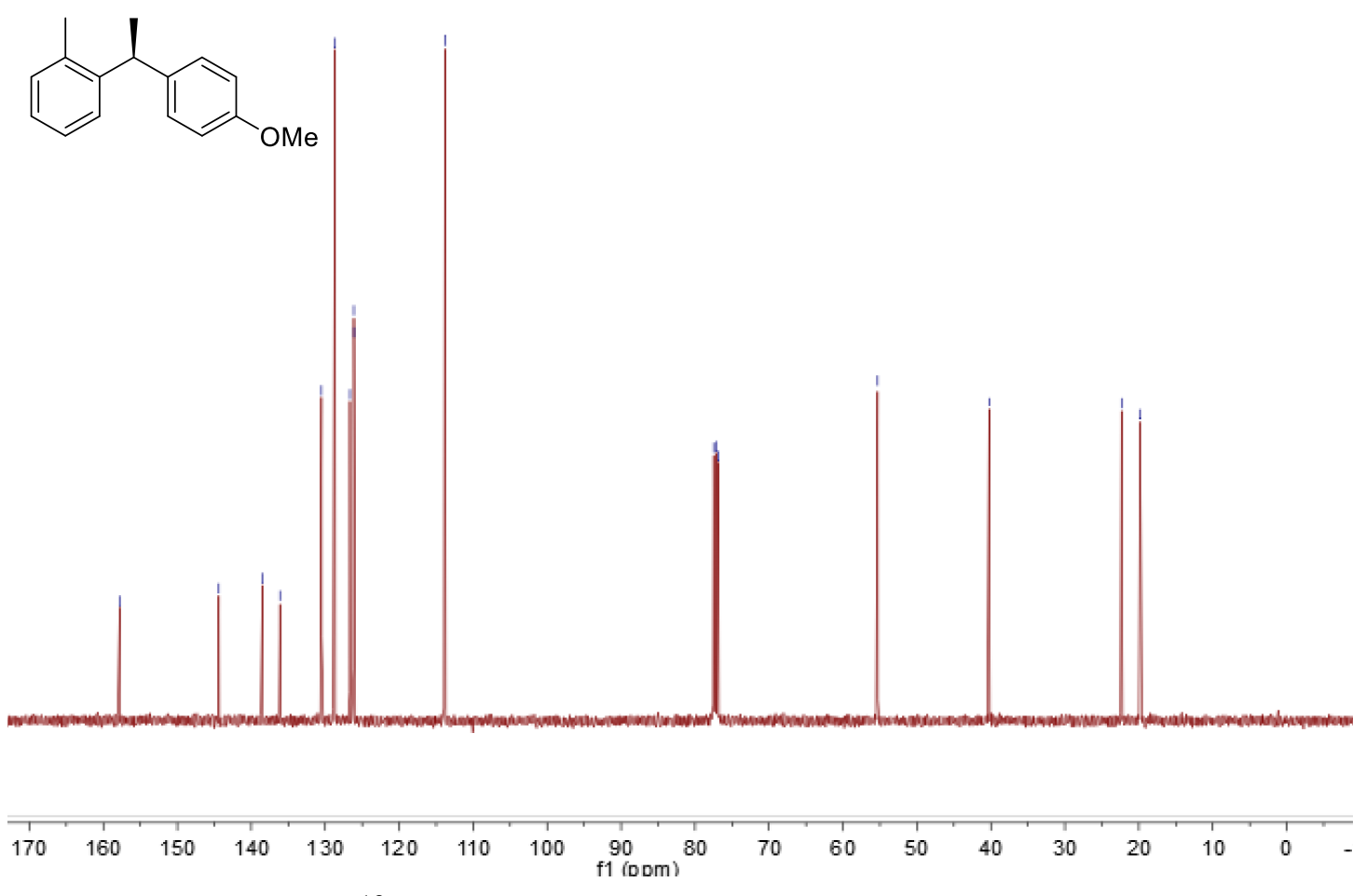

${ }^{13} \mathrm{C}$ NMR $\left(101 \mathrm{M}, \mathrm{CDCl}_{3}\right)$ spectrum of $\mathbf{8 r}$

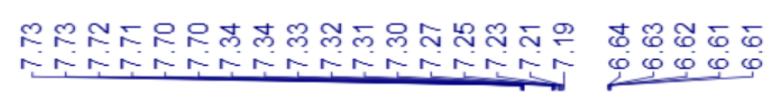
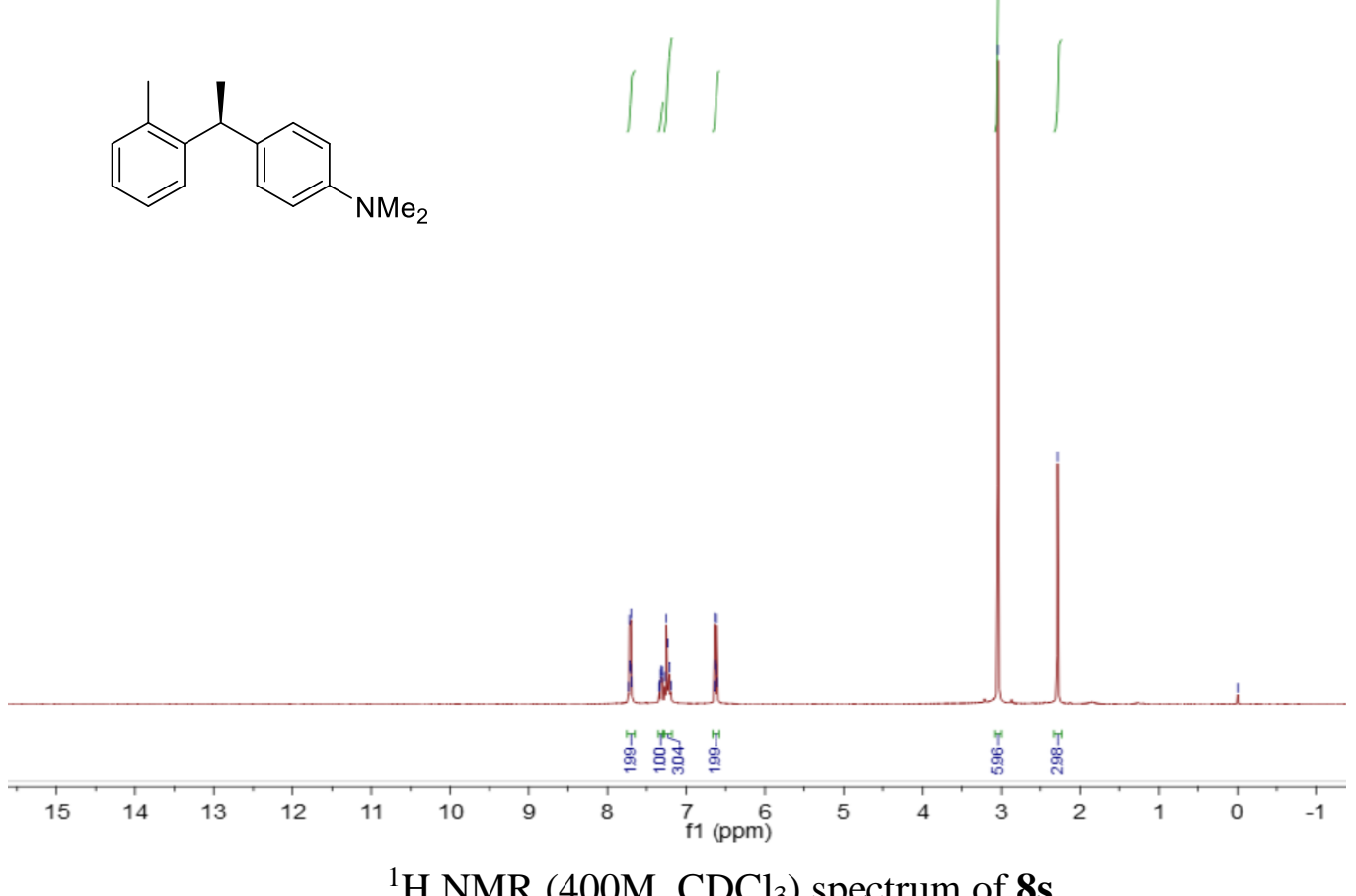

${ }^{1} \mathrm{H}$ NMR (400M, $\mathrm{CDCl}_{3}$ ) spectrum of $\mathbf{8 s}$

S128 

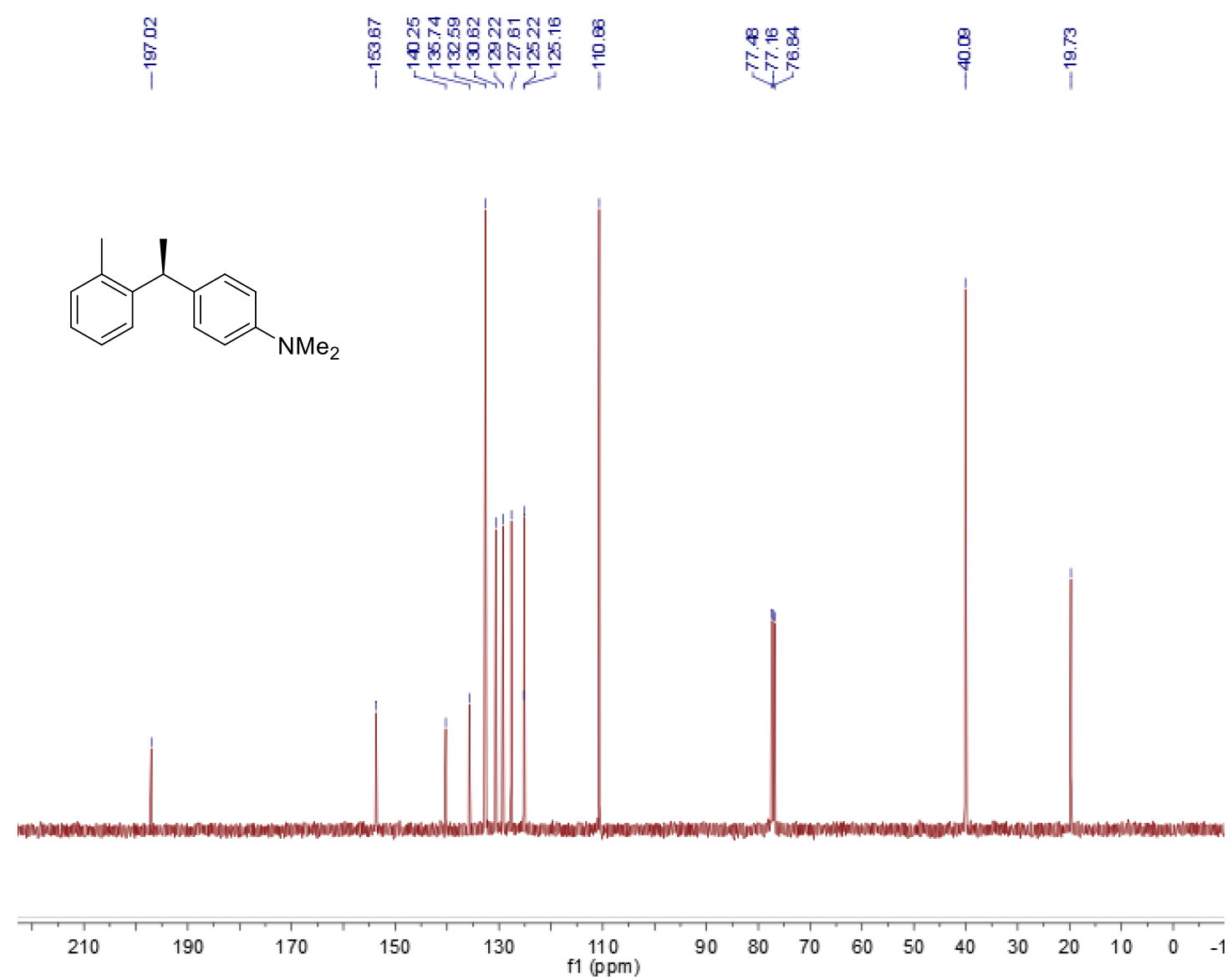

${ }^{13} \mathrm{C}$ NMR $\left(101 \mathrm{M}, \mathrm{CDCl}_{3}\right)$ spectrum of $\mathbf{8 s}$

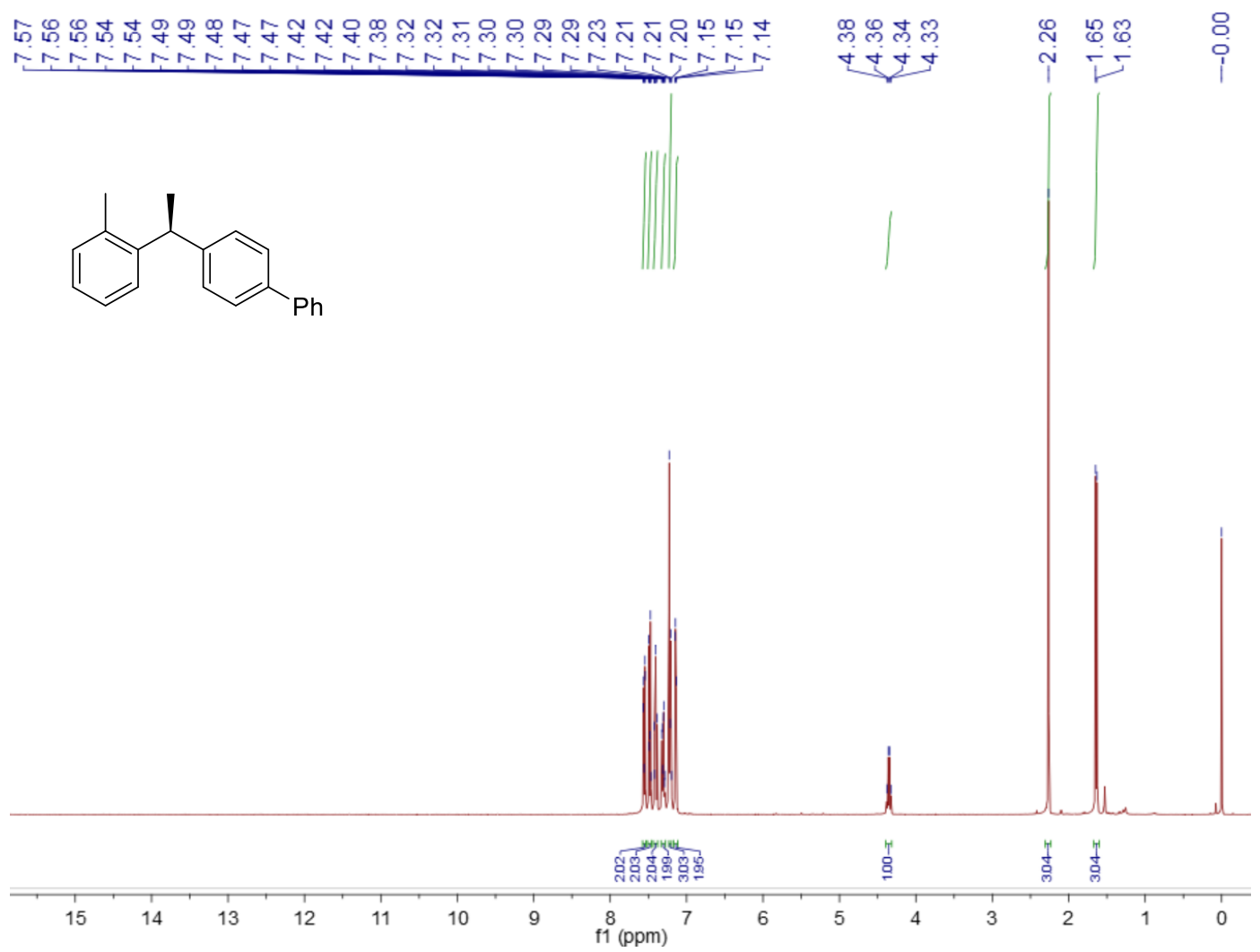


${ }^{1} \mathrm{H}$ NMR (400M, $\left.\mathrm{CDCl}_{3}\right)$ spectrum of $\mathbf{8 t}$

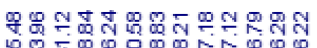

守年

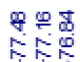

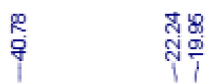

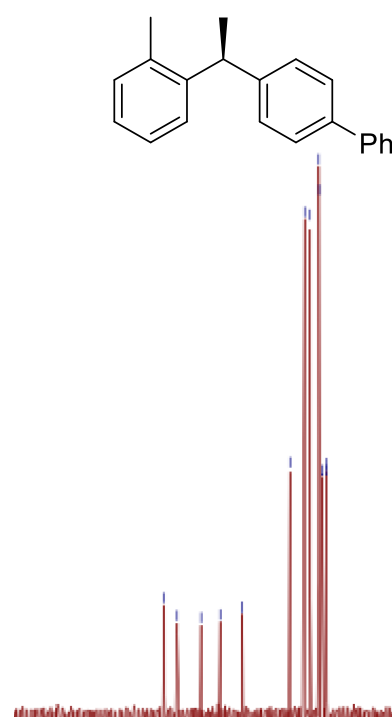

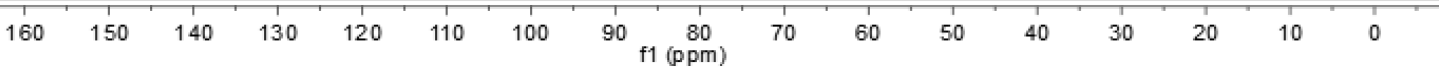

${ }^{13} \mathrm{C} \mathrm{NMR}\left(101 \mathrm{M}, \mathrm{CDCl}_{3}\right)$ spectrum of $\mathbf{8 t}$

\begin{tabular}{|c|c|}
\hline 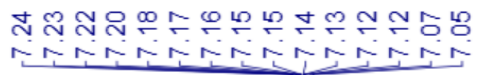 & 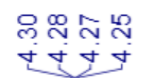 \\
\hline
\end{tabular}<smiles>Cc1ccccc1C(C)c1ccc(Cl)c(I)c1</smiles>

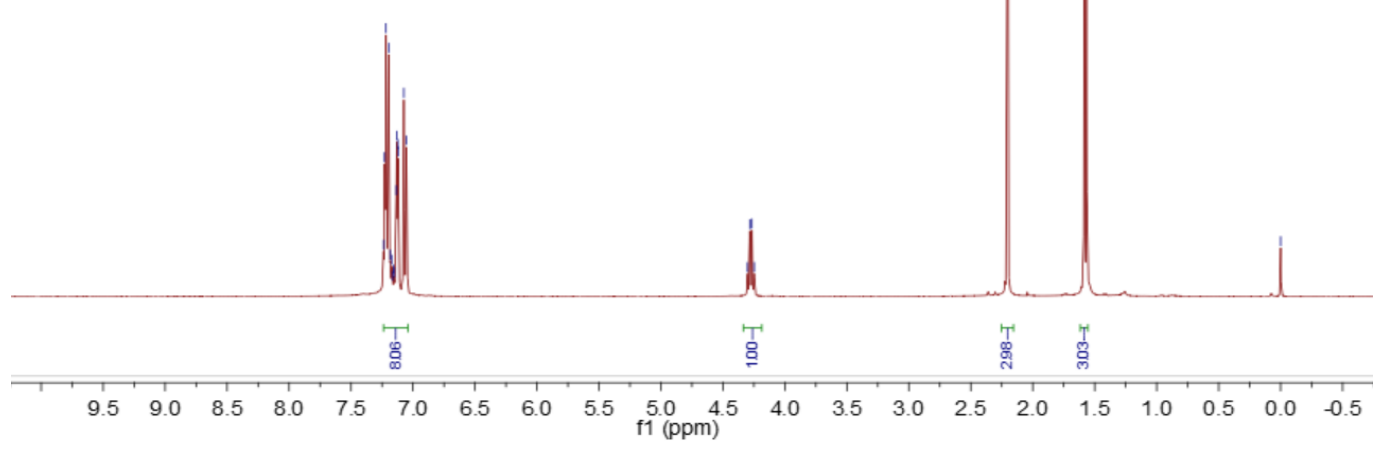

${ }^{1} \mathrm{H}$ NMR (400M, $\mathrm{CDCl}_{3}$ ) spectrum of $\mathbf{8 u}$ 

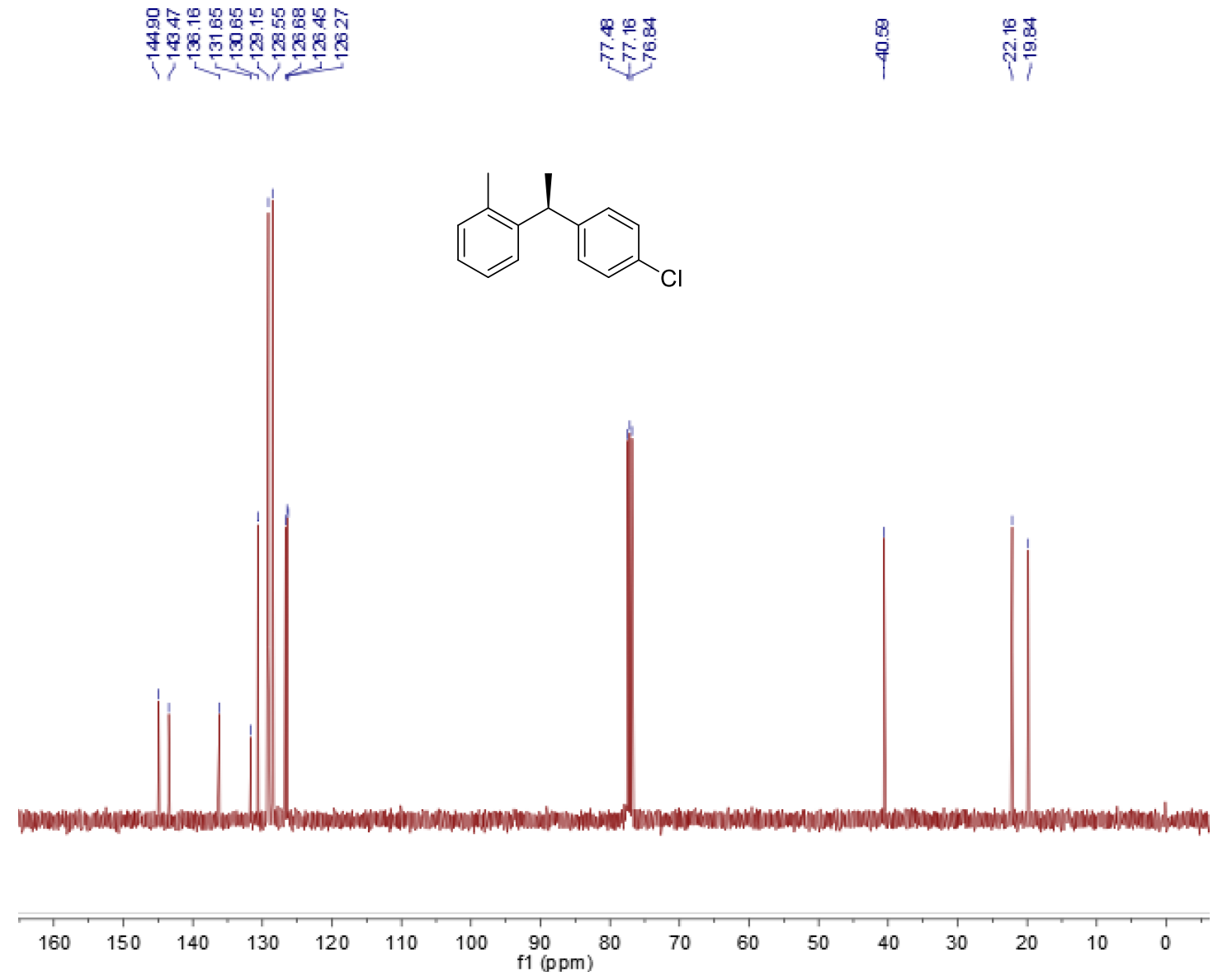

${ }^{13} \mathrm{C}$ NMR $\left(101 \mathrm{M}, \mathrm{CDCl}_{3}\right)$ spectrum of $\mathbf{8 u}$
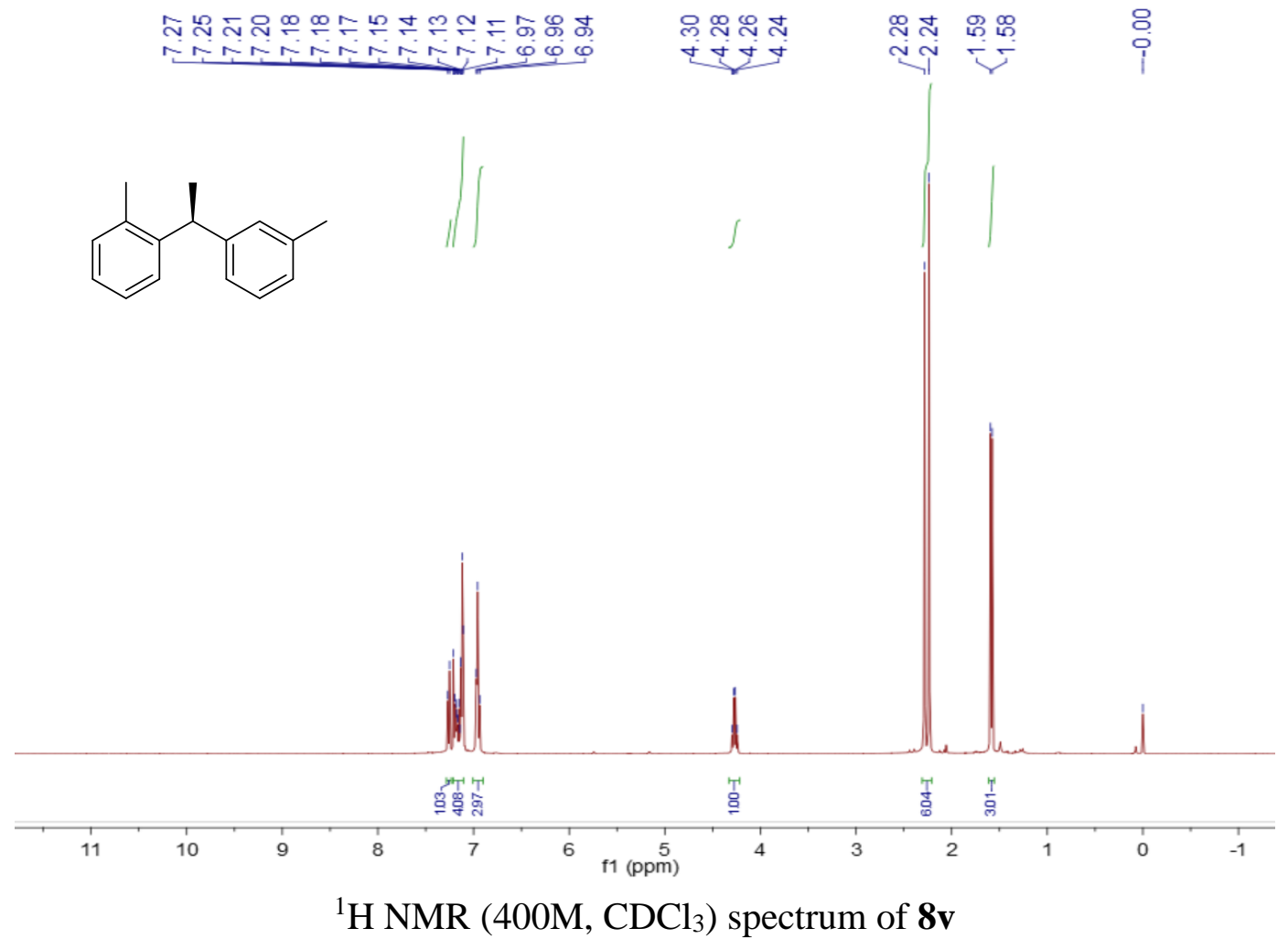

S131 


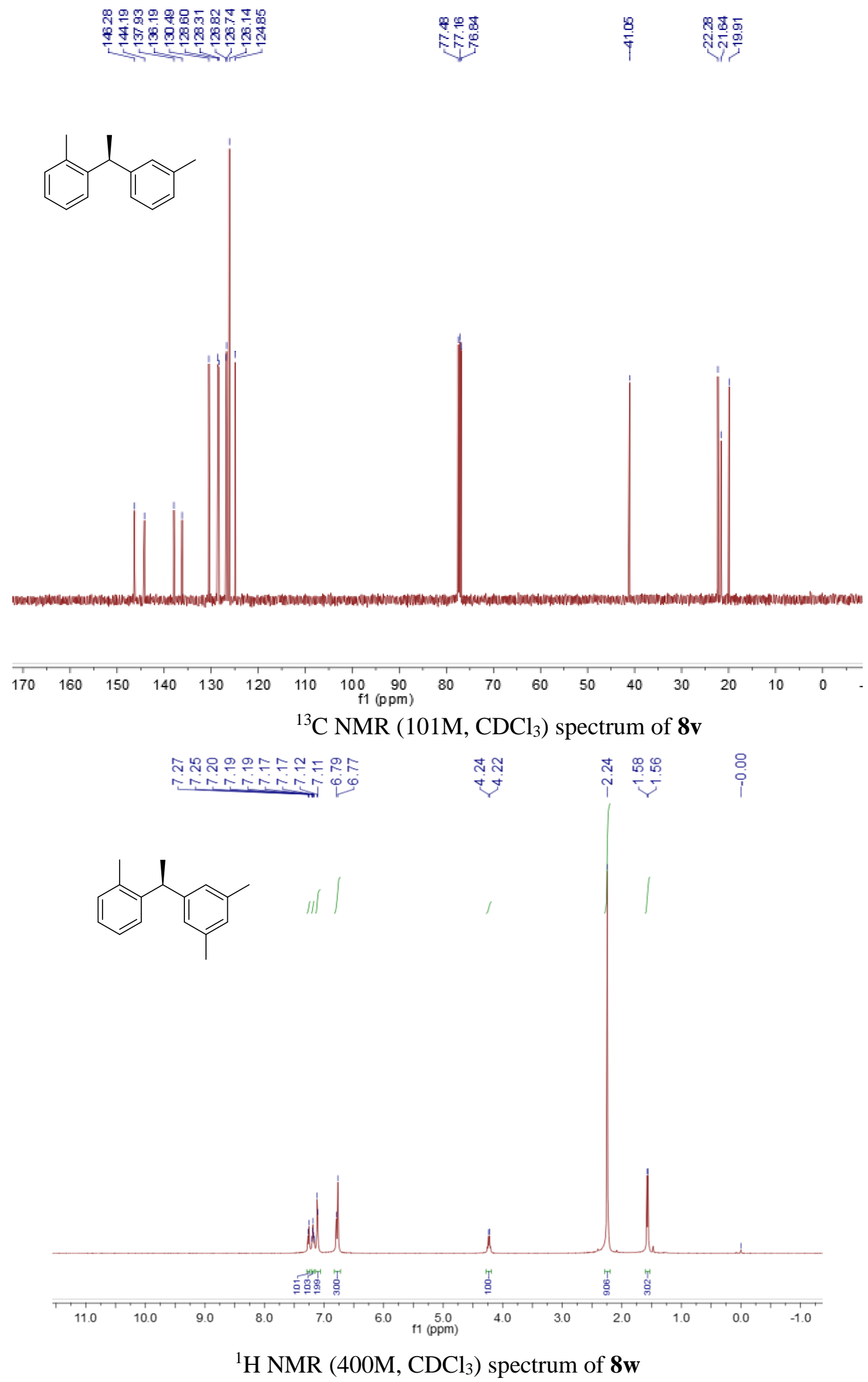



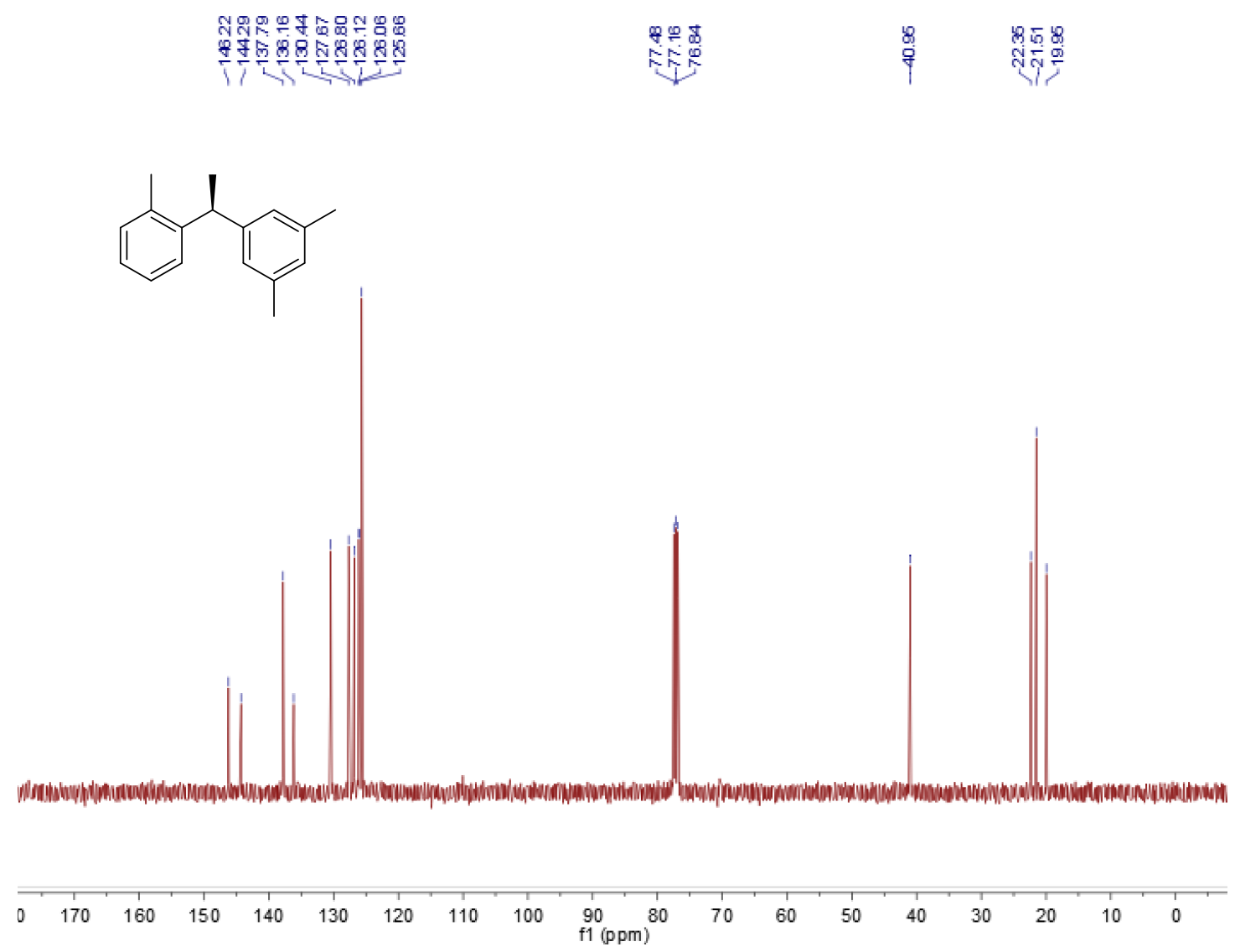

${ }^{13} \mathrm{C}$ NMR $\left(101 \mathrm{M}, \mathrm{CDCl}_{3}\right)$ spectrum of $\mathbf{8 w}$

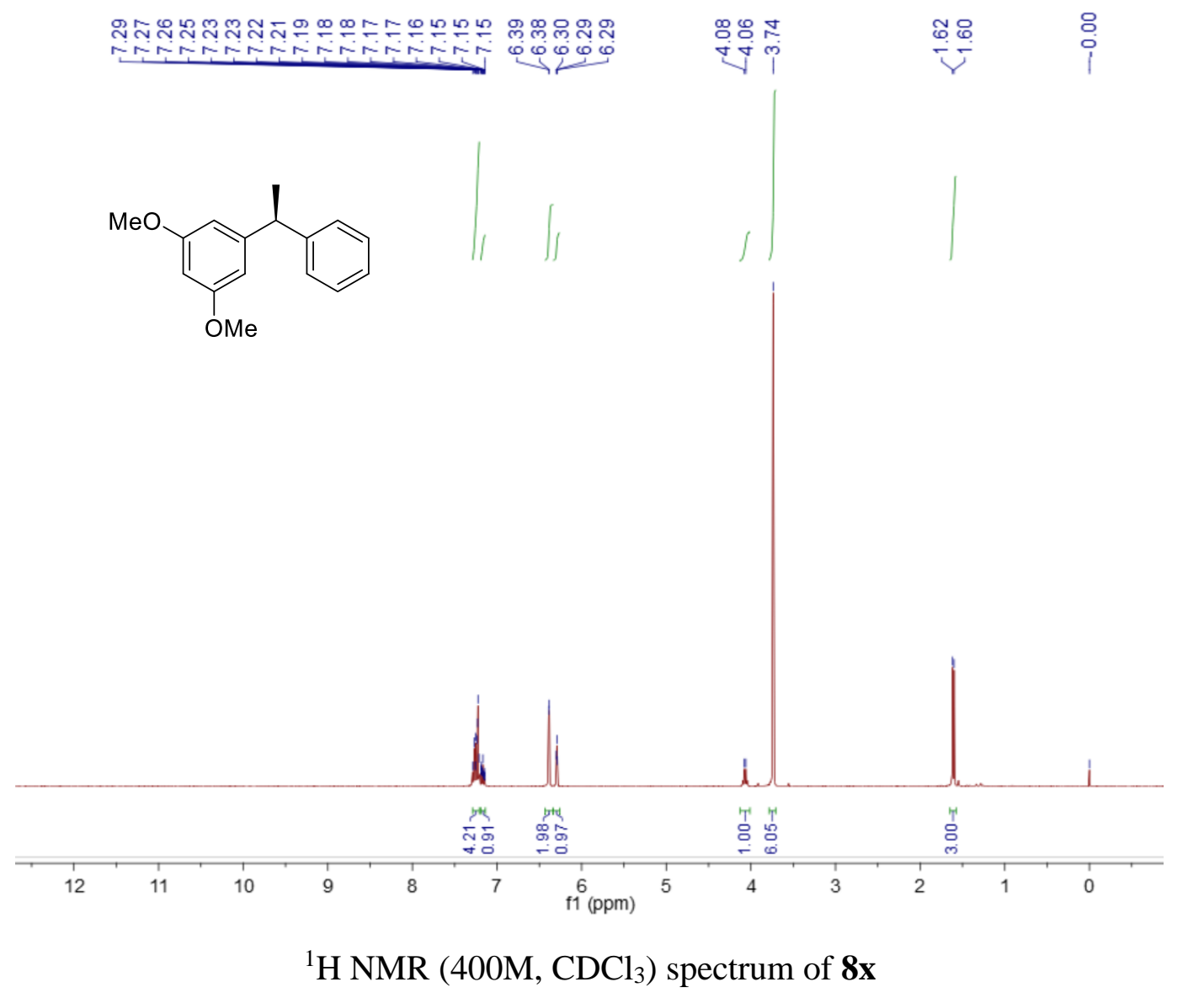




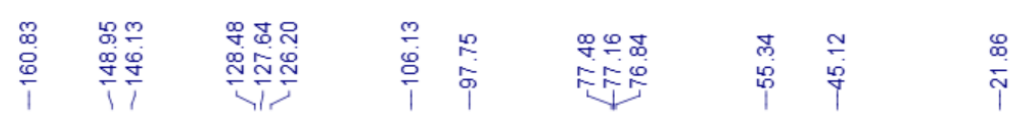
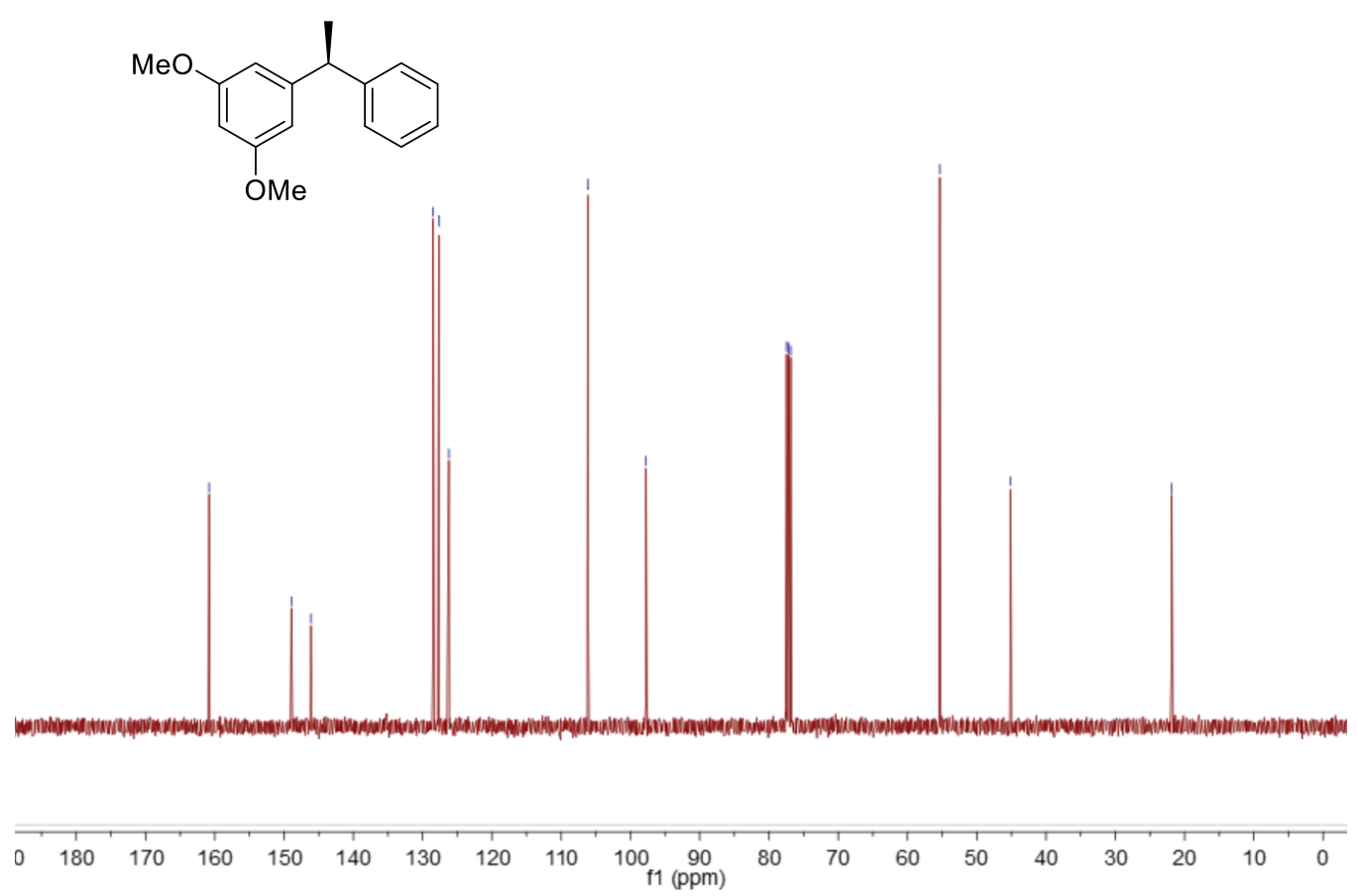

${ }^{13} \mathrm{C}$ NMR $\left(101 \mathrm{M}, \mathrm{CDCl}_{3}\right)$ spectrum of $\mathbf{8 x}$
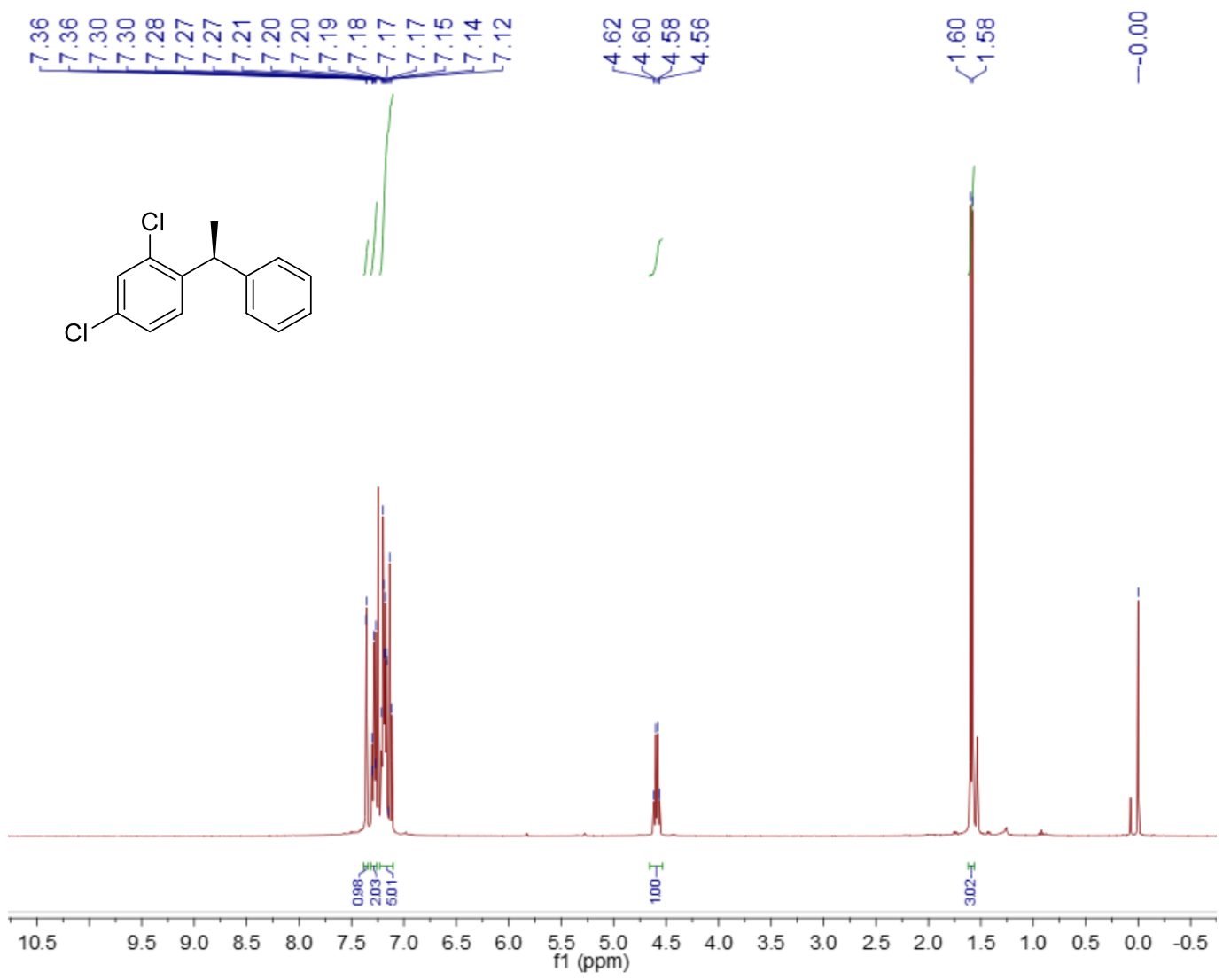

${ }^{1} \mathrm{H}$ NMR (400M, $\mathrm{CDCl}_{3}$ ) spectrum of $\mathbf{8 y}$

S134 
<smiles>CC(c1ccccc1)c1ccc(Cl)cc1Cl</smiles>
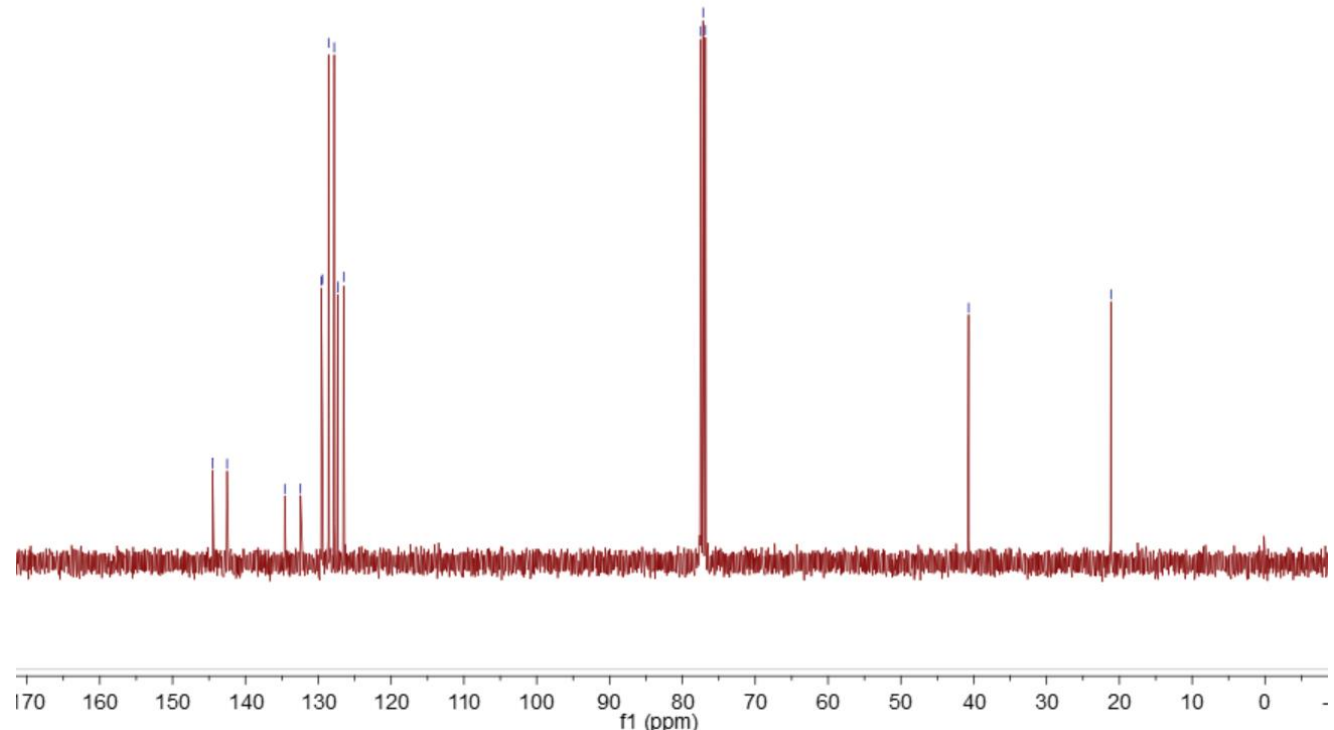

${ }^{13} \mathrm{C} \mathrm{NMR}\left(101 \mathrm{M}, \mathrm{CDCl}_{3}\right)$ spectrum of $\mathbf{8 y}$

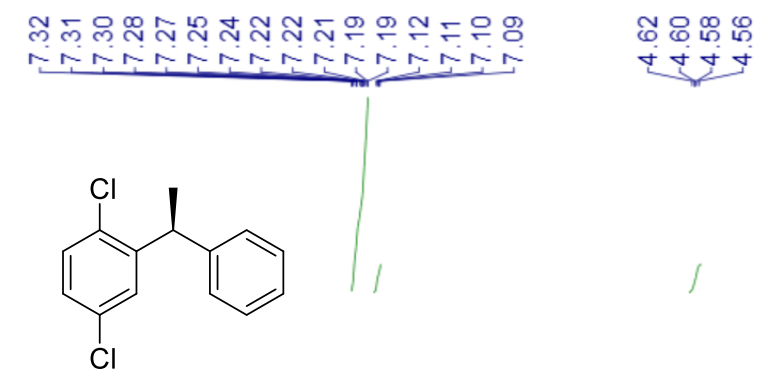

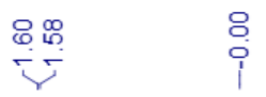

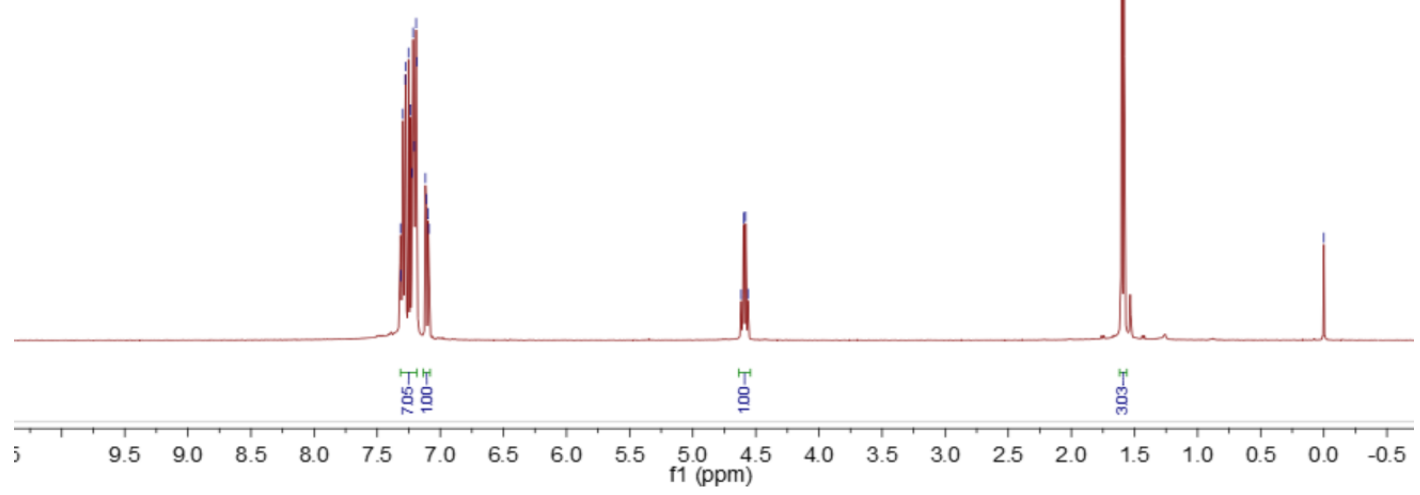

${ }^{1} \mathrm{H}$ NMR $\left(400 \mathrm{M}, \mathrm{CDCl}_{3}\right)$ spectrum of $\mathbf{8 z}$ 


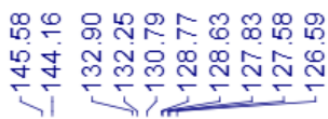

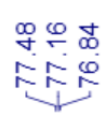

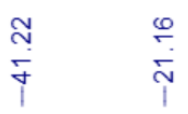
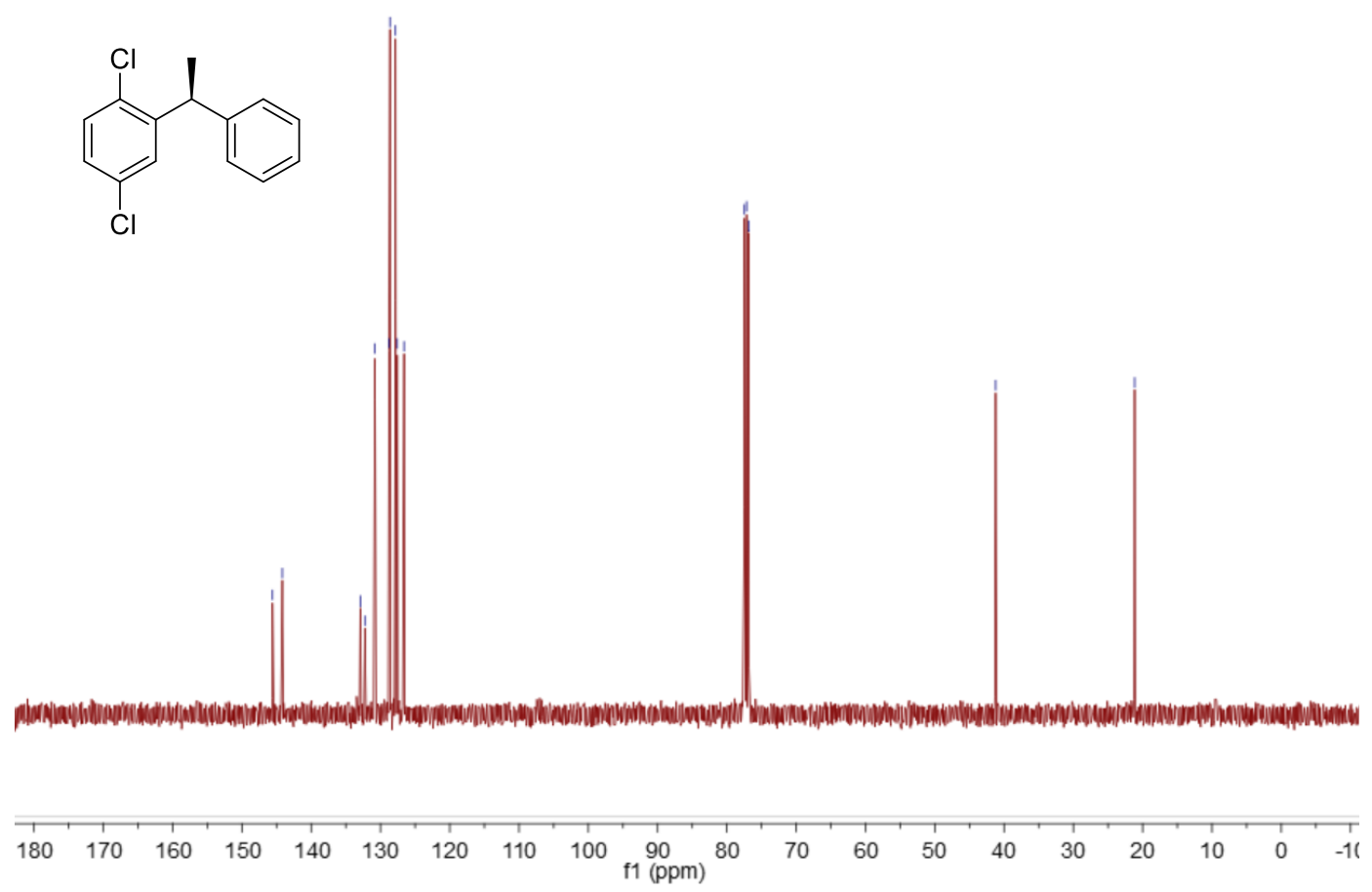

${ }^{13} \mathrm{C}$ NMR (101M, $\mathrm{CDCl}_{3}$ ) spectrum of $\mathbf{8 z}$

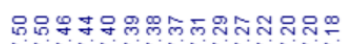

高皆昌

通

$\stackrel{\circ}{i}$
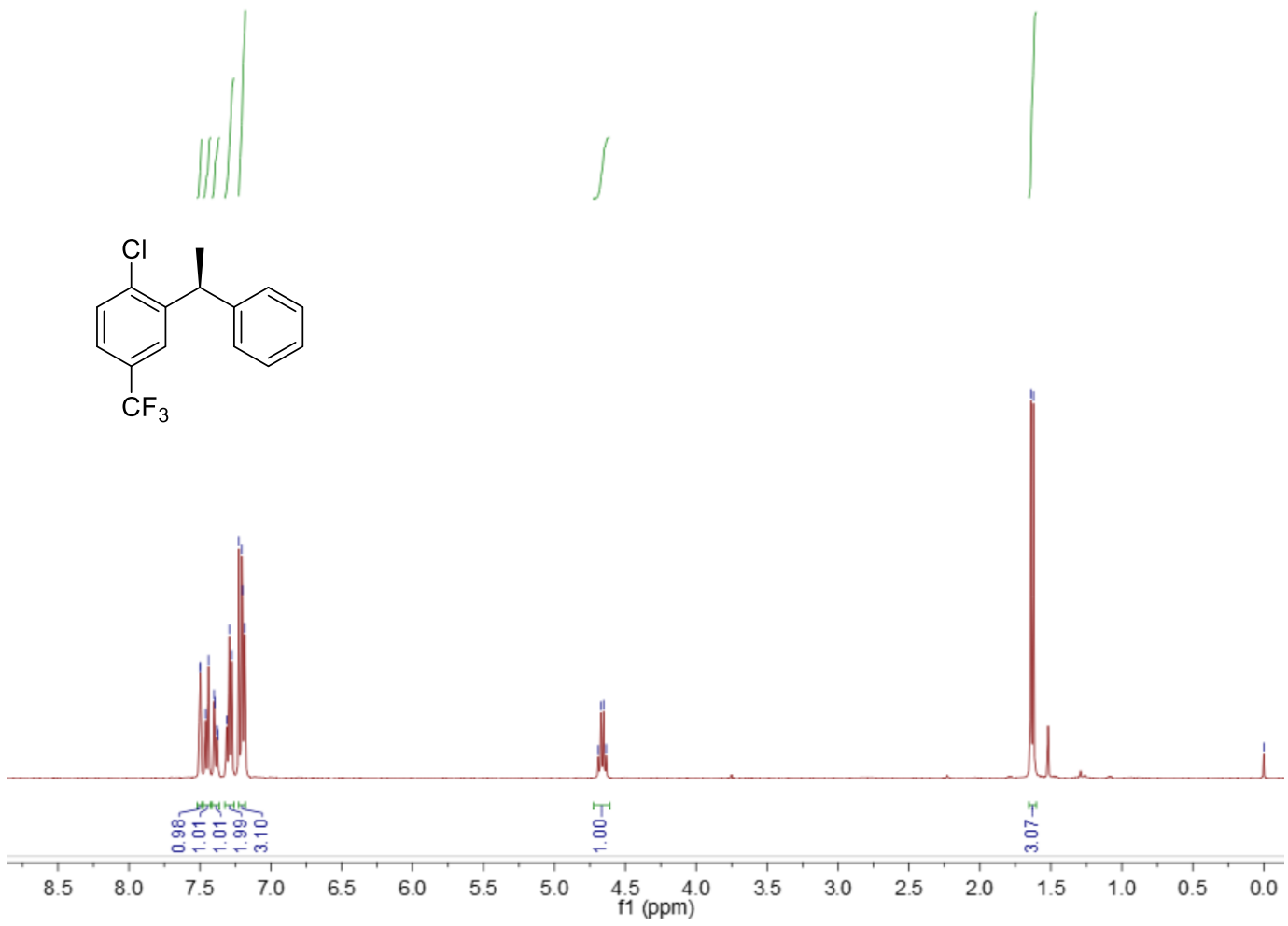

${ }^{1} \mathrm{H}$ NMR (400M, $\mathrm{CDCl}_{3}$ ) spectrum of $\mathbf{8 a b}$

S136 


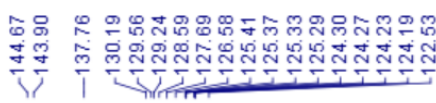

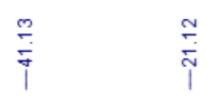
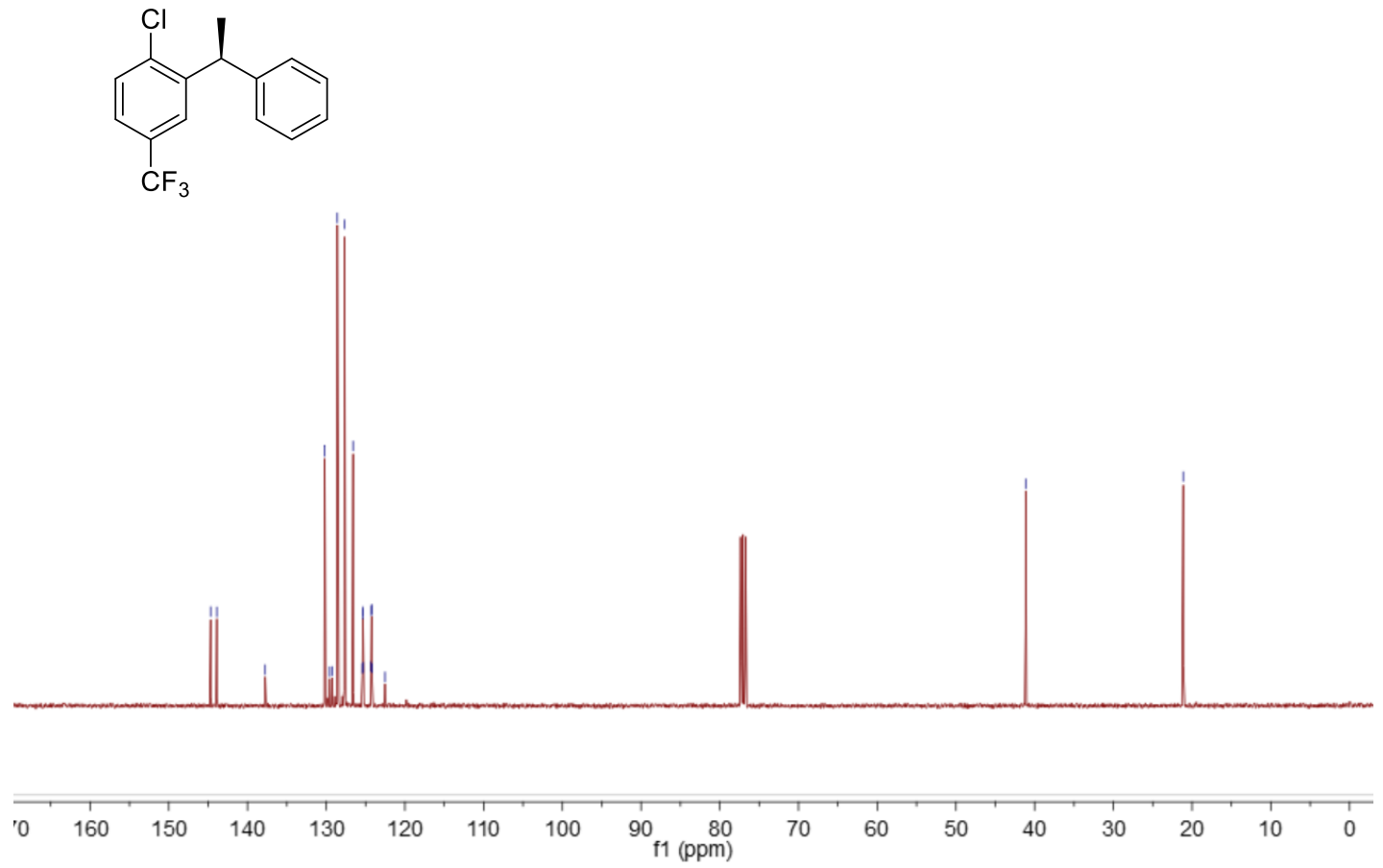

${ }^{13} \mathrm{C}$ NMR $\left(101 \mathrm{M}, \mathrm{CDCl}_{3}\right)$ spectrum of 8ab

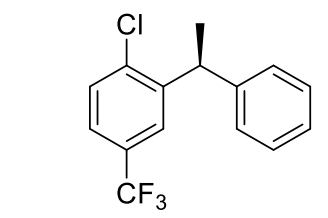

尔

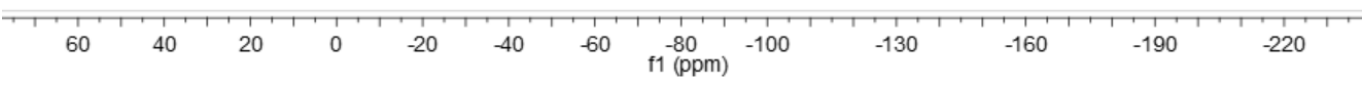

${ }^{19}$ F NMR (101M, $\left.\mathrm{CDCl}_{3}\right)$ spectrum of 8ab

S137 


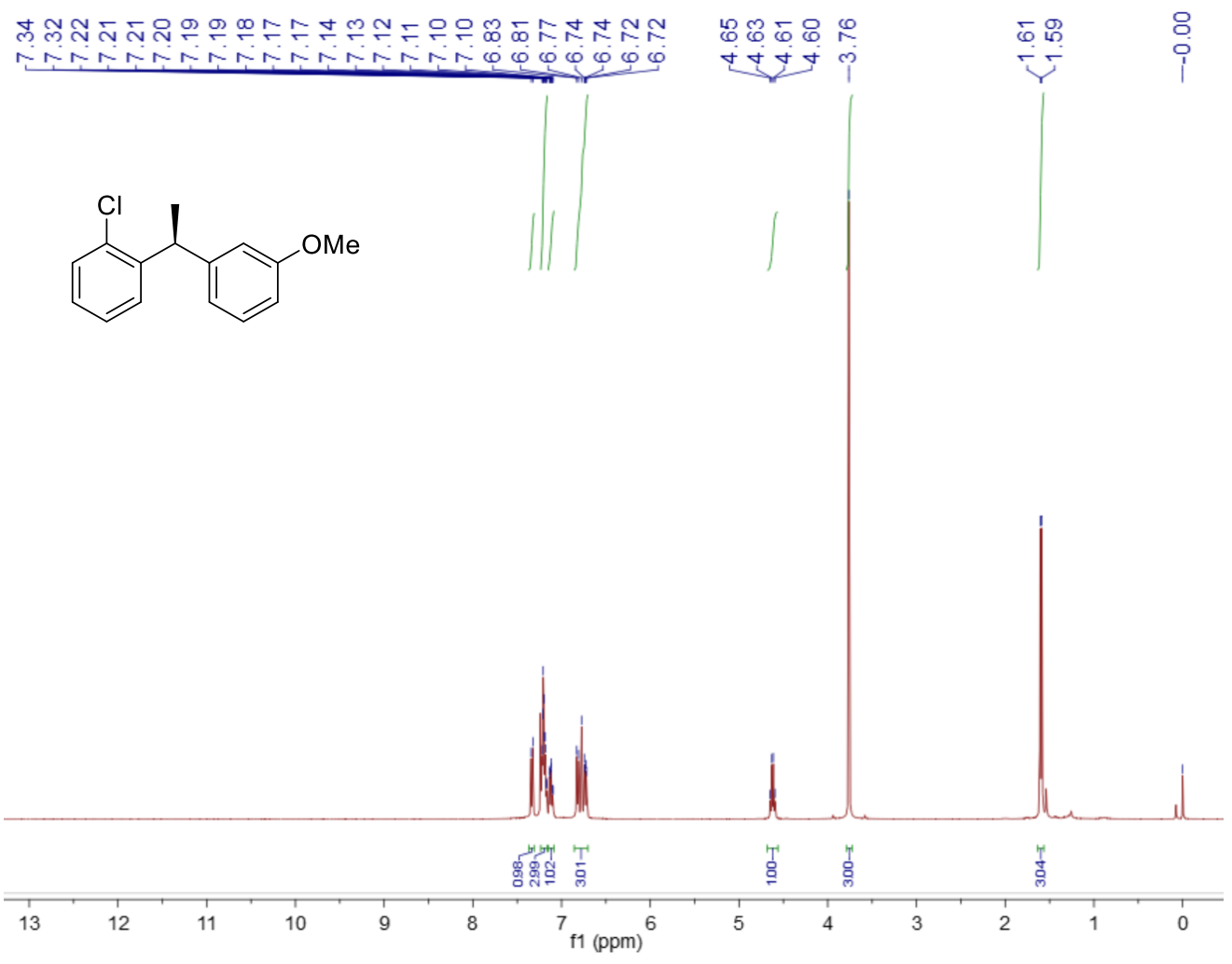

${ }^{1} \mathrm{H}$ NMR $\left(400 \mathrm{M}, \mathrm{CDCl}_{3}\right)$ spectrum of $\mathbf{8 a c}$

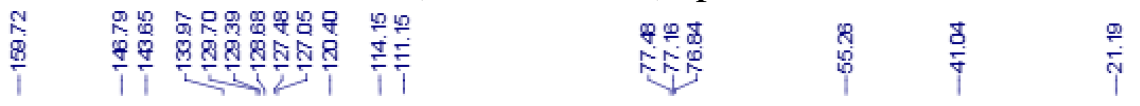<smiles>COc1cccc([C@H](C)c2ccccc2Cl)c1</smiles>

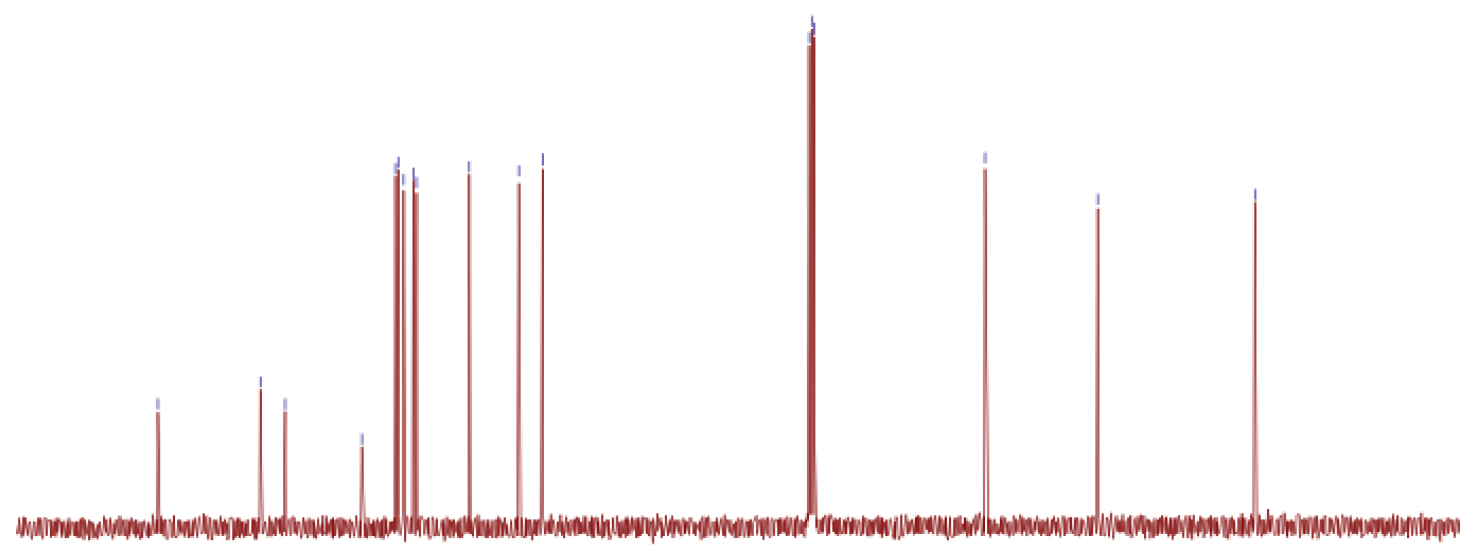

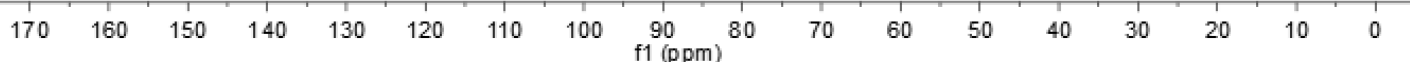

${ }^{13} \mathrm{C}$ NMR $\left(101 \mathrm{M}, \mathrm{CDCl}_{3}\right)$ spectrum of $\mathbf{8 a c}$ 

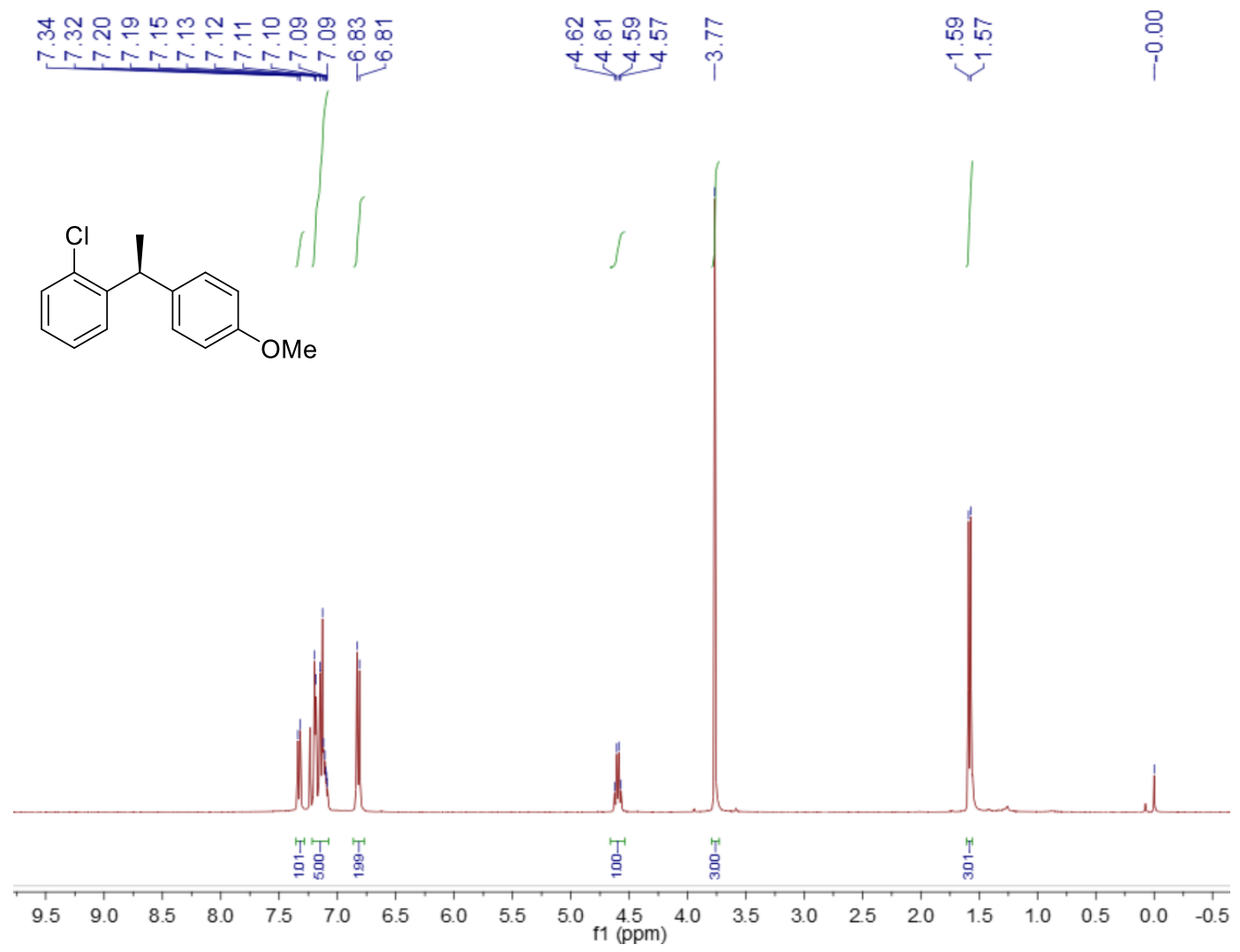

${ }^{1} \mathrm{H}$ NMR (400M, $\mathrm{CDCl}_{3}$ ) spectrum of $8 \mathbf{a d}$

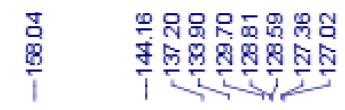

然尊 营

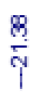
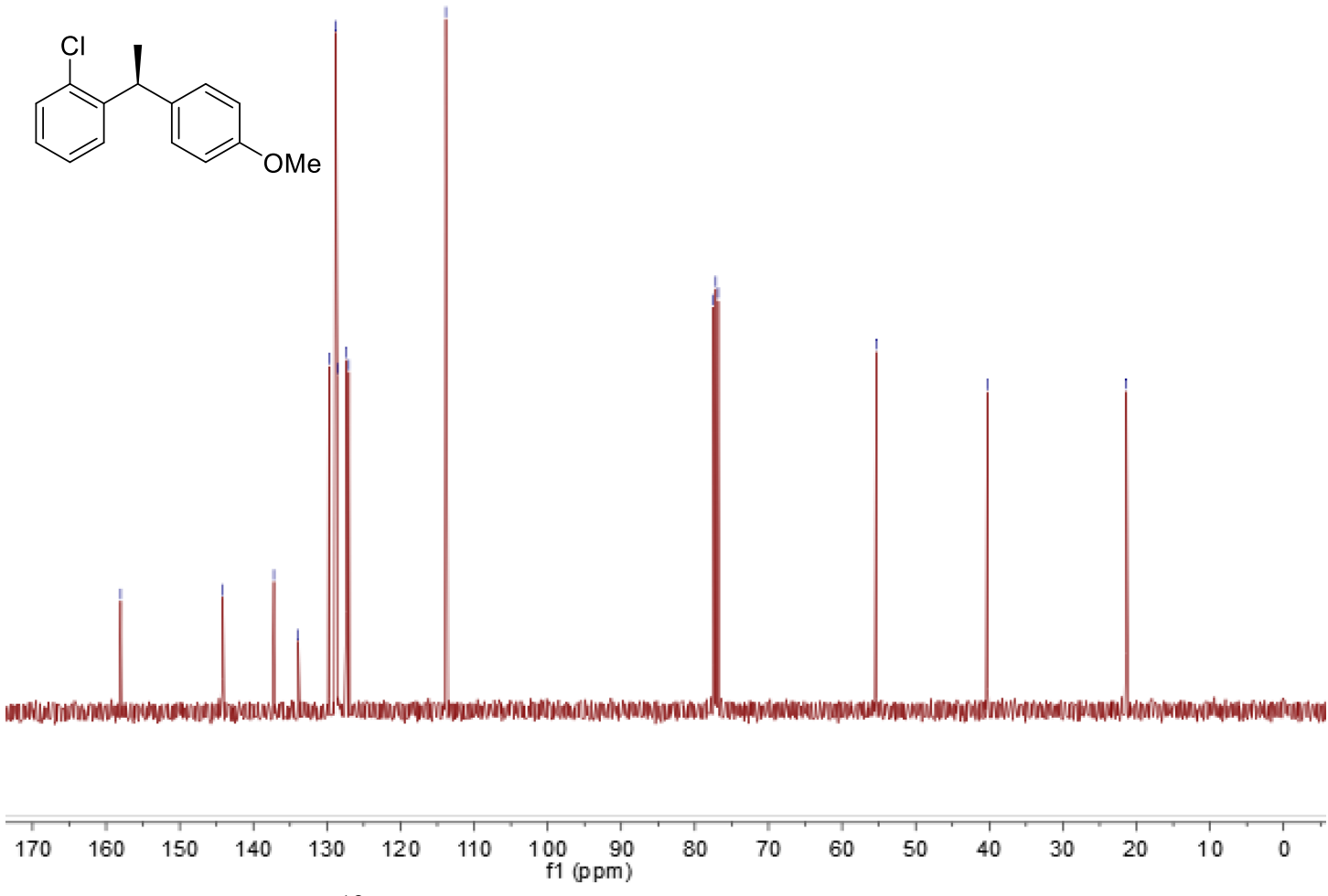

${ }^{13} \mathrm{C}$ NMR $\left(101 \mathrm{M}, \mathrm{CDCl}_{3}\right)$ spectrum of $\mathbf{8 a d}$

S139 


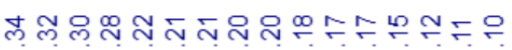
vivitivitutivitia
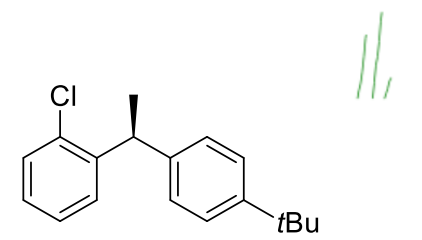

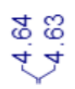

운

i
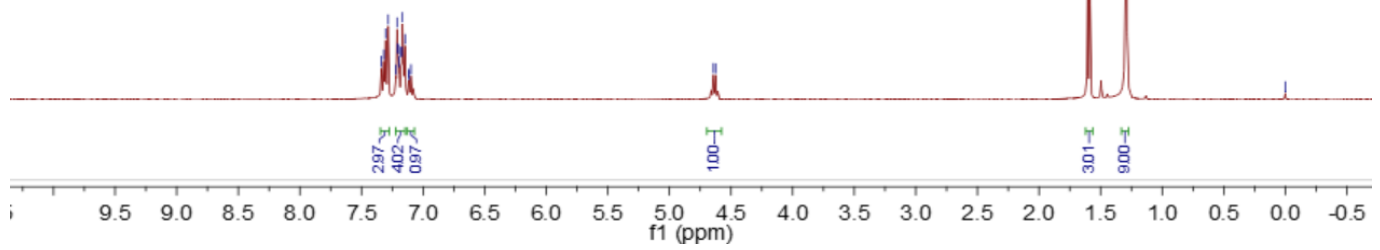

${ }^{1} \mathrm{H}$ NMR (400M, $\left.\mathrm{CDCl}_{3}\right)$ spectrum of $8 \mathbf{a e}$

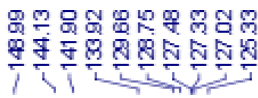
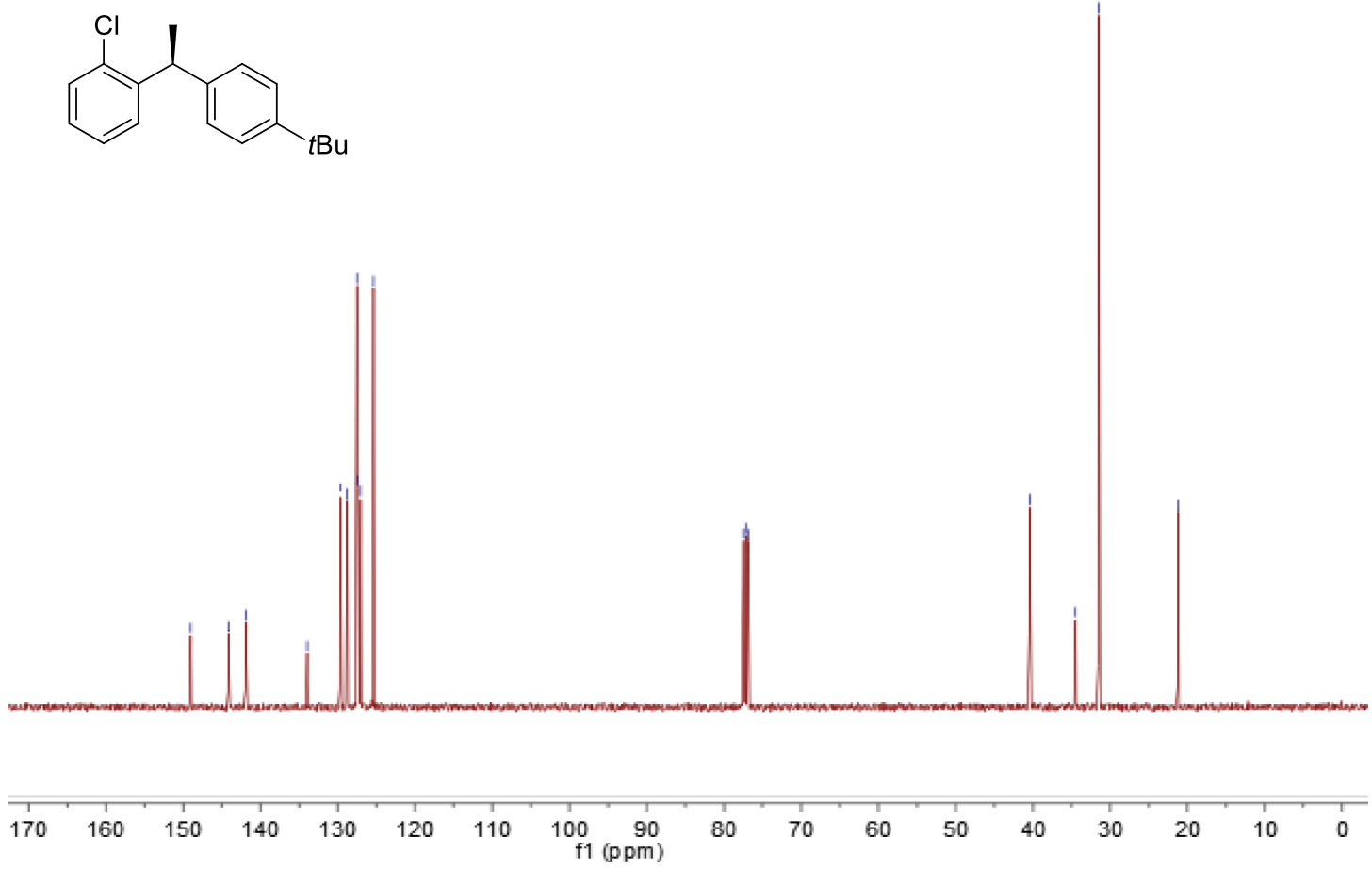

${ }^{13} \mathrm{C}$ NMR $\left(101 \mathrm{M}, \mathrm{CDCl}_{3}\right)$ spectrum of $\mathbf{8 a e}$

S140 


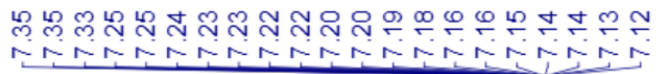

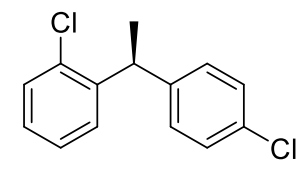

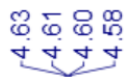

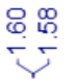

i
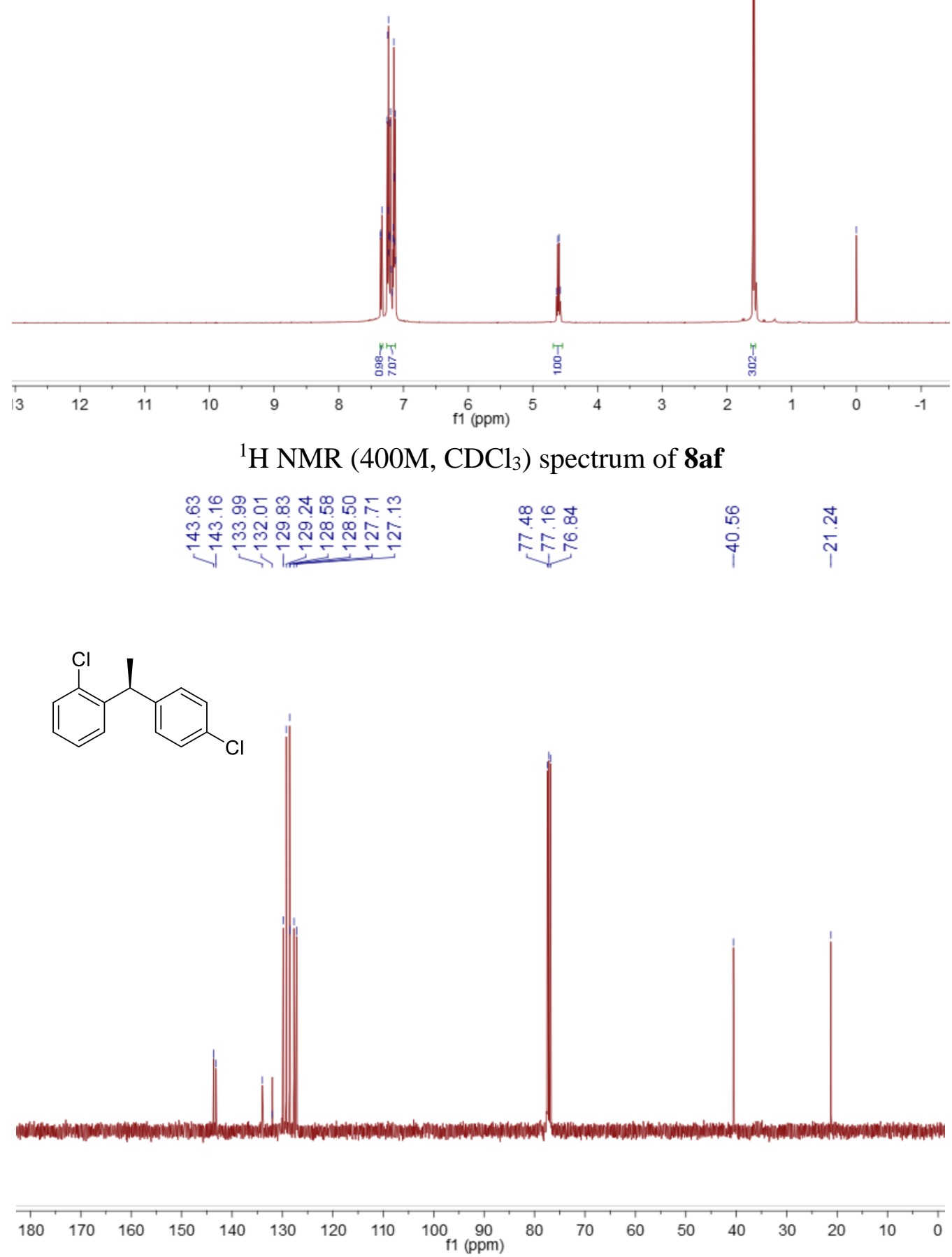

${ }^{13} \mathrm{C}$ NMR (101M, $\left.\mathrm{CDCl}_{3}\right)$ spectrum of 8af

S141 


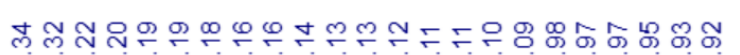
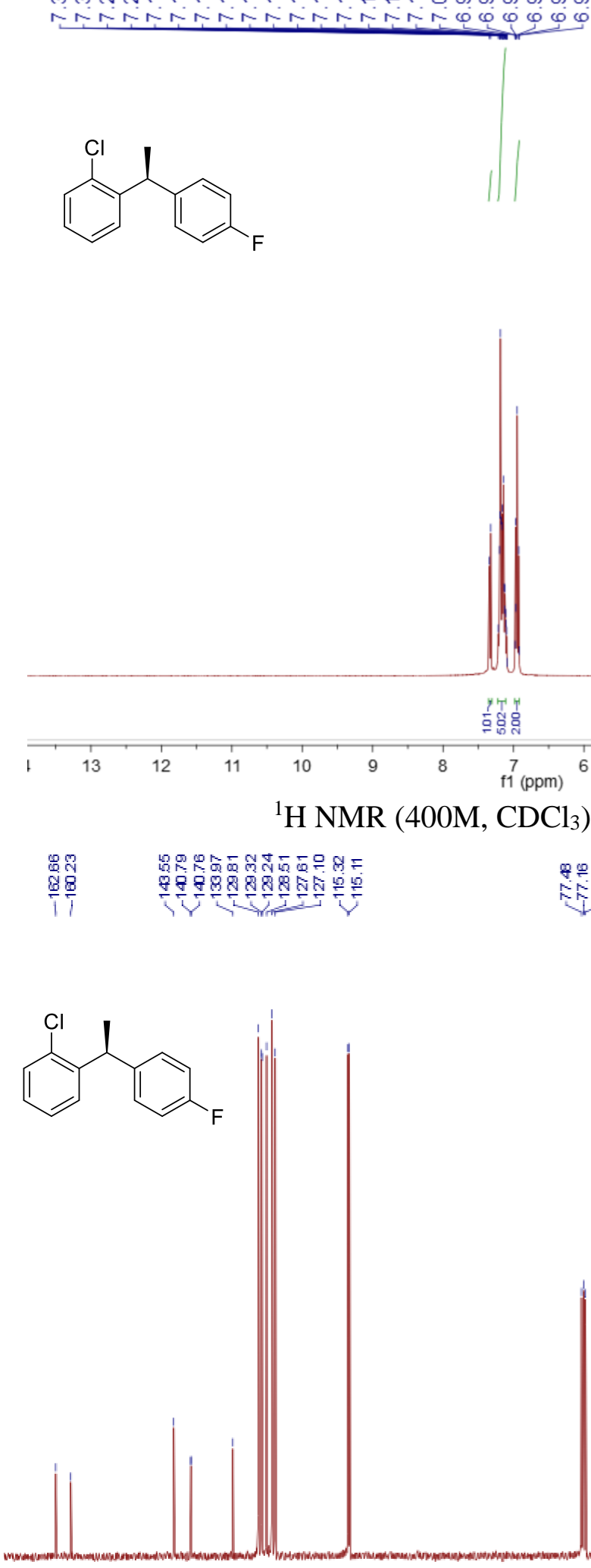

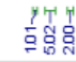

年哭要
षั

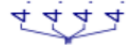

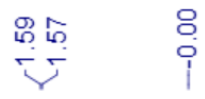
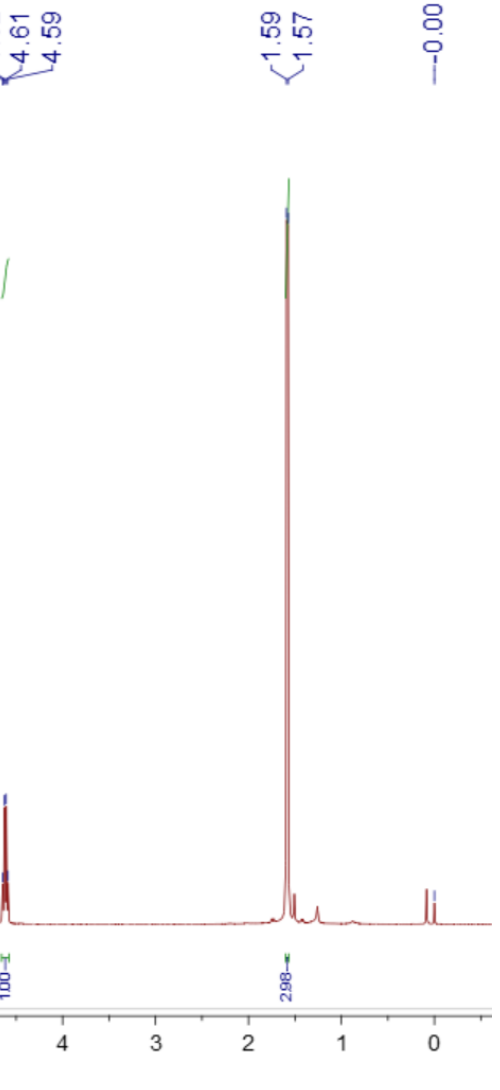

$\left.\mathrm{M}, \mathrm{CDCl}_{3}\right)$ spectrum of $\mathbf{8 a g}$

号

$\stackrel{7}{9}$

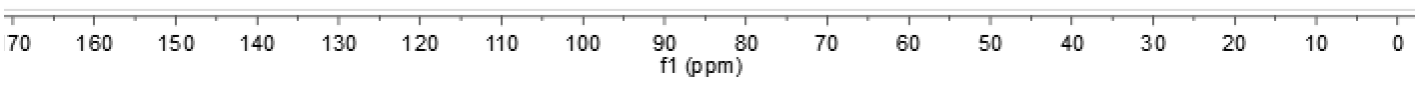

${ }^{13} \mathrm{C}$ NMR $\left(101 \mathrm{M}, \mathrm{CDCl}_{3}\right)$ spectrum of $\mathbf{8 a g}$

S142 


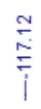
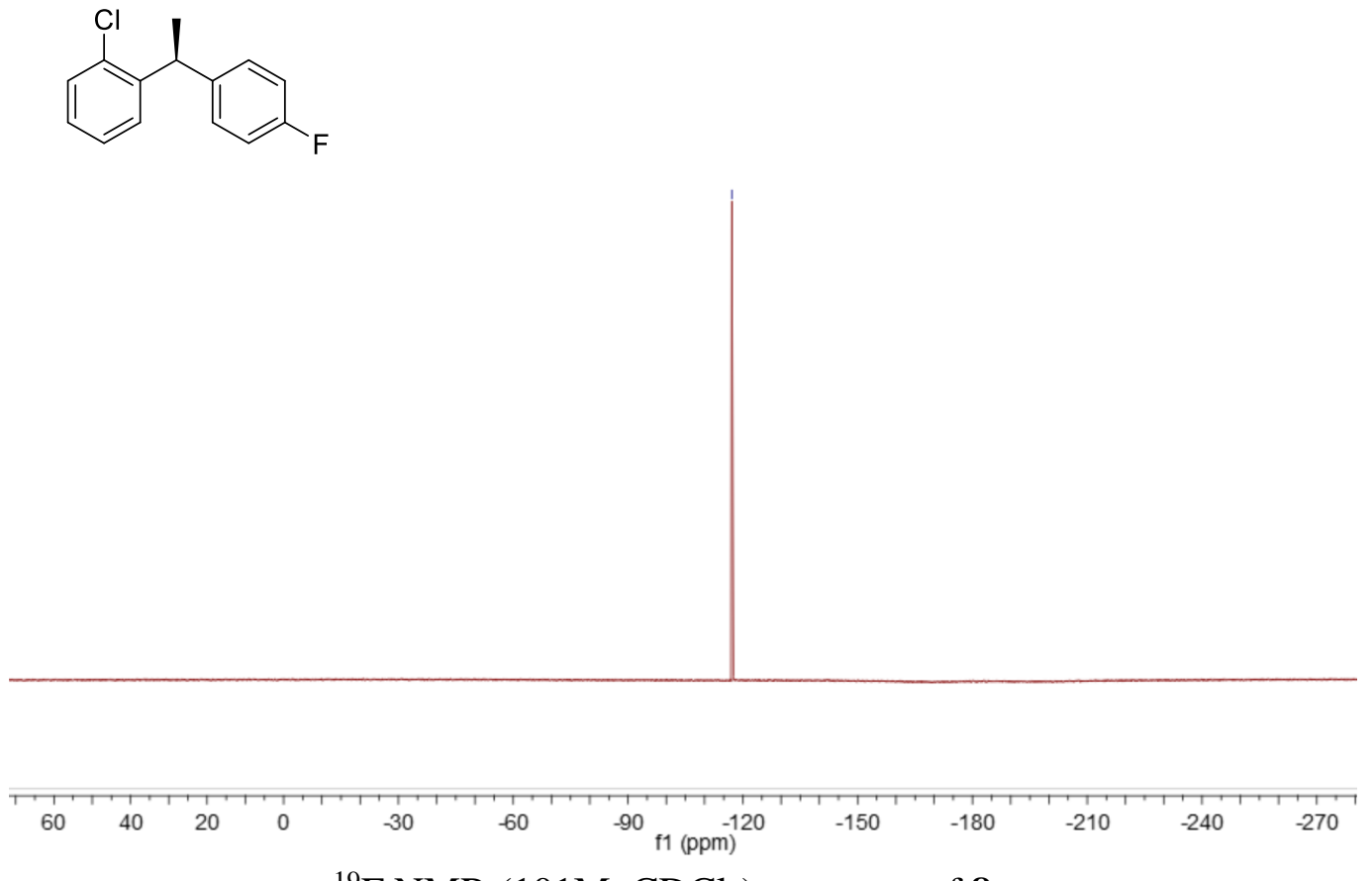

${ }^{19} \mathrm{~F}$ NMR $\left(101 \mathrm{M}, \mathrm{CDCl}_{3}\right)$ spectrum of $\mathbf{8 a g}$
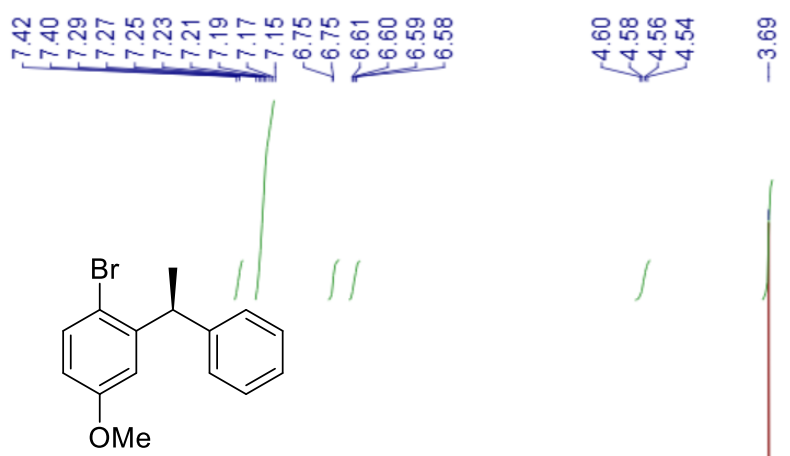

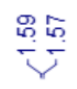

$\stackrel{8}{i}$

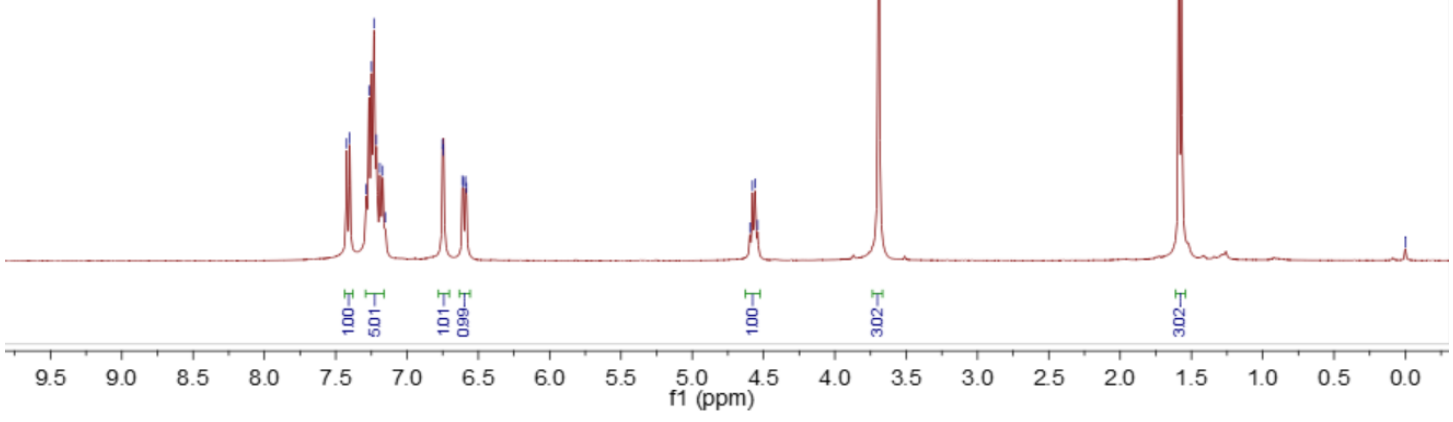

${ }^{1} \mathrm{H}$ NMR (400M, $\mathrm{CDCl}_{3}$ ) spectrum of Bah

S143 


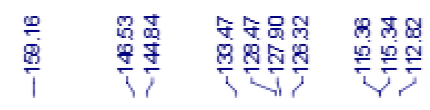

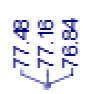

5
9

$\frac{8}{1}$
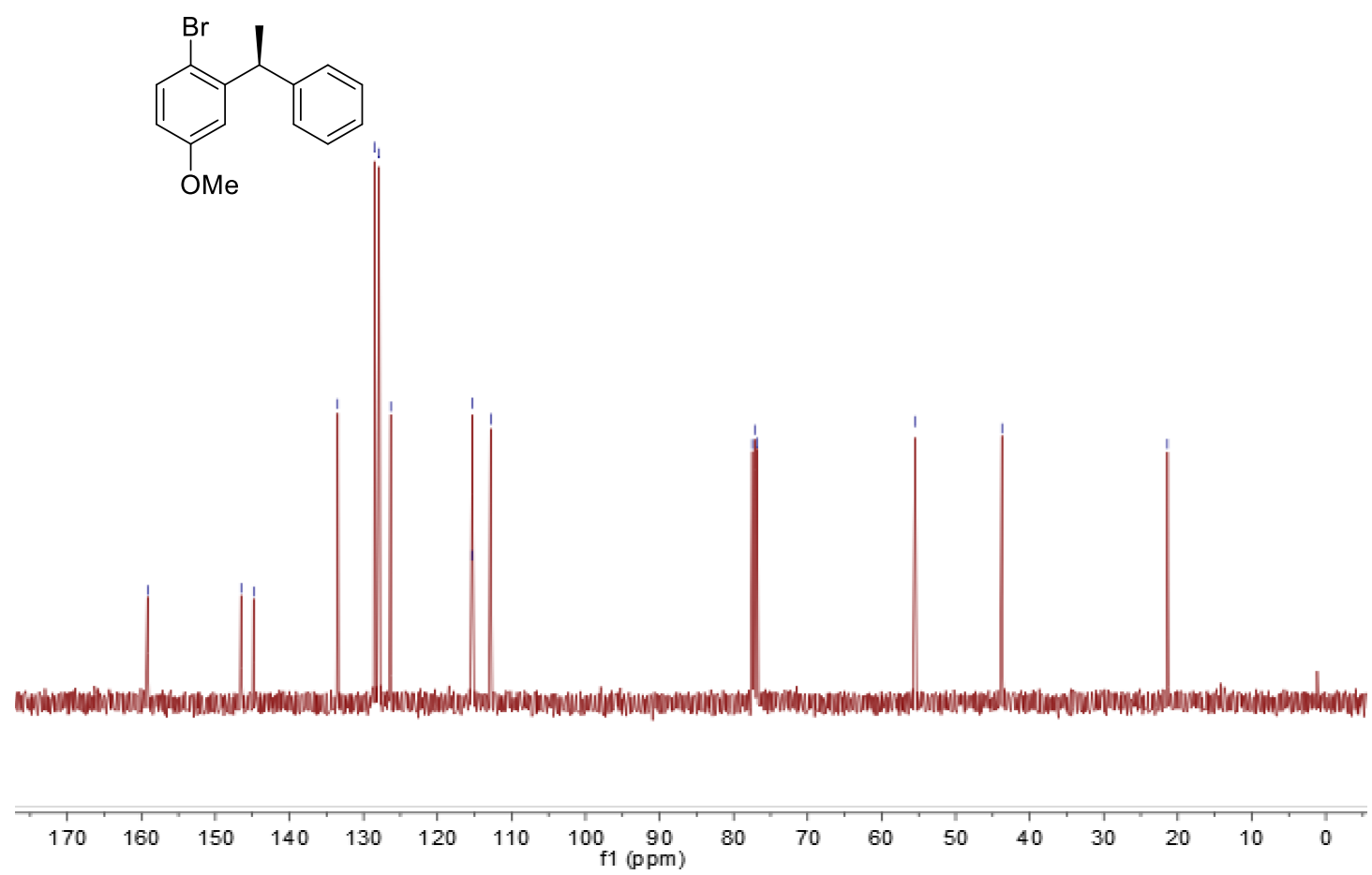

${ }^{13} \mathrm{C}$ NMR $\left(101 \mathrm{M}, \mathrm{CDCl}_{3}\right)$ spectrum of $\mathbf{8 a h}$
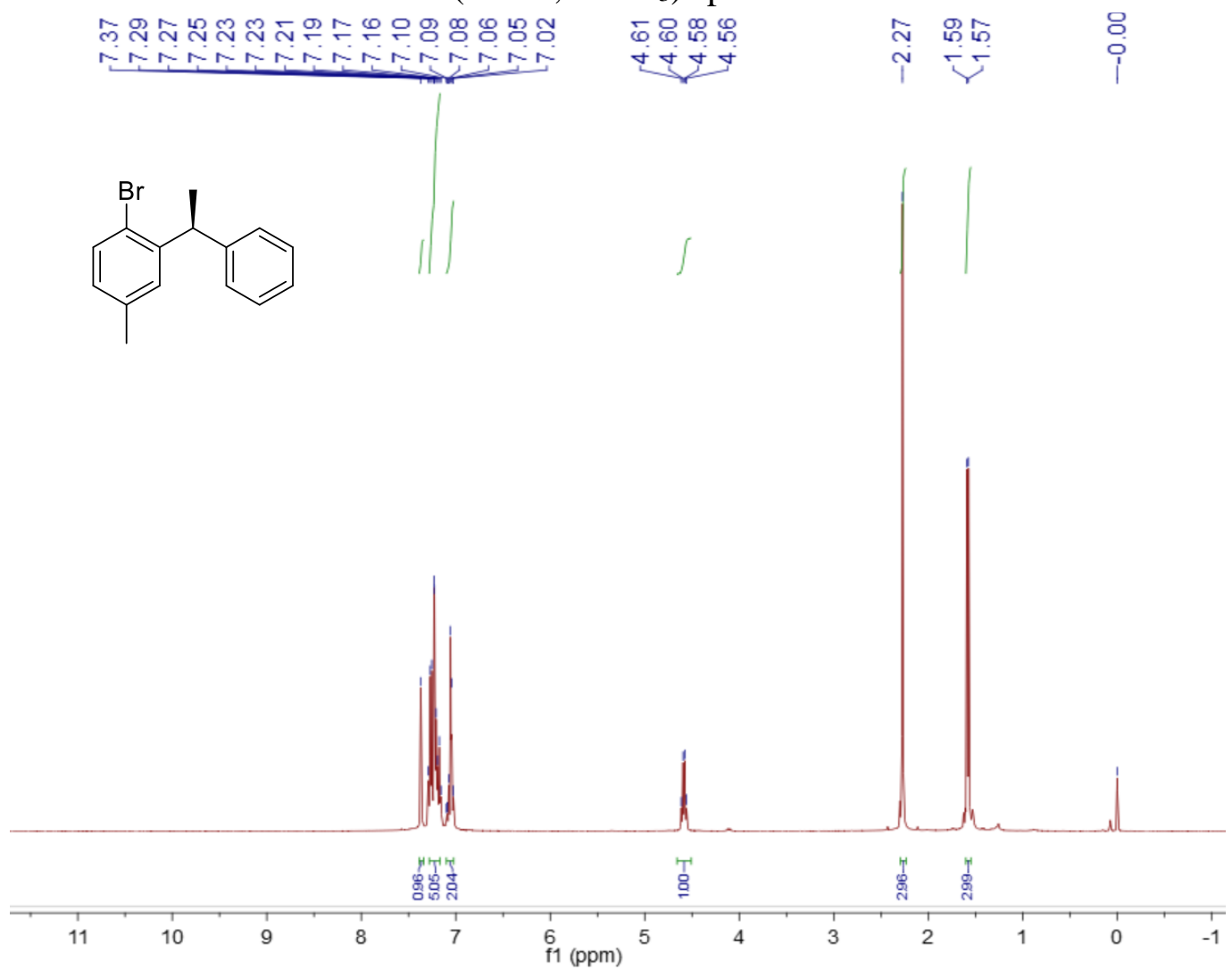

${ }^{1} \mathrm{H}$ NMR (400M, $\mathrm{CDCl}_{3}$ ) spectrum of ai

S144 


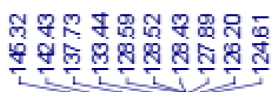

野总

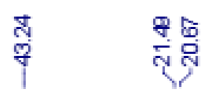
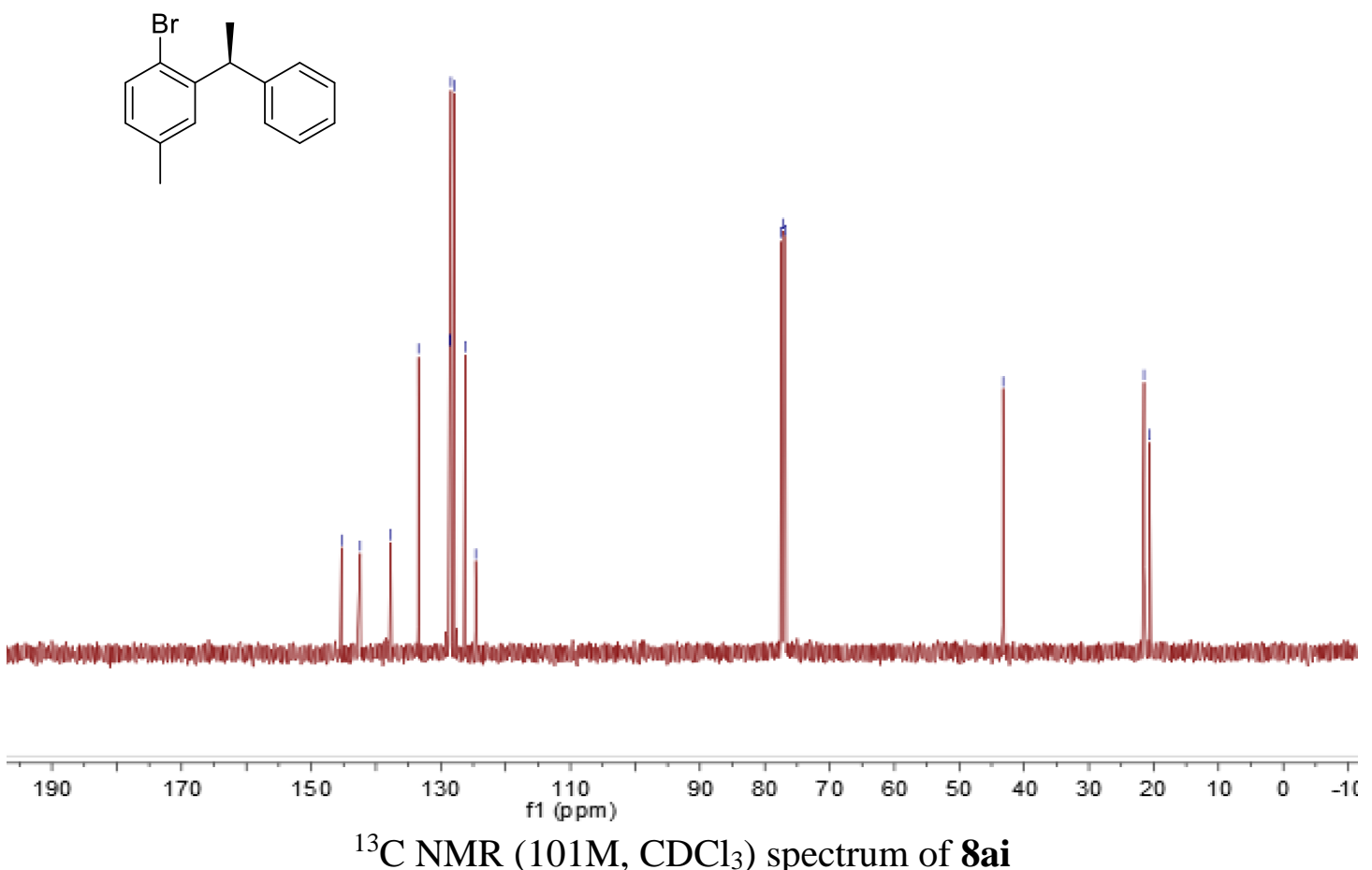

${ }^{13} \mathrm{C} \mathrm{NMR}\left(101 \mathrm{M}, \mathrm{CDCl}_{3}\right)$ spectrum of 8ai
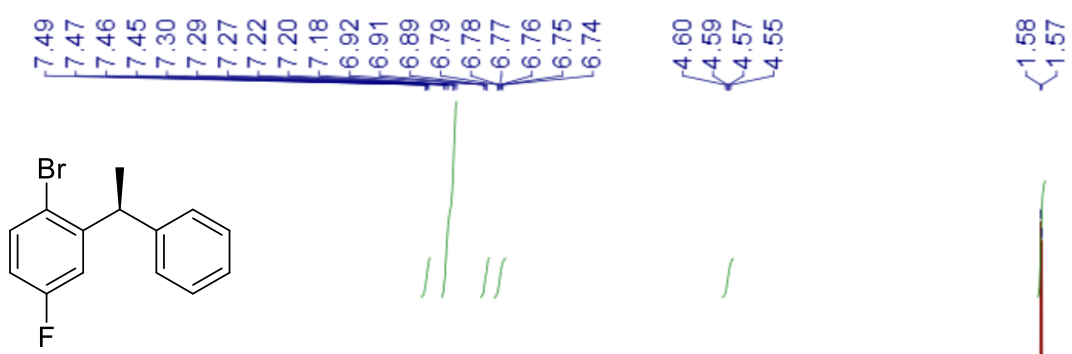

i

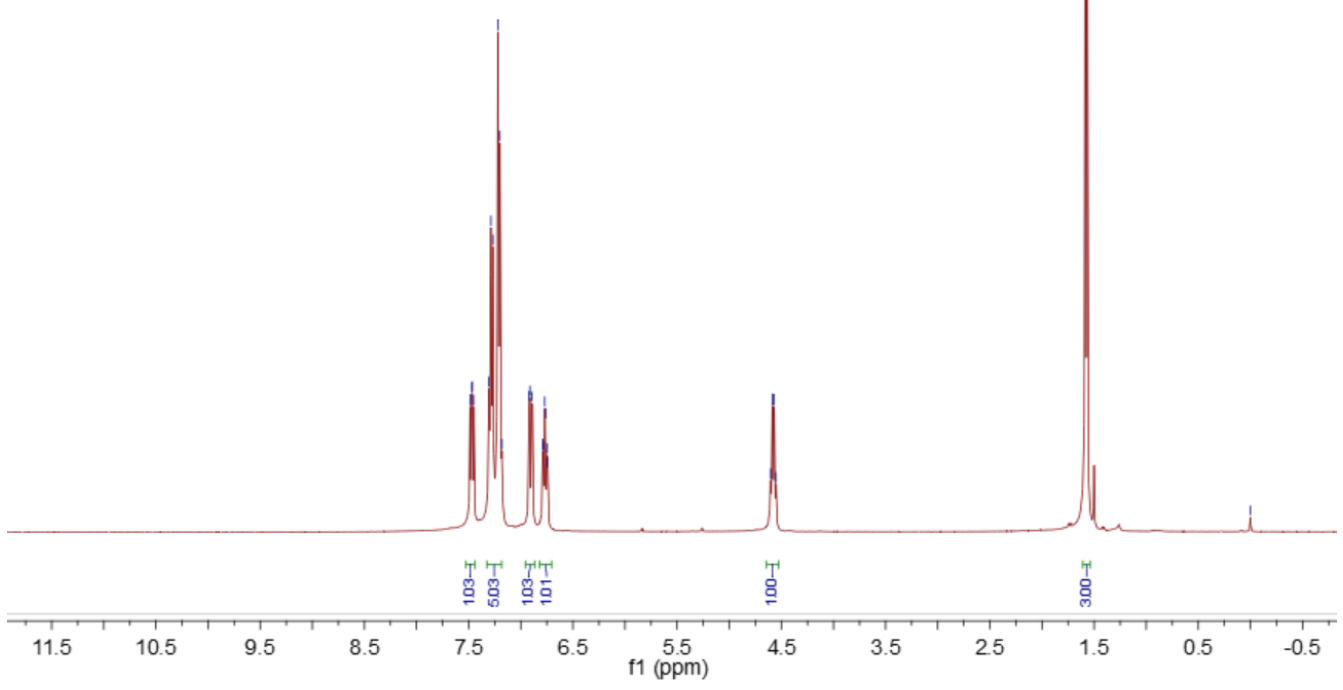

${ }^{1} \mathrm{H}$ NMR $\left(400 \mathrm{M}, \mathrm{CDCl}_{3}\right)$ spectrum of $\mathbf{8 a j}$

S145 


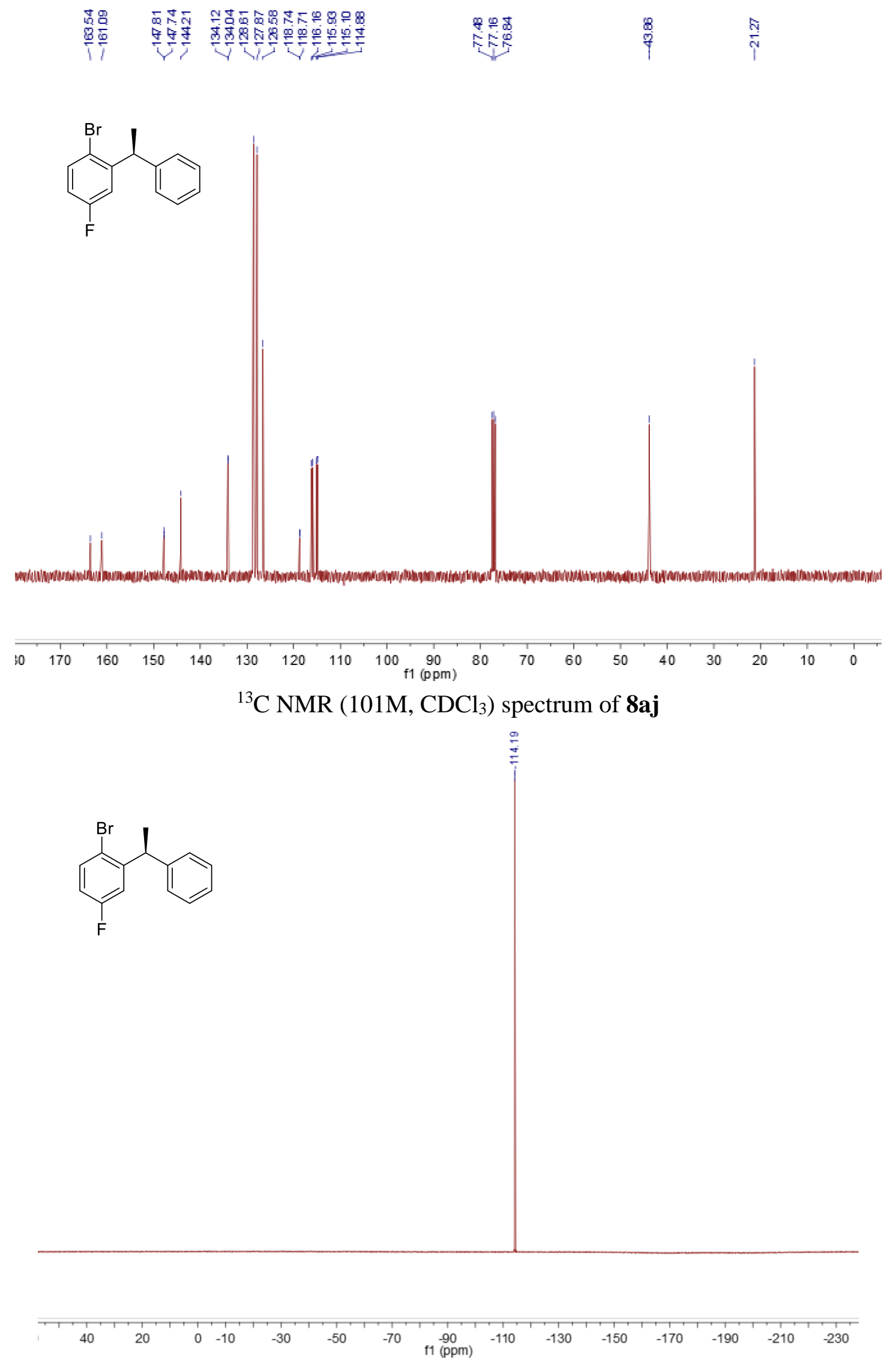

${ }^{19} \mathrm{~F}$ NMR (101M, $\left.\mathrm{CDCl}_{3}\right)$ spectrum of 8aj 

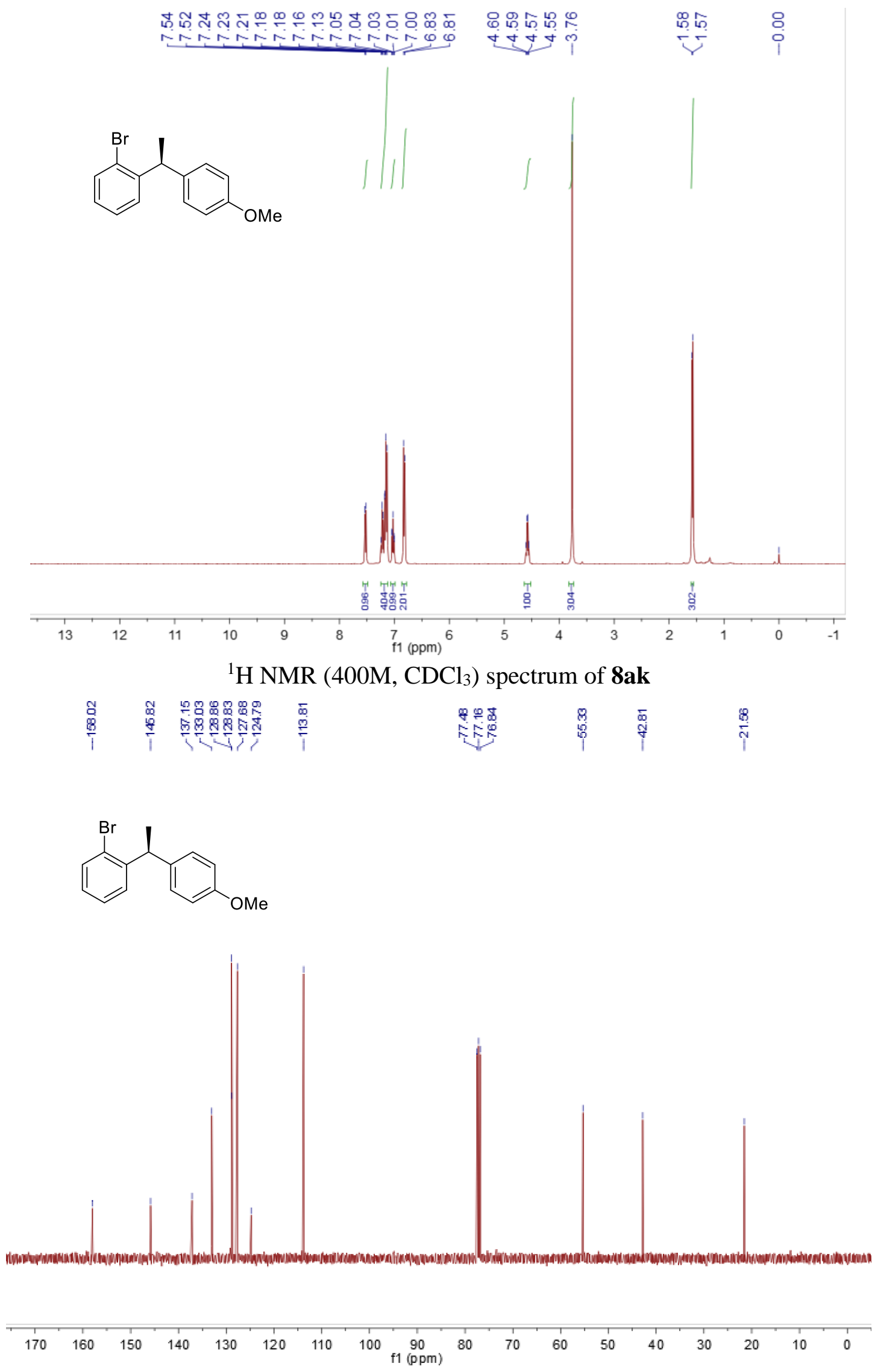

${ }^{13} \mathrm{C}$ NMR (101M, $\left.\mathrm{CDCl}_{3}\right)$ spectrum of 8ak

S147 


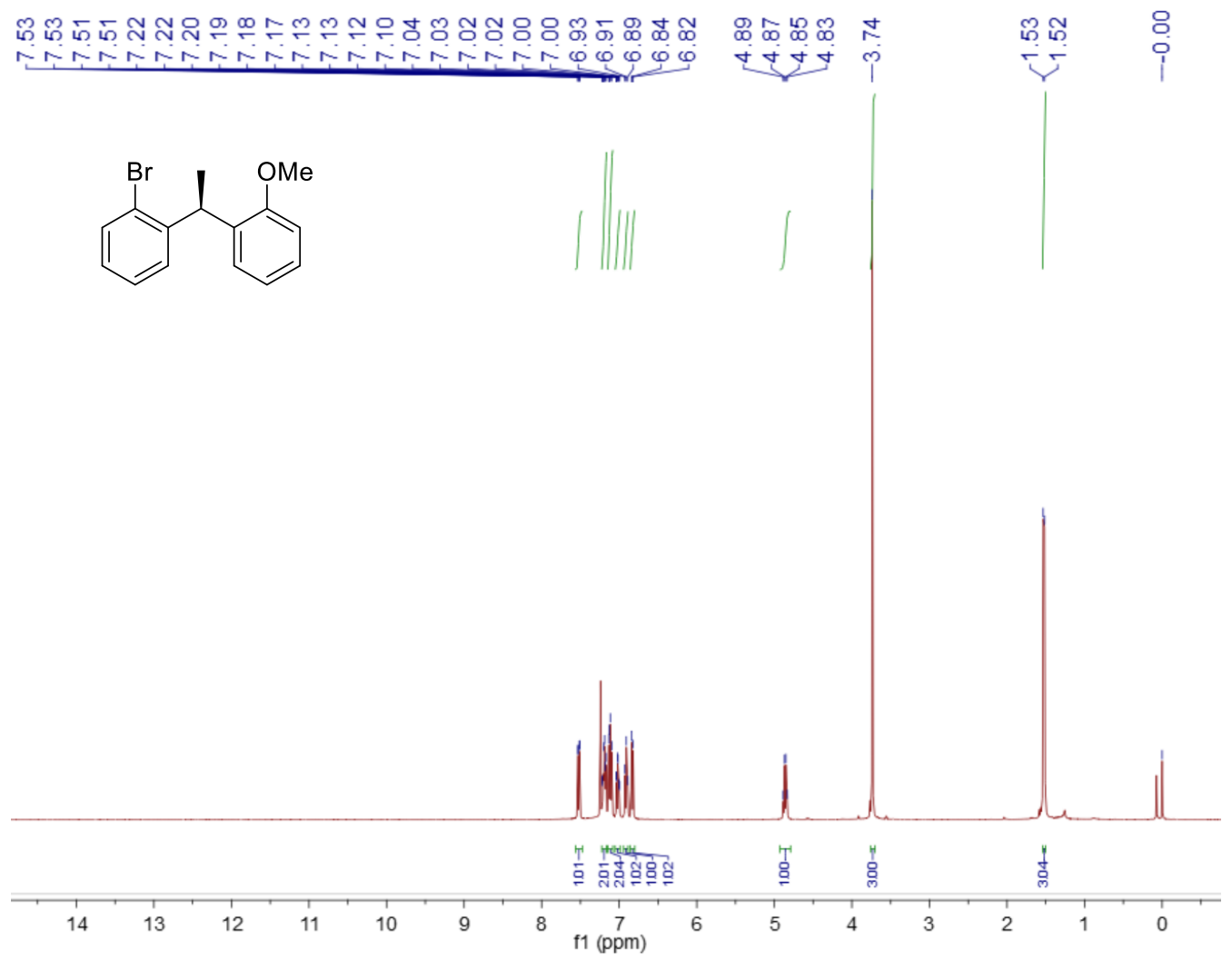

${ }^{1} \mathrm{H}$ NMR $\left(400 \mathrm{M}, \mathrm{CDCl}_{3}\right)$ spectrum of 8al

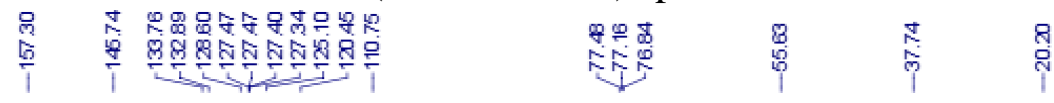<smiles>COc1ccccc1C(C)c1ccccc1Br</smiles>

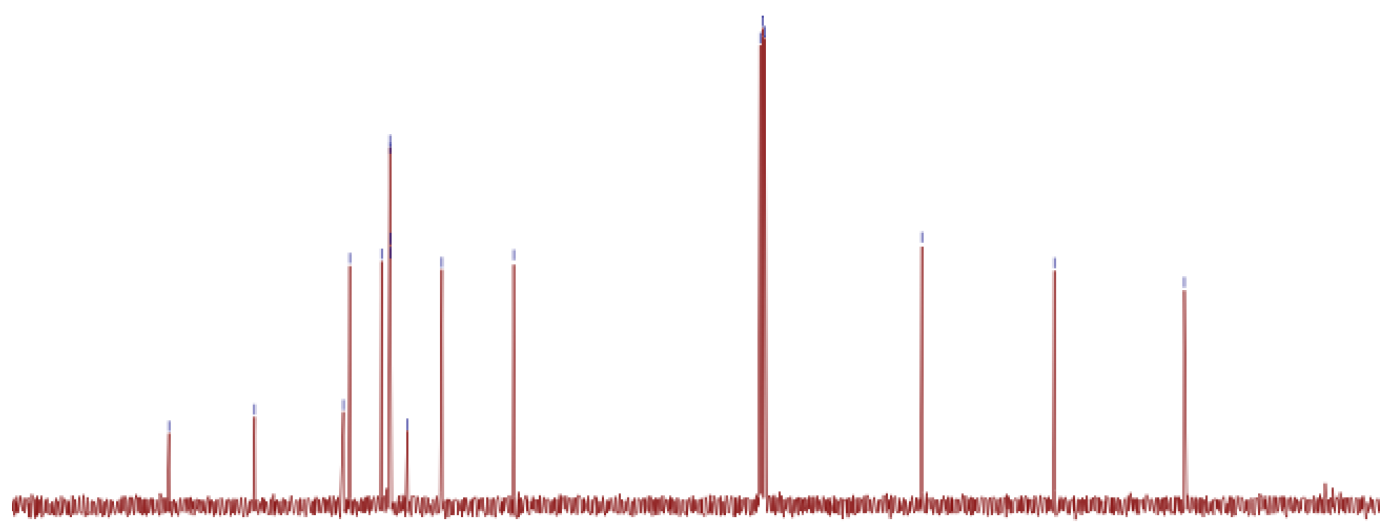

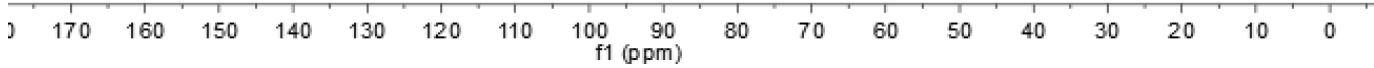
${ }^{13} \mathrm{C}$ NMR $\left(101 \mathrm{M}, \mathrm{CDCl}_{3}\right)$ spectrum of $\mathbf{8 a l}$ 
<smiles>COc1cc([C@H](C)c2ccc3ccccc3c2)c(Br)c(OC)c1OC</smiles>
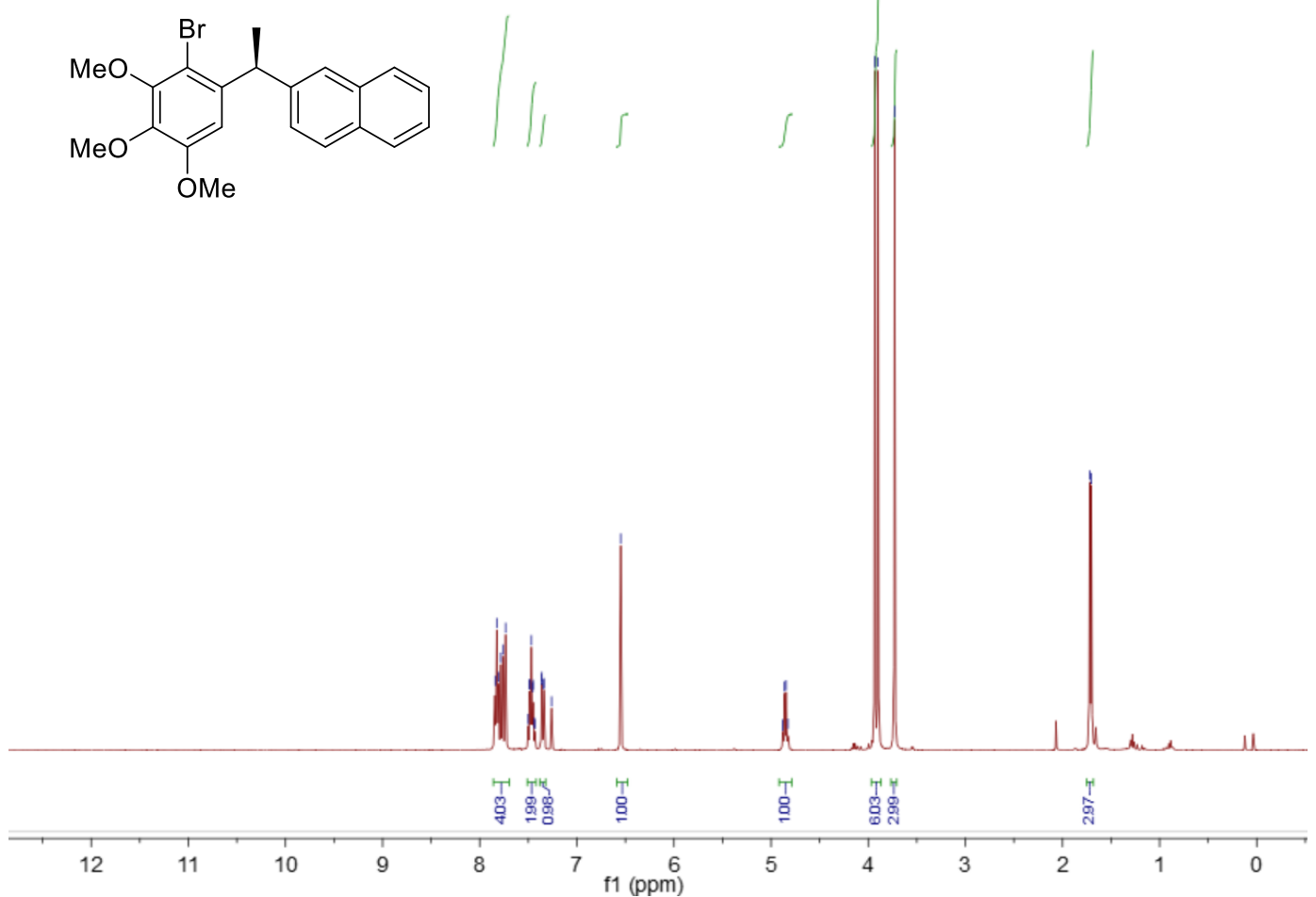

${ }^{1} \mathrm{H} \mathrm{NMR}\left(400 \mathrm{M}, \mathrm{CDCl}_{3}\right)$ spectrum of $8 \mathbf{a m}$

界罢<smiles>COc1cc([C@H](C)c2ccc3ccccc3c2)c(Br)c(OC)c1OC</smiles>
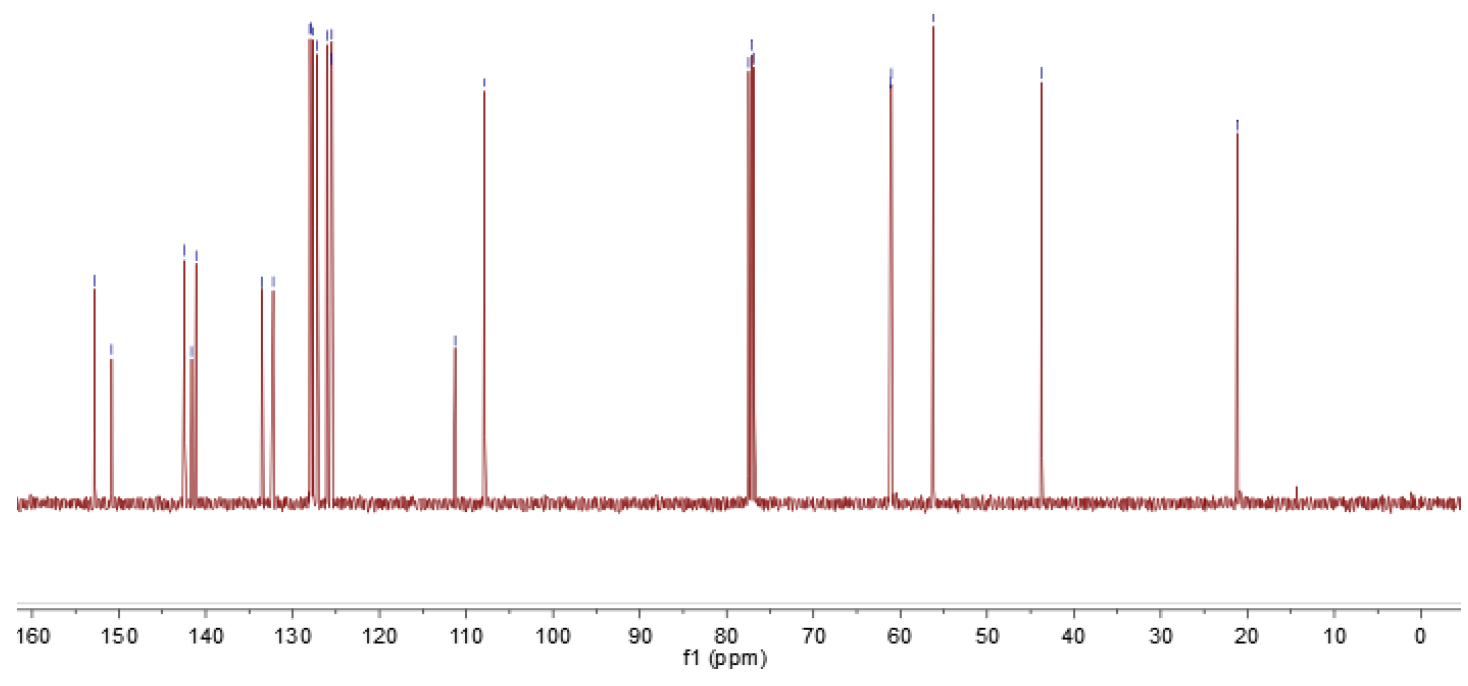

${ }^{13} \mathrm{C}$ NMR (101M, $\left.\mathrm{CDCl}_{3}\right)$ spectrum of 8am 


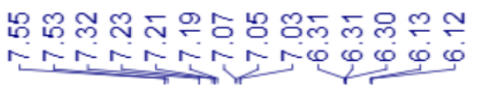

$\int \| \int \mid \int$

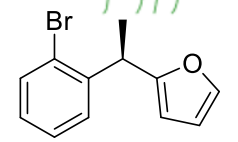

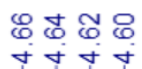

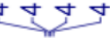

这莫

o

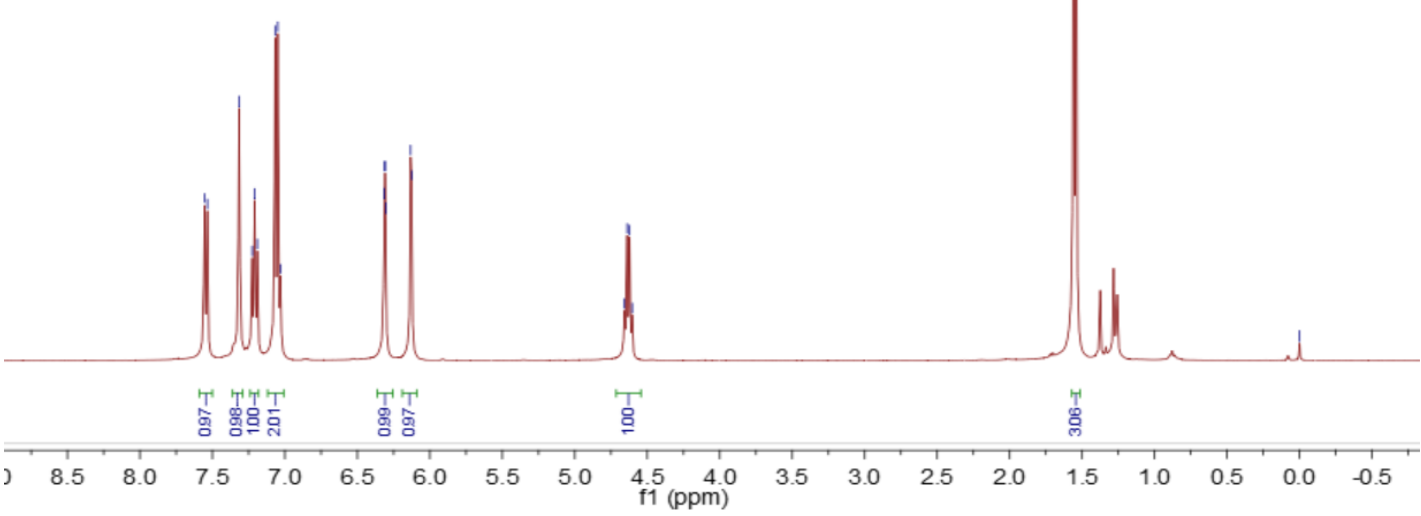

${ }^{1} \mathrm{H}$ NMR (400M, $\mathrm{CDCl}_{3}$ ) spectrum of $\mathbf{8 a n}$

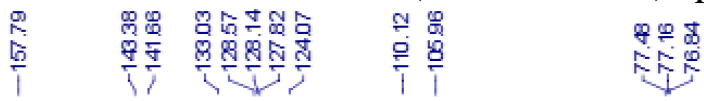

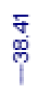

$\underset{5}{\overline{5}}$
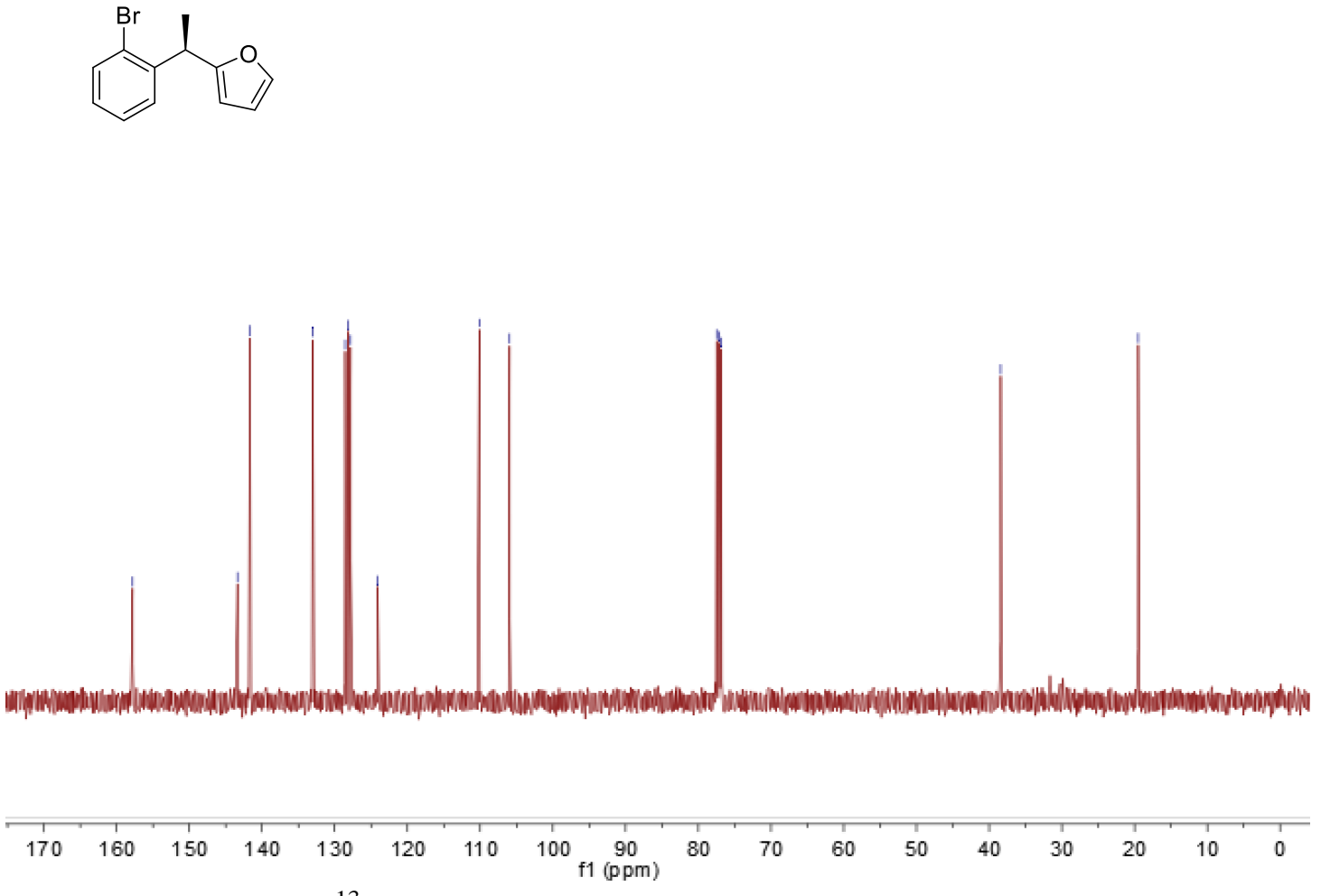

${ }^{13} \mathrm{C}$ NMR $\left(101 \mathrm{M}, \mathrm{CDCl}_{3}\right)$ spectrum of 8an

S150 


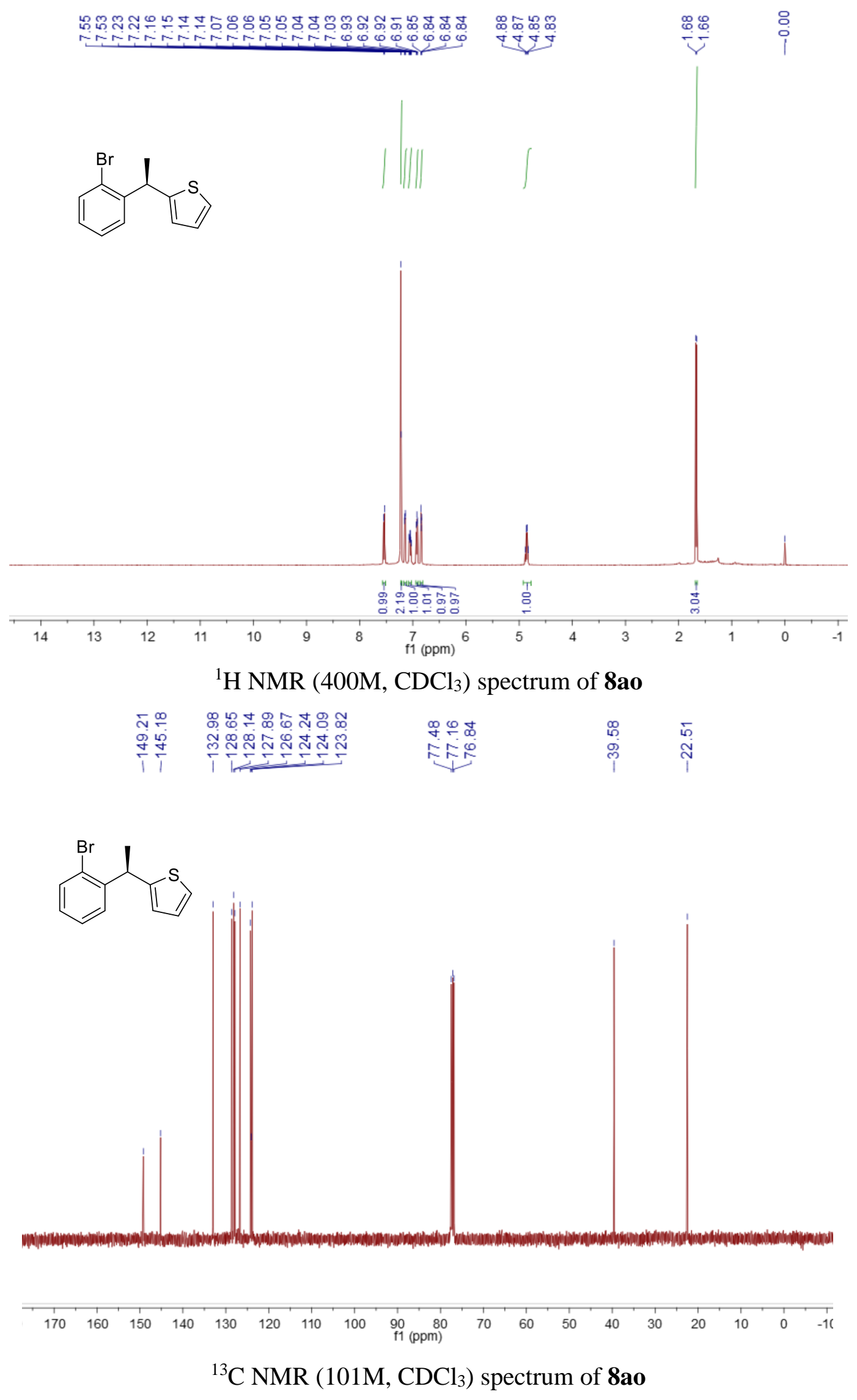



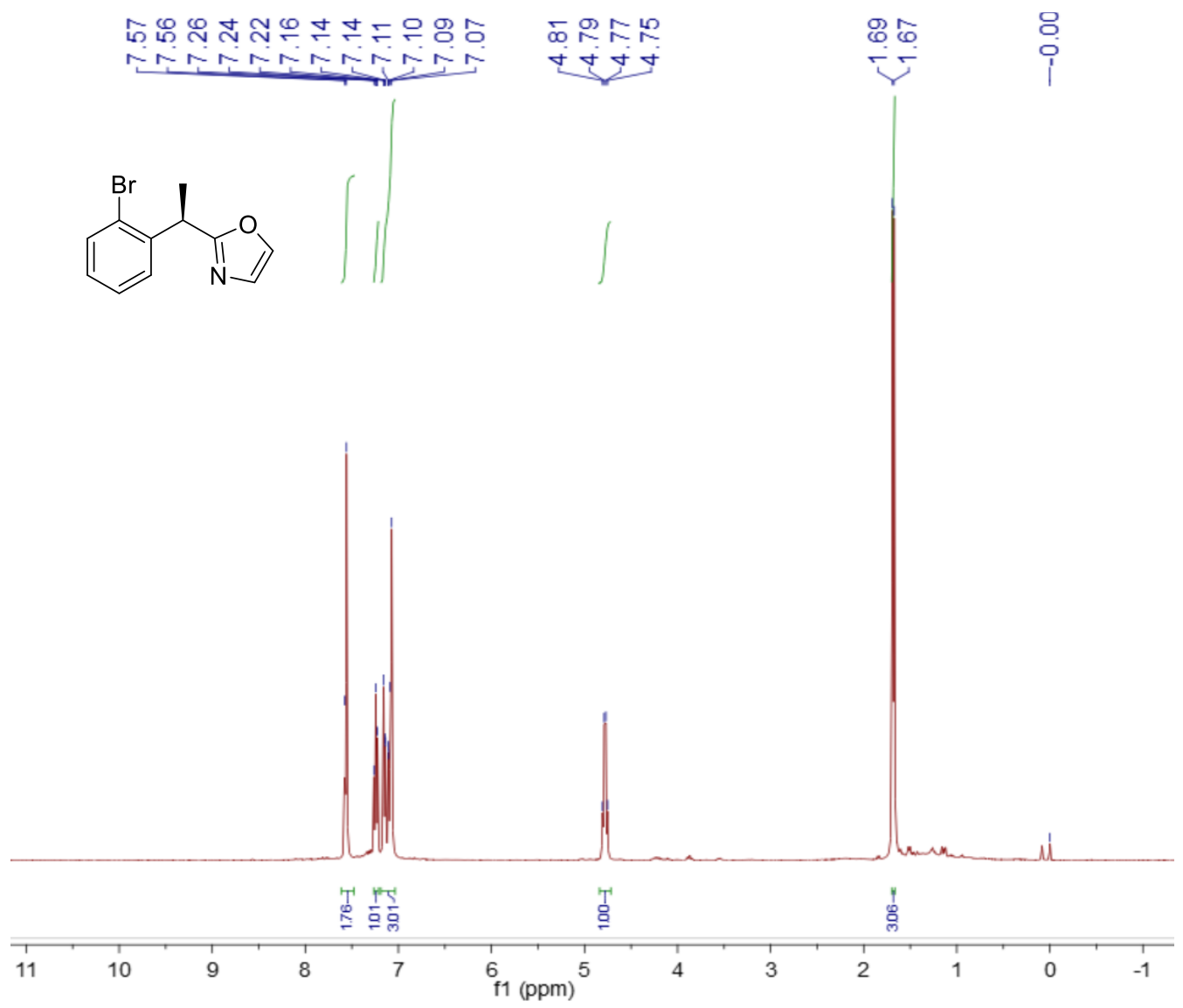

${ }^{1} \mathrm{H}$ NMR (400M, $\mathrm{CDCl}_{3}$ ) spectrum of 8ap

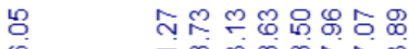

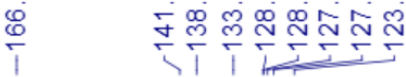

$\stackrel{\infty}{\infty} \stackrel{\infty}{\infty}$

슨

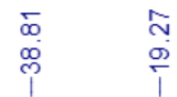<smiles>C[C@@H](c1ncco1)c1ccccc1Br</smiles>

$\begin{array}{llllllllll}190 & 180 & 170 & 160 & 150 & 140 & 130 & 120 & 110 & \begin{array}{l}100 \\ \mathrm{f} 1(\mathrm{ppm})\end{array}\end{array}$

${ }^{13} \mathrm{C}$ NMR $\left(101 \mathrm{M}, \mathrm{CDCl}_{3}\right)$ spectrum of 8ap 
<smiles>C[C@H](c1nccs1)c1ccccc1Br</smiles><smiles>C1#CCCC1</smiles>

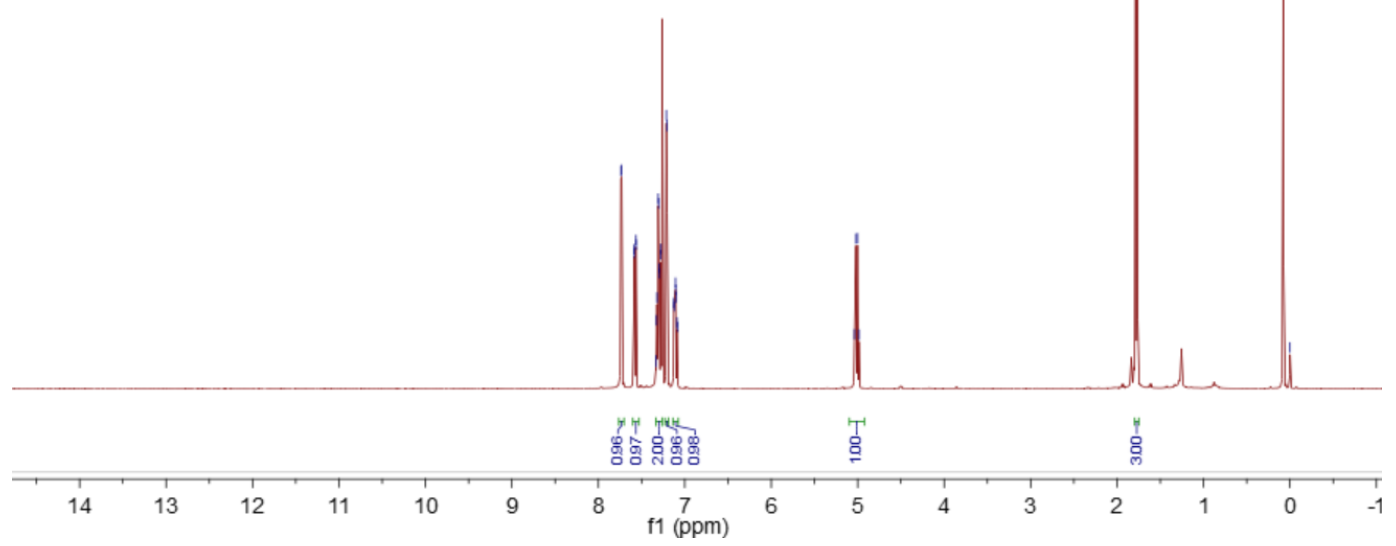

${ }^{1} \mathrm{H}$ NMR (400M, $\mathrm{CDCl}_{3}$ ) spectrum of $\mathbf{8 a q}$

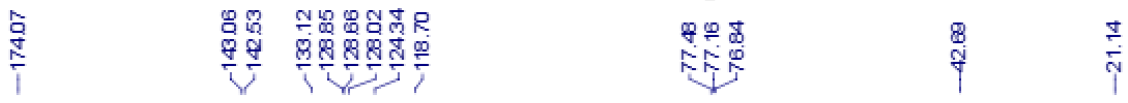<smiles>CC(c1nccs1)c1ccccc1Br</smiles>

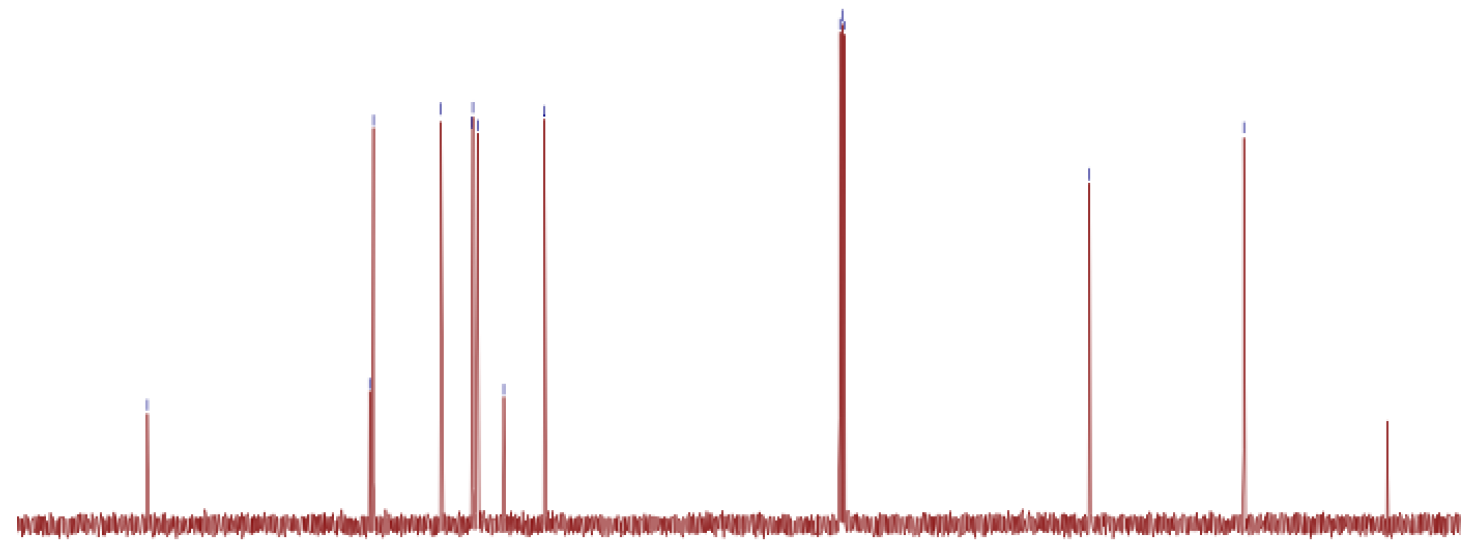

$190 \quad 180 \quad 170 \quad 160 \quad 150$

${ }^{13} \mathrm{C}$ NMR $\left(101 \mathrm{M}, \mathrm{CDCl}_{3}\right)$ spectrum of $\mathbf{8 a q}$ 


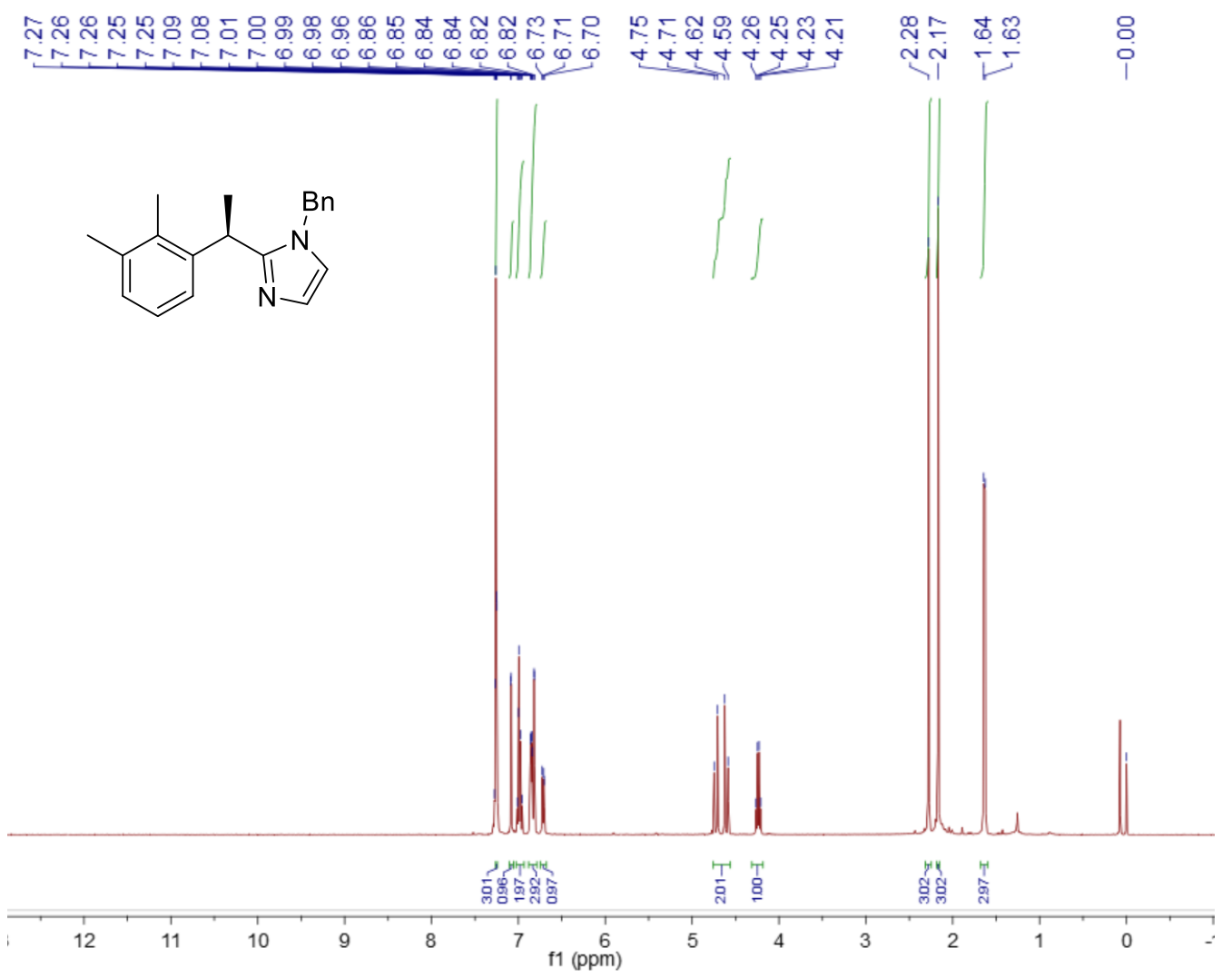

${ }^{1} \mathrm{H}$ NMR (400M, $\mathrm{CDCl}_{3}$ ) spectrum of 8ar

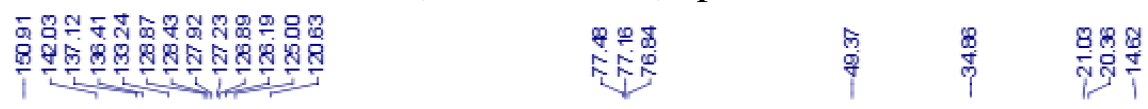

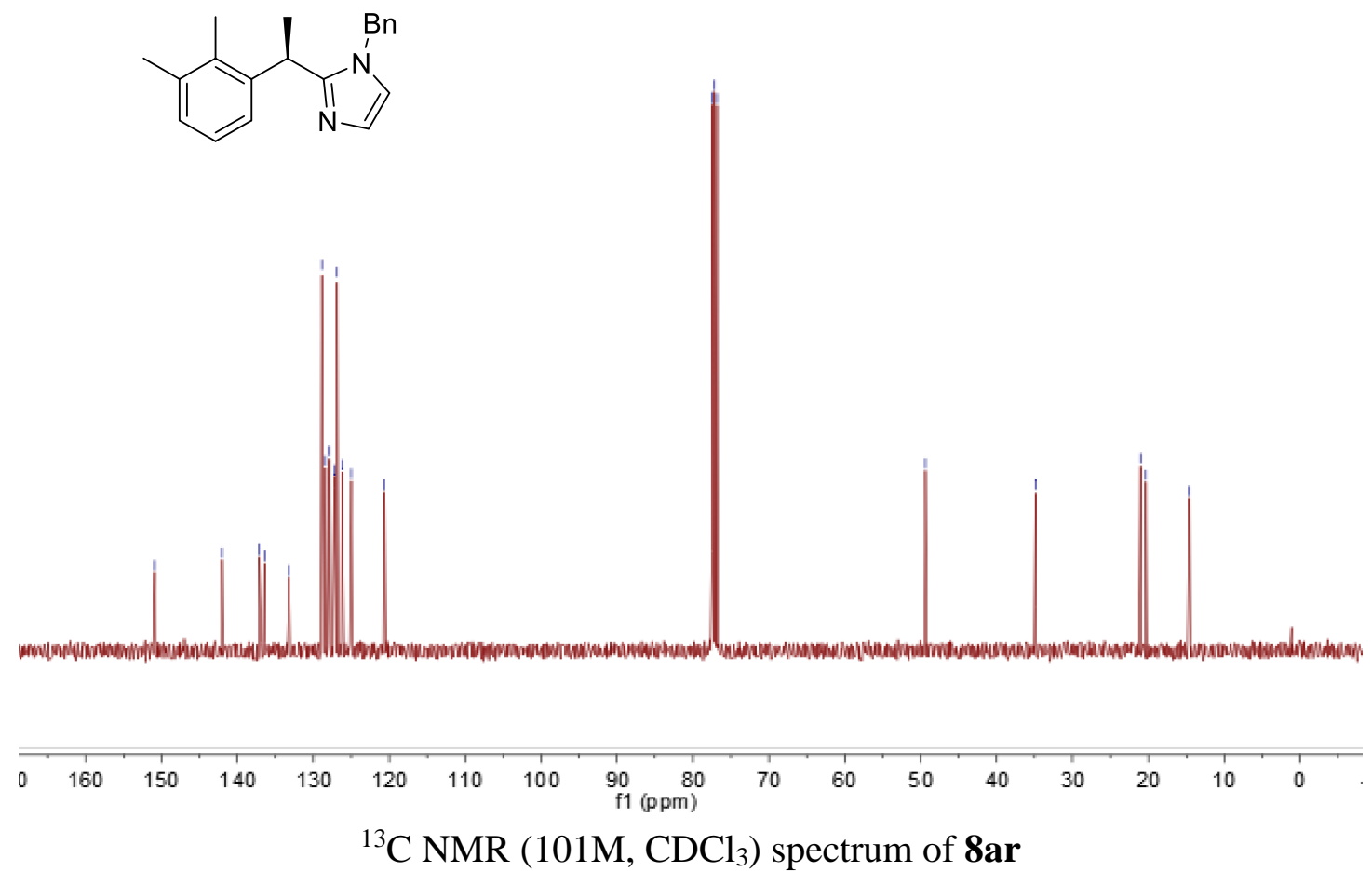




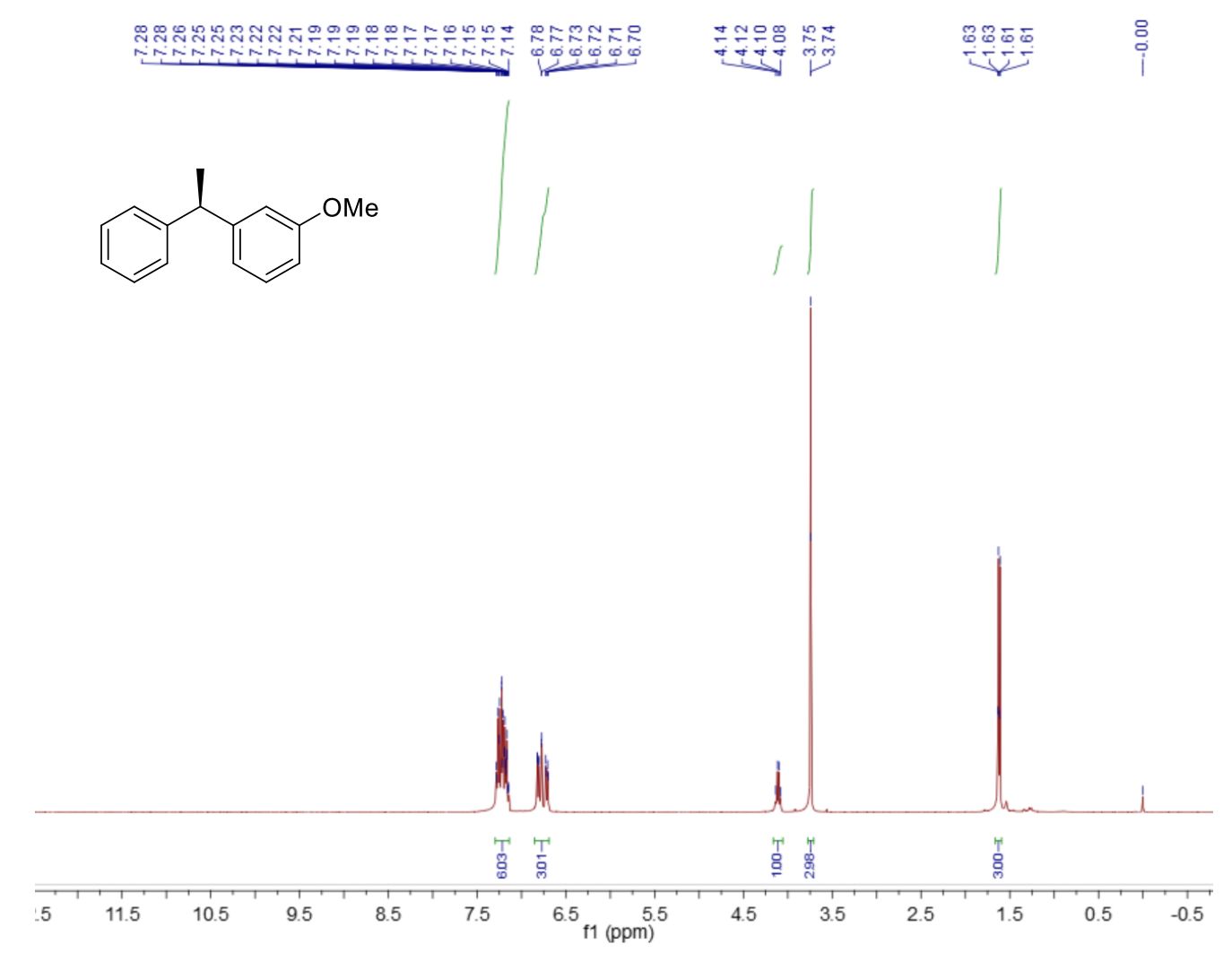

${ }^{1} \mathrm{H}$ NMR (400M, $\mathrm{CDCl}_{3}$ ) spectrum of 9ah

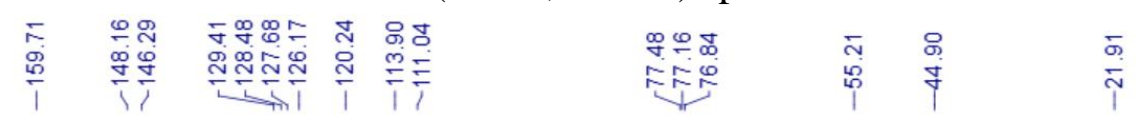<smiles>COc1cccc(C(C)c2ccccc2)c1</smiles>
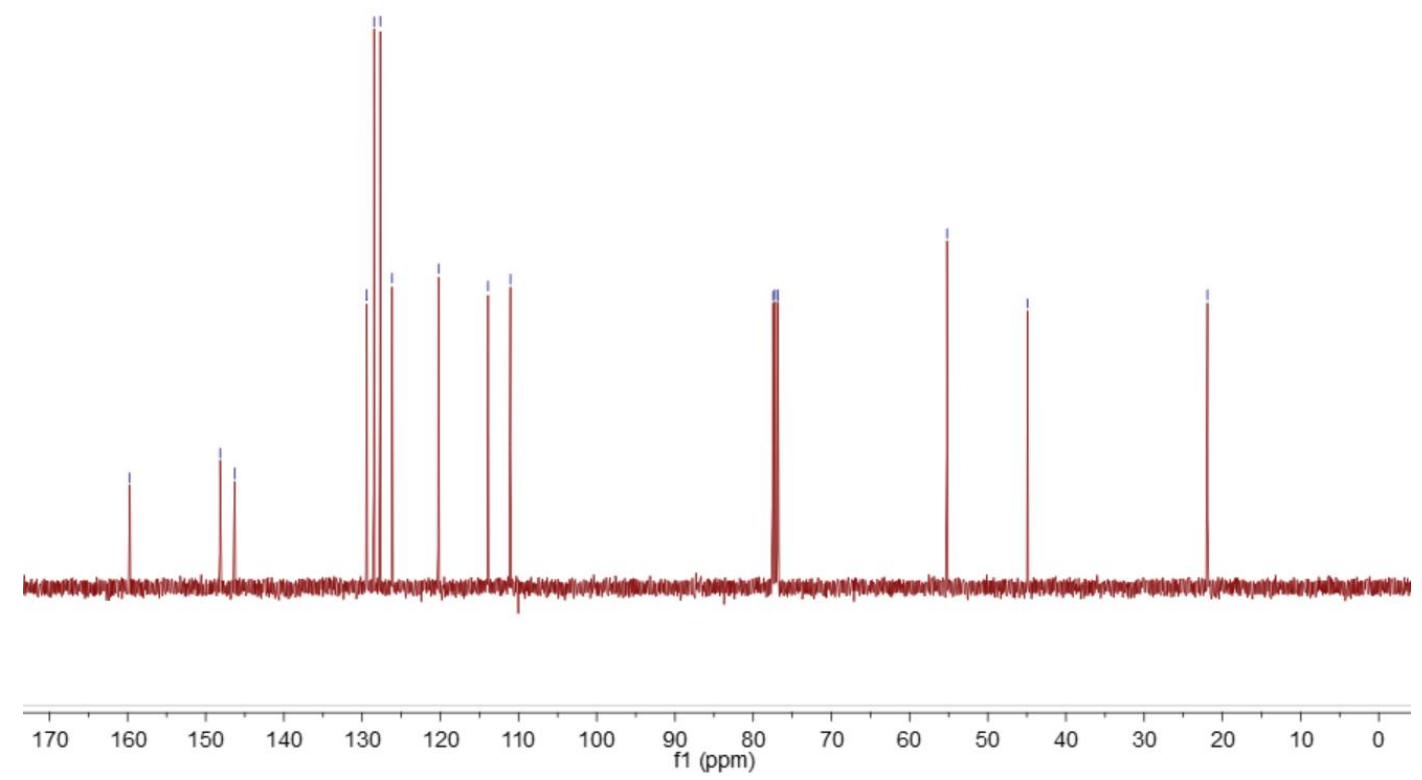

${ }^{13} \mathrm{C}$ NMR $\left(101 \mathrm{M}, \mathrm{CDCl}_{3}\right)$ spectrum of 9ah 


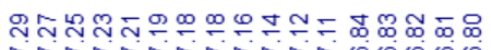
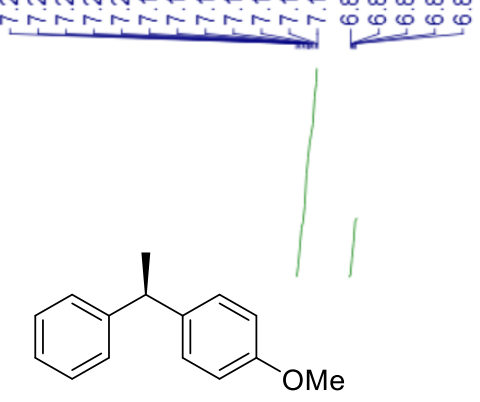

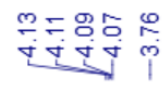

$\stackrel{\square}{\square}$

i

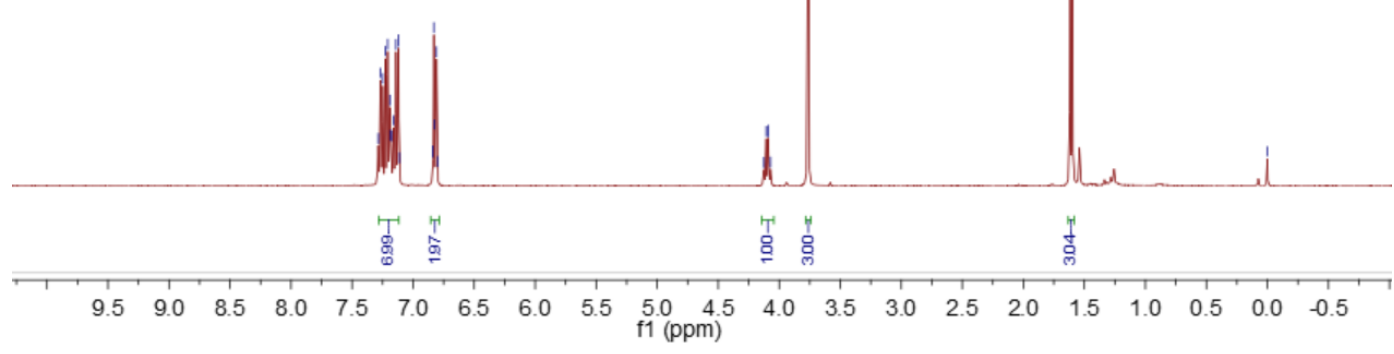

${ }^{1} \mathrm{H}$ NMR (400M, $\mathrm{CDCl}_{3}$ ) spectrum of 9ak

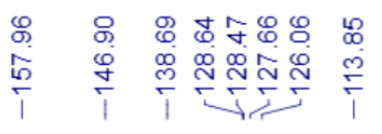

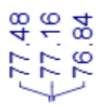

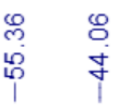

$\stackrel{\stackrel{9}{i j}}{i}$
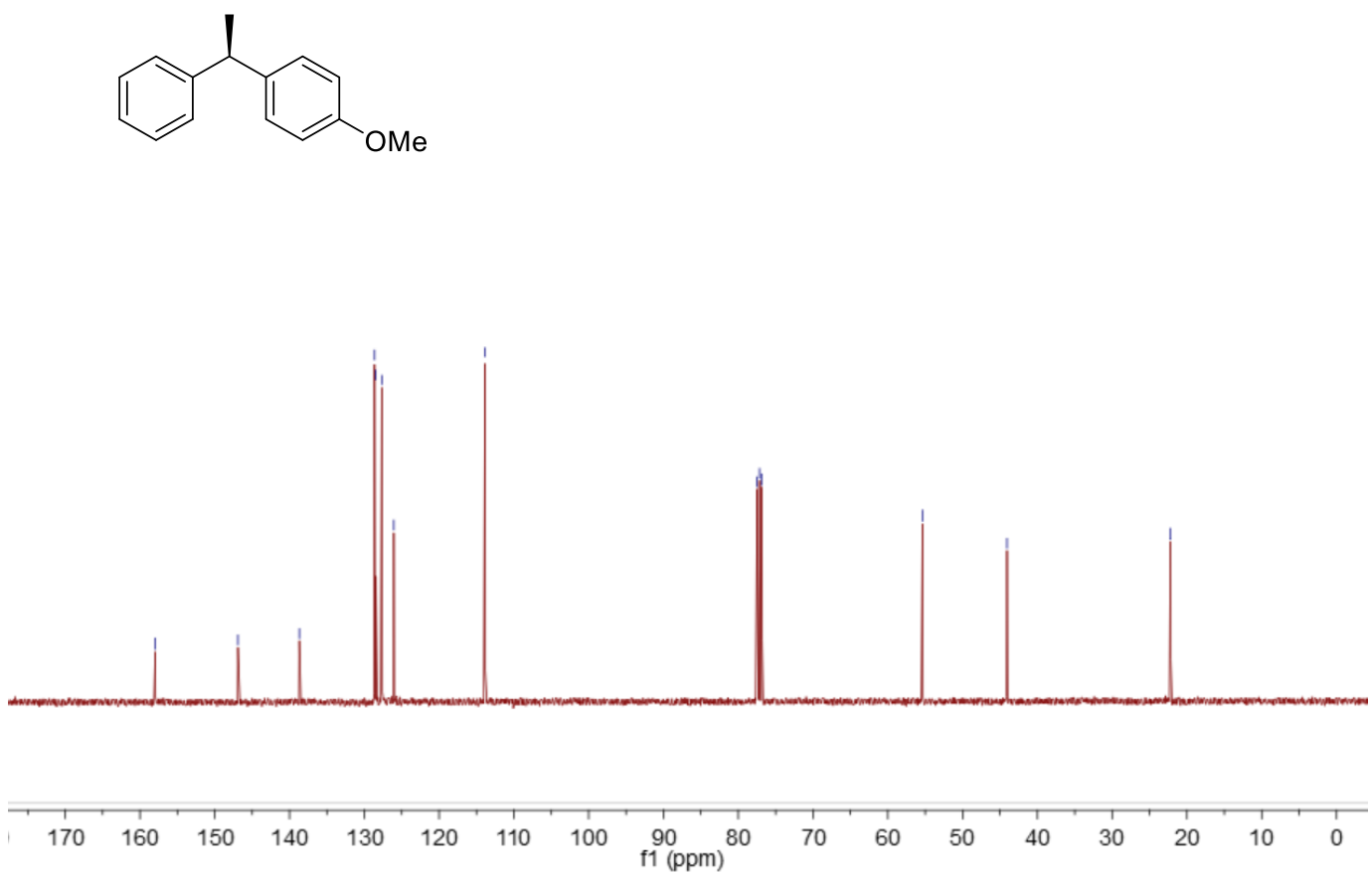

${ }^{13} \mathrm{C}$ NMR $\left(101 \mathrm{M}, \mathrm{CDCl}_{3}\right)$ spectrum of 9ak

S156 


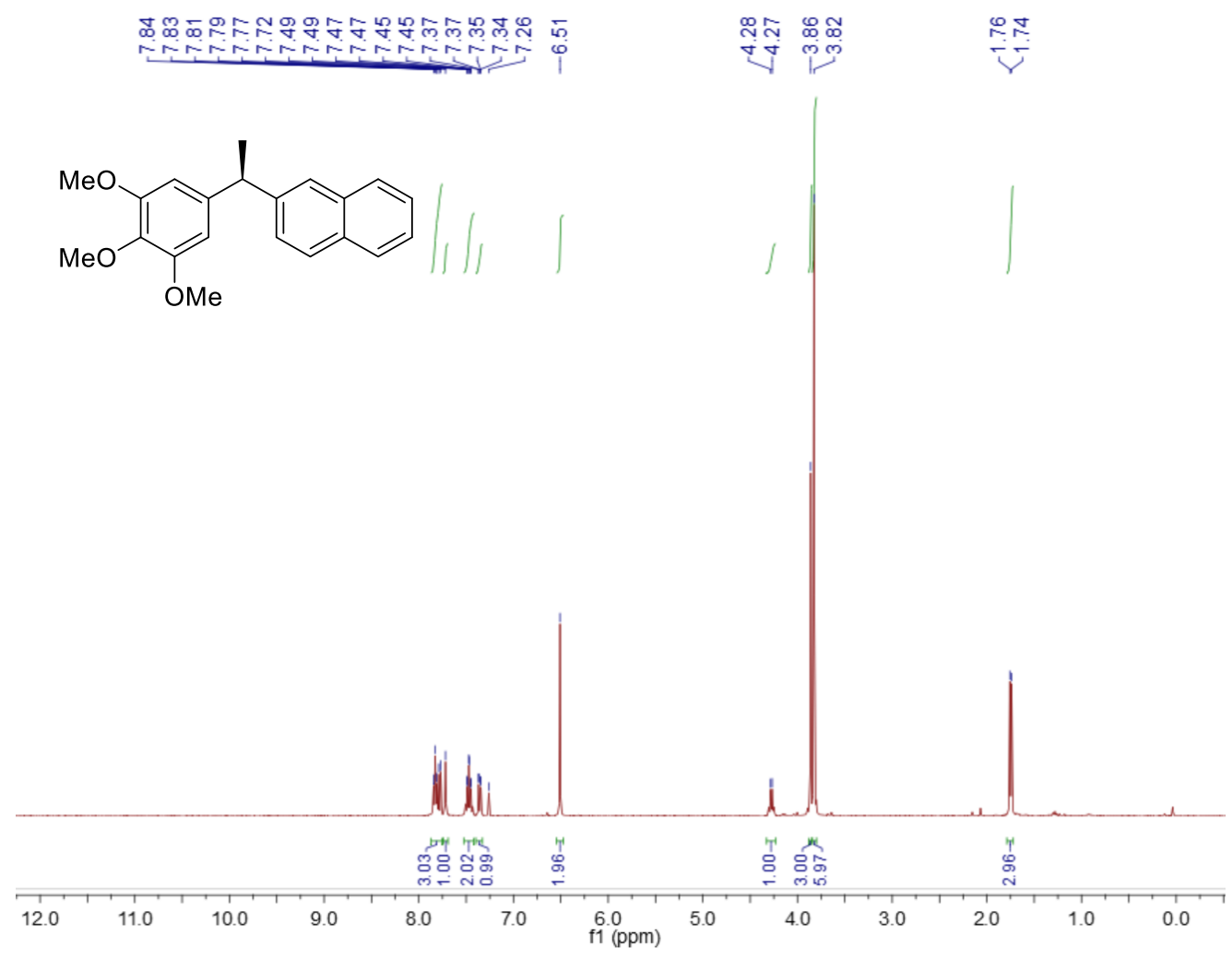

${ }^{1} \mathrm{H}$ NMR (400M, $\mathrm{CDCl}_{3}$ ) spectrum of 9am

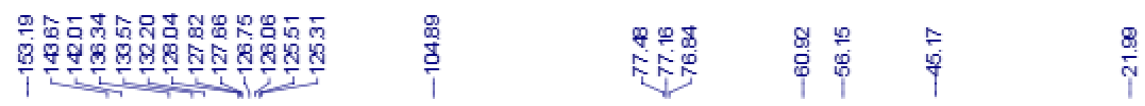<smiles>COc1cc([C@H](C)c2ccc3ccccc3c2)cc(OC)c1OC</smiles>
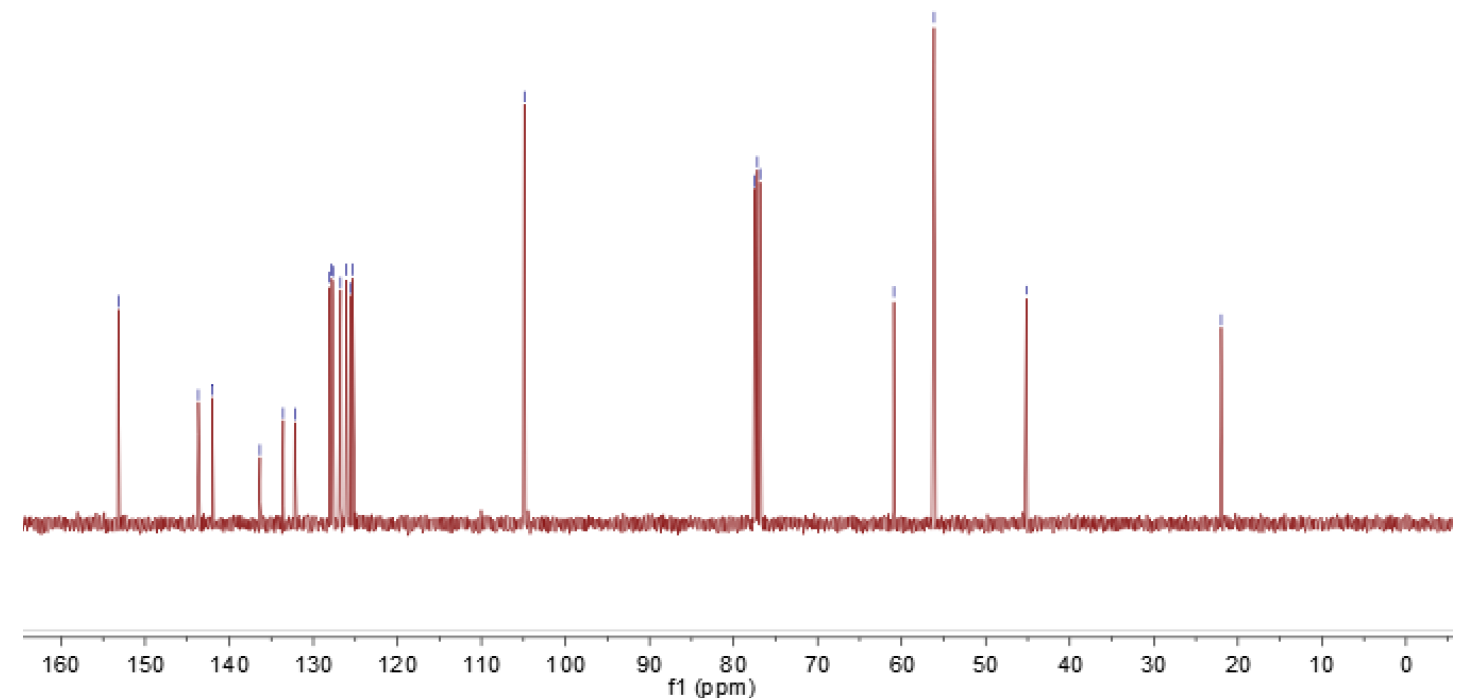

${ }^{13} \mathrm{C}$ NMR $\left(101 \mathrm{M}, \mathrm{CDCl}_{3}\right)$ spectrum of 9am 


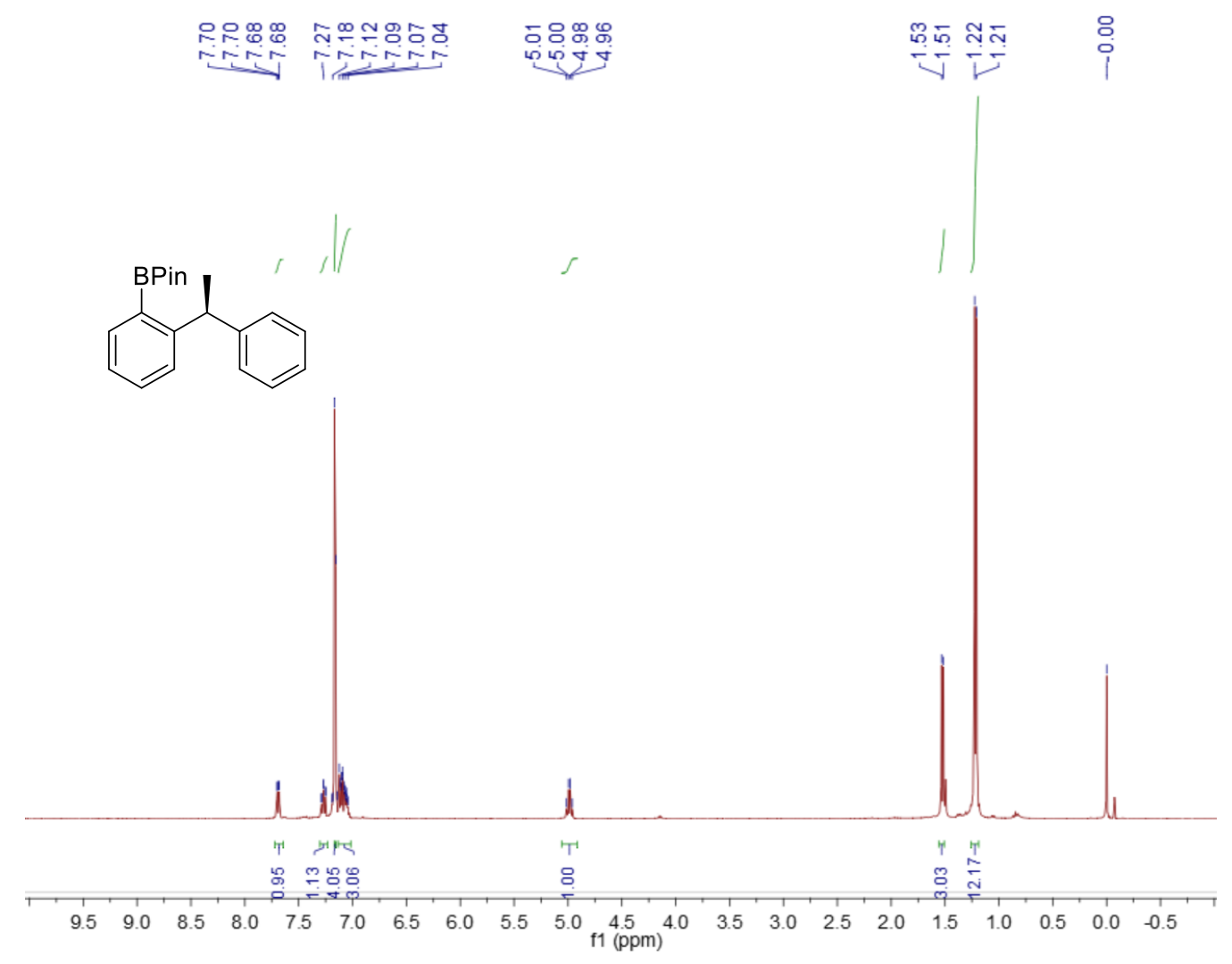

${ }^{1} \mathrm{H}$ NMR $\left(400 \mathrm{M}, \mathrm{CDCl}_{3}\right)$ spectrum of $\mathbf{1 0}$

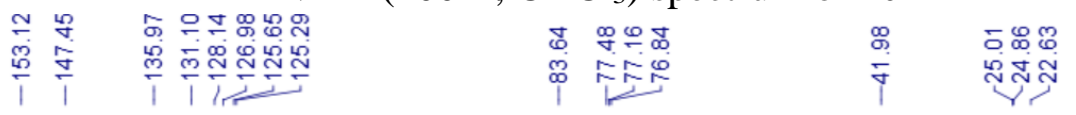<smiles>CC(c1ccccc1)c1ccccc1C(C)c1ccccc1</smiles>
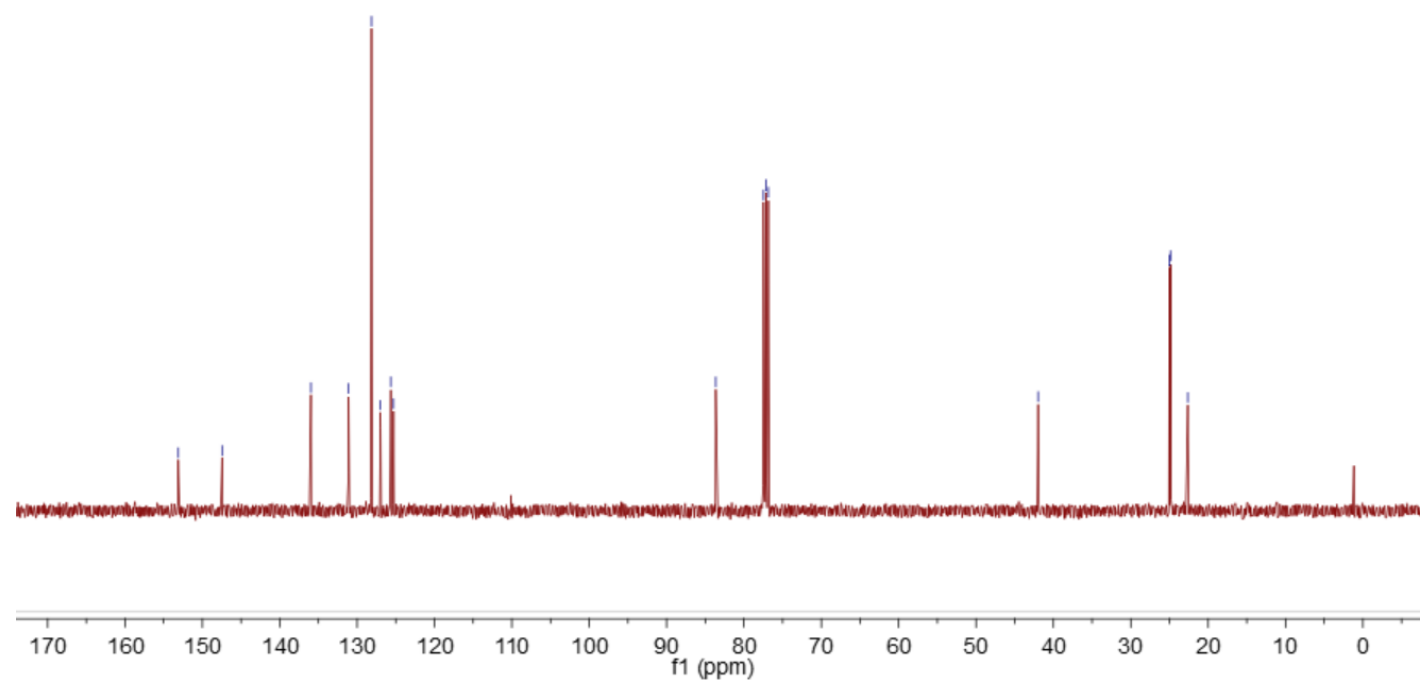

${ }^{13} \mathrm{C} \mathrm{NMR}\left(101 \mathrm{M}, \mathrm{CDCl}_{3}\right)$ spectrum of $\mathbf{1 0}$ 


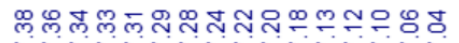
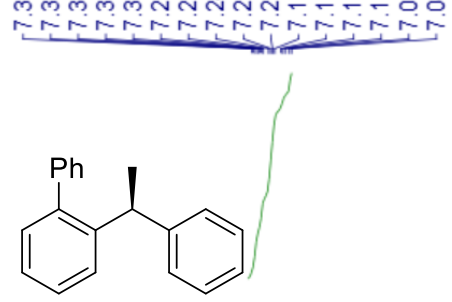

过过王

㒖

i

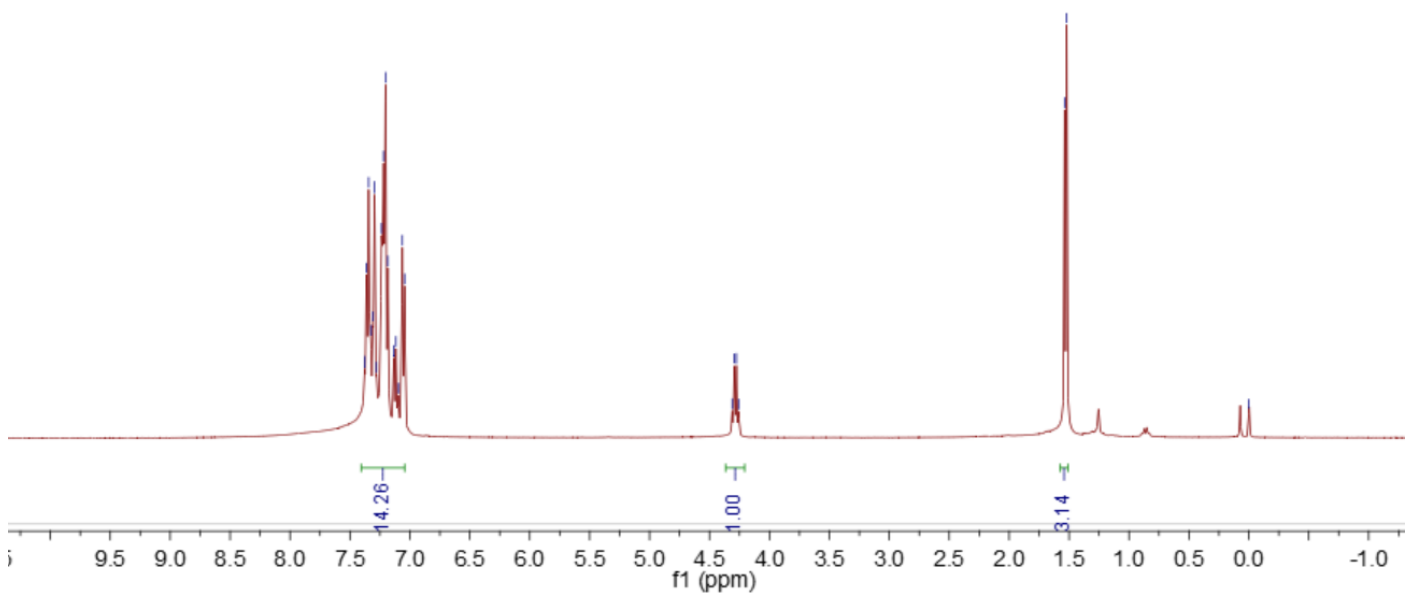

${ }^{1} \mathrm{H}$ NMR (400M, $\mathrm{CDCl}_{3}$ ) spectrum of $\mathbf{1 1}$

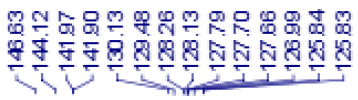

然幂

ฟู
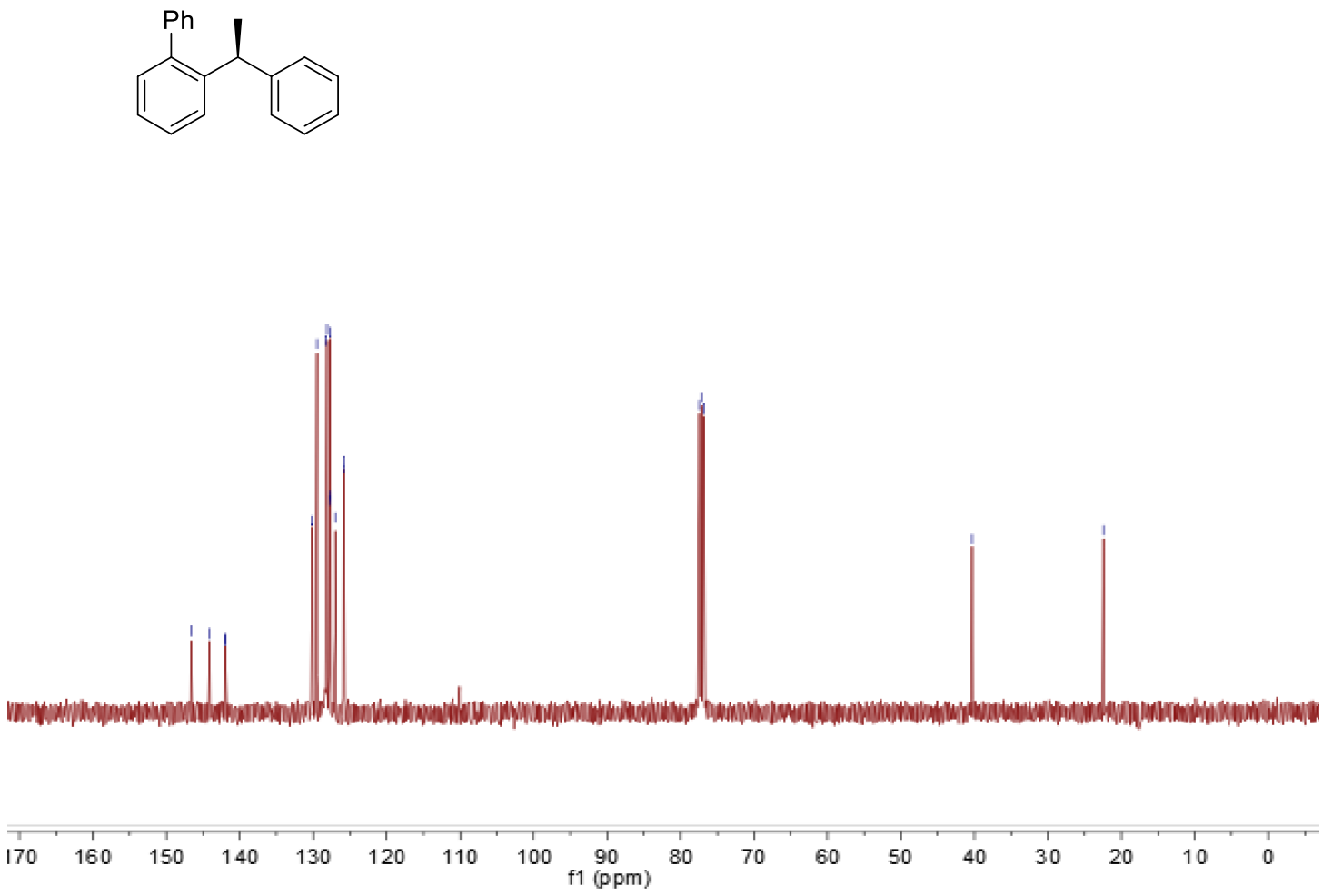

${ }^{13} \mathrm{C}$ NMR $\left(101 \mathrm{M}, \mathrm{CDCl}_{3}\right)$ spectrum of $\mathbf{1 1}$

S159 


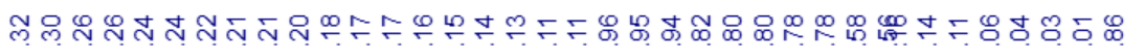

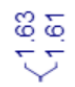

$\stackrel{8}{i}$

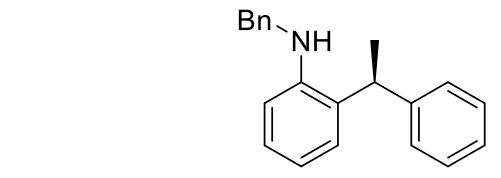

$\int / 1$

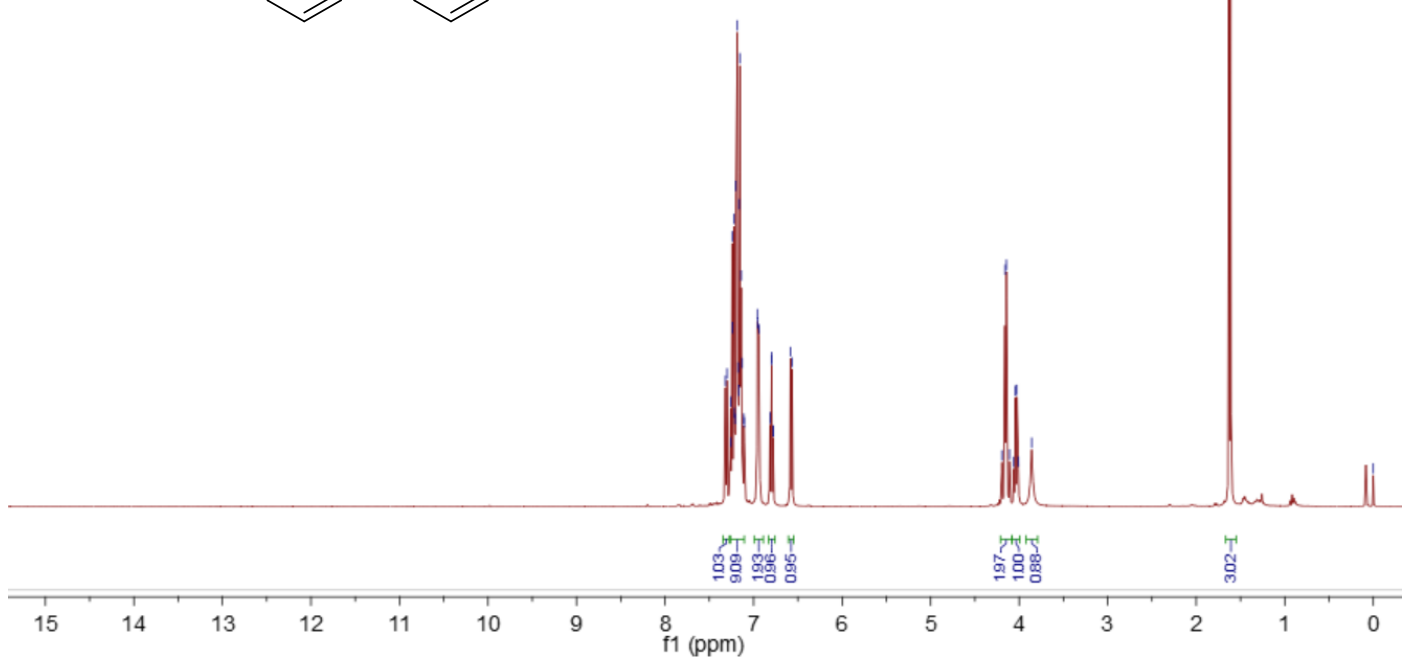

${ }^{1} \mathrm{H}$ NMR (400M, $\mathrm{CDCl}_{3}$ ) spectrum of $\mathbf{1 2}$

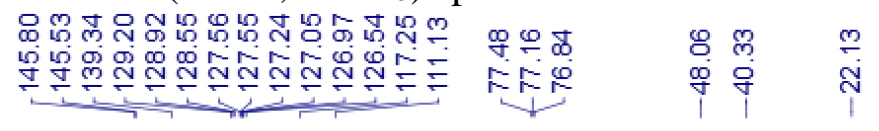
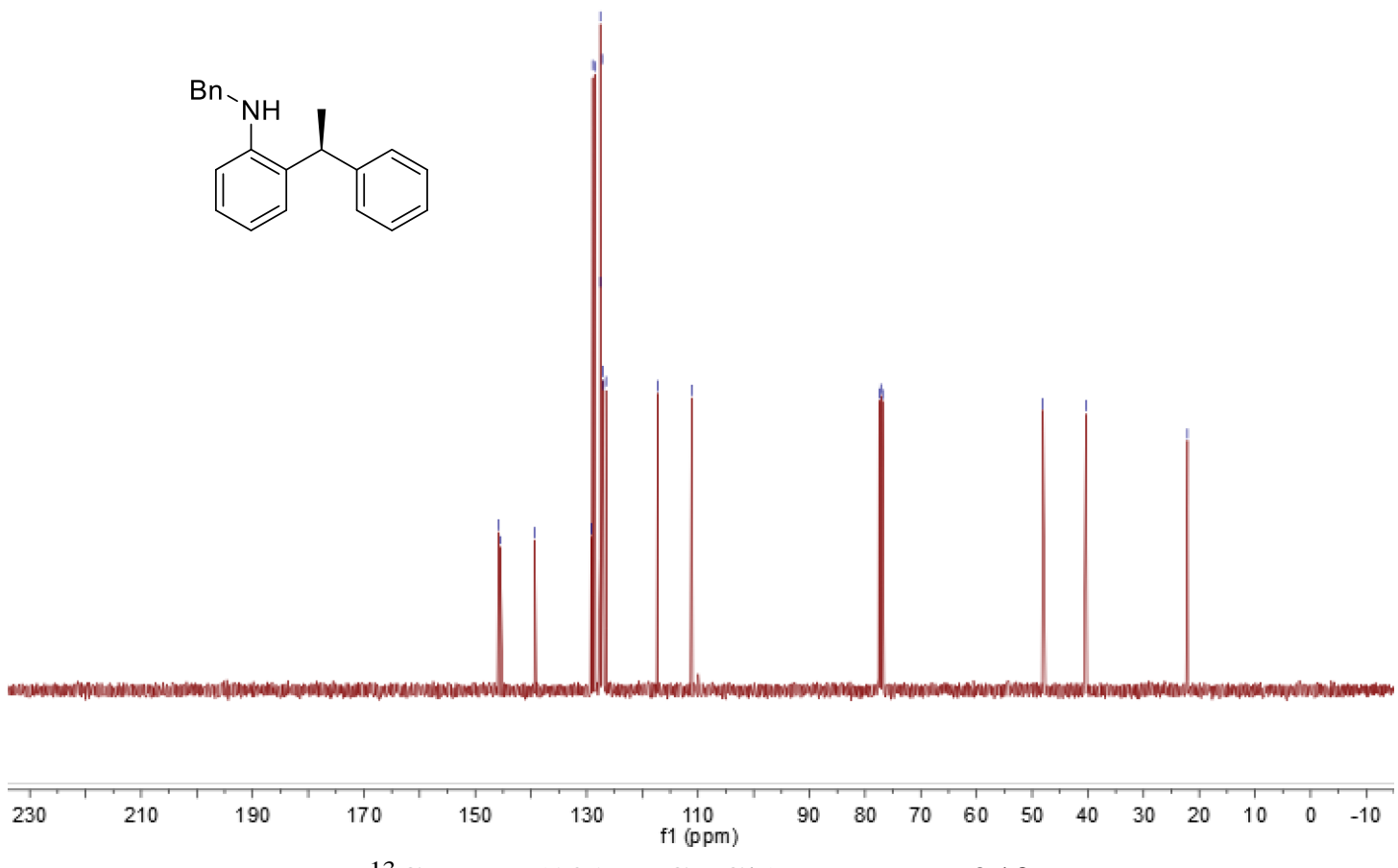

${ }^{13} \mathrm{C}$ NMR $\left(101 \mathrm{M}, \mathrm{CDCl}_{3}\right)$ spectrum of $\mathbf{1 2}$

S160 


\section{Copies of HPLC and SFC Spectra}

\section{(S)-1-methyl-2-(1-phenylethyl)benzene (8a)}

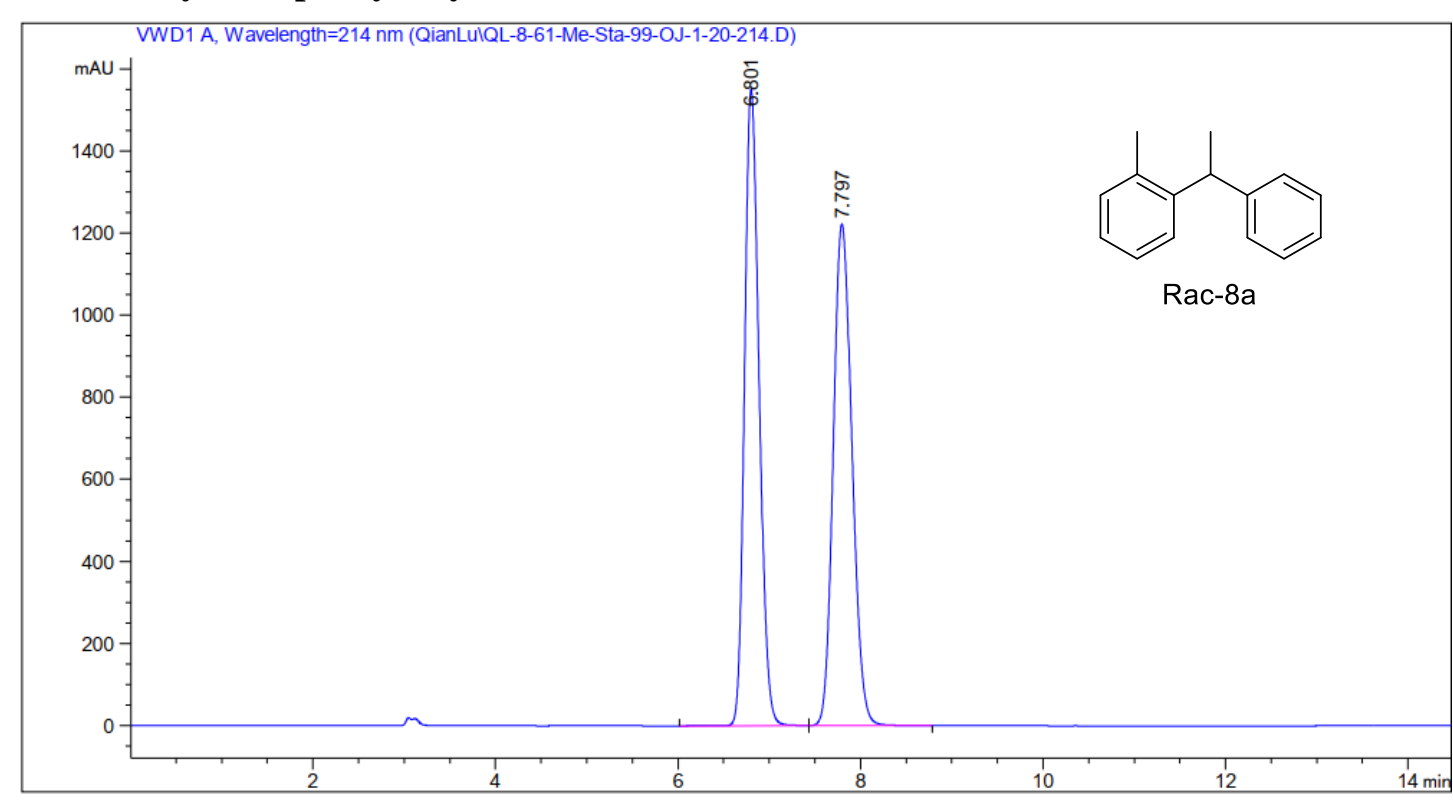

\begin{tabular}{|c|c|c|c|c|c|}
\hline $\begin{array}{c}\text { Peak } \\
\#\end{array}$ & $\begin{array}{l}\text { RetTime Type } \\
\text { [min] }\end{array}$ & $\begin{array}{l}\text { Width } \\
\text { [min] }\end{array}$ & $\begin{array}{c}\text { Area } \\
{\left[\mathrm{mAU}^{*} \mathrm{~s}\right]}\end{array}$ & $\begin{array}{l}\text { Height } \\
\text { [mAU] }\end{array}$ & $\begin{array}{c}\text { Area } \\
\%\end{array}$ \\
\hline & & & & & \\
\hline 1 & 6.801 & 0 & $.72809 \mathrm{e} 4$ & 1551.23938 & 49.8269 \\
\hline 2 & 7.797 BB & 1 & $1.74010 \mathrm{e} 4$ & 1222.11719 & 50.1731 \\
\hline
\end{tabular}

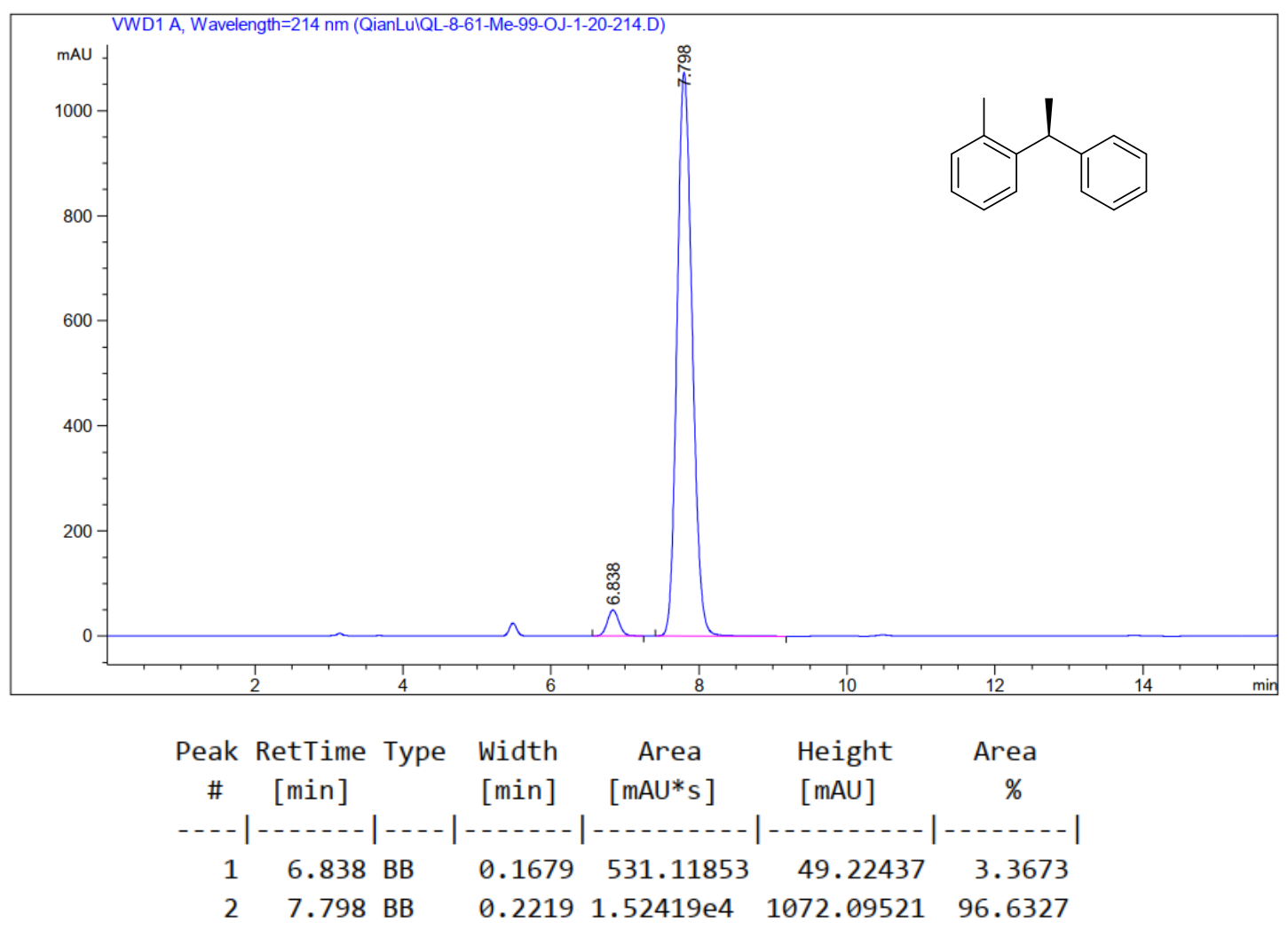


(S)-1-ethyl-2-(1-phenylethyl)benzene (8b)
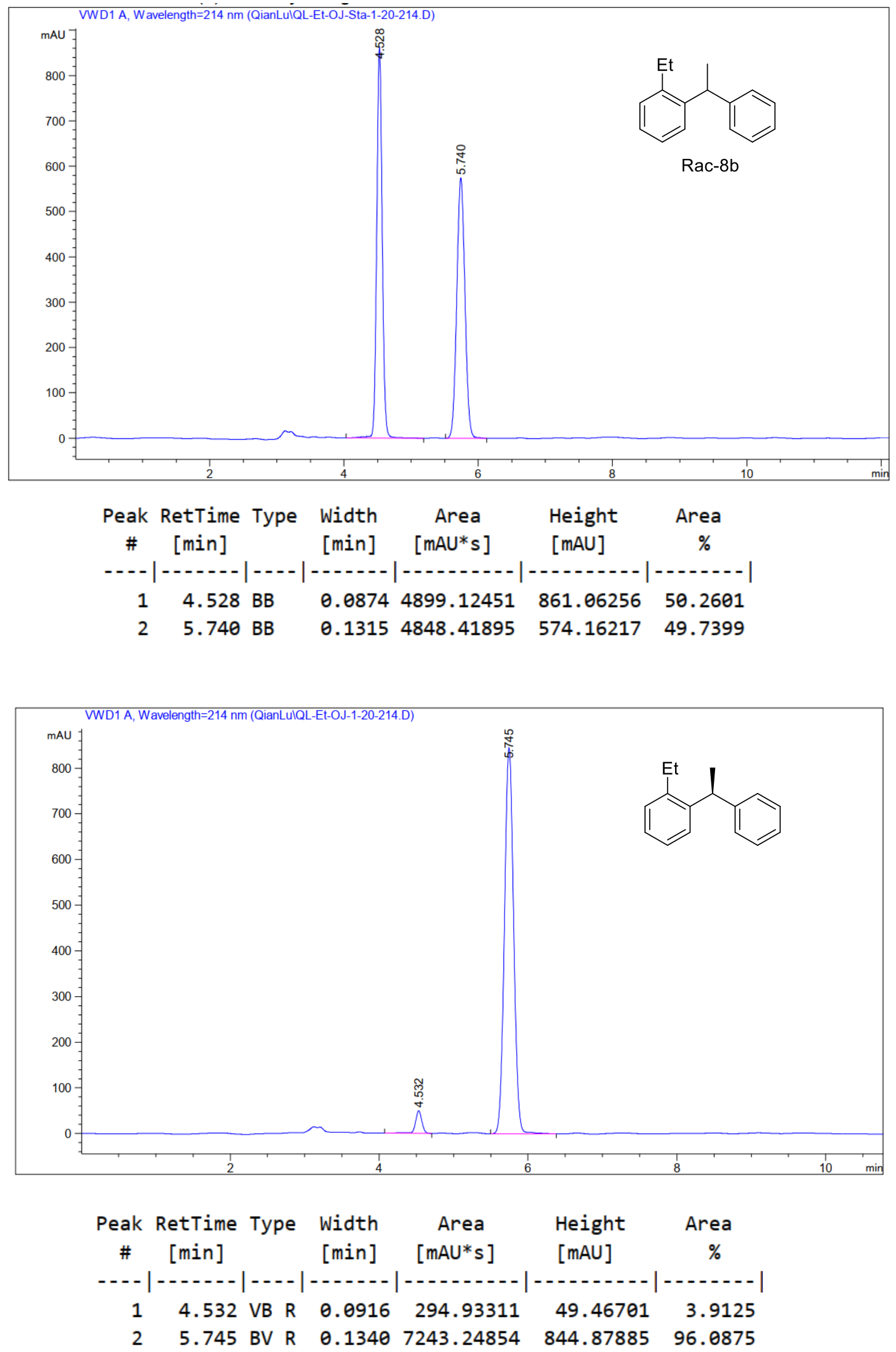
(S)-1-fluoro-2-(1-phenylethyl)benzene (8c)
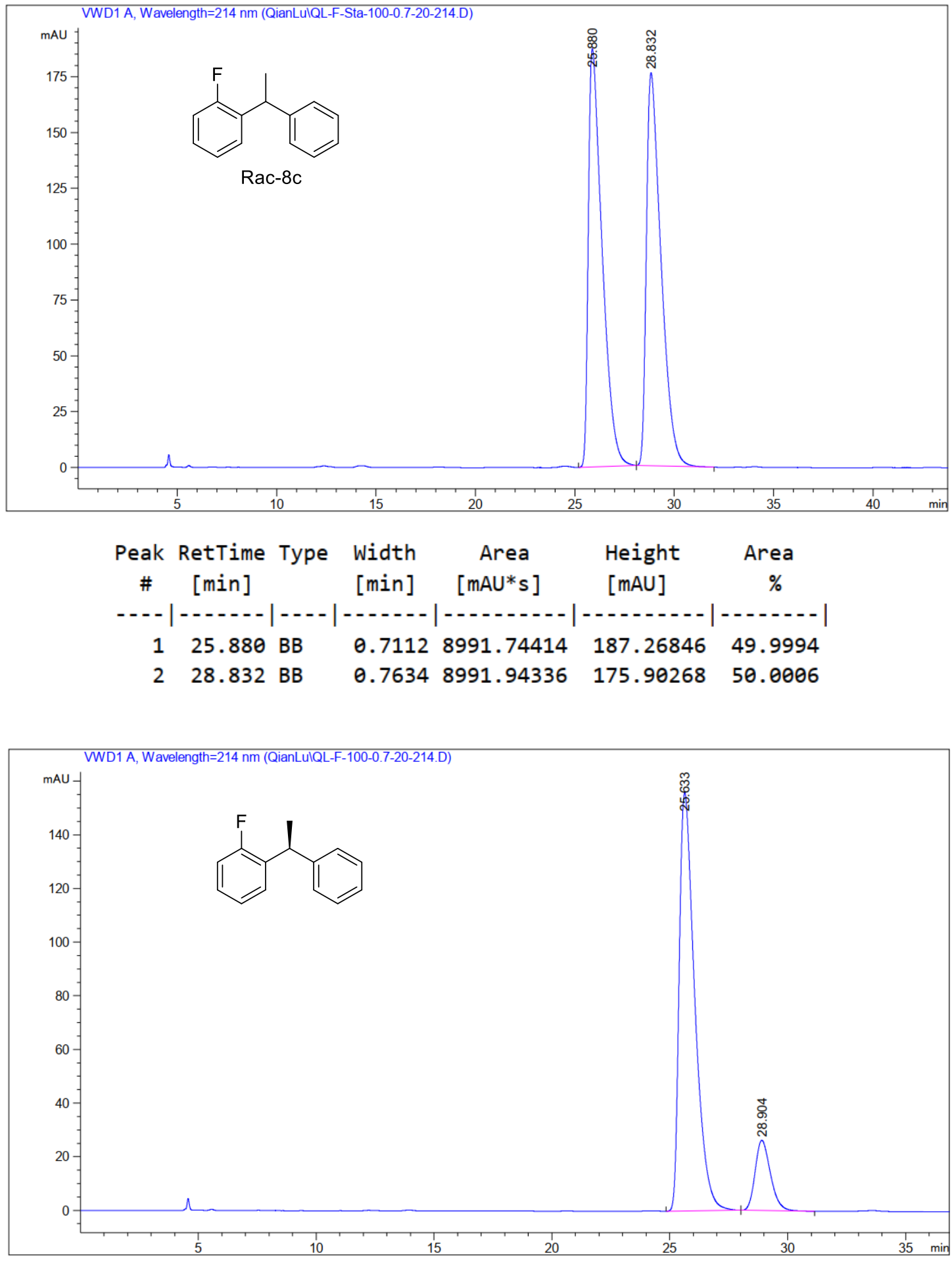

\begin{tabular}{cccccc}
$\begin{array}{c}\text { Peak RetTime Type } \\
\text { \# }\end{array}$ [min] & $\begin{array}{c}\text { Width } \\
\text { [min] }\end{array}$ & $\begin{array}{c}\text { Area } \\
\text { [mAU*s] }\end{array}$ & $\begin{array}{c}\text { Height } \\
\text { [mAU] }\end{array}$ & $\begin{array}{c}\text { Area } \\
\%\end{array}$ \\
\hline 1 & 25.633 BB & 0.6918 & 7120.73535 & 155.91840 & 85.7508 \\
2 & 28.904 BB & 0.6896 & 1183.24988 & 26.21512 & 14.2492
\end{tabular}


(S)-1-chloro-2-(1-phenylethyl)benzene (8d)
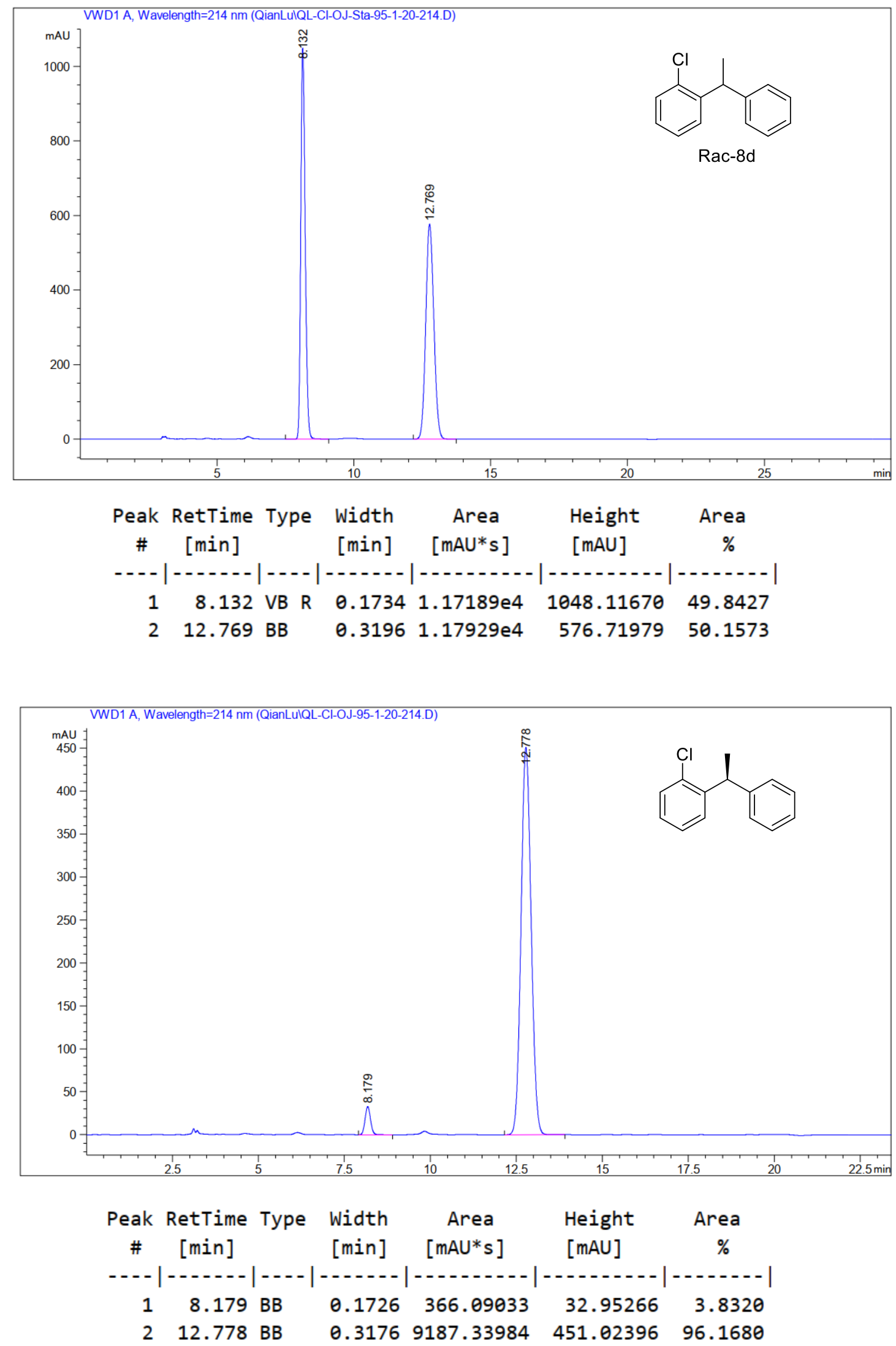
(S)-1-bromo-2-(1-phenylethyl)benzene (8e)
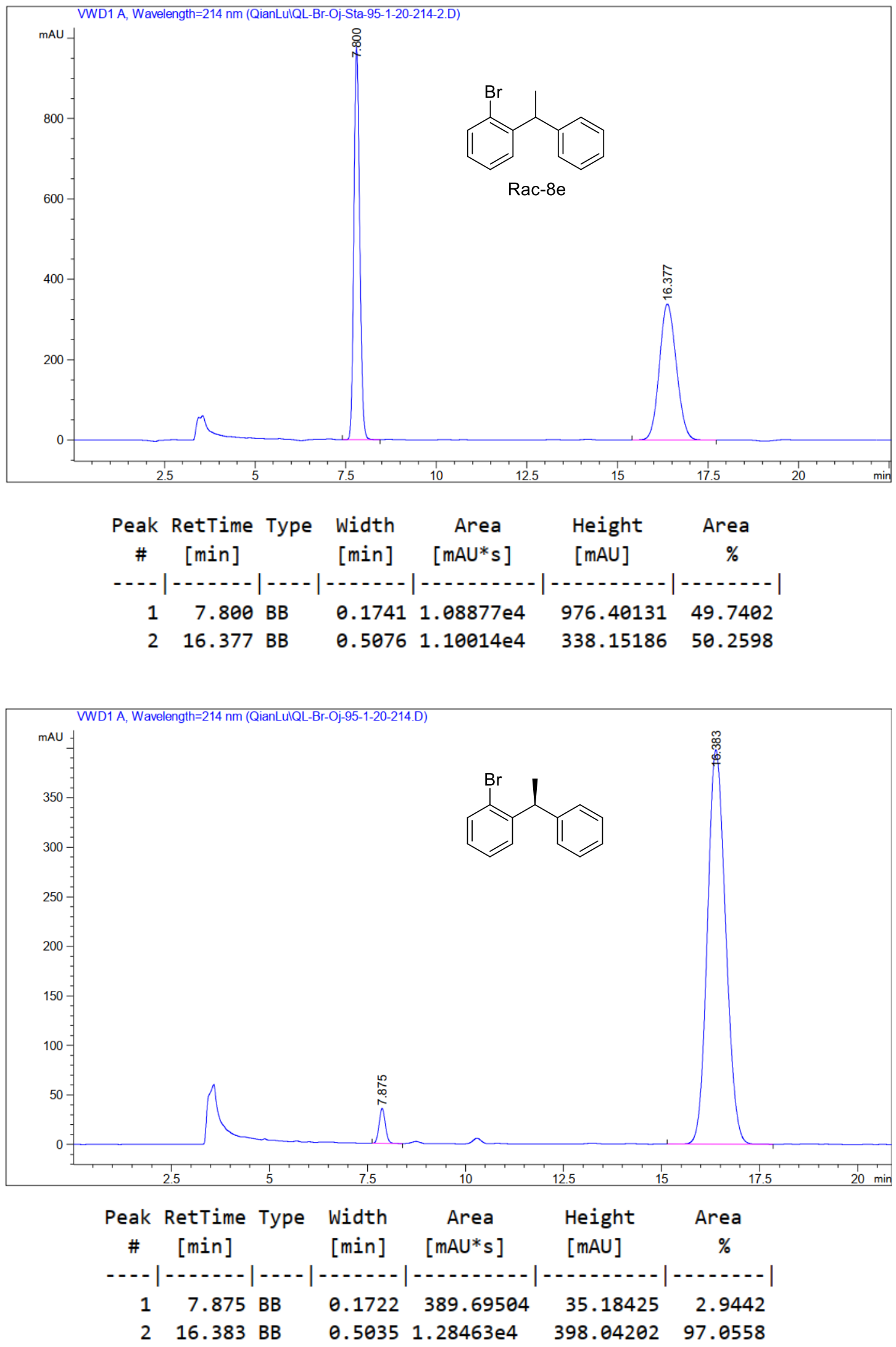
(S)-1-methoxy-2-(1-phenylethyl)benzene (8f)
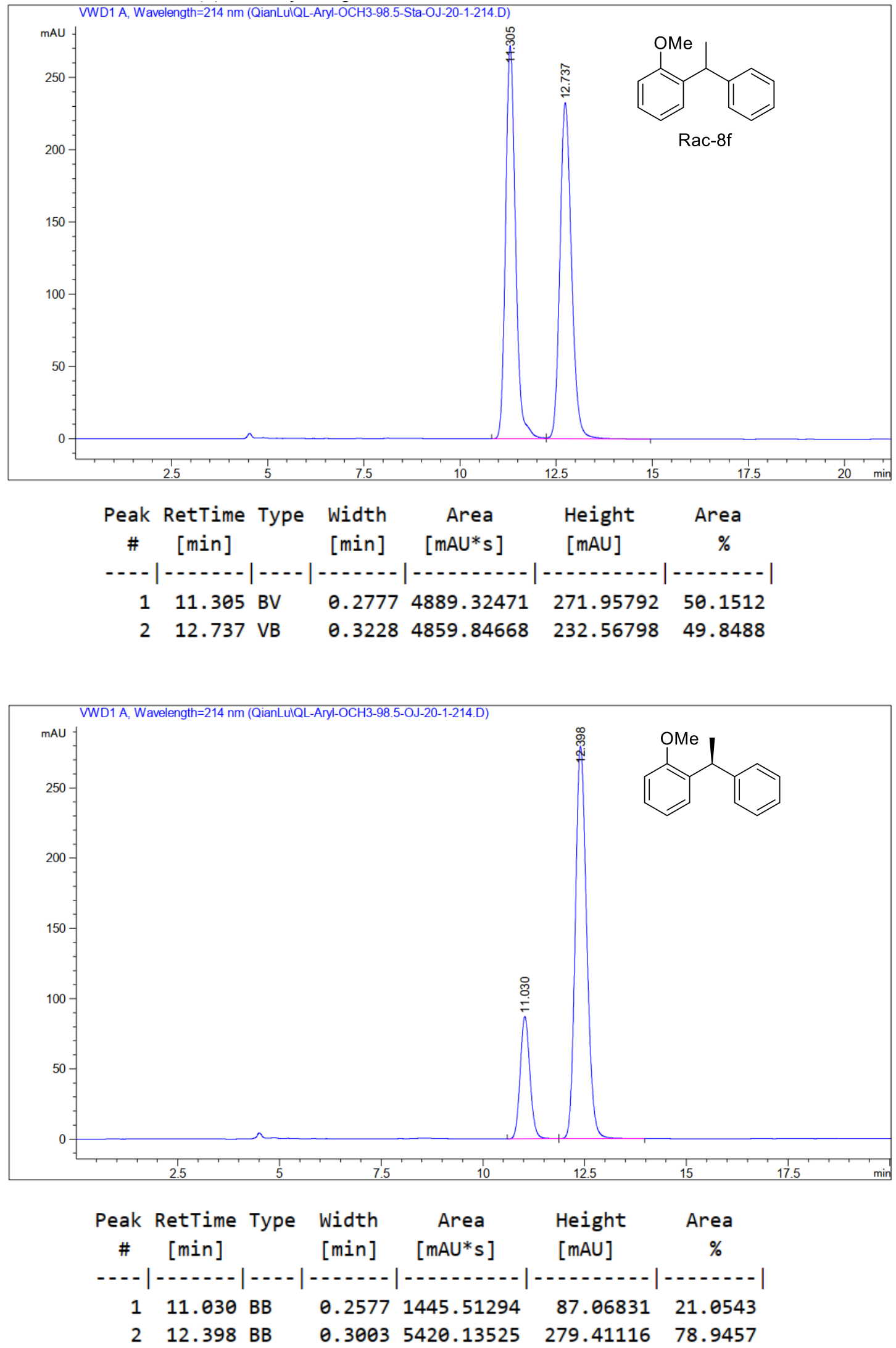
Ethyl (S)-2-(1-phenylethyl)benzoate (8g)
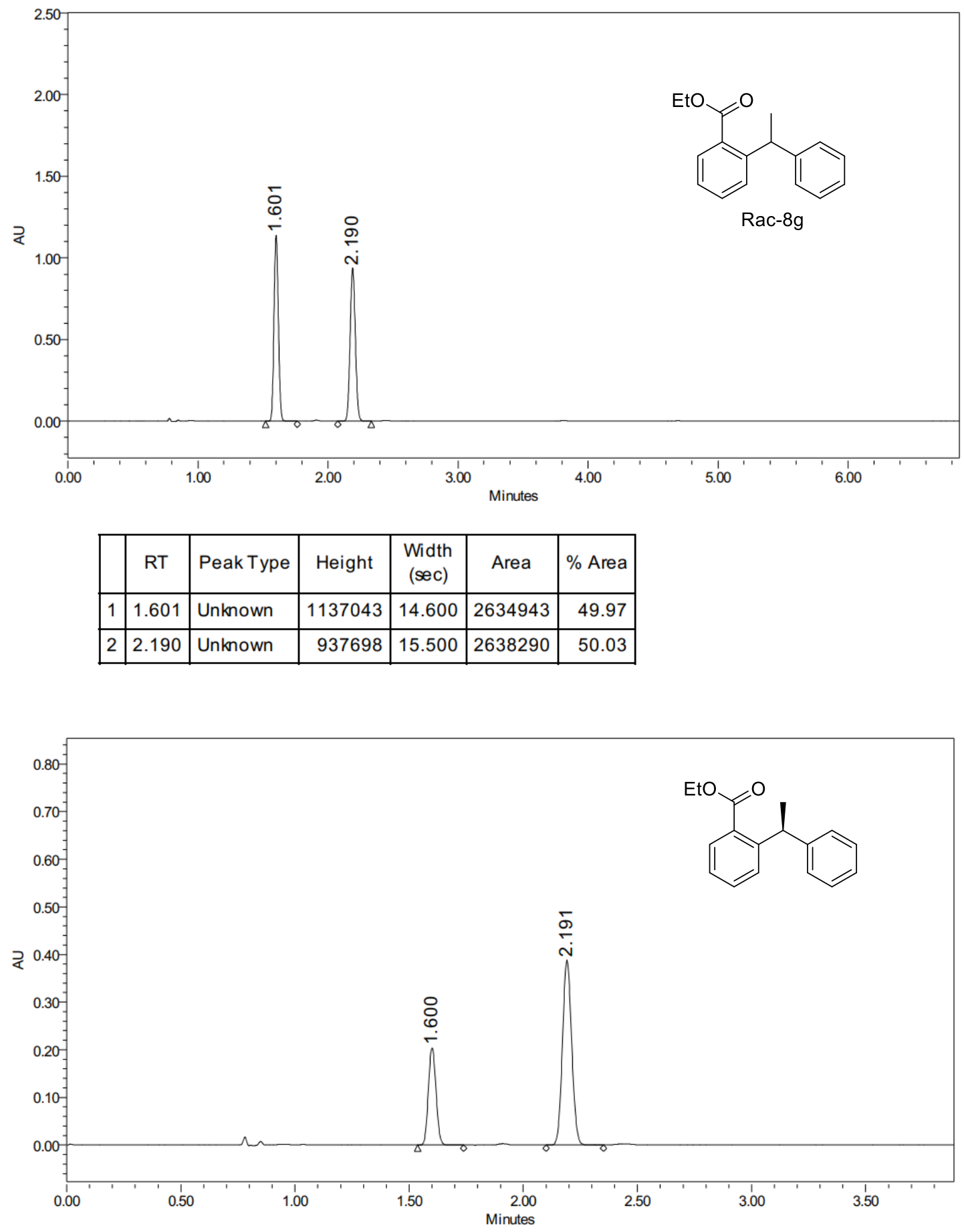

\begin{tabular}{|c|c|l|c|c|c|c|}
\hline & RT & Peak Type & Height & $\begin{array}{c}\text { Width } \\
(\mathrm{sec})\end{array}$ & Area & $\%$ Area \\
\hline 1 & 1.600 & Unknown & 203819 & 12.100 & 472301 & 30.12 \\
\hline 2 & 2.191 & Unknown & 388163 & 15.100 & 1095522 & 69.88 \\
\hline
\end{tabular}


(S)-1-(1-phenylethyl)naphthalene (8h)
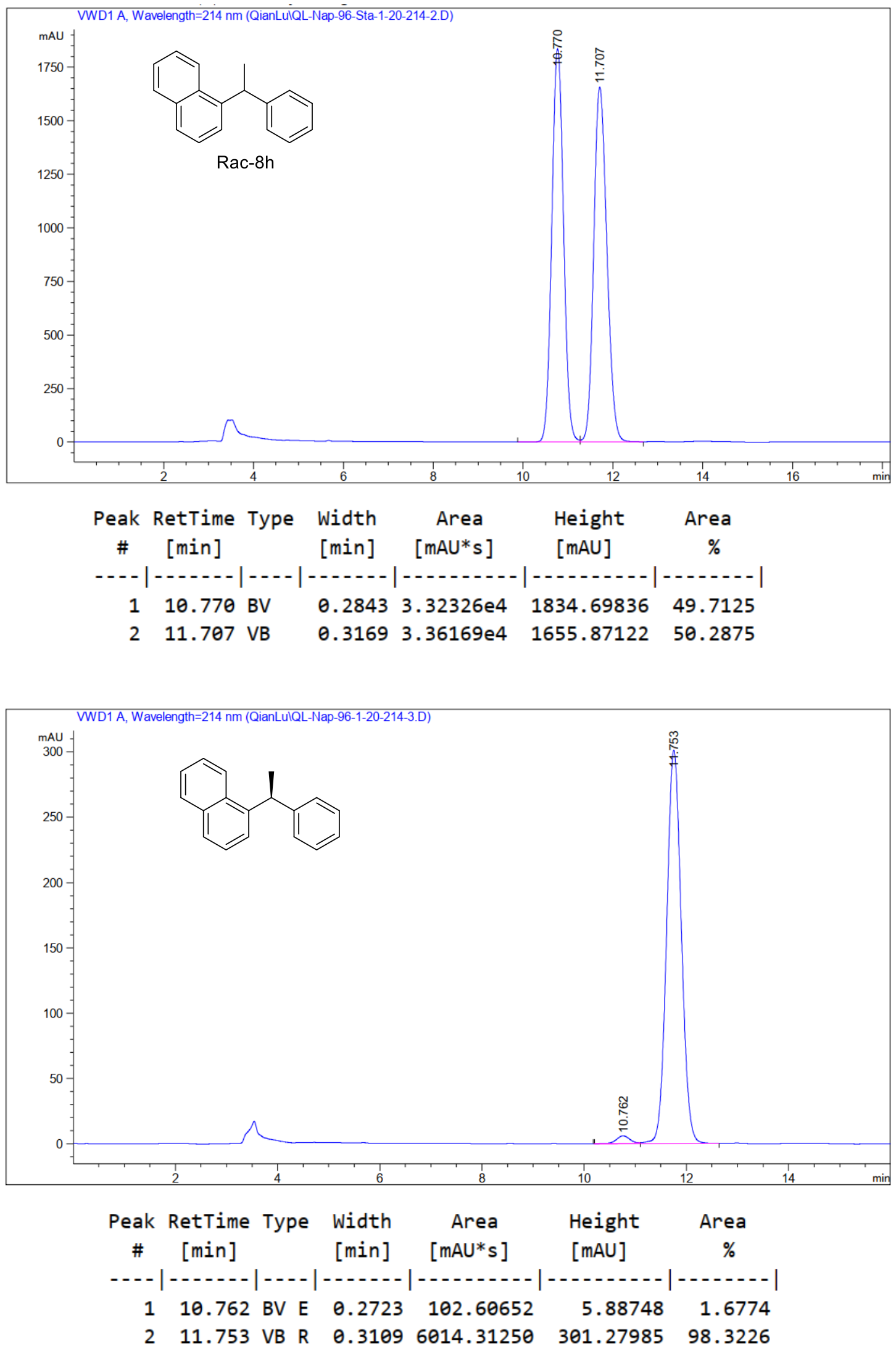
(S)-1-fluoro-2-methyl-3-(1-phenylethyl)benzene (8i)

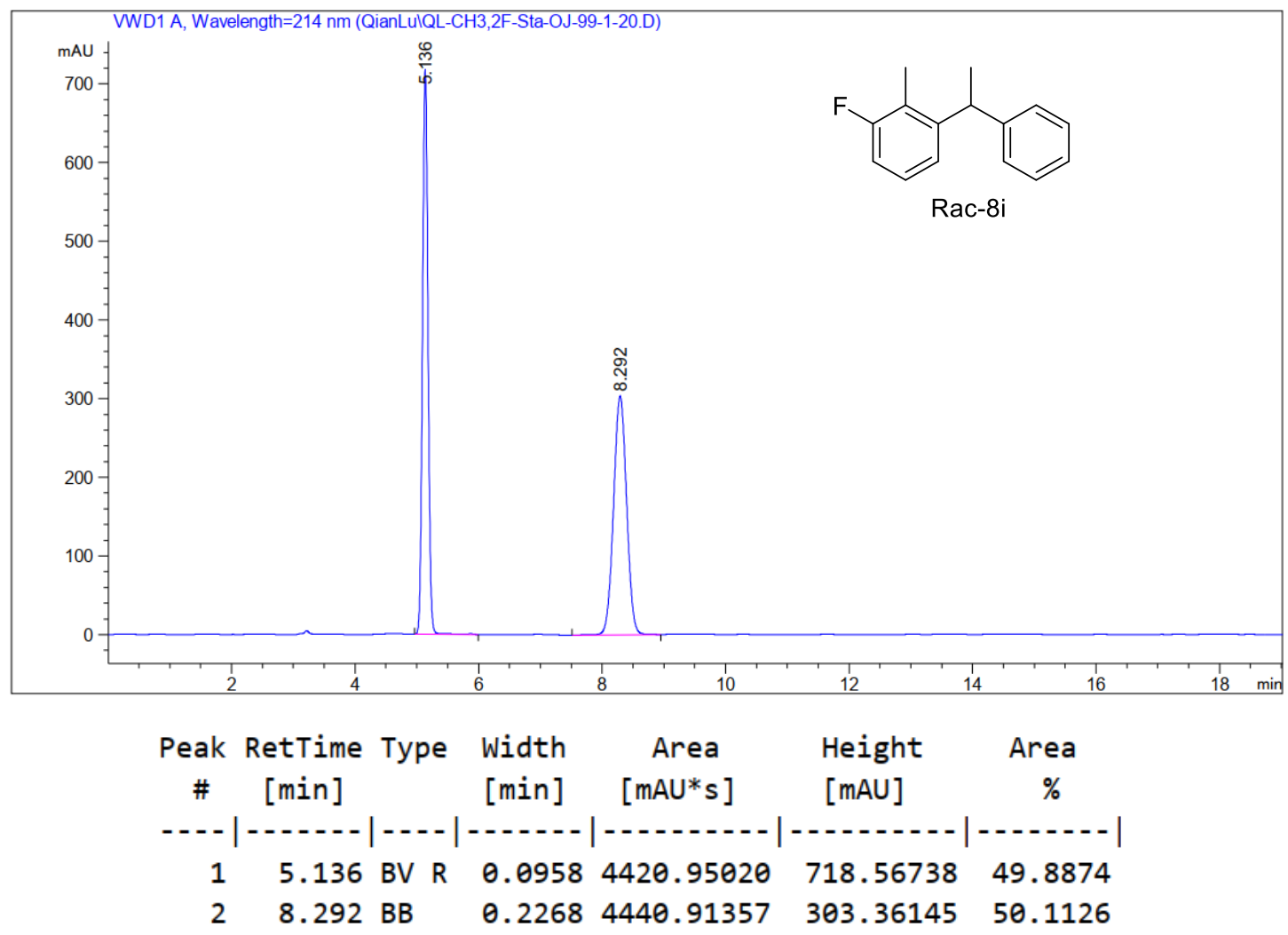

$\mathrm{n}$

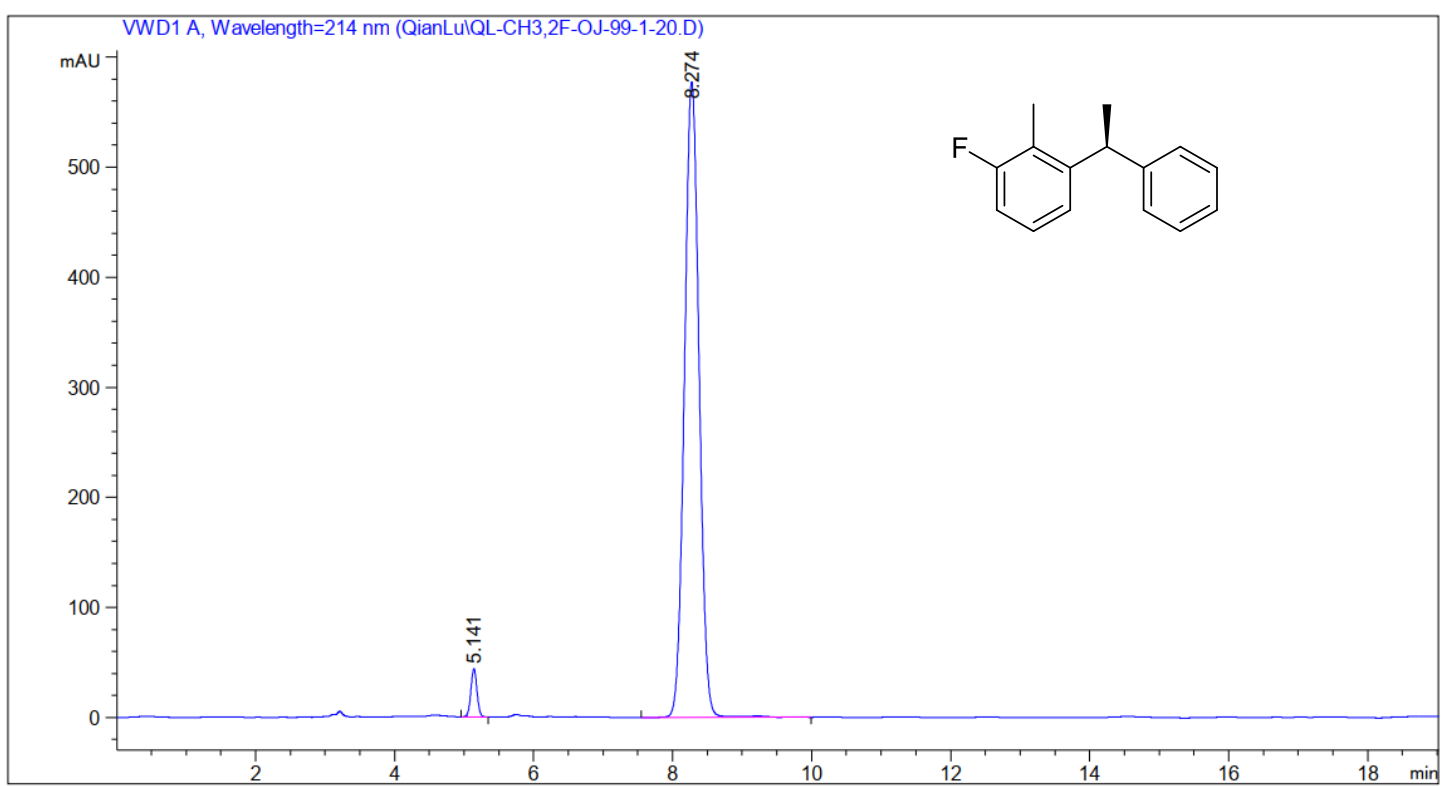

\begin{tabular}{cccccc}
$\begin{array}{c}\text { Peak RetTime Type width } \\
\text { [min] }\end{array}$ & $\begin{array}{c}\text { Area } \\
\text { [min] }\end{array}\left[\begin{array}{c}\text { Height } \\
\text { [mAUs }\end{array}\right.$ & \multicolumn{1}{c}{$\begin{array}{c}\text { Area } \\
\text { [mAU] }\end{array}$} & \multicolumn{1}{c}{$\%$} \\
\hline 1 & 5.141 BB & 0.0949 & 266.12952 & 43.81913 & 3.0498 \\
2 & 8.274 BV R & 0.2279 & 8459.95313 & 577.51935 & 96.9502
\end{tabular}


(S)-4-fluoro-2-methyl-1-(1-phenylethyl)benzene (8j)
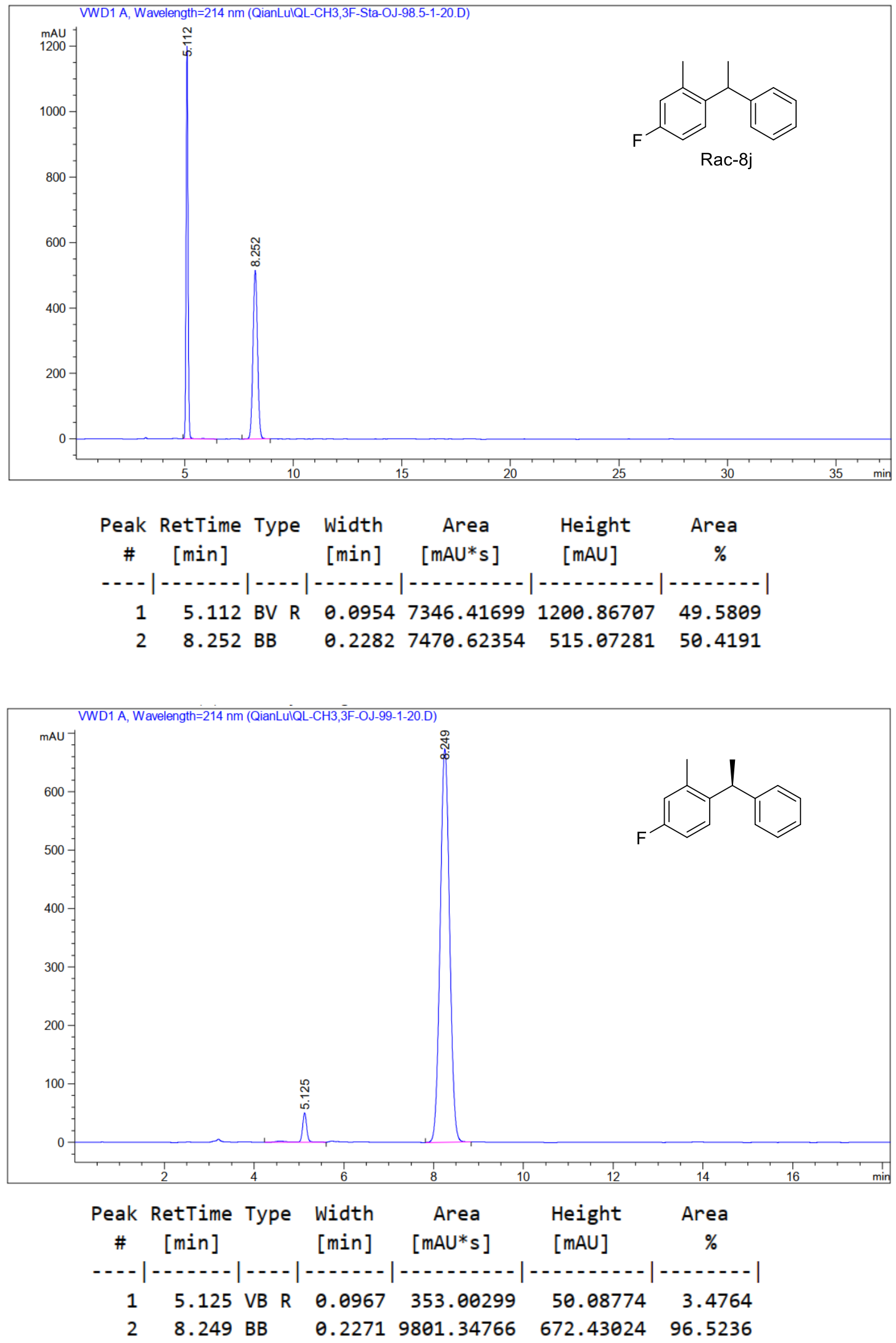
(S)-4-fluoro-1-methyl-2-(1-phenylethyl)benzene (8k)

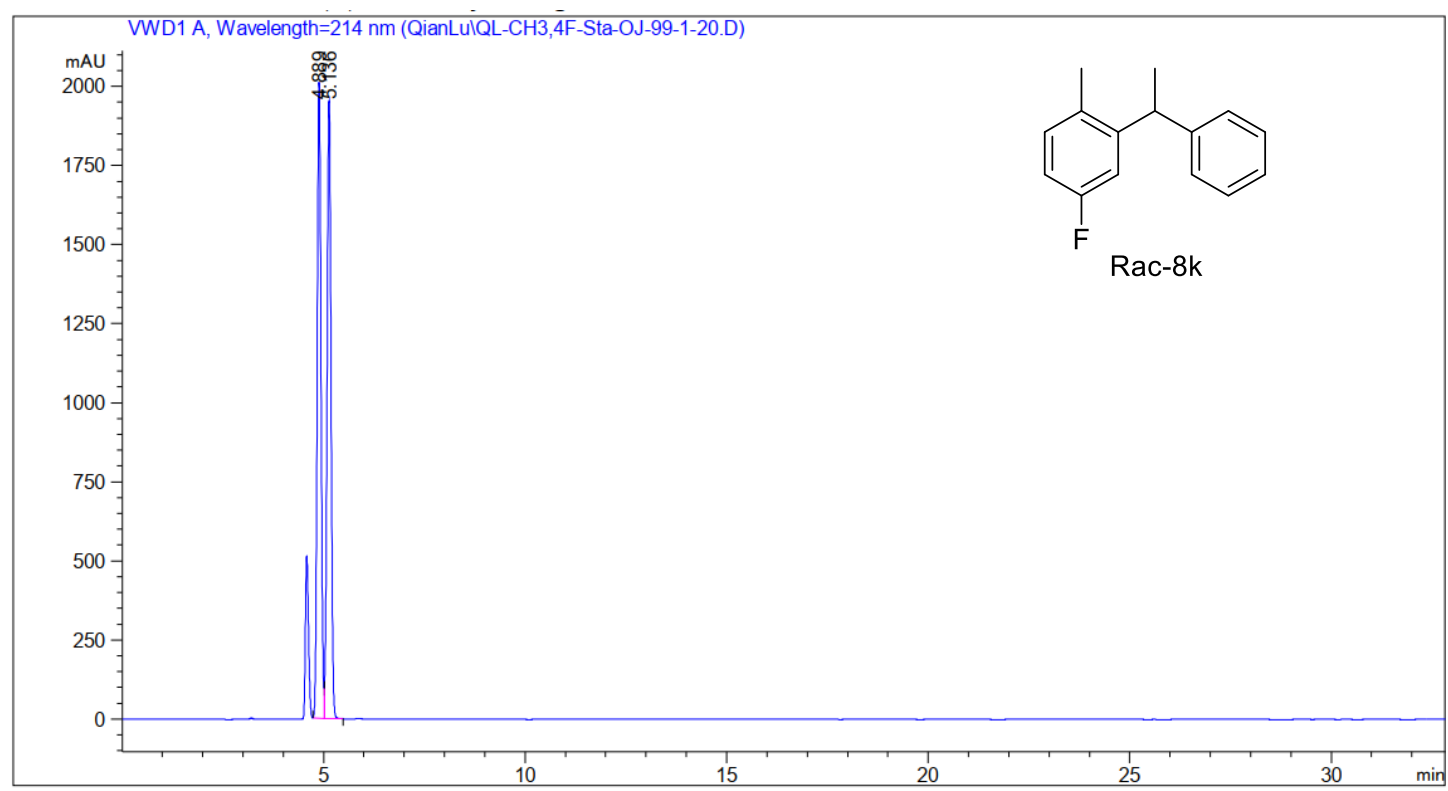

\begin{tabular}{cccccc}
$\begin{array}{c}\text { Peak RetTime Type } \\
\text { [min] }\end{array}$ & $\begin{array}{c}\text { Width } \\
\text { [min] }\end{array}$ & $\begin{array}{c}\text { Area } \\
{[\text { mAU*s] }}\end{array}$ & $\begin{array}{c}\text { Height } \\
{[\mathrm{mAU}]}\end{array}$ & $\begin{array}{c}\text { Area } \\
\%\end{array}$ \\
\hdashline 1 & 4.889 BV & 0.1033 & $1.31473 \mathrm{e} 4$ & 2010.79407 & 49.7085 \\
2 & 5.136 VB & 0.1065 & $1.33015 \mathrm{e} 4$ & 1952.69507 & 50.2915
\end{tabular}

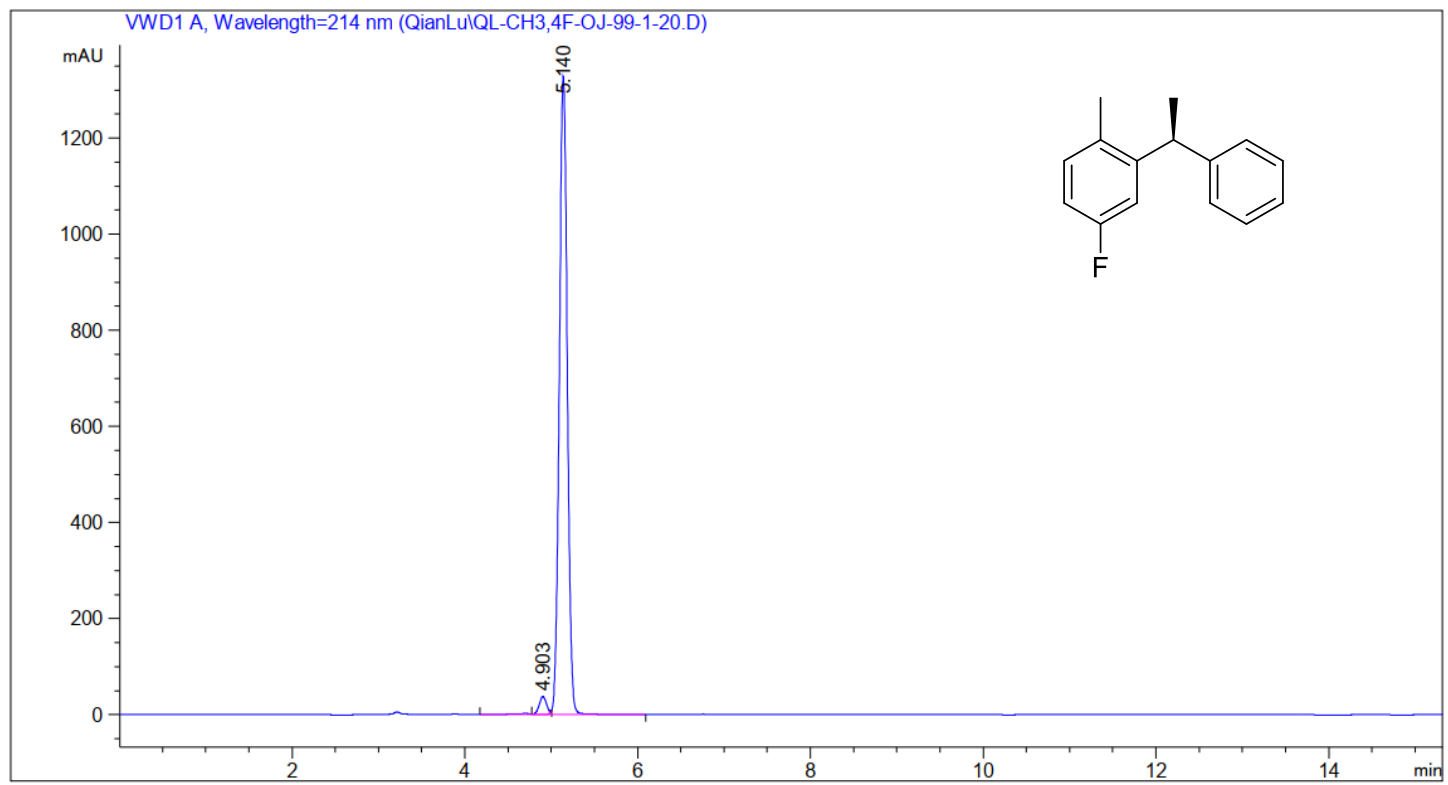

\begin{tabular}{cccccc}
$\begin{array}{c}\text { Peak RetTime Type } \\
\text { \# } \\
\text { [min] }\end{array}$ & $\begin{array}{c}\text { Width } \\
\text { [min] }\end{array}$ & $\begin{array}{c}\text { Area } \\
\text { [mAU* }]\end{array}$ & \multicolumn{1}{l}{$\begin{array}{l}\text { Height } \\
\text { [mAU] }\end{array}$} & \multicolumn{1}{c}{$\begin{array}{c}\text { Area } \\
\%\end{array}$} \\
\hdashline 1 & 4.903 VV E & 0.0911 & 219.89171 & 37.64965 & 2.4995 \\
2 & 5.140 VV R & 0.1002 & 8577.38965 & 1327.73718 & 97.5005
\end{tabular}


(S)-1,2-dimethyl-3-(1-phenylethyl)benzene (8I)
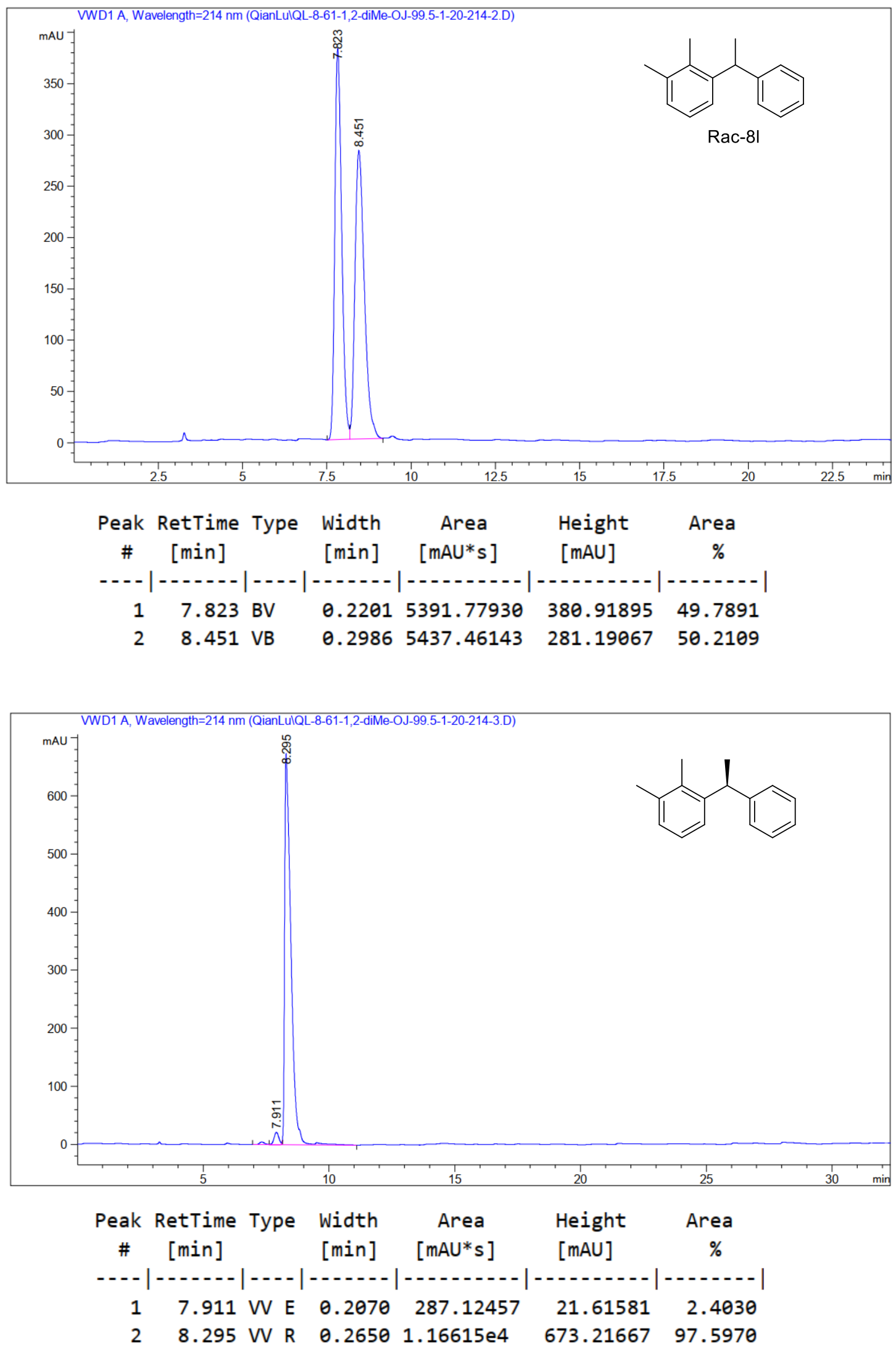
(S)-2,4-dimethyl-1-(1-phenylethyl)benzene (8m)
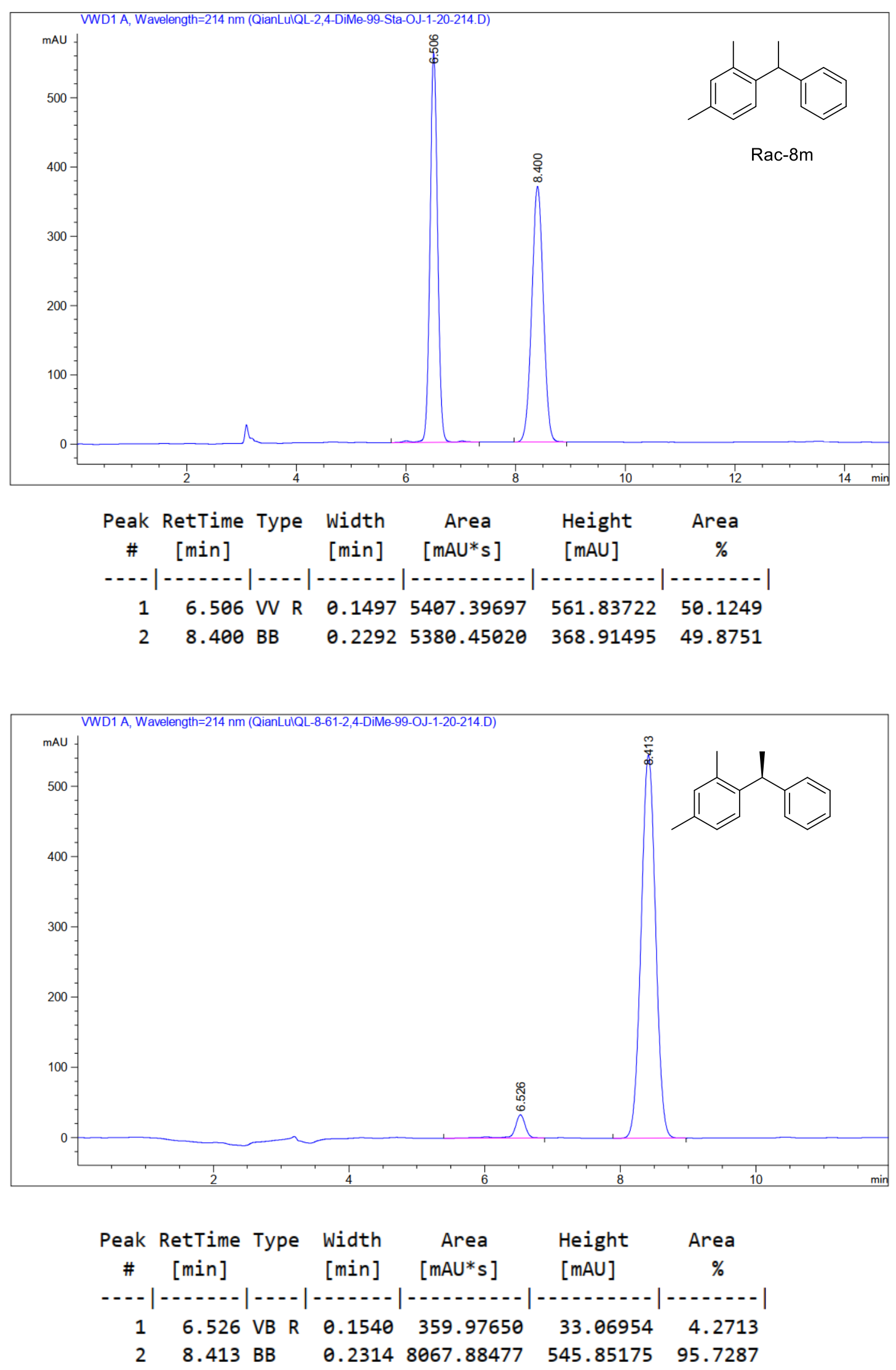
(S)-1,4-dimethyl-2-(1-phenylethyl)benzene (8n)
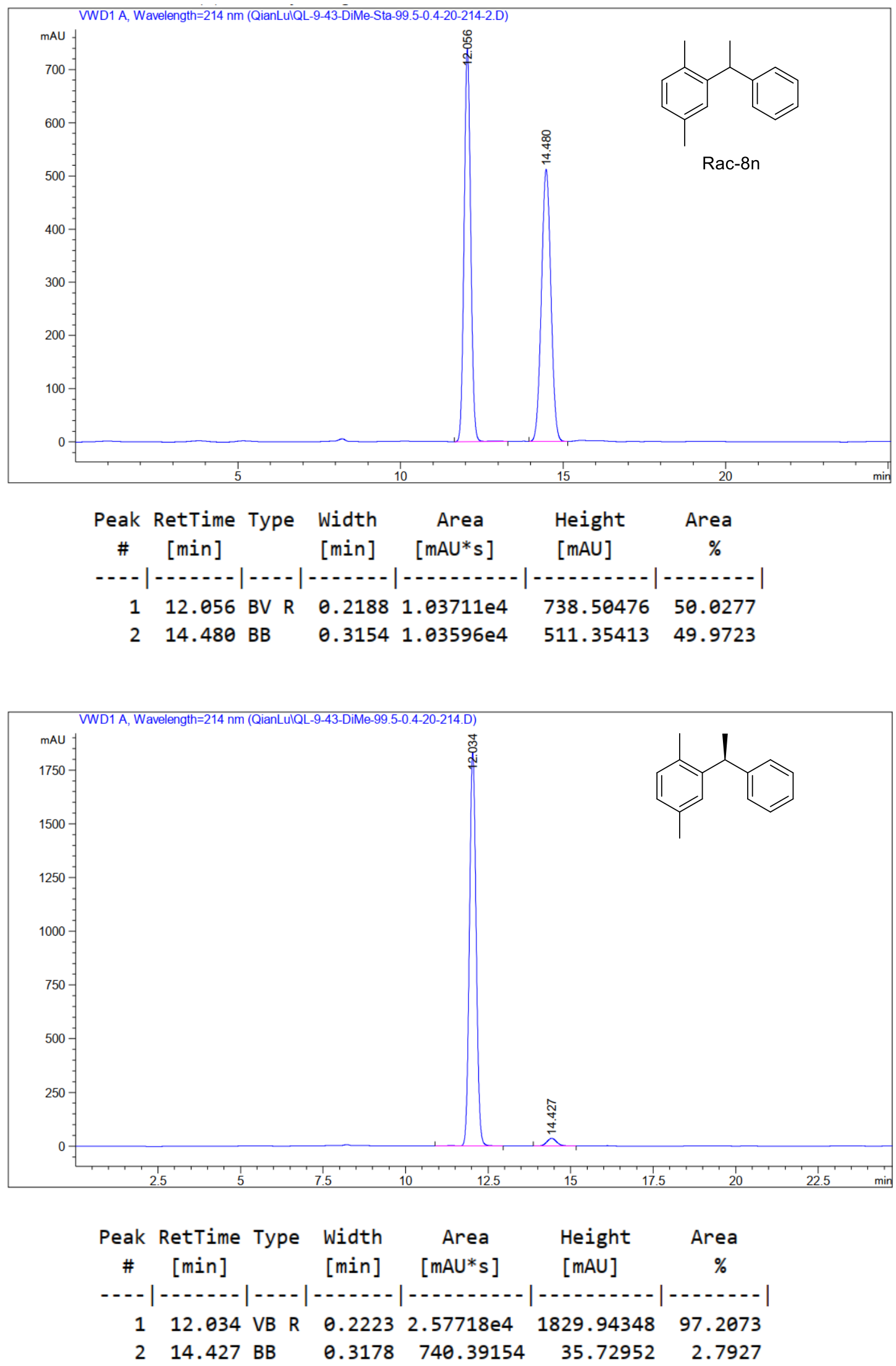
(S)-4-chloro-2-methyl-1-(1-phenylethyl)benzene (80)
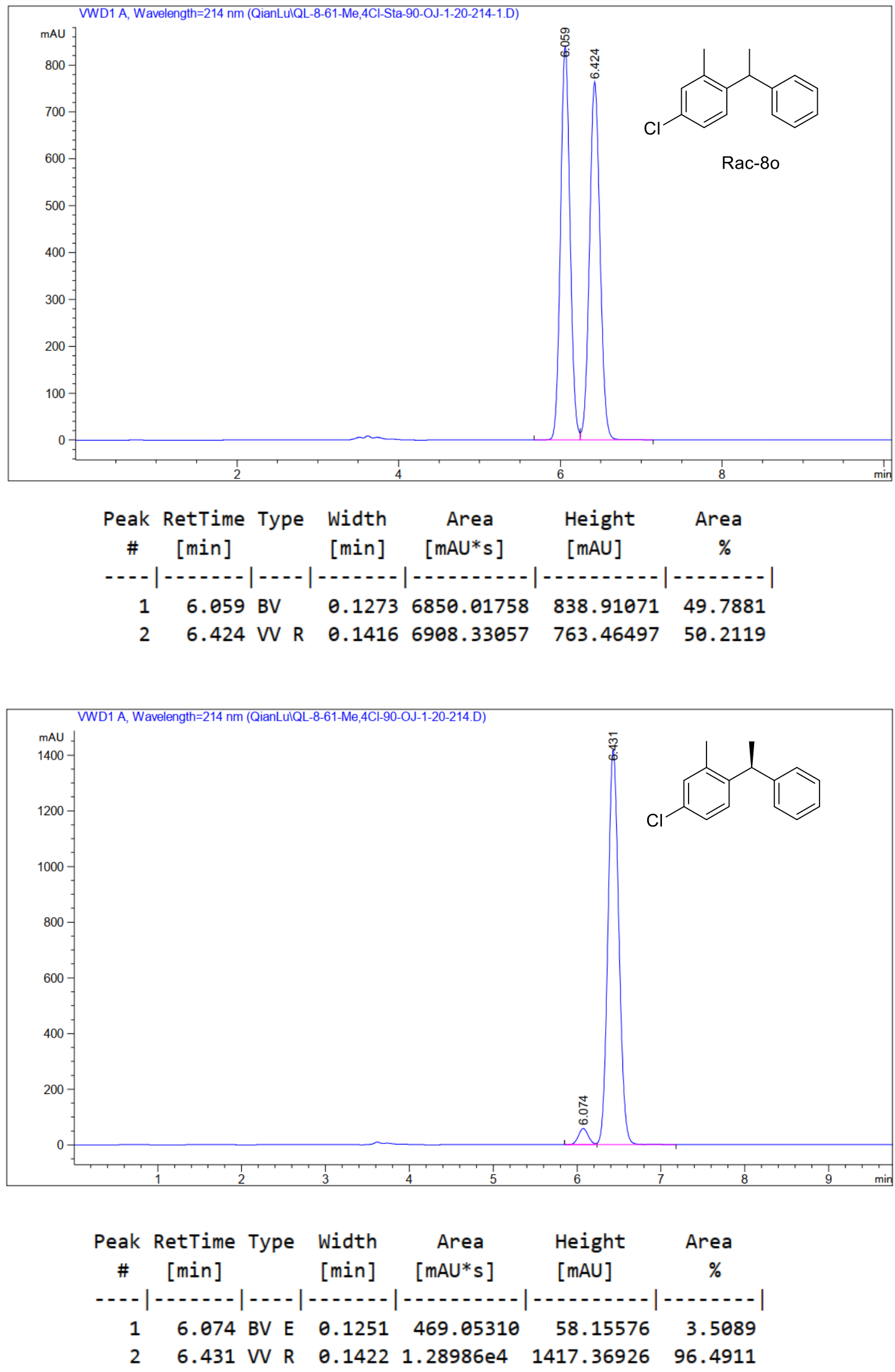
(S)-1,2,4-trimethyl-5-(1-phenylethyl)benzene (8p)

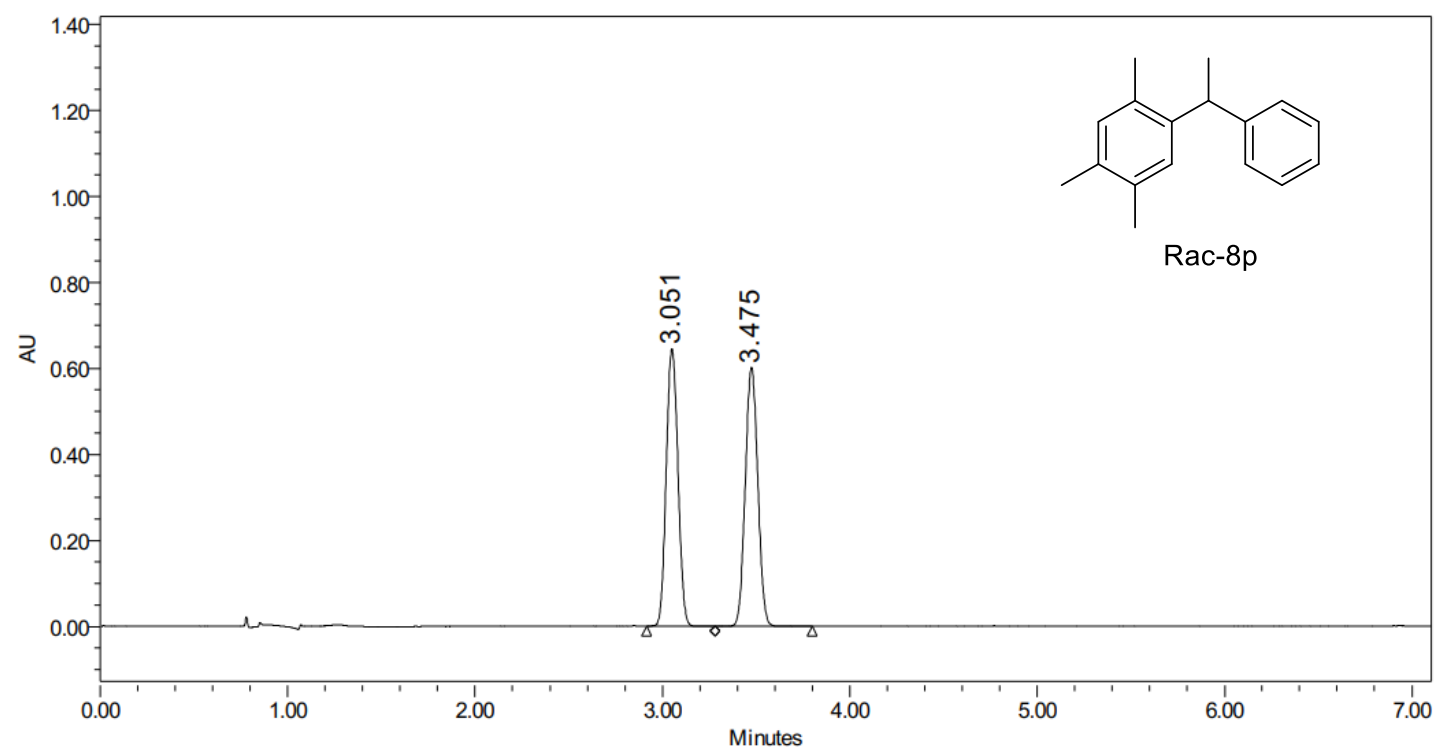

\begin{tabular}{|c|c|l|c|c|c|c|}
\hline & RT & Peak Type & Height & $\begin{array}{c}\text { Width } \\
(\mathrm{sec})\end{array}$ & Area & $\%$ Area \\
\hline 1 & 3.051 & Unknown & 644480 & 21.800 & 2797962 & 49.95 \\
\hline 2 & 3.475 & Unknown & 601830 & 31.200 & 2803455 & 50.05 \\
\hline
\end{tabular}

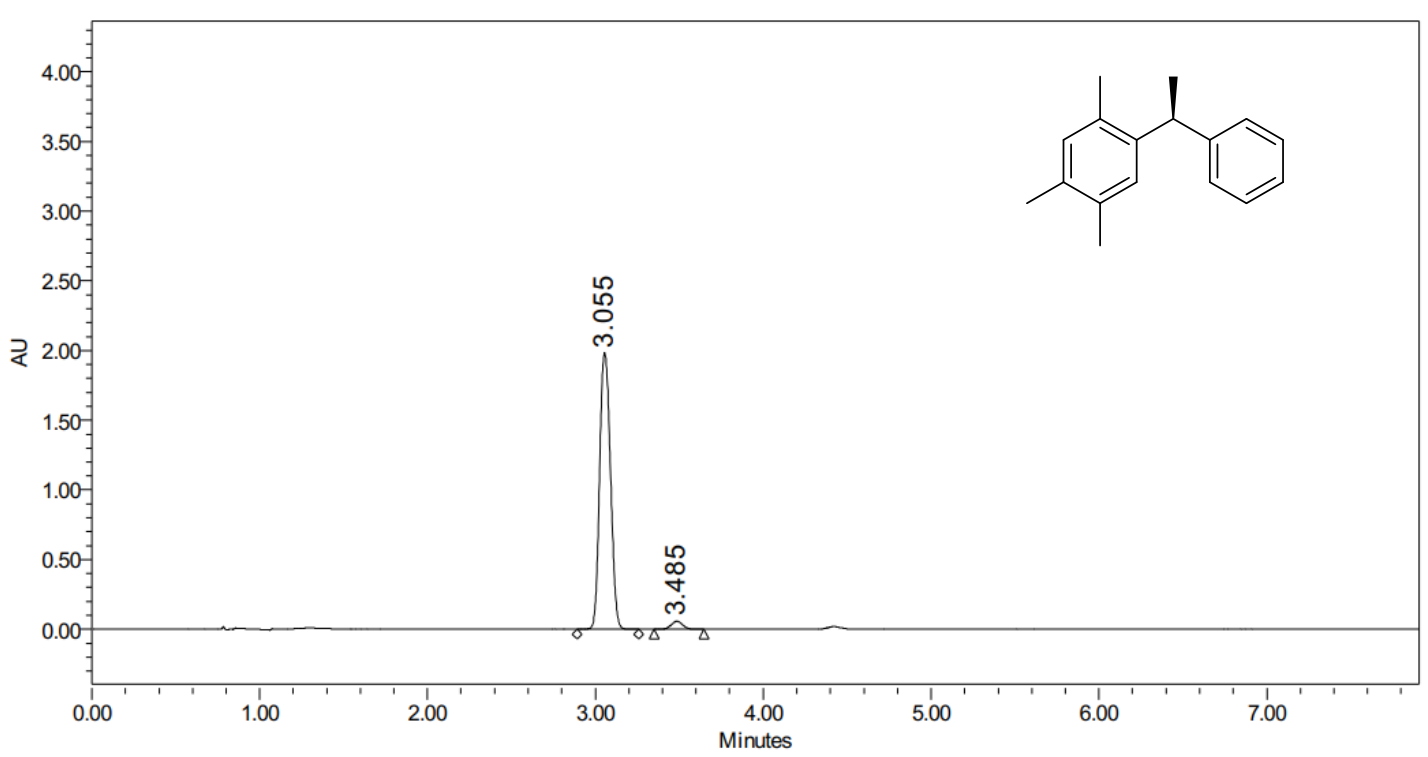

\begin{tabular}{|r|c|l|r|r|r|r|}
\hline & RT & Peak Type & Height & $\begin{array}{c}\text { Width } \\
(\text { sec })\end{array}$ & Area & $\%$ Area \\
\hline 1 & 3.055 & Unknown & 1983623 & 22.000 & 8964923 & 97.10 \\
\hline 2 & 3.485 & Unknown & 57074 & 17.800 & 267274 & 2.90 \\
\hline
\end{tabular}


(S)-1-(1-(3-methoxyphenyl)ethyl)-2-methylbenzene (8q)
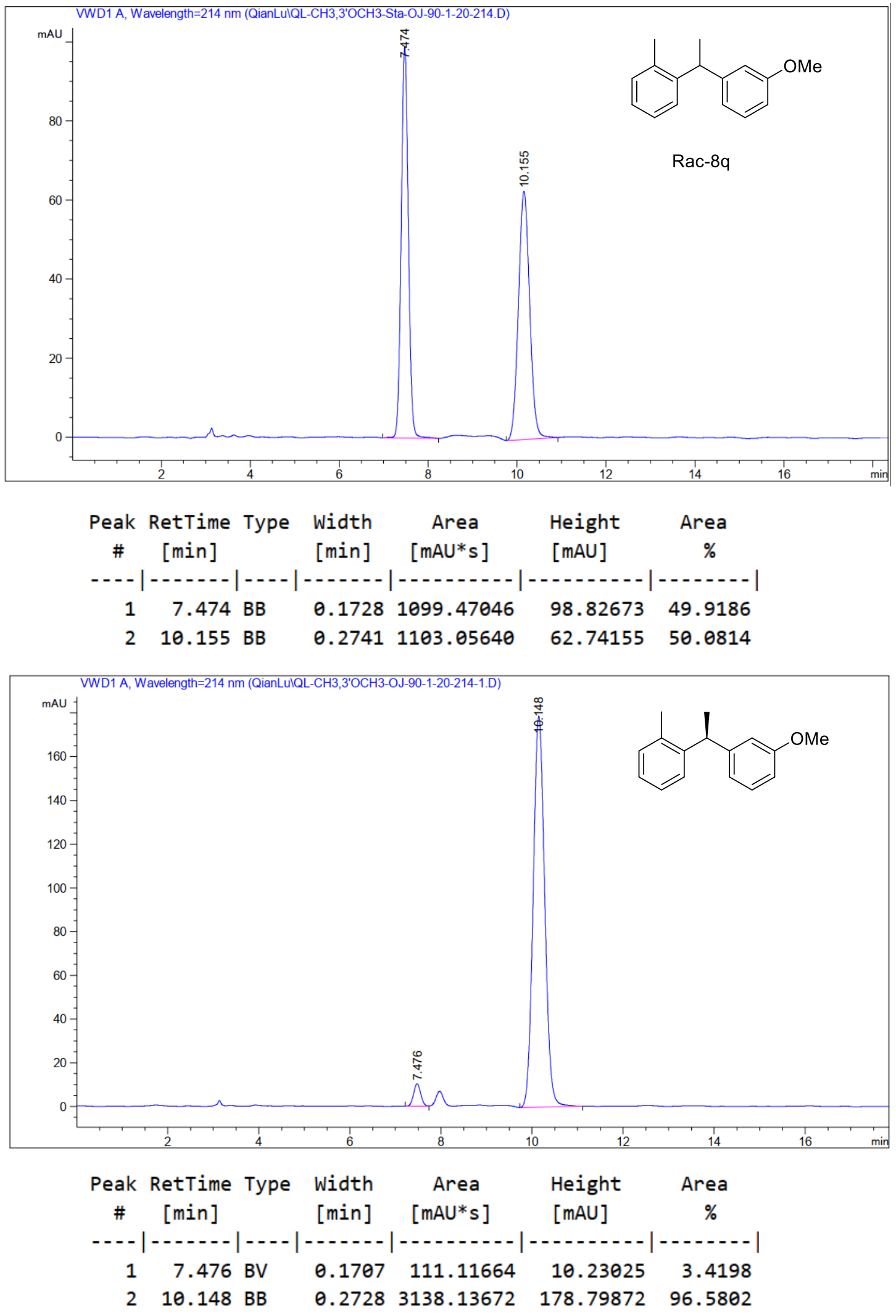
(S)-1-(1-(4-methoxyphenyl)ethyl)-2-methylbenzene (8r)
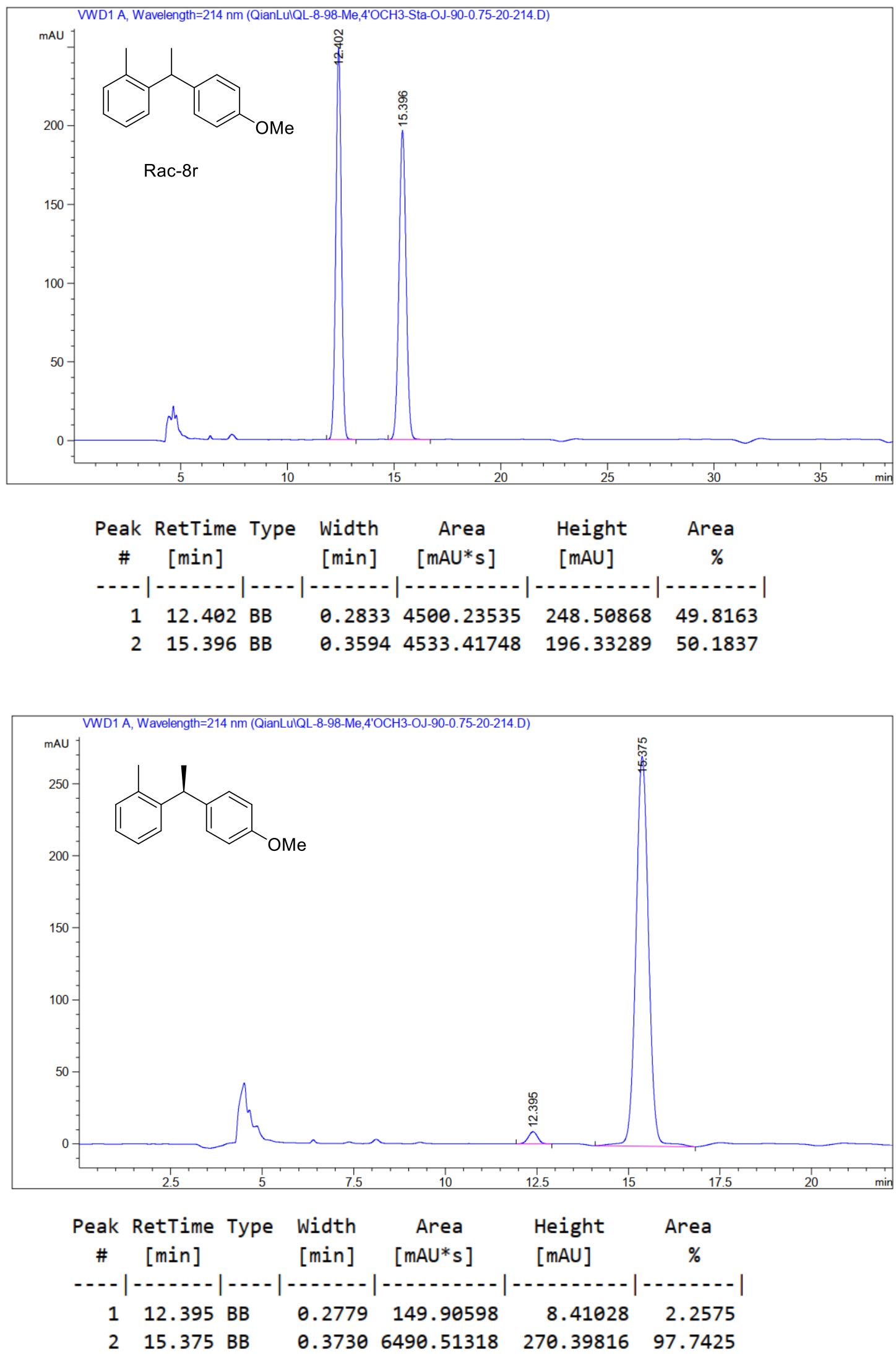
(S)-N,N-dimethyl-4-(1-(o-tolyl)ethyl)aniline (8s)
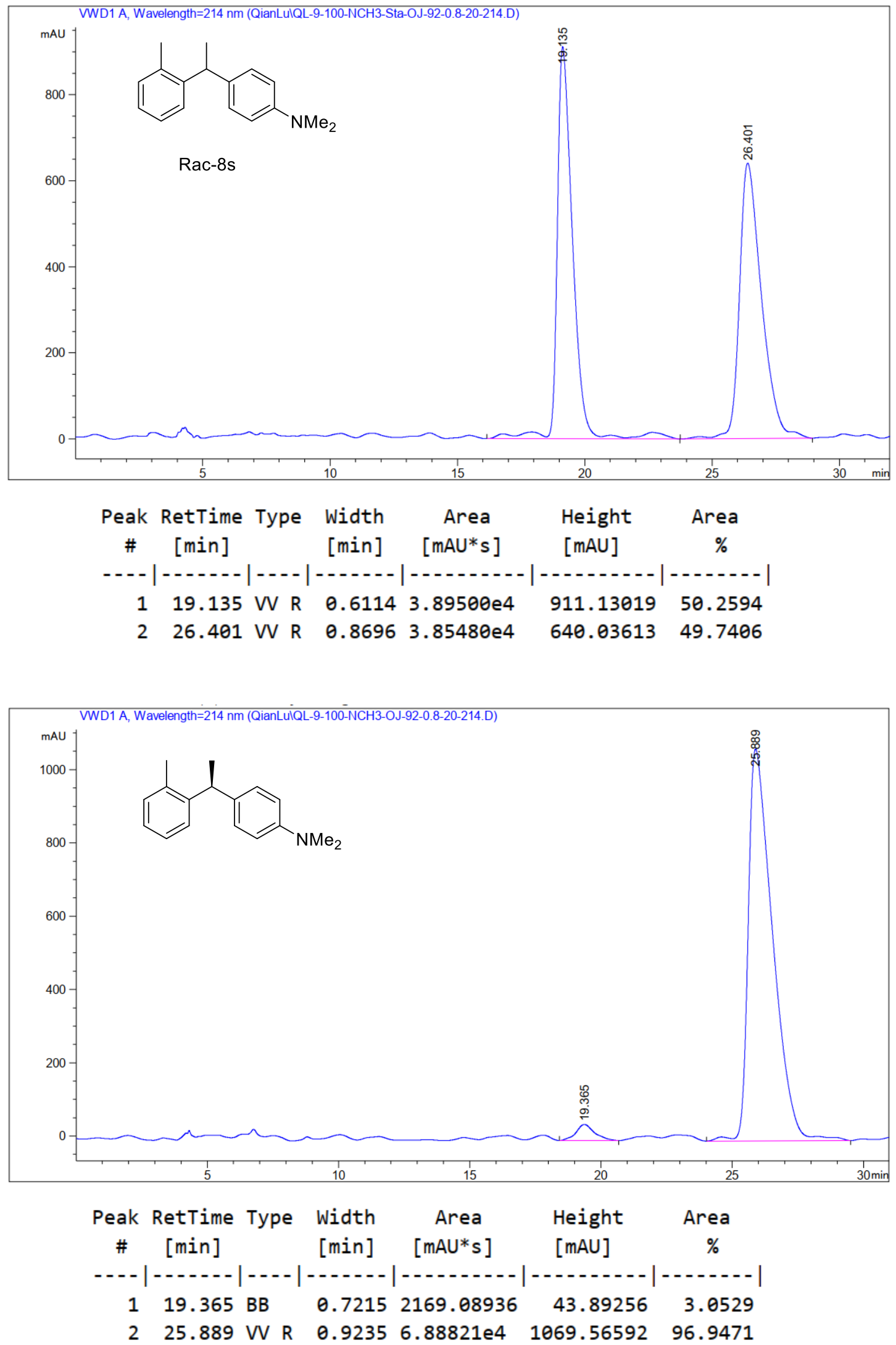
(S)-4-(1-(o-tolyl)ethyl)-1,1'-biphenyl (8t)
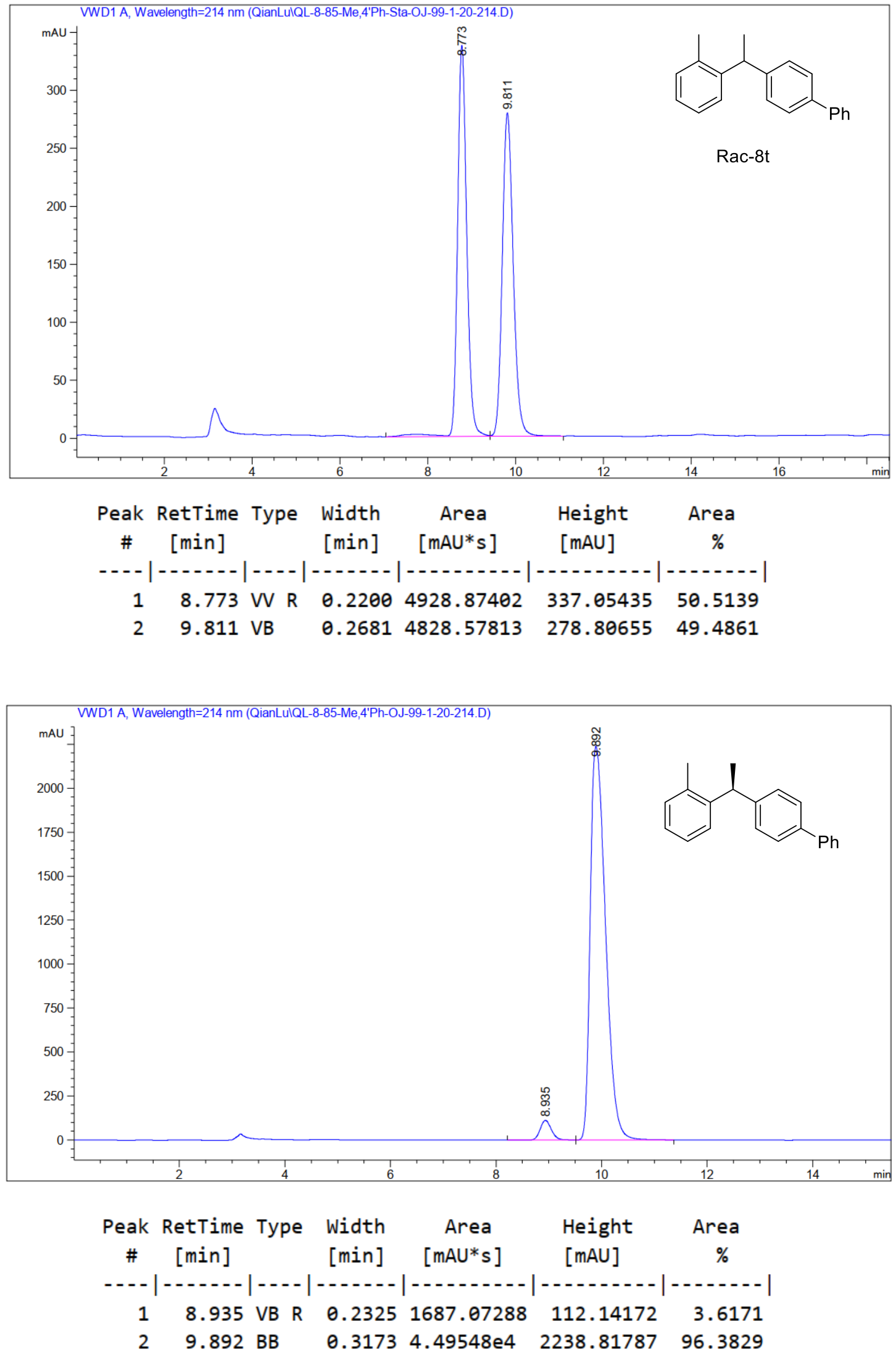
(S)-1-(1-(4-chlorophenyl)ethyl)-2-methylbenzene (8u)
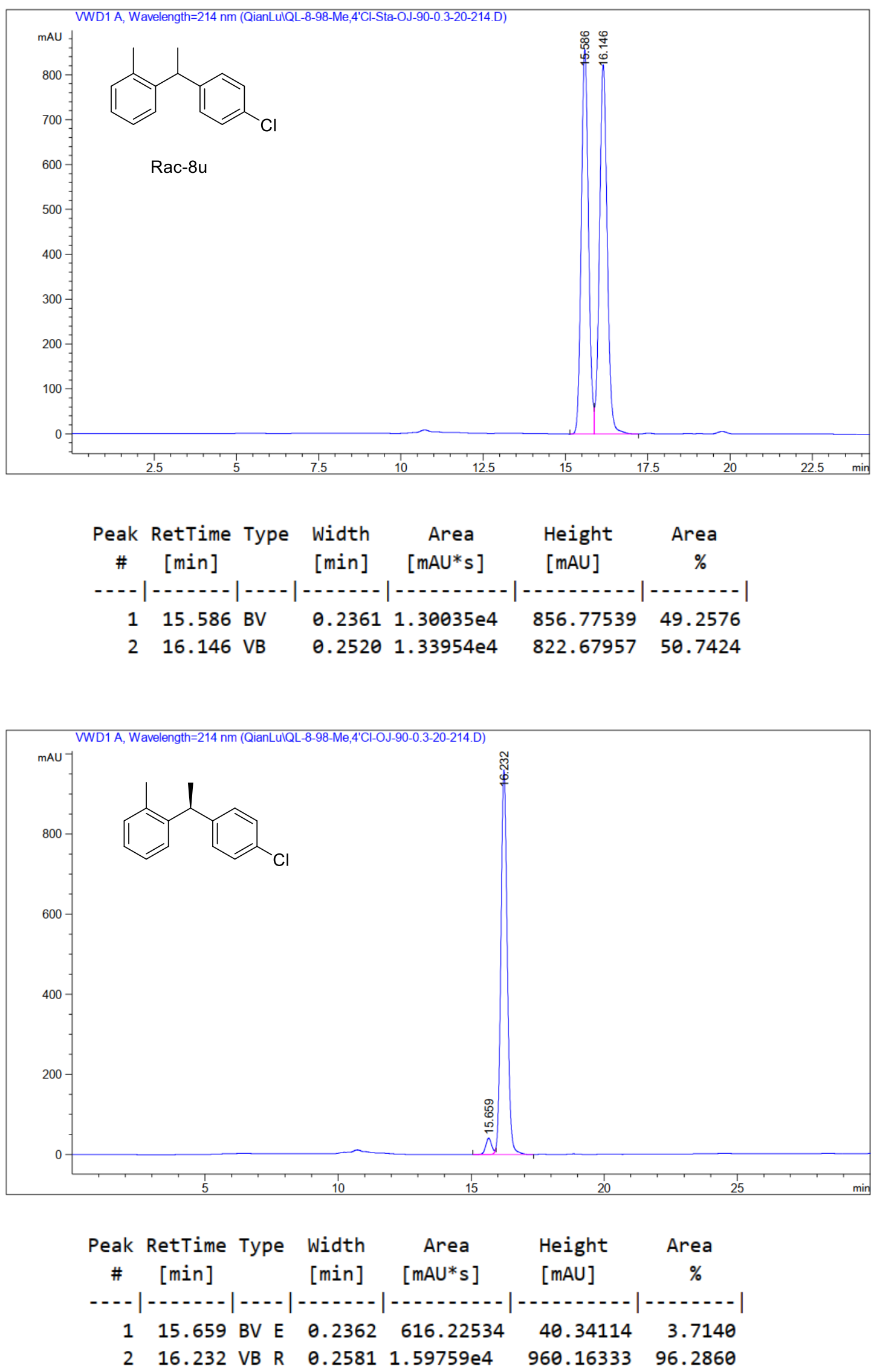
(S)-1-methyl-2-(1-(m-tolyl)ethyl)benzene (8v)

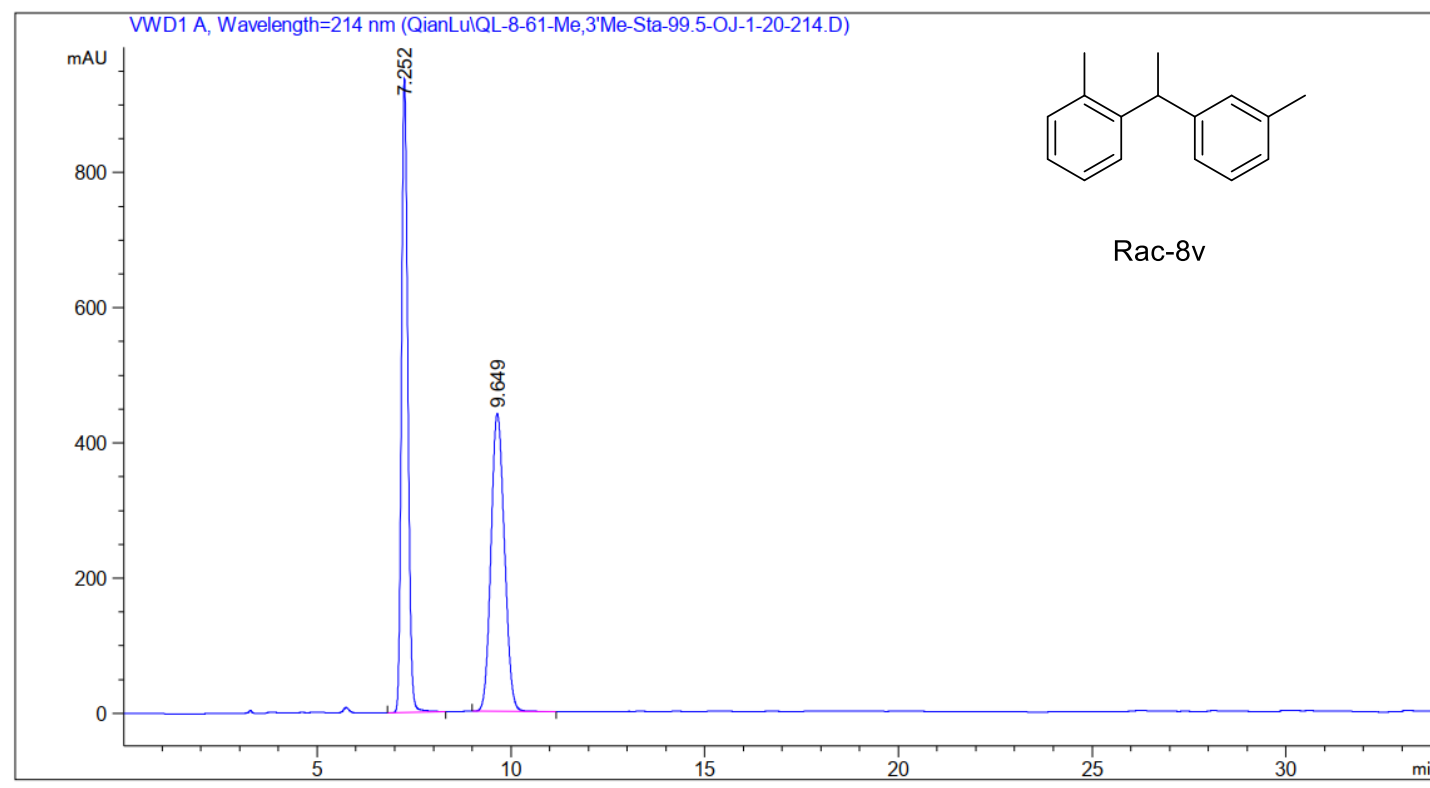

\begin{tabular}{cccccc}
$\begin{array}{c}\text { Peak RetTime Type Width } \\
\text { [min] }\end{array}$ & $\begin{array}{c}\text { Area } \\
{[\text { min] }}\end{array}\left[\begin{array}{c}\text { Height } \\
{[\text { mAU* }]}\end{array}\right.$ & $\begin{array}{c}\text { Area } \\
{[\text { mAU] }}\end{array}$ & $\%$ \\
\hline 1 & 7.252 BB & 0.1777 & $1.06693 \mathrm{e} 4$ & 937.76038 & 50.0203 \\
2 & 9.649 BB & 0.3680 & $1.06607 e 4$ & 441.09042 & 49.9797
\end{tabular}

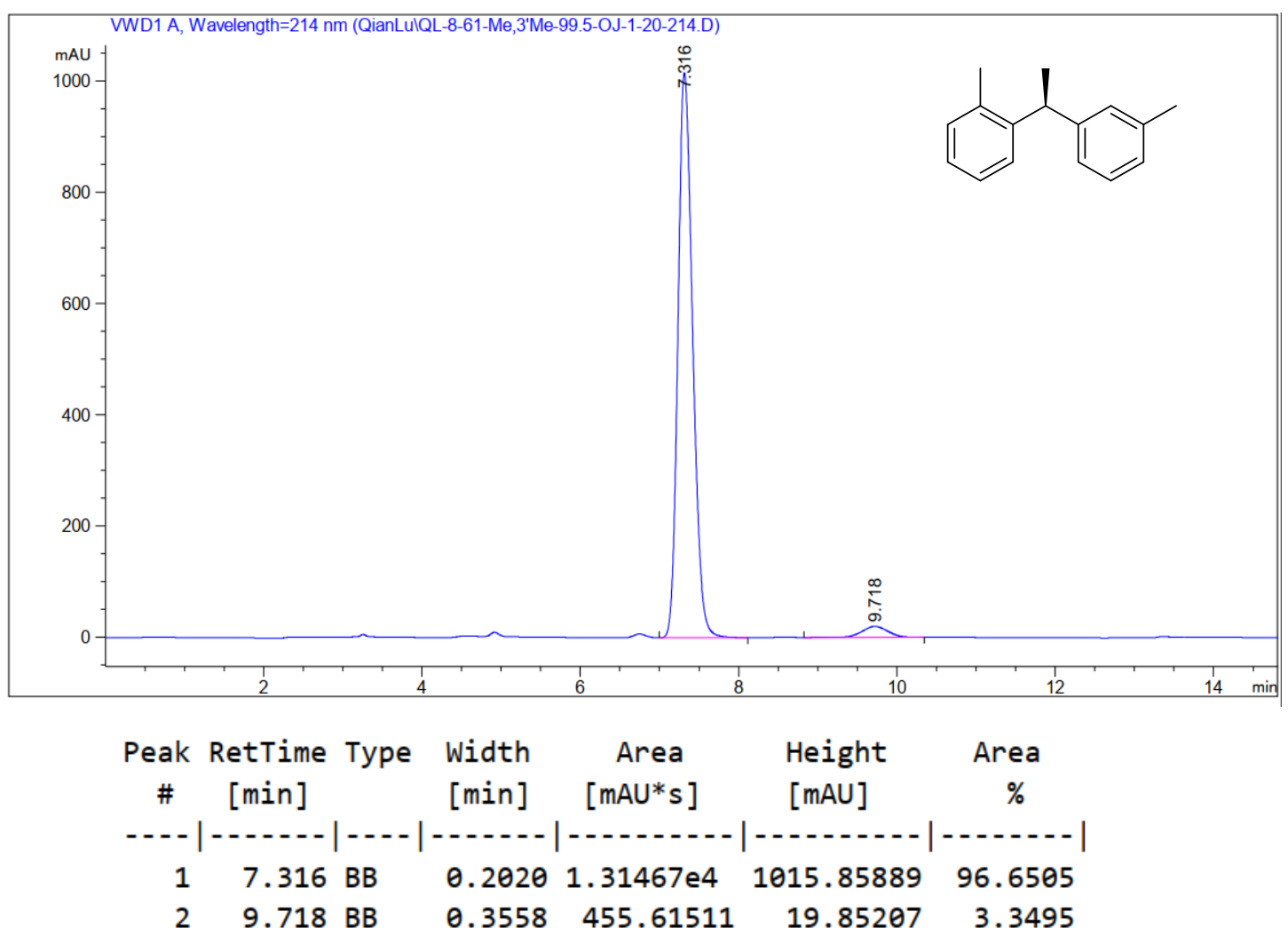


(S)-1,3-dimethyl-5-(1-(o-tolyl)ethyl)benzene (8w)
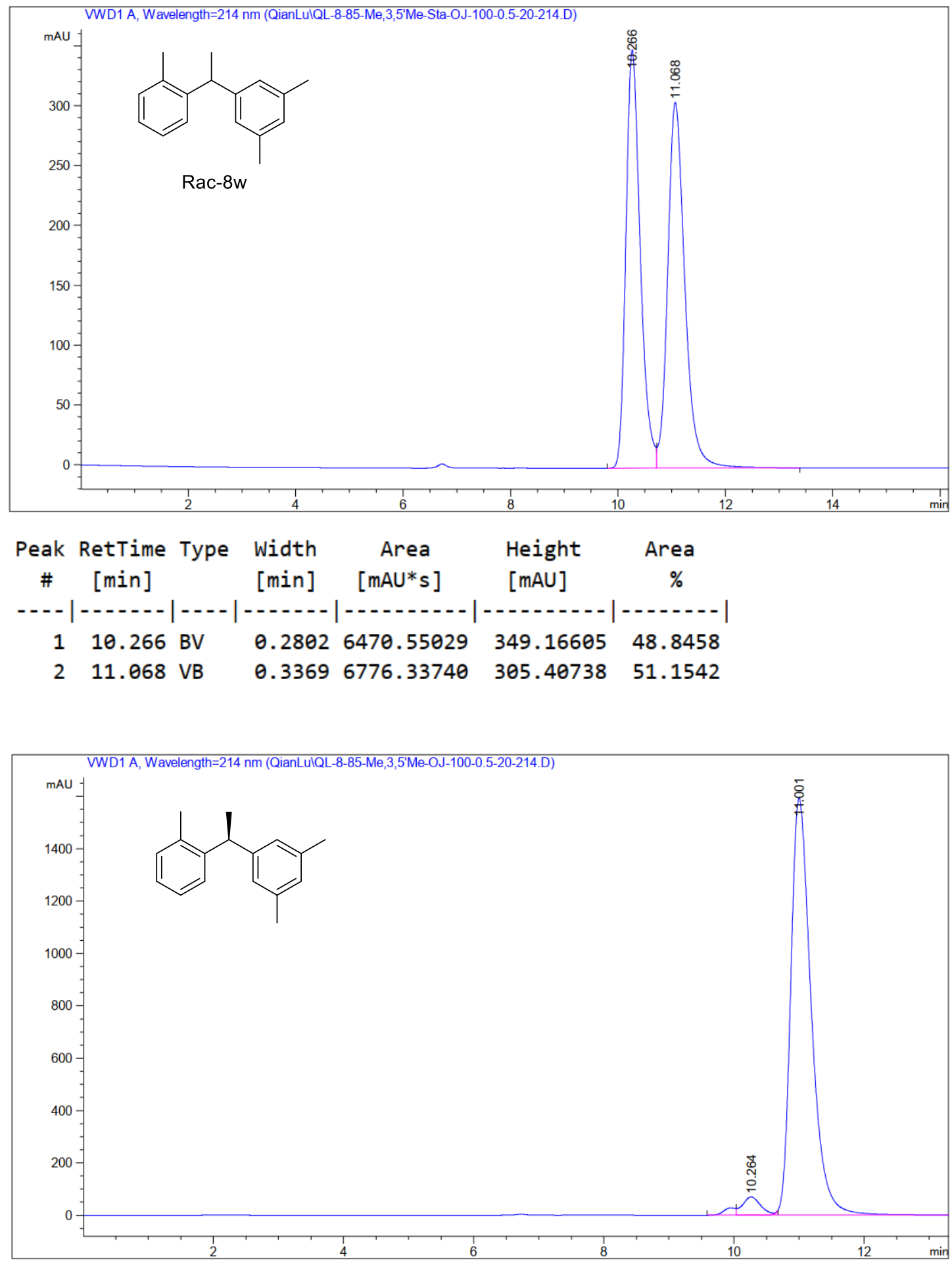

\begin{tabular}{|c|c|c|c|c|c|}
\hline $\begin{array}{l}\text { eak } \\
\text { \# }\end{array}$ & $\begin{array}{l}\text { RetTime Type } \\
\text { [min] }\end{array}$ & $\begin{array}{l}\text { Width } \\
\text { [min] }\end{array}$ & $\begin{array}{c}\text { Area } \\
{[\mathrm{mAU} * \mathrm{~s}]}\end{array}$ & $\begin{array}{l}\text { Height } \\
{[\mathrm{mAU}]}\end{array}$ & $\begin{array}{c}\text { Area } \\
\%\end{array}$ \\
\hline & & & 2 & 85 & \\
\hline & & & $19779 \mathrm{e} 4$ & & \\
\hline
\end{tabular}


(S)-1,3-dimethoxy-5-(1-phenylethyl)benzene (8x).

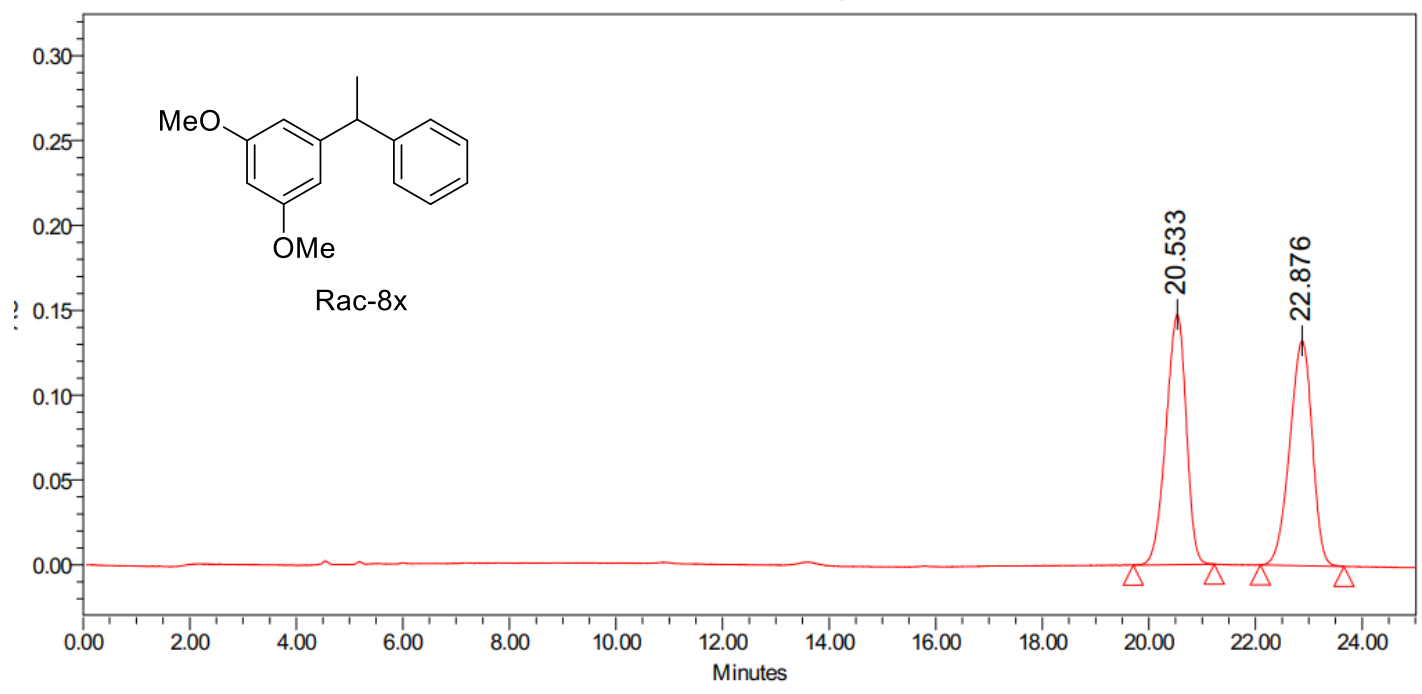

Peak Results

\begin{tabular}{|l|l|l|l|l|l|}
\hline QL-14-73-RAC & 20.533 & 91.000 & 147396 & 3888185 & 50.04 \\
\hline QL-14-73-RAC & 22.876 & 94.200 & 132522 & 3881899 & 49.96 \\
\hline
\end{tabular}

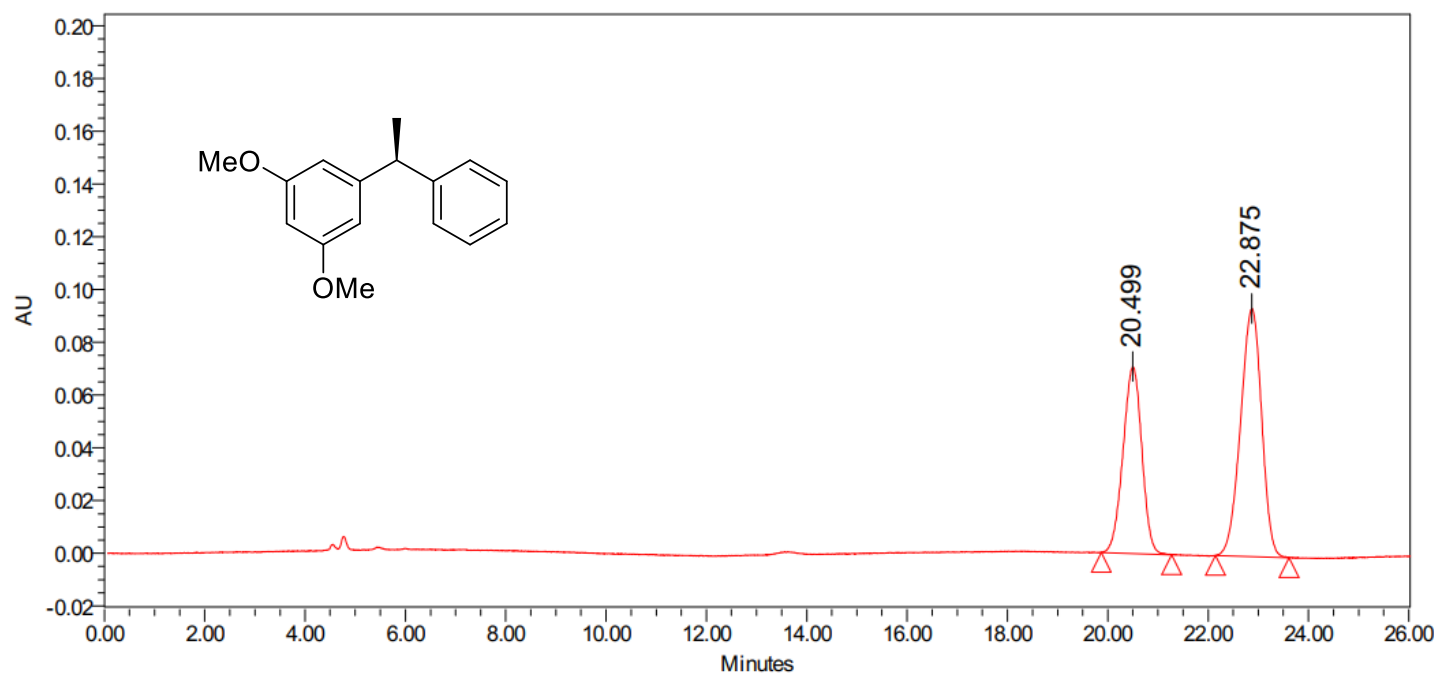

\section{Peak Results}

\begin{tabular}{|l|l|l|l|l|r|}
\hline QL-14-73-CHIRAL & 20.499 & 84.000 & 70857 & 1847749 & 40.23 \\
\hline QL-14-73-CHIRAL & 22.875 & 88.300 & 94079 & 2745595 & 59.77 \\
\hline
\end{tabular}


(S)-2,4-dichloro-1-(1-phenylethyl)benzene (8y)
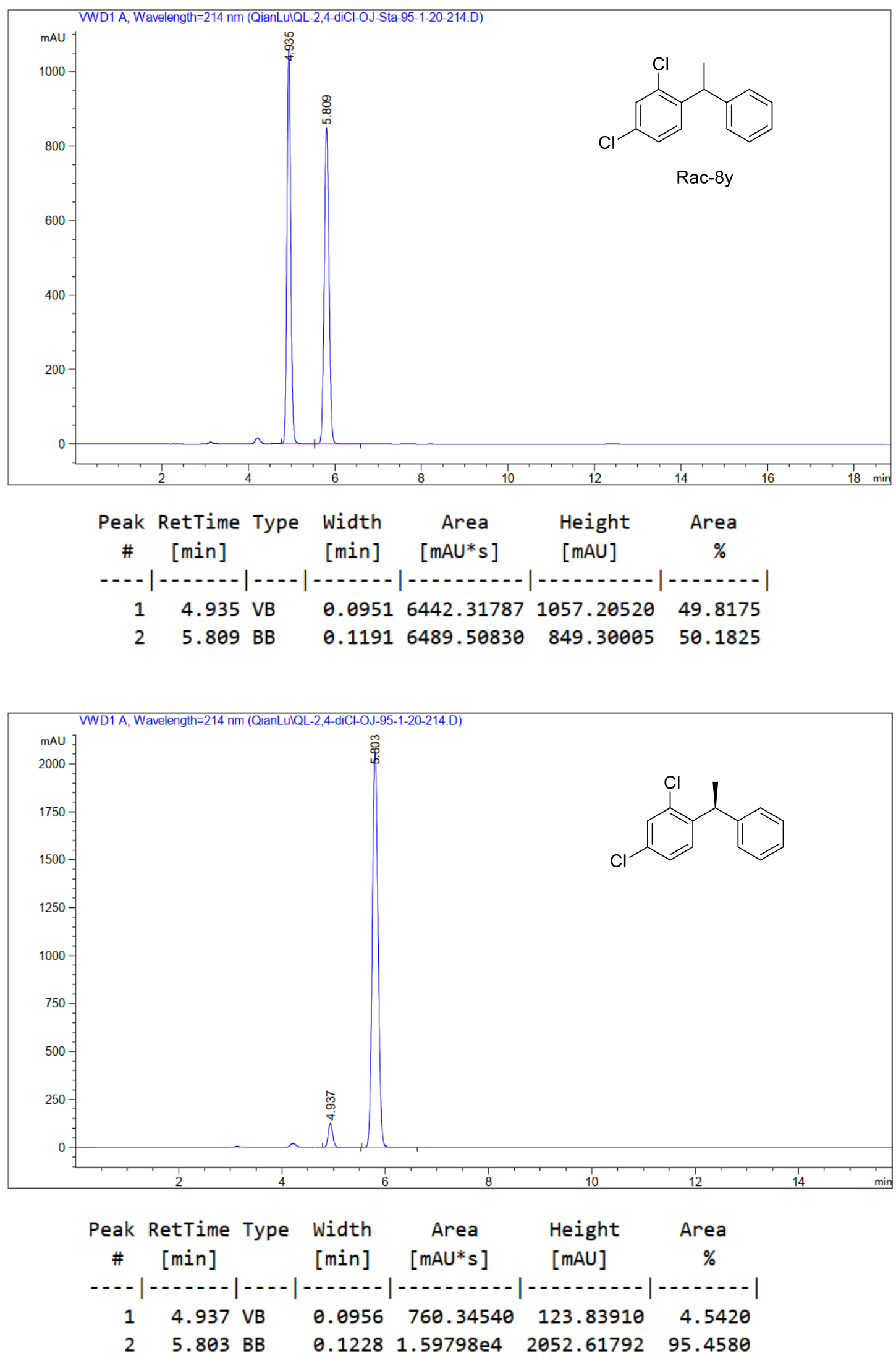
(S)-1,4-dichloro-2-(1-phenylethyl)benzene (8z)
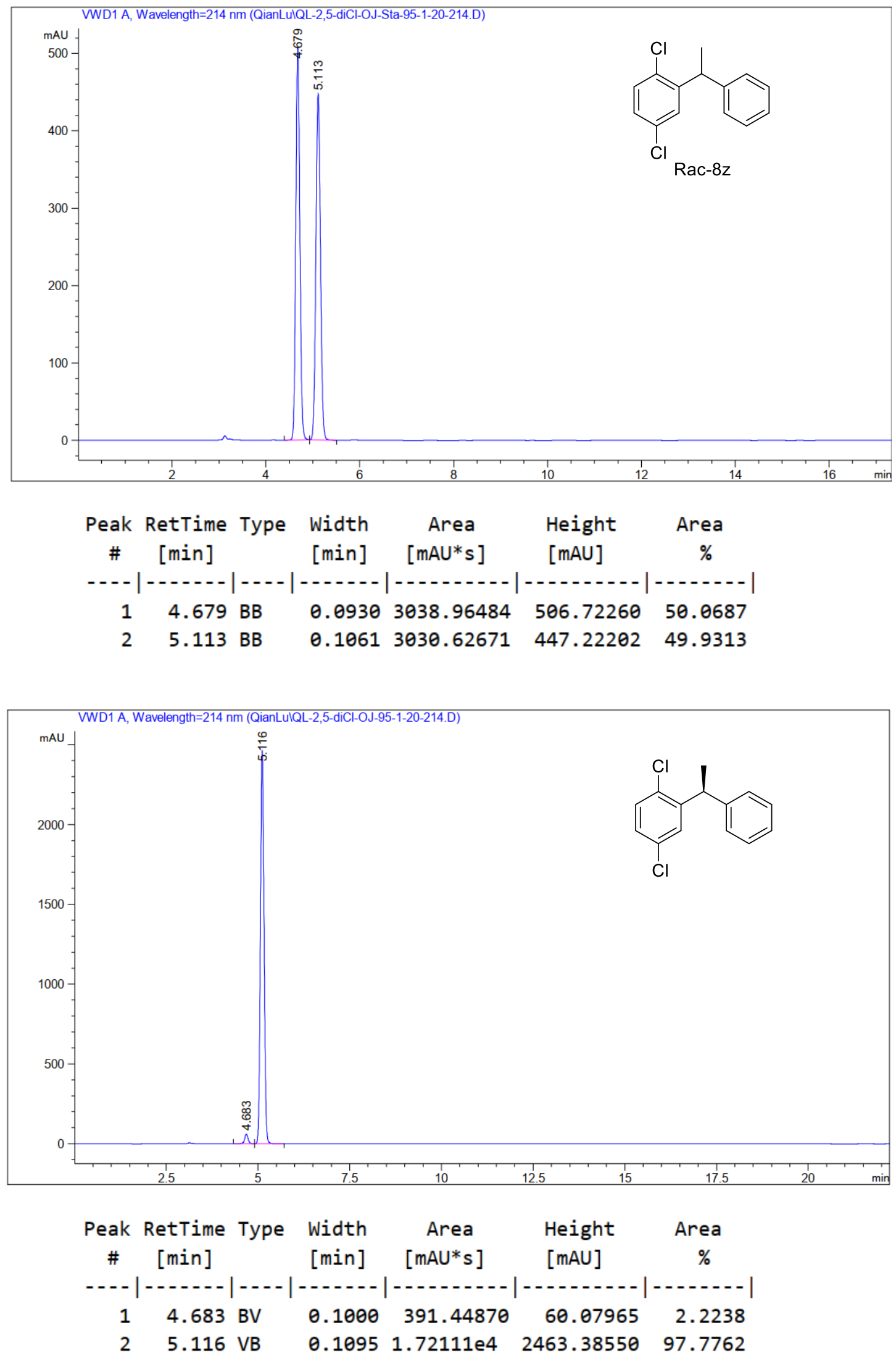
(S)-1-chloro-2-(1-phenylethyl)-4-(trifluoromethyl)benzene (8ab)
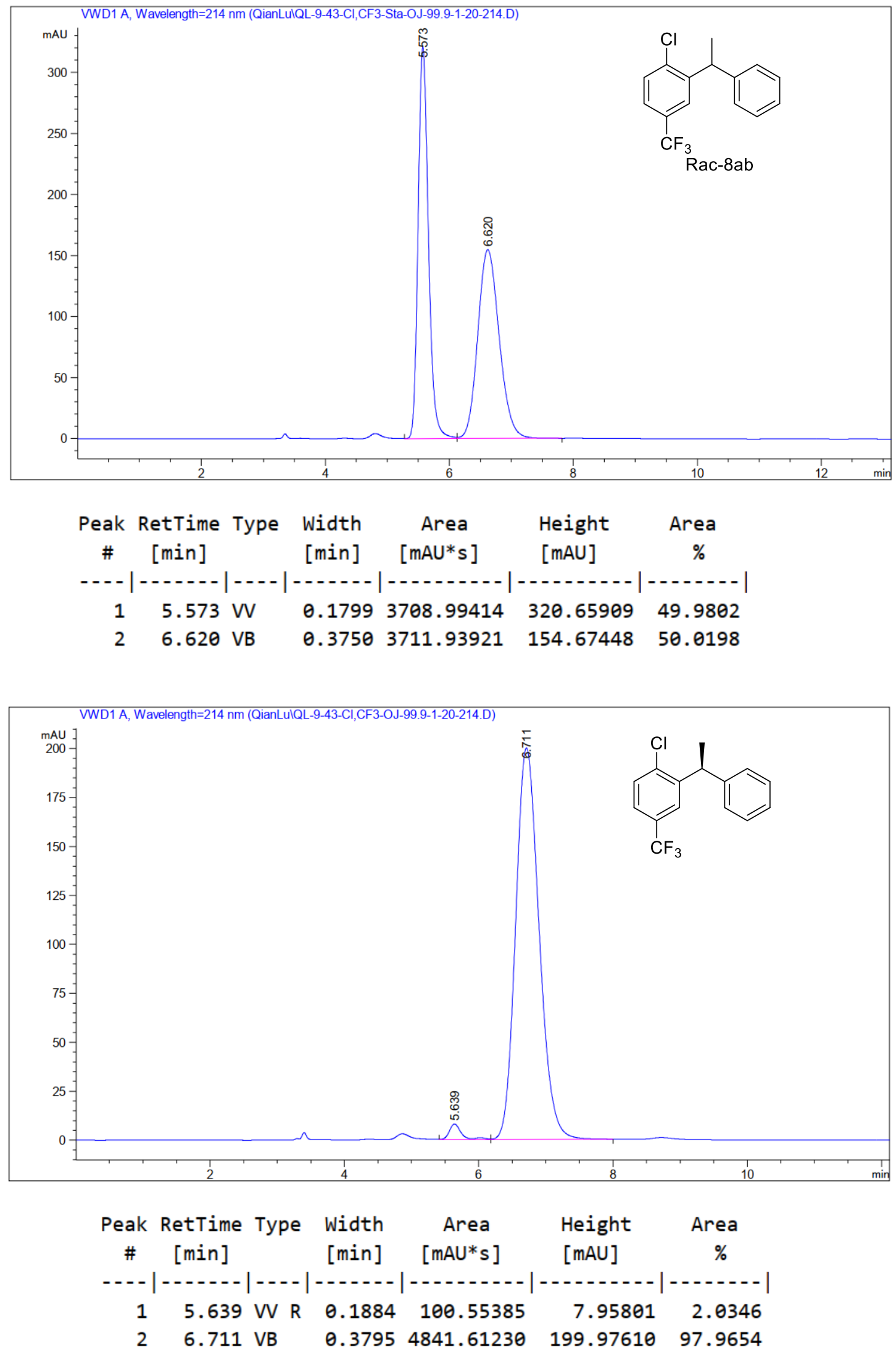
(S)-1-chloro-2-(1-(3-methoxyphenyl)ethyl)benzene (8ac)
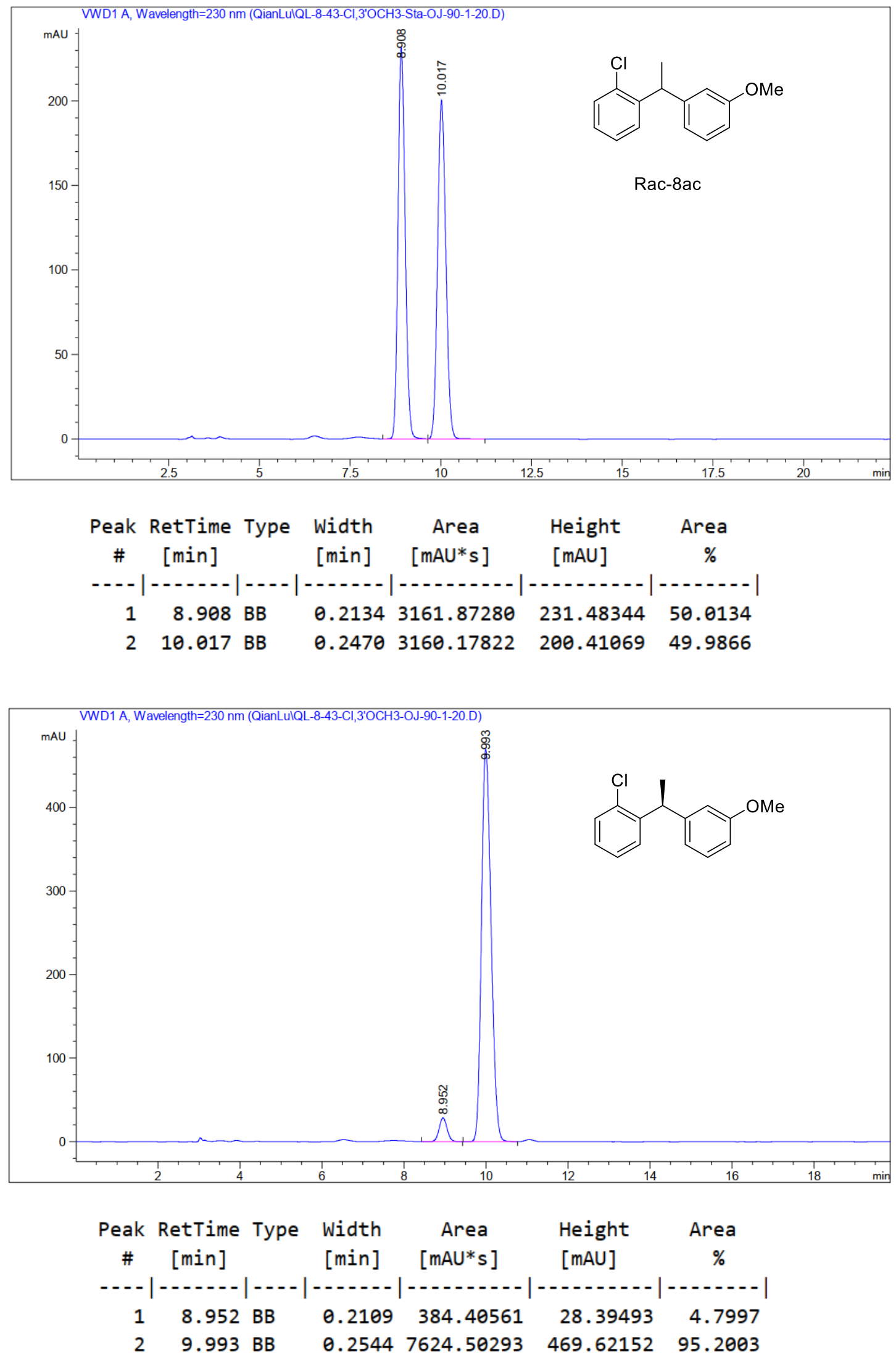
(S)-1-chloro-2-(1-(4-methoxyphenyl)ethyl)benzene (8ad)
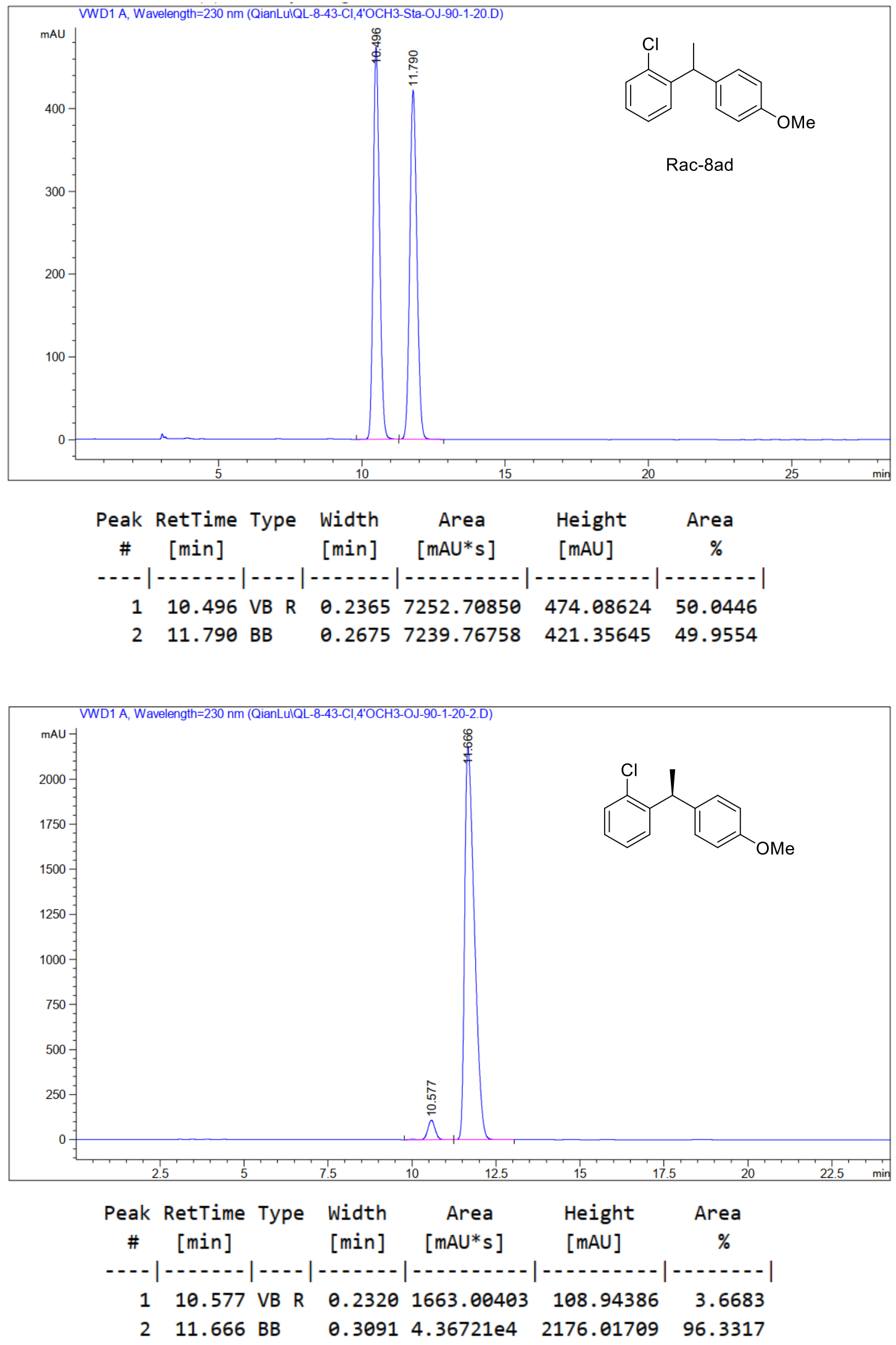
(S)-1-(1-(4-(tert-butyl)phenyl)ethyl)-2-chlorobenzene (8ae)
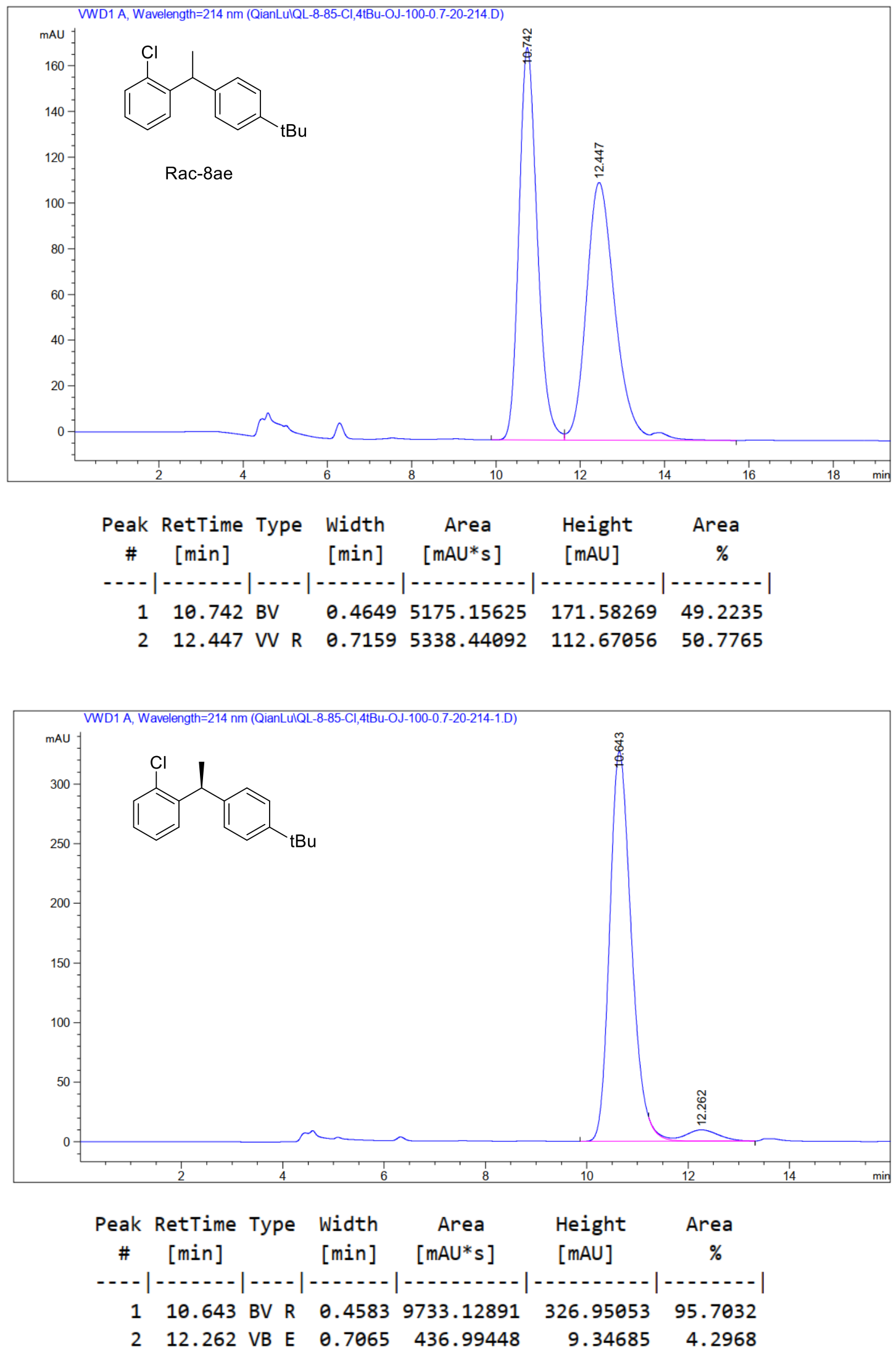
(S)-1-chloro-2-(1-(4-chlorophenyl)ethyl)benzene (8af)

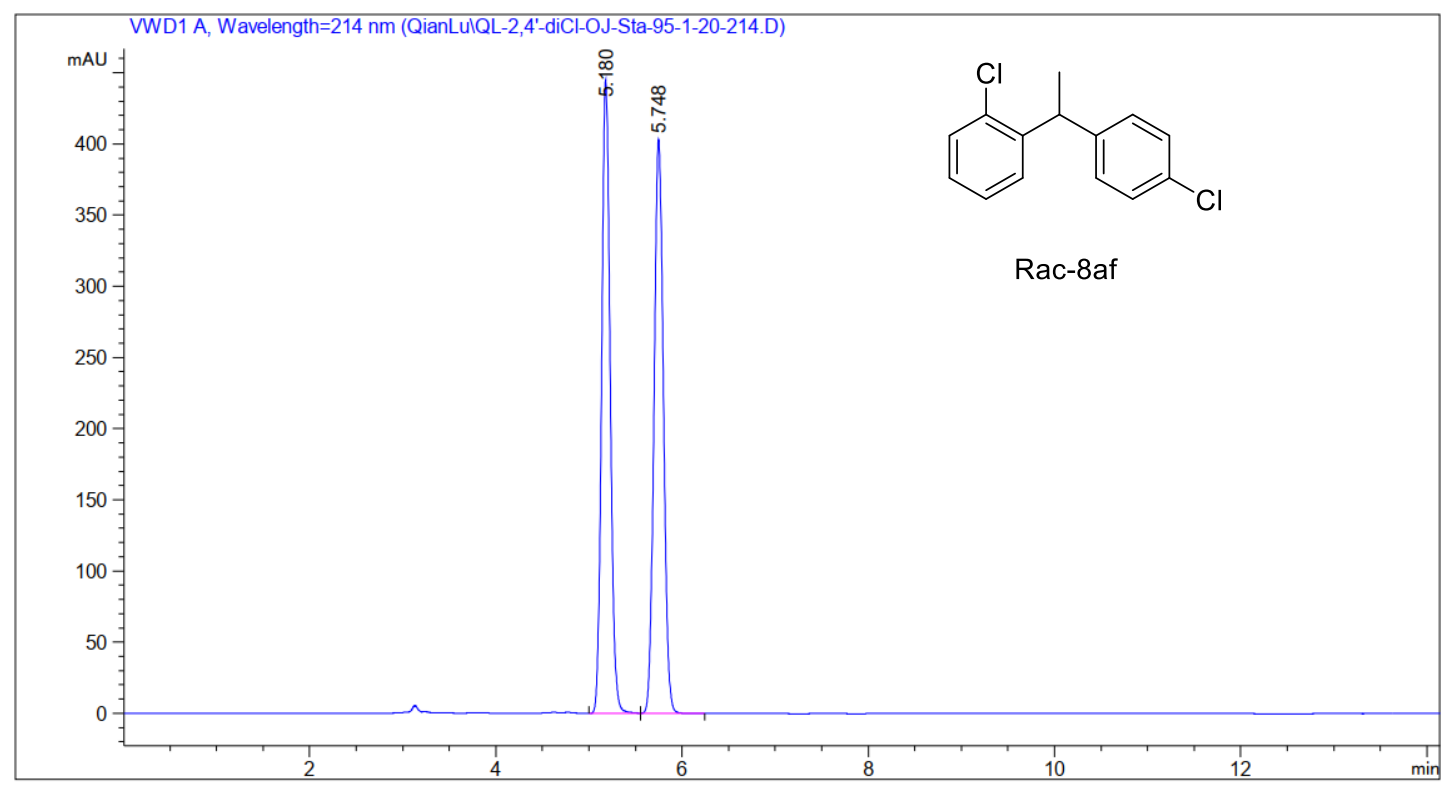

\begin{tabular}{|c|c|c|c|c|c|c|}
\hline $\begin{array}{c}\text { Peak } \\
\#\end{array}$ & $\begin{array}{c}\text { RetTime } \\
\text { [min] }\end{array}$ & Type & $\begin{array}{l}\text { Width } \\
\text { [min] }\end{array}$ & $\begin{array}{c}\text { Area } \\
\text { [mAU*s] }\end{array}$ & $\begin{array}{l}\text { Height } \\
{[\mathrm{mAU}]}\end{array}$ & $\begin{array}{c}\text { Area } \\
\%\end{array}$ \\
\hline & & & & & & \\
\hline 1 & 5.180 & BB & 0.0993 & 2833.21899 & 444.93237 & 50.0084 \\
\hline 2 & 5.748 & BB & 0.1099 & 2832.27173 & 403.62823 & 49.9916 \\
\hline
\end{tabular}

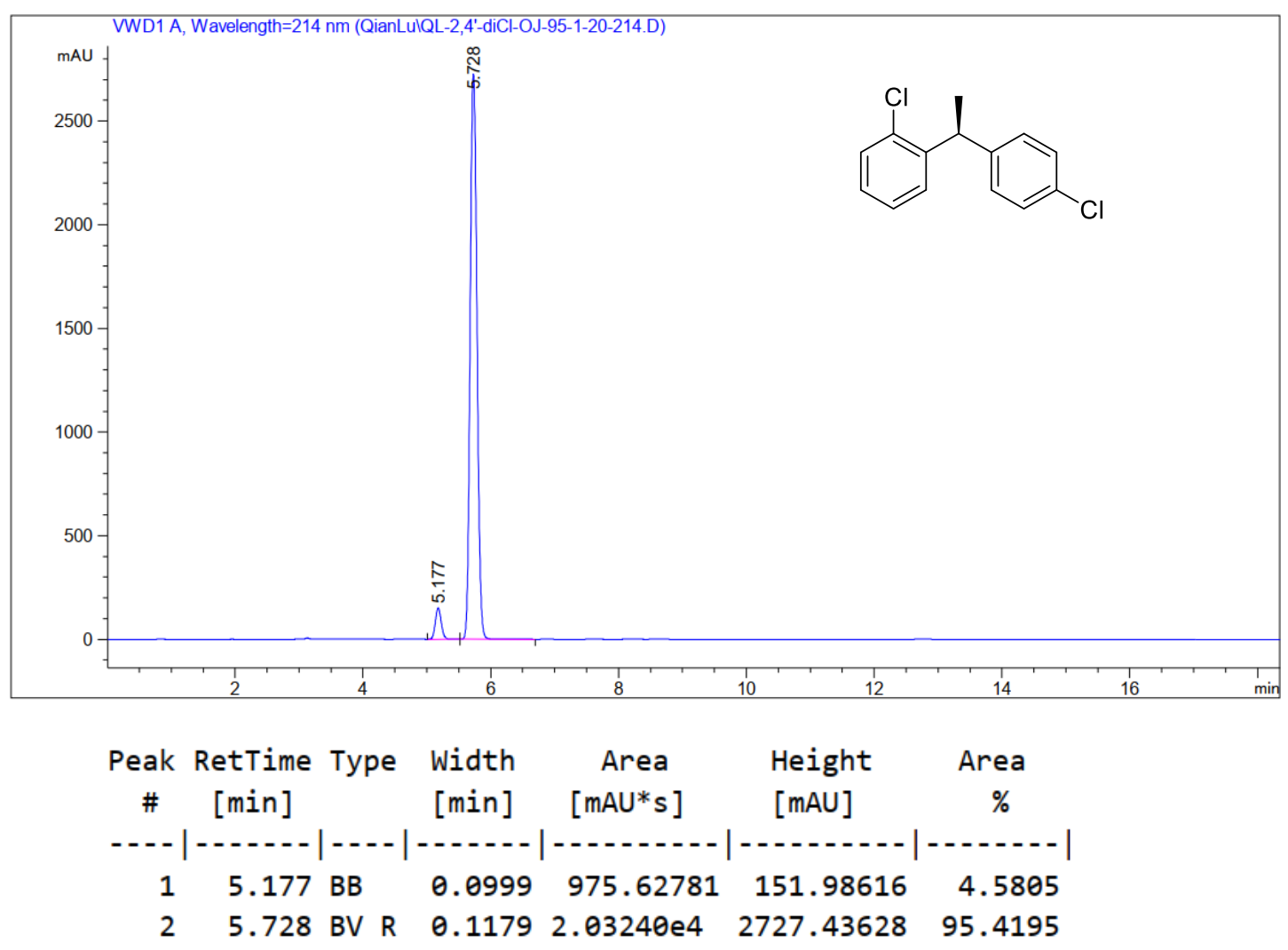


(S)-1-chloro-2-(1-(4-fluorophenyl)ethyl)benzene(8ag)
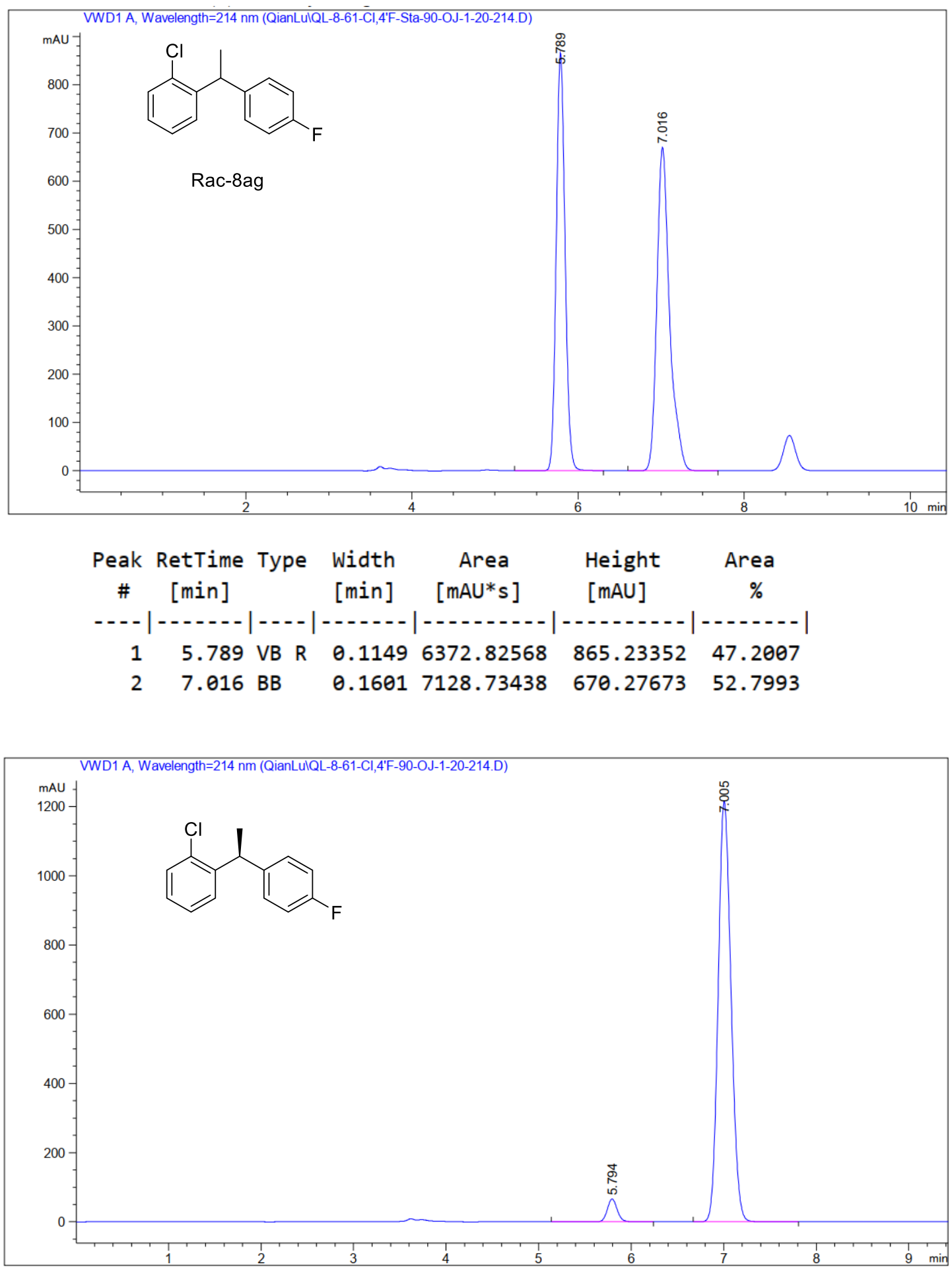

\begin{tabular}{cccccc}
$\begin{array}{c}\text { Peak RetTime Type } \\
\text { [min] }\end{array}$ & $\begin{array}{c}\text { Width } \\
\text { [min] }\end{array}$ & $\begin{array}{c}\text { Area } \\
{[\text { mAU*s] }}\end{array}$ & $\begin{array}{c}\text { Height } \\
\text { [mAU] }\end{array}$ & \multicolumn{1}{c}{$\begin{array}{c}\text { Area } \\
\%\end{array}$} \\
\hline 1 & 5.794 VB R & 0.1150 & 491.76349 & 65.93414 & 4.0257 \\
2 & 7.005 BB & 0.1506 & $1.17238 \mathrm{e} 4$ & 1214.58679 & 95.9743
\end{tabular}


(S)-1-bromo-4-methoxy-2-(1-phenylethyl)benzene (8ah)

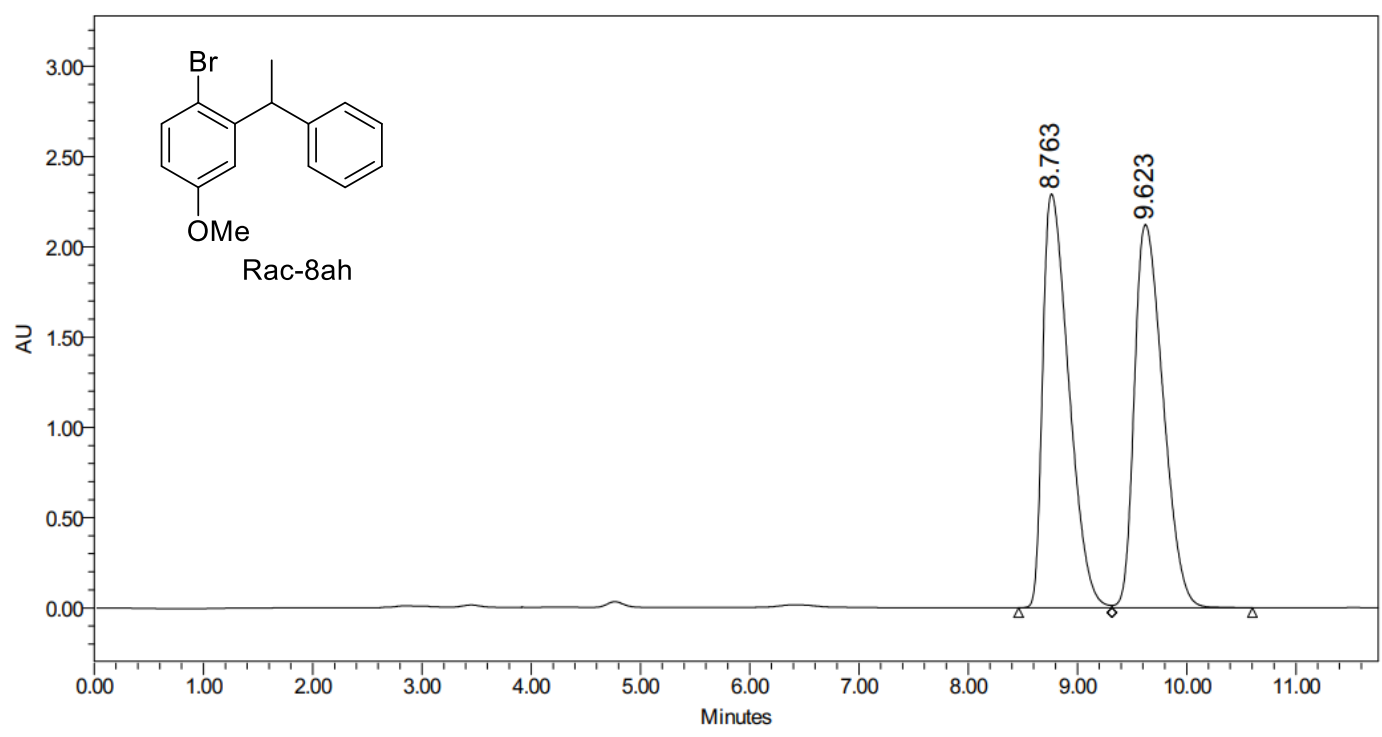

\begin{tabular}{|c|c|c|c|c|r|}
\hline & SampleName & RT & Height & Area & $\%$ Area \\
\hline 1 & QL-9-43-Br-OCH3-STA & 8.763 & 2291744 & 37979642 & 49.58 \\
\hline 2 & QL-9-43-Br-OCH3-STA & 9.623 & 2122516 & 38623029 & 50.42 \\
\hline
\end{tabular}

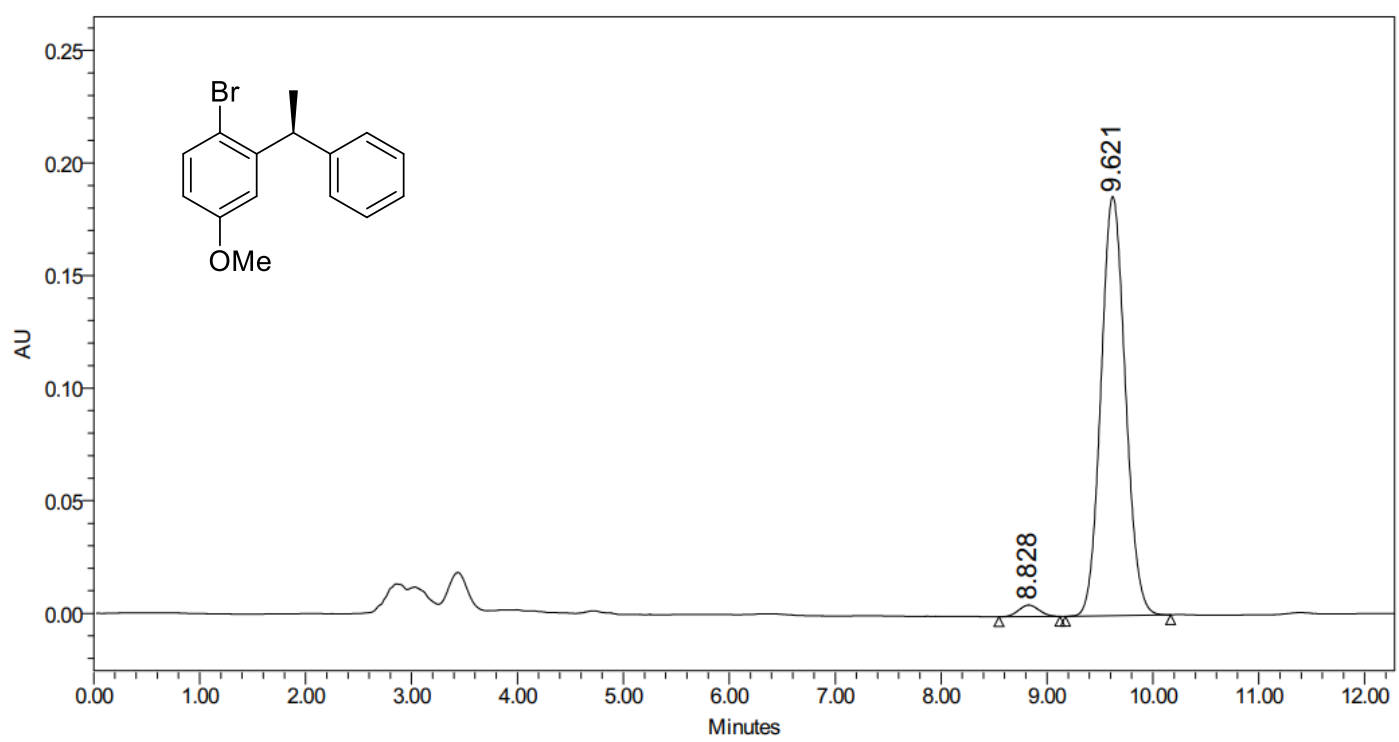

\begin{tabular}{|c|c|c|r|r|r|}
\hline & SampleName & RT & Height & Area & $\%$ Area \\
\hline 1 & QL-9-43-Br-OCH3-CHIRAL & 8.828 & 5076 & 68092 & 2.24 \\
\hline 2 & QL-9-43-Br-OCH3-CHIRAL & 9.621 & 186219 & 2976991 & 97.76 \\
\hline
\end{tabular}


(S)-1-bromo-4-methyl-2-(1-phenylethyl)benzene (8ai)
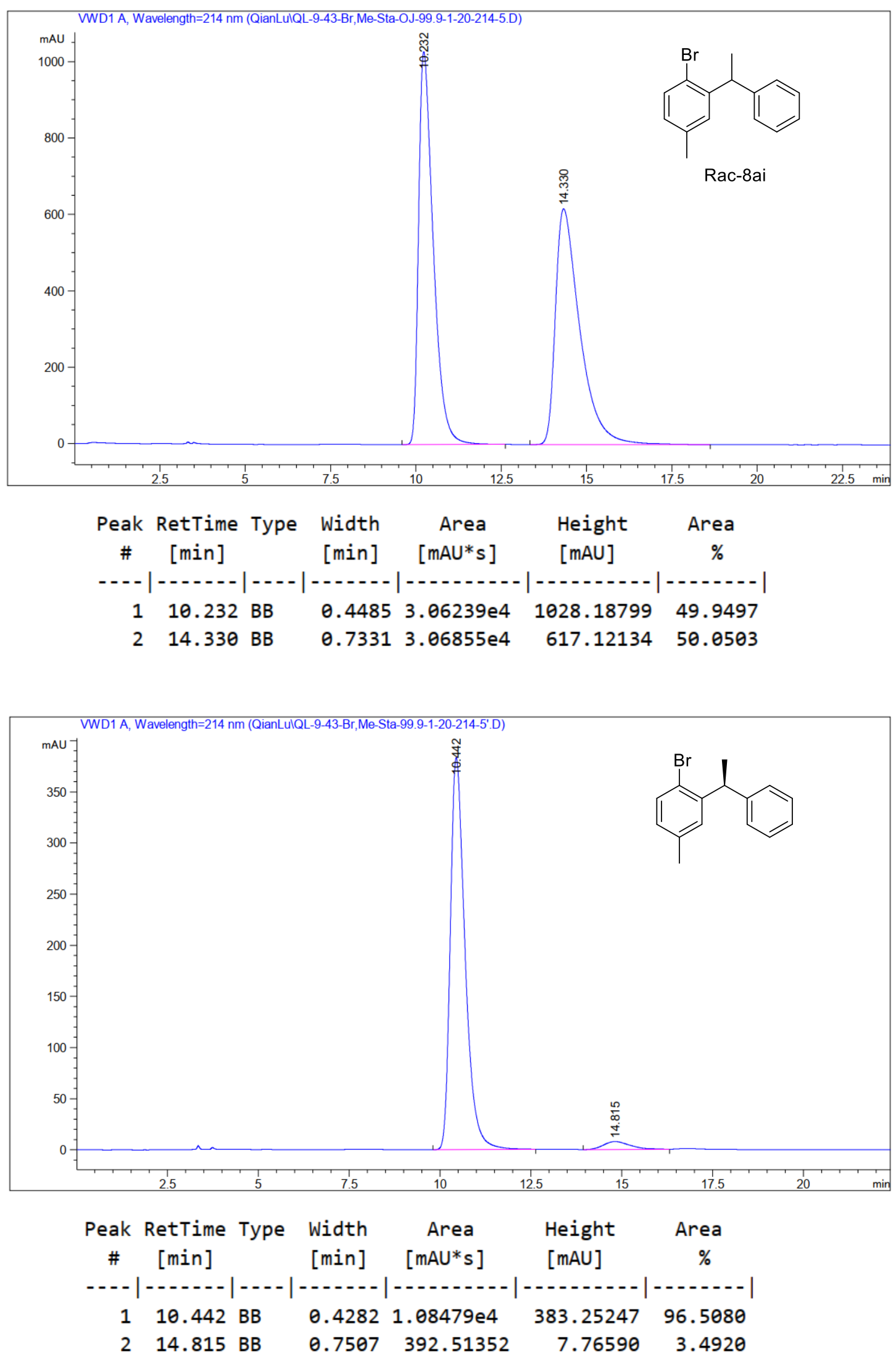
(S)-1-bromo-4-fluoro-2-(1-phenylethyl)benzene (8aj)

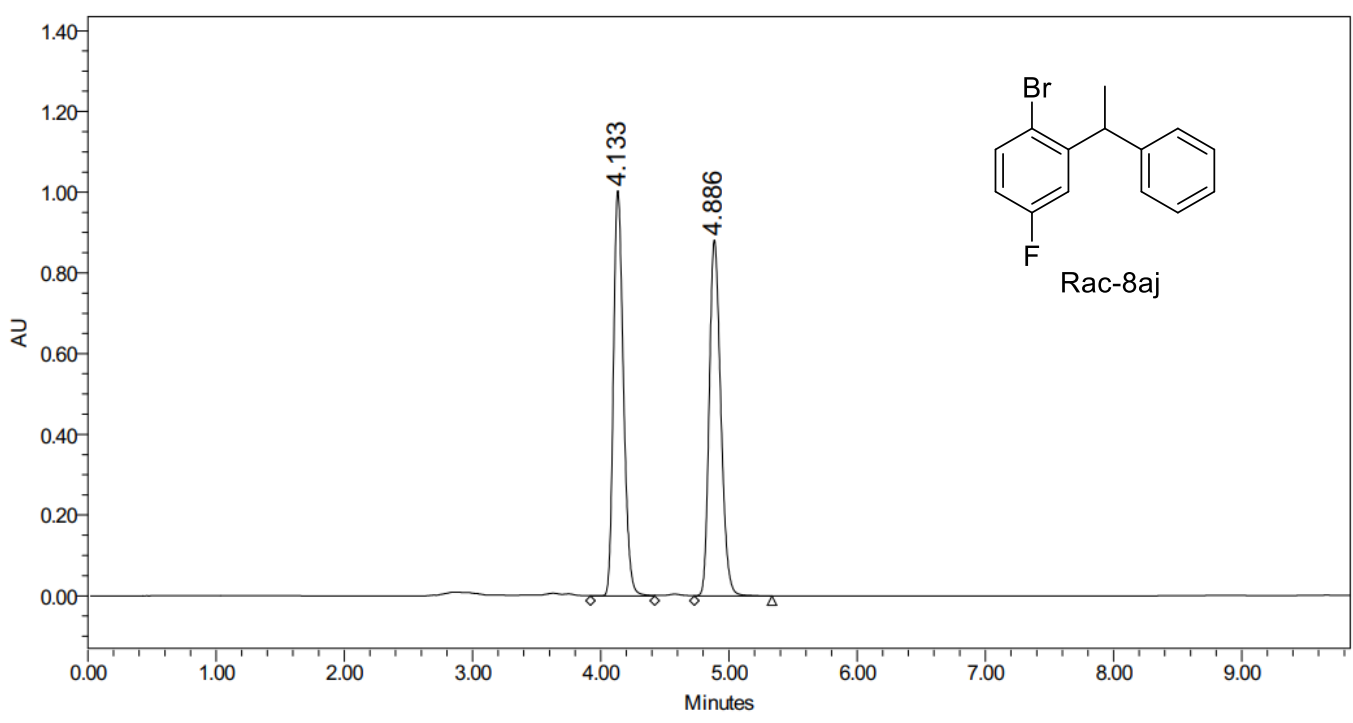

\begin{tabular}{|c|c|c|c|c|r|}
\hline & SampleName & RT & Height & Area & $\%$ Area \\
\hline 1 & QL-9-43-Br-F-STA & 4.133 & 1003615 & 5466567 & 49.82 \\
\hline 2 & QL-9-43-Br-F-STA & 4.886 & 881477 & 5505769 & 50.18 \\
\hline
\end{tabular}

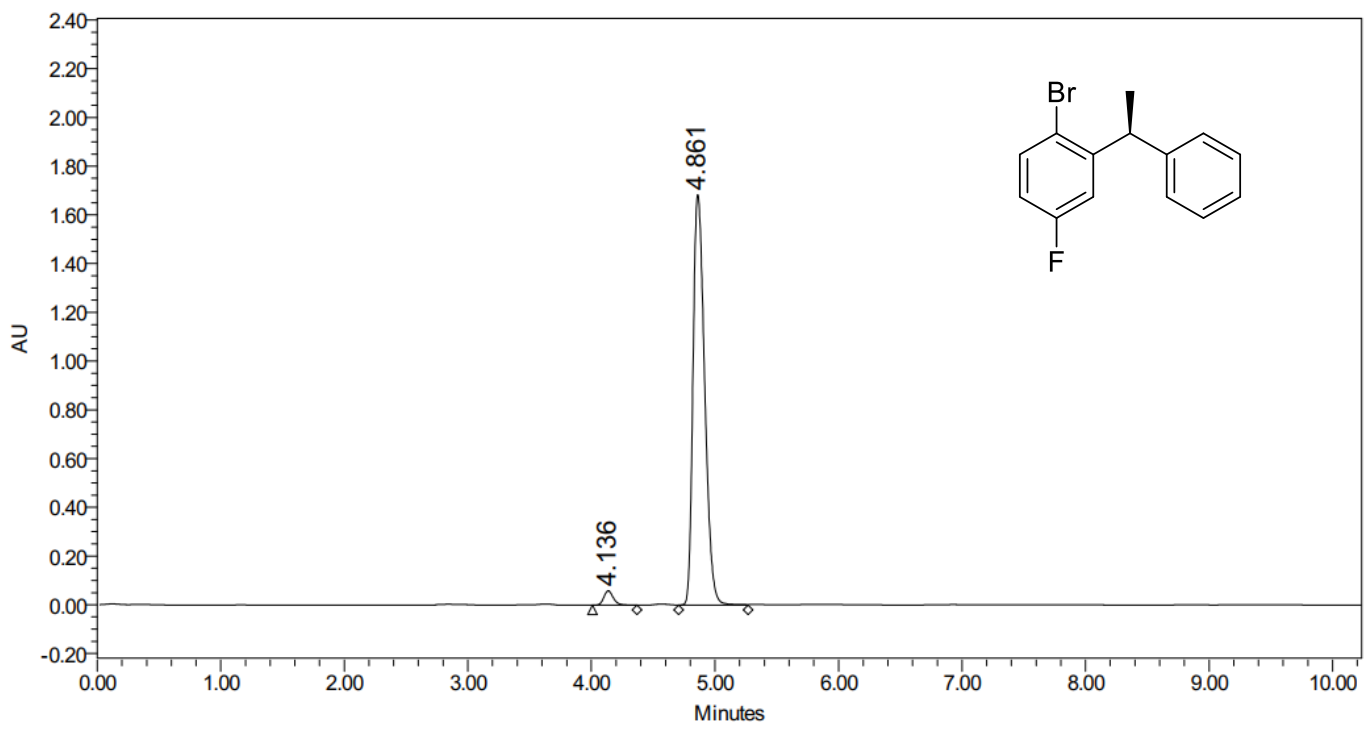

\begin{tabular}{|c|c|c|r|r|r|}
\hline & SampleName & RT & Height & \multicolumn{1}{c|}{ Area } & $\%$ Area \\
\hline 1 & QL-9-43-Br-F-CHIRAL & 4.136 & 58997 & 306763 & 2.69 \\
\hline 2 & QL-9-43-Br-F-CHIRAL & 4.861 & 1683416 & 11099266 & 97.31 \\
\hline
\end{tabular}


(S)-1-bromo-2-(1-(4-methoxyphenyl)ethyl)benzene (8ak)
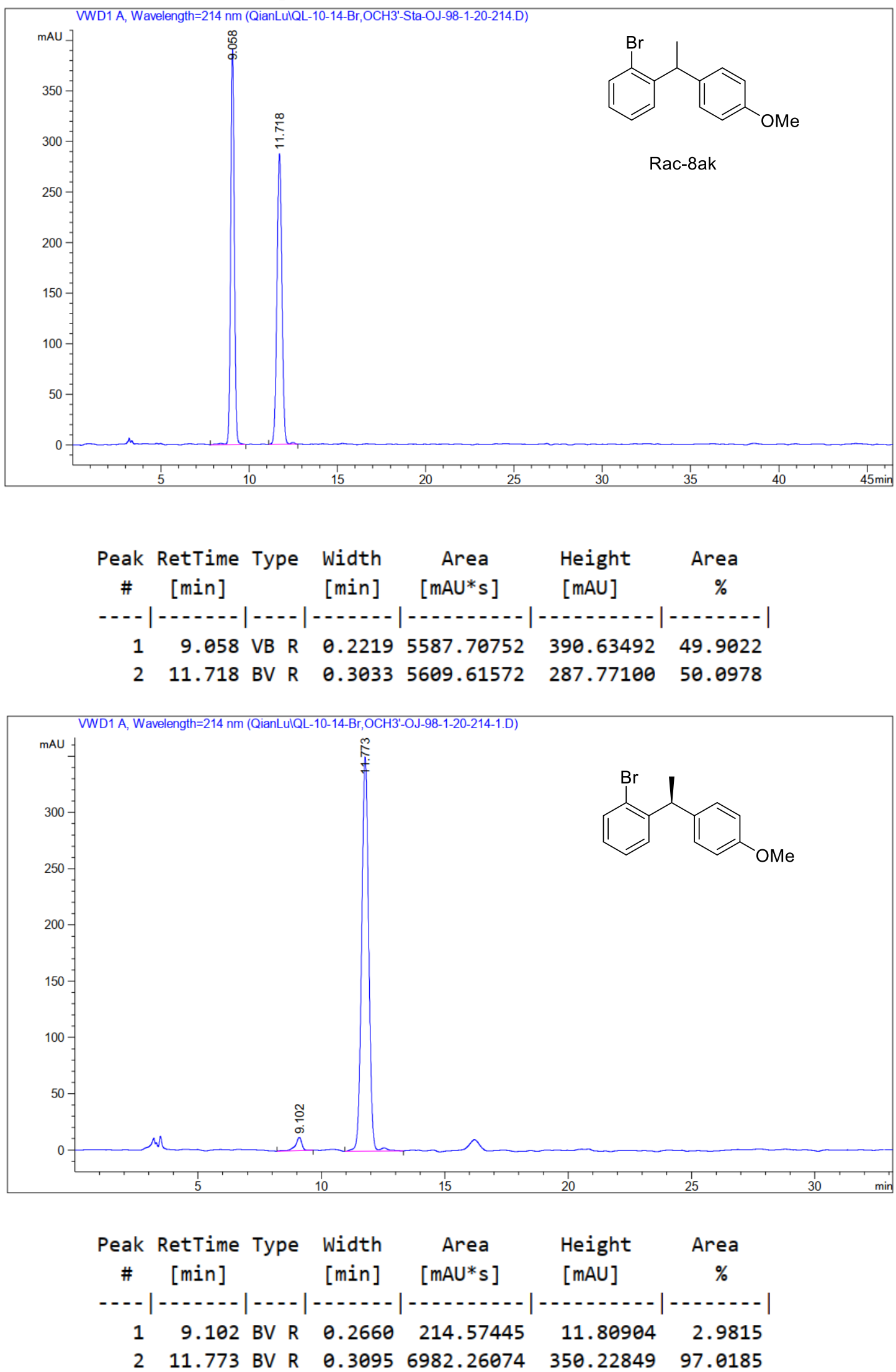
(S)-1-bromo-2-(1-(2-methoxyphenyl)ethyl)benzene (8al)

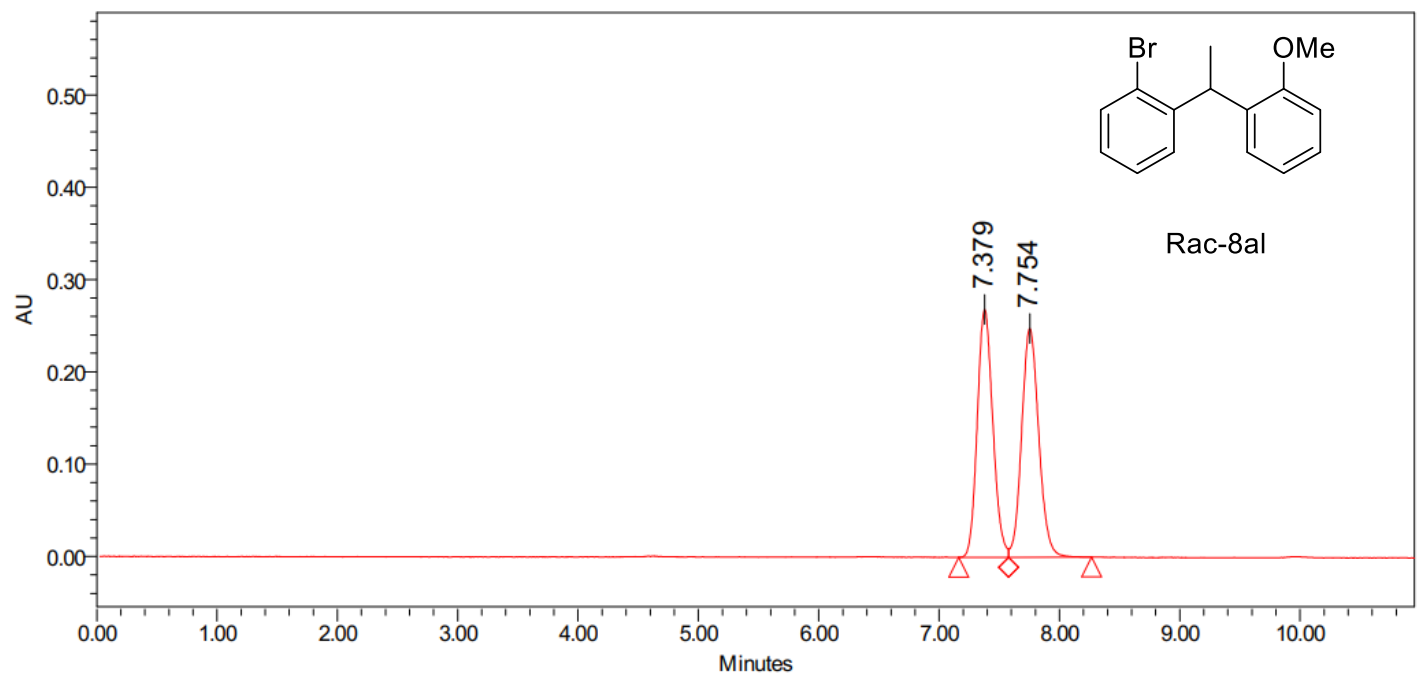

Peak Results

\begin{tabular}{|l|l|l|l|l|l|l|r|}
\hline ql-11-34-Br-rac & 7 & 7.379 & 7.379 & 24.900 & 268712 & 2408757 & 49.55 \\
\hline ql-11-34-Br-rac & 7 & 7.754 & 7.754 & 41.400 & 247942 & 2452181 & 50.45 \\
\hline
\end{tabular}

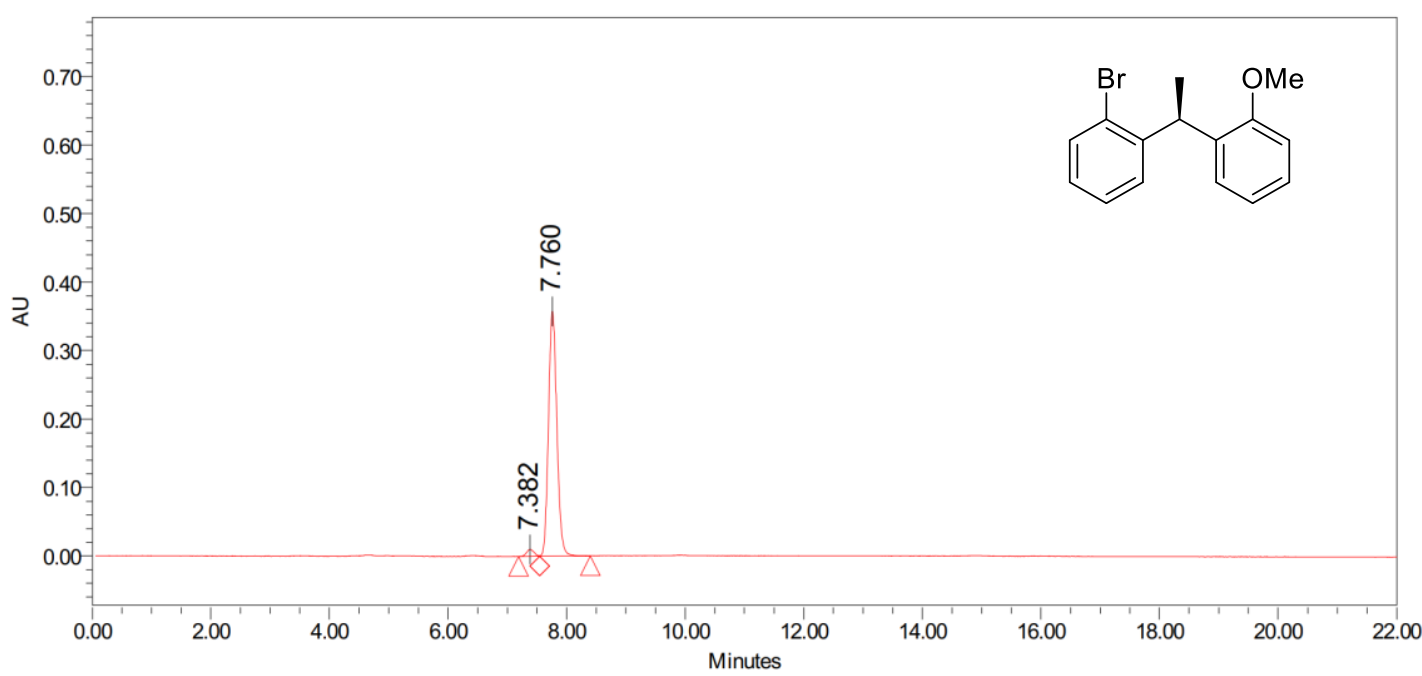

Peak Results

\begin{tabular}{|l|l|l|r|r|r|r|r|}
\hline ql-11-34-Br-chiral & 8 & 7.382 & 7.382 & 21.000 & 10269 & 90697 & 2.51 \\
\hline ql-11-34-Br-chiral & 8 & 7.760 & 7.760 & 51.500 & 357680 & 3528061 & 97.49 \\
\hline
\end{tabular}


(S)-2-(1-(2-bromo-3,4,5-trimethoxyphenyl)ethyl)naphthalene (8am)
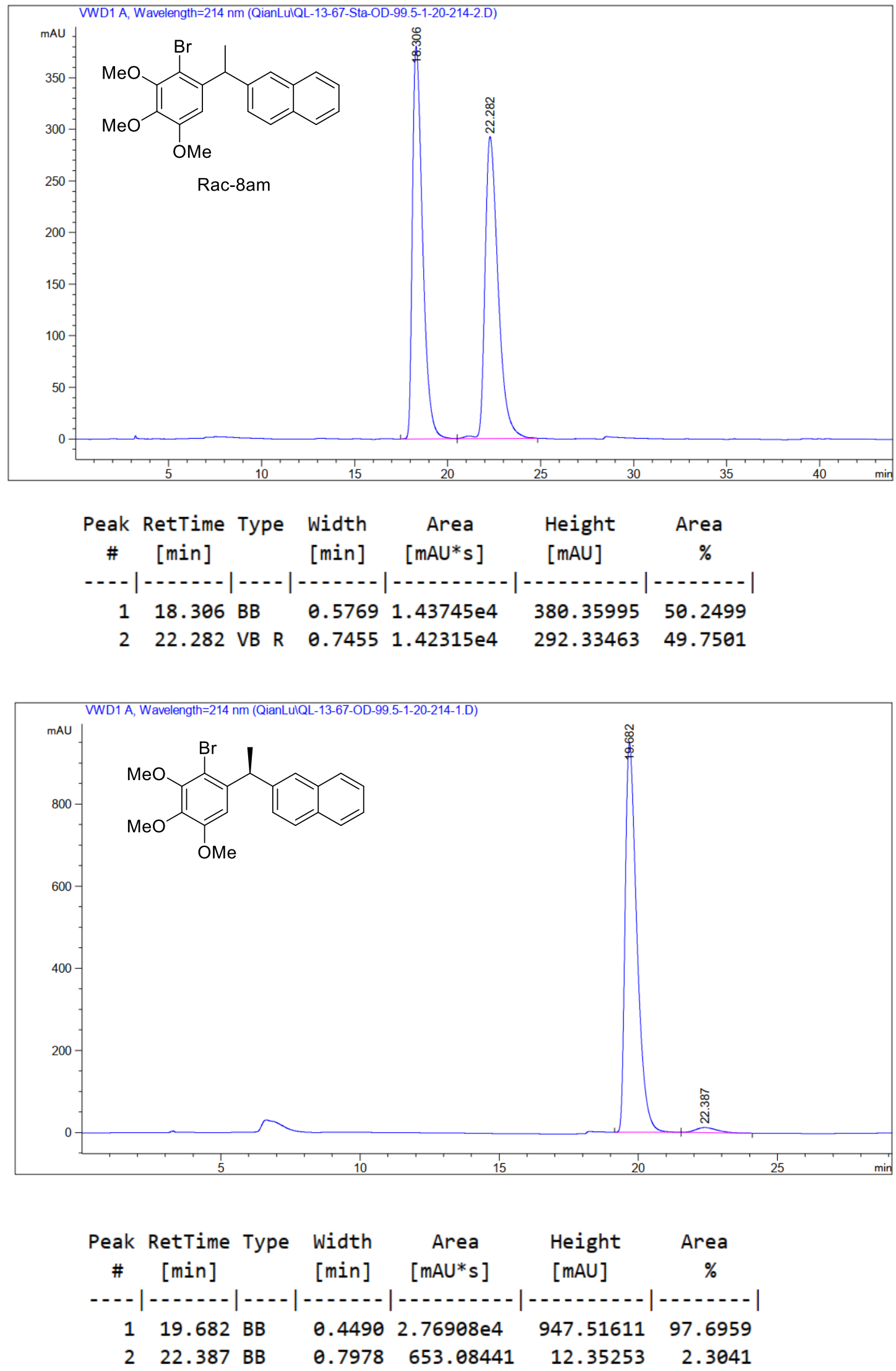
(R)-2-(1-(2-bromophenyl)ethyl)furan (8an)
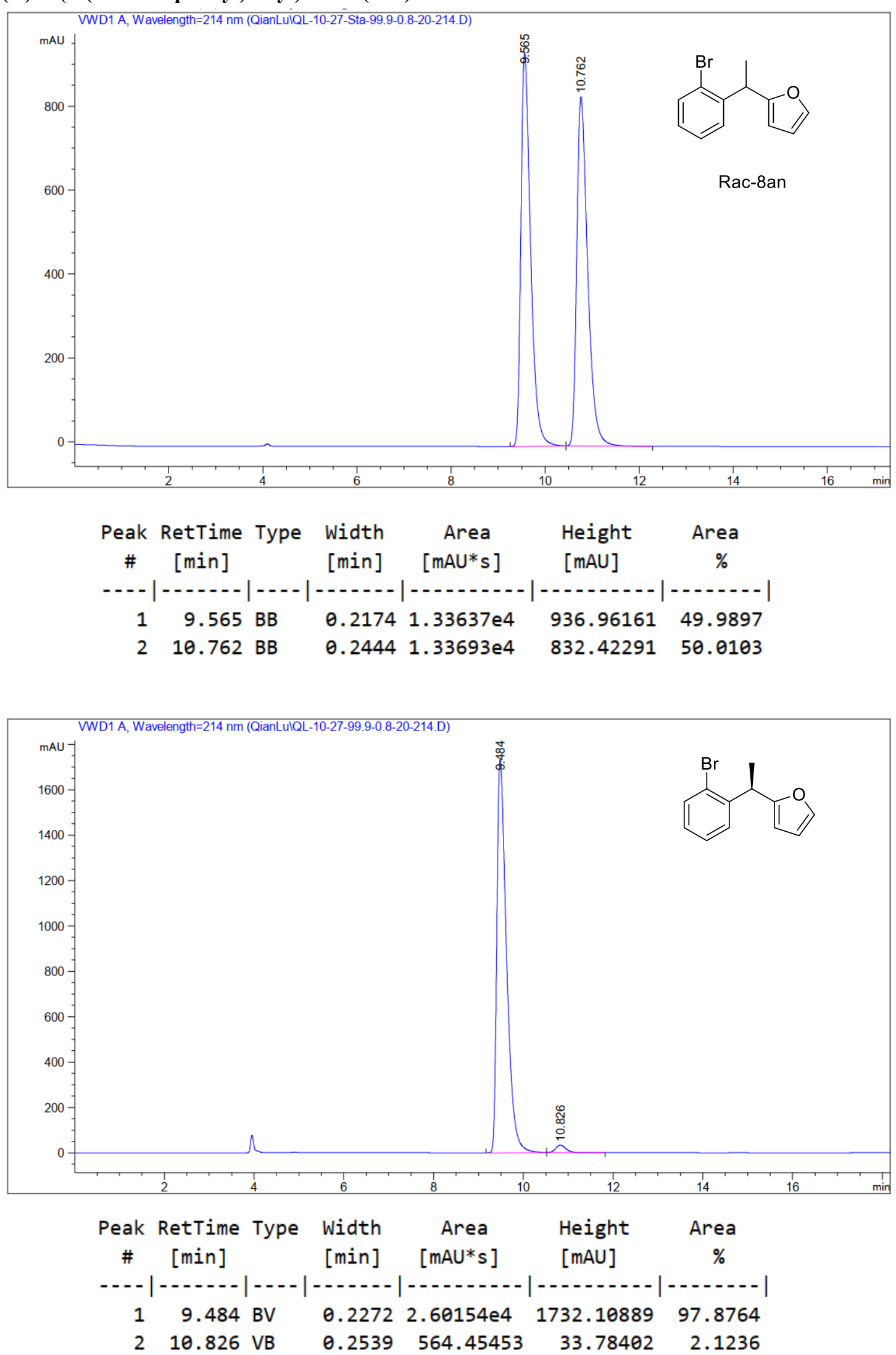
(R)-2-(1-(2-bromophenyl)ethyl)thiophene (8ao)
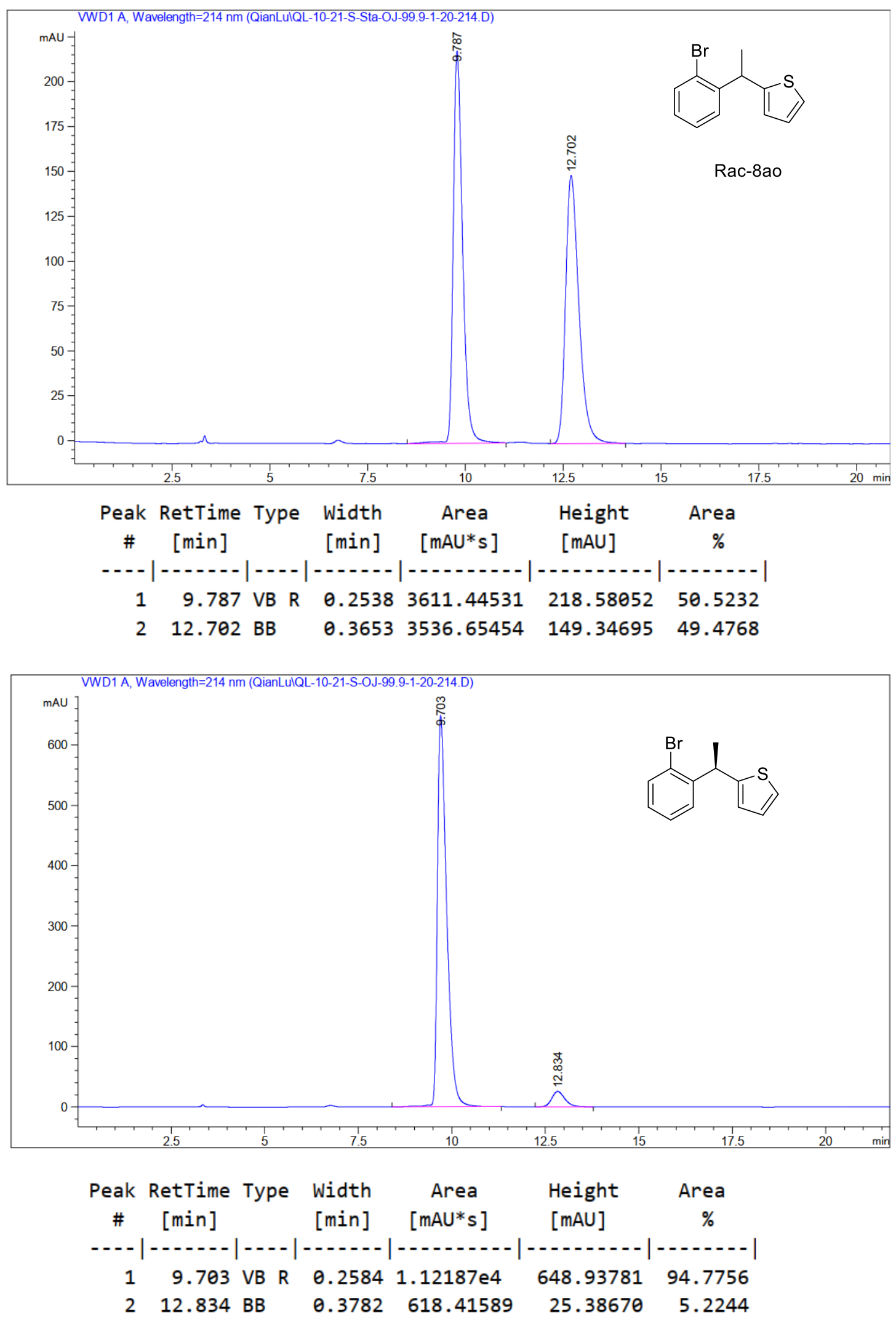
(R)-2-(1-(2-bromophenyl)ethyl)oxazole (8ap)

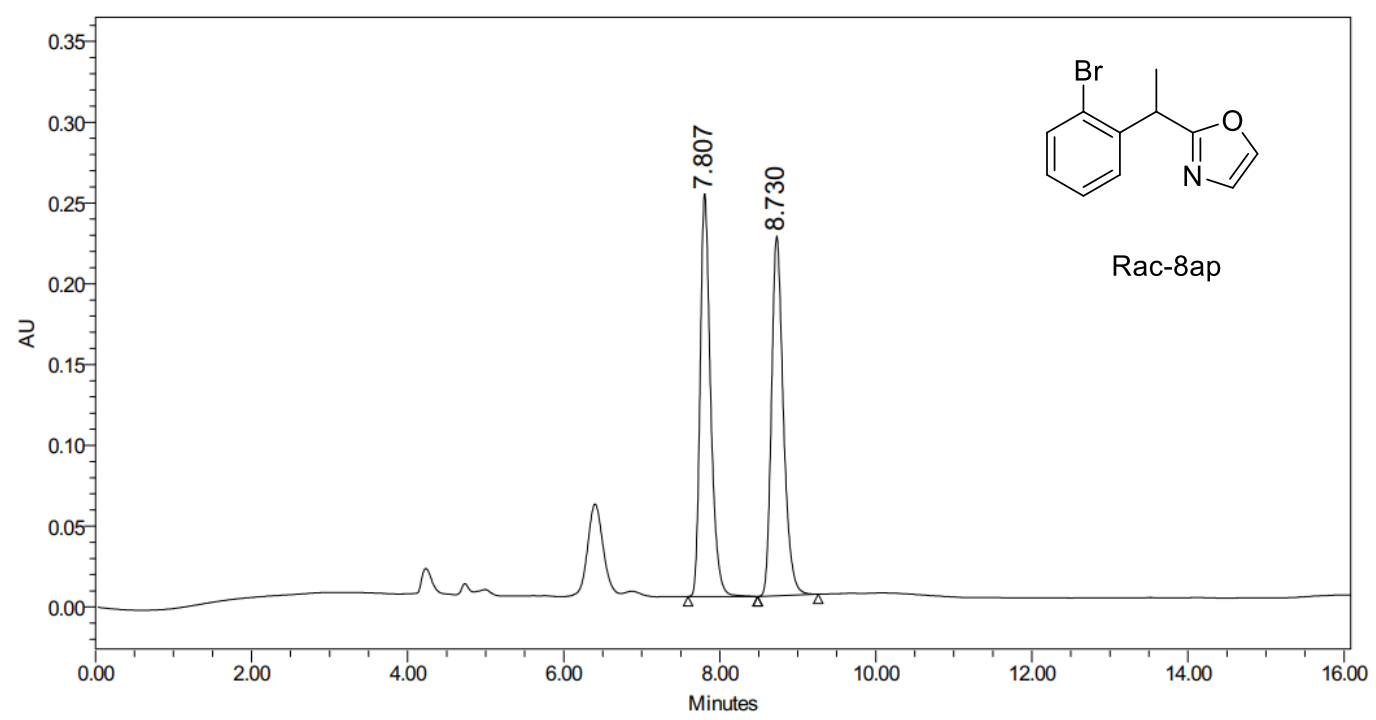

\begin{tabular}{|c|c|c|c|c|r|}
\hline & SampleName & RT & Height & Area & $\%$ Area \\
\hline 1 & QL-12-54-O-RAC & 7.807 & 249156 & 2323364 & 49.68 \\
\hline 2 & QL-12-54-O-RAC & 8.730 & 222551 & 2353289 & 50.32 \\
\hline
\end{tabular}

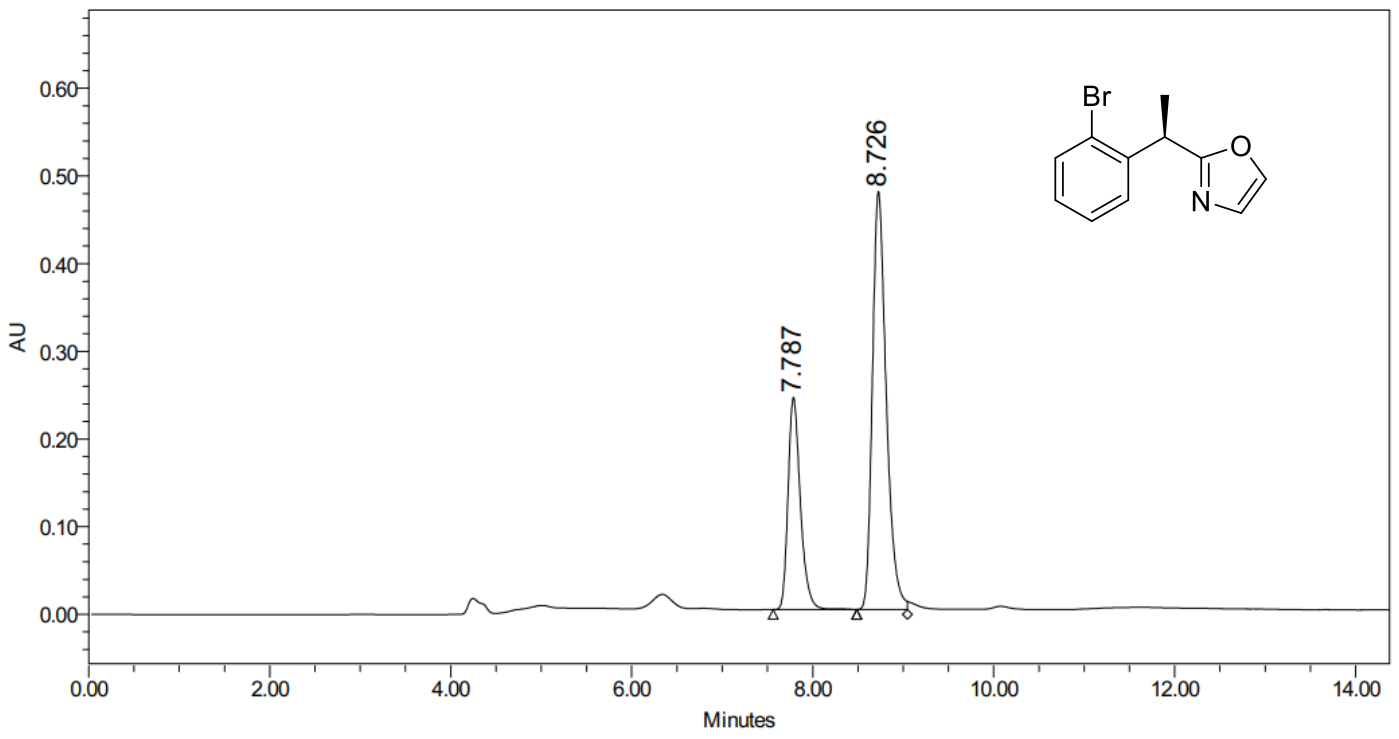

\begin{tabular}{|c|c|c|c|c|r|}
\hline & SampleName & RT & Height & Area & $\%$ Area \\
\hline 1 & QL-12-54-O-CHIRAL & 7.787 & 241573 & 2288237 & 31.11 \\
\hline 2 & QL-12-54-O-CHIRAL & 8.726 & 476523 & 5066665 & 68.89 \\
\hline
\end{tabular}


(R)-2-(1-(2-bromophenyl)ethyl)thiazole (8aq)

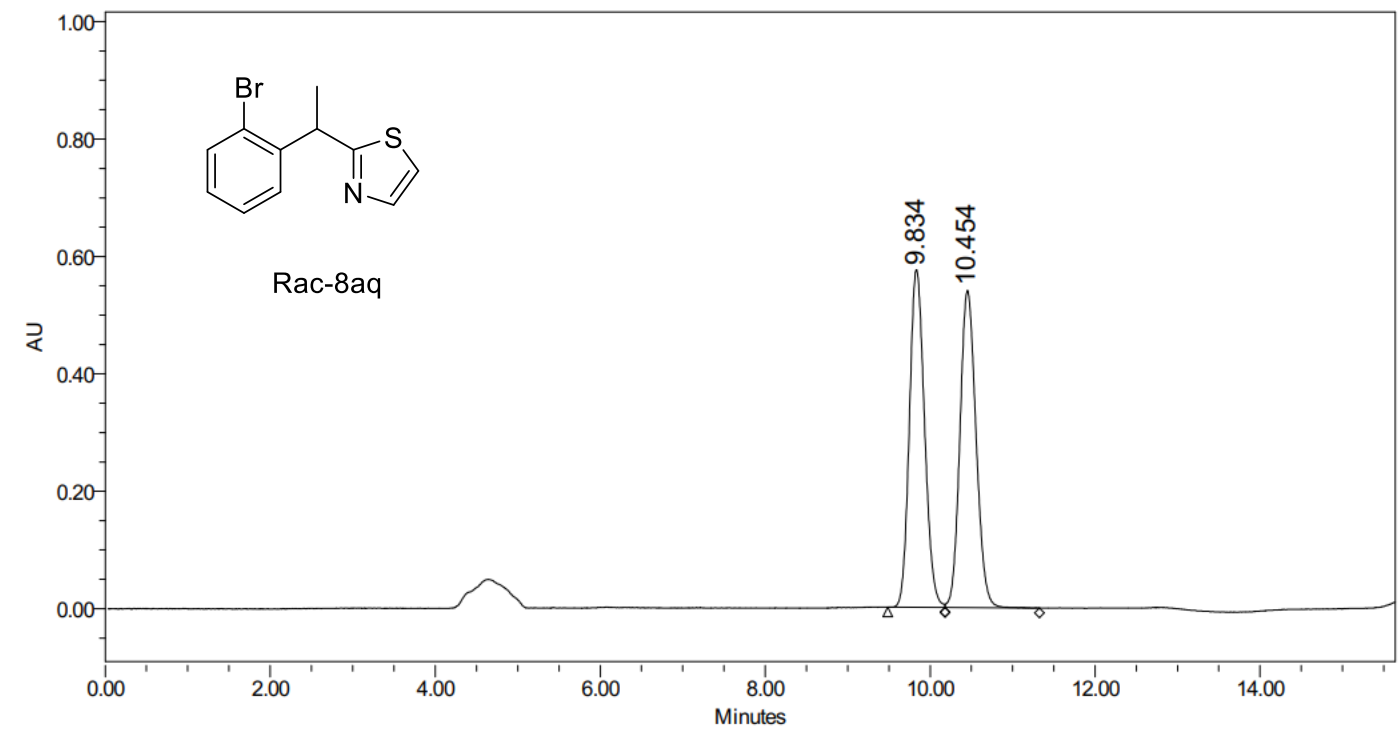

\begin{tabular}{|c|c|c|c|r|}
\hline & RT & Height & Area & $\%$ Area \\
\hline 1 & 9.834 & 575264 & 7349098 & 49.95 \\
\hline 2 & 10.454 & 540547 & 7363981 & 50.05 \\
\hline
\end{tabular}

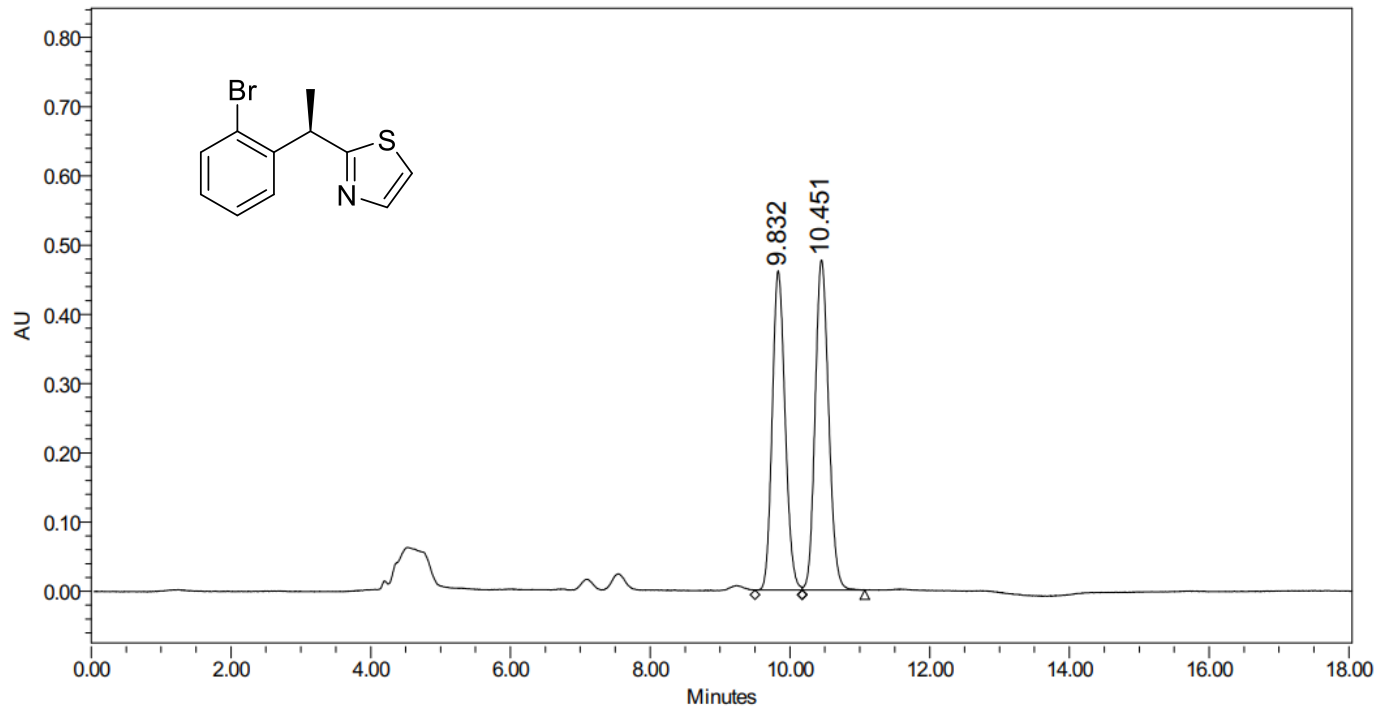

\begin{tabular}{|c|c|c|c|r|}
\hline & RT & Height & Area & $\%$ Area \\
\hline 1 & 9.832 & 461238 & 5925347 & 47.98 \\
\hline 2 & 10.451 & 476797 & 6425045 & 52.02 \\
\hline
\end{tabular}


(R)-1-benzyl-2-(1-(2,3-dimethylphenyl)ethyl)-1H-imidazole (8ar)

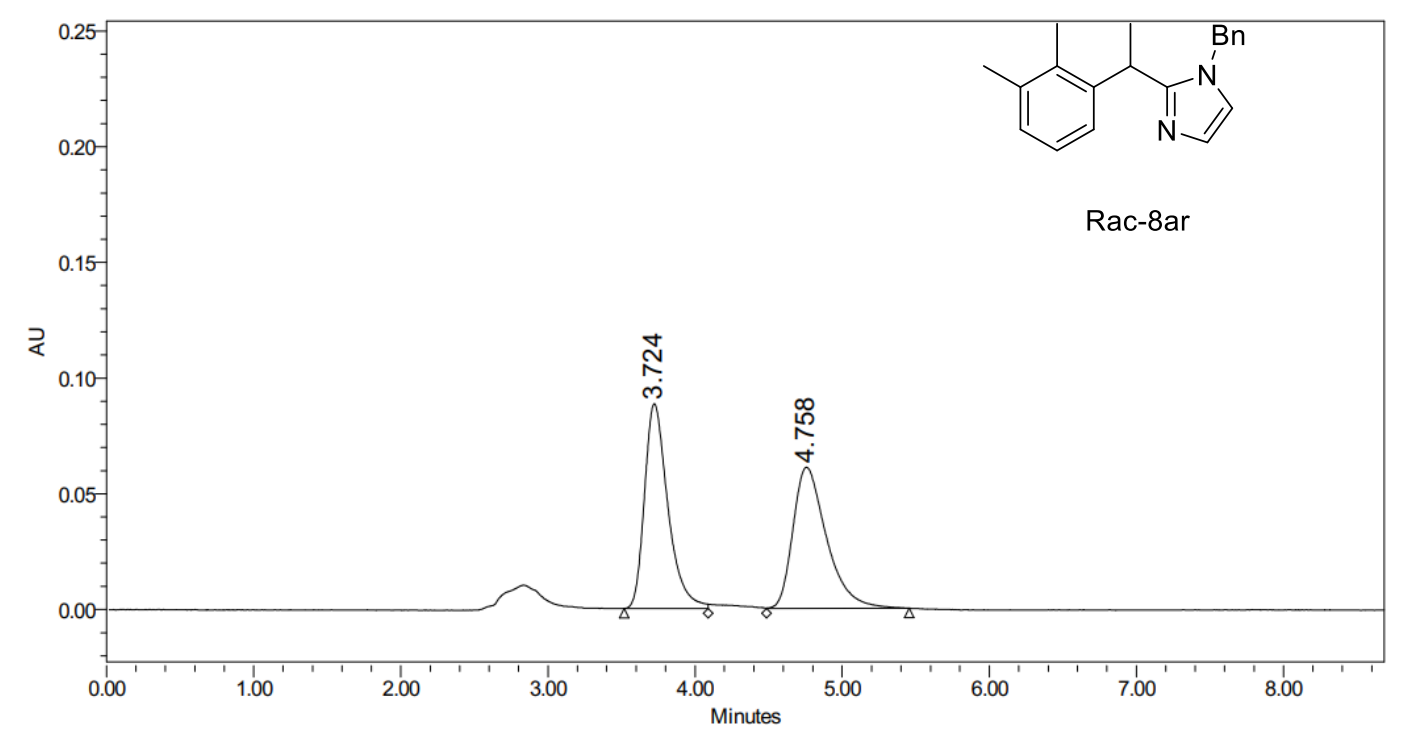

\begin{tabular}{|c|c|c|c|l|c|r|}
\hline & RT & $\begin{array}{c}\text { Width } \\
(\mathrm{sec})\end{array}$ & Height & Peak Type & Area & $\%$ Area \\
\hline 1 & 3.724 & 34.200 & 88515 & Unknown & 979126 & 50.25 \\
\hline 2 & 4.758 & 58.300 & 61001 & Unknown & 969380 & 49.75 \\
\hline
\end{tabular}

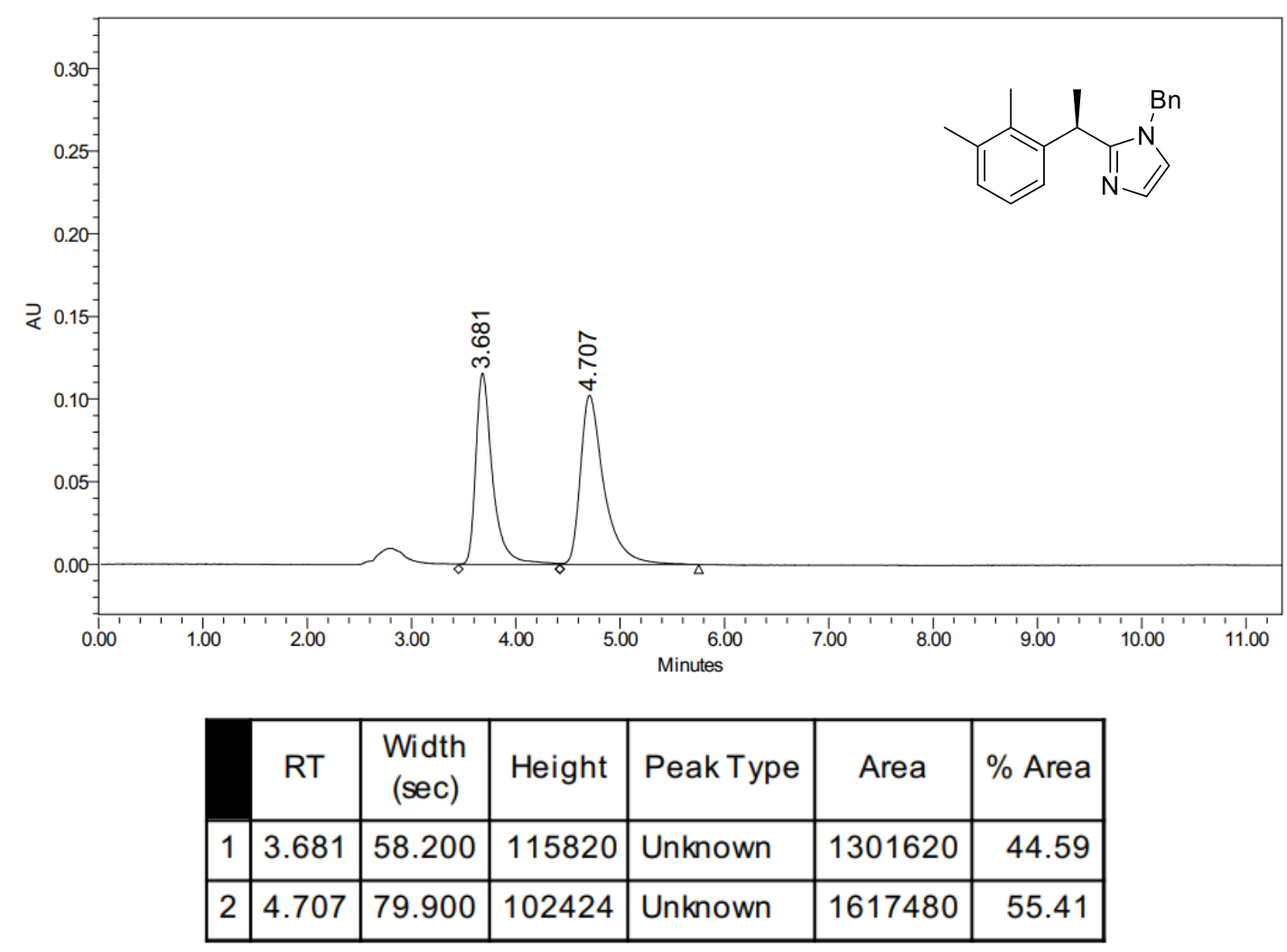


(S)-1-methoxy-3-(1-phenylethyl)benzene (9ah)
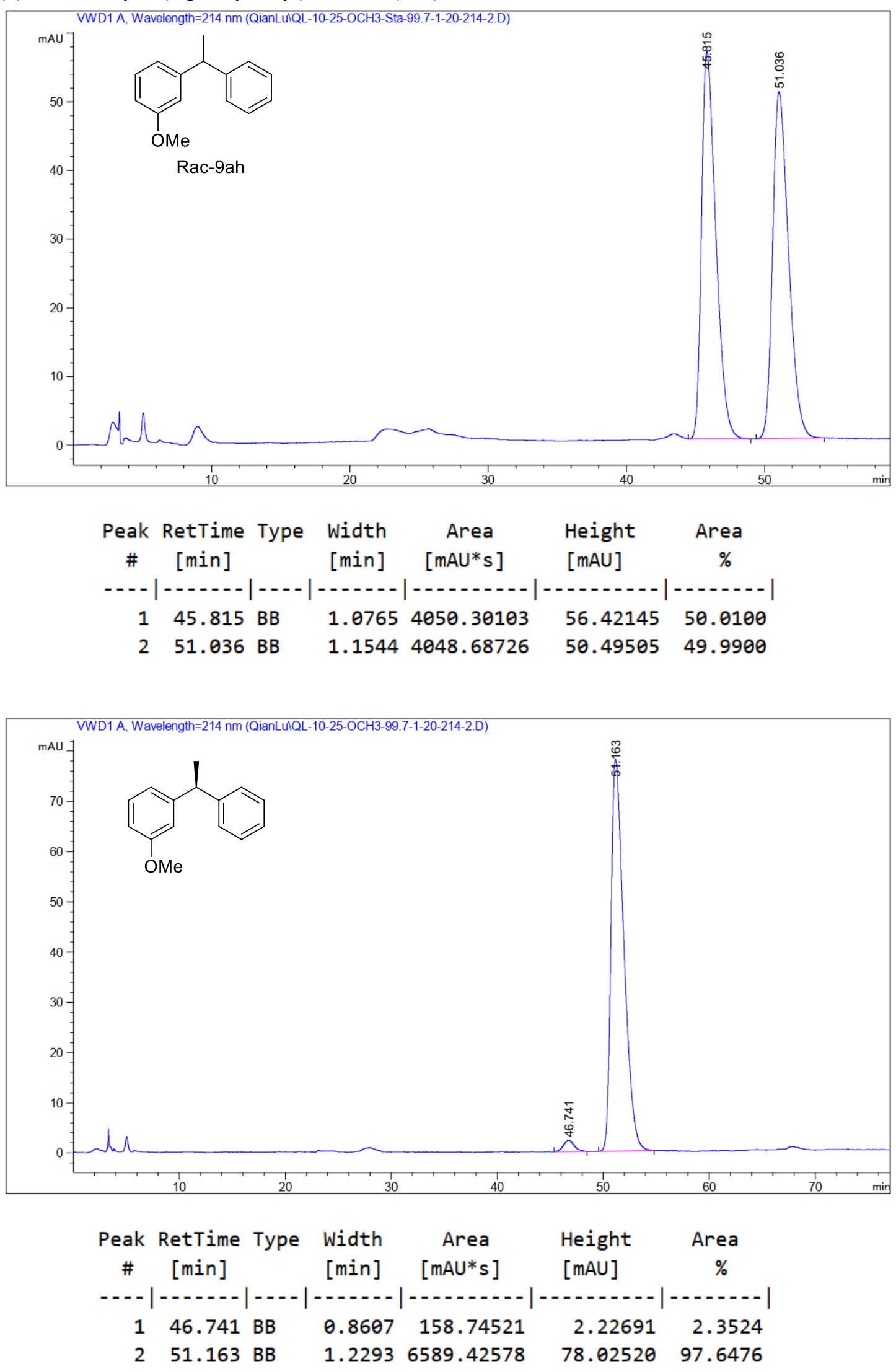
(R)-1-methoxy-4-(1-phenylethyl)benzene (9ak)
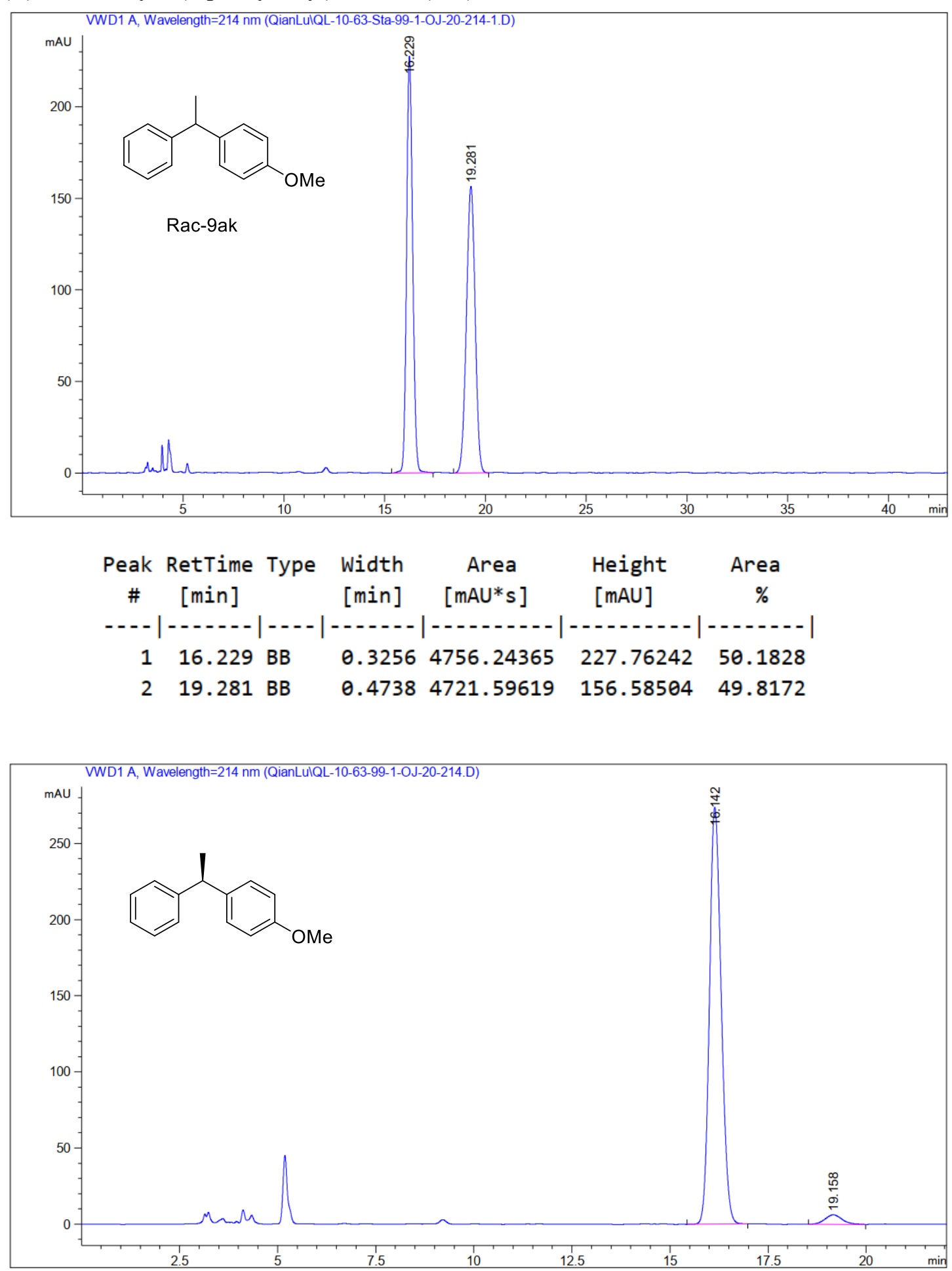

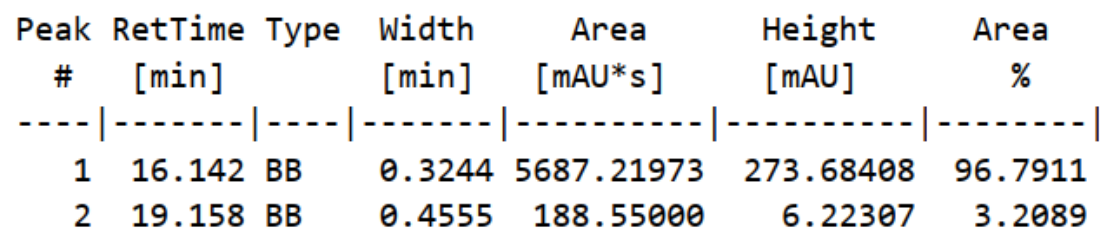


(S)-2-(1-(3,4,5-trimethoxyphenyl)ethyl)naphthalene (9am)
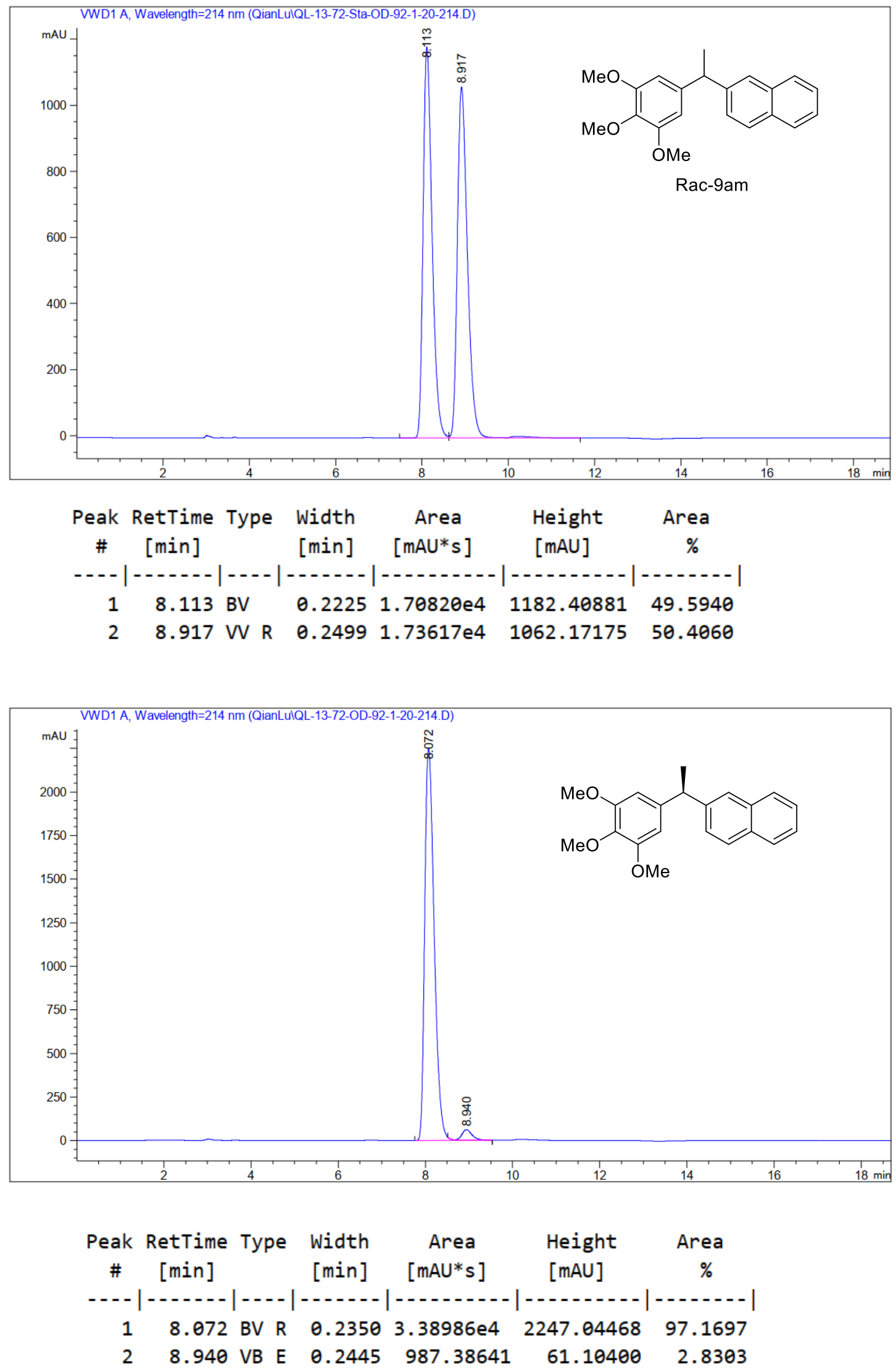
(S)-4,4,5,5-tetramethyl-2-(2-(1-phenylethyl)phenyl)-1,3,2-dioxaborolane (10).
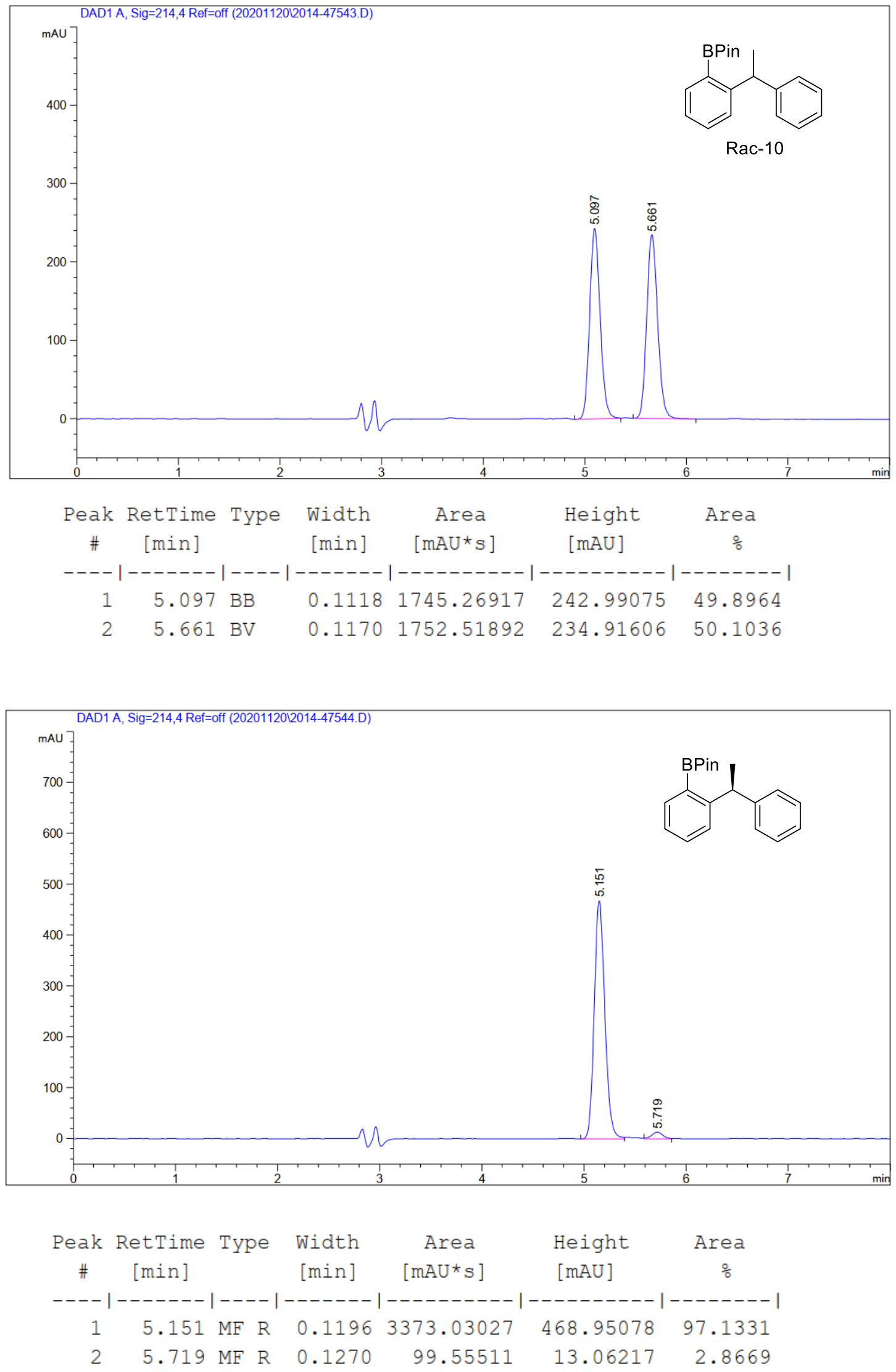
(S)-2-(1-phenylethyl)-1,1'-biphenyl (11)
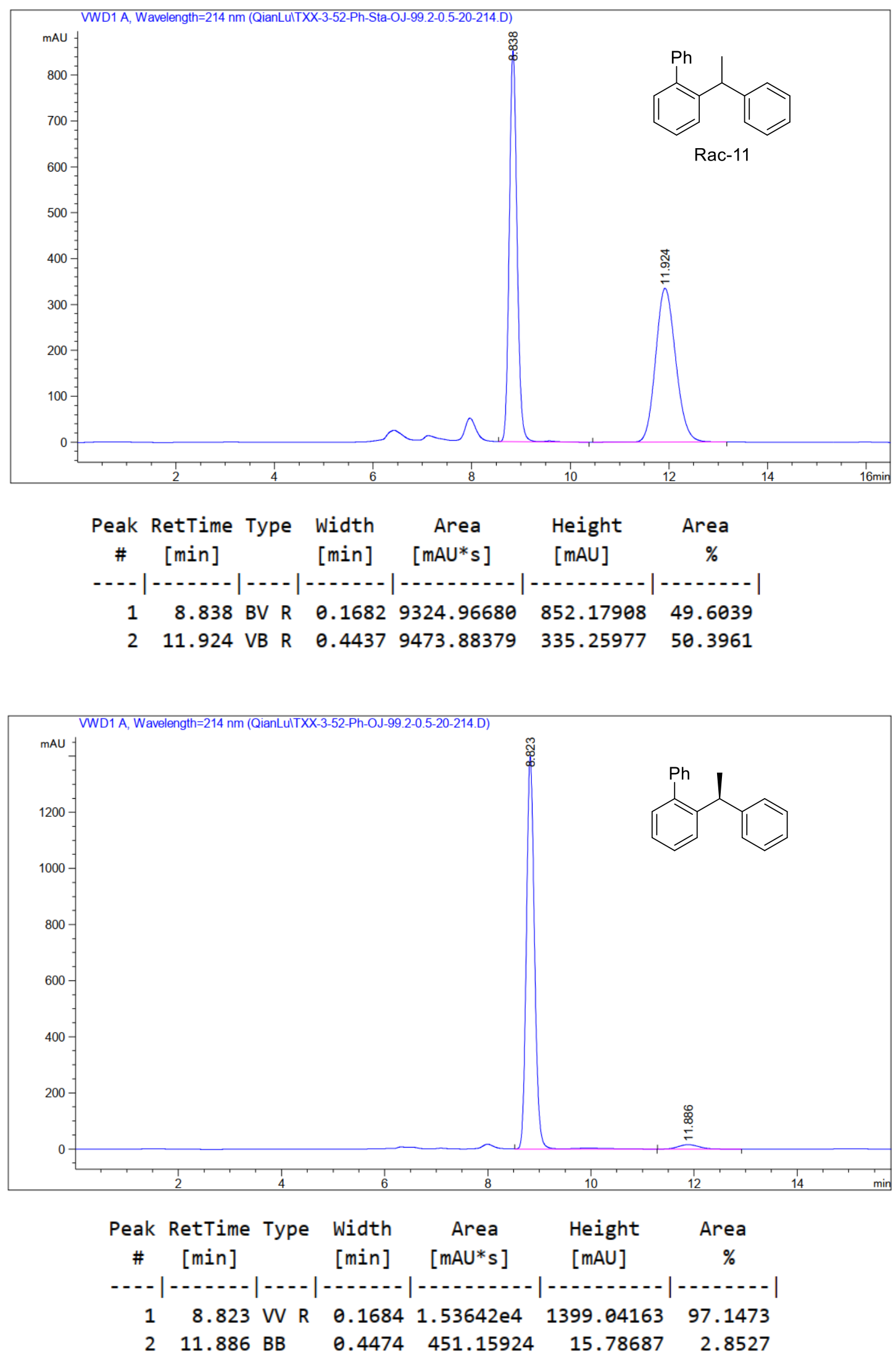
(S)-N-benzyl-2-(1-phenylethyl)aniline (12).

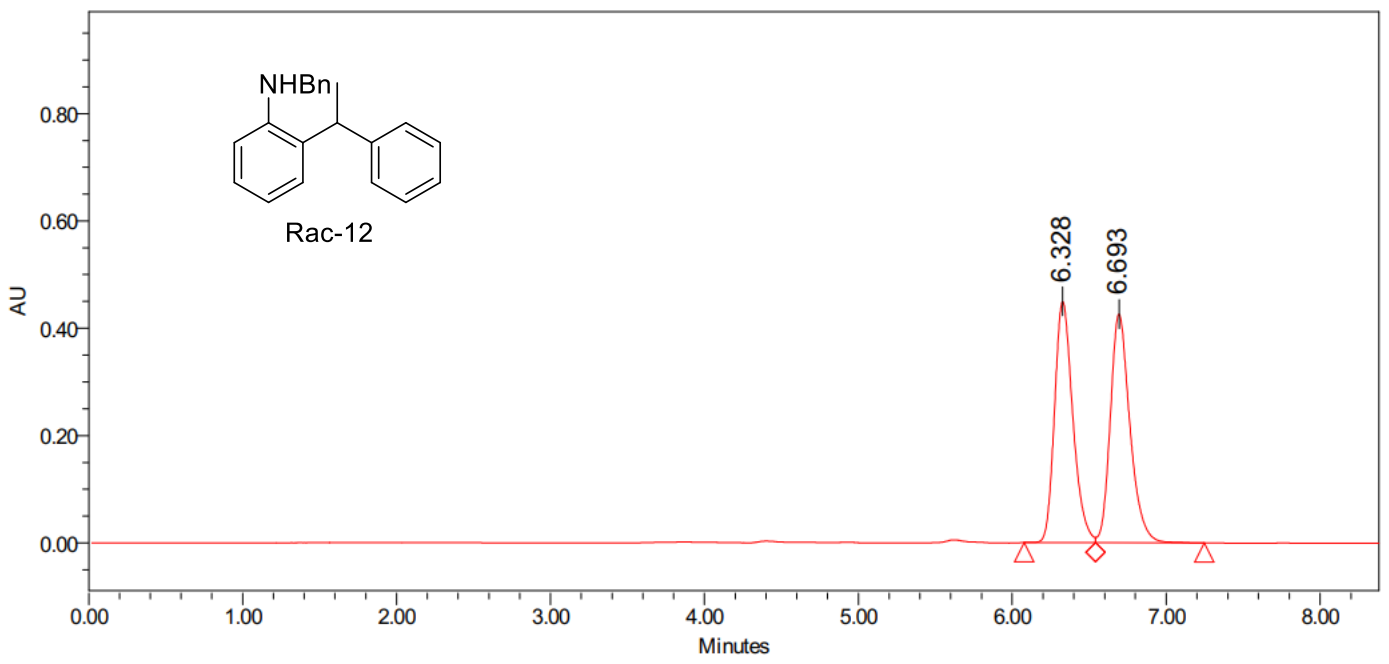

Peak Results

\begin{tabular}{|l|l|l|l|l|l|}
\hline QL-14-77-RAC-OD-9-1 & 6.328 & 27.900 & 449298 & 3700704 & 49.47 \\
\hline QL-14-77-RAC-OD-9-1 & 6.693 & 42.400 & 426037 & 3780545 & 50.53 \\
\hline
\end{tabular}

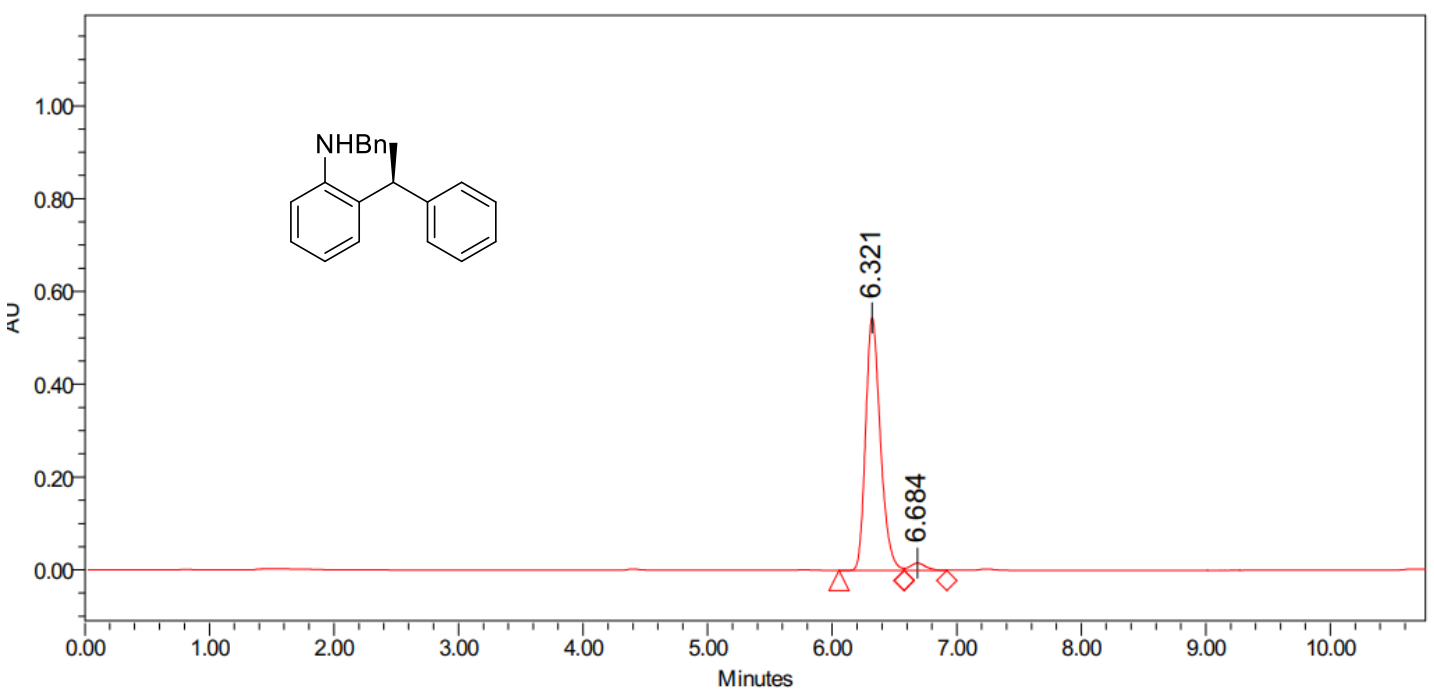

Peak Results

\begin{tabular}{|l|r|r|r|r|r|}
\hline QL-14-77-CHIRAL & 6.321 & 31.400 & 544470 & 4490809 & 96.91 \\
\hline QL-14-77-CHIRAL & 6.684 & 20.500 & 15844 & 143040 & 3.09 \\
\hline
\end{tabular}

S209 


\section{X-Ray Crystallographic Data}
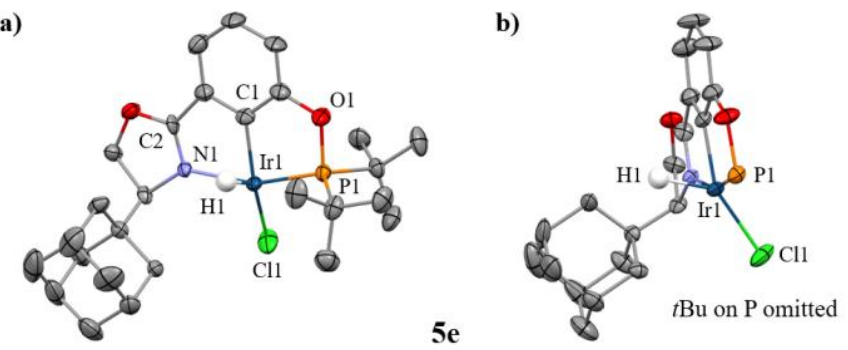

Figure S1. Crystal structure of complex $5 \mathbf{e}$ viewed from two different directions. The $\mathrm{H}$-atoms, except the hydride, were omitted for clarity. The $t \mathrm{Bu}$ groups on the $\mathrm{P}$-atom in b) were omitted as well. Thermal ellipsoids are set at the $50 \%$ probability level.

Table S1. Crystal data and structure refinement for $5 \mathbf{e}$.

Identification code

Empirical formula

Formula weight

Temperature

Wavelength

Crystal system

Space group

Unit cell dimensions

Volume

$\mathrm{Z}$

Density (calculated)

Absorption coefficient

$\mathrm{F}(000)$

Crystal size

Theta range for data collection

Index ranges

Reflections collected

Independent reflections

Completeness to theta $=53.594^{\circ}$

Absorption correction

Max. and min. transmission

Refinement method

Data / restraints / parameters

Goodness-of-fit on $\mathrm{F}^{2}$

Final $\mathrm{R}$ indices [I $>2 \operatorname{sigma}(\mathrm{I})]$

$\mathrm{R}$ indices (all data)

Absolute structure parameter
$5 e$

C27 H40 Cl Ir N O2 P

669.22

$169.99 \mathrm{~K}$

$1.34139 \AA$

Orthorhombic

P212121

$\mathrm{a}=8.6025(8) \AA$

$\alpha=90^{\circ}$.

$\mathrm{b}=11.2414(11) \AA$

$\beta=90^{\circ}$.

$\mathrm{c}=27.909(3) \AA$

$\mathrm{g}=90^{\circ}$

2699.0(4) $\AA^{3}$

4

$1.647 \mathrm{Mg} / \mathrm{m}^{3}$

$7.736 \mathrm{~mm}^{-1}$

1336

$0.05 \times 0.03 \times 0.03 \mathrm{~mm}^{3}$

5.634 to $54.983^{\circ}$

$-10<=\mathrm{h}<=10,-13<=\mathrm{k}<=13,-29<=\mathrm{l}<=33$

22595

$5029[\mathrm{R}(\mathrm{int})=0.0513]$

$98.8 \%$

Semi-empirical from equivalents

0.7508 and 0.4715

Full-matrix least-squares on $\mathrm{F}^{2}$

5029 / 0 / 309

1.094

$\mathrm{R} 1=0.0289, \mathrm{wR} 2=0.0784$

$\mathrm{R} 1=0.0292, \mathrm{wR} 2=0.0787$

$0.147(19)$ 
Extinction coefficient

Largest diff. peak and hole $\mathrm{n} / \mathrm{a}$

0.988 and -1.568 e. $\AA^{-3}$

Table S2. Selected bond length and angles for complex 5e

\begin{tabular}{cc}
\hline Selected bond length & Distance $(\AA)$ \\
Ir1-H1 & $1.66(14)$ \\
Ir1-C11 & $2.3728(19)$ \\
Ir1-P1 & $2.2062(19)$ \\
Ir1-C1 & $1.977(7)$ \\
Ir1-N1 & $2.121(6)$ \\
O1-P1 & $1.673(5)$ \\
N1-C2 & $1.301(10)$ \\
Selected bond angles & $\left({ }^{\circ}\right)$ \\
C1-Ir1-C11 & $150.6(4)$ \\
C1-Ir1-H1 & $70(5)$ \\
C1-Ir1-N1 & $78.8(3)$ \\
C1-Ir1-P1 & $81.2(2)$ \\
\hline
\end{tabular}

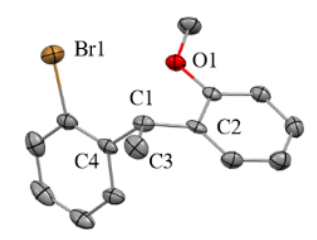

Figure S2. Crystal structure of complex 8al. Thermal ellipsoids are set at the $40 \%$ probability level.

Table S3. Crystal data and structure refinement for $\mathbf{8 a l}$.

Identification code
Empirical formula
Formula weight
Temperature
Wavelength
Crystal system
Space group
Unit cell dimensions

Volume

Z 8al

C15 H15 Br O

291.18

$192.99 \mathrm{~K}$

$1.34139 \AA$

Orthorhombic

P212121

$\mathrm{a}=7.6949(2) \AA$

$\alpha=90^{\circ}$.

$\mathrm{b}=9.6817(2) \AA$

$\beta=90^{\circ}$.

$\mathrm{c}=17.8988(4) \AA$

$\gamma=90^{\circ}$. 
Density (calculated)

Absorption coefficient

$\mathrm{F}(000)$

Crystal size

Theta range for data collection

Index ranges

Reflections collected

Independent reflections

Completeness to theta $=53.594^{\circ}$

Absorption correction

Max. and min. transmission

Refinement method

Data / restraints / parameters

Goodness-of-fit on $\mathrm{F}^{2}$

Final R indices [I $>2 \operatorname{sigma}(\mathrm{I})]$

$\mathrm{R}$ indices (all data)

Absolute structure parameter

Extinction coefficient

Largest diff. peak and hole
$1.450 \mathrm{Mg} / \mathrm{m}^{3}$

$2.778 \mathrm{~mm}^{-1}$

592

$0.15 \times 0.08 \times 0.06 \mathrm{~mm}^{3}$

4.298 to $55.019^{\circ}$.

$-7<=\mathrm{h}<=9,-11<=\mathrm{k}<=11,-21<=\mathrm{l}<=21$

13949

$2546[\mathrm{R}(\mathrm{int})=0.0955]$

$100.0 \%$

Semi-empirical from equivalents

0.7508 and 0.3949

Full-matrix least-squares on $\mathrm{F}^{2}$

2546 / 0 / 156

1.056

$\mathrm{R} 1=0.0440, \mathrm{wR} 2=0.0988$

$\mathrm{R} 1=0.0486, \mathrm{wR} 2=0.1020$

$0.05(2)$

$\mathrm{n} / \mathrm{a}$

0.334 and -1.100 e. $\AA^{-3}$

Table S4. Selected bond length and angles for 8al

\begin{tabular}{cc}
\hline Selected bond length & Distance $(\AA)$ \\
C1-C2 & $1.519(6)$ \\
C1-C3 & $1.544(6)$ \\
C1-C4 & $1.511(7)$ \\
Selected bond angles & $\left({ }^{\circ}\right)$ \\
C2-C1-C3 & $113.5(4)$ \\
C2-C1-C4 & $111.3(4)$ \\
C3-C1-C4 & $109.7(4)$ \\
\hline
\end{tabular}

